\title{
諅
}

Volume 1

Sensors for Gait,

Posture, and

Health Monitoring

Edited by

Thurmon Lockhart

Printed Edition of the Special Issue Published in Sensors 
Sensors for Gait, Posture, and Health Monitoring 



\section{Sensors for Gait, Posture, and Health Monitoring}

\section{Volume 1}

Special Issue Editor

Thurmon Lockhart 
Special Issue Editor

Thurmon Lockhart

Arizona State University

USA

Editorial Office

MDPI

St. Alban-Anlage 66

4052 Basel, Switzerland

This is a reprint of articles from the Special Issue published online in the open access journal Sensors (ISSN 1424-8220) from 2017 to 2019 (available at: https:/ /www.mdpi.com/journal/sensors/special_ issues/Gait_Recognition).

For citation purposes, cite each article independently as indicated on the article page online and as indicated below:

LastName, A.A.; LastName, B.B.; LastName, C.C. Article Title. Journal Name Year, Article Number, Page Range.

Volume 1

ISBN 978-3-03936-342-1 (Pbk)

ISBN 978-3-03936-343-8 (PDF)
Volume 1-3

ISBN 978-3-03936-348-3 (Pbk)

ISBN 978-3-03936-349-0 (PDF)

(C) 2019 by the authors. Articles in this book are Open Access and distributed under the Creative Commons Attribution (CC BY) license, which allows users to download, copy and build upon published articles, as long as the author and publisher are properly credited, which ensures maximum dissemination and a wider impact of our publications.

The book as a whole is distributed by MDPI under the terms and conditions of the Creative Commons license CC BY-NC-ND. 


\title{
Contents
}

About the Special Issue Editor $\ldots \ldots \ldots \ldots \ldots \ldots \ldots \ldots \ldots \ldots \ldots \ldots \ldots \ldots$

Preface to "Sensors for Gait, Posture, and Health Monitoring" . . . . . . . . . . . . xi

Gema Chamorro-Moriana, Antonio José Moreno and José Luis Sevillano

Technology-Based Feedback and Its Efficacy in Improving Gait Parameters in Patients with Abnormal Gait: A Systematic Review

Reprinted from: Sensors 2018, 18, 142, doi:10.3390/s18010142 . . . . . . . . . . . . . . 1

Youngsu Cha, Hojoon Kim and Doik Kim

Flexible Piezoelectric Sensor-Based Gait Recognition

Reprinted from: Sensors 2018, 18, 468, doi:10.3390/s18020468 . . . . . . . . . . . . . . . . 24

Rosaria Rucco, Antonietta Sorriso, Marianna Liparoti, Giampaolo Ferraioli, Pierpaolo Sorrentino, Michele Ambrosanio and Fabio Baselice

Type and Location of Wearable Sensors for Monitoring Falls during Static and Dynamic Tasks in Healthy Elderly: A Review

Reprinted from: Sensors 2018, 18, 1613, doi:10.3390/s18051613 . . . . . . . . . . . . . . 3

\begin{abstract}
Ahmed Nait Aicha, Gwenn Englebienne, Kimberley S. van Schooten, Mirjam Pijnappels and Ben Kröse

Deep Learning to Predict Falls in Older Adults Based on Daily-Life Trunk Accelerometry

Reprinted from: Sensors 2018, 18, 1654, doi:10.3390/s18051654 . . . . . . . . . . . . . . .
\end{abstract}

Mario Muñoz-Organero and Ramona Ruiz-Blázquez

Detecting Steps Walking at very Low Speeds Combining Outlier Detection, Transition Matrices and Autoencoders from Acceleration Patterns

Reprinted from: Sensors 2017, 17, 2274, doi:10.3390/s17102274 . . . . . . . . . . . . . . 68

Masataka Hori, Akihito Nakai and Isao Shimoyama

Three-Axis Ground Reaction Force Distribution during Straight Walking

Reprinted from: Sensors 2017, 17, 2431, doi:10.3390/s17102431

Jianning Wu, Jiajing Wang, Yun Ling and Haidong $\mathrm{Xu}$

An Advanced Hybrid Technique of DCS and JSRC for Telemonitoring of Multi-Sensor Gait Pattern

Reprinted from: Sensors 2017, 17, 2764, doi:10.3390/s17122764 . . . . . . . . . . . . . . 91

Christian Mitschke, Pierre Kiesewetter and Thomas L. Milani

The Effect of the Accelerometer Operating Range on Biomechanical Parameters: Stride Length, Velocity, and Peak Tibial Acceleration during Running

Reprinted from: Sensors 2018, 18, 130, doi:10.3390/s18010130 . . . . . . . . . . . . . . . 110

Lena Carcreff, Corinna N. Gerber, Anisoara Paraschiv-Ionescu, Geraldo De Coulon, Christopher J. Newman, Stéphane Armand and Kamiar Aminian

What is the Best Configuration of Wearable Sensors to Measure Spatiotemporal Gait Parameters in Children with Cerebral Palsy?

Reprinted from: Sensors 2018, 18, 394, doi:10.3390/s18020394 . . . . . . . . . . . . . . . 122 
Xinyao Hu, Jun Zhao, Dongsheng Peng, Zhenglong Sun and Xingda Qu

Estimation of Foot Plantar Center of Pressure Trajectories with Low-Cost Instrumented Insoles

Using an Individual-Specific Nonlinear Model

Reprinted from: Sensors 2018, 18, 421, doi:10.3390/s18020421 . . . . . . . . . . . . . . . . 139

\section{Hanatsu Nagano and Rezaul K. Begg}

Shoe-Insole Technology for Injury Prevention in Walking

Reprinted from: Sensors 2018, 18,1468, doi:10.3390/s18051468 . . . . . . . . . . . . . . . . . 155

Jing Tang, Jianbin Zheng, Yang Wang, Lie Yu, Enqi Zhan and Qiuzhi Song

Self-Tuning Threshold Method for Real-Time Gait Phase Detection Based on Ground Contact

Forces Using FSRs

Reprinted from: Sensors 2018, 18,481, doi:10.3390/s18020481 . . . . . . . . . . . . . . . . . . 171

Andrew Brueck, Tashfin Iftekhar, Alicja B. Stannard, Kumar Yelamarthi and Tolga Kaya

A Real-Time Wireless Sweat Rate Measurement System for Physical Activity Monitoring

Reprinted from: Sensors 2018, 18,533, doi:10.3390/s18020533 . . . . . . . . . . . . . . . . . 186

Kai Wang, Xianmin Zhang, Jun Ota and Yanjiang Huang

Estimation of Handgrip Force from SEMG Based on Wavelet Scale Selection

Reprinted from: Sensors 2018, 18,663, doi:10.3390/s18020663 . . . . . . . . . . . . . . . . . . 198

Arif Reza Anwary, Hongnian Yu and Michael Vassallo

An Automatic Gait Feature Extraction Method for Identifying Gait Asymmetry Using Wearable Sensors

Reprinted from: Sensors 2018, 18,676, doi:10.3390/s18020676 . . . . . . . . . . . . . . . . . . 213

Srinivas Pandit, Anoop Kant Godiyal, Amit Kumar Vimal, Upinderpal Singh, Deepak Joshi and Dinesh Kalyanasundaram

An Affordable Insole-Sensor-Based Trans-Femoral Prosthesis for Normal Gait

Reprinted from: Sensors 2018, 18, 706, doi:10.3390/s18030706

Mohammad Al-Amri, Kevin Nicholas, Kate Button, Valerie Sparkes, Liba Sheeran and Jennifer L Davies

Inertial Measurement Units for Clinical Movement Analysis: Reliability and Concurrent Validity

Reprinted from: Sensors 2018, 18,719, doi:10.3390/s18030719 . . . . . . . . . . . . . . . . 259

He Zhou, Hyoki Lee, Jessica Lee, Michael Schwenk and Bijan Najafi

Motor Planning Error: Toward Measuring Cognitive Frailty in Older Adults Using Wearables

Reprinted from: Sensors 2018, 18, 926, doi:10.3390/s18030926 . . . . . . . . . . . . . . . . 288

Kun Liu, Yong Liu, Jianchao Yan and Zhenyuan Sun

Nondestructive Estimation of Muscle Contributions to STS Training with Different Loadings

Based on Wearable Sensor System

Reprinted from: Sensors 2018, 18,971, doi:10.3390/s18040971 . . . . . . . . . . . . . . . . . . . 302

Yanshun Zhang, Yingyue Li, Chuang Peng, Dong Mou, Ming Li and Wei Wang

The Height-Adaptive Parameterized Step Length Measurement Method and Experiment Based on Motion Parameters

Reprinted from: Sensors 2018, 18, 1039, doi:10.3390/s18041039 . . . . . . . . . . . . . . . . 318 
Saba Rezvanian, Thurmon Lockhart, Christopher Frames, Rahul Soangra and Abraham Lieberman

Motor Subtypes of Parkinson's Disease Can Be Identified by Frequency Component of Postural Stability

Reprinted from: Sensors 2018, 18, 1102, doi:10.3390/s18041102

José Antonio Santoyo-Ramón, Eduardo Casilari and José Manuel Cano-García

Analysis of a Smartphone-Based Architecture with Multiple Mobility Sensors for Fall Detection with Supervised Learning

Reprinted from: Sensors 2018, 18,1155, doi:10.3390/s18041155 . . . . . . . . . . . . . . . . . . 341

Dinesh John, Alvin Morton, Diego Arguello, Kate Lyden and David Bassett

"What Is a Step?" Differences in How a Step Is Detected among Three Popular Activity Monitors That Have Impacted Physical Activity Research

Reprinted from: Sensors 2018, 18, 1206, doi:10.3390/s18041206 . . . . . . . . . . . . . . . . 370

Rob C. van Lummel, Jordi Evers, Martijn Niessen, Peter J. Beek and Jaap H. van Dieën

Older Adults with Weaker Muscle Strength Stand up from a Sitting Position with More Dynamic Trunk Use

Reprinted from: Sensors 2018, 18,1235, doi:10.3390/s18041235 . . . . . . . . . . . . . . . . . 385 



\section{About the Special Issue Editor}

Thurmon Lockhart is Professor in the Biomedical Engineering and Biological Design program in the School of Biological Health and Systems Engineering at Arizona State University, Tempe, AZ. He is also Adjunct Professor at the Barrow Neurological Institute, Research Affiliate at the Mayo Clinic College of Medicine, Division of Endocrinology, and Guest Professor at Ghent University in Belgium. His research focuses on the identification of injury mechanisms and the quantification of sensorimotor deficits and movement disorders associated with aging and neurological disorders on fall accidents. His academic grounding in biomechanical modeling, nonlinear dynamics, human postural control, gait mechanics, and wearable biosensor design underscore a fundamental capacity to provide unique clinical solutions to injury prevention utilizing both engineering and biomedical principles. He has translated research findings into practice by reaching a significant number of external organizations and individuals. His outreach efforts have impacted several organizations, including UPS, the US Navy, Los Alamos National Security, the DOE, GE, and BP. In recognition of these scientific achievements, Prof. Lockhart and co-workers were awarded the Alexander C. Williams, Jr., Design Award from the Human Factors and Ergonomics Society in 2008. His research was recently featured on the PBS NOVA ScienceNow and Good Morning America programs and in the Fortune, AgingWell, Men's Health, and Discover magazines. 



\section{Preface to "Sensors for Gait, Posture, and Health Monitoring"}

The acquisition of gait and postural characteristics during active and passive movements provides important information about limb propulsion and postural control strategies and provides insight into performance and risk of injury. These measures were traditionally assessed by utilizing motion capture systems and force plates. Although modern motion capture laboratories collect precise gait and posture data, they are expensive and immobile and require serial (single person at-a-time data capture) and clustered data collection, limiting the use of motion capture in the field to obtain more realistic motion profiles that may be applicable to various interventions.

As such, in recent years, many technologies for gait and posture assessments have emerged. Wearable sensors, active and passive in-house monitors, and many combinations thereof all promise to provide accurate measures of gait and posture parameters. The objective of this Special Issue is to address and disseminate the latest gait and posture monitoring systems as well as various mathematical models/methods that characterize mobility functions.

This Special Issue explores the core scientific issues associated with the use of custom-designed, wearable, wireless sensor nodes for continuous, non-invasive gait-posture-activity monitoring and analysis in order to accurately study the relationship between these monitoring variables and physical and psychological health conditions to predict adverse medical events in a variety of populations. This type of assessment will dramatically expand the clinical usefulness of these analyses and pave the way for identifying potential adverse health conditions and appropriate interventions for those most at risk.

Thurmon Lockhart

Special Issue Editor 



\title{
Technology-Based Feedback and Its Efficacy in Improving Gait Parameters in Patients with Abnormal Gait: A Systematic Review
}

\author{
Gema Chamorro-Moriana ${ }^{1}$, Antonio José Moreno ${ }^{1}$ and José Luis Sevillano ${ }^{2, *}$ \\ 1 Department of Physiotherapy, Universidad de Sevilla, 41009 Sevilla, Spain; gchamorro@us.es (G.C.M.), \\ toni.moreno.martin@gmail.com (A.J.M.) \\ 2 Department of Computer Technology and Architecture, Universidad de Sevilla, 41012 Sevilla, Spain \\ * Correspondence: sevi@atc.us.es; Tel.: +34-954-556-142
}

Received: 11 October 2017; Accepted: 2 January 2018; Published: 6 January 2018

\begin{abstract}
This systematic review synthesized and analyzed clinical findings related to the effectiveness of innovative technological feedback for tackling functional gait recovery. An electronic search of PUBMED, PEDro, WOS, CINAHL, and DIALNET was conducted from January 2011 to December 2016. The main inclusion criteria were: patients with modified or abnormal gait; application of technology-based feedback to deal with functional recovery of gait; any comparison between different kinds of feedback applied by means of technology, or any comparison between technological and non-technological feedback; and randomized controlled trials. Twenty papers were included. The populations were neurological patients (75\%), orthopedic and healthy subjects. All participants were adults, bar one. Four studies used exoskeletons, 6 load platforms and 5 pressure sensors. The breakdown of the type of feedback used was as follows: $60 \%$ visual, $40 \%$ acoustic and $15 \%$ haptic. $55 \%$ used terminal feedback versus $65 \%$ simultaneous feedback. Prescriptive feedback was used in $60 \%$ of cases, while $50 \%$ used descriptive feedback. $62.5 \%$ and $58.33 \%$ of the trials showed a significant effect in improving step length and speed, respectively. Efficacy in improving other gait parameters such as balance or range of movement is observed in more than $75 \%$ of the studies with significant outcomes. Conclusion: Treatments based on feedback using innovative technology in patients with abnormal gait are mostly effective in improving gait parameters and therefore useful for the functional recovery of patients. The most frequently highlighted types of feedback were immediate visual feedback followed by terminal and immediate acoustic feedback.
\end{abstract}

Keywords: feedback technology; gait; rehabilitation; motor control

\section{Introduction}

The basic motor functions of the human being, such as gait, can be altered because of a wide range of traumatalogical, neurological, rheumatic, etc. pathologies [1,2]. Hip arthrosis [3], knee osteoarthritis [4], strokes, hemiparesis [5-7], or lower-limb amputations [8], all produce important alterations to gait patterns.

Developments in technology and information technology (IT) have enabled the development of new techniques for gait re-training based on feedback supplied by electronic devices. This has been demonstrated by authors such as Druzbicki et al. [5], Basta et al. [9], Zanoto et al. [10] and Segal et al. [11].

The basic principle of feedback is the ability to voluntarily control and change certain bodily functions or biological processes when information is provided about them [12]. The main advantage of feedback is the supply of information about a specific biological process about which the patient does not consciously have information [13]. 
Currently, technology is developing towards facilitating the functional recovery of the patient, sometimes even without the physiotherapist. These treatments incorporate: robot assisted movement [10,14-16], virtual reality technology [17] and inertial monitoring devices [18,19] amongst others. Some of these systems use visual $[5,11,20]$, acoustic $[15,21]$ and/or haptic $[22,23]$ feedback in a coherent and detailed way, adapted to each user's individual needs [24]. New technologies based on feedback are extremely useful in the area of rehabilitation for re-educating an altered function or teaching a new one $[2,25]$. These aspects represent the main objectives of physiotherapy $[13,25]$.

However, technological systems are frequently adopted in clinical practice without their efficacy having been proven. Researchers need to focus on providing clinical findings [24]. Therefore, the effects of these novel devices need to be measured $[26,27]$ on different study populations, considering gait parameters, therapeutic guidelines adopted, clinical results obtained, systems of assessment used, etc. Similarly, we need to analyze the efficacy of different types of extrinsic feedback, in other words, that coming from an external source [28]. In this case, electronic devices will provide concurrent or immediate feedback, that is, feedback received simultaneously with the action (for example, during the foot support phase, the patient knows the amount of vertical reaction force of the floor on the limb or during walking the patient knows his/her speed); terminal or retarded feedback, or feedback received when the action is finished (for example, at the end of a tour the patient knows information about his/her progress, length of the steps, speed, kinematic of the knee, etc.); acoustic (e.g., beep, oral, etc.), visual (e.g., video cameras, displays, etc.) or haptic information (usually vibrations in some body area such as the soles of the feet) [29]; etc. Finally, this study also considers whether extrinsic feedback offers knowledge of performance (KP), in other words, characteristics of performance (e.g., if the foot bears the right direction, if the trunk remains erect during the action, etc.); or knowledge of result (KR) [30] (correct or incorrect action, score, etc.); whether this is descriptive (description of errors) or also prescriptive (how to correct errors) [24] (for example, we describe an error in walking saying that the patient is dragging the foot during the swing phase of the step. However, to correct it, we ask the patient to flex the hip and knee more when taking the step, so that the foot does not touch the ground).

Hence, the need to review, synthesize and analyze clinical findings related to the use of different kinds of technology-based feedback and their effectiveness in improving certain parameters in functional gait recovery.

\section{Materials and Methods}

The method was based on the PRISMA protocol [31].

\subsection{Data Sources and Search Strategy}

An electronic search of PUBMED, PEDro, WOS, CINAHL, and DIALNET was carried out from January 2011 to December 2016. In addition to this, we checked the reference lists of the included studies. Mesh terms (Medical Subject Headings) for English language or Decs Terms (Descriptores en Ciencias de la Salud) for Spanish database and search strategies are shown in Tables 1 and 2.

Table 1. Mesh and Decs Terms put into groups by mean.

\begin{tabular}{|c|c|}
\hline Terms and Strategies & Identifier \\
\hline feedback or biofeedback or neurofeedback or proprioception & 1 \\
\hline $\begin{array}{c}\text { treatment or program * or exercise * or rehabilit * or training or educat * or "stimulation } \\
\text { training" or teaching or learning }\end{array}$ & 2 \\
\hline software or program * or technology or "biomedical technology" or system & 3 \\
\hline gait or walking or ambulation or locomotion or "stair navigation" & 4 \\
\hline Randomiza * or study or "clinical trial" & 5 \\
\hline Trata * or program * or rehabilit* & 6 \\
\hline feedback or biofeedback or neurofeedback or retroalimentación & 7 \\
\hline marcha or ambul * or locomoción & 8 \\
\hline
\end{tabular}


Table 2. Search strategy.

\begin{tabular}{ccc}
\hline Database & Search Strategy & Simplified Strategy \\
\hline PubMed & $\begin{array}{c}\text { (treatment or program * or exercise * or rehabilit * or training or } \\
\text { educat * or "stimulation training" or teaching or learning) and } \\
\text { (feedback or biofeedback or neurofeedback or proprioception) } \\
\text { and (gait or walking or ambulation or locomotion or "stair } \\
\text { navigation") and (software or program * or technology or } \\
\text { "biomedical technology" or system) }\end{array}$ & 2 and 1 and 4 and 3 \\
\hline PEDro & $\begin{array}{c}\text { feedback and gait } \\
\text { (feedback or biofeedback or neurofeedback or proprioception) } \\
\text { navigation") and (software or program * or technology or }\end{array}$ & 1 and 4 and 3 and 5 5 \\
\hline "biomedical technology" or system) and (randomiza * or study or \\
"clinical trial")
\end{tabular}

\subsection{Study Selection and Inclusion Criteria}

The papers included in this review had to meet the following criteria:

- Population: Mainly patients with a modified or abnormal gait (i.e., spatiotemporal gait parameters) due to a pathology such as cerebral palsy, hip orthoprosthesis, lower member amputation, knee ligamentoplasty, etc.

- Interventions: application of technology-based feedback (haptic and/or visual and/or acoustic) to assist functional gait recovery as much as possible. The feedback had to be received by the patient directly (external feedback).

- Comparisons: Any comparison between different kinds of feedback (visual, haptic, immediate/concurrent, retarded/terminal, etc.) applied using technology. Or any comparison between technological and non-technological feedback, usual care or an alternative exercise therapy/intervention not based on feedback.

- Outcomes: Any validated measures of parameters or aspects associated to gait, such as: pain, functionality, balance, unload weight bearing, spatiotemporal parameters (speed, cadence, step length), kinematic data (range of movement-ROM) and score by specific gait assessment test or scale (i.e., Up and Go, chair-stand time).

- $\quad$ Study design: Randomised controlled trials (RCTs).

- $\quad$ Measure of methodological quality of RCT: A minimum of 4 points according to PEDro Scale. That is, "fair" and "high" quality studies [32] (see Quality Appraisal).

- Language: Studies reported in English or Spanish.

- $\quad$ Setting: Not limited to a particular setting.

The titles and abstracts of the search results were screened to check if a study met the pre-established inclusion criteria. We obtained the full text article of those studies which met the criteria, and documented the causes for any exclusions at this stage.

\subsection{Data Extraction}

Data extraction was carried out by one reviewer (A.J.M.) and checked for accuracy by a second reviewer (G.C.M.), using a table designed to detail information on study features, participant characteristics, feedback modality, technology employed (for feedback and assessment), interventions, comparisons, and outcome measurements. 


\subsection{Quality Appraisal}

Apposite studies were assessed for methodological quality using the Physiotherapy Evidence Database (PEDro) critical appraisal tool [33]. This method was valid and reliable for assessing the internal validity of a study (criteria 2-9). We also evaluated the adequacy of the statistical information for interpreting the results (criteria 10-11) [34-36]. PEDro consists of 11 criteria overall; although criterion 1 refers to the external validity of the trial and is not included in the final score [34]. Each criterion could be Yes (one point) or No (0 points), with a maximum score out of ten. Only "fair" (scores $4 / 5$ ) and "high" (scores $\geq 6 / 10$ ) quality studies [32] were included in this review.

\section{Results}

\subsection{Search Results}

We found 884 articles in the electronic databases. Most of them in Pubmed (404), and the rest in PEDro (61), WOS (16), Cinahl (339) and Dialnet (64). Following the removal of duplicates, 776 articles were screened by title, abstract and full-text, due to: not including feedback technology, not applying the feedback directly to the patient, not being RCT, not using feedback for gait functional recovery, not having $\geq 4$ score in PEDro Scale. After the screening, 20 studies were left for inclusion in this review.

Figure 1 shows the search and study selection process, which was based on PRISMA [37] guidelines.

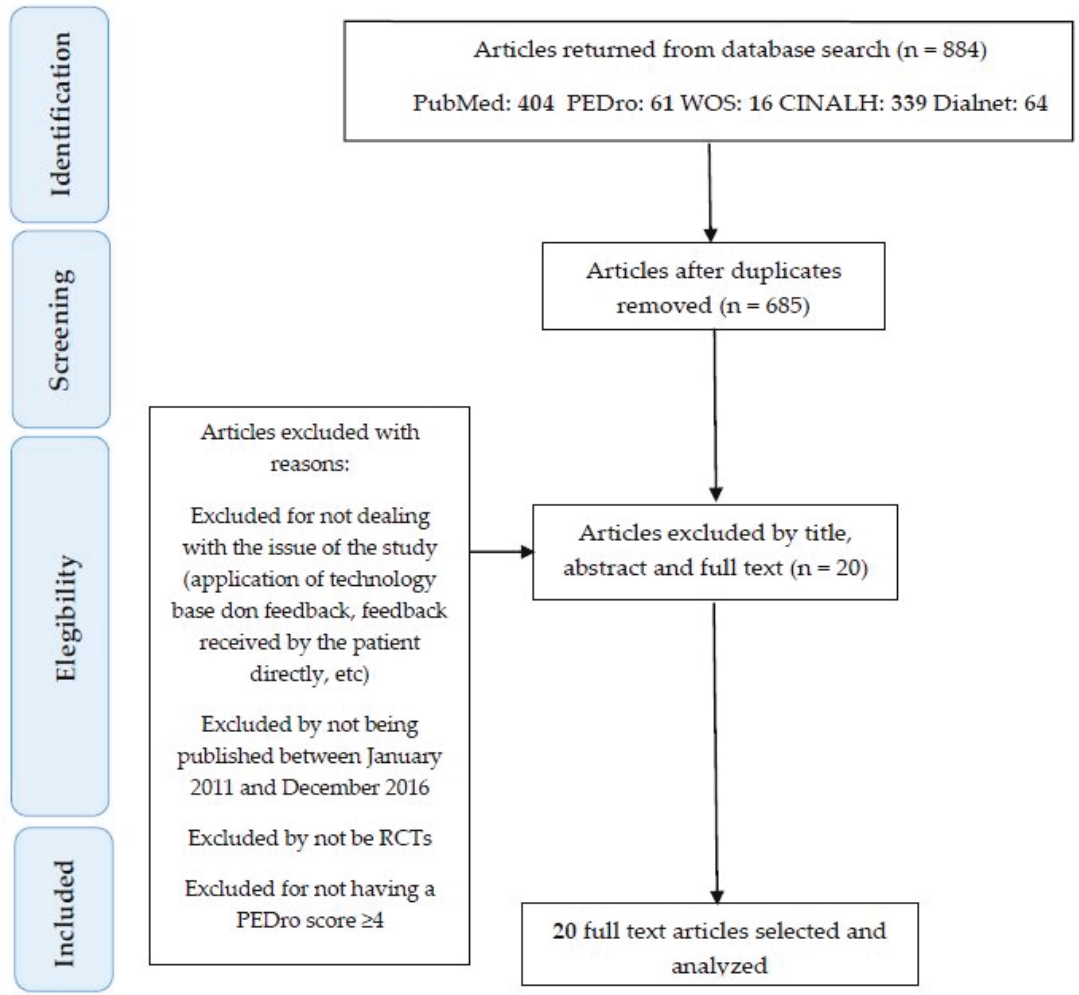

Figure 1. Research method of this study.

\subsection{Characteristics of Included Studies}

A detailed summary of the features and results of each selected study is shown in Table 3. 


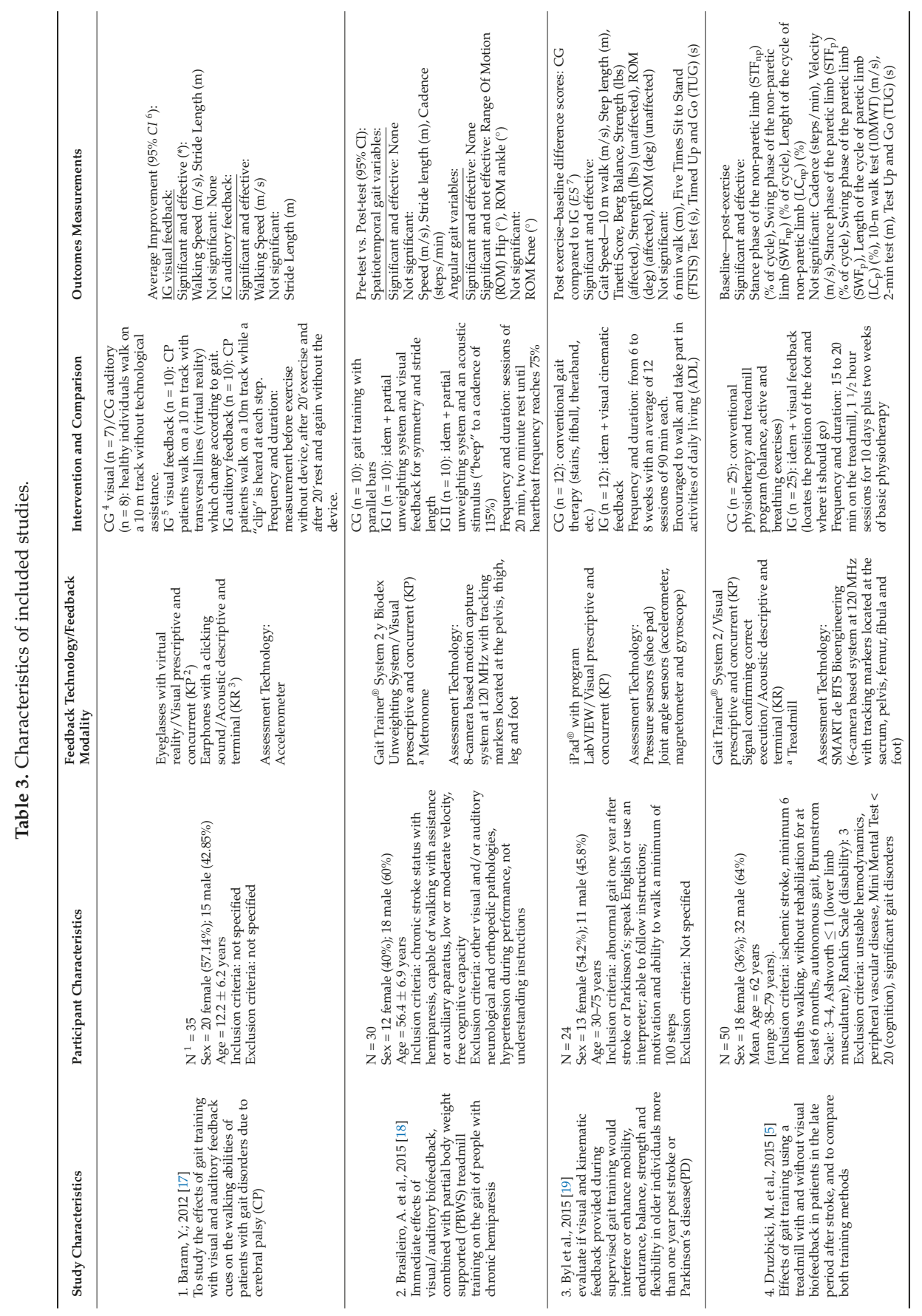




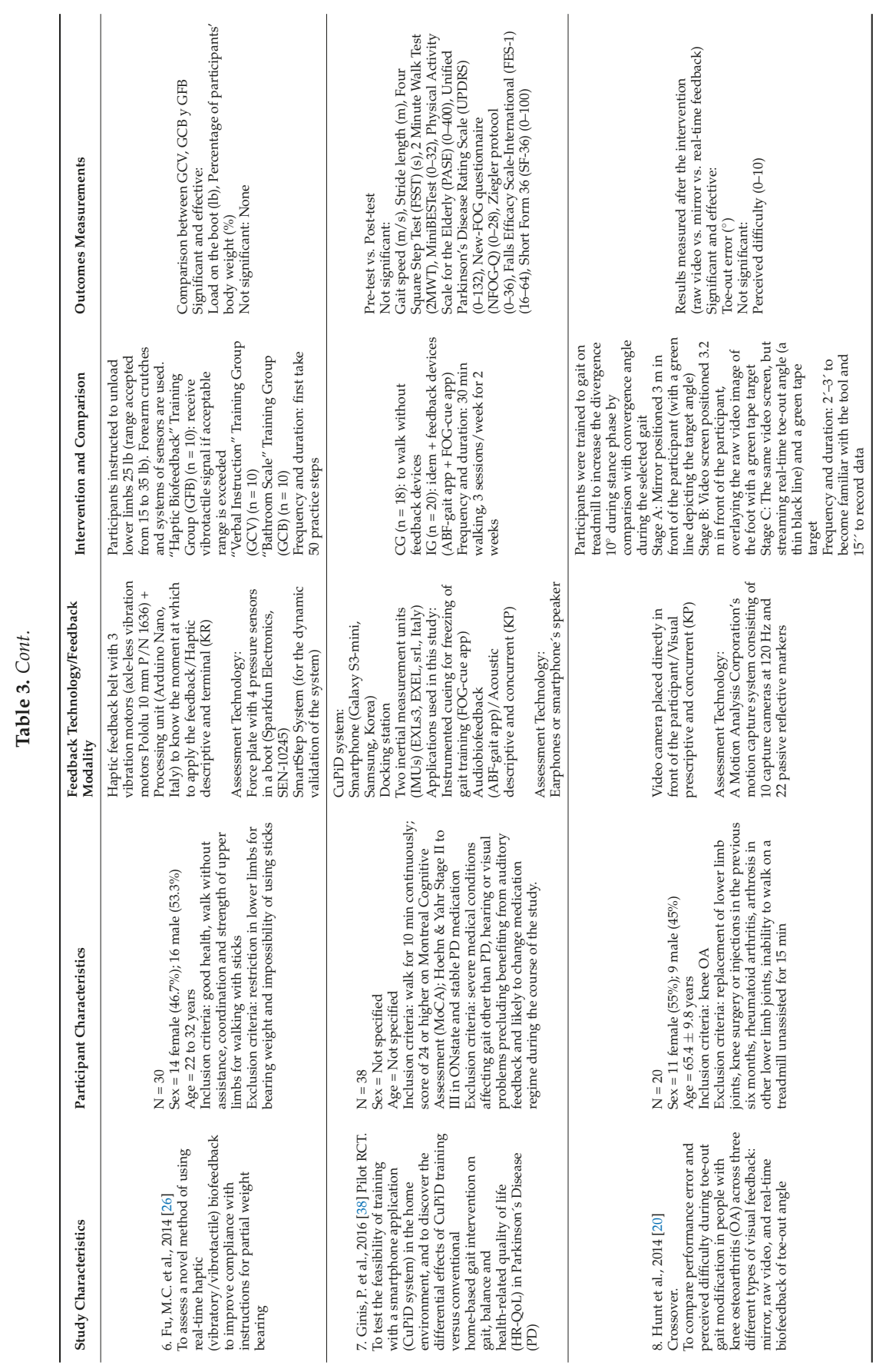




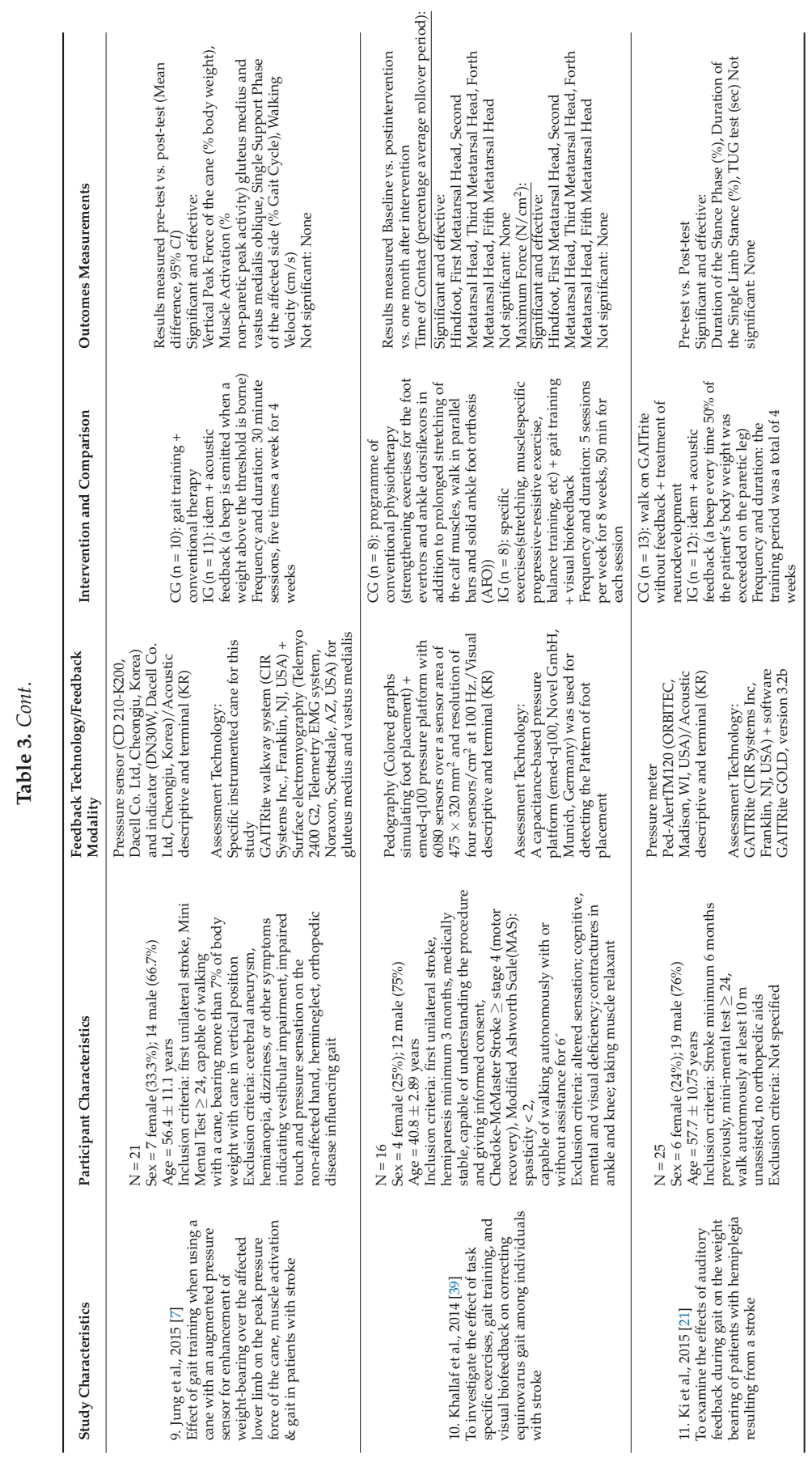




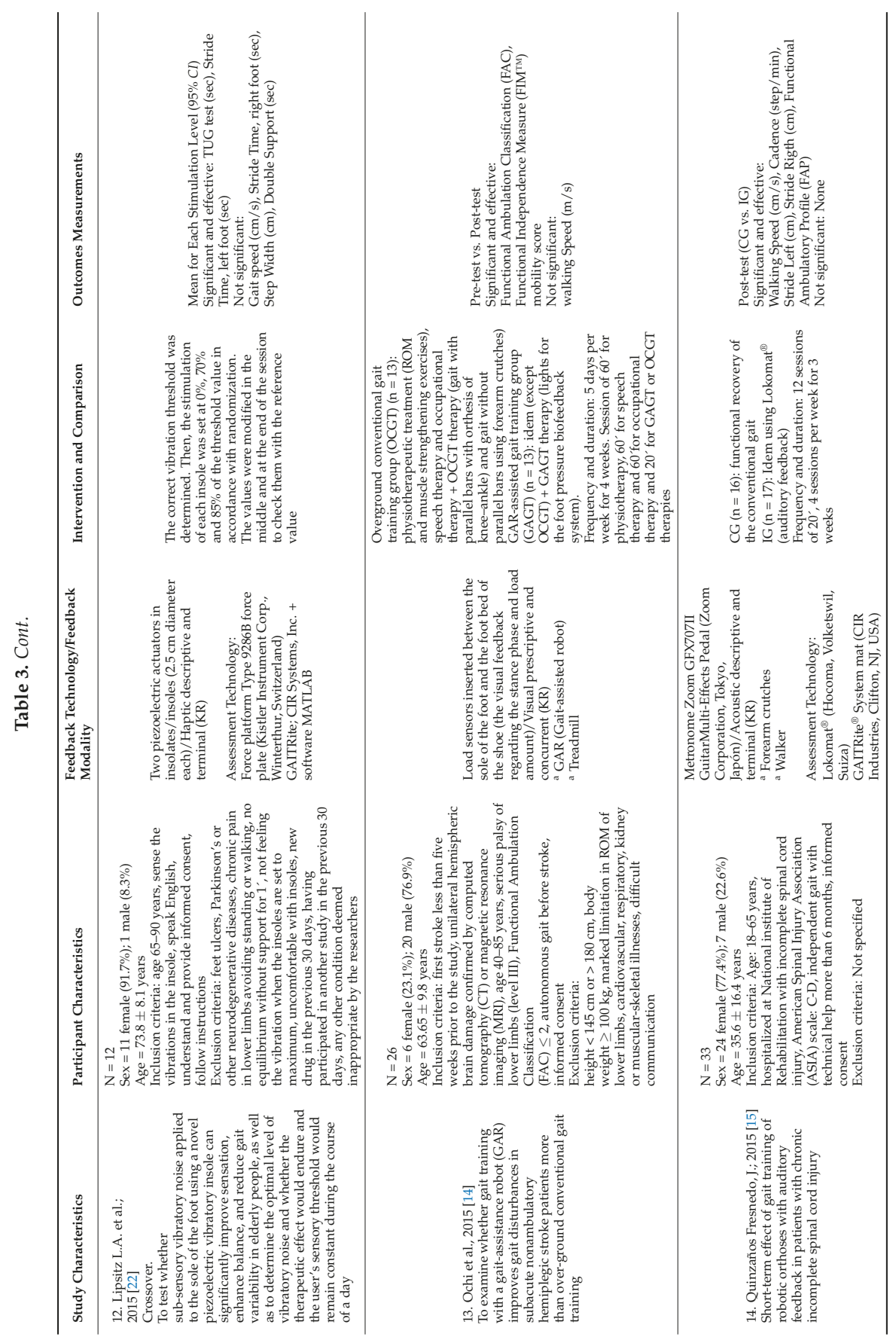




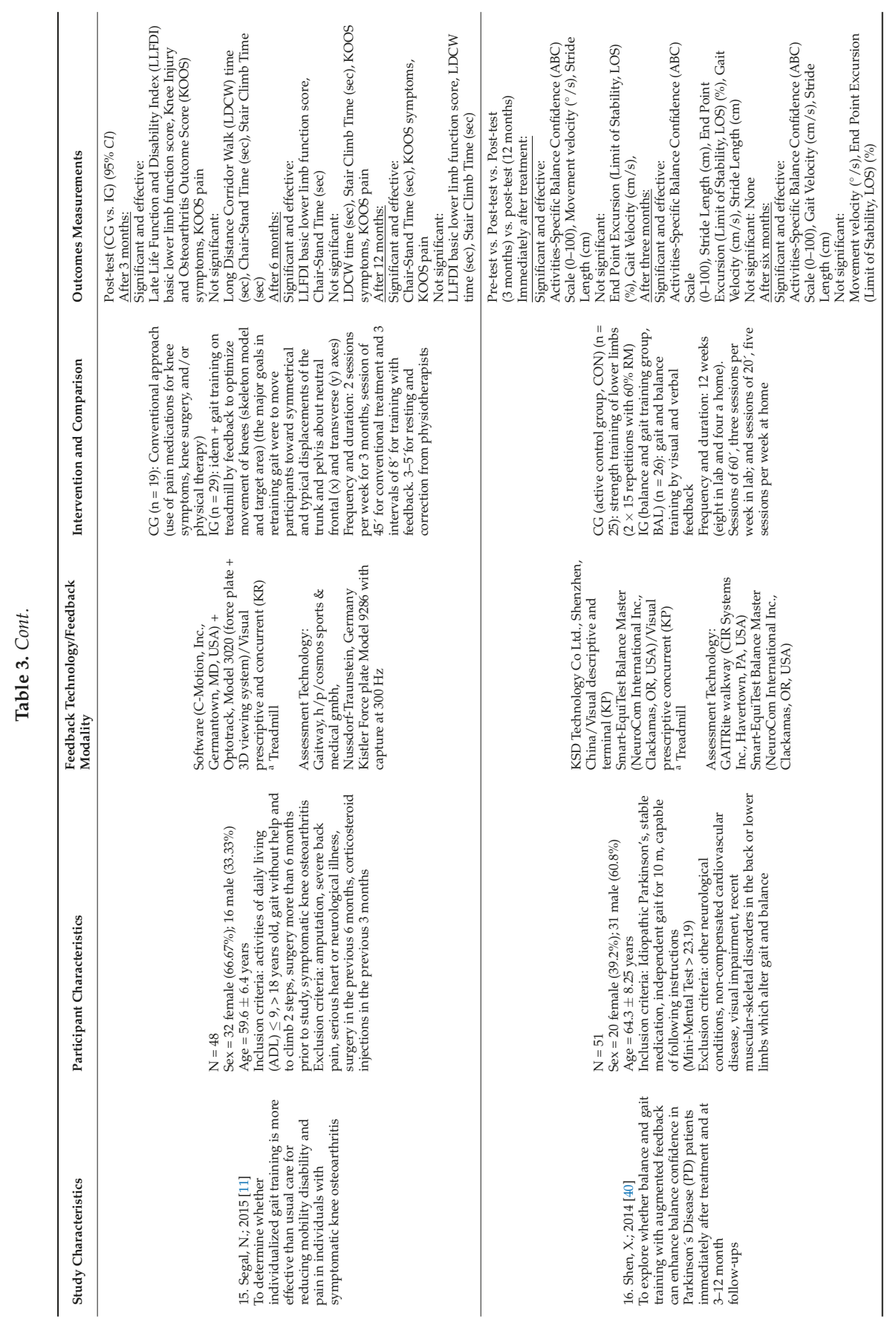




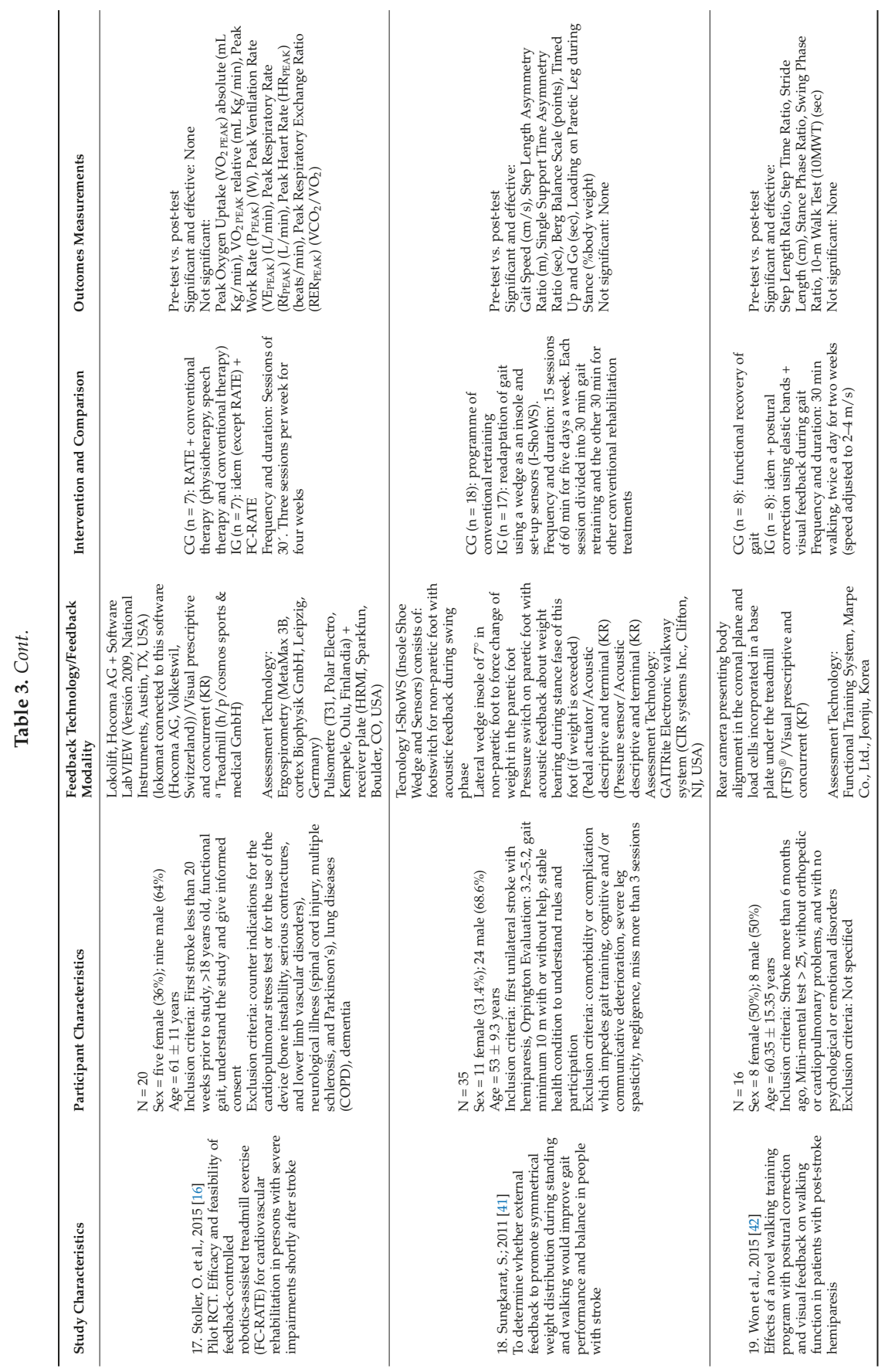




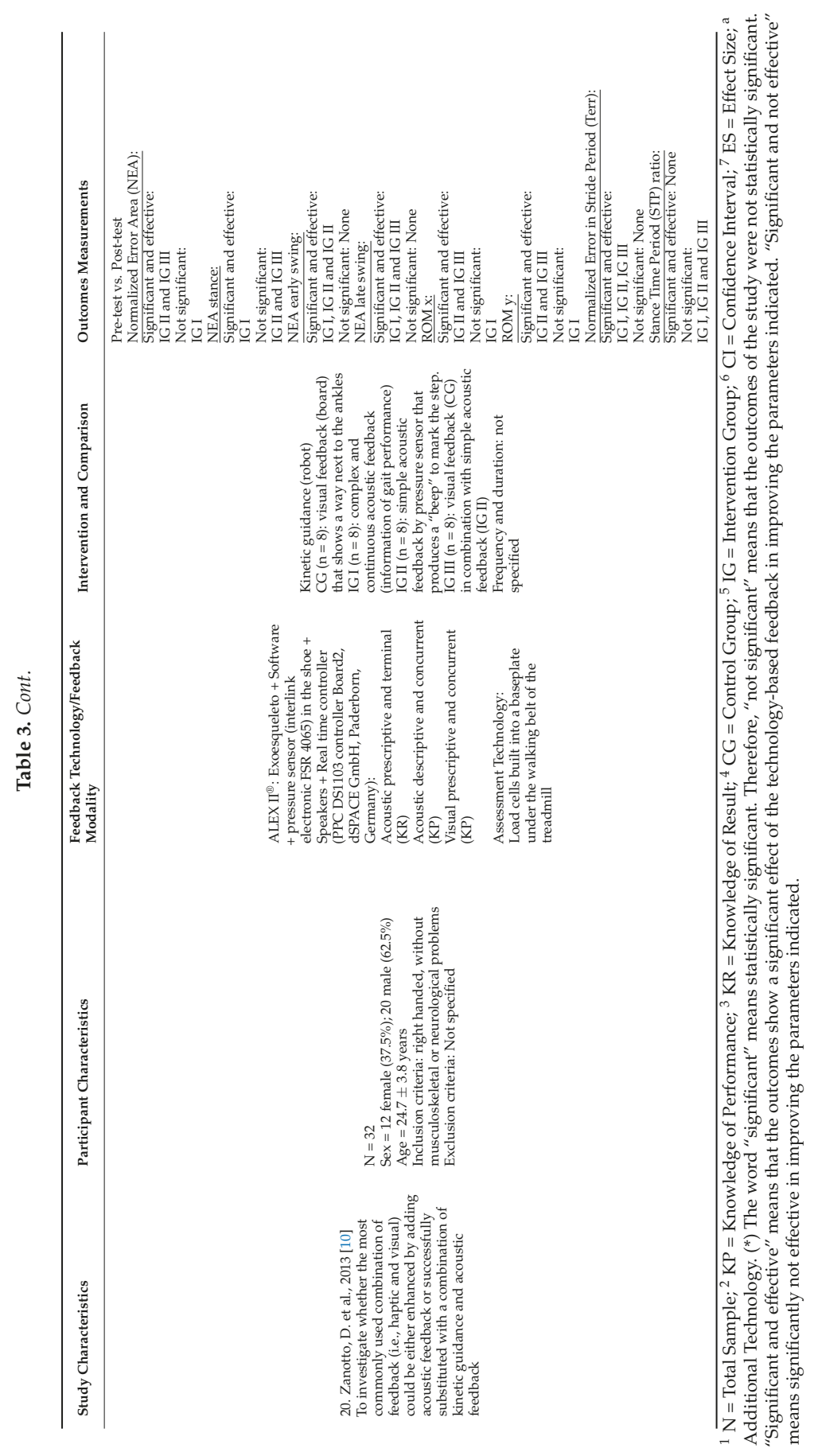




\subsection{Quality Assessment}

The results of the PEDro scoring are shown in Table 4. All the selected papers rated "fair" and "high" quality ( $\geq 4$ points).

Table 4. Completed PEDro quality appraisal.

\begin{tabular}{|c|c|c|c|c|c|c|c|c|c|c|c|c|}
\hline \multirow{2}{*}{ Study } & \multicolumn{11}{|c|}{ Criteria } & \multirow[t]{2}{*}{ Total Score } \\
\hline & 1 & 2 & 3 & 4 & 5 & 6 & 7 & 8 & 9 & 10 & 11 & \\
\hline 1. Baram, Y. et al., 2012 [17] & $x$ & $\checkmark$ & $x$ & $\checkmark$ & $x$ & $x$ & $x$ & $\checkmark$ & $\checkmark$ & $x$ & $x$ & 4 \\
\hline 2. Brasileiro, A. et al., 2015 [18] & $x$ & $\checkmark$ & $x$ & $\checkmark$ & $x$ & $x$ & $x$ & $\checkmark$ & $\sqrt{ }$ & $\checkmark$ & $\checkmark$ & 6 \\
\hline 3. Byl, N. et al., 2015 [19] & $\checkmark$ & $\checkmark$ & $x$ & $x$ & $x$ & $x$ & $X$ & $\checkmark$ & $x$ & $\checkmark$ & $\checkmark$ & 4 \\
\hline 4. Drużbicki, M. et al., 2015 [5] & $\checkmark$ & $\checkmark$ & $\checkmark$ & $\checkmark$ & $x$ & $x$ & $\checkmark$ & $\checkmark$ & $\checkmark$ & $\checkmark$ & $\checkmark$ & 8 \\
\hline 5. El-Tamawy, M. et al., 2012 [23] & $\checkmark$ & $\checkmark$ & $x$ & $\checkmark$ & $x$ & $x$ & $\checkmark$ & $\checkmark$ & $x$ & $\checkmark$ & $\checkmark$ & 6 \\
\hline 6. Fu, M.C. et al., 2014 [26] & $\checkmark$ & $\checkmark$ & $x$ & $x$ & $x$ & $x$ & $x$ & $\checkmark$ & $\checkmark$ & $\checkmark$ & $X$ & 4 \\
\hline 7. Ginis, P. et al., 2016 [38] & $\checkmark$ & $\checkmark$ & $x$ & $\checkmark$ & $x$ & $x$ & $x$ & $\checkmark$ & $\checkmark$ & $\checkmark$ & $\checkmark$ & 6 \\
\hline 8. Hunt, M.A. et al., 2014 [20] & $\checkmark$ & $\checkmark$ & $x$ & $\checkmark$ & $x$ & $x$ & $x$ & $\checkmark$ & $\checkmark$ & $\checkmark$ & $\checkmark$ & 6 \\
\hline 9. Jung, K. et al., 2015 [7] & $\checkmark$ & $\checkmark$ & $\checkmark$ & $\checkmark$ & $x$ & $x$ & $\checkmark$ & $\checkmark$ & $x$ & $\checkmark$ & $\checkmark$ & 7 \\
\hline 10. Khallaf, M.E. et al., 2014 [39] & $\checkmark$ & $\checkmark$ & $\checkmark$ & $\checkmark$ & $x$ & $x$ & $x$ & $x$ & $x$ & $\checkmark$ & $\checkmark$ & 5 \\
\hline 11. Ki, K. et al., 2015 [21] & $\checkmark$ & $\checkmark$ & $x$ & $\checkmark$ & $x$ & $x$ & $x$ & $\checkmark$ & $x$ & $\checkmark$ & $\checkmark$ & 5 \\
\hline 12. Lipsitz, L.A. et al., 2015 [22] & $\checkmark$ & $\checkmark$ & $\checkmark$ & $x$ & $x$ & $x$ & $\checkmark$ & $x$ & $x$ & $\checkmark$ & $x$ & 4 \\
\hline 13. Ochi, M. et al., 2015 [14] & $\checkmark$ & $\checkmark$ & $\checkmark$ & $\checkmark$ & $x$ & $x$ & $\checkmark$ & $x$ & $X$ & $\checkmark$ & $\checkmark$ & 6 \\
\hline 14. Quinzaños Fresnedo, J. et al., 2015 [15] & $\checkmark$ & $\checkmark$ & $x$ & $\checkmark$ & $x$ & $x$ & $x$ & $\checkmark$ & $\checkmark$ & $\checkmark$ & $\checkmark$ & 6 \\
\hline 15. Segal, N.A. et al., 2015 [11] & $\checkmark$ & $\checkmark$ & $\checkmark$ & $\checkmark$ & $x$ & $x$ & $x$ & $x$ & $X$ & $\checkmark$ & $\checkmark$ & 5 \\
\hline 16. Shen, X. et al., 2014 [40] & $\checkmark$ & $\checkmark$ & $x$ & $\checkmark$ & $x$ & $x$ & $\checkmark$ & $\checkmark$ & $\checkmark$ & $\checkmark$ & $\checkmark$ & 7 \\
\hline 17. Stoller, O. et al., 2015 [16] & $\checkmark$ & $\checkmark$ & $\checkmark$ & $\checkmark$ & $x$ & $x$ & $\checkmark$ & $x$ & $\checkmark$ & $\checkmark$ & $\checkmark$ & 7 \\
\hline 18. Sungkarat, S. et al., 2011 [41] & $\checkmark$ & $\checkmark$ & $\checkmark$ & $\checkmark$ & $x$ & $x$ & $\sqrt{ }$ & $\checkmark$ & $X$ & $\checkmark$ & $\checkmark$ & 7 \\
\hline 19. Won, S.H. et al., 2015 [42] & $\checkmark$ & $\checkmark$ & $\checkmark$ & $\checkmark$ & $x$ & $x$ & $x$ & $\sqrt{ }$ & $\checkmark$ & $\checkmark$ & $\checkmark$ & 7 \\
\hline 20. Zanotto, D. et al., 2013 [10] & $\checkmark$ & $\checkmark$ & $X$ & $\checkmark$ & $x$ & $x$ & $x$ & $\checkmark$ & $\checkmark$ & $\checkmark$ & $\checkmark$ & 6 \\
\hline
\end{tabular}

Criteria: ${ }^{1}$ Eligibility criteria were specified (not used for score); ${ }^{2}$ Subjects were randomly allocated to groups;

${ }^{3}$ Allocation was concealed; ${ }^{4}$ Groups were similar at baseline regarding the most important prognostic indicators;

${ }^{5}$ There was blinding of all subjects; ${ }^{6}$ There was blinding of all therapists who administered the therapy; ${ }^{7}$ There was blinding of all assessors who measured at least one key outcome; ${ }^{8}$ Measures of at least one key outcome were obtained from more than $85 \%$ of the subjects initially allocated to groups; ${ }^{9}$ All subjects for whom outcome measures were available received the treatment or control condition as allocated or, where this was not the case, data for at least one key outcome was analyzed by 'intention-to-treat'; ${ }^{10}$ The results of between-group statistical comparisons are reported for at least one key outcome; ${ }^{11}$ The study provides both point measures and measures of variability for at least one key outcome). $\boldsymbol{V}=$ criteria met; $X=$ criteria not met.

The item "Subjects were randomly allocated to groups" (2) was scored by all papers because it was an inclusion criterion. Besides, the items "Eligibility criteria were specified" (1) and "The results of between-group statistical comparisons are reported for at least one key outcome" (10) were scored in all studies apart from 2.

Although the studies were considered to be of "fair" and "high" quality, there were two items with 0 scores: "Blinding of all subjects" (5) and "Blinding of all therapists who administered the therapy" (6).

\subsection{Participant Characteristics}

Relative to the population in this review, neurological patients were found in 15 out of 20 papers (75\%). That is: 8 of stroke [5,7,16,19,21,39,41,42]; 1 of cerebral palsy [17]; 2 of hemiparesis [14,18]; 4 of Parkinson's [19,23,38,40]; and 1 with incomplete spinal cord injury [15]. Byl et al. [19] include stroke and Parkinson's in the same research. Besides, 2 studies were found with patients in the orthopaedic area $[11,20]$; and 3 more with healthy subjects $[10,22,26]$.

All participants were adults bar one [17].

\subsection{Feedback Technology}

Four studies [10,14-16] stood out due to their use of exoskeletons, although only 2 of them produced feedback, Alex II [10] and Lokomat [16]. The others used complementary technology which only assists gait: Gar [14] and Lokomat [15] in this case without feedback. 
Six studies were based on load platforms [5,14,18,22,40,42], such as Smart Equitest ${ }^{\circledR}$ [40], Gait Trainer ${ }^{\circledR}[5,18]$ and Functional Trainer System ${ }^{\circledR}[42]$; and 5 on pressure sensors $[11,19,22,26,39]$ for example Emed-Q100 ${ }^{\circledR}[39]$ or Ped-Alert TM120 ${ }^{\circledR}[21]$.

The feedback technology was supplemented with other tools in 8 papers: treadmills [5,11,14,16,23,40], exoskeletons [14,15], forearm crutches [15], and metronome [18]. Figure 2 summarizes the use of technologies.

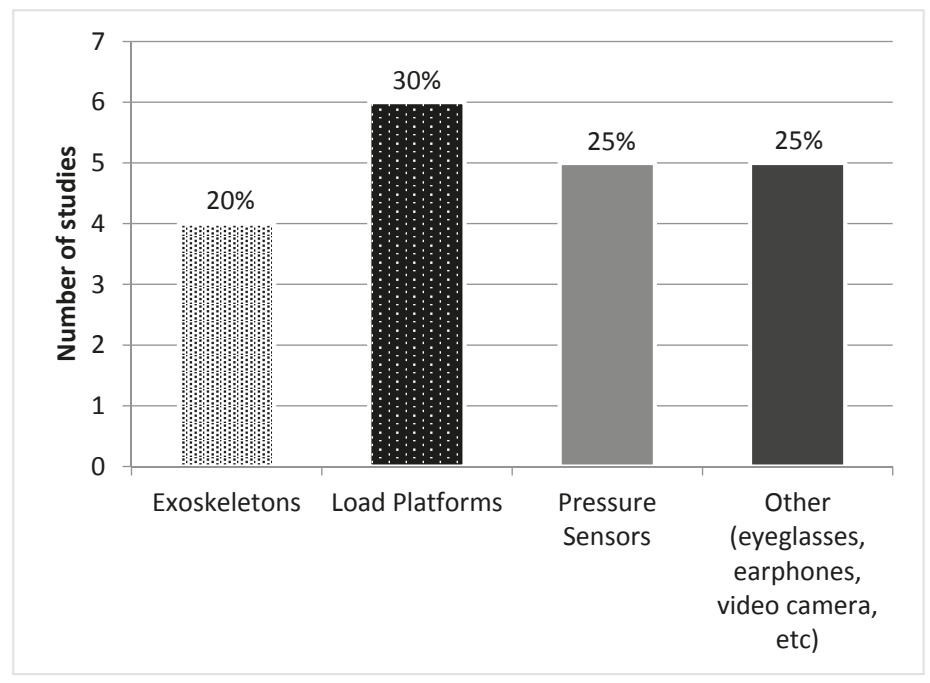

Figure 2. Feedback technologies.

\subsection{Feedback Modalities}

The studies used different types of feedback: visual, acoustic and haptic; terminal/retarded and concurrent/immediate; descriptive and prescriptive; with both KR and KP. Visual feedback was used in $60 \%$ of the papers, acoustic in $40 \%$ and haptic in $15 \%$. Terminal/retarded feedback was used in $55 \%$ and concurrent/immediate in $65 \%$. Descriptive feedback was used in 50\% of cases, with prescriptive in $60 \%$. KP was featured in $45 \%$ and $\mathrm{KR}$ in $70 \%$ (Table 5). 
Sensors 2018, 18, 142

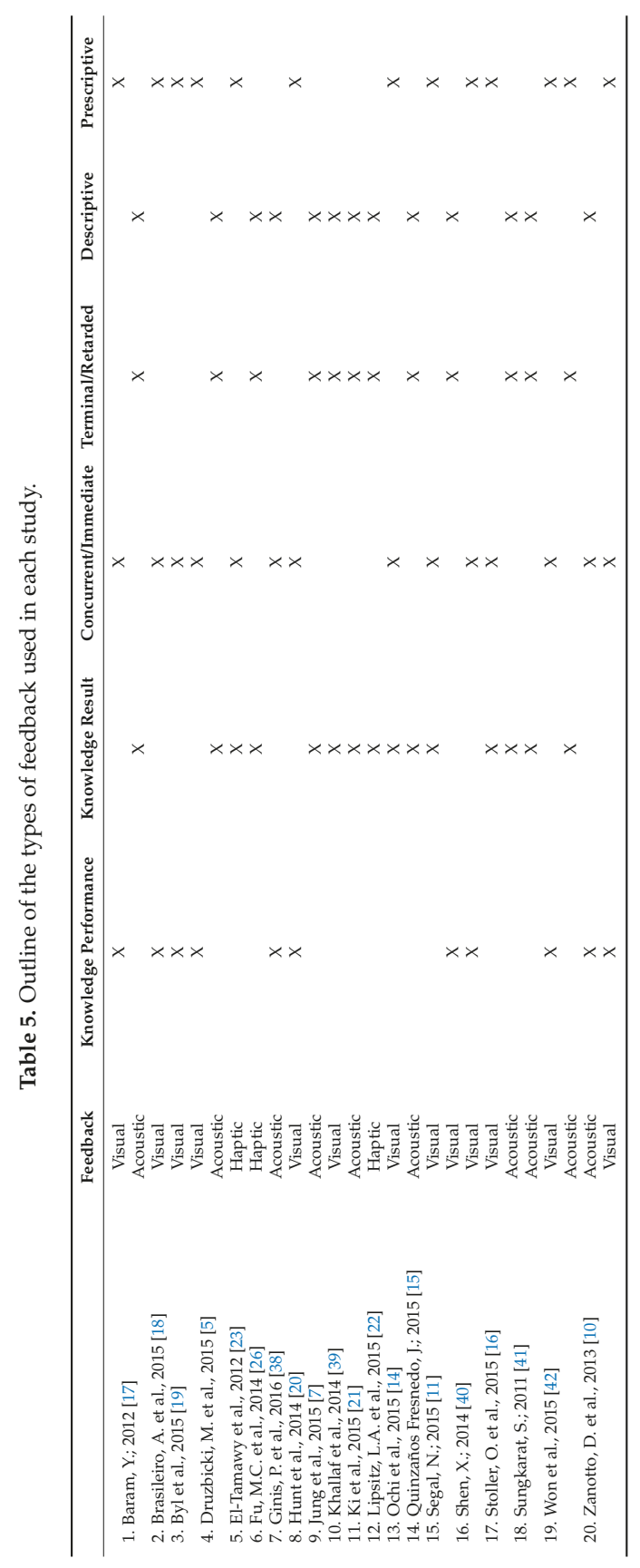


The combination of types of feedback used in descending order was: $55 \%$ visual, concurrent/immediate and prescriptive feedback [5,10,11,14,16-20,40,42];30\% acoustic, terminal/retarded and descriptive $[5,7,15,17,21,41] ; 10 \%$ haptic, terminal/retarded and descriptive [22,26], acoustic, concurrent/immediate and descriptive [10,38] and visual, terminal/retarded and descriptive feedback [39,40]; 5\% combined haptic, concurrent/immediate and prescriptive feedback [23] (Figure 3).

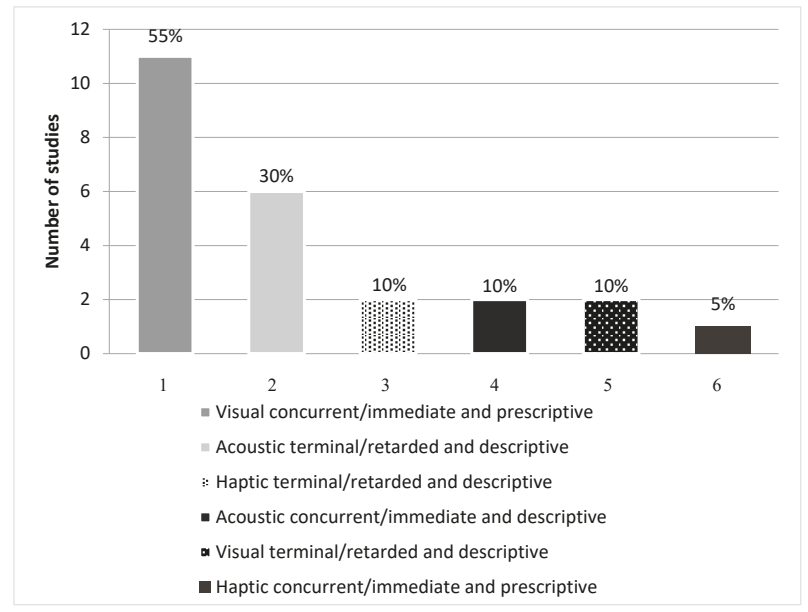

Figure 3. Types of feedback.

\subsection{Assessment Technology}

The technology used to assess gait in the selected studies was as follows: 3D movement analysis systems [5,18,20,23]; platform or treadmill force sensors [10,11,22,26,40]; pressure sensors in insoles [19], platforms [26] and parallel bars [7,15,21,22,40,41], pulsometer and ergospirometry [16]; functional training system [42]; exoskeleton [15]; and Gaitway [11].

\subsection{Interventions and Comparators}

In six studies the application of the feedback systems lasted $20 \min [5,11,14,15,18,40]$, although some took up to $90 \mathrm{~min}$ [19]. Results also included some complementary treatments to technological feedback, such as balance [5], strength training [19], postural correction [23], stretching [7,40], speech therapy [16] and medications [11].

\subsection{Outcome Measures and Results}

The measurements taken in the studies were in descending order of frequency: speed, $75 \%[5,7,14,15,17-19,22,23,38,40,41] ;$ step length, 50\% [17-19,23,38,40,42]; Up and Go Test, $20 \%$ [19,21,22,41]; cadence, 20\% [5,15,18,23]; ROM, 10\% [18,23]; 10MWT 10\% [5,42]; Berg Scale $10 \%[19,41]$ and 2 MWT $10 \%[5,38]$. Other parameters approached to a lesser degree were: IQR [5], peak respiratory rate [16], peak heart rate [16], etc.

For the most frequently considered parameters (speed, step length, Up and Go Test, Cadence, ROM, 10MWT and Berg Scale) the studies with significant outcomes were: $58.33 \%$ for speed $[7,15,17,19,23,40,41] ; 62.5 \%$ for step length [17,19,23,40,42]; $75 \%$ for TUG [21,22,41], 50\% for cadence [15,23], 100\% for ROM [18,23], 50\% for 10MWT [42] and 100\% for Berg Scale [19,41]. The clinical interventions of these studies with significant outcomes, except one [18], were effective in improving the parameters indicated. Table 6 summarizes these studies. 
Sensors 2018, 18, 142

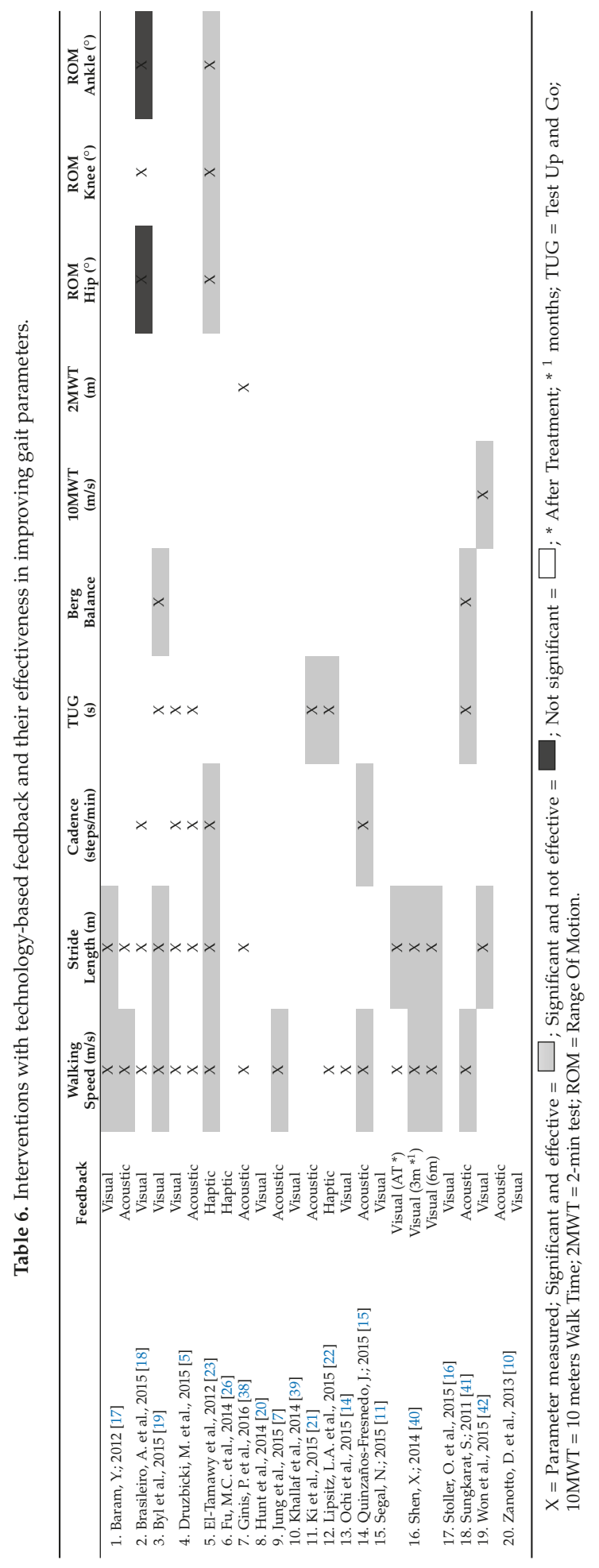




\section{Discussion}

The aim of this review was to synthesize clinical findings regarding the effectiveness of technological feedback in assisting functional gait recovery. Studies defending such effectiveness versus non-technological feedback include: Baram et al. [17], Ki et al. [21], El-Tamawy et al. [23] and Sungkarat et al. [41] amongst others. The authors of this study defend the use of technological feedback but not at the cost of usual care such as: mirror therapy [7], assisted gait [7] or verbal feedback [19], etc. In other words, technological feedback and traditional physiotherapy complement each other in assisting the functional recovery of the patient. To a lesser degree, other authors such as Brasileiro et al. [18], Byl et al. [19] or Hunt et al. [20], state that technological feedback did not obtain positive, or at least significant, results, in relation to other treatments.

In Physiotherapy, the current trend is to improve treatments using new technologies adapted as much as possible to the user needs. Furthermore, it is not only the system that must be individualized, but also the type of feedback used. To exemplify this trend, consider the GCH Control System [27], an instrumented forearm crutch that controls the loads exerted on the crutch when the patient has to partially discharge his/her affected limb. It includes a feedback mechanism to send information about these loads to both the physiotherapist and the patient. When the patient has deficiencies in their coordination skills, the first sessions are usually started with indirect feedback. That is, the therapist receives feedback from the system and verbalizes it to the patient. The patient finds it easier to understand the information through the physiotherapist, who verbally adapts it to their individual conditions (e.g., "Load a little more", "Try to keep that same load", "Be careful that you load more with the right stick than with the left", etc.). The system also has the possibility of adapting the type of feedback (immediate, delayed, visual, auditory, etc.) according to the user's needs. For instance, based on our experience, the use of immediate feedback is easier for the patient and leads to a faster but less lasting result, so it is used when the patient has fewer skills. The delayed feedback is, on the contrary, more complex for the patient and the results come later, although they are more durable [43]. On the other hand, in the case of the GCH System the visual feedback is much simpler than the auditory feedback, which can only be used when the user completely dominates the former.

The articles analyzed in this review highlight how the feedback used when the subject is healthy is more complex $[10,22]$ than when he/she is sick $[7,15,17]$. Also, in the present review, it is observed how there are parameters such as the cadence that can be easily corrected by means of a sound signal such as that emitted by a digital metronome or a more complex one by means of an exoskeleton [15,21,41]. On the other hand, deviations from the center of gravity are better worked by means of images $[11,25]$.

However, it is worth mentioning that, again according to our experience, current technological systems have the tendency to personalize their treatments but without even nuancing the exact needs of the patient. It will be the therapist who makes the decision to use the technology in one way or another, always based on an initial and continuous assessment of the process and taking into consideration the coordinating, proprioceptive abilities of the user. The feedback received by the therapist for decision-making will be not only through technological means, but also through observational analysis. Both assessments, the technological and the visual or manual, are again complementary in the process of functional recovery of gait.

The technological devices, based on feedback, used by the different authors range from the complex to the basic. The complex group would include, for example: Biodex [5,18], Gaitway [11], GAR [14] or LOKOMAT [15]. The specific characteristics of each device means they each have pros and contras in terms of functionality. For example, LOKOMAT requires much more preparation time than GAR [14]. The basic devices include: heel switches [23], virtual glasses (used as computer monitor) and headphones [17], or a cane with a step-counting sensor [7]. The latter has been rendered obsolete as it has been superseded by other canes $[27,44]$ with much more advanced technology and functions. These devices even have their own software designed specifically for functional gait recovery [27].

On the other hand, the high cost of these devices means that their everyday use is unfeasible despite their effectiveness [20]. Many authors [10,20,26], including those writing this article, favour 
efficiency versus the effectiveness of clinical technology in relation to financial, spatiotemporal and human resources [45]. In other words, clinical professionals require assessment and treatment systems which are feasible for everyday clinical practice, allowing adequate development of a process of functional $[1,22]$ gait recovery. For instance, Quinzaños et al. [15] highlight the efficacy of the acoustic stimulus for re-training gait cadence and symmetry. As a result, a basic metronome [18] can be highly useful for functional gait recovery.

As this paper's introduction shows, there are many different classifications of feedback. For example, depending on the sense used, it will be acoustic, visual or haptic [28]. Relating to the moment of the stimulus, there is immediate/concurrent or retarded/terminal feedback. Finally, if the information provides data about performance or result we would be talking about KP or KR [30]. The results of this review show that authors do not just use one isolated type of feedback, instead they sometimes prefer to combine them. The one used most on its own is visual feedback $[5,10,11,14,16-20,39,40,42]$, which is also concurrent $[5,10,11,14,16-20,23,38,40,42]$. In contrast, combined, we find four articles with visual and acoustic feedback at the same time $[5,10,17,38]$ : prescriptive and again concurrent visual feedback; and descriptive, concurrent or terminal, acoustic feedback. Summing up, of the RCTs selected in this review, $55 \%$ of the articles featured prescriptive and concurrent visual feedback $[5,10,11,14,16-20,40,42]$, and 30\% descriptive and terminal acoustic feedback $[5,7,10,15,17,21,41]$. Although many of the devices used in the clinical trials had more types of feedback available (for example, haptic $[23,26]$ ), the authors opted for concurrent feedback, either terminal acoustic or concurrent visual which are the most effective according to Agresta et al. [6]. Thus, it has been demonstrated that concurrent feedback produces the best short-term results [24], while retarded feedback obtains the best results in the long-term [46,47]. However, other authors such as Parker et al. [24] or Salmoni et al. [48] stress that feedback can be counterproductive for learning a complex task if the procedure is applied in too detailed a manner. In other words, detailed feedback can make it more difficult for the participant to understand or process other sensory information.

We must clarify that this statement refers especially to short-term learning, particularly if complex information is offered to patients with limited coordination skills. If we consider a long-term learning the patient has more time to assume complex information although the authors of this study advocate the progression in difficulty based on a continuous assessment of the process. Another handicap of complex and prolonged feedback is the creation of the patient's dependence on receiving feedback. In this sense, the patient responds to feedback automatically in a specific task but does not integrate the learning so it is unable to extrapolate it to other similar tasks [49].

On the other hand, all the information received by the patient can be descriptive (it simply states and describes the error) or prescriptive (it provides data on how to correct the error) [24]. When the correction is simple like in the aforementioned case of the instrumented forearm crutch, just by describing the load exerted the patient knows that he/she must exert more or less force. In other cases, the description and prescription of the correction are not so obvious. When a patient touches the ground with the foot in the swing phase of a step, the correction depends on the cause and this is multifactorial (kinematics, poor coordination, etc.). The patient may not flex the hip, knee or ankle sufficiently, either due to joint limitation or muscle weakness of the tibialis anterior in the case of dorsiflexion of the ankle, hamstrings for knee flexion or iliopsoas and anterior rectus of the quadriceps in the case of the hip. Another cause would be the lack of proprioception of the patient that prevents her/him from making the gesture or even carrying it out simultaneously (step and triple flexion of the lower limb at a time). In this case, the prescription must be offered by the physiotherapist based on the causes, in a progressive and individualized manner. Selective muscle strengthening exercises, manual therapy to gain range of motion in some joint or working the patient's balance independently to the walking session may be prescribed.

Another example is arm movement during gait. Error detection and description can be easily implemented using technology. On the contrary, the prescription for its correction is usually more complex because again the causes are multiple: lack of integration of the arms in the body scheme, 
lack of dissociation between the scapular and pelvic waists, lack of mobility of the glenohumeral joint, etc. Deepening further, the patient can brace but not fluidly, i.e., without rotation of the shoulder girdle and without transferring the energy from proximal (trunk) to distal (arms), which would be incorrect. Even the patient may not swing arms in an opposing direction with respect to the lower limb, which would lead to an erroneous walking. Again the prescription must be made by the physiotherapist based on the cause and of course on a rigorous initial and continuous assessment.

Other authors such as Sigrist [49] affirm that to provide the idea of a movement, the feedback should be in principle prescriptive. Eventually, when the subject has internalized the action, descriptive feedback may be applied to make the correction more effective. Similarly, Sulzenbruck [50] states that, before the skill is acquired, prescriptive feedback is more effective than descriptive feedback. Still, there are authors such as Ki et al. [21] who use descriptive feedback (a beep to indicate that the weight load has been exceeded in the paretic limb) while others such as Segal et al. [11] opt for prescriptive feedback in his RCT (a graphic representation of the subject by means of a skeleton, on a screen, informs him how the optimal knee movement should be made).

Overall, the selected articles obtained significantly positive results in relation to the use of technological feedback. Even so, it should be noted that some specific parameters were not particularly significant. That is the case of stride speed or time $[5,14,17,18,22,40]$, which can be influenced by complex robotized systems or exoskeletons, treadmills, supports etc., and the focus of the user's attention on other parameters of interest. These show an improvement in overall gait despite not actually increasing speed.

As for the populations covered, most of the technological feedback applications were applied in the neurological field. The results of this review show that $75 \%$ came from that area $[5,7,14-19,21,23,38-42]$. Hence, feedback is capable of changing motor strategies in patients with neurological lesions [18], with the application of this type of treatment being more appropriate during early stages of rehabilitation [24]. As for other clinical areas, this review has only included 2 articles $(10 \%)$ based on muscular-skeletal lesions [11,20]. They outlined the limitation of traditional physiotherapy in the recovery of lower-limb functions [51]. Only 3 articles (15\%) used a sample of healthy subjects $[10,22,26]$. Despite being an RCT, it is sometimes necessary to perform research with healthy subjects to ascertain the efficacy of a new technological system before using it with patients requiring treatment. Continuing with the study population, it should be noted that $95 \%$ of the reviewed articles included samples of adult subjects $[5,7,10,11,14-16,18-23,26,38-42]$. Only $5 \%$ of the subjects were under 18 [17]. For this reason, we believe more scientific findings need to be generated in other clinical areas and in young population samples.

The following gait parameters were assessed in the selected RCTs, in descending order of frequency: speed (cm/s) $[7,15,17-19,22,23,38,40,41]$, step length (m) $[15,17-19,22,23,38,41,42]$, and cadence (steps $/ \mathrm{min}$ ) $[5,15,18,23]$. These parameters were chosen because the unit of gait is the step and time-space parameters are essential for its assessment [2,52-55]. The measurement devices were in some cases also those providing the feedback $[7,10,14,19,26,39-42]$. The majority measured short-term effects $[5,7,14-16,18-21,23,41,42]$. The few which measured long-term effects did not obtain conclusive results $[11,39,40]$, which underlines the need for prospective studies.

As a final reflection, the authors of this study recognize that technological progress has led to the development of highly useful tools in the field of physiotherapy which complement conventional therapy. In no case are these technologies considered substitute media, in contrast to the opinion of Parker et al. [24]. Despite the multiple benefits which new technologies offer, a physiotherapist's face-to-face treatment of a patient cannot be equaled by technological means. The personalized and intuitive adaptation of the health-care professional is the key to successful treatment.

\section{Conclusions}

Treatment based on feedback using innovative technology in patients with abnormal gait is mostly effective in improving gait parameters and therefore of use in the functional recovery of a patient. 
Concurrent/immediate visual is the most frequently used type of feedback, followed by terminal/retarded acoustic. Also, prescriptive feedback and knowledge of result are the most frequent alternatives.

Most of the systems used are based on force and pressure sensors, normally accompanied by complementary software.

Walking speed is the most frequently evaluated parameter, with the majority of studies reporting significant improvements (in one study the changes were only significant after 3 months). The positive effect on the stride length is also found significant in most cases. In general, the number of studies with significant outcomes for the other parameters (such as balance or range of movement) is too low.

Acknowledgments: Part of this work was supported by the Telefonica Chair "Intelligence in Networks" of the Universidad de Sevilla, Spain.

Author Contributions: Gema Chamorro-Moriana and José Luis Sevillano conceptualised the idea. Antonio José Moreno and Gema Chamorro-Moriana carried out the study selection, data extraction and manuscript drafting. Gema Chamorro-Moriana, Antonio José Moreno and José Luis Sevillano have been involved in critically revising for important intellectual contents. All authors contributed to the final version and approved the final paper for publication.

Conflicts of Interest: The authors declare no conflict of interest.

\begin{tabular}{|c|c|}
\hline 10MWT & 10-m Walk Test \\
\hline $\mathrm{ABC}$ & Activities-Specific Balance Confidence \\
\hline ADL & Activities of Daily Living \\
\hline $\mathrm{AFO}$ & Ankle Foot Orthosis \\
\hline CG & Control Group \\
\hline $\mathrm{CI}$ & Confidence Interval \\
\hline COPD & Chronic Obstructive Pulmonary Disease \\
\hline $\mathrm{CP}$ & Cerebral Palsy \\
\hline CT & Computed Tomography \\
\hline ES & Effect Size \\
\hline FAC & Functional Ambulation Classification \\
\hline FC-RATE & Feedback Controlled Robotics Assisted Treadmill Exercise \\
\hline FIM $^{\mathrm{TM}}$ & Functional Independence Measure \\
\hline FTS $^{(1)}$ & Functional Training System \\
\hline FTSTS test & Five Times Sit To Stand \\
\hline GAGT & GAR-Assisted Gait Training Group \\
\hline GAR & Gait-Assistance Robot \\
\hline GCB & "Bathroom Scale" Training Group \\
\hline GCV & "Verbal Instruction" Training Group \\
\hline GFB & "Haptic Biofeedback" Training Group \\
\hline HRpeak & Peak Heart Rate \\
\hline IG & Intervention Group \\
\hline $\mathrm{IQR}$ & Barthel Index \\
\hline IT & Information Technology \\
\hline KOOS & Knee Injury and Osteoarthritis Outcome Score \\
\hline KP & Knowledge of Performance \\
\hline KR & Knowledge of Result \\
\hline LCnp & Length of the Cycle of Non-Paretic Limb \\
\hline LCp & Length of the Cycle of Paretic Limb \\
\hline LDCW & Long Distance Corridor Walk \\
\hline LLFDI & Late Life Function and Disability Index \\
\hline LOS & Limit Of Stability \\
\hline MAS & Modified Ashworth Scale \\
\hline MRI & Magnetic Resonance Imaging \\
\hline $\mathrm{N}$ & Total Sample \\
\hline NEA & Normalized Error Area \\
\hline $\mathrm{OA}$ & Osteoarthritis \\
\hline OCGT & Overground Conventional Gait Training Group \\
\hline Ppeak & Peak Work Rate \\
\hline PBWS & Partial Body Weight Supported \\
\hline PD & Parkinson's Disease \\
\hline RATE & Robotics Assisted Treadmill Exercise \\
\hline RCTs & Randomised Controlled Trials \\
\hline RERpeak & Peak Respiratory Exchange Ratio \\
\hline Rfpeak & Peak Respiratory Rate \\
\hline ROM & Range of Movement \\
\hline SD & Standar Deviation \\
\hline
\end{tabular}




$\begin{array}{ll}\text { STFnp } & \text { Stance Phase of the Non-Paretic Limb } \\ \text { STFp } & \text { Stance Phase of the Paretic Limb } \\ \text { STP } & \text { Stance Time Period } \\ \text { SWFnp } & \text { Swing Phase of the Non-Paretic Limb } \\ \text { SWFp } & \text { Swing Phase of the Paretic Limb } \\ \text { Terr } & \text { Normalized Error in the Stride Period } \\ \text { TUG test } & \text { Timed Up and Go } \\ \text { UPDRS } & \text { United Parkinson's Disease Rating Scale } \\ \text { VEpeak } & \text { Peak Ventilation Rate }\end{array}$

\section{References}

1. Chamorro-Moriana, G.; Ridao-Fernández, C.; Ojeda, J.; Benítez-Lugo, M.; Sevillano, J.L. Reliability and validity study of the Chamorro Assisted Gait Scale for people with sprained ankles, walking with forearm crutches. PLoS ONE 2016, 11, 1-12. [CrossRef] [PubMed]

2. Chamorro-Moriana, G.; Rebollo-Roldán, J.; Jiménez-Rejano, J.J.; Chillón-Martínez, R.; Suárez-Serrano, C. Design and validation of GCH System 1.0 which measures the weight-bearing exerted on forearm crutches during aided gait. Gait Posture 2013, 37, 564-569. [CrossRef] [PubMed]

3. Whittle, M.W. Gait Analysis: An Introduction, 3rd ed.; Butterworth-Heinemann: Oxford, MS, USA, 2003; pp. 140-142, ISBN 9780702039225.

4. Van Den Noort, J.C.; Steenbrink, F. Real time visual feedback for gait retraining: Toward application in knee osteoarthritis. Med. Biol. Eng. Comput. 2015, 53, 275-286. [CrossRef] [PubMed]

5. Druzbicki, M.; Guzik, A.; Przysada, G.; Kwolek, A.; Brzozowska-Magoń, A. Efficacy of gait training using a treadmill with and without visual biofeedback in patients after stroke: A randomized study. J. Rehabil. Med. 2015, 47, 419-425. [CrossRef] [PubMed]

6. Agresta, C.; Hall, J. Gait Retraining for Injured and Healthy Runners using Augmented Feedback: A Systematic Literature Review. J. Orthop. Sports Phys. Ther. 2015, 45, 576-584. [CrossRef] [PubMed]

7. Jung, K.; Kim, Y.; Cha, Y.; In, T.; Hur, Y.; Chung, Y. Effects of gait training with a cane and an augmented pressure sensor for enhancement of weight bearing over the affected lower limb in patients with stroke: A randomized controlled pilot study. Clin. Rehabil. 2015, 29, 135-142. [CrossRef] [PubMed]

8. Isakov, E. Gait rehabilitation: A new biofeedback device for monitoring and enhancing weight-bearing over the affected lower limb. Eura Medic. 2007, 43, 21-26.

9. Basta, D.; Rossi-Izquierdo, M.; Soto-Varela, A.; Greters, M.E.; Bittar, R.S.; Steinhagen-Thiessen, E.; Eckardt, R.; Harada, T.; Goto, F.; Ogawa, K.; et al. Efficacy of a vibrotactile neurofeedback training in stance and gait conditions for the treatment of balance deficits: A double-blind, placebo-controlled multicenter study. Otol. Neurotol. 2011, 32, 1492-1499. [CrossRef] [PubMed]

10. Zanotto, D.; Rosati, G.; Spagnol, S.; Stegall, P.; Agrawal, S.K. Effects of Complementary Auditory Feedback in Robot-Assisted Lower Extremity Motor Adaptation. IEEE Trans. Neural Syst. Rehabil. Eng. 2013, 21, 775-786. [CrossRef] [PubMed]

11. Segal, N.A.; Glass, N.A.; Teran-Yengle, P.; Singh, B.; Wallace, R.B.; Yack, H.J. Intensive Gait Training for Older Adults with Symptomatic Knee Osteoarthritis. Am. J. Phys. Med. Rehabil. 2015, 94, 848-858. [CrossRef] [PubMed]

12. Nanhoe-Mahabier, W.; Allum, J.H.; Pasman, E.P.; Overeem, S.; Bloem, B.R. The effects of vibrotactile biofeedback training on trunk sway in Parkinson's disease patients. Parkinsonism Relat. Disord. 2012, 18, 1017-1021. [CrossRef] [PubMed]

13. Fernández, R.; Rodríguez, B.; Barcia, B.; Souto, S.; Chouza, M.; Martínez, S. Generalidades sobre Feedback (o retroalimentación). Fisioterapia 1998, 20, 3-11.

14. Ochi, M.; Wada, F.; Saeki, S.; Hachisuka, K. Gait training in subacute non-ambulatory stroke patients using a full weight-bearing gait-assistance robot: A prospective, randomized, open, blinded-endpoint trial. J. Neurol. Sci. 2015, 353, 130-136. [CrossRef] [PubMed]

15. Quinzaños Fresnedo, J.; Sahagún Olmos, R.C.; León Hernández, S.R.; Pérez Zavala, R.; Quiñones Uriostegui, I.; Solano Salazar, C.J.; Cruz Lira, R.T.; Tinajero Santana, M.C. Efectos a corto plazo del entrenamiento de la marcha en una órtesis robótica (Lokomat ${ }^{\circledR}$ ) con retroalimentación auditiva en pacientes con lesión medular incompleta crónica. Rehabilitacion 2015, 49, 30-37. [CrossRef]

16. Stoller, O.; de Bruin, E.D.; Schindelholz, M.; Schuster-Amft, C.; de Bie, R.A.; Hunt, K.J. Efficacy of Feedback-Controlled Robotics-Assisted Treadmill Exercise to Improve Cardiovascular Fitness Early After Stroke. J. Neurol. Phys. Ther. 2015, 39, 156-165. [CrossRef] [PubMed] 
17. Baram, Y.; Lenger, R. Gait Improvement in Patients with Cerebral Palsy by Visual and Auditory Feedback. Neuromodulation: Technol. Neural Interface 2012, 15, 48-52. [CrossRef] [PubMed]

18. Brasileiro, A.; Gama, G.; Trigueiro, L.; Ribeiro, T.; Silva, E.; Galvão, É.; Lindquist, A. Influence of visual and auditory biofeedback on partial body weight support treadmill training of individuals with chronic hemiparesis: A randomized controlled clinical trial. Eur. J. Phys. Rehabil. Med. 2015, 51, 49-58. [PubMed]

19. Byl, N.; Zhang, W.; Coo, S.; Tomizuka, M. Clinical impact of gait training enhanced with visual kinematic biofeedback: Patients with Parkinson's disease and patients stable post stroke. Neuropsychologia 2015, 79, 332-343. [CrossRef] [PubMed]

20. Hunt, M.A.; Takacs, J.; Hart, K.; Massong, E.; Fechko, K.; Biegler, J. Comparison of mirror, raw video, and real-time visual biofeedback for training toe-out gait in individuals with knee osteoarthritis. Arch. Phys. Med. Rehabil. 2014, 95, 1912-1917. [CrossRef] [PubMed]

21. Ki, K.I.; Kim, M.S.; Moon, Y.; Choi, J.D. Effects of auditory feedback during gait training on hemiplegic patients' weight bearing and dynamic balance ability. J. Phys. Ther. Sci. 2015, 27, 1267-1269. [CrossRef] [PubMed]

22. Lipsitz, L.A.; Lough, M.; Niemi, J.; Travison, T.; Howlett, H.; Manor, B. A shoe insole delivering subsensory vibratory noise improves balance and gait in healthy elderly people. Arch. Phys. Med. Rehabil. 2015, 96, 432-439. [CrossRef] [PubMed]

23. El-Tamawy, M.; Darwish, M.; Khallaf, M. Effects of augmented proprioceptive cues on the parameters of gait of individuals with Parkinson's disease. Ann. Indian Acad. Neurol. 2012, 15, 267. [CrossRef] [PubMed]

24. Parker, J.; Mountain, G.; Hammerton, J. A review of the evidence underpinning the use of visual and auditory feedback for computer technology in post-stroke upper-limb rehabilitation. Disabil. Rehabil. Assist. Technol. 2011, 6, 465-472. [CrossRef] [PubMed]

25. Thikey, H.; Grealy, M.; van Wijck, F.; Barber, M.; Rowe, P. Augmented visual feedback of movement performance to enhance walking recovery after stroke: Study protocol for a pilot randomised controlled trial. Trials 2012, 13, 1. [CrossRef] [PubMed]

26. Fu, M.C.; DeLuke, L.; Buerba, R.; Fan, R.E.; Zheng, Y.J.; Leslie, M.P.; Baumgaertner, M.R.; Grauer, J.N. Haptic biofeedback for improving compliance with lower-extremity partial weight bearing. Orthopedics 2014, 37, e993-e998. [CrossRef] [PubMed]

27. Chamorro-Moriana, G.; Sevillano, J.L.; Ridao-Fernández, C. A compact forearm crutch based on force sensors for aided gait: Reliability and validity. Sensors 2016, 16, 925. [CrossRef] [PubMed]

28. Mortensen, D.H.; Bech, S.; Begault, D.R.; Adelstein, B.D. The relative importance of visual, auditory, and haptic information for the user's experience of mechanical switches. Perception 2009, 38, 1560-1571. [CrossRef] [PubMed]

29. Lefmann, S.; Russo, R.; Hillier, S. The effectiveness of robotic-assisted gait training for paediatric gait disorders: Systematic review. J. Neuroeng. Rehabil. 2017, 14, 1. [CrossRef] [PubMed]

30. Sharma, D.A.; Chevidikunnan, M.F.; Khan, F.R.; Gaowgzeh, R.A. Effectiveness of knowledge of result and knowledge of performance in the learning of a skilled motor activity by healthy young adults. J. Phys. Ther. Sci. 2016, 28, 1482-1486. [CrossRef] [PubMed]

31. Liberati, A.; Altman, D.G.; Tetzlaff, J.; Mulrow, C.; Gøtzsche, P.C.; Ioannidis, J.P.A. The PRISMA statement for reporting systematic reviews and meta-analyses of studies that evaluate health care interventions: Explanation and elaboration. J. Clin. Epidemiol. 2009, 62, e1-e34. [CrossRef] [PubMed]

32. Moseley, A.M.; Herbert, R.D.; Sherrington, C.; Maher, C.G. Evidence for physiotherapy practice: A survey of the Physiotherapy Evidence Database (PEDro). Aust. J. Physiother. 2002, 48, 43-49. [CrossRef]

33. Yamato, T.P.; Maher, C.; Koes, B.; Moseley, A. The PEDro scale had acceptably high convergent validity, construct validity, and interrater reliability in evaluating methodological quality of pharmaceutical trials. J. Clin. Epidemiol. 2017. [CrossRef] [PubMed]

34. Sherrington, C.; Herbert, R.; Maher, C.; Moseley, A. PEDro. A database of randomized trials and systematic reviews in physiotherapy. Man Ther. 2000, 5, 223-226. [CrossRef] [PubMed]

35. Maher, C.G.; Sherrington, C.; Herbert, R.D.; Moseley, A.M. Reliability of the PEDro scale for rating quality of randomized controlled trials. Phys. Ther. 2003, 83, 713-721. [PubMed]

36. De Morton, N.A. The PEDro scale is a valid measure of the methodological quality of clinical trials: A demographic study. Aust. J. Physiother. 2009, 55, 129-133. [CrossRef] 
37. Moher, D.; Liberati, A.; Tetzlaff, J.; Altman, D.G. Academia and Clinic Annals of Internal Medicine Preferred Reporting Items for Systematic Reviews and Meta-Analyses: The PRISMA Statement. Annu. Intern. Med. 2009, 151, 264-269. [CrossRef]

38. Ginis, P.; Nieuwboer, A.; Dorfman, M.; Ferrari, A.; Gazit, E.; Canning, C.G.; Rocchi, L.; Chiari, L.; Hausdorff, J.M.; Mirelman, A. Feasibility and effects of home-based smartphone-delivered automated feedback training for gait in people with Parkinson's disease: A pilot randomized controlled trial. Parkinsonism Relat. Disord. 2016, 22, 28-34. [CrossRef] [PubMed]

39. Khallaf, M.E.; Gabr, A.M.; Fayed, E.E. Effect of Task Specific Exercises, Gait Training, and Visual Biofeedback on Equinovarus Gait among Individuals with Stroke: Randomized Controlled Study. Neurol. Res. Int. 2014. [CrossRef] [PubMed]

40. Shen, X.; Mak, M.K.Y. Balance and Gait Training with Augmented Feedback Improves Balance Confidence in People with Parkinson's Disease. Neurorehabil. Neural Repair 2014, 28, 524-535. [CrossRef] [PubMed]

41. Sungkarat, S.; Fisher, B.E.; Kovindha, A. Efficacy of an insole shoe wedge and augmented pressure sensor for gait training in individuals with stroke: A randomized controlled trial. Clin. Rehabil. 2011, 25, 360-369. [CrossRef] [PubMed]

42. Won, S.H.; Kim, J.C.; Oh, D.W. Effects of a novel walking training program with postural correction and visual feedback on walking function in patients with post-stroke hemiparesis. J. Phys. Ther. Sci. 2015, 27, 2581-2583. [CrossRef] [PubMed]

43. Tzetzis, G.; Votsis, E.; Kourtessis, T. The effect of different corrective feedback methods on the outcome and self confidence of young athletes. J. Sports Sci. Med. 2008, 7, 371-378. [PubMed]

44. Sardini, E.; Serpelloni, M.; Lancini, M. Wireless Instrumented Crutches for Force and Movement Measurements for Gait Monitoring. IEEE Trans. Instrum. Meas. 2015, 64, 3369-3379. [CrossRef]

45. Tuttle, N.; Jacuinde, G. Design and Construction of a Novel Low-Cost Device to Provide Feedback on Manually Applied Forces. J. Orthop. Sport Phys. Ther. 2011. [CrossRef] [PubMed]

46. Winstein, C.J.; Pohl, P.S.; Cardinale, C.; Green, A.; Scholtz, L.; Waters, C. Learning a partial-weight-bearing skill: Effectiveness of two forms of feedback. Phys. Ther. 1996, 76, 985-993. [CrossRef] [PubMed]

47. Warren, C.G.; Lehmann, J. Training procedures and biofeedback methods to achieve controled partial weight bearing: An assessment. Arch. Phys. Med. Rehabil. 1975, 56, 449-455. [PubMed]

48. Salmoni, A.W.; Schmidt, R.A. Knowledge of results and motor learning: A review and critical reappraisal. Psychol. Bull. 1984, 95, 355-386. [CrossRef] [PubMed]

49. Sigrist, R.; Rauter, G.; Riener, R.; Wolf, P. Augmented visual, auditory, haptic, and multimodal feedback in motor learning: A review. Psychon. Bull. Rev. 2013, 20, 21-53. [CrossRef] [PubMed]

50. Sülzenbrück, S.; Heuer, H. Type of visual feedback during practice influences the precision of the acquired internal model of a complex visuo-motor transformation. Ergonomics 2011, 54, 34-46. [CrossRef] [PubMed]

51. Li, J.; Wu, T.; Xu, Z.; Gu, X. A pilot study of post-total knee replacement gait rehabilitation using lower limbs robot-assisted training system. Eur. J. Orthop. Surg. Traumatol. 2014, 24, 203-208. [CrossRef] [PubMed]

52. Kloos, A.D.; Kegelmeyer, D.A.; White, S. The impact of different types of assistive devices on gait measures and safety in Huntington's disease. PLoS ONE 2012. [CrossRef] [PubMed]

53. Thomas, K.S.; Russell, D.M.; Van Lunen, B.L.; Colberg, S.R.; Morrison, S. The impact of speed and time on gait dynamics. Hum. Mov. Sci. 2017, 54, 320-330. [CrossRef] [PubMed]

54. Figueiredo, P.R.P.; Silva, P.L.P.; Avelar, B.S.; Chagas, P.S.C.; Oliveira, L.C.P.; Mancini, M.C. Assessment of gait in toddlers with normal motor development and in hemiplegic children with mild motor impairment: A validity study. Brazilian J. Phys. Ther. 2013, 17, 359-366. [CrossRef]

55. Yang, C.C.; Hsu, Y.L.; Shih, K.S.; Lu, J.M. Real-time gait cycle parameter recognition using a wearable accelerometry system. Sensors 2011, 11, 7314-7326. [CrossRef] [PubMed]

Sample Availability: All primary data were extracted from the referenced sources. Full search strategy available from the authors on request.

(C) 2018 by the authors. Licensee MDPI, Basel, Switzerland. This article is an open access article distributed under the terms and conditions of the Creative Commons Attribution (CC BY) license (http:/ / creativecommons.org/licenses/by/4.0/). 
Article

\title{
Flexible Piezoelectric Sensor-Based Gait Recognition
}

\author{
Youngsu Cha ${ }^{1, *}$, Hojoon Kim ${ }^{1,2}$ and Doik Kim ${ }^{1}$ \\ 1 Center for Robotics Research, Korea Institute of Science and Technology, Seoul 02792, Korea; \\ hojun5266@korea.ac.kr (H.K.); doikkim@kist.re.kr (D.K.) \\ 2 School of Electrical Engineering, Korea University, Seoul 02841, Korea \\ * Correspondence: givemong@kist.re.kr; Tel.: +82-2-958-6949
}

Received: 2 January 2018; Accepted: 3 February 2018; Published: 5 February 2018

\begin{abstract}
Most motion recognition research has required tight-fitting suits for precise sensing. However, tight-suit systems have difficulty adapting to real applications, because people normally wear loose clothes. In this paper, we propose a gait recognition system with flexible piezoelectric sensors in loose clothing. The gait recognition system does not directly sense lower-body angles. It does, however, detect the transition between standing and walking. Specifically, we use the signals from the flexible sensors attached to the knee and hip parts on loose pants. We detect the periodic motion component using the discrete time Fourier series from the signal during walking. We adapt the gait detection method to a real-time patient motion and posture monitoring system. In the monitoring system, the gait recognition operates well. Finally, we test the gait recognition system with 10 subjects, for which the proposed system successfully detects walking with a success rate over $93 \%$.
\end{abstract}

Keywords: flexible sensor; gait recognition; piezoelectric material; wearable

\section{Introduction}

Recent advancements in wearable sensors have promoted major scientific and technological developments in the field of human activity recognition [1,2]. Wearable sensors, for this purpose, have significant benefits over external sensors in terms of privacy, pervasiveness, and complexity [1]. Most people would be averse to being continuously monitored by external cameras [1,3]. It is also difficult to obtain full body imagery all the time. Moreover, image processing to recognize human activities is computationally complex.

Gait recognition and analysis are one of the most important research areas, because walking is a common human physical activity [2,4]. The gait recognition system can be utilized for therapeutic and diagnostic applications $[5,6]$. In addition, the sensing part can be combined to gait assistant system $[7,8]$. Various wearable sensors, such as inertial measurement units (IMU), pressure sensors, force sensitive resistors, accelerometers, and gyroscopes, are utilized for gait recognition and analysis [2,4,9]. These sensors are positioned on hips, thighs, knees, shanks, and feet in the lower body; they measure angles and contacts during walking [4]. For example, a piezoelectric gyroscope on the thigh of one leg has been used for detecting stride length and walking velocity [10]. Piezoresistive accelerometers have been studied in ambulatory monitoring [11]. A real-time gait phase detection system with a gyroscope and force sensitive resistors installed inside a shoe insole has also been presented [12]. Bending type piezoelectric accelerometers have been utilized for footstep detection [13]. A method of measuring joint angle for gait using a combination of accelerometers and gyroscopes has been presented [14]. A step counting method, using tri-axial accelerometers on the ankles, thigh, and waist has been proposed [15]. In most research, wearable sensors have been tightly attached to the body for accurate sensing. However, tight attachment can cause inconvenience for users. 
In this paper, we introduce a flexible piezoelectric sensor-based gait recognition system available in loose clothing. The flexible sensor is made of a piezoelectric material: polyvinylidene fluoride (PVDF) [16-18]. PVDF is one of the most flexible piezoelectric materials, outputting voltage signals when it experiences strain [19-21]. Four flexible piezoelectric sensors are attached to the knee and hip parts of the loose clothing. We measure the sensor outputs during human walking by analyzing the raw signals, signal processed values, and fast Fourier transform (FFT) magnitudes. Furthermore, we demonstrate a gait detection system based on the outputs of the flexible piezoelectric sensors. Specifically, we add the gait detection method to the real-time patient motion and posture monitoring system reported in [22]. The integration of the gait detection to the monitoring system presents that the proposed method can be combined as other posture detections or system. In addition, the monitoring system can have an important update about gait detection of patients through the integration. The monitoring system recognizes five postures including walking and eight transitions between postures. We show the operation of the monitoring system in a demo scenario. Additionally, we conduct user tests of gait detection in the monitoring system.

This paper is organized as follows. In Section 2, we introduce the experimental setup of the flexible piezoelectric sensor, the module for collecting the sensor's signal, and walking test on a treadmill. In Section 3, we present an investigation of the sensor outputs during the walking motion. The decision method for gait recognition using the sensor outputs during walking is described in Section 4. In Section 5, we present the demonstration of gait recognition with a real-time patient motion and posture monitoring system. The conclusions are summarized in the final section.

\section{Experimental Setup}

We use similar patient cloth with flexible sensors as [22] for our experiments. In this work, the four sensors are embedded in both knee and hip parts on the patient's pants (see Figure 1). The sensors are composed of PVDF, produced by Measurement Specialties, and Mylar [17,22]. A PVDF sheet is glued on a Mylar sheet with 3M DP460 epoxy. The sensor size is $(L) 75 \times(W) 25 \times(T) 0.3 \mathrm{~mm}^{3}$. We sense electrical signals from the flexible sensors using conductive adhesive copper tape electrodes, produced by $3 \mathrm{M}$, attached on both surface of PVDF. The copper electrodes are connected to a sensing module through wires. The voltages from the sensors, carried by the wires, are digitized using the analog-digital converters in the sensing module. The signals are then transmitted by Bluetooth wireless communication. The sensing frequency is approximately $100 \mathrm{~Hz}$.

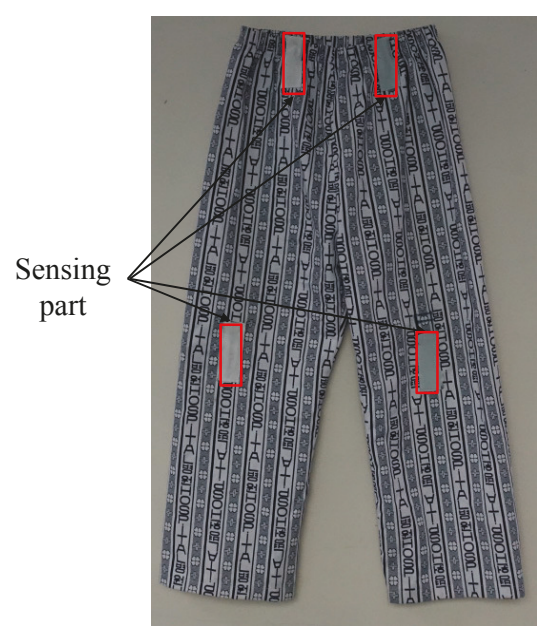

Figure 1. Patient clothing for four flexible sensors. 
A KTS 7500TS treadmill is utilized to test walking at standard speed. In the tests, a male student (age: 25 years, height: $170 \mathrm{~cm}$, weight: $70 \mathrm{~kg}$ ) wears the patient clothes with flexible sensors and walks on the treadmill. The test walking speed varies from 0.5 to $6 \mathrm{~km} / \mathrm{h}$, which, if exceeded, requires running. When the student walks on the treadmill, we record the sensor outputs via the sensing system.

\section{Sensor Output for Walking}

We analyze the sensor outputs during walking. Figure 2 shows the voltage outputs from the sensors during walking at $4 \mathrm{~km} / \mathrm{h}$ on the treadmill. The signal amplitudes at both knee sensors are bigger than those from the hip sensors because of several peaks. However, all signal wave forms of the hip sensors are more periodic than the knee sensors. The differences can be attributed to the loose patient cloth. During walking, the knee has a larger bending angle motion than the hip [23]. With tight clothes, the electric signal from the piezoelectric sensor attached to the knee will be larger than the signal at the hip [24]. With the loose clothing of this test, the knee sensors do not perfectly capture the periodic motions of the knees during walking. Conversely, the hip signals are sensed well during walking, because the waist band of the pants fits more tightly. Thus, the hip sensors are attached more closely than the knee sensors.

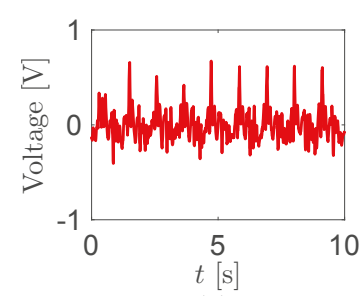

(a)

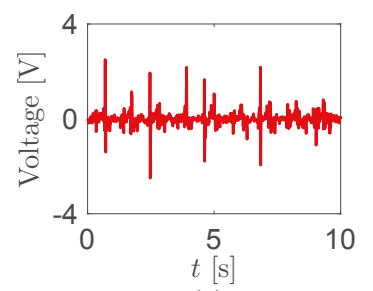

(c)

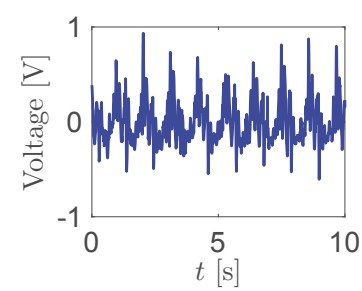

(b)

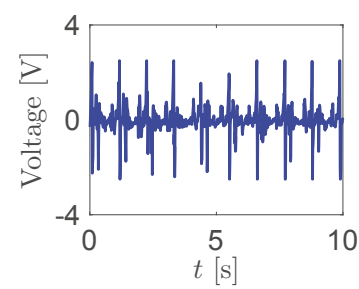

(d)

Figure 2. Sensor voltage outputs during walking at the (a) left hip; (b) right hip; (c) left knee; and (d) right knee. The test walking velocity is $4 \mathrm{~km} / \mathrm{h}$.

The sensor voltages correlate to the angular velocities of their bending [22]. We process data to acquire the bending motions from the sensor voltages, following the methods of [22]. Briefly, the sensor voltage outputs are processed through removing their voltage offsets and integrating as the time. We sense clearer periodic signals during walking (see Figure 3). Similarly, the processed values at the hip are more periodic than at the knee. To clarify the periodicity between the processed values at the hip and knee, we conduct FFT in MATLAB using the processed values. Figure 4 displays the FFT results from the signal processed values of Figure 3. The magnitudes of the main walking frequency in all FFT results are the largest. However, the results at the knee show that the higher harmonic frequencies still have big amplitudes. 


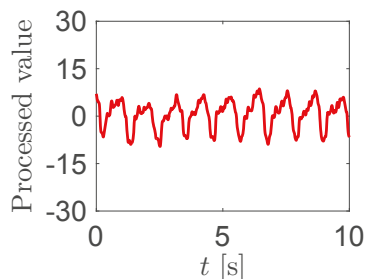

(a)

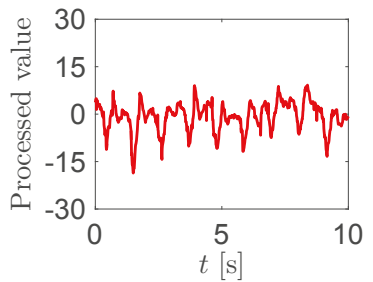

(c)

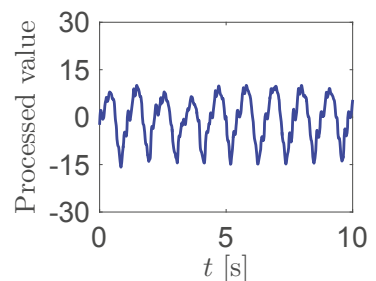

(b)

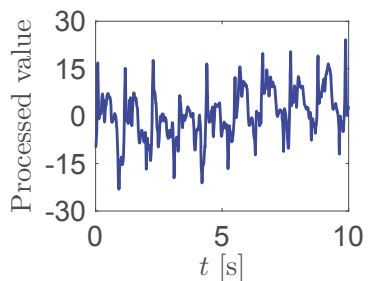

(d)

Figure 3. Signal processed values from the sensor outputs during walking at the (a) left hip; (b) right hip; (c) left knee; and (d) right knee. The test walking velocity is $4 \mathrm{~km} / \mathrm{h}$.

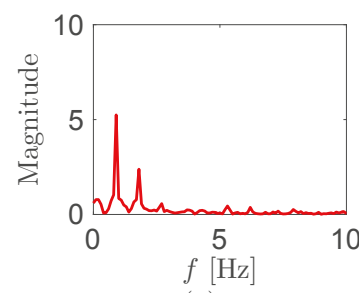

(a)

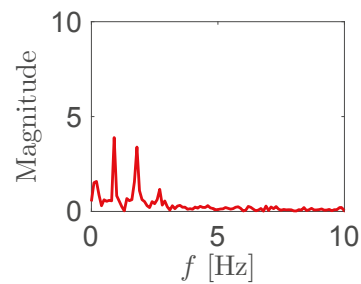

(c)

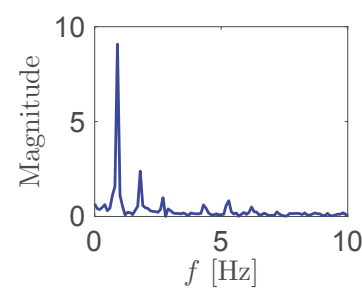

(b)

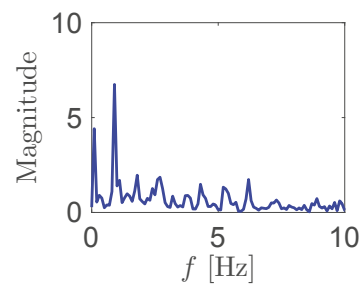

(d)

Figure 4. FFT magnitude spectra of the signal processed values from the sensor outputs during walking at the (a) left hip; (b) right hip; (c) left knee; and (d) right knee. The test walking velocity is $4 \mathrm{~km} / \mathrm{h}$.

\section{Gait Recognition}

We decide to use only left and right hip sensors to recognize walking motion, based on the findings in Section 3. We perform additional tests at various walking speeds $(0.5,1,2,3,4,5$ and $6 \mathrm{~km} / \mathrm{h})$ on the treadmill. Figure 5 displays the FFT magnitude spectra at $0.5,1,3$ and $6 \mathrm{~km} / \mathrm{h}$. We can detect the main walking frequency by finding the biggest magnitude in each plot. We observe that the main walking frequencies increase with the walking speeds. The walking frequency as the speed of the treadmill is presented in Table 1. Herein, we focus on normal walking speed [25], which is approximately $1.1 \mathrm{~m} / \mathrm{s}$ $(\sim 4 \mathrm{~km} / \mathrm{h})$. At that walking speed, the main frequency is between 0.9 and $1 \mathrm{~Hz}$. 
For detecting the walking frequency in real-time system, we use discrete time Fourier series instead of FFT to reduce computing time. In the same context, we obtain the series coefficients of the frequency, $1 \mathrm{~Hz}$, and the second harmonic. Specifically, the coefficients are obtained every half second. When the average of the two coefficients is over a decision value, we make a decision about the gait recognition. Herein, we select the decision value of 0.8 from preliminary tests. When we use the gait recognition method with the decision value, the detection rate is over $90 \%$ at $3-6 \mathrm{~km} / \mathrm{h}$.

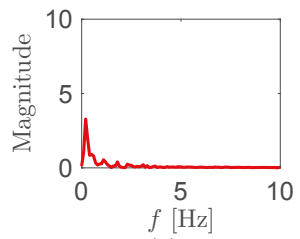

(a)

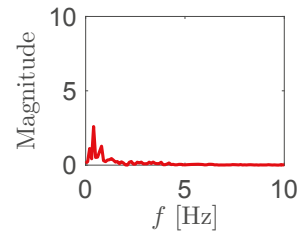

(c)

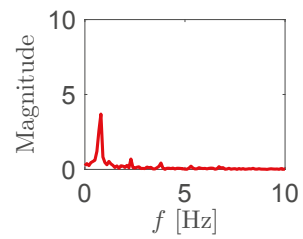

(e)

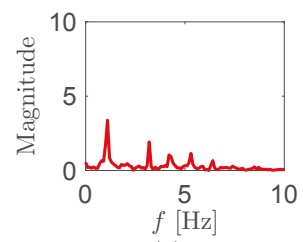

(g)

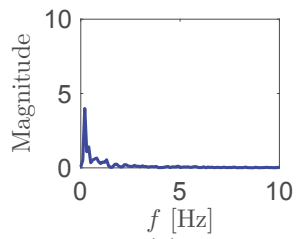

(b)

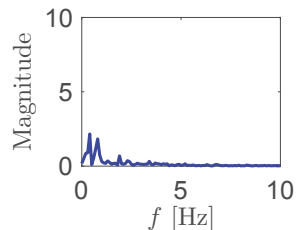

(d)

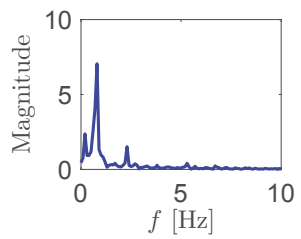

(f)

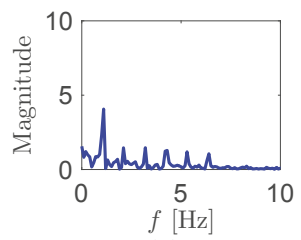

(h)

Figure 5. FFT magnitude spectra during walking of (a) left and (b) right hip at $0.5 \mathrm{~km} / \mathrm{h}$, (c) left and (d) right hip at $1 \mathrm{~km} / \mathrm{h}$, (e) left and (f) right hip at $3 \mathrm{~km} / \mathrm{h}$, and $(\mathrm{g})$ left and (h) right hip at $6 \mathrm{~km} / \mathrm{h}$.

Table 1. Walking speeds, frequencies, and detection rate on the treadmill.

\begin{tabular}{ccc}
\hline Speed $[\mathrm{km} / \mathrm{h}]$ & Frequency $[\mathrm{Hz}]$ & Detection Rate $[\%]$ \\
\hline 0.5 & 0.25 & 19 \\
1 & 0.41 & 36 \\
2 & 0.61 & 69 \\
3 & 0.79 & 98 \\
4 & 0.92 & 100 \\
5 & 1.04 & 100 \\
6 & 1.14 & 100 \\
\hline
\end{tabular}




\section{System Integration}

We adapt the gait detection to the patient monitoring system of [22]. The patient monitoring system was developed for motion and posture recognitions of patients; the gait detection method is also programmed for the system. Figure 6 displays the test condition for the patient motion and posture monitoring system. Specifically, the flexible sensor data is collected and processed in an external computer for monitoring; the monitoring program shows the decision about the motion and posture of the patient. In the monitoring program, we can detect a total of eight transitions and five postures. The five postures are (i) walking, (ii) standing, (iii) sitting, (iv) sitting knee extension, and (v) supine. We also predict the motions during the transitions between the postures (see Figure 7). To avoid incorrect operations, the walking is only transited from and to the standing pose. We note that the total four sensors' data are monitored in the system, the two hip sensors' data are utilized for the gait detection, and the two left sensors' data are used for capturing the transitions between standing, sitting, sitting knee extension, and supine. We comment that the system follows [22] about the detailed procedures for the decision between the postures of standing, sitting, sitting knee extension, and supine.

Figure 8 displays the captured computer screen image of the patient motion and posture monitoring system. At the left of the screen, it displays the processed values from the sensors of the left knee and hip and the right knee and hip. At the right of the screen, the detected patient posture is shown with a green outline. A demonstration of the patient motion and posture monitoring system is shown in Figure 9. Therein, the patient performs the transitions: (1) standing $\rightarrow$ (2) sitting on the bed $\rightarrow$ (3) sitting knee extension on the bed $\rightarrow$ (4) supine on the bed $\rightarrow$ (5) sitting knee extension on the bed $\rightarrow$ (6) sitting on the bed $\rightarrow$ (7) standing up $\rightarrow$ (8) walking to the wheelchair $\rightarrow$ (9) stopping in front of the wheelchair $\rightarrow$ (10) sitting on the wheelchair $\rightarrow$ (11) moving on the wheelchair $\rightarrow$ (12) standing up. All patient motion and posture monitoring of Figure 9 are displayed in Video S1 as Supplementary Material.

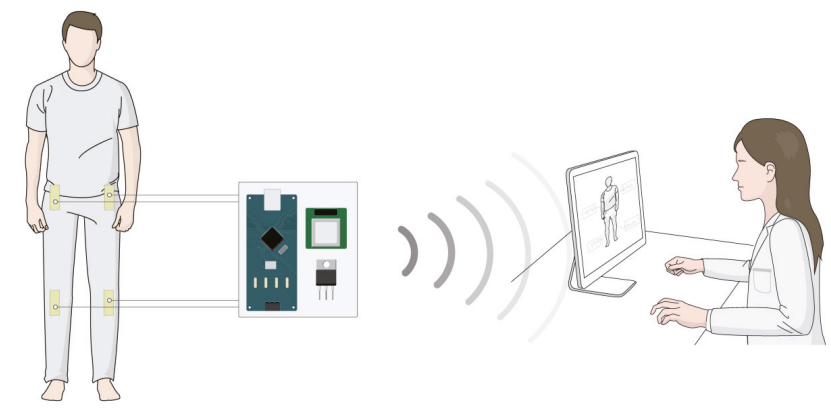

Figure 6. Experimental setup for testing the patient motion and posture monitoring system. 


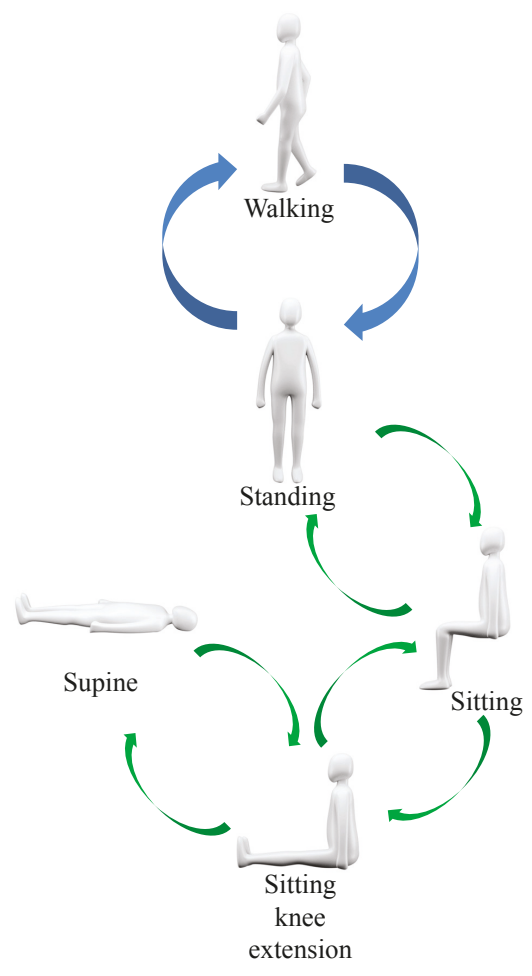

Figure 7. Flow chart for motion and posture decisions.

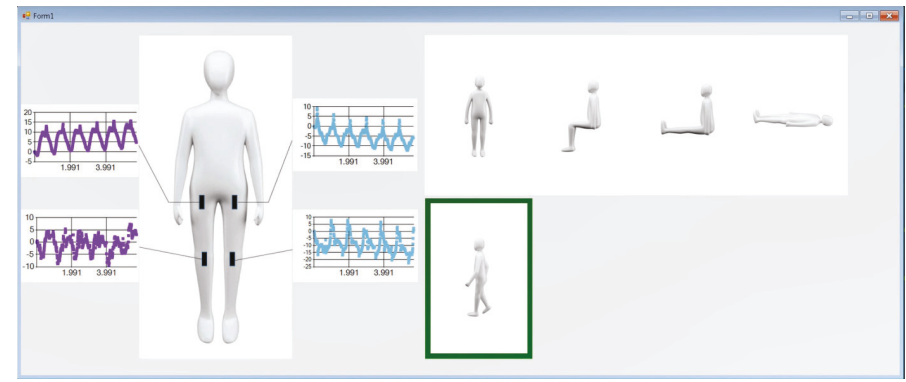

Figure 8. Captured computer screen showing patient walking detection and signal processed values from the four sensor outputs. 


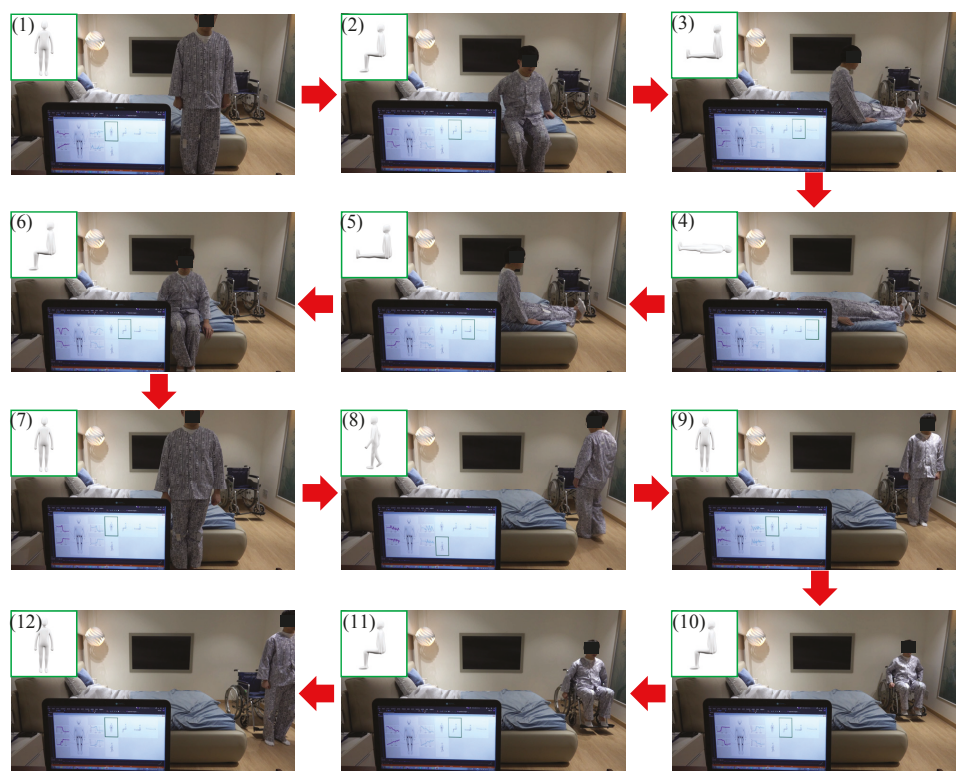

Figure 9. Test of the patient motion and posture monitoring system. (1-7) The patient lies down on the bed, and then stands up. (7-9) He moves to a wheelchair. (9-12) He sits on the wheelchair, moves via the wheelchair, and stands up from the wheelchair.

Additionally, we conduct gait detection tests from the other 10 users wearing the patient clothing. Specifically, the users walk and stop 50 times for a total of 1000 tests, 100 tests per user. We give the users simple orders about walking and stopping without specifying speed. Table 2 shows the success rates from the user test of the patient motion and posture monitoring system. The success rates are over $93 \%$, and the total average is $97.5 \%$. Several failed cases missed the changes or falsely detected sitting. In addition, most errors happen at taller users than the subject with the treadmill test. These errors can be attributed to the slow walking speeds of users and the low sensor outputs of the loose patient clothing. The success rate can be improved by selecting the gait detection frequency through personal statistics. For example, when we use the monitoring system for weak patients, they can have low walking speed. In this case, we need to adjust the gait detection frequency as lower value.

Table 2. Success rate of the gait recognition in the patient motion and posture monitoring system.

\begin{tabular}{cccccc}
\hline Subject Number & Gender & Age [Years] & Weight [kg] & Height [cm] & Success Rate [\%] \\
\hline$\# 1$ & M & 25 & 60 & 174 & 96 \\
$\# 2$ & F & 29 & 51 & 161 & 96 \\
$\# 3$ & M & 26 & 70 & 175 & 100 \\
$\# 4$ & M & 25 & 63 & 173 & 95 \\
$\# 5$ & M & 33 & 77 & 166 & 100 \\
$\# 6$ & M & 26 & 64 & 170 & 100 \\
$\# 7$ & M & 25 & 70 & 177 & 93 \\
$\# 8$ & M & 33 & 72 & 173 & 100 \\
$\# 9$ & M & 28 & 68 & 178 & 95 \\
$\# 10$ & F & 28 & 48 & 159 & 100 \\
\hline
\end{tabular}




\section{Conclusions}

In this paper, we introduced a gait recognition system with flexible piezoelectric sensors in loose clothing. The flexible sensors were attached to the knee and hip parts on the pants. We analyzed the sensor outputs of a user during walking. Specifically, we investigated raw signals from the sensors, signal processed signals from integration, and FFT magnitude spectra. From the analysis, we found that the signals of the hip sensors are more suitable for gait detection. We demonstrated the gait detection method in a real-time patient motion and posture monitoring system. The monitoring system was utilized to detect five postures including walking and eight transitions between the postures. We showed the successful operation of the monitoring system in a demo video, which consisted of motions and postures around a bed and wheelchair. Additionally, our gait detection system was tested on 10 subjects, and the success rates were over $93 \%$. The successful performance of our system shows the feasibility of gait detection from loose clothes using flexible sensors. We anticipate that the same methodology can be utilized to explore gait recognition using alternative flexible sensors. Moreover, it can provide an insight into motion recognition using smart suits or textiles.

Supplementary Materials: The following are available online at www.mdpi.com/1424-8220/18/2/468/s1.

Acknowledgments: This work was supported by the Korea Institute of Science and Technology (KIST) flagship program "Connected Active Space for X" (Project No. 2E28250).

Author Contributions: Youngsu Cha led the experiment, performed data analysis, and drafted the manuscript. Hojoon Kim conducted the experiment. Doik Kim contributed to the setup of the experiment.

Conflicts of Interest: The authors declare no conflict of interest.

\section{References}

1. Lara, O.D.; Labrador, M.A. A survey on human activity recognition using wearable sensors. IEEE Commun. Surv. Tutor. 2013, 15, 1192-1209.

2. Sprager, S.; Juric, M.B. Inertial sensor-based gait recognition: A review. Sensors 2015, 15, 22089-22127.

3. Sathyanarayana, S.; Satzoda, R.K.; Sathyanarayana, S.; Thambipillai, S. Vision-based patient monitoring: A comprehensive review of algorithms and technologies. J. Ambient Intell. Humaniz. Comput. 2015, 1-27, doi:10.1007/s12652-015-0328-1.

4. Rueterbories, J.; Spaich, E.G.; Larsen, B.; Andersen, O.K. Methods for gait event detection and analysis in ambulatory systems. Med. Eng. Phys. 2010, 32, 545-552.

5. Moore, S.T.; MacDougall, H.G.; Gracies, J.; Cohen, H.S.; Ondo, W.G. Long-term monitoring of gait in Parkinson's disease. Gait Posture 2007, 26, 200-207.

6. Bae, J.; Kong, K.; Byl, N.; Tomizuka, M. A mobile gait monitoring system for abnormal gait diagnosis and rehabilitation: A pilot study for Parkinson disease patients. J. Biomech. Eng. 2011, 133, 041005.

7. Kloos, A.D.; Kegelmeyer, D.A.; White, S.E.; Kostyk, S.K. The impact of different types of assistive devices on gait measures and safety in Huntington's disease. PloS ONE 2012, 7, e30903.

8. Quinlivan, B.T.; Lee, S.; Malcolm, P.; Rossi, D.M.; Grimmer, M.; Siviy, C.; Karavas, N.; Wagner, D.; Asbeck, A.; Galiana, I.; et al. Assistance magnitude versus metabolic cost reductions for a tethered multiarticular soft exosuit. Sci. Robot. 2017, 2, eaah4416.

9. Kavanagh, J.J.; Menz, H.B. Accelerometry: A technique for quantifying movement patterns during walking. Gait Posture 2008, 28, 1-15.

10. Miyazaki, S. Long-term unrestrained measurement of stride length and walking velocity utilizing a piezoelectric gyroscope. IEEE Trans. Biomed. Eng. 1997, 44, 753-759.

11. Foerster, F; Smeja, M.; Fahrenberg, J. Detection of posture and motion by accelerometry: A validation study in ambulatory monitoring. Comput. Hum. Behav. 1999, 15, 571-583.

12. Pappas, I.P.I.; Keller, T.; Mangold, S.; Popovic, M.R.; Dietz, V.; Morari, M. A reliable gyroscope-based gait-phase detection sensor embedded in a shoe insole. IEEE Sens. J. 2004, 4, 268-274.

13. Mazarakis, G.P.; Avaritsiotis, J.N. A prototype sensor node for footstep detection. In Proceedings of the Second European Workshop on Wireless Sensor Networks, Istanbul, Turkey, 2 February 2005; pp. 415-418. 
14. Dejnabadi, H.; Jolles, B.M.; Aminian, K. A new approach to accurate measurement of uniaxial joint angles based on a combination of accelerometers and gyroscopes. IEEE Trans. Biomed. Eng. 2005, 52, 1478-1484.

15. Fortune, E.; Lugade, V.; Morrow, M.; Kaufman, K. Validity of using tri-axial accelerometers to measure human movement-Part II: Step counts at a wide range of gait velocities. Med. Eng. Phys. 2014, 36, 659-669.

16. Broadhurst, M.G.; Davis, G.T.; McKinney, J.E.; Collins, R.E. Piezoelectricity and pyroelectricity in polyvinylidene fluoride-A model. J. Appl. Phys. 1978, 49, 4992-4997.

17. Akaydin, H.D.; Elvin, N.; Andreopoulos, Y. Energy Harvesting from Highly Unsteady Fluid Flows using Piezoelectric Materials. J. Intell. Mater. Syst. Struct. 2010, 21, 1263-1278.

18. Cha, Y.; Hong, J.; Lee, J.; Park, J.M.; Kim, K. Flexible Piezoelectric Energy Harvesting from Mouse Click Motions. Sensors 2016, 16, 1045.

19. Shen, D. Piezoelectric Energy Harvesting Devices for Low Frequency Vibration Applications; ProQuest: Ann Arbor, MI, USA, 2009.

20. Farinholt, K.M.; Pedrazas, N.A.; Schluneker, D.M.; Burt, D.W.; Farrar, C.R. An Energy Harvesting Comparison of Piezoelectric and Ionically Conductive Polymers. J. Intell. Mater. Syst. Struct. 2009, 20, 633-642.

21. Tiwari, R.; Kim, K.J. IPMC as a mechanoelectric energy harvester: tailored properties. Smart Mater. Struct. 2013, 22, 015017.

22. Cha, Y.; Nam, K.; Kim, D. Patient Posture Monitoring System Based on Flexible Sensors. Sensors 2017, 17,584 .

23. Riemer, R.; Shapiro, A. Biomechanical energy harvesting from human motion: theory, state of the art, design guidelines, and future directions. J. Neuroeng. Rehabilit. 2011, 8, 22.

24. Proto, A.; Penhaker, M.; Bibbo, D.; Vala, D.; Conforto, S.; Schmid, M. Measurements of Generated Energy /Electrical Quantities from Locomotion Activities Using Piezoelectric Wearable Sensors for Body Motion Energy Harvesting. Sensors 2016, 16, 524.

25. Liu, M.Q.; Anderson, F.C.; Schwartz, M.H.; Delp, S.L. Muscle contributions to support and progression over a range of walking speeds. J. Biomech. 2008, 41, 3243-3252.

(C) 2018 by the authors. Licensee MDPI, Basel, Switzerland. This article is an open access article distributed under the terms and conditions of the Creative Commons Attribution (CC BY) license (http:/ / creativecommons.org/licenses/by/4.0/). 


\title{
Type and Location of Wearable Sensors for Monitoring Falls during Static and Dynamic Tasks in Healthy Elderly: A Review
}

\author{
Rosaria Rucco ${ }^{1,2, *}$, Antonietta Sorriso ${ }^{3}$, Marianna Liparoti ${ }^{1,2}$, Giampaolo Ferraioli ${ }^{4}$, \\ Pierpaolo Sorrentino ${ }^{2,3}$, Michele Ambrosanio ${ }^{3}$ and Fabio Baselice ${ }^{3}$ \\ 1 Department of Motor Sciences and Wellness, University of Naples "Parthenope", 80133 Naples, Italy; \\ marianna.liparoti@uniparthenope.it \\ 2 IDC Hermitage Capodimonte, 80133 Naples, Italy; pierpaolo.sorrentino@uniparthenope.it \\ 3 Department of Engineering, University of Naples "Parthenope", 80133 Naples, Italy; \\ antonietta.sorriso@uniparthenope.it (A.S.); michele.ambrosanio@uniparthenope.it (M.A.); \\ fabio.baselice@uniparthenope.it (F.B.) \\ 4 Department of Science and Technologies, University of Naples "Parthenope", 80133 Naples, Italy; \\ giampaolo.ferraioli@uniparthenope.it \\ * Correspondence: rosaria.rucco@uniparthenope.it; Tel.: +39-081-748-3511
}

Received: 29 January 2018; Accepted: 15 May 2018; Published: 18 May 2018

\begin{abstract}
In recent years, the meaning of successful living has moved from extending lifetime to improving the quality of aging, mainly in terms of high cognitive and physical functioning together with avoiding diseases. In healthy elderly, falls represent an alarming accident both in terms of number of events and the consequent decrease in the quality of life. Stability control is a key approach for studying the genesis of falls, for detecting the event and trying to develop methodologies to prevent it. Wearable sensors have proved to be very useful in monitoring and analyzing the stability of subjects. Within this manuscript, a review of the approaches proposed in the literature for fall risk assessment, fall prevention and fall detection in healthy elderly is provided. The review has been carried out by using the most adopted publication databases and by defining a search strategy based on keywords and boolean algebra constructs. The analysis aims at evaluating the state of the art of such kind of monitoring, both in terms of most adopted sensor technologies and of their location on the human body. The review has been extended to both dynamic and static analyses. In order to provide a useful tool for researchers involved in this field, the manuscript also focuses on the tests conducted in the analyzed studies, mainly in terms of characteristics of the population involved and of the tasks used. Finally, the main trends related to sensor typology, sensor location and tasks have been identified.
\end{abstract}

Keywords: falls in healthy elderly; fall risk assessment; fall prevention; fall detection; wearable sensors

\section{Introduction}

Falls and related accidents are a common and serious problem not only in a pathologic condition like Parkinson's Disease [1], stroke [2] or multiple sclerosis [3], in which they are due to the motor and cognitive characteristics of the specific disease, but also for healthy people aged 65 and over [4]. A fall is defined as coming to rest on the ground or floor or other lower level, suddenly and involuntarily $[5,6]$.

Many epidemiological studies have reported a fall frequency of $28-35 \%$ in adults aged 65 and over [7-9], highlighting the need of developing effective and inexpensive ways to predict and prevent risk factors. $[5,6,10]$. 
Although some falls are probably unavoidable, most of them are due to the combination of intrinsic and extrinsic risk factors [11]. Intrinsic risk factors are related to the subject's characteristics, which include immutable biological features, sedentary lifestyle, concomitant presence of pathologies and use of medicines, age-related changes such as cognitive impairment, gait pattern alterations, and inability to maintain postural stability [12]. Musculoskeletal weakness [13] and decline of cognitive functions are known to be correlated with falls risk [14]. More specifically, a close relationship between motor and cognitive functions in both healthy elders and cognition-compromised subjects has been observed $[15,16]$.

Nevertheless, falls may also depend upon extrinsic factors, including environmental, ergonomic and organizational aspects (inadequate housing, insufficient illumination, hazards in domestic and public places, lack of social and health care services and restricted social interaction) [12].

As reported in [17], in most cases, falls occur as the result of the individual's inability to adapt to environmental conditions. For example, increasing the slipperiness of a floor surface (i.e., from dry to wet) creates a high risk of slipping. In fact, the forces of the foot that are normally generated during gait require friction to counteract the shear forces and maintain balance. When the friction available at the level of the shoe can not meet the biomechanical requirements, a slip becomes more likely, and the consequent risk of injury with it.

In the case of highly predisposing risk factors, the frequency of falls increases, leading to a growth in the mortality rate and in the probability of causing/worsening disabilities [18]. Fractures of the femur, head injuries, damages to the lower and upper limbs and post-fall syndrome are fall-related events, which could cause a loss of confidence, hesitation and, consequently, a reduction of the quality of life $[4,6,19]$. The incidence of fall-related mortality is greater in men [6] than in women, although the latter show a higher prevalence of falls. The incidence of mortality is higher in men probably due to more comorbidities or to the tendency to not seek medical care until the condition becomes severe, or to delay to the access to prevention and management of diseases [6]. Furthermore, men are usually involved in intense and dangerous physical activity and risky behaviours that predispose to major risk of fall. Many epidemiological studies predict twice as many injuries up to the year $2030[6,20]$.

Fall-related costs can be categorized in direct costs (which include medical and diagnostic examinations, rehabilitation treatments) and indirect costs (which have an impact on family economy) [4]. Some injuries related to falls, in addition to physical and psychological consequences on the subject, require a rehabilitative or surgical treatment, resulting in an increased hospitalization time, which has a significant economic impact on the healthcare system [6,21]. Indeed, this results in the highest direct costs, which represent about $50 \%$ of the total share in the case of falls, as shown by epidemiological data from the World Health Organization (WHO) [6].

Given the high incidence of falls in healthy elderly, in order to prevent them, it is necessary to identify predisposing risk factors, to analyze subject specific needs and to use a targeted preventive strategy, like behavioral changes [6] or promoting a healthy lifestyle [22], despite some evidence showing the opposite [23,24]. In addition, in order to make elderly people less predisposed to falls, it would be useful to plan age-friendly environments through the installation of staircases, adequate lighting and non-slip rugs [25].

The interest in the studying of mechanisms of balance and body-orientation control has increased in the last years since it has proved to be an effective tool for fall monitoring. Different techniques of measurement and assessment have been used to quantify the postural stability in elderly individuals both in static and dynamic conditions [26,27].

Human posture can be defined as "the position of one or many body segments in relation to one another and their orientation in space" [28]. Head, trunk, pelvis, lower limbs and feet are known as body "segments", whereas spinal, hips, knees, ankle and shoulder joints are denoted as body "linkages" [29]. Human posture depends on a great number of factors-among them, we cite the muscle tone [30], the orientation and the equilibrium [31], which are involved in the control of the body position with respect to the walking surface (the relationship between the Center of Mass (CoM) and 
Base of Support (BoS) [32]), and in the control of alignment of Center of Pressure (CoP) with respect to the Center of Gravity (CoG) [26].

According to a biomechanical approach, the falls could be due to a change in position of the CoM projection beyond the BoS without any correction [10]. Even when standing, the body sways constantly and for this reason such posture is called static standing or stand still. The most common way to study the balance in static standing is through the trajectories of the CoP with respect to the CoG [33-35], although nonlinear measures have been proposed in some studies [36-38]. The assessment of the degree of postural sway during static standing could be useful to evaluate the ability to preserve balance control. For this reason, participants are commonly asked to perform a static upright task, on a force platform, in open- and closed-eyes condition, with both arms along the body [39,40]. During this test, it is also possible to analyze the relationship between the CoM and CoP, it being useful for fall prediction [39] and risk assessment [40].

One of the major health issues for an older adult is the loss of stability or a slip and the subsequent fall during walking. For this reason, the evaluation of dynamic stability, the assessment of activity and mobility in daily living conditions (for example, through ADLs) or through tasks such as simple walk, "time up and go" (TUG) test [41] and the 6-minute walk test [42], could provide useful motor outcomes to detect the early signs of balance alteration and then provide key information about fall risk [43]. The latter results could, however, be capitalized in a predictive fashion so as to accomplish fall detection/prediction. Many articles in the literature are focused on defining gait characteristics in the elderly with the aim of identifying the causes of fall. In the recent review article by [27], the authors tried to evaluate the sensitivity of biomechanical measures that quantify gait stability in older populations. In detail, they analyzed different approaches to study gait stability based on two different classes: (i) linear variability of temporal measures (such as swing and stance time, step width, stride velocity), and (ii) (nonlinear) orbital or local stability measures (such as Floquet multipliers or Lyapunov exponents). They concluded that, although some biomechanical approaches for determining specific parameters of mobility can assess the function and stability in the elderly, they hardly have been taken up in clinical settings because of their unclear sensitivity and specificity, together with the time and effort required for their use. Within this framework, a key role is played by the adopted sensing technologies, both in terms of hardware and software approaches.

Recently, the interest in falls is increased not only in healthy subjects, but also in pathological conditions such as Parkinson's disease [1,44,45], multiple sclerosis [3,46], stroke [2,47] and Alzheimer's disease $[48,49]$. However, this review investigates the studies published in the last two decades in the field of stability control for fall risk assessment, prevention and detection based on wearable sensors in healthy elderly people only. This is because, in a pathologic condition, the stability, and therefore the fall risk, strongly depends on the motor and cognitive characteristics of the disease.

An interesting review can be found in [50], where the authors focus their attention on wearable inertial sensors for fall risk assessment in geriatric populations. Within this manuscript, we extend the analysis to other sensor typologies, such as pressure sensors, electromyographic sensors and cameras, and to fall detection and prevention. Therefore, the aim of our work is to provide an overview of the most adopted sensing technologies in these fields, with a focus on the type of sensors (rather than algorithms), their position on the body and the kind of tasks they are used in. The existing literature has been found by defining a search criteria and considering some of the most important publication databases. In more detail, the search criterion is reported in Section 2, and the search results are reported in Section 3. Starting from these outcomes, some trends are identified and reported in Section 4. Finally, conclusions are drawn in Section 5.

\section{Methods}

This section is focused on the definition of the research criteria and strategy. After the introduction of the keywords, which are required in order to correctly understand the flow chart of the selection 
procedure, the framework of the research strategy is proposed and motivated. Finally, an overview of the results is briefly summarized in the last subsection.

\subsection{Keywords Definition}

In this section, a brief description of the main keywords is provided in order to clarify the search criteria.

"Aged": with this term, we considered all those works whose studies either take into account the stability analysis for subjects aged over 64 years or consider a younger population for a preliminary assessment but with an intended application to old people.

"Postural control": in biomechanics, it refers to the ability of maintaining the line of gravity (vertical line from centre of mass) of a body within the base of support with minimal postural sway. In order to classify the different articles, we adopted the terms reported in Figure 1 both for the dynamic analysis (i.e., "gait" and "walk" words) as well as the statical analysis (i.e., "static", "stationary", "standing" and "stance" words). The former refers to the study of a walking subject, while the latter investigates the function of the balance system during quiet stance.

"Fall detection": it refers to the drop from a standing position or during an activity. Falls are a major public health problem among old people and the use of a fall detection system as an assistive device is important to alert when an event of fall occurs.

"Fall prevention": it refers to all those articles that analyze strategies aimed at preventing the fall of older people, including doing exercises to improve muscle strength and balance, and making simple changes to home or wearing sensible shoes.

"Fall risk assessment": it refers to those manuscripts that focus on the cause of falls, such as environmental issues, age, mental state and mobility.

"Wearable sensor systems": national and international guidelines on fall prevention all reinforce the need to screen and assess older people, also with an attempt to identify the ones with increased risk of falling. In order to achieve this goal, one of the adopted solutions is to use wearable or portable sensors systems.

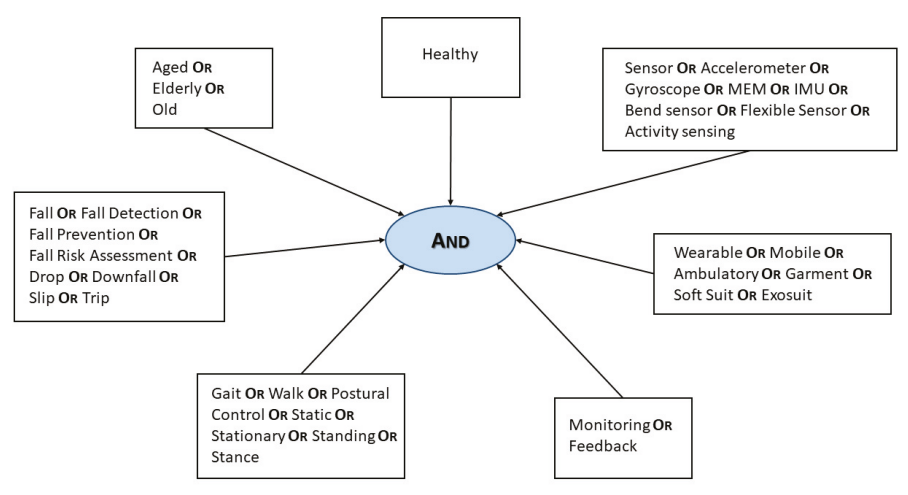

Figure 1. Block scheme of the adopted search strategy for the papers selection.

\subsection{Literature Research Strategy}

The aim of the manuscript is to provide an overview of the type and location of wearable sensors for the monitoring and assessment of falls during static and dynamic tasks (i.e., walking and standing) in healthy elderly people [51-53]. For this purpose, a bibliographical research on the most important scientific publication databases was performed. In particular, an approach similar to the one proposed 
in [54] in case of the newborns has been followed. We focused on IEEE Xplore, SpringerLink, Science Direct and PubMed databases. Articles related to both dynamic and static stability analysis have been taken into account, since, as previously reported, there is a close relationship between falls and static (i.e., [26,55-58]) or dynamic (i.e., [27,59-62]) tasks. In order to perform this research, the set of keywords previously introduced has been applied. Figure 1 illustrates the flow chart of the selection process. The key point is represented by the AND block, which merges the other peripheral branches, in which each word is related to its synonyms by means of an OR condition. By doing so, the research is carried out via the intersection of at least one word per each peripheral block, otherwise the result of the query is null.

\subsection{Study Selection and Screening Process}

After a preliminary search, 675 results have been found for the dynamic task and 4064 articles for the static task, as shown in Figure 2. In the first step, based on paper titles and abstracts, the following exclusion criteria have been identified:

- $\quad$ all the studies that do not include healthy aged population as target;

- $\quad$ articles not including a wearable sensor technology;

- all the manuscripts not related to "fall risk assessment", "fall prevention", "fall detection" and "standing analysis";

- $\quad$ all the review articles.

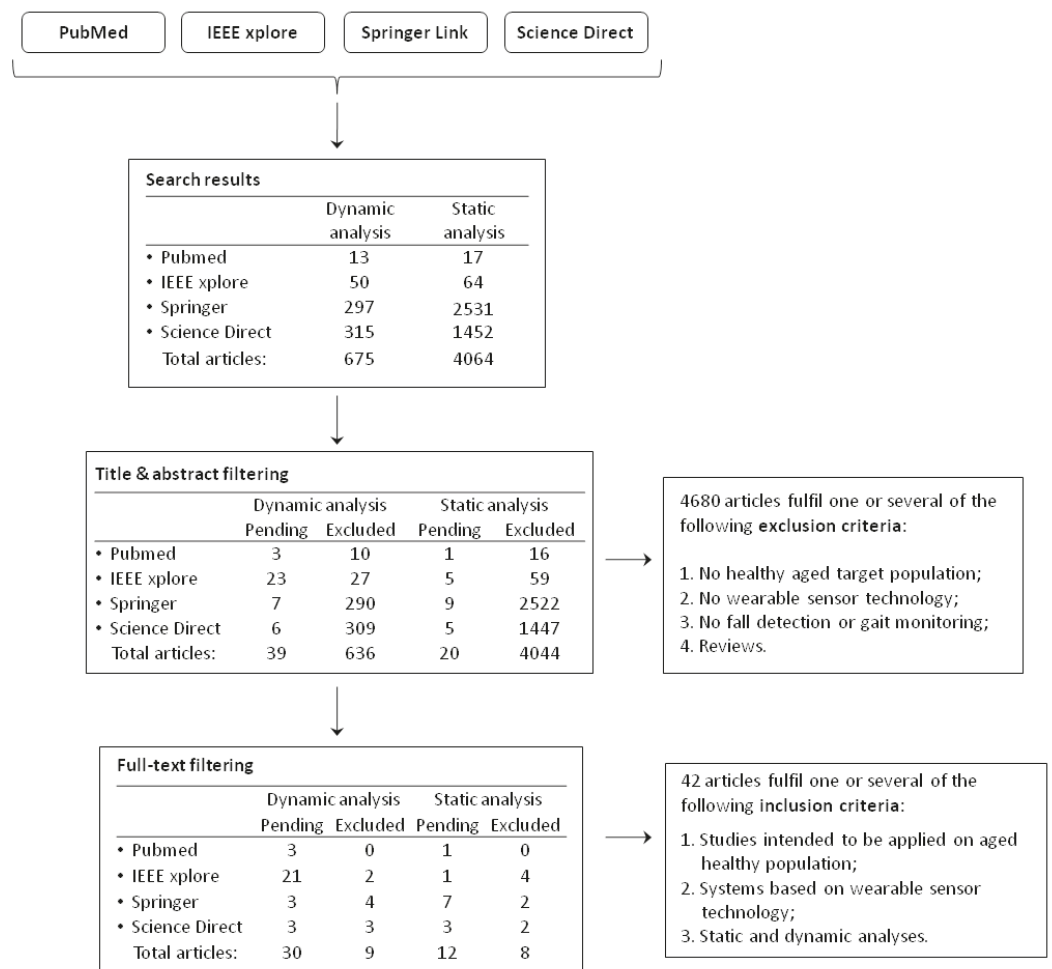

Figure 2. Adopted research methodology. The flow chart illustrates the two steps of the selection procedure (title and abstract filtering and full-text reading). 
This selection drove into the exclusion of 4680 articles. Regarding the remaining 59 manuscripts, a second refinement step has been performed, which consists of a full-text reading of the articles. This filtering process was based on the following inclusion criteria:

- $\quad$ all studies on healthy aged people or intended to be applied on an aged population;

- $\quad$ all systems based on a wearable sensor technology;

- $\quad$ gait and standing analysis have been included.

Finally, 42 articles remained and were considered in this work. In more detail, we started the selection procedure by considering all the previous databases for collecting the articles, in order to provide more information regarding their distribution among the databases, although most of the papers were present on Pubmed. Therefore, we decided to indicate with the "Pubmed" label all those articles not present on the other databases.

Figure 2 provides an overview of the whole selection process and the results of the literature research are reported in Table 1. In addition in Table 2 are reported the acronyms for the tasks, in Table 3 the acronyms for the sensor positions while in Table 4 the acronyms for the sensors types, used in Table 1. Starting from these results, the quantity of works published in last years, both in conference proceedings and journals, the number and age distribution of participants, the number and typology of sensors and the types of conducted tasks have been reported as graphs. In Figure 3, the distribution of the manuscripts of Table 1 is shown. In order to make the trend more clear, a five point moving average has been applied, resulting in the red dotted line of Figure 3. It is evident that the topics of fall risk assessment, falls monitoring and falls preventing in elderly people have gained increasing interest in last years, with an almost linear growth of the published manuscripts.

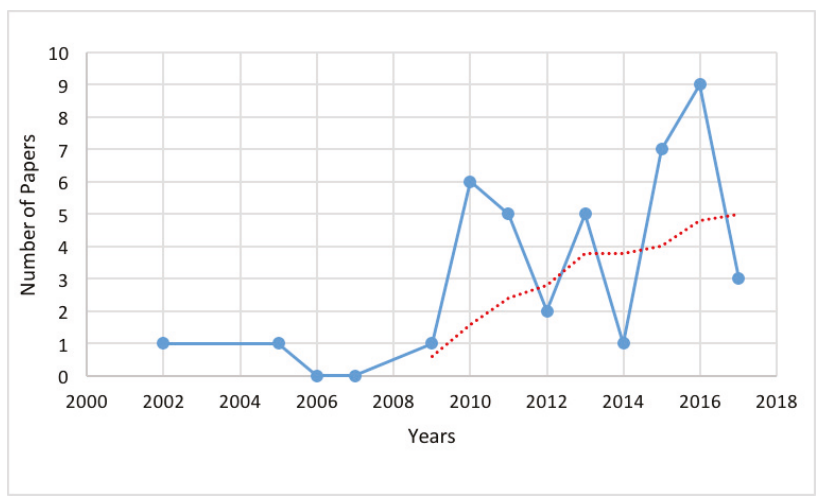

Figure 3. Number of found manuscripts that have been published in the last 15 years (blue continuous line) and its average (red dotted line).

The manuscripts resulting from the selection have been critically analyzed in order to provide an overview of the general trends and of the most adopted technologies in the field of falls detection, falls risk assessment and falls prevention in healthy elderly. The analysis has been carried out according to three aspects: the characteristics of the participants involved in each study, the approaches for sensing and the typology of tasks that the cohort has been asked to perform. 


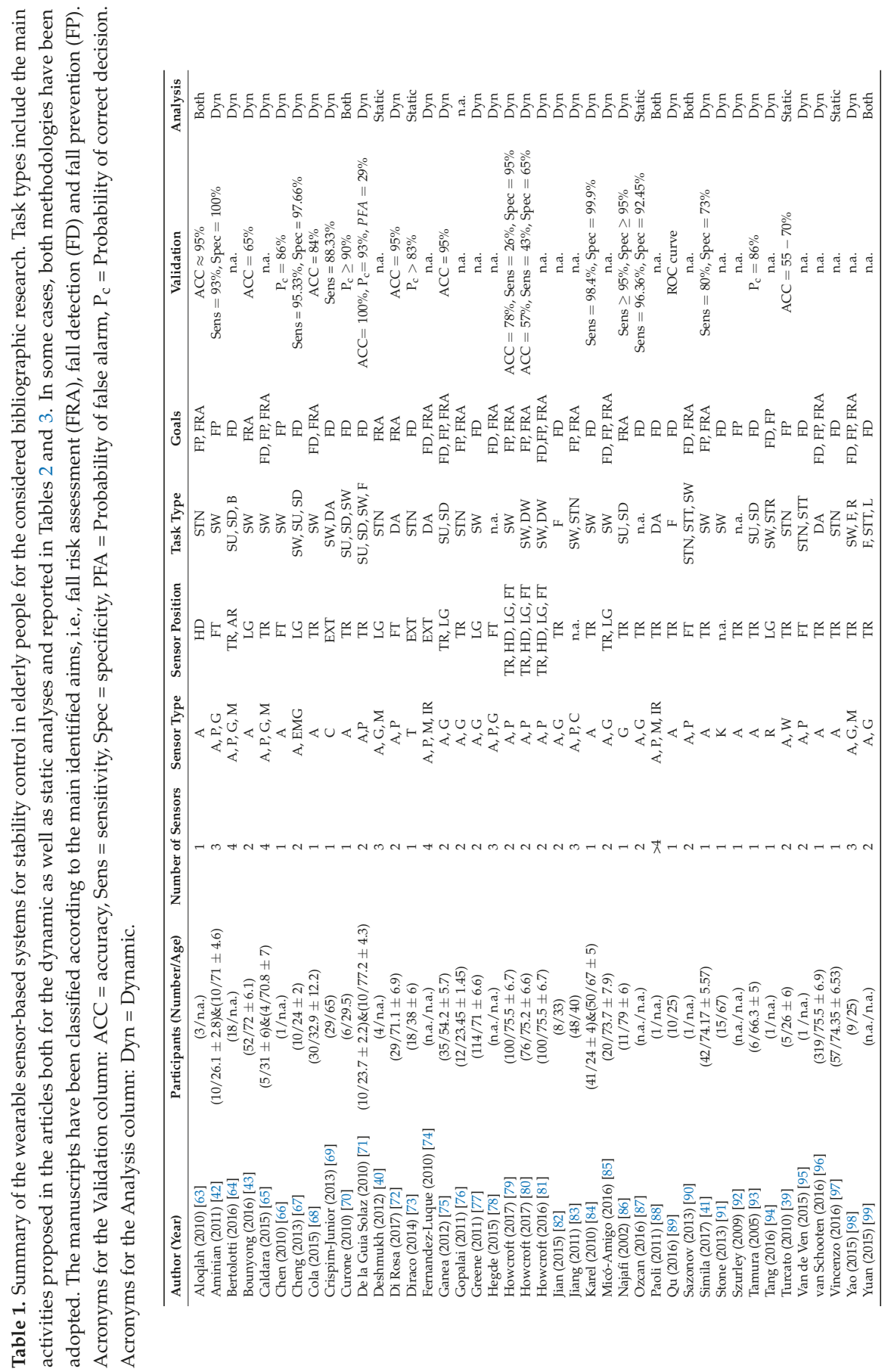


Table 2. Legend of acronyms for the tasks in Table 1.

\begin{tabular}{lc}
\hline Task Type & Acronym \\
\hline Standing & STN \\
Single Task Walking & SW \\
Dual Task Walking & DW \\
Standing Up & SU \\
Sitting Down & SD \\
Bending & B \\
Daily Activities & DA \\
Falling & F \\
Running & R \\
Sitting & STT \\
Lying & L \\
Stairs & STR \\
\hline
\end{tabular}

Table 3. Legend of acronyms for the sensor positions in Table 1.

\begin{tabular}{llc}
\hline Sensor Position & Anatomical Location & Acronym \\
\hline Head & & HD \\
Foot & Shoes, heel & FT \\
Trunk & L3, L5, sternum, waist, pelvis, neck, chest & TR \\
Arm & Wrist, forearm & AR \\
Leg & Thigh, cruris, ankle, shank, knee & LG \\
External & & EXT \\
\hline
\end{tabular}

Table 4. Legend of acronyms for the sensors types in Table 1.

\begin{tabular}{lc}
\hline Sensor Type & Acronym \\
\hline Accelerometer & $\mathrm{A}$ \\
Gyroscope & $\mathrm{G}$ \\
Pressure sensors & $\mathrm{P}$ \\
Magnetometer & $\mathrm{M}$ \\
Radar & $\mathrm{R}$ \\
Time-of-flight (TOF) Camera & $\mathrm{T}$ \\
Kinect console & $\mathrm{K}$ \\
Wii console & $\mathrm{W}$ \\
Electromyography & EMG \\
Infrared sensors & $\mathrm{IR}$ \\
\hline
\end{tabular}

\section{Results of the Bibliographic Research}

The papers resulting from the selection are summarized in Table 1 in a compact way. We chose to categorize the papers according to three main aspects: the participants involved in the study, the adopted sensors and the body portion under focus. The assessment of the participants gives an idea of the target of the study in terms of subjects, and defines if one is dealing mainly with a technical experiment of with clinical research. The second aspect allows to identify the technical features of the research and its costs. The analysis of the body parts mainly involved in the study allows to define the main biomechanical aspects considered in the work. Relevant information about such parameters can be found in the columns of the table. In more details, papers have been organized according to the following aspects:

- Author (year): the family name of the first author is reported, and the publication year between brackets; 
- Participants (number/age): the number of volunteers considered in the study. For each population, the number of the subjects involved and their average age is reported;

- Number of sensors: how many sensors are used (simultaneously or not) within the study. In the case of compound sensors, each of the elementary sensing technology has been accounted for;

- Sensor type: the typology of sensors adopted in the study. Their acronyms are reported in Table 4 . In case smartphones have been employed, we only reported the name of the actual sensors;

- Sensor placement: sensor attachment location;

- Task: the (one or more) task the participants have been asked to perform for the validation of the study. We chose to include activities implying oscillations of the center of mass, as they make falls more likely;

- Analysis: the type of performed analysis, i.e., static, dynamic or both.

\section{Discussion}

\subsection{Participants}

In Figure 4, we report the number of participants for each study (grouped in four categories) for the considered articles, excluding the ones that do not specify the population age and/or participants' number, and that discuss a specific sensor/methodology only qualitatively, without any population involved. From the inspection of the figure, the most frequent bins are very different from a numerical viewpoint. Additionally, only a few articles have more than 100 participants in the study, reflecting the difficulty in recruiting a wide audience for these studies.

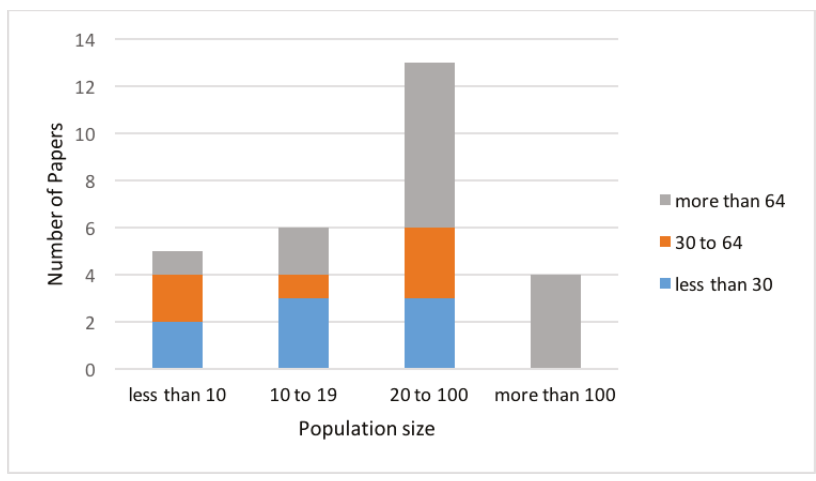

Figure 4. Participant age distribution in the case of different population sizes.

From the conducted review of the literature, it emerges that there are mainly two lines of research: (a) studies concerning the comparison between old versus young subjects $[42,65,71,84]$ and (b) works aiming at underlining some peculiar differences between groups of elderly fallers and non-fallers [43,79-81]. In both cases, there is a prevalent presence of healthy elderly groups. This observation is confirmed by Figure 4, where it is shown that most of the participants in all studies are 64 years old or more, which is not surprising provided that this was used as an inclusion criterion. On the other hand, the prevalence of the young and middle (30 to 64) groups in studies with less than 10 subjects reflects the need of a fast validation in the case of a novel instrumental technology.

\subsection{Typology of Sensors}

By considering the sensors adopted in the studies, a first important consideration can be drawn. Specifically, most of the proposed sensing methodologies are simple from a technological point of view, as a significant share of the works adopt a few sensor technologies for monitoring the subjects: 
16 papers use only one sensor and 20 papers use two sensors. On the other hand, only a small percentage of the papers proposes the joint use of three or more sensors types for improving the monitoring performance. Given the above numerical categorization (e.g. single sensor, two sensors and $\geq 3$ sensors, respectively), we proceed in what follows with category-specific observations.

\subsubsection{Single Sensor}

From Figure 5, it can be seen that the accelerometer is by far the most adopted sensor in the group of single-sensor type paper with a percentage of adoption of about $70 \%$. This is probably due to the low-cost and to the plethora of devices available on the market, most of them with integrated wireless communication system, small size, weight and long-lasting batteries. For example, Cola et al. [68] propose a method requiring the use of a single waist-mounted accelerometer to achieve a continuous monitoring of deviation in the gait of elderly people. Among the single sensor approaches, about one fourth of the papers adopts a wide range of sensor technologies, both commercial products (e.g. Kinect, Wii, cameras) and prototypes. For instance, Diraco et al. [73] propose a non-invasive technique for posture classification suitable to be used in several in-home scenarios. This procedure exploits 3D point cloud sequences acquired by using a single time-of-flight sensor to classify the posture hierarchy by using a support vector machine (SVM) approach. Conversely, from the cameras proposed in [73], Najavi et al. [86] propose a commercial prototype. A more recent technology can also be employed for gait analysis and fall detection. Stone et al. [91] monitored a population of aged people (67-97 years old) for a few months using a Microsoft Kinect sensor (Redmond, WA, USA) in order to evaluate fall risk by means of the standard methodologies timed-up-and-go (TUG) time and habitual gait speed (HGS) tests.

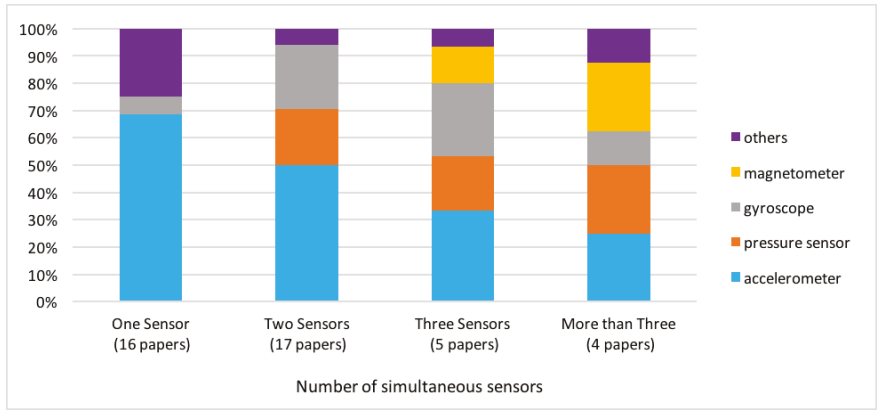

Figure 5. Sensor typologies in the case of papers exploiting one, two, three or more than three sensors.

\subsubsection{Two Sensors}

By focusing on the papers that use two sensors, two families can be identified: approaches that combine the accelerometer with a pressure sensor (generally placed within the shoes), and methodologies that jointly use the accelerometer and the gyroscope sensors, usually placed on the same electronic board. For instance, the study proposed in [75] introduces a new robust classifier for sit-to-stand (SiSt) and stand-to-sit (StSi) detection in daily activity based on both gyroscope and accelerometer. The monitoring system consists of a single inertial sensor placed on the trunk. By using dynamic time warping, the trunk acceleration patterns of SiSt and StSi are classified based on their similarity to specific templates. Gopalai et al. [76] also use accelerometers and gyroscopes to improve postural control and shorten rehabilitation periods among the young and the elderly. Beyond such "standard" sensors, the use of some peculiar devices can be explored for the stability control of elderly people. Greene et al. [77] investigate the gait variable Minimum Ground Clearance (MGC) using shank-mounted inertial sensors containing both a tri-axial accelerometer and an add-on tri-axial 
gyroscope. The aim is to estimate clinically meaningful parameters, which may be used in the screening for falls risk.

\subsubsection{Three or More Sensors}

In the case of three or more devices, other sensing technologies are adopted, including magnetometer, camera or electromyography (EMG), as described in [64], where Bertolotti et al. have designed and built an autonomous wearable 9-degrees-of-freedom system embedding three axial accelerometers, three-axial gyroscopes, and three-axial magnetometers. In [88], a support system for detecting falls of an elder person is presented. This system is an AAL (Ambient Assisted Living) system that allows to infer a potential fall by the combination of a wearable wireless sensor node, based on an accelerometer, and a static wireless non-intrusive sensory device, based on heterogeneous sensor nodes. The sensors network would spread throughout the environment, in any room of the house, routing and linking nodes to the base station. The wearable node is not intended for determining a falling situation, but to advise the reasoner layer about specific acceleration patterns that could eventually imply a fall.

\subsection{Position of Sensors}

Regarding the position of the sensors, 25 studies monitored two or three points of the body (Figure 6). Most importantly, the placement of sensors on the human body is mostly on the trunk.

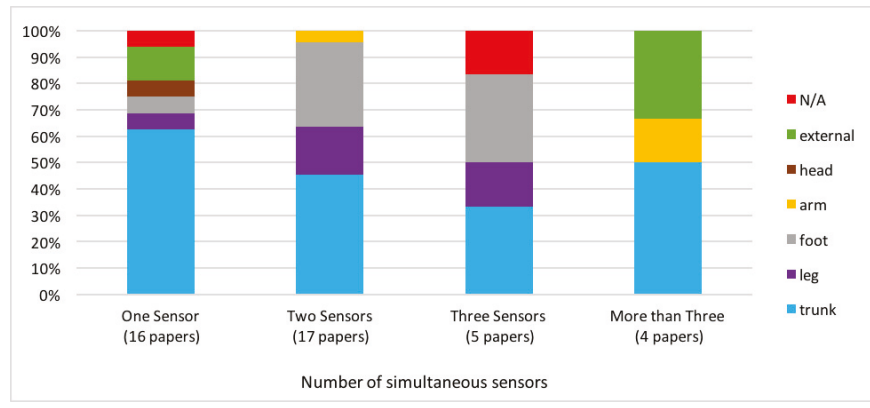

Figure 6. Sensor locations in case of papers exploiting one, two, three or more than three sensors.

Among all, the work of Curone et al. [70] needs to be mentioned, who developed a processing methodology to monitor the posture and detect the activity level of the subject based on data from an accelerometer. The sensor is placed on the upper trunk, inside a garment similar to a jacket. Moreover, the algorithm is able to associate a reliability value in order to launch alarms only in case of effectively dangerous conditions. The methodology exhibits a very high accuracy in task classification (about $96 \%$ ), although the evaluation has been done on a very small cohort (6 participants). In [86], authors present a method for evaluating postural transitions. The algorithm analyzes the parameters in the wavelet domain, and related them to the falling risk of the subject. In addition, Ref. [68] monitors the trunk for its analysis, reaching a very interesting accuracy value (84\%) in the case of a 30 people cohort. Unfortunately, no evaluation is done in the case of older subjects.

Besides the trunk, sensors are usually placed on the foot (almost $30 \%$ of all selected studies, corresponding to pressure sensors within the shoes) and the leg, when one to three sensors are considered. In addition, the inertial device presented by Bertolotti [64] provides objective measurements of limb movements for the assessment of motor and balance control. It can be adopted for the fall risk assessment, quantifying sports exercise, studying people habits, and monitoring the elderly. Several acquisition configurations are presented as the sensors can be positioned on the trunk, on the thighs, on the arms and also on the head. In [75], the authors adopt inertial sensors positioned on 
the trunk and on the leg to monitor subjects, reaching high accuracies in classifying postural transitions (up to $95 \%$ ).

Very few works adopt not-body worn sensors for monitoring subjects, although such kind of systems have the advantage of being totally non invasive. In particular, in [69], a video system is proposed for monitoring Alzheimer's patients. A software module has been developed in order to recognize both physical tasks and instrumental activities of daily living. The proposed video processing algorithm is quite effective in the physical task recognition, achieving an F- score of 93. The approach proposed in [73] uses time of flight sensors for collecting 3D point cloud sequences and classifying the subject's activities. Such methodology is interesting and capable of good performances, at least without any partial occlusion of the subject.

The reason why the trunk is the most used segment for the sensor location is tightly coupled to the upright gait, being a human characteristic that requires the ability to preserve the upper body balance during walking. Interestingly, in 1992, Perry [100] defined gait analysis as centered on the lower limbs, in particular on hip, knee and ankle joint kinematics, while defining the upper body as a mere "static passenger unit" of the locomotor apparatus. Later, empirical evidence greatly changed this point of view. Indeed, in recent years, many studies have moved their focus and confirmed that the trunk plays a fundamental role both in static and in dynamic stability, see for example the study of Bertolotti et al. [64]. Furthermore, in [68], the authors used a single waist-mounted accelerometer in order to assess frailty and risk of falls in the elderly. Curone [70] designed an algorithm that analyzes, in real time, the signals produced by one three-axial accelerometer placed on the trunk, in order to classify human activities and posture transitions. In addition, Karel et al. [84] used a a triaxial accelerometer worn at the level of the sacrum to evaluate the incidence of stumbling in the elderly in daily life.

A loss of gait stability in older people is documented in many studies and the corresponding literature shows that different aging factors, such as the loss of muscle strength [101], the decline in vision and the peripheral and vestibular sensations [102], directly hamper the ability to keep the upper-body stable during walking. According to these results, the scientific interest in the trunk increased in recent years, and the development of wearable sensor technologies, which enable reliable measurements of trunk movements, has contributed to such increased interest.

As observed earlier, there is a significant adoption of pressure sensors within the shoes. The reason is that monitoring the CoP both in static and dynamic conditions, together with measuring spatio-temporal gait variability, can identify the fall risk, and help reducing it. In particular, Aminina [42] proposed a 3D method for gait analysis using the foot orientation and trajectory during each cycle based on two inertial modules worn on each foot. Using data transmitted by the modules during long distance walking periods, a dedicated algorithm provides relevant gait parameters for the evaluation of the outcome of the rehabilitation program for fall prevention in elderly subjects. In addition, Di Rosa [72] used a pair of electronic insoles, for the "Wireless Insole for Independent and Safe Elderly Living" project, in order to assess the risk of falls through a novel Fall Risk Index based on multiple gait parameters and gait pattern recognition.

\subsection{Tasks}

The assessment of the ability to maintain the balance in static and dynamic conditions and the estimation of the risk factors of falls in older people has been done by asking the participants to perform one or more tasks, whether simple or complex. In Figure 7, the distribution of the different types of task in case of one, two or more required activities is reported. We have divided the "Task" paragraph accordingly. 


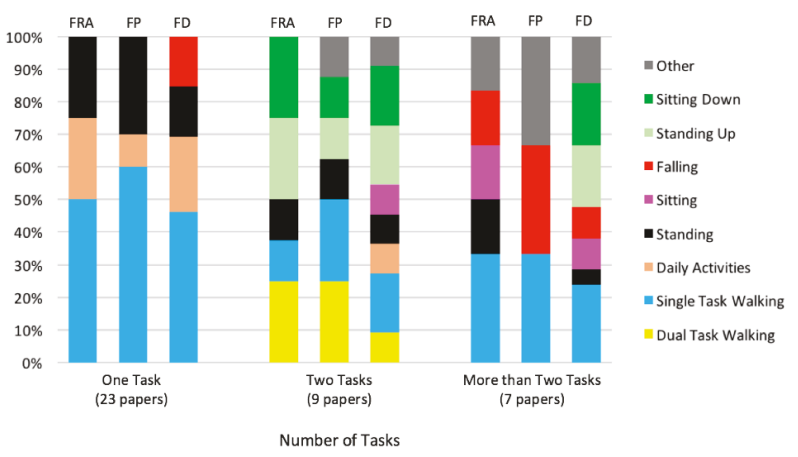

Figure 7. The type of tasks the participants have been asked to perform is case of one, two or more activities for fall risk assessment (FRA), fall prevention (FP) and fall detection (FD). Notice that (a) three out of all the articles listed in Table 1 were not reported in the figure (because they did not perform any test) and (b) to avoid an excessive number of categories, less investigated activities were clustered within "other" label.

\subsubsection{One Task}

Among the papers selected in this study, roughly 23 works include in the experimental protocol the execution of one task only. As it can be see in Table 1, 15 papers focus on the analysis of dynamics using one task, such as a walk at self-selected velocity, the six minute walk test, falling test or the "time up and go". These tests are simple to manage, facilitate patients collaboration by being tolerable and also reflect most of the activities that are frequently performed by elderly people. Through the use of sensors, such as accelerometers or pressure sensors, the simple walk allows not only to detect the functional mobility of elderly people, but also to quantify their spatio-temporal parameters (speed, walking time, length and width stride etc.), the joint range of motion, and to identify a small set of features for the classification of falls. Indeed, Howcroft et al. [79] proposed the use of accelerometers and plantar pressure sensors during the $7.62 \mathrm{~m}$ ( 25 feet) test to detect, from a broader set of features, a smaller one to be used for falls classification, which could further improve falls classification. Although a greater walking distance might be a more suitable indicator of everyday walking activities, the assessment of brief gait distance is commonly used in clinical practice and could allow to find a small set of features in order to improve the clinical fall risk prediction. In addition, Similä et al. [41] suggested that gait characteristics can be used to estimate the outcome of clinical assessment of dynamic stability and predict balance decline. Furthermore, walking monitored by wearable sensors could be a useful screening tool to identify people with early signs of balance deficit [103]. As shown in Figure 7, 28\% of the papers includes the analysis of static condition by employing a single task, such as standing in an anatomical reference position, with open or closed eyes. These tasks allow for detecting age-related postural changes in antero-posterior or mid-lateral directions. In Turcato et al. [39], an inertial sensor has been useful to detect the angular velocity and the linear accelerations of trunk in the static condition. The results of the study highlight that it is possible to reliably predict, with a resolution by up to $400 \mathrm{~ms}$, the higher frequency CoM displacements. Since the loss of static balance occurs when the CoM goes beyond the BoS, the approach described can be useful to develop wearable devices able to warn subject of the risk of falling, so that they can implement strategies to keep the balance. Furthermore, as suggested by Gopalai et al. [76], with vibrotactile feedback tools, the static evaluation of body allows to monitor and correct the postural control. In fact, they found that the use of an intelligent biofeedback system integrated by a vibrating platform is useful to improve postural control.

Additionally, as it can be seen in Table 1, two studies implement daily activities and two other studies analyze falling task. Specifically, Di Rosa et al. [72] evaluated the performance of free-activities 
of daily life, in order to develop a self-learning and wearable system to identify the walking decline and to detect the risks of falls at home. Furthermore, a recent paper [82] proposed a portable device able to detect falls with accuracy and to provide monitoring and timely help for the the elderly.

\subsubsection{Two Tasks}

Of all papers included in this review, nine of them consider two tasks, as reported in Figure 7. In this case, the most adopted tests are (single and dual task) walking, sitting down and standing up. Some studies $[80,81]$ reported in our review have explored the use of wearable sensors, the motor control and the risk of falls during the performance of a single task (walking for $7.62 \mathrm{~m}$ ) and dual task (walking for $7.62 \mathrm{~m}$ with a cognitive load). These studies found that wearable sensors, such as accelerometers and pressure-sensitive insoles, can be useful, during the performance of two tasks, to detect alterations of the motor functions such as gait variability, which is indicative of greater instability and is predictive of the risk of falls. Activities like SiSt or StSi carried out daily by old people allow for detecting postural transitions, analyzing the adopted postural strategy and preventing the fall events, as reported in [75]. In [86], the authors ask to perform two tasks in order to develop a device that can assess quantitatively the degree of mobility in old people and monitor risk factors for falls.

\subsubsection{More than Two Tasks}

In this review, seven selected papers include more than two activities. The most adopted tasks are walking, sitting down and standing up as can be seen in Figure 7. In order to improve the quality of life of elderly people, for the authors in [67], both biomechanical evaluation and detection by sensors of multiple consecutive activities are necessary, in order to reflect the static and dynamic transition period, which could predispose to a greater risk of falling if not adequately controlled. Indeed, three types of tasks (walking, standing up and falling down) were analyzed in order to monitor and to detect fall events.

Finally, five works have not been included in Figure 7 as they only provide an overview of the importance of using sensors to assess the risk of falls in the elderly, without specifying the task used for the analysis.

\section{Conclusions}

Within this manuscript, a literature review of monitoring technologies for fall risk assessment, fall prevention and fall detection in case of healthy elderly people is provided. The analysis showed that several methodologies have been proposed in the literature, and it is very difficult to find a general rule. From the perspective of sensors, most of the considered methodologies uses two sensors at maximum. Among them, accelerometers and gyroscopes are the most widespread technologies, probably because they can combine low cost and informative signals. Nevertheless, some works adopt unconventional sensors, such as radar or cameras, with the aim of investigating the effectiveness of other sensing technologies for monitoring issues. Most of them only show some preliminary results, and still require a more complete statistical validation.

Regarding the position of the sensors, the trunk is the most used segment because it plays a fundamental role both in static and dynamic stability.

By looking at the task standpoint, in case of static stability assessment, the majority of the works adopt a quiet standing test with open or closed eyes, allowing for detecting age-related postural changes in antero-posterior or mid-lateral directions. Instead, for dynamic evaluations, a walking task or a sit-to-stand test is usually adopted to detect postural variations.

Regarding the validation of sensors, the information arising from the scientific literature is reported in Table 1 . However, the information about sensibility, specificity and accuracy of the considered methodologies are too diverse and do not allow for evaluating the impact of parameters such as sampling rate or sensor precision. From the analysis, there is a need for the definition of one 
(or more) gold standards in terms of sensors (both types and location) and tasks to be performed in order to face the extremely high variety of proposed approaches.

Funding: This research received no external funding.

Conflicts of Interest: The authors declare no conflict of interest.

\author{
Abbreviations \\ The following abbreviations are used in this manuscript: \\ BoS: Base of Support \\ CoG: Center of Gravity \\ CoM: Center of Mass \\ CoP: Center of Pressure
}

\title{
References
}

1. Amboni, M.; Iuppariello, L.; Lista, I.; Rucco, R.; Varriale, P.; Picillo, M.; Iavarone, A.; Sorrentino, G.; Barone, P. Gait patterns associated with freezing of gait in patients with Parkinson's disease. Mov. Disord. 2015, 30, S29-S30.

2. Teasell, R.; McRae, M.; Foley, N.; Bhardwaj, A. The incidence and consequences of falls in stroke patients during inpatient rehabilitation: Factors associated with high risk. Arch. Phys. Med. Rehabil. 2002, 83, 329-333. [CrossRef] [PubMed]

3. Matsuda, P.N.; Shumway-Cook, A.; Bamer, A.M.; Johnson, S.L.; Amtmann, D.; Kraft, G.H. Falls in multiple sclerosis. PM R 2011, 3, 624-632. [CrossRef] [PubMed]

4. Lord, S.R.; Sherrington, C.; Menz, H.B. FALLS in Older People; NICE: London, UK, 2001.

5. Tinetti, M.E.; Speechley, M.; Ginter, S.F. Risk factors for falls among elderly persons living in the community. N. Engl. J. Med. 1988, 319, 1701-1707. [CrossRef] [PubMed]

6. World Health Organizatio (WHO). Global Report on Falls: Prevention in Older Age; WHO: Geneva, Switzerland, 2007.

7. Blake, A.; Morgan, K.; Bendall, M.; Dallosso, H.; Ebrahim, S.; Arie, T.; Fentem, P.; Bassey, E. Falls by elderly people at home: Prevalence and associated factors. Age Ageing 1988, 17, 365-372. [CrossRef] [PubMed]

8. Prudham, D.; Evans, J.G. Factors associated with falls in the elderly: A community study. Age Ageing 1981, 10, 141-146. [CrossRef] [PubMed]

9. Campbell, A.J.; Reinken, J.; Allan, B.; Martinez, G. Falls in old age: A study of frequency and related clinical factors. Age Ageing 1981, 10, 264-270. [CrossRef] [PubMed]

10. Isaacs, B. Clinical and laboratory studies of falls in old people. Prospects for prevention. Clin. Geriatr. Med. 1985, 1, 513-524.

11. Kannus, P.; Sievänen, H.; Palvanen, M.; Järvinen, T.; Parkkari, J. Prevention of falls and consequent injuries in elderly people. Lancet 2005, 366, 1885-1893. [CrossRef]

12. Ambrose, A.F.; Paul, G.; Hausdorff, J.M. Risk factors for falls among older adults: A review of the literature. Maturitas 2013, 75, 51-61. [CrossRef] [PubMed]

13. Moreland, J.D.; Richardson, J.A.; Goldsmith, C.H.; Clase, C.M. Muscle weakness and falls in older adults: A systematic review and meta-analysis. J. Am. Geriatr. Soc. 2004, 52, 1121-1129. [CrossRef] [PubMed]

14. Holtzer, R.; Friedman, R.; Lipton, R.B.; Katz, M.; Xue, X.; Verghese, J. The relationship between specific cognitive functions and falls in aging. Neuropsychology 2007, 21, 540-548. [CrossRef] [PubMed]

15. Kearney, F.C.; Harwood, R.H.; Gladman, J.R.; Lincoln, N.; Masud, T. The relationship between executive function and falls and gait abnormalities in older adults: A systematic review. Dement. Geriatr. Cogn. Disord. 2013, 36, 20-35. [CrossRef] [PubMed]

16. Rucco, R.; Agosti, V.; Jacini, F.; Sorrentino, P.; Varriale, P.; De Stefano, M.; Milan, G.; Montella, P.; Sorrentino, G. Spatio-temporal and kinematic gait analysis in patients with Frontotemporal dementia and Alzheimer's disease through 3D motion capture. Gait Posture 2017, 52, 312-317. [CrossRef] [PubMed]

17. Hanson, J.P.; Redfern, M.S.; Mazumdar, M. Predicting slips and falls considering required and available friction. Ergonomics 1999, 42, 1619-1633. [CrossRef] [PubMed] 
18. Nevitt, M.C.; Cummings, S.R.; Hudes, E.S. Risk factors for injurious falls: A prospective study. J. Gerontol. 1991, 46, M164-M170. [CrossRef] [PubMed]

19. Oliver, D.; Daly, F.; Martin, F.C.; McMurdo, M.E. Risk factors and risk assessment tools for falls in hospital in-patients: A systematic review. Age Ageing 2004, 33, 122-130. [CrossRef] [PubMed]

20. Kannus, P.; Palvanen, M.; Niemi, S.; Parkkari, J. Alarming rise in the number and incidence of fall-induced cervical spine injuries among older adults. J. Gerontol. Ser. A Biol. Sci. Med. Sci. 2007, 62, 180-183. [CrossRef]

21. Davis, J.; Robertson, M.; Ashe, M.; Liu-Ambrose, T.; Khan, K.; Marra, C. International comparison of cost of falls in older adults living in the community: A systematic review. Osteoporosi. Int. 2010, 21, 1295-1306. [CrossRef] [PubMed]

22. Peel, N.M.; McClure, R.J.; Hendrikz, J.K. Health-protective behaviours and risk of fall-related hip fractures: a population-based case-Control study. Age Ageing 2006, 35, 491-497. [CrossRef] [PubMed]

23. Gillespie, L.D.; Robertson, M.C.; Gillespie, W.J.; Sherrington, C.; Gates, S.; Clemson, L.M.; Lamb, S.E. Interventions for Preventing Falls in Older People Living in The Community; The Cochrane Library: London, UK, 2012.

24. Cameron, I.D.; Murray, G.R.; Gillespie, L.D.; Robertson, M.C.; Hill, K.D.; Cumming, R.G.; Kerse, N. Interventions for preventing falls in older people in nursing care facilities and hospitals. Cochrane Database Syst. Rev. 2010, 1. [CrossRef]

25. Society, A.G.; Society, G.; Prevention, O.F.; Panel, O.S. Guideline for the prevention of falls in older persons. J. Am. Geriatr. Soc. 2001, 49, 664-672. [CrossRef]

26. Duarte, M.; Freitas, S.M. Revision of posturography based on force plate for balance evaluation. Braz. J. Phys. Ther. 2010, 14, 183-192. [CrossRef]

27. Hamacher, D.; Singh, N.; Van Dieen, J.; Heller, M.; Taylor, W. Kinematic measures for assessing gait stability in elderly individuals: A systematic review. J. R. Soc. Interface 2011, 8, 1682-1698. [CrossRef] [PubMed]

28. Ham, R.; Aldersea, P.; Porter, D. Wheelchair Users and Postural Seating: A Clinical Approach; WB Saunders Company: Philadelphia, PA, USA, 1998.

29. Pope, P. Posture management and special seating. Neurological Physiotherapy; Churchill Livingstone: London, UK, 2002.

30. Terrier, P.; Reynard, F. Effect of age on the variability and stability of gait: A cross-sectional treadmill study in healthy individuals between 20 and 69 years of age. Gait Posture 2015, 41, 170-174. [CrossRef] [PubMed]

31. Horak, F.B. Postural orientation and equilibrium: What do we need to know about neural control of balance to prevent falls? Age Ageing 2006, 35, ii7-ii11. [CrossRef] [PubMed]

32. Lugade, V.; Lin, V.; Chou, L.S. Center of mass and base of support interaction during gait. Gait Posture 2011, 33, 406-411. [CrossRef] [PubMed]

33. Baltich, J.; von Tscharner, V.; Nigg, B.M. Degradation of postural control with aging. Proc. Instit. Mech. Eng. Part H J. Eng. Med. 2015, 229, 638-644. [CrossRef] [PubMed]

34. Porter, S.; Nantel, J. Older adults prioritize postural stability in the anterior-Posterior direction to regain balance following volitional lateral step. Gait Posture 2015, 41, 666-669. [CrossRef] [PubMed]

35. Batista, W.O.; Junior, A.; de Drummond, E.; Porto, F.; Pereira, F.D.; Santana, R.F.; Gurgel, J.L. Influence of the length of institutionalization on older adults' postural balance and risk of falls: A transversal study. Rev. Lat.-Am. Enferm. 2014, 22, 645-653. [CrossRef]

36. Liu, K.; Wang, H.; Xiao, J. The multivariate largest Lyapunov exponent as an age-related metric of quiet standing balance. Comput. Math. Methods Med. 2015, 2015, 309756. [CrossRef] [PubMed]

37. Yang, F.; Pai, Y.C. Can stability really predict an impending slip-related fall among older adults? J. Biomech. 2014, 47, 3876-3881. [CrossRef] [PubMed]

38. Lizama, L.E.C.; Pijnappels, M.; Rispens, S.M.; Reeves, N.P.; Verschueren, S.M.; van Dieën, J.H. Mediolateral balance and gait stability in older adults. Gait Posture 2015, 42, 79-84. [CrossRef] [PubMed]

39. Turcato, A.; Ramat, S. Studying postural sway using wearable sensors: Fall prediction. In Proceedings of the XII Mediterranean Conference on Medical and Biological Engineering and Computing, Chalkidiki, Greece, 27-30 May 2010; pp. 620-623.

40. Deshmukh, P.M.; Russell, C.M.; Lucarino, L.E.; Robinovitch, S.N. Enhancing clinical measures of postural stability with wearable sensors. Conf. Proc. IEEE Eng. Med. Biol. Soc. 2012, 2012, 4521-4524. [PubMed]

41. Similä, H.; Immonen, M.; Ermes, M. Accelerometry-based assessment and detection of early signs of balance deficits. Comput. Biol. Med. 2017, 85, 25-32. [CrossRef] [PubMed] 
42. Aminian, K.; Mariani, B.; Paraschiv-Ionescu, A.; Hoskovec, C.; Büla, C.; Penders, J.; Tacconi, C.; Marcellini, F. Foot worn inertial sensors for gait assessment and rehabilitation based on motorized shoes. Conf. Proc. IEEE Eng. Med. Biol. Soc. 2011, 2011, 5820-5823. [PubMed]

43. Bounyong, S.; Adachi, S.; Ozawa, J.; Yamada, Y.; Kimura, M.; Watanabe, Y.; Yokoyama, K. Fall risk estimation based on co-contraction of lower limb during walking. In Proceedings of the IEEE International Conference on Consumer Electronics (ICCE), Las Vegas, NV, USA, 7-11 January 2016; pp. 331-332.

44. Canning, C.G.; Paul, S.S.; Nieuwboer, A. Prevention of falls in Parkinson's disease: A review of fall risk factors and the role of physical interventions. Neurodegener. Dis. Manag. 2014, 4, 203-221. [CrossRef] [PubMed]

45. Okuma, Y. Freezing of gait and falls in Parkinson's disease. J. Parkinson Dis. 2014, 4, 255-260.

46. Gunn, H.J.; Newell, P.; Haas, B.; Marsden, J.F.; Freeman, J.A. Identification of risk factors for falls in multiple sclerosis: A systematic review and meta-analysis. Phys. Ther. 2013, 93, 504. [CrossRef] [PubMed]

47. Nyberg, L.; Gustafson, Y. Patient falls in stroke rehabilitation: A challenge to rehabilitation strategies. Stroke 1995, 26, 838-842. [CrossRef] [PubMed]

48. Horikawa, E.; Matsui, T.; Arai, H.; Seki, T.; Iwasaki, K.; Sasaki, H. Risk of falls in Alzheimer's disease: A prospective study. Intern. Med. 2005, 44, 717-721. [CrossRef] [PubMed]

49. Sheridan, P.L.; Solomont, J.; Kowall, N.; Hausdorff, J.M. Influence of executive function on locomotor function: Divided attention increases gait variability in Alzheimer's disease. J. Am. Geriatr. Soc. 2003, 51, 1633-1637. [CrossRef] [PubMed]

50. Howcroft, J.; Kofman, J.; Lemaire, E.D. Review of fall risk assessment in geriatric populations using inertial sensors. J. Neuroeng. Rehabil. 2013, 10, 91. [CrossRef] [PubMed]

51. Scanaill, C.N.; Carew, S.; Barralon, P.; Noury, N.; Lyons, D.; Lyons, G.M. A review of approaches to mobility telemonitoring of the elderly in their living environment. Ann. Biomed. Eng. 2006, 34, 547-563. [CrossRef] [PubMed]

52. Shany, T.; Redmond, S.; Marschollek, M.; Lovell, N. Assessing fall risk using wearable sensors: A practical discussion. Z. Gerontol. Geriatr. 2012, 45, 694-706. [CrossRef] [PubMed]

53. Igual, R.; Medrano, C.; Plaza, I. Challenges, issues and trends in fall detection systems. Biomed. Eng. Online 2013, 12, 66. [CrossRef] [PubMed]

54. Chen, H.; Xue, M.; Mei, Z.; Bambang Oetomo, S.; Chen, W. A Review of Wearable Sensor Systems for Monitoring Body Movements of Neonates. Sensors 2016, 16, 2134. [CrossRef] [PubMed]

55. Baloh, R.W.; Fife, T.D.; Zwerling, L.; Socotch, T.; Jacobson, K.; Bell, T.; Beykirch, K. Comparison of static and dynamic posturography in young and older normal people. J. Am. Geriatr. Soc. 1994, 42, 405-412. [CrossRef] [PubMed]

56. Buatois, S.; Gueguen, R.; Gauchard, G.C.; Benetos, A.; Perrin, P.P. Posturography and risk of recurrent falls in healthy non-institutionalized persons aged over 65. Gerontology 2006, 52, 345-352. [CrossRef] [PubMed]

57. Whitney, S.L.; Marchetti, G.F.; Schade, A.I. The relationship between falls history and computerized dynamic posturography in persons with balance and vestibular disorders. Arch. Phys. Med. Rehabil. 2006, 87, 402-407. [CrossRef] [PubMed]

58. Ricci, N.A.; de Faria Figueiredo Gonçalves, D.; Coimbra, A.M.V.; Coimbra, I.B. Sensory interaction on static balance: A comparison concerning the history of falls of community-dwelling elderly. Geriatr. Gerontol. Int. 2009, 9, 165-171. [CrossRef] [PubMed]

59. Maki, B.E. Gait changes in older adults:maki1997gait predictors of falls or indicators of fear? J. Am. Geriatr. Soc. 1997, 45, 313-320. [CrossRef] [PubMed]

60. Shumway-Cook, A.; Brauer, S.; Woollacott, M. Predicting the probability for falls in community-dwelling older adults using the Timed Up \& Go Test. Phys. Ther. 2000, 80, 896-903. [PubMed]

61. Kahle, J.T.; Highsmith, M.J.; Hubbard, S.L. Comparison of nonmicroprocessor knee mechanism versus C-Leg on Prosthesis Evaluation Questionnaire, stumbles, falls, walking tests, stair descent, and knee preference. J. Rehabil. Res. Dev. 2008, 45, 1-14. [CrossRef] [PubMed]

62. Kang, H.G.; Dingwell, J.B. Dynamic stability of superior vs. inferior segments during walking in young and older adults. Gait Posture 2009, 30, 260-263. [CrossRef] [PubMed]

63. Aloqlah, M.; Lahiji, R.; Loparo, K.; Mehregany, M. A headband for classifying human postures. In Proceedings of the Annual International Conference of the IEEE Engineering in Medicine and Biology Society (EMBC), Buenos Aires, Argentina, 31 August-4 September 2010 ; pp. 382-385. 
64. Bertolotti, G.; Cristiani, A.; Colagiorgio, P.; Romano, F.; Bassani, E.; Caramia, N.; Ramat, S. A Wearable and Modular Inertial Unit for Measuring Limb Movements and Balance Control Abilities. IEEE Sens. J. 2016, 16, 3496-3499. [CrossRef]

65. Caldara, M.; Locatelli, P.; Comotti, D.; Dellerma, N.; Corenzi, A.; Pessione, M. Application of a wireless BSN for gait and balance assessment in the elderly. In Proceedings of the IEEE 12th International Conference on Wearable and Implantable Body Sensor Networks (BSN), Cambridge, MA, USA, 9-12 June 2015.

66. Chen, Y.C.; Lin, Y.W. Indoor RFID gait monitoring system for fall detection. In Proceedings of the 2nd International Symposium on Aware Computing (ISAC), Tainan, Taiwan, 1-4 November 2010; pp. 207-212.

67. Cheng, J.; Chen, X.; Shen, M. A Framework for Daily Activity Monitoring and Fall Detection Based on Surface Electromyography and Accelerometer Signals. IEEE J. Biomed. Health Inform. 2013, 17, 38-45. [CrossRef] [PubMed]

68. Cola, G.; Avvenuti, M.; Vecchio, A.; Yang, G.; Lo, B. An On-Node Processing Approach for Anomaly Detection in Gait. IEEE Sens. J. 2015, 15, 6640-6649. [CrossRef]

69. Crispim, C.; Bathrinarayanan, V.; Fosty, B.; Konig, A.; Romdhane, R.; Thonnat, M.; Bremond, F. Evaluation of a monitoring system for event recognition of older people. In Proceedings of the 10th IEEE International Conference on Advanced Video and Signal Based Surveillance, Krakow, Poland, 27-30 August 2013.

70. Curone, D.; Bertolotti, G.M.; Cristiani, A.; Secco, E.L.; Magenes, G. A real-time and self-calibrating algorithm based on triaxial accelerometer signals for the detection of human posture and activity. IEEE Trans. Inf. Technol. Biomed. 2010, 14, 1098-1105. [CrossRef] [PubMed]

71. De la Guia Solaz, M.; Bourke, A.; Conway, R.; Nelson, J.; ÓLaighin, G. Real-time low-energy fall detection algorithm with a Programmable Truncated MAC. Conf. Proc. IEEE Eng. Med. Biol. Soc. 2010, 2010, 2423-2426. [PubMed]

72. Di Rosa, M.; Hausdorff, J.M.; Stara, V.; Rossi, L.; Glynn, L.; Casey, M.; Burkard, S.; Cherubini, A. Concurrent validation of an index to estimate fall risk in community dwelling seniors through a wireless sensor insole system: A pilot study. Gait Posture 2017, 55, 6-11. [CrossRef] [PubMed]

73. Diraco, G.; Leone, A.; Siciliano, P. In-home hierarchical posture classification with a time-of-flight 3D sensor. Gait Posture 2014, 39, 182-187. [CrossRef] [PubMed]

74. Fernandez-Luque, F.J.; Zapata, J.; Ruiz, R. A system for ubiquitous fall monitoring at home via a wireless sensor network. In Proceedings of the annual International Conference of the IEEE Engineering in Medicine and Biology Society (EMBC), Buenos Aires, Argentina, 1-4 September 2010; pp. 2246-2249.

75. Ganea, R.; Paraschiv-lonescu, A.; Aminian, K. Detection and classification of postural transitions in real-world conditions. IEEE Trans. Neural Syst. Rehabil. Eng. 2012, 20, 688-696. [CrossRef] [PubMed]

76. Gopalai, A.A.; Senanayake, S.A.A. A wearable real-time intelligent posture corrective system using vibrotactile feedback. IEEE/ASME Trans. Mechatron. 2011, 16, 827-834. [CrossRef]

77. Greene, B.; McGrath, D.; Foran, T.; Doheny, E.; Caulfield, B. Body-worn sensor based surrogates of minimum ground clearance in elderly fallers and controls. In Proceedings of the Annual International Conference of the IEEE Engineering in Medicine and Biology Society, EMBC, Honolulu, HI, USA, 30 August-3 September 2011; pp. 6499-6502.

78. Hegde, N.; Sazonov, E.S. SmartStep 2.0-A completely wireless, versatile insole monitoring system. In Proceedings of the IEEE International Conference on Bioinformatics and Biomedicine (BIBM), Washington, DC, USA, 9-12 November 2015; pp. 746-749.

79. Howcroft, J.; Kofman, J.; Lemaire, E.D. Feature selection for elderly faller classification based on wearable sensors. J. Neuroeng. Rehabil. 2017, 14, 47. [CrossRef] [PubMed]

80. Howcroft, J.; Kofman, J.; Lemaire, E. Prospective fall-risk prediction models for older adults based on wearable sensors. IEEE Trans. Neural Syst. Rehabil. Eng. 2017, 25, 1812-1820. [CrossRef] [PubMed]

81. Howcroft, J.; Kofman, J.; Lemaire, E.D.; McIlroy, W.E. Analysis of dual-task elderly gait in fallers and non-fallers using wearable sensors. J. Biomech. 2016, 49, 992-1001. [CrossRef] [PubMed]

82. Jian, H.; Chen, H. A portable fall detection and alerting system based on k-NN algorithm and remote medicine. China Commun. 2015, 12, 23-31. [CrossRef]

83. Jiang, S.; Zhang, B.; Wei, D. The elderly fall risk assessment and prediction based on gait analysis. In Proceedings of the IEEE 11th International Conference on Computer and Information Technology (CIT), Pafos, Cyprus, 31 August-2 September 2011; pp. 176-180. 
84. Karel, J.M.; Senden, R.; Janssen, J.E.; Savelberg, H.; Grimm, B.; Heyligers, I.; Peeters, R.; Meijer, K. Towards unobtrusive in vivo monitoring of patients prone to falling. In Proceedings of the Annual International Conference of the IEEE Engineering in Medicine and Biology, Buenos Aires, Argentina, 31 August-4 September 2010; pp. 5018-5021.

85. Micó-Amigo, M.E.; Kingma, I.; Ainsworth, E.; Walgaard, S.; Niessen, M.; van Lummel, R.C.; van Dieën, J.H. A novel accelerometry-based algorithm for the detection of step durations over short episodes of gait in healthy elderly. J. Neuroeng. Rehabil. 2016, 13, 38. [CrossRef] [PubMed]

86. Najafi, B.; Aminian, K.; Loew, F.; Blanc, Y.; Robert, P.A. Measurement of stand-sit and sit-stand transitions using a miniature gyroscope and its application in fall risk evaluation in the elderly. IEEE Trans. Biomed. Eng. 2002, 49, 843-851. [CrossRef] [PubMed]

87. Ozcan, K.; Velipasalar, S. Wearable camera-and accelerometer-based fall detection on portable devices. IEEE Embed. Syst. Lett. 2016, 8, 6-9. [CrossRef]

88. Paoli, R.; Fernández-Luque, F.J.; Doménech, G.; Martínez, F.; Zapata, J.; Ruiz, R. A system for ubiquitous fall monitoring at home via a wireless sensor network and a wearable mote. Expert Syst. Appl. 2012, 39, 5566-5575. [CrossRef]

89. Qu, W.; Lin, F.; Wang, A.; Xu, W. Evaluation of a low-complexity fall detection algorithm on wearable sensor towards falls and fall-alike activities. In Proceedings of the IEEE Signal Processing in Medicine and Biology Symposium (SPMB), Philadelphia, PA, USA, 13 December 2014; pp. 1-6.

90. Sazonov, E.S.; Hegde, N.; Tang, W. Development of SmartStep: An insole-based physical activity monitor. In Proceedings of the 35th Annual International Conference of the IEEE Engineering in Medicine and Biology Society (EMBC), Osaka, Japan, 3-7 July 2013; pp. 7209-7212.

91. Stone, E.E.; Skubic, M. Unobtrusive, continuous, in-home gait measurement using the Microsoft Kinect. IEEE Trans. Biomed. Eng. 2013, 60, 2925-2932. [CrossRef] [PubMed]

92. Szurley, J.; Druzgalski, C. A novel approach in remote monitoring and assessment of patient balance. In Proceedings of the Pan American Health Care Exchanges (PAHCE), Mexico City, Mexico, 16-20 March 2009; p. 143.

93. Tamura, T. Wearable accelerometer in clinical use. In Proceedings of the IEEE-EMBS 27th Annual International Conference of the Engineering in Medicine and Biology Society, Shanghai, China, 17-18 January 2006; pp. 7165-7166.

94. Tang, Y.; Ran, L.; Li, C. A feasibility study on human gait monitoring using a wearable K-band radar. In Proceedings of the 46th European Microwave Conference (EuMC), London, UK, 4-6 Octomber 2016; pp. 918-921.

95. Van de Ven, P.; O’Brien, H.; Nelson, J.; Clifford, A. Unobtrusive monitoring and identification of fall accidents. Med. Eng. Phys. 2015, 37, 499-504. [CrossRef] [PubMed]

96. van Schooten, K.S.; Pijnappels, M.; Rispens, S.M.; Elders, P.J.; Lips, P.; Daffertshofer, A.; Beek, P.J.; van Dieen, J.H. Daily-life gait quality as predictor of falls in older people: A 1-year prospective cohort study. PLoS ONE 2016, 11, e0158623. [CrossRef] [PubMed]

97. Vincenzo, J.L.; Glenn, J.M.; Gray, S.M.; Gray, M. Balance measured by the sway balance smart-device application does not discriminate between older persons with and without a fall history. Aging Clin. Exp. Res. 2016, 28, 679-686. [CrossRef] [PubMed]

98. Yao, M.; Zhang, Q.; Li, M.; Li, H.; Ning, Y.; Xie, G.; Zhao, G.; Ma, Y.; Gao, X.; Jin, Z. A wearable pre-impact fall early warning and protection system based on MEMS inertial sensor and GPRS communication. In Proceedings of the IEEE 12th International Conference on Wearable and Implantable Body Sensor Networks (BSN), Cambridge, MA, USA, 9-12 June 2015; pp. 1-6.

99. Yuan, X.; Yu, S.; Dan, Q.; Wang, G.; Liu, S. Fall detection analysis with wearable MEMS-based sensors. In Proceedings of the 16th International Conference on Electronic Packaging Technology (ICEPT), Changsha, China, 11-14 August 2015; pp. 1184-1187.

100. Perry, J.; Davids, J.R. Gait analysis: Normal and pathological function. J. Pediatr. Orthop. 1992, $12,815$. [CrossRef]

101. Doherty, T.J. Invited review: Aging and sarcopenia. J. Appl. Physiol. 2003, 95, 1717-1727. [CrossRef] [PubMed] 
102. Menz, H.B.; Lord, S.R.; Fitzpatrick, R.C. Acceleration patterns of the head and pelvis when walking on level and irregular surfaces. Gait Posture 2003, 18, 35-46. [CrossRef]

103. Schwenk, M.; Mohler, J.; Wendel, C.; D”Huyvetter, K.; Fain, M.; Taylor-Piliae, R.; Najafi, B. Wearable sensor-based in-home assessment of gait, balance, and physical activity for discrimination of frailty status: Baseline results of the Arizona frailty cohort study. Gerontology 2015, 61, 258-267. [CrossRef] [PubMed]

(C) 2018 by the authors. Licensee MDPI, Basel, Switzerland. This article is an open access article distributed under the terms and conditions of the Creative Commons Attribution (CC BY) license (http:/ / creativecommons.org/licenses/by/4.0/). 


\title{
Deep Learning to Predict Falls in Older Adults Based on Daily-Life Trunk Accelerometry
}

\author{
Ahmed Nait Aicha ${ }^{1, *}$, Gwenn Englebienne ${ }^{2}$, Kimberley S. van Schooten ${ }^{3}$, Mirjam Pijnappels ${ }^{4}$ \\ and Ben Kröse ${ }^{1,5}$ \\ 1 Department of Computer Science, Amsterdam University of Applied Sciences, 1091 GM Amsterdam, \\ The Netherlands; b.j.a.krose@hva.nl \\ 2 Human Media Interaction, University of Twente, 7522 NH Enschede, The Netherlands; \\ englebienne@gmail.com \\ 3 Neuroscience Research Australia, University of New South Wales, Sydney 2031, Australia; \\ k.vanschooten@neura.edu.au \\ 4 Department of Human Movement Sciences, Vrije Universiteit Amsterdam, 1081 HV Amsterdam, \\ The Netherlands; m.pijnappels@vu.nl \\ 5 Informatics Institute, University of Amsterdam, 1098 XH Amsterdam, The Netherlands \\ * Correspondence: a.nait.aicha@hva.nl
}

Received: 31 March 2018; Accepted: 18 May 2018; Published: 22 May 2018

\begin{abstract}
Early detection of high fall risk is an essential component of fall prevention in older adults. Wearable sensors can provide valuable insight into daily-life activities; biomechanical features extracted from such inertial data have been shown to be of added value for the assessment of fall risk. Body-worn sensors such as accelerometers can provide valuable insight into fall risk. Currently, biomechanical features derived from accelerometer data are used for the assessment of fall risk. Here, we studied whether deep learning methods from machine learning are suited to automatically derive features from raw accelerometer data that assess fall risk. We used an existing dataset of 296 older adults. We compared the performance of three deep learning model architectures (convolutional neural network (CNN), long short-term memory (LSTM) and a combination of these two (ConvLSTM)) to each other and to a baseline model with biomechanical features on the same dataset. The results show that the deep learning models in a single-task learning mode are strong in recognition of identity of the subject, but that these models only slightly outperform the baseline method on fall risk assessment. When using multi-task learning, with gender and age as auxiliary tasks, deep learning models perform better. We also found that preprocessing of the data resulted in the best performance (AUC $=0.75$ ). We conclude that deep learning models, and in particular multi-task learning, effectively assess fall risk on the basis of wearable sensor data.
\end{abstract}

Keywords: accidental falls; older adults; machine learning; neural networks; convolutional neural network; long short-term memory; accelerometry

\section{Introduction}

Falls among older adults are one of the major health problems that lead to a decreased quality of life and increased morbidity and mortality. In addition, falls pose high costs to the public health service. Risk factors for falls include weak muscles, unsteady gait, cognitive decline, and psychoactive medications. Early detection and monitoring of fall risk factors can significantly reduce the risk of future falls [1,2]. Among these factors, history of falls and of gait and balance disorders have been identified as strong predictors [3].

Fall risk assessment is a process in which the probability of a future fall is estimated, usually within a time frame of 6-12 months. In many intervention programs proposed for fall prevention, 
fall risk assessment is performed as the initial step to identify persons at highest risk. The assessment of fall risk is commonly conducted in a clinical setting and based on questionnaires and functional tests of mobility such as the Timed Up and Go (TUG) [4], the Performance Oriented Mobility Assessment (POMA) [5], or the Berg Balance Scale test [6]. Although these tests provide a good indication of one's optimal mobility and performance, their predictive ability for prospective falls is limited (e.g., [7]), possibly because this optimal ability might not be representative of one's use in daily life behavior.

In previous research, we studied the use of ambient sensors for the continuous monitoring of human activities in their natural environment [8,9]. In this paper, we focus on body-worn inertial sensors that are used in many research studies on the ambulatory monitoring of humans in daily life providing reliable insight into an individual's daily activities and gait quality characteristics [10].

Much research is done in the characterization of the quantity of movement of subjects, including the duration of low-, moderate-, and high-intensity activities, the total number of daily steps, and the daily percentage of time spent lying, sitting, standing, and walking [10]. Recent research showed the added value of the characterization of one's quality of movement in the determination of fall risk in older adults [11]. These studies revealed that biomechanical features such as gait stability, variability, and smoothness [12,13], but also mean turn duration [14] and the number of abnormal sit-to-stand transitions [15], are associated with fall risk. However, estimation of these features often requires event detection, which is in need of improvement, and may not currently exploit the wealth of information that has been collected. On the other hand, deep learning allows for the data-driven generation of features and does not suffer from these shortcomings.

In machine learning, deep convolutional and long short-term memory (LSTM) recurrent neural networks have shown to be successful for the recognition of activities [16] and gait patterns [17] from inertial sensor data. However, the assessment of fall risk with such models has not been done before. The contributions of this paper are (a) a comparison of the performance of deep learning models for the assessment of fall risk with a baseline model based on biomechanical features using a large data set of 296 subjects and (b) the extension and testing of these models with multi-task learning to improve their performance.

\section{Sensor Data}

The data used in this paper were collected between March 2011 and January 2014 as part of the fall risk assessment in older adults (FARAO) cohort study performed at the Vrije Universiteit Amsterdam. The FARAO study collected data on fall risk factors in older adults with questionnaires, physical tests, and wearable sensors. Participants in the cohort were between 65 and 99 years of age, had a mini mental state examination score (MMSE [18]) between 19 and 30, and were able to walk at least $20 \mathrm{~m}$ with the aid of an assistive device, if needed. We re-analyzed the data described in [21], which consisted of a population of 296 older adults. These participants wore a triaxial accelerometer (Dynaport MoveMonitor, McRoberts) on their lower back, which registered 3D trunk accelerations at $100 \mathrm{~Hz}$ and \pm 6 G, for 1 week. During a 6-month follow-up period in which fall incidences and descriptions were obtained monthly, 101 subjects $(34.1 \%)$ had experienced at least one fall and were identified as fallers. Table 1 provides an overview of the descriptive characteristics of the population. A detailed description of the population and the methods for data collection can be found in $[11,19,21]$.

Table 1. Descriptive statistics of the population.

\begin{tabular}{ccccc}
\hline & Male (\%) & Age (Years) & Weight $\mathbf{( k g )}$ & Height (cm) \\
\hline Mean & 74.1 & 75.3 & 49.2 & 170.6 \\
Standard deviation & - & 6.8 & 13.3 & 8.8 \\
25\% Quantile & - & 70.0 & 64.0 & 165.0 \\
75\% Quantile & - & 80.0 & 81.8 & 176.0 \\
\hline
\end{tabular}


Participants were instructed to wear the accelerometer with an elastic belt around their lower back at all times, except during aquatic activities such as showering. The distribution of the total time that the sensor was worn for fallers and non-fallers was similar. Bouts of non-wearing, locomotion, sitting, lying, and standing were identified using the manufacturer's activity classification algorithm [20]. Only the locomotion bouts were analyzed in the current study. For each locomotion bout, the acceleration in three directions (i.e., anteroposterior (AP), mediolateral (ML), and vertical (VT)) was recorded. Figure 1 shows two examples of locomotion bouts lasting $10 \mathrm{~s}$ each.

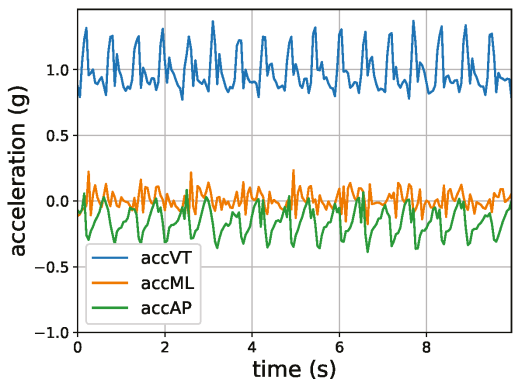

(a)

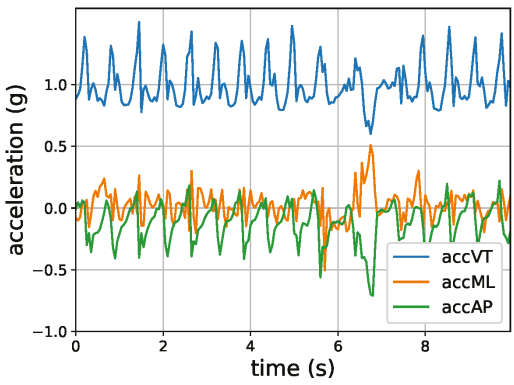

(b)

Figure 1. An example of two locomotion samples. (a) A typical walking sample; (b) A walking sample interwoven with a turning activity at $7 \mathrm{~s}$. accAP: acceleration in the anteroposterior direction; accML: acceleration in the mediolateral direction; accVT: acceleration in the vertical direction.

\section{Approach}

On the basis of this data set containing bouts of accelerometer data from 296 participants and the identification of the participants into fall or non-fall categories, a model was made that predicts falls from accelerometer data. In van Schooten et al. [21], a linear model was used, based on biomechanical features from the accelerometer data. In this paper, we used deep neural networks. Deep learning allows for the creation of computational models that are composed of multiple processing layers and learn representations of data with multiple levels of abstraction [22]. This can result in more powerful models, because the complexity of the feature computations are dictated directly by the data and by the quality of the model predictions, rather than by the preconceptions of the operator. On the other hand, no prior knowledge is leveraged in the creation of the model, so it is useful to compare deep learning approaches to traditional machine learning methods.

We evaluated two types of deep neural network (DNN) for the analysis of fall risk. First, we considered the convolutional neural network (CNN), which constrains the number of parameters by sharing parameter values in different parts of the network. It has been used with great success in speech recognition [23] and in the detection, segmentation, and recognition of objects and regions in images [24,25]. We then looked at the long short-term memory (LSTM) model, a specific type of recurrent neural network (RNN). RNNs specifically model sequential inputs such as speech and language [26,27]. In this work, we used a model that combines convolutional and recurrent models, which we refer to as the "ConvLSTM".

We trained the model parameters and evaluated the resulting models by minimizing the loss, a function that expresses how many prediction errors the model makes, and evaluated the models for different values of their so-called "hyper-parameters", which include the number of layers and the number of nodes in each layer, based on their the receiver operating characteristic (ROC) curves. The models can make different types of errors, as well as false positive and false negative predictions, and a single model can be tweaked to minimize one type of error at the expense of the other. The ROC curve shows the model's performance for multiple choices of this trade-off. The area under the ROC 
curve (AUC) is a robust metric of a model's performance. The training, validation, and testing of the DNN was performed on a Distributed ASCI Supercomputer 5 (DAS-5) server [28].

\section{Deep Learning Neural Network Models}

\subsection{Feed-Forward Neural Networks}

Deep neural networks (DNNs) consist of large numbers of simple processing modules, the "neurons", which compute a fixed function-the "activation function" - of the weighted sum of their inputs and are organized in separate layers. The simplicity of the neurons make network training possible, while the large number of nodes and their organization in a large number of layers allows them to perform complex tasks. DNNs have the ability to learn representations of the training data and relate them to the output variable(s) that we train them to predict. An example of a DNN consisting of two hidden layers is given in Figure 2. The number of nodes in the input layer is determined by the dimensionality of the data, while the number of nodes in the output layer is determined by the chosen representation of the intended prediction. The structure of the network is determined by the complexity of the task being predicted. In addition to the number of nodes and layers, the connections between layers affect the complexity of the network. In a dense layer, each neuron is connected to all neurons of the previous layer and has its own set of weights. In a convolutional layer, a neuron is connected to a subset of the neurons in the previous layer, and shares its weights with the other neurons of that layer.

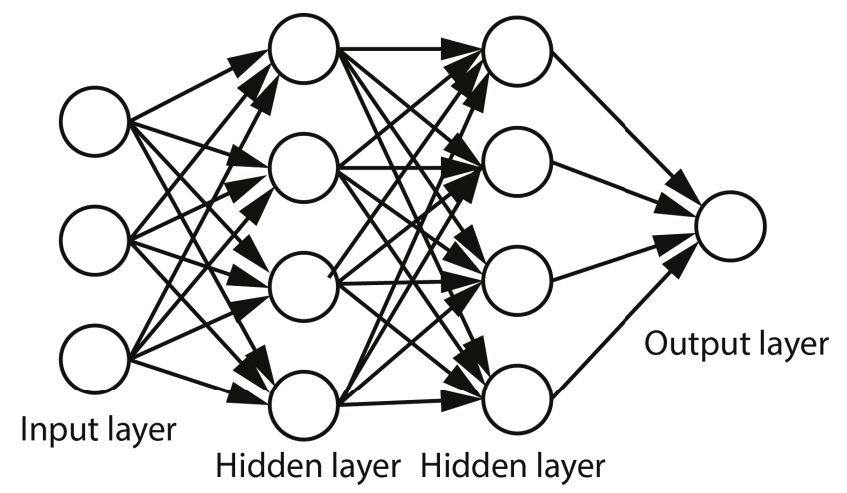

Figure 2. A three-layer neural network with three input neurons, two hidden layers of four neurons each, and one output layer.

\subsection{Long Short-Term Memory (LSTM) Network}

Recurrent neural networks are a type of neural network where inputs are organized sequentially, and the output at time $t$ is connected to all inputs from time 0 to $t$ (Figure 3a). Such a network is still a feed-forward network, but the number of layers between an output and previous inputs increases as the time difference increases. In practice, the training of recurrent neural networks (RNNs) with long-term temporal dependencies can be problematic because the gradient of the loss function decays exponentially with the number of layers and, therefore, with time [29]. LSTM networks, introduced by Hochreiter and Schmidhuber [30], are a type of RNN that uses special units to solve this so-called vanishing gradient problem by "gating" the propagation of information over time. They extend RNNs with memory blocks (Figure $3 \mathrm{~b}$ ) to store information, easing the learning of temporal relationships over long time periods. 


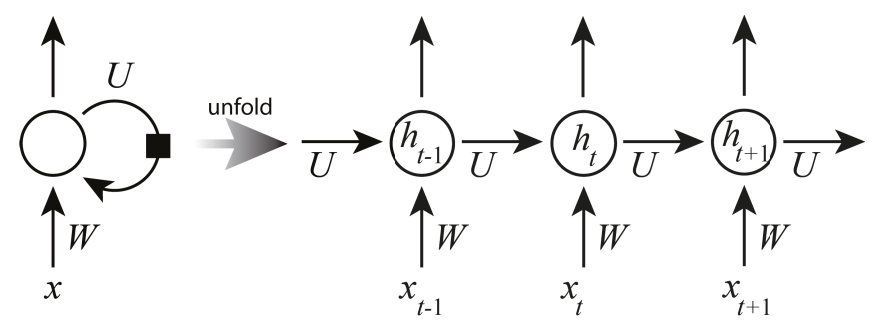

(a) RNN

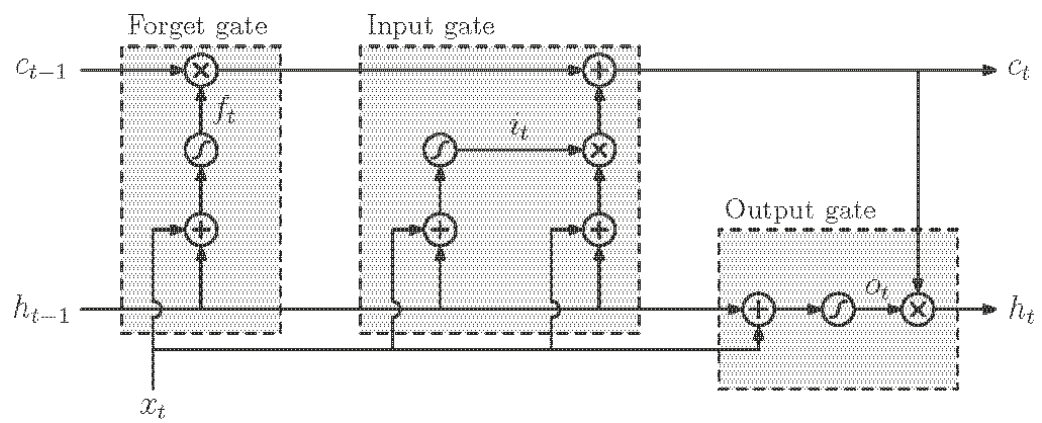

(b) LSTM

Figure 3. (a) A cyclic connection of a recurrent neural network (RNN) folded and unfolded. (b) An long short-term memory (LSTM) memory block consisting of one cell at time $t$ and the three gates $\left(i_{t}, o_{t}\right.$, and $\left.f_{t}\right)$ which control the activation of the cell $c_{t}$ and its output $h_{t}$.

\subsection{Multi-Task Learning}

Multi-task learning (MTL) has been proposed by Caruana [31] to learn several related tasks by a single model. Having a network learn multiple tasks increases the complexity of the function it computes, but when the tasks are related, the models can share parameters. The complexity of a network performing multiple tasks is then lower than the complexity of multiple networks learning the tasks separately. In addition, the fact that tasks essentially compete for the resources of the network tends to force the network to avoid modeling non-essential aspects of the problem, thereby also improving the performance on the individual tasks.

\section{Experiments and Results}

We conducted a set of five experiments to evaluate the presented approach. In the first experiment, we compared deep neural networks (DNNs) with the current state-of-the-art model described in Section 5.1, which relies on manually engineered feature extraction. In the second experiment, we investigated the performance of DNNs in the prediction of fall status at the sample level (i.e., when allowing the model to train and test on different data from the same person), and show drastically improved results. In the third experiment, we explored whether these improvements are due to the model learning to identify people from their gait, rather than from better modeling of fall risk. We observed that the model is capable of identifying people from their gait, but that this does not by itself explain all of the performance increase. In the fourth experiment, we therefore explored how person-specific but not fall-related information can improve the model. We showed that multi-task learning improved fall prediction. Finally, in the fifth experiment, we showed how improving the focus of the model on cleaner data further improved the overall prediction performance. To train 
a model and calculate its performance, the complete dataset was split into a training and a validation set $(90 \%)$ and a test set $(10 \%)$.

\subsection{Experiment 1}

We compared the performance of three types of DNN using raw inputs to the performance of the state-of-the-art model. This base model was previously described by van Schooten et al. [21], and is based on a dataset of ten-second gait samples from which several features such as walking speed, variability, smoothness, and complexity were extracted. Principal components analysis (PCA) was applied to these features (as well as other parameters obtained from questionnaires and tests), keeping 18 principal components, and a multivariate model was developed to predict time to prospective falls. The median of a person's ten-second segments' predictions provided that person's risk assessment. This base model resulted in a performance of AUC $=0.67$ ( $95 \%$ confidence interval $[0.59,0.73])$ at 6 months [21].

The same complete dataset was randomly split into three subsets (training, validation, and testing) at the subject level, where all the 10-s samples of a subject $A$ occur in a single subset. The ratio of fallers to non-fallers was approximately the same in these three sets. The DNNs were given 10-s samples $x$ and the corresponding faller/non-faller label $y \in\{0,1\}$ for training and testing. For each sample $x$, the predicted value using a DNN architecture was denoted by $\hat{y}$. The median of the predicted values for all of a subject's samples was used as the predicted value for that subject. The subjects' predicted values and their actual values (label) were used to plot the ROC and to calculate the corresponding AUC. Figure 4 shows an illustration of predicted values $(\hat{y})$ for multiple 10-s sequences grouped by subject.

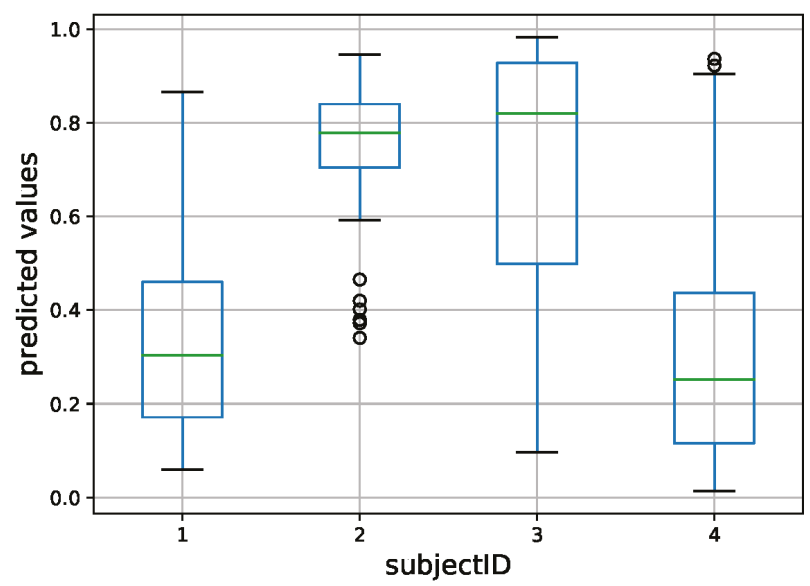

Figure 4. Example boxplots of the normalized predicted values $(\hat{y})$ for multiple 10 -s sequences, grouped by subject. Subjects 1 and 4 were non-fallers and the other two were fallers. The final prediction per subject was given by the median of the predictions, as per van Schooten et al. [21]. The green line inside the box represents the median, the box represents the range of first and third quartile and circles represent outliers.

As described in Section 3, three types of DNN architectures (CNN, LSTM, and ConvLSTM) were applied to a small set of the data to determine the best-fitting model. The models were trained by minimizing the binary cross-entropy loss function (Figure 5a), and evaluated in terms of the area under the ROC curve for each subject (Figure 6a). The corresponding AUC was used to measure the performance of the models (Figure 7a).

From these, we can conclude that the LSTM and ConvLSTM architectures resulted in a slightly better performance than the CNN architecture ( $p$-values were, respectively, 0.056 and 0.022 ) and that 
there was no significant difference in the performance between LSTM and ConvLSTM $(p=0.480)$. The time needed for the training of the LSTMs was very long compared to the ConvLSTM architecture (Table 2), because two or more LSTM layers were used in the LSTM architecture while the ConvLSTM architecture was set to have exactly one LSTM layer. For this reason, we selected a ConvLSTM architecture and its corresponding hyper parameters to be trained on larger datasets. Table 3 illustrates the architecture of the ConvLSTM type used. The AUC and the corresponding training time of this architecture are given in Table 4.

We compared the performance, in terms of average AUC, of the best-fitting model to the base model using a $z$-test, and found no statistically significant difference $(p=0.209)$. In addition, the results also showed a poor generalization ability of the DNN model when trained at the subject level, as indicated by the gap between the two loss functions in Figure 5a. Perhaps the model learned concepts from the training data that did not apply to the test data and therefore negatively impacted the performance of the model. For the investigation of the cause of this generalization problem, we conducted a second experiment, where we applied the same types of DNNs on different training and testing subsets.

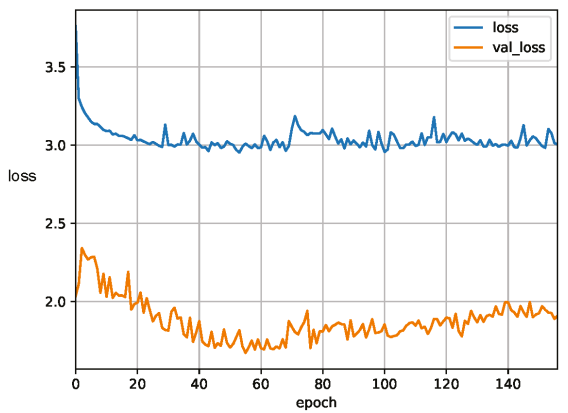

(a) subject level

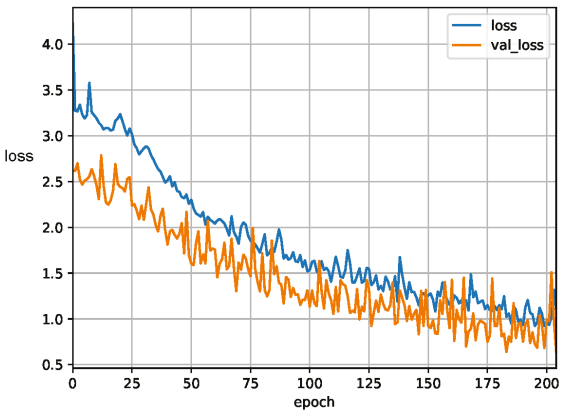

(b) sample level

Figure 5. A typical loss versus epoch graph during the training of a deep neural network (DNN). The data has been split at (a) subject level or (b) sample level. Loss is the training loss and val_loss is the validation loss. The gap between the training and validation loss indicates the amount of over-fitting.

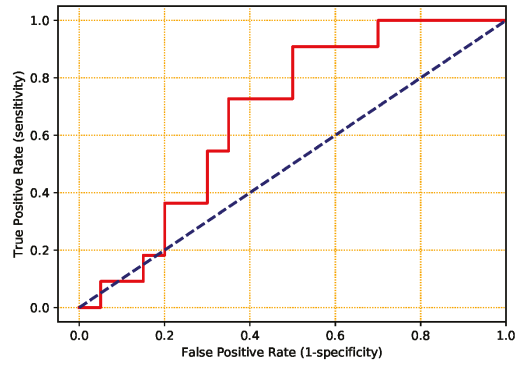

(a) Subject level, $\mathrm{AUC}=0.65$, chance $=0.50$

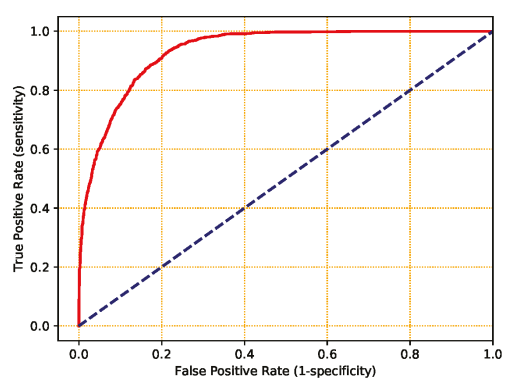

(b) Sample level, $\mathrm{AUC}=0.94$, chance $=0.50$

Figure 6. Examples of the receiver operating characteristic (ROC) curves (solid red lines) and their corresponding area under the curve (AUC) values obtained using a ConvLSTM model. The dashed blue line represents the ROC for chance. The dataset was split at (a) the subject level and (b) the sample level. 
Table 2. Average AUC (standard deviation) and corresponding average training time per neural network (NN) architecture type for a subset of the data. The difference in training time between the ways of splitting the data is due to the slower convergence when splitting at the sample level.

\begin{tabular}{ccccc}
\hline & \multicolumn{2}{c}{ Subject Level } & \multicolumn{2}{c}{ Sample Level } \\
\cline { 2 - 5 } & AUC & Time (h) & AUC & Time (h) \\
\hline CNN & $0.52(0.07)$ & 6 & $0.74(0.07)$ & 7 \\
LSTM & $0.61(0.10)$ & 160 & $0.91(0.06)$ & 180 \\
ConvLSTM & $0.60(0.09)$ & 35 & $0.90(0.05)$ & 40 \\
\hline
\end{tabular}

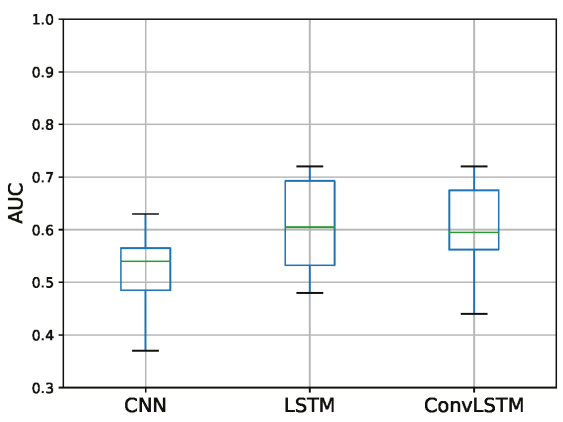

(a) subject level

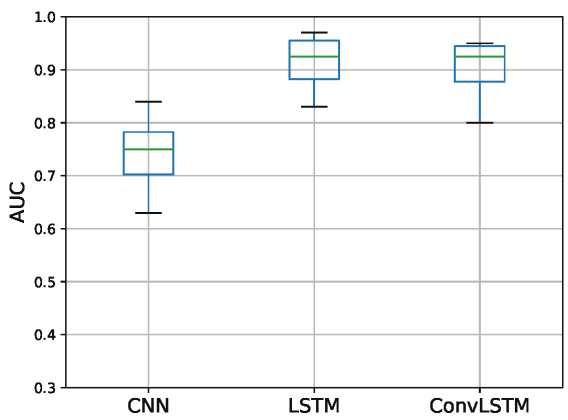

(b) sample level

Figure 7. A boxplot of the AUCs of different DNN architectures. For the LSTM architecture, at least two LSTM layers were involved, while for the ConvLSTM architecture, only one LSTM layer was involved. The dataset was split at (a) the subject level and (b) the sample level.

Table 3. The ConvLSTM architecture (ConvLSTM is our proposed model that combines convolutional and recurrent models). To keep the architecture clear, we omitted the input layer (layer 00) and the dropout layers (the even layer indices) applied after each convolutional neural network (CNN) layer. $N$ was set to 128 .

\begin{tabular}{cccccccc}
\hline Layer Index & $\mathbf{0 1}$ & $\mathbf{0 3}$ & $\mathbf{0 5}$ & $\mathbf{0 7}$ & $\mathbf{0 9}$ & $\mathbf{1 1}$ & $\mathbf{1 2}$ \\
\hline type of filter & $\mathrm{CNN}$ & $\mathrm{CNN}$ & $\mathrm{CNN}$ & $\mathrm{CNN}$ & $\mathrm{CNN}$ & LSTM & Dense \\
number of filters & $N$ & $N$ & $N$ & $\frac{3}{4} N$ & $\frac{3}{4} N$ & $N$ & 2 \\
\hline
\end{tabular}

Table 4. Average AUC, training duration, and number of folds obtained when applying the ConvLSTM model to different dataset sizes. The dataset was cut into three subsets at the subject level.

\begin{tabular}{cccccc}
\hline & \multicolumn{5}{c}{ Dataset Size in Minutes } \\
\cline { 2 - 6 } & $\mathbf{1 0}$ & $\mathbf{3 0}$ & $\mathbf{6 0}$ & $\mathbf{1 2 0}$ & Complete Dataset \\
\hline Average AUC & 0.61 & 0.63 & 0.65 & 0.65 & 0.65 \\
Training duration (h) & 35 & 90 & 150 & 250 & 350 \\
Number of folds & 10 & 10 & 10 & 2 & 1 \\
\hline
\end{tabular}

\subsection{Experiment 2}

For this experiment, the complete dataset was randomly split into three subsets (training, validation, and testing) again, but now at the sample level. As a consequence, there was only a small chance that all of the 10-s samples of a single subject were allocated to only one subset. As in the first experiment, we tested three DNN architectures on a small set of the data to identify the best-performing architecture. The ConvLSTM architecture again resulted in the best trade-off between performance and 
training time (Figures $6 \mathrm{~b}$ and $7 \mathrm{~b}$ ). A $t$-test showed that both LSTM and ConvLSTM had a significantly better performance than CNN $(p<0.002)$, and there was no significant difference between LSTM and ConvLSTM (0.580). Furthermore, this experiment resulted in a better performance than the previous experiment, as shown in Table 5.

Table 5. Average AUC and the corresponding standard deviation when splitting at sample or subject levels.

\begin{tabular}{lcc}
\hline & \multicolumn{2}{c}{ AUC } \\
\cline { 2 - 3 } & Average & Standard Deviation \\
\hline subject level & 0.65 & 0.09 \\
sample level & 0.94 & 0.07 \\
\hline
\end{tabular}

The high AUC when splitting the data at the sample level compared to the subject level can be explained by the smaller within-subject, compared to between-subject, variability of gait. However, another explanation may be that the model learns to identify subjects better than it recognizes characteristics indicating fall risk (since the same subjects were present in training and testing sets, the model could map their identity to fall risk). In the third experiment, we checked the model's ability to identify subjects' gait signatures.

\subsection{Experiment 3}

We again split the dataset into three subsets at the sample level. To learn subject signatures together with their fall risk, we used multi-task learning (MTL): fall risk was the main task, while the identity of the subject was the auxiliary task. We used the same ConvLSTM architecture as in Table 3, because of its good trade-off between performance and learning time in the previous experiments, with an additional dense output layer (connected to Layer 11) for the auxiliary task. The overall loss of the network is a weighted sum of the losses on the main and auxiliary tasks. Ten-fold cross-validation was used to calculate the performance of both the main and auxiliary tasks. The performance of the auxiliary task, which identified the person out of the 296 in the dataset, was evaluated with a plot of the ROC for each subject in a one-versus-all approach. The ROC of the main task and, for clarity, a random sample of the ROCs for the auxiliary task are shown in Figure 8.

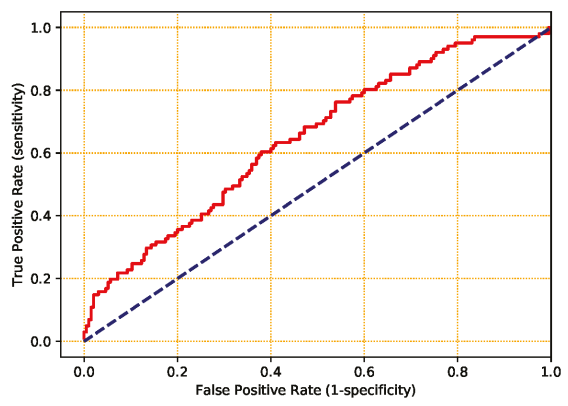

(a) Main task, $\mathrm{AUC}=0.63$, chance $=0.50$

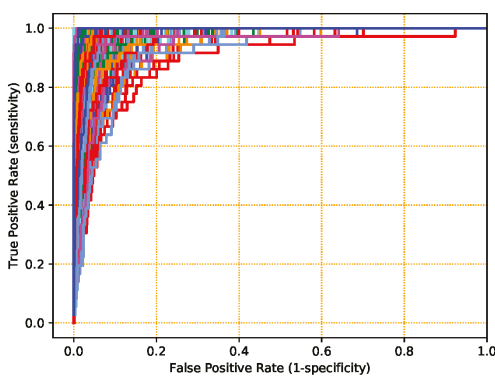

(b) Auxiliary task, average AUC $=0.97$

Figure 8. Cont. 


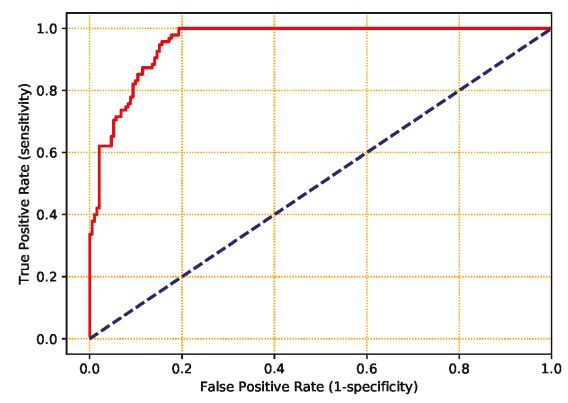

(c) Main task, $\mathrm{AUC}=0.96$, chance $=0.50$

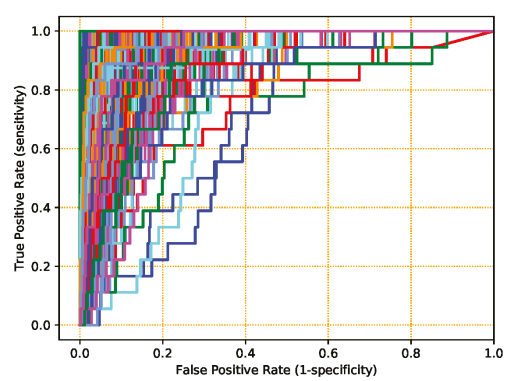

(d) Auxiliary task, average AUC $=0.91$

Figure 8. A sample of obtained ROCs for multi-task deep learning (MTDL) with fall status as the main task and subject identity as the auxiliary task. For the auxiliary task, the ROCs were computed using one-versus-all. The corresponding average AUC is reported. For $(\mathbf{a}, \mathbf{b})$, the main and auxiliary losses were given the same weight $(1: 1)$; for $(\mathbf{c}, \mathbf{d})$, the main loss function was given higher weight $\left(10^{4}: 1\right)$ than the auxiliary loss function. The dashed blue lines in $(\mathbf{a}, \mathbf{c})$ represent the chance ROC.

As we can see, when both tasks were given the same weight (Figure 8a,b), the network was exceedingly good at recognizing identities, but not as good at predicting fall risk. The network had the information to learn the mapping from identity to risk, but not the information capacity to learn this mapping. Therefore, when we increase the weight of the main task, the network becomes better at predicting fall risk at the expense of the identification task (Figure 8c,d). From this, we could conclude that there were important differences between subjects, but that there were other informative patterns in the data, which led us to our next experiment.

\subsection{Experiment 4}

In this experiment, we investigated the effect of MTDL on the model performance. When we split the data at the subject level, it makes no sense to use subject ID as the auxiliary task (since the IDs in the test set are never seen during training), but other subject characteristics can form an informative auxiliary task. The experimental setup is similar to the previous experiment, except that the data was split at the subject level and the auxiliary task was one of the following subject characteristics: age, gender, weight, and height. Table 6 shows the average AUC and the corresponding standard deviation of the main task (fall status). We can conclude that MTDL consistently resulted in improved performance compared to the single-task learning used in the first experiment. However, the improvement was not significant when compared to the base model.

Table 6. Average AUC and the corresponding standard deviation of the main task (fall status), obtained when the ConvLSTM is applied to the test set. The $p$-value was obtained using the $z$-test to test the difference in the performance to the base model.

\begin{tabular}{ccccc}
\hline \multirow{2}{*}{ Characteristic } & \multicolumn{2}{c}{ AUC Main Task (std dev) } & $p$-Value Diff to Base Model \\
\cline { 2 - 5 } & Experiment 4 & Experiment 5 & Experiment 4 & Experiment 5 \\
\hline Gender & $0.70(0.06)$ & $0.75(0.05)$ & 0.070 & $<0.001$ \\
Age & $0.70(0.05)$ & $0.74(0.05)$ & 0.082 & $<0.001$ \\
Weight & $0.68(0.05)$ & $0.72(0.05)$ & 0.306 & 0.005 \\
Height & $0.63(0.06)$ & $0.65(0.06)$ & 0.987 & 0.897 \\
\hline
\end{tabular}




\subsection{Experiment 5}

In the previous experiments, we used the exact same 10-s data segments as found in van Schooten et al. [21], which consist of samples of locomotion as identified by the accelerometer manufacturer's algorithm [20]. As the data were collected in a daily living environment, the locomotion bouts may contain some "non-gait" data samples, which may have negatively affected the performance of the DNNs. Visual inspection of the data indeed suggested the presence of such data samples. These data samples correspond to cyclic accelerations of the trunk without taking clear steps (e.g., when riding a bike) or involve only a few steps (e.g., when moving in the kitchen while preparing a meal). The objective of this experiment was to investigate the effect of conservatively selecting gait data samples on the performance of the models. To do so, 10-s data samples having a very low dominant frequency in the vertical direction (VT-axis) $(\leq 0.2 \mathrm{~Hz})$ were removed from the data, resulting in approximately $20 \%$ discarded data. An example of such included and excluded samples is shown in Figure 9. A procedure similar to Experiment 4 was followed to train, test, and calculate the performance of the ConvLSTM model. Table 6 shows the obtained average AUC and the corresponding standard deviation of the main task. It should be noted that, although different data were used for both training and testing, the results are per subject. Therefore, for the same subjects, they are comparable. Comparing these results with those of Experiment 4, we may conclude that the excluded samples did have a negative effect on the performance of the DNNs. The obtained results of the z-test showed that this model resulted in a significant improvement compared to the performance of the base model. These results suggest that improvement in fall prediction based on accelerometry is not only warranted on the modeling side, but also on the input (or activity classification) side.

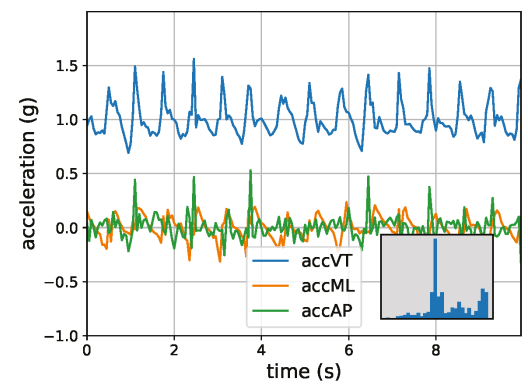

(a) gait

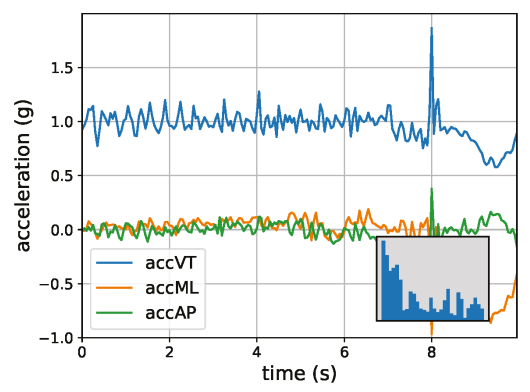

(b) non-gait

Figure 9. (a) An example of a 10-s data sample included in the training and testing set and (b) an example of a data sample excluded in Experiment 5 due to the low dominant frequencies in the VT-axis. In the bottom-right corners, histograms of the VT-frequencies up to $3 \mathrm{~Hz}$ are depicted. Both examples were included in the first 4 experiments.

\section{Discussion and Conclusions}

In this paper, we studied the use of deep learning neural networks to model fall risk on the basis of accelerometer data. Our aim was to compare the performance of deep learning on raw acceleration data with the performance of a base model that uses biomechanical features extracted from the data. For this comparison, we used the same dataset. We did not compare our approach with other work done on different tasks such as activity recognition [16] or age-related differences in walking [17].

In our first experiment, we selected the ConvLSTM neural model based on its trade-off between performance and training time. However, although we found that this architecture was best in modeling the training data, it generalized poorly over subjects. This was confirmed in Experiment 2, where we achieved a very good performance (AUC $=0.94)$ when the training and validation sets contained data, split at samples, from all 296 subjects. The very good performance in this case may have 
been caused by the network learning identities of subjects from gait data and using these implicitly to model individual fall risk. In Experiment 3, we studied an MTL network that simultaneously modeled fall risk and identity. We inferred the need to control for subject-specific factors, since training for both fall risk and identity improved the model's performance considerably. In Experiment 4, we studied an MTL approach where as auxiliary task we chose more general characteristics such as age or weight as secondary tasks that were still related to the subject, but were not the subject itself. We found an improvement of the performance of the ConvLSTM model on the validation set of new subjects if we used gender and age as auxiliary outputs. When we compared the performance of our MTL ConvLSTM with the base model of van Schooten et al. [11], we saw a slightly higher performance. However, this was not significant. Nevertheless, our results indicate that deep learning methods provide similar high accuracy of fall risk prediction compared to biomechanical models, with the advantage that they do not require painstakingly crafted features.

The performance of a model relies on the model architecture used and on the input data. In Experiment 5, we therefore studied an approach where we selectively ignored some of the data samples based on a spectral analysis. We found a significantly better performance. These results suggest that a stricter gait classification algorithm may result in more accurate identification of an individual's gait signature and therefore improve model performance. Another option is to use the dynamics in the data over periods longer than $10 \mathrm{~s}$. This can be done by using the entire locomotion bout as input for the ConvLSTM network. Another method is adopting hierarchical methods [32].

In conclusion, this work shows that machine learning on accelerometer data acquired in the home environment provides comparable accuracy to conventional models in the assessment of fall risk of older adults, with the advantage that they do not rely on handcrafted extracted features. We believe that this approach will contribute to the societal challenge of healthy and active aging in the home environment.

Author Contributions: Conceptualization, A.N.A., G.E., K.S.v.S., M.P. and B.K.; Methodology, A.N.A., G.E., K.S.v.S., M.P. and B.K.; Software, A.N.A. and K.S.v.S.; Validation, A.N.A.; Formal Analysis, A.N.A., G.E. and B.K.; Investigation, A.N.A., G.E., K.S.v.S. and M.P.; Resources, K.S.v.S., M.P. and B.K.; Data Curation, K.S.v.S. and M.P.; Writing-Original Draft Preparation, A.N.A., G.E., K.S.v.S., M.P. and B.K.; Writing-Review \& Editing, A.N.A., G.E., K.S.v.S., M.P. and B.K.; Visualization, A.N.A. and G.E.; Supervision, G.E., K.S.v.S., M.P. and B.K.; Project Administration, A.N.A., K.S.v.S., M.P. and B.K.; Funding Acquisition, K.S.v.S., M.P. and B.K.

Acknowledgments: This work is part of the research project BRAVO, which is (partly) financed by the the Dutch research funding organization SIA. The authors would like to thank the participants of the project.

Conflicts of Interest: The authors declare no conflict of interest.

\section{References}

1. Ambrose, A.F.; Paul, G.; Hausdorff, J.M. Risk factors for falls among older adults: A review of the literature. Maturitas 2013, 75, 51-61. [CrossRef] [PubMed]

2. Rubenstein, L.Z. Falls in older people: Epidemiology, risk factors and strategies for prevention. Age Ageing 2006, 35, ii37-ii41. [CrossRef] [PubMed]

3. Deandrea, S.; Lucenteforte, E.; Bravi, F.; Foschi, R.; La Vecchia, C.; Negri, E. Review Article: Risk Factors for Falls in Community-dwelling Older People: A Systematic Review and Meta-analysis. Epidemiology 2010, 21, 658-668. [CrossRef] [PubMed]

4. Podsiadlo, D.; Richardson, S. The Timed Up \& Go: A Test of Basic Functional Mobility for Frail Elderly Persons. J. Am. Geriatr. Soc. 1991, 39, 142-148. [PubMed]

5. Tinetti, M.E. Performance-Oriented Assessment of Mobility Problems in Elderly Patients. J. Am. Geriatr. Soc. 1986, 34, 119-126. [CrossRef] [PubMed]

6. Berg, K.O.; Wood-Dauphinee, S.L.; Williams, J.I.; Maki, B. Measuring balance in the elderly: Validation of an instrument. Physiother. Can. 1989, 41, 304-311. [CrossRef]

7. Barry, E.; Galvin, R.; Keogh, C.; Horgan, F.; Fahey, T. Is the Timed Up and Go test a useful predictor of risk of falls in community dwelling older adults: A systematic review and meta-analysis. BMC Geriatr. 2014, 14, 14. [CrossRef] [PubMed] 
8. Ordonez, F.; Englebienne, G.; de Toledo, P.; van Kasteren, T.; Sanchis, A.; Kröse, B. Bayesian Inference in Hidden Markov Models for In-Home Activity Recognition. IEEE Pervasive Comput. 2014, 13, 67-75. [CrossRef]

9. Nait Aicha, A.; Englebienne, G.; Kröse, B. Continuous measuring of the indoor walking speed of older adults living alone. J. Ambient Intell. Hum. Comput. 2017, 1-11. [CrossRef]

10. Howcroft, J.; Kofman, J.; Lemaire, E.D. Review of fall risk assessment in geriatric populations using inertial sensors. J. Neuroeng. Rehabil. 2013, 10, 91. [CrossRef] [PubMed]

11. Van Schooten, K.S.; Pijnappels, M.; Rispens, S.M.; Elders, P.J.; Lips, P.; van Dieën, J.H. Ambulatory fall-risk assessment: Amount and quality of daily-life gait predict falls in older adults. J. Gerontol. Ser. A Biomed. Sci. Med. Sci. 2015, 70, 608-615. [CrossRef] [PubMed]

12. Rispens, S.M.; van Schooten, K.S.; Pijnappels, M.; Daffertshofer, A.; Beek, P.J.; van Dieën, J.H. Identification of fall risk predictors in daily life measurements: Gait characteristics' reliability and association with self-reported fall history. Neurorehabil. Neural Repair 2015, 29, 54-61. [CrossRef] [PubMed]

13. Weiss, A.; Brozgol, M.; Dorfman, M.; Herman, T.; Shema, S.; Giladi, N.; Hausdorff, J.M. Does the evaluation of gait quality during daily life provide insight into fall risk? A novel approach using 3-day accelerometer recordings. Neurorehabil. Neural Repair 2013, 27, 742-752. [CrossRef] [PubMed]

14. Mancini, M.; Schlueter, H.; El-Gohary, M.; Mattek, N.; Duncan, C.; Kaye, J.; Horak, F.B. Continuous monitoring of turning mobility and its association to falls and cognitive function: A pilot study. J. Gerontol. Ser. A Biol. Sci. Med. Sci. 2016, 71, 1102-1108. [CrossRef] [PubMed]

15. Najafi, B.; Aminian, K.; Loew, F.; Blanc, Y.; Robert, P.A. Measurement of stand-sit and sit-stand transitions using a miniature gyroscope and its application in fall risk evaluation in the elderly. IEEE Trans. Biomed. Eng. 2002, 49, 843-851. [CrossRef] [PubMed]

16. Ordónez, F.J.; Roggen, D. Deep convolutional and lstm recurrent neural networks for multimodal wearable activity recognition. Sensors 2016, 16, 115. [CrossRef] [PubMed]

17. Hu, B.; Dixon, P.; Jacobs, J.; Dennerlein, J.; Schiffman, J. Machine learning algorithms based on signals from a single wearable inertial sensor can detect surface-and age-related differences in walking. J. Biomech. 2018, 71, 37-42. [CrossRef] [PubMed]

18. Folstein, M.F.; Folstein, S.E.; McHugh, P.R. Mini-mental state: A practical method for grading the cognitive state of patients for the clinician. J. Psychiatr. Res. 1975, 12, 189-198. [CrossRef]

19. Rispens, S.M.; van Schooten, K.S.; Pijnappels, M.; Daffertshofer, A.; Beek, P.J.; van Dieën, J.H. Do extreme values of daily-life gait characteristics provide more information about fall risk than median values? JMIR Res. Protoc. 2015, 4, e4. [CrossRef] [PubMed]

20. Dijkstra, B.; Kamsma, Y.; Zijlstra, W. Detection of gait and postures using a miniaturised triaxial accelerometerbased system: Accuracy in community-dwelling older adults. Age Ageing 2010, 39, 259-262. [CrossRef] [PubMed]

21. Van Schooten, K.S.; Pijnappels, M.; Rispens, S.M.; Elders, P.J.M.; Lips, P.; Daffertshofer, A.; Beek, P.J.; van Dieën, J.H. Daily-life gait quality as predictor of falls in older people: A 1-year prospective cohort study. PLoS ONE 2016, 11, e0158623. [CrossRef] [PubMed]

22. LeCun, Y.; Bengio, Y.; Hinton, G. Deep learning. Nature 2015, 521, 436-444. [CrossRef] [PubMed]

23. Abdel-Hamid, O.; Mohamed, A.R.; Jiang, H.; Penn, G. Applying convolutional neural networks concepts to hybrid NN-HMM model for speech recognition. In Proceedings of the 2012 IEEE International Conference on Acoustics, Speech and Signal Processing (ICASSP), Kyoto, Japan, 25-30 March 2012; pp. 4277-4280.

24. Krizhevsky, A.; Sutskever, I.; Hinton, G.E. Imagenet classification with deep convolutional neural networks. In Proceedings of the 25th International Conference on Neural Information Processing Systems, Lake Tahoe, NV, USA, 3-6 December 2012; pp. 1097-1105.

25. Toshev, A.; Szegedy, C. Deeppose: Human pose estimation via deep neural networks. In Proceedings of the IEEE Conference on Computer Vision and Pattern Recognition, Columbus, OH, USA, 24-27 June 2014; pp. 1653-1660.

26. Sutskever, I.; Vinyals, O.; Le, Q.V. Sequence to sequence learning with neural networks. In Proceedings of the Advances in Neural Information Processing Systems, Montreal, QC, Canada, 8-13 December 2014; pp. 3104-3112.

27. Graves, A. Generating sequences with recurrent neural networks. arXiv 2013, arXiv:1308.0850. 
28. Bal, H.; Epema, D.; de Laat, C.; van Nieuwpoort, R.; Romein, J.; Seinstra, F.; Snoek, C.; Wijshoff, H. A Medium-Scale Distributed System for Computer Science Research: Infrastructure for the Long Term. Computer 2016, 49, 54-63. [CrossRef]

29. Bengio, Y.; Simard, P.; Frasconi, P. Learning long-term dependencies with gradient descent is difficult. IEEE Trans. Neural Netw. 1994, 5, 157-166. [CrossRef] [PubMed]

30. Hochreiter, S.; Schmidhuber, J. Long Short-Term Memory. Neural Comput. 1997, 9, 1735-1780. [CrossRef] [PubMed]

31. Caruana, R. Multitask Learning: A Knowledge-Based Source of Inductive Bias. In Proceedings of the Tenth International Conference on Machine Learning, Amherst, MA, USA, 27-29 June 1993; pp. 41-48.

32. Van Kasteren, T.L.; Englebienne, G.; Kröse, B.J. Hierarchical activity recognition using automatically clustered actions. In Proceedings of the International Joint Conference on Ambient Intelligence, Amsterdam, The Netherlands, 16-18 November 2011; pp. 82-91.

(C) 2018 by the authors. Licensee MDPI, Basel, Switzerland. This article is an open access article distributed under the terms and conditions of the Creative Commons Attribution (CC BY) license (http://creativecommons.org/licenses/by/4.0/). 
Article

\title{
Detecting Steps Walking at very Low Speeds Combining Outlier Detection, Transition Matrices and Autoencoders from Acceleration Patterns
}

\author{
Mario Muñoz-Organero ${ }^{1,2, *}$ and Ramona Ruiz-Blázquez ${ }^{1}$ \\ 1 Telematics Engineering Department, Carlos III University of Madrid, 28903 Getafe, Spain; \\ raruizb@it.uc3m.es \\ 2 UC3M-BS Institute of Financial Big Data, Carlos III University of Madrid, 28903 Getafe, Spain \\ * Correspondence: munozm@it.uc3m.es; Tel.: +34-91-624-8801
}

Received: 30 August 2017; Accepted: 4 October 2017; Published: 5 October 2017

\begin{abstract}
In this paper, we develop and validate a new algorithm to detect steps while walking at speeds between 30 and 40 steps per minute based on the data sensed from a single tri-axial accelerometer. The algorithm concatenates three consecutive phases. First, an outlier detection is performed on the sensed data based on the Mahalanobis distance to pre-detect candidate points in the acceleration time series that may contain a ground contact segment of data while walking. Second, the acceleration segment around the pre-detected point is used to calculate the transition matrix in order to capture the time dependencies. Finally, autoencoders, trained with data segments containing ground contact transition matrices from acceleration series from labeled steps are used to reconstruct the computed transition matrices at each pre-detected point. A similarity index is used to assess if the pre-selected point contains a true step in the $30-40$ steps per minute speed range. Our experimental results, based on a database from three different participants performing similar activities to the target one, are able to achieve a recall $=0.88$ with precision $=0.50$ improving the results when directly applying the autoencoders to acceleration patterns (recall $=0.77$ with precision $=0.50$ ).
\end{abstract}

Keywords: step detection; machine learning; outlier detection; transition matrices; autoencoders

\section{Introduction}

The automatic recognition of human activities and movements using wearable sensor data is able to provide contextual information to many areas of application. Some examples are ambient-assisted living [1], the self-management and monitoring of health parameters for patients with chronic conditions [2], training in sports [3], or entertainment and security [4]. By using the data extracted from sensors such as accelerometers, physiological sensors, Global Position System (GPS), or environmental sensors, several movement related features can be computed and machine learning algorithms can be trained to classify different activities and movements [4].

One particular application of using sensor data to detect human movements is for counting steps while walking at very slow speeds. People with physical disabilities or medical long-term conditions may find it difficult to walk at normal speeds [5]. However, physical activity is normally recommended to this particular set of users in order to promote or regain a healthy lifestyle. Counting steps is a supporting mechanism for accurately measuring physical activity for these users. Among the available wearable sensors, accelerometers and pedometers are commonly used for tracking ambulatory physical activity in clinical populations. By using the sensor display, information such as the number of steps walked and/or the number of calories burnt could be shown to the user, which are useful in motivating patients to increase their activity levels [5]. However, the accuracy of accelerometers for gait analysis tends to decrease (underestimating the real number of steps) in slow walking conditions [6]. 
Some examples of of-the-shelf sensors and devices that underestimate strides at slower walking speeds can be found in [7]. The authors in [7] found that the number of steps counted by two off-the shelf devices while walking at a walking cadence around 67 steps / minute was around $90 \%$ of the real value. This degradation would increase for slower cadences.

In this paper, we propose a novel mechanism to detect and count steps at slow speeds by processing acceleration data from a wearable device. We validate the proposed algorithm by using the data from three different participants executing slow-walking segments in the middle of several related activities such as sliding (walking without lifting the feet from the ground) at slow speeds, walking in circles at slow speeds, or walking at moderate speeds. The algorithm combines outlier detection for pre-selecting and aligning candidate steps, transition matrices to capture time dependences in the sensed time series, and autoencoders to assess the similarity of pre-selected segments with real steps used for training. Outlier detection from acceleration time series will identify segments of interest that exhibit particular stochastic properties, such as the ground contact instant while walking.

\section{Related Work}

Physical and mental health conditions benefit from physical activity. A healthy lifestyle improves both physical and mental health aspects [8]. In order to monitor the physical activity of a particular user, the counting of steps is one of the most used measures, and therefore it is important that activity-monitoring devices are both specific and sensitive in estimating the actual number of steps walked (discriminating real steps from non-stepping body movements) [8]. Many off-the-shelf devices register a significant number of false positive steps per minute when executing non-walking activities [8] and fail to count steps while walking at slow speeds [7]. Some previous studies have reviewed the accuracy of accelerometers for gait analysis in slow walking, such as $[6,7,9,10]$, finding that off-the-shelf sensors tend to underestimate strides (significantly in some cases) at slower walking speeds (more specifically for patients of severe medical conditions which affect the gait) [11].

Different approaches and algorithms have been previously used for counting steps in different scenarios. The authors in [12] used a threshold based algorithm, characterized by a linear computational time, trying to improve the real-time monitoring and real-time analysis of the walking behaviour of animals such as dairy cows. The research in [13] proposed an algorithm based on the use of an Android smartphone accelerometer, Fast Fourier Transform (FFT), and thresholding for detecting steps. The algorithm was not validated for slow walking and achieved poor results for running segments of data. The study in [14] used a wrist worn accelerometer to estimate the walking cadence and speed in daily life walking in several environments. The study in [15] proposed the use of some particular points in the acceleration time series in order to detect steps at normal speeds.

There are some preliminary results for detecting steps while walking at slow speeds based on raw acceleration data $[6,16]$. The slow execution of movements can contribute to increasing the difficulty in the process of detecting steps from acceleration data and in order to minimize the false positives from other activities.

Outlier detection has attracted a significant interest in several different areas. The authors in [17] applied outlier detection to estimate peak ground accelerations in seismic data. An algorithm for collision and hazard detection for motorcycles via inertial measurements based on outlier detection was presented in [18]. The authors in [19] made use of univariate outlier detection techniques in order to detect unusual sleep patterns. The authors in [20] used a density-based outlier detection method by measuring the LOF (Local Outlier Factor) on a projected PCA (Principal Component Analysis) domain from real world spatiotemporal traffic signals to detect traffic data outliers, which are errors in data and traffic anomalies in real situations, such as accidents and congestions. The research in [21] also used an outlier detection algorithm in a traffic system as a basis for alerting the transport department and drivers about some abnormal traffic conditions, such as traffic accidents or traffic congestion.

Outlier detection has also been previously used for the recognition of human movements. By using different sensor technologies, some human movements can be studied in terms of sequential data 
analysis. The authors in [22] used models that utilize sequential data as a measure to determine if the executions of a particular activity are close enough to a pre-defined specification of the activity or if they should be considered as executed in a wrong way. The authors used Hidden Markov Models (HMM) for time series characterization. The research study in [23] tackled the problem of fall detection by using a combination of outlier detectors. Using HMM for outlier detection and its application to fall detection has been studied in [24].

The research in this paper combines outlier detection from acceleration time series with time dependencies characterization via a transition matrix to feed a detection algorithm based on the similarity between the input and output of an autoencoder. Data from real steps while walking at slow speeds are used to train the autoencoder. The trained autoencoder is used to reconstruct the transition matrices at pre-selected points based on a Mahalanobis distance outlier detection mechanism. The Pearson correlation index is used to assess the similarity between the input and output of the autoencoder in order to determine if the pre-selected point is a step at slow speed walking.

The rest of the paper is organized as follows. Section 3 is dedicated to presenting the mechanism used to obtain the input data from the acceleration time series. Section 4 describes the outlier detection algorithm used in this paper. Section 5 is dedicated to present the mechanism used to estimate the transition matrices. The way we use autoencoders is shown in Section 6. Section 7 details the experimental results and Section 8 captures the main conclusions of this research.

\section{Sensor and Data Series}

Smart mobile devices such as smartphones or tablets contain several sensors that are able to automatically monitor and track some user related information. In our case, the proposed algorithm in this paper is based on the use of the accelerometer and gravity sensors. Combing the output of these sensors, we obtain acceleration time series that are both gravity free and geo-referenced in order to characterize the user's movements.

Figure 1 shows the device used to record the raw acceleration data and the three axes as defined by the Android operating system to provide accelerometer and gravity information. The device used was a Nexus 6 Android mobile device, which contains an accelerometer sensor and is able to estimate the gravity force vector using the combination of the accelerometer, gyroscope, and magnetic field sensors. The device is able to obtain 50 samples per second from these two sensors.

The data gathering process performs a series of steps. First, we remove the gravity component from the acceleration time series provided by the accelerometer sensor by subtracting the output from the gravity sensor to the accelerometer sensor output, as captured in Equation (1). $\vec{a}$ represents the gravity free acceleration, $\overrightarrow{a_{c}}$ contains the output of the accelerometer sensor, and $\vec{g}$ represents the output of the gravity sensor.

$$
\vec{a}=\overrightarrow{a_{c}}-\vec{g}=\left(a_{x}, a_{y}, a_{z}\right),
$$

After calculating $\vec{a}$ (which is referenced to the axes in Figure 1) we can project the acceleration component in the gravity direction (perpendicular to the Earth's surface), which is independent of the relative position of the mobile device and the relative movements of the device while walking (when the device is carried not tight to the human body). Equation (2) captures the computation of the acceleration component in the gravity direction.

$$
\overrightarrow{a_{g}}=\frac{\vec{a} \cdot \vec{g}}{\|\vec{g}\|^{2}} \vec{g}
$$


The value of $a_{g}=\overrightarrow{a_{g}} \cdot \frac{\vec{g}}{\|\vec{g}\|}$ provides the vertical acceleration while walking. In order to improve the detection accuracy, the acceleration information in the horizontal plane has also been used. In particular, we have used the $a_{H x}$ component from Equation (3):

$$
\overrightarrow{a_{H}}=\vec{a}-\overrightarrow{a_{g}}=\left(a_{H x}, a_{H y}, a_{H z}\right),
$$

The raw data used as the input to the step detection algorithm will be the time sequence of $\left(a_{g}, a_{\mathrm{Hx}}\right)$, sampled at $50 \mathrm{~Hz}$ when the mobile device is carried in the user's trouser pocket. Placing the sensor device close to the hip is a normal practice in step detection [5].

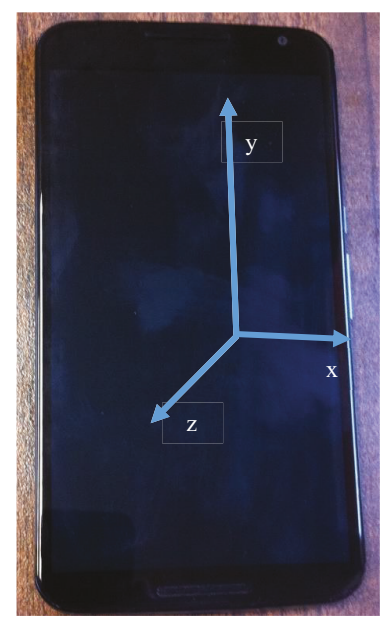

Figure 1. Nexus 6 mobile device and accelerometer axes.

\section{Outlier Pre-Detection}

In order to save computation energy and optimize the search for candidate time segments that may contain a step, and at the same time provide an alignment for the time sequence, an outlier detection technique based on the Mahalanobis distance is proposed in this paper. Gravity compensated step related acceleration patterns have a prominent activity in the milliseconds after the ground contact of each foot. In order to detect steps of up to 60 steps per minute, a time window of up to $1 \mathrm{~s}$ should be used in order to detect outliers in the $\left(a_{g}, a_{H x}\right)$ sequence. Outliers are potential candidates that may contain a ground contact in a step.

In order to train the algorithm, a labeled sequence of steps at low speeds (at 30 and 40 steps per minute) from three different users sampled at $50 \mathrm{~Hz}$ is used. The outlier detection provides a set of candidate points that are post-filtered using the labeled information. The result is a set of outliers associated with ground contact instants of real steps executed at slow speeds. These steps will be used to compute the transition matrices and then train the final autoencoders (one for $a_{g}$ and another for $\left.a_{H x}\right)$ for steps at slow speed detection.

\section{Transition Matrices}

The transition matrix computed from a time series captures the probability of moving from each state-a at instant " $t$ " to each state-b in the next sample at " $t+1$ ". In our case, we have two acceleration time series $a_{g}(t), a_{H x}(t)$. A transition matrix is computed for the acceleration samples around each pre-detected outlier. For each outlier at $t_{i}$, the transition matrices are computed form $a_{g}\left(t_{i-N}, \ldots, t_{i}, \ldots, t_{i+N}\right)$ and $a_{H x}\left(t_{i-N}, \ldots, t_{i}, \ldots, t_{i+N}\right)$. Each sample is assigned to a state according 
to the distance to the mean value of the series in terms of the standard deviation of the series. We have used eight states as follows (for the particular case of $a_{g}(t)$ ):

$$
\begin{aligned}
& \mathrm{m}=\text { mean value of }\left(a_{g}\left(t_{i-N}, \ldots, t_{i}, \ldots, t_{i+N}\right)\right) \\
& \mathrm{std}=\text { standard deviation of }\left(a_{g}\left(t_{i-N}, \ldots, t_{i}, \ldots, t_{i+N}\right)\right) \\
& \mathrm{S}_{1} \text { if } a_{g}(t)-\mathrm{m} \leq-1.5^{*} \text { std } \\
& \mathrm{S}_{2} \text { if } a_{g}(t)-\mathrm{m} \leq- \text { std and } a_{g}(t)-\mathrm{m}>-1.5^{*} \text { std } \\
& \mathrm{S}_{3} \text { if } a_{g}(t)-\mathrm{m} \leq-0.5^{*} \text { std and } a_{g}(t)-\mathrm{m}>- \text { std } \\
& \mathrm{S}_{4} \text { if } a_{g}(t)-\mathrm{m} \leq 0 \text { and } a_{g}(t)-\mathrm{m}>-0.5^{*} \text { std } \\
& \mathrm{S}_{5} \text { if } a_{g}(t)-\mathrm{m} \leq 0.5^{*} \text { std and } a_{g}(t)-\mathrm{m}>0 \\
& \mathrm{~S}_{6} \text { if } a_{g}(t)-\mathrm{m} \leq \operatorname{std} \text { and } a_{g}(t)-\mathrm{m}>0.5^{*} \text { std } \\
& \mathrm{S}_{7} \text { if } a_{g}(t)-\mathrm{m} \leq 1.5^{*} \text { std and } a_{g}(t)-\mathrm{m}>\text { std } \\
& \mathrm{S}_{8} \text { if } a_{g}(t)-\mathrm{m}>1.5^{*} \text { std }
\end{aligned}
$$

The state vectors for outlier " $i$ ", $s_{g}\left(t_{i-N}, \ldots, t_{i}, \ldots, t_{i+N}\right)$ and $s_{H x}\left(t_{i-N}, \ldots, t_{i}, \ldots, t_{i+N}\right)$, are used to calculate the transition matrix at $t_{\mathrm{i}}$. The transition matrix counts the number of times that being at state $\mathrm{j}$ goes to state $\mathrm{k}$ in the next instant of time. A final regularization is performed to convert counts to probabilities by dividing the cumulative count of each row, as captured in Equation (4), where the component in row $j$ and column $\mathrm{k}$ of the transition matrix is calculated.

$$
T(j, k)=\frac{\operatorname{Count}(j \rightarrow k)}{\sum_{l} \operatorname{Count}(j \rightarrow l)},
$$

In order to use the transition matrices at each outlier as the input to the autoencoder, described in the next section, the matrix is converted into a vector by concatenating each row in the matrix one after the other.

\section{Autoencoders}

A method based on training two different autoencoders, one for the $s_{g}$ transition matrices at each step and a similar one based on $s_{H x}$, transition matrices, have been used in order to detect steps from pre-selected outliers. The Pearson correlation index is used to calculate the similarity of the reconstructed transition matrix at each pre-selected outlier in the detection time series with the input one. If the Pearson correlation index for both autoencoders is above a certain threshold, than the pre-selected point is detected as a step. A description on how autoencoders work can be found in [25].

The design of an autoencoder tries to minimize the error between the reconstructed output and the input following Equation (5).

$$
\varepsilon(x, x \prime)=\left\|x-f_{2}\left(W^{\prime}\left(f_{1}(W x+b)\right)+b \prime\right)\right\|^{2},
$$

where $x^{\prime}$ is the reconstructed signal after an encoder and decoder functions $\left(f_{1}\right.$ and $f_{2}$ are activation functions such as the sigmoid function). In our case, $x$ corresponds to the vector calculated from serializing the transition matrix $T$, as described in the previous section.

\section{Results}

This section presents the results of the conducted experiment. The set-up for the experiment is presented first (both in terms of the data gathered as well as the implemented algorithm details). A first sub-section is dedicated to present the experiment set-up and the database gathered. A second sub-section is dedicated to explaining the internal details of the implemented algorithm. Then, the obtained results are captured in two different sub-sections. A first sub-section shows the results achieved when directly applying the autoencoders to the acceleration time series around the pre-selected outliers. A second sub-section uses the transition matrices instead of the acceleration 
time series as the input to the final autoencoders in order to capture the time dependencies. The idea is not only to present the results of the proposed algorithm in terms of accuracy and recall, but also to assess the gain in these figures when using transition matrices instead of acceleration time series, as other previous research has presented.

As a mechanism to compare the detection results provided in each case, we have used the obtained precision, recall, and $F$ score. If we define $t p$ as the number of "steps walking at slow speeds" that are correctly detected, Tp as the total number of "steps walking at slow speeds" present in the validation sequence, and $f p$ as the total number of "non-steps walking at slow speeds" that are detected as positive samples, the precision, the recall, and the F score can be defined, as shown in Equation (6).

$$
\begin{aligned}
& \text { precision }=\frac{t p}{(t p+f p)} \\
& \text { recall }=\frac{t p}{T p} \\
& F=2 \cdot \frac{\text { precision } \cdot \text { recall }}{\text { precision }+ \text { recall }},
\end{aligned}
$$

\subsection{Experiment Set-up and Database}

A group of three volunteers have recorded the output of both the acceleration and gravity sensors when wearing a Nexus 6 Android smart phone in the pocket of the trousers (close to the participant's hip, a common place that has been widely used in previous literature for step detection and counting). The demographic details for the participants are captured on Table 1.

Table 1. Participant demographics.

\begin{tabular}{cccc}
\hline Participant ID & Age & Gender & Normal Walk \\
\hline 1 & 24 & $\mathrm{M}$ & $\mathrm{Y}$ \\
2 & 41 & $\mathrm{~F}$ & $\mathrm{Y}$ \\
3 & 45 & $\mathrm{M}$ & $\mathrm{Y}$ \\
\hline
\end{tabular}

The implemented algorithm combining the techniques presented in Sections 4-6 should be trained with data that will characterize the objective class to be detected. For the case of this paper, the objective of the algorithm has been set to detect the steps executed at cadences between 30 and 40 steps per minute. Our objective is to maximize the number of true positives (the number of true steps detected) while minimizing the false positives (segments of other activities that are detected as members of the target class). Previous studies, such as [7], have shown how the reported number of steps by off-the-shelf devices tend to underestimate the real value for speeds around 67 steps per minute. In our research, we have designed an algorithm that could be trained to detect steps at even smaller cadences. Moreover, we have added the study of how the algorithm discriminates data from similar activities (which has been many times overlooked in previous studies such as [7]). We have generated a dataset that includes segments of data in the target class, as well as three other activities that may present similar acceleration patterns (sitting down and up, walking in circles at 30 steps per minute, and walking at 60 steps per minute). In order to generate the required data, each participant was asked to execute the following sequence of movements:

- $\quad$ to stand still for $5 \mathrm{~s}$ (this information will be used to validate the calibration of the gravity sensor and to mark the start of the data);

- walk at a speed of 60 steps per minute during $60 \mathrm{~s}$;

- $\quad$ to stand still for $5 \mathrm{~s}$ (this information will be used to validate the calibration of the gravity sensor and facilitate the automatic split of the recorded data into segments of single activities);

- walk at a speed of 30 steps per minute during $60 \mathrm{~s}$;

- $\quad$ to stand still for $5 \mathrm{~s}$;

- walk at a speed of 40 steps per minute during $60 \mathrm{~s}$; 
- $\quad$ to stand still for $5 \mathrm{~s}$;

- $\quad$ slide (walk without separating the feet from the ground) at a speed of 30 steps per minute during $60 \mathrm{~s}$;

- to stand still for $5 \mathrm{~s}$;

- $\quad$ sit down and up 10 times;

- to stand still for $5 \mathrm{~s}$;

- walk around a chair (in circles) at a speed of 30 steps per minute during $60 \mathrm{~s}$; and,

- to stand still for $5 \mathrm{~s}$.

The movements contained in the database are all related to the target class of steps walking at speeds of 30 to 40 steps per minute. Walking without lifting the feet from the ground (sliding) at slow speeds is expected to generate similar $a_{H x}(t)$ sequences than walking at the same speeds, but the algorithm should detect the differences in the $a_{g}(t)$ component. On the opposite site, sitting down and standing up could generate similar acceleration patterns in the gravity axis $a_{g}(t)$ but different ones in $a_{H x}(t)$. Walking in circles around a chair will change the direction of the movement and so the acceleration components. Finally, walking at moderate speeds (60 steps per minute) will generate similar patterns for some of the steps. In the real application of counting steps for monitoring physical activities, all of the steps would count for a positive output. In the case of this research, we want to test the discrimination rate of the proposed algorithm for the particular case of walking at slow speeds from some similar classes.

\subsection{Implemented Algorithm Details}

The techniques presented in Sections 4-6 have been combined to implement two "steps at slow speeds" detection algorithms. This sub-section captures the design and implementation details for these algorithms. The experimental results for these algorithms using the data gathered as described in the previous sub-section are captured in Sections 7.3 and 7.4.

The first algorithm will combine outlier detection techniques and autoencoders in order to detect "steps at slow speeds" from acceleration data. The algorithm details are:

- Define the maximum and minimum cadences of steps to be detected $\left(c_{\max }\right.$ and $\left.c_{\min }\right)$ in steps per minute $\rightarrow$ in our case $c_{\max }=40$ and $\mathrm{c}_{\min }=30$

- Set the outlier detection window to $T_{\text {out }}=\frac{60}{c_{\max }} \mathrm{s}$

- For each $\mathrm{T}_{\text {out }}=1.5 \mathrm{~s}$ of $a_{g}(t), a_{H x}(t)$ centered at $t_{c}$, calculate the Mahalanobis distance $\mathrm{md}\left(t_{c}\right)$ from $\left(a_{g}\left(t_{c}\right), a_{H x}\left(t_{c}\right)\right)$ and $\left[\left(a_{g}\left(t_{c}-0.75\right), a_{H x}\left(t_{c}-0.75\right)\right), \ldots,\left(a_{g}\left(t_{c}+0.75\right), a_{H x}\left(t_{c}+0.75\right)\right)\right]$

- For all $t_{c}$ in $\left(0: t_{\max }\right)$, if $\operatorname{md}\left(t=t_{c}\right)>$ th then consider $t_{c}$ an outlier (th $=3$ has been empirically selected).

- For each $t_{c}$. corresponding to an outlier use $\left[\left(a_{g}\left(t_{c}-0.12\right), a_{H x}\left(t_{c}-0.12\right)\right), \ldots\right.$, $\left.\left(a_{g}\left(t_{c}+0.12\right), a_{H x}\left(t_{c}+0.12\right)\right)\right]$ to feed an autoencoder with a single hidden layer with 5 hidden units.

- Calculate the Pearson correlation index between the input and output of the autoencoder as a similarity index to decide if the outlier corresponds to a step.

The second algorithm will try to improve the results of the previous algorithm by characterizing the time sequences around each pre-detected outlier in terms of its transition matrix. The algorithm details are:

- Define the maximum and minimum cadences of steps to be detected ( $c_{\max }$ and $\left.c_{\min }\right)$ in steps per minute $\rightarrow$ in our case $c_{\max }=40$ and $\mathrm{c}_{\min }=30$

- Set the outlier detection window to $T_{\text {out }}=\frac{60}{c_{\max }} \mathrm{s}$

- For each $\mathrm{T}_{\text {out }}=1.5 \mathrm{~s}$ of $a_{g}(t), a_{H x}(t)$ centered at $t_{c}$, calculate the Mahalanobis distance $\operatorname{md}\left(t_{c}\right)$ from $\left(a_{g}\left(t_{c}\right), a_{H x}\left(t_{c}\right)\right)$ and $\left[\left(a_{g}\left(t_{c}-0.75\right), a_{H x}\left(t_{c}-0.75\right)\right), \ldots,\left(a_{g}\left(t_{c}+0.75\right), a_{H x}\left(t_{c}+0.75\right)\right)\right]$ 
- For all $t_{c}$ in $\left(0: t_{\max }\right)$, if $\mathrm{md}\left(t=t_{c}\right)>$ th then consider $t_{c}$ an outlier (th $=3$ has been empirically selected).

- For each $t_{c}$ corresponding to an outlier use $\left[\left(a_{g}\left(t_{c}-0.12\right), a_{H x}\left(t_{c}-0.12\right)\right), \ldots\right.$, $\left.\left(a_{g}\left(t_{c}+0.12\right), a_{H x}\left(t_{c}+0.12\right)\right)\right]$ in order to estimate the transition matrix as described in Section 5 (being $N=6$ ). The values of $a_{g}(t)$ and $a_{H x}(t)$ are mapped into 8 different states (this number has been empirically selected) as described in Section 5. The states are assigned depending on the distance of each pair $a_{g}(t), a_{H x}(t)$ to the mean values of $a_{g}[T]$ and $a_{H x}[T]$ in the previously selected $240 \mathrm{~ms}$ time window centered at each outlier in terms of their standard deviation. This normalization is required in order to compensate different user weights.

- Use the transition matrices to feed an autoencoder with a single hidden layer with five hidden units.

- Calculate the Pearson correlation index between the input and output of the autoencoder as a similarity index to decide if the outlier corresponds to a step.

Both of the algorithms have been implemented in Matlab. The mahal function has been used in order to calculate the Mahalanobis distance. The trainAutoencoder [26], encode and decode functions have been used for the autoencoder.

\subsection{Autoencoders Based on Acceleration Data around Outlier Pre-Detected Points}

The data from the segments of slow walking ( 30 and 40 steps per minute) from two out of the three participants were isolated in order to train the autoencoders. Each step was detected by using the outlier pre-detection phase previously described in Section 4 . The time location of each detected outlier was compared with a predefined vector manually introduced containing the ground truth (manual labels) for each step. The outlier pre-detection phase was needed in order to automatically align the segments of the acceleration data associated with each step (the manual labeling process did not require therefore the exact location of the ground contact instant but a visual approximation, eliminating therefore the human error when labeling the data). Only two participants have been selected for training to implement a leave one out validation approach.

In order to generate the samples of acceleration, windows of $240 \mathrm{~ms}$ of raw data (both accelerometer and gravity data) have been used around each pre-detected outlier corresponding to each labeled step. The size of $240 \mathrm{~ms}$. has been empirically selected in order to include all of the acceleration patterns corresponding to the ground contact instant. Figure 2 captures some of the acceleration samples $a(t)$ corresponding to outliers associated with labeled steps.

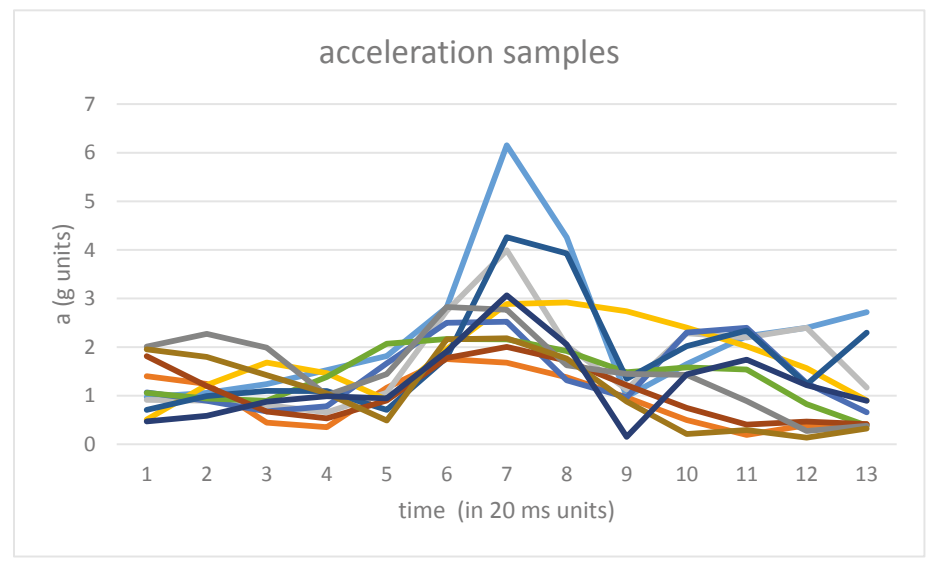

Figure 2. Acceleration samples around outliers corresponding to slow walking steps. Each color represents a different sample. 
For each window of pre-selected data, the $a_{g}(t), a_{H x}(t)$ components were computed. The samples of each component were used to train a different autoencoder.

After finishing with the training of the autoencoders, the validation phase used all of the recorded data from the third participant in order to detect steps (the leave-one-out process was repeated with all tree participants). Windows of acceleration data were computed for each pre-detected outlier in a similar way, but now for the entire time series containing all of the movements for each of the three participants. A Pearson correlation index was used to compare the output and the input of the autoencoders and different detection thresholds have been used to assess the obtained results. The idea is that the acceleration sequences computed for similar steps to those used in the training of the algorithm will show high values for the Pearson correlation index. However, acceleration sequences computed for other movements from those used in the training of the algorithm will show small values for the Pearson correlation index.

The results obtained for the recall, precision, and F-score for different similarity thresholds are captured in Table 2. The optimal value for the $F$-score is achieved when using a similarity threshold of 0.6. The recall in this case is 0.67 and the precision is 0.59 . A recall $=0.67$ means that we are able to detect $67 \%$ of all steps executed at slow speeds (30 and 40 steps per minute). A precision $=0.59$ means that we detect $41 \%$ of false positives (other activities detected as slow walking steps). The recall of the algorithm could be improved by detecting slow walking segments in which missing intermediate walking steps could be incorporated into the output of the algorithm. The idea is to estimate the walking cadence as the inverse of the difference in time between two consecutive detected steps. When the cadence shows a relatively small variation among consecutive steps for the last steps and the time between two consecutive detected steps suddenly approximates the double of previous steps, we could estimate that one more step has been executed. In our case, we have not used this detection feature since we only had a limited number of walking segments and the results will be close to the optimal value of recall $=1$ since it is known that the test person did not perform intermediate stops (except for the steps missing at the beginning of a waking segment).

Table 2. Results for different similarity thresholds.

\begin{tabular}{cccc}
\hline Sim Thr & Recall & Precision & F Score \\
\hline 0.40 & 0.77 & 0.50 & 0.61 \\
0.50 & 0.75 & 0.53 & 0.62 \\
0.60 & 0.67 & 0.59 & 0.63 \\
0.70 & 0.50 & 0.65 & 0.56 \\
0.80 & 0.33 & 0.73 & 0.46 \\
0.90 & 0.02 & 0.50 & 0.04 \\
\hline
\end{tabular}

The average distribution of false positives among the different movements is captured in Table 3. No outlier in the acceleration time series for the sit down and stand up segments is classified as walking at slow speeds. The horizontal acceleration component is different and there is no confusion for this movement. Only a moderate $6.83 \%$ of walking in circles steps are detected as walking at slow speeds. The horizontal component is again different. The majority of false positives are related to sliding sections. In this case, the horizontal component is similar to the target class and some of the misclassified segments show a similar vertical acceleration pattern despite the fact that the foot is not separated from the ground (the sensor is located in the pocket of the participant which captures some vertical acceleration activity). Finally, some of the walking at moderate speed steps are also generating false positives and showing similar patterns as those steps executed at slower speeds.

Table 3. Percentage of false positives.

\begin{tabular}{ccccc}
\hline & Sit down & Walk in Circles & Slide & Walk 60 spm \\
\hline$\%$ detected as $\rightarrow$ & 0.00 & 6.83 & 55.82 & 37.36 \\
\hline
\end{tabular}




\subsection{Autoencoders Based on Transition Matrices around Outlier Pre-Detected Points.}

In this sub-section, the same computations are performed but using the transition matrices instead of the acceleration time series in order to train the autoencoders and perform step detection. Transition matrices capture the temporal dependencies from adjacent samples in acceleration segments. We use the method proposed in Section 5 to compute the transition matrices and to convert them into vectors in order to feed the final autoencoders.

The results obtained in this case for the recall, precision, and F-score for different similarity thresholds are captured in Table 4 . The recall, precision, and $F$ scores improve as compared to the previous section. For a value of the similarity threshold of 0.4 , a recall of 0.88 is achieved, meaning that $88 \%$ of the steps walking at slow speeds are counted (with $50 \%$ of false positives among the related movements). The optimal value for the $F$-score is achieved when using a similarity threshold of 0.8 . The optimal $F$-score is in this case 0.67 , improving from 0.63 in the case of using acceleration time series instead of transition matrices. A balanced result for both recall and precision is achieved for a similarity threshold of 0.7 , in which approximately $2 / 3$ of the steps walking at slow speeds are detected (without using the post-detection estimation of missing steps previously described) and $2 / 3$ of true positives are detected (1/3 of false positives).

Table 4. Results for different similarity thresholds.

\begin{tabular}{cccc}
\hline Sim Thr & Recall & Precision & F Score \\
\hline 0.40 & 0.88 & 0.50 & 0.64 \\
0.50 & 0.79 & 0.54 & 0.64 \\
0.60 & 0.73 & 0.57 & 0.64 \\
0.70 & 0.67 & 0.64 & 0.65 \\
0.80 & 0.60 & 0.74 & 0.67 \\
0.90 & 0.44 & 0.78 & 0.56 \\
\hline
\end{tabular}

Figure 3 captures a visual comparison for the recall achieved by both methods for different similarity thresholds. Using the transition matrices to characterize the time dependencies improves the results obtained for all of the similarity threshold values. The results do not decay so fast when the threshold moves close to 1 .

The results for the F-score for the different similarity thresholds for both methods are captured in Figure 4. Using the transition matrices instead of time acceleration segments generates a more constant F-score along the different similarity thresholds (the lost in recall is compensated by a similar gain in precision).

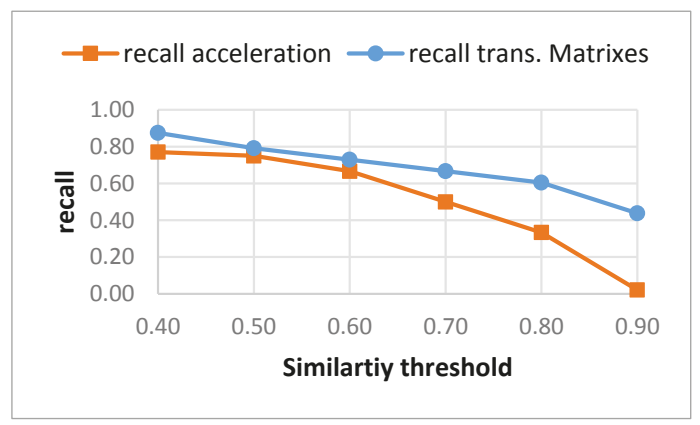

Figure 3. Recall for different similarity threshold for both methods. 


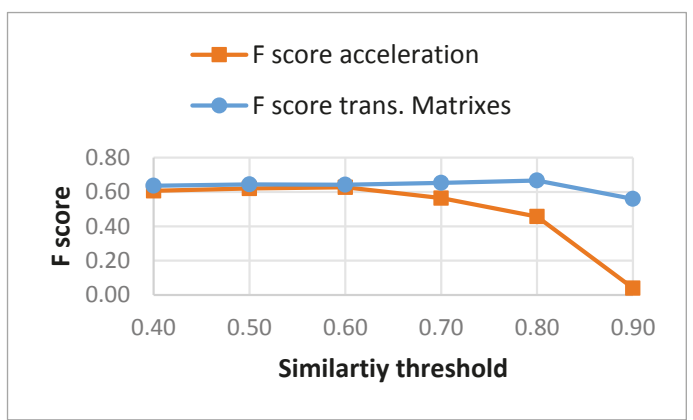

Figure 4. F-score for different similarity threshold for both methods.

The average distribution of false positives among the different movements for the case of using transition matrices to capture the time dependencies is shown in Table 5. Similar results to those presented in Table 3 are obtained. In this case, there is a small decrement in the percentage of steps at 60 steps per minute detected as walking at slower speeds, which is compensated by the increment in slide segments detected, as walking at slow speeds.

Table 5. Percentage of false positives.

\begin{tabular}{ccccc}
\hline & Sit down & Walk in Circles & Slide & Walk 60 spm \\
\hline$\%$ detected as $\rightarrow$ & 0.00 & 5.07 & 46.20 & 48.73 \\
\hline
\end{tabular}

\section{Conclusions}

This study has proposed and validated a new method to detect steps while walking at slow speeds. The algorithm combines the use of outlier detection in acceleration time series, sensor random movements compensation, time dependencies modelling in acceleration series, and a final detection phase. We have used the Mahalanobis distance to detect outliers in the time series. A threshold has been used so that the ground contact instant in steps walking at slow speeds is detected as an outlier in at least $95 \%$ of the recorded data. Random movements of the sensor when not tightly worn to the participant's body and initial placement compensation has been conducted by using the gravity sensor to estimate the direction of the gravity force. Temporal dependencies have been modeled using transition matrices. A comparison when acceleration time series are used instead of transition matrices is also presented in the paper. Finally, we have used autoencoders and a similarity function based on the Pearson correlation index in order to perform the final step detection.

The results have been validated using a new database that we have recorded using three participants executing a series of movements that are similar in terms of acceleration patterns to the target class that we want to detect (walking at slow speeds). Using acceleration time series as the input to the final autoencoders achieves an optimal $F$ score of 0.63 for the data in the recorded database. Using transition matrices to model the time dependencies improves the results to a $F$ score value of 0.67 .

Similar movements have been introduced in the recorded database to validate the proposed algorithm in a pessimistic scenario. The results show that around half of the false positives are for steps executed at higher speeds, which show similar patterns around the ground contact instant to steps at slower speeds. These false positives will contribute to positive results for the generic approach of detecting steps while walking at any speed in applications to motivate the physical exercise to people in need. 
As a future work, the study will include other parts of the body to which to attach the accelerometer sensor and using devices from different manufacturers in order to generalize results. Moreover, participants suffering long-term conditions, such as COPD, will also be added in order to validate the applicability of the proposed algorithms for different types of users.

Acknowledgments: The research leading to these results has received funding from the from the "HERMES-SMART DRIVER" project TIN2013-46801-C4-2-R (MINECO), funded by the Spanish Agencia Estatal de Investigación (AEI), and the "ANALYTICS USING SENSOR DATA FOR FLATCITY" project TIN2016-77158-C4-1-R (MINECO/ ERDF, EU) funded by the Spanish Agencia Estatal de Investigación (AEI) and the European Regional Development Fund (ERDF).

Author Contributions: The main author, Mario Muñoz-Organero, conceived and developed the algorithm. The authors Mario Muñoz-Organero and Ramona Ruiz-Blazquez participated in the experiment and validated the experimental results.

Conflicts of Interest: The authors declare no conflict of interest.

\section{References}

1. Wang, A.; Chen, G.; Yang, J.; Zhao, S.; Chang, C. A Comparative Study on Human Activity Recognition Using Inertial Sensors in a Smartphone. IEEE Sens. J. 2016, 16, 4566-4578. [CrossRef]

2. Hassanalieragh, M.; Page, A.; Soyata, T.; Sharma, G.; Aktas, M.; Mateos, G.; Kantarci, B.; Andreescu, S. Health Monitoring and Management Using Internet-of-Things (IoT) Sensing with Cloud-based Processing: Opportunities and Challenges. In Proceedings of the 2015 IEEE International Conference on Services Computing (SCC), New York, NY, USA, 27 June-2 July 2015.

3. Avci, A.; Bosch, S.; Marin-Perianu, M.; Marin-Perianu, R.; Havinga, P. Activity recognition using inertial sensing for healthcare, wellbeing and sports applications: A survey. In Proceedings of the $201023 \mathrm{rd}$ International Conference on Architecture of Computing Systems (ARCS); VDE: Hannover, Germany, 2010.

4. Lara, O.D.; Labrador, M.A. A survey on human activity recognition using wearable sensors. IEEE Commun. Surv. Tutor. 2013, 15, 1192-1209. [CrossRef]

5. Bassett, D.R., Jr; John, D. Use of pedometers and accelerometers in clinical populations: Validity and reliability issues. Phys. Ther. Rev. 2013, 15, 135-142. [CrossRef]

6. $\quad$ Feng, Y.; Wong, C.K.; Janeja, V.; Kuber, R.; Mentis, H.M. Comparison of tri-axial accelerometers step-count accuracy in slow walking conditions. Gait Posture 2017, 53, 11-16. [CrossRef] [PubMed]

7. Sandroff, B.M.; Motl, R.W.; Pilutti, L.A.; Learmonth, Y.C.; Ensari, I.; Dlugonski, D.; Klaren, R.E.; Balantrapu, S.; Riskin, B.J. Accuracy of StepWatch ${ }^{\mathrm{TM}}$ and ActiGraph Accelerometers for Measuring Steps Taken among Persons with Multiple Sclerosis. PLoS ONE 2014, 9, e93511. [CrossRef] [PubMed]

8. O'Connell, S.; ÓLaighin, G.; Quinlan, L.R. When a Step Is Not a Step! Specificity Analysis of Five Physical Activity Monitors. PLoS ONE 2017, 12, e0169616. [CrossRef] [PubMed]

9. Motl, R.W.; Snook, E.M.; Agiovlasitis, S. Does an accelerometer accurately measure steps taken under controlled conditions in adults with mild multiple sclerosis? Disabil. Health J. 2011, 4, 52-57. [CrossRef] [PubMed]

10. Korpan, S.M.; Schafer, J.L.; Wilson, K.C.; Webber, S.C. Effect of ActiGraph GT3X+ Position and Algorithm Choice on Step Count Accuracy in Older Adults. J. Aging Phys. Act. 2015, 23, 377-382. [CrossRef] [PubMed]

11. Moon, Y.; McGinnis, R.S.; Seagers, K.; Motl, R.W.; Sheth, N.; Wright, J.A., Jr; Ghaffari, R.; Sosnoff, J.J. Monitoring gait in multiple sclerosis with novel wearable motion sensors. PLoS ONE 2017, 12, e0171346. [CrossRef] [PubMed]

12. Arcidiacono, C.; Porto, S.M.; Mancino, M.; Cascone, G. A threshold-based algorithm for the development of inertial sensor-based systems to perform real-time cow step counting in free-stall barns. Biosyst. Eng. 2017, 153, 99-109. [CrossRef]

13. DIrican, A.C.; Aksoy, S. Step Counting Using Smartphone Accelerometer and Fast Fourier Transform. Sigma J. Eng. Nat. Sci. 2017, 8, 175-182.

14. Fasel, B.; Duc, C.; Dadashi, F.; Bardyn, F.; Savary, M.; Farine, P.A.; Aminian, K. A wrist sensor and algorithm to determine instantaneous walking cadence and speed in daily life walking. Med. Biol. Eng. Comput. 2017, 55, 1773-1785. [CrossRef] [PubMed] 
15. Munoz-Organero, M.; Lotfi, A. Human movement recognition based on the stochastic characterisation of acceleration data. Sensors 2016, 16, 1464. [CrossRef] [PubMed]

16. Godfrey, A.; Morris, R.; Hickey, A.; Del Din, S. Beyond the front end: Investigating a thigh worn accelerometer device for step count and bout detection in Parkinson's disease. Med. Eng. Phys. 2016, 38, 1524-1529. [CrossRef] [PubMed]

17. Yuen, K.V.; Ortiz, G.A. Outlier detection and robust regression for correlated data. Comput. Methods Appl. Mech. Eng. 2017, 313, 632-646. [CrossRef]

18. Selmanaj, D.; Corno, M.; Savaresi, S.M. Hazard Detection for Motorcycles via Accelerometers: A Self-Organizing Map Approach. IEEE Trans. Cybern. 2016, PP, 1-12. [CrossRef] [PubMed]

19. Liang, Z.; Martell, M.A.C.; Nishimura, T. A Personalized Approach for Detecting Unusual Sleep from Time Series Sleep-Tracking Data. In Proceedings of the 2016 IEEE International Conference on Healthcare Informatics (ICHI), Chicago, IL, USA, 4-7 October 2016; pp. 18-23.

20. Ma, M.X.; Ngan, H.Y.; Liu, W. Density-based Outlier Detection by Local Outlier Factor on Largescale Traffic Data. In ISET International Symposium on Electronic Imaging Science and Technology 2016: Image Processing: Machine Vision Applications IX; Society for Imaging Science and Technology (IS\&T): Springfield, VA, USA, 2016; pp. 1-4.

21. Tang, J.; Ngan, H.Y. Traffic Outlier Detection by Density-Based Bounded Local Outlier Factors. Inf. Technol. Ind. 2016, 4, 6-18.

22. Palma, C.; Salazar, A.; Vargas, F. Automatic Detection of Deviations in Human Movements Using HMM: Discrete vs. Continuous. In International Symposium on Visual Computing; Springer International Publishing: Cham, Switzerland, 2016; pp. 534-543.

23. Medrano, C.; Igual, R.; García-Magariño, I.; Plaza, I.; Azuara, G. Combining novelty detectors to improve accelerometer-based fall detection. Med. Biol. Eng. Comput. 2017, 55, 1849-1858. [CrossRef] [PubMed]

24. Khan, S.S.; Karg, M.E.; Kulić, D.; Hoey, J. Detecting falls with X-Factor Hidden Markov Models. Appl. Soft Comput. 2017, 55, 168-177. [CrossRef]

25. Le, Q.V. A Tutorial on Deep Learning Part 2: Autoencoders, Convolutional Neural Networks and Recurrent Neural Networks. Google Brain. 2015. Available online: http://www.cs.mcgill.ca/ dprecup/courses/ML/ Materials/dl-tutorial2.pdf (accessed on 28 September 2017).

26. MathWorks Documentation. The Autoencoder class in Matlab. Available online: https://es.mathworks. com/help/nnet/ref/autoencoder-class.html (accessed on 28 September 2017).

(C) 2017 by the authors. Licensee MDPI, Basel, Switzerland. This article is an open access article distributed under the terms and conditions of the Creative Commons Attribution (CC BY) license (http:/ / creativecommons.org/licenses/by/4.0/). 
Article

\title{
Three-Axis Ground Reaction Force Distribution during Straight Walking
}

\author{
Masataka Hori, Akihito Nakai and Isao Shimoyama * \\ Department of Mechano-Informatics, Graduate School of Information Science and Technology, \\ The University of Tokyo, 7-3-1 Hongo, Bunkyo-ku, Tokyo 113-8656, Japan; hori@leopard.t.u-tokyo.ac.jp (M.H.); \\ nakai@leopard.t.u-tokyo.ac.jp (A.N.) \\ * Correspondence: isao@leopard.t.u-tokyo.ac.jp; Tel.: +81-3-5841-6318; Fax: +81-3-3818-0835
}

Received: 30 August 2017; Accepted: 19 October 2017; Published: 24 October 2017

\begin{abstract}
We measured the three-axis ground reaction force (GRF) distribution during straight walking. Small three-axis force sensors composed of rubber and sensor chips were fabricated and calibrated. After sensor calibration, 16 force sensors were attached to the left shoe. The three-axis force distribution during straight walking was measured, and the local features of the three-axis force under the sole of the shoe were analyzed. The heel area played a role in receiving the braking force, the base area of the fourth and fifth toes applied little vertical or shear force, the base area of the second and third toes generated a portion of the propulsive force and received a large vertical force, and the base area of the big toe helped move the body's center of mass to the other foot. The results demonstrate that measuring the three-axis GRF distribution is useful for a detailed analysis of bipedal locomotion.
\end{abstract}

Keywords: ground reaction force (GRF); micro electro mechanical systems (MEMS); gait; walk; bipedal locomotion; 3-axis force sensor; shoe; force distribution

\section{Introduction}

Bipedal locomotion is one of the most remarkable features of humans. Many researchers have focused on the relationship between bipedal locomotion and the ground reaction force (GRF) [1-4]. Recently, force plates have enabled us to measure the three-axis resultant forces while walking on such a plate in limited laboratory settings. For walking action outdoors, only the normal force distribution can be measured by use of a piezoelectric film sensor inserted into the insole of a shoe [5-9]. However, researchers cannot obtain shear force distribution data from these methods, which is necessary for evaluating forward and lateral motion.

A large amount of the shear force distribution data with normal force distribution data obtained during walking is useful for analyzing bipedal locomotion in more detail. The force distribution data can be used to determine the locations at which large shear and vertical forces are applied during walking. This result will be helpful for optimizing the positions of stud pins in athletic shoes and for determining the best shoe cushion design. Thus, this knowledge is beneficial for shoe design, sports science, and medical treatment. Unfortunately, it is impossible to obtain the shear force distribution data from multiple steps using the current commercially available methods. Therefore, we fabricated a new force sensor and obtained the three-axis force distribution data.

In this paper, we measured the three-axis GRF distribution during walking using a small three-axis force sensor attached to a shoe. The small three-axis force sensor was composed of rubber and a sensor chip fabricated using a micro-electro-mechanical system (MEMS). These force sensors were attached to the shoe after being fixed in a rubber frame without the use of a metal plate. Using this shoe-sensor combination, the three-axis GRF distribution was measured during straight walking on a treadmill. After measurement, the local features of the applied three-axis force under the shoe were analyzed. 


\section{Materials and Methods}

\subsection{Three-Axis Force Sensor and Rubber Frame}

The three-axis force sensor used for the measurements consisted of a sensor chip, a flexible cable, and thermoplastic rubber. The fabrication method, force detection principal, and a detailed feature of the sensor chip were described in [10]. Briefly, the dimensions of the sensor chip were $2 \times 2 \times 0.3 \mathrm{~mm}$, and three pairs of $\mathrm{Si}$-doped beams were fabricated on the sensor using a MEMS. These beams were used as the sensing elements for the applied three-axis force, and canceled out the effect of temperature change. This sensor chip was attached to the flexible cable substrate, and was electrically connected using conductive paste (XA-874, FUJIKURA KASEI Co., Ltd., Tokyo, Japan). After finishing the electrical connection, the sensor chip was covered with thermoplastic rubber (TSE3431-H(A) and TSE3431-H(E), Momentive Performance Materials Japan LLC, Tokyo, Japan). The covering rubber was $8 \mathrm{~mm}$ in diameter. The total dimensions of the three-axis force sensor were $11 \times 11 \times 3.5 \mathrm{~mm}$, and the weight of the sensor was $0.6 \mathrm{~g}$ (Figure 1a).

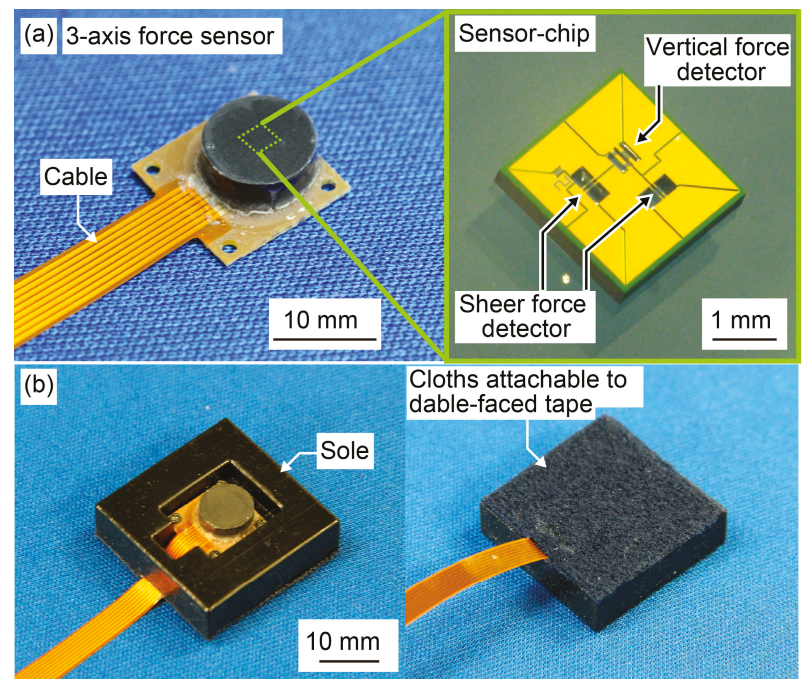

Figure 1. (a) Sensor chip and three-axis force sensor; (b) Top and bottom of the three-axis force sensor embedded in rubber material used as a sole.

Before the force sensor was used for measurement, it was fixed in a rubber frame. The frame was made of rubber (TangoBlack, Stratasys Ltd., Rehovot, Israel) and was constructed using a 3D printer (EDEN260V, Stratasys Ltd., Rehovot, Israel). The Shore A hardness of the rubber material was 61, which is within the hardness range of the soles of commercially available shoes. The dimensions of the frame were $25 \times 25 \times 7 \mathrm{~mm}$. To fix the three-axis force sensor, a $11.5 \times 13 \times 3.5 \mathrm{~mm}$ depression was made in the center of the sole, and a hole was created near the depression to allow the force sensor cable to pass through. The three-axis force sensor was fixed in the depression using clinchers. To attach the sensor to the shoe, a cloth was bonded to the backside of the frame using glue. The cloth was able to be detached via a hook-and-loop fastener (Figure 1b). The total weight of the three-axis force sensor and the frame was $15 \mathrm{~g}$.

Vertical and shear forces were applied to the three-axis force sensor for calibration. A commercially available six-axis force sensor (SI-130-10, ATI Industrial Automation, Inc., Apex, NC, USA) was used as a reference device for the three-axis force sensor in the calibration experiment. The six-axis force sensor was fixed on the stage, and our three-axis force sensor that was fixed in the frame was attached 
to the top of the six-axis force sensor using a hook-and-loop fastener. An acrylic plate was fixed over the three-axis force sensor. In the calibration procedure, the stage was moved upward until our sensor touched the acrylic plate, and then vertical force was applied. Then, the stage was moved horizontally so that shear forces were applied to our sensor. The relationship between the applied force and the sensor outputs was determined from this calibration, and the result was used to measure the three-axis GRF distribution during walking (Figure 2).
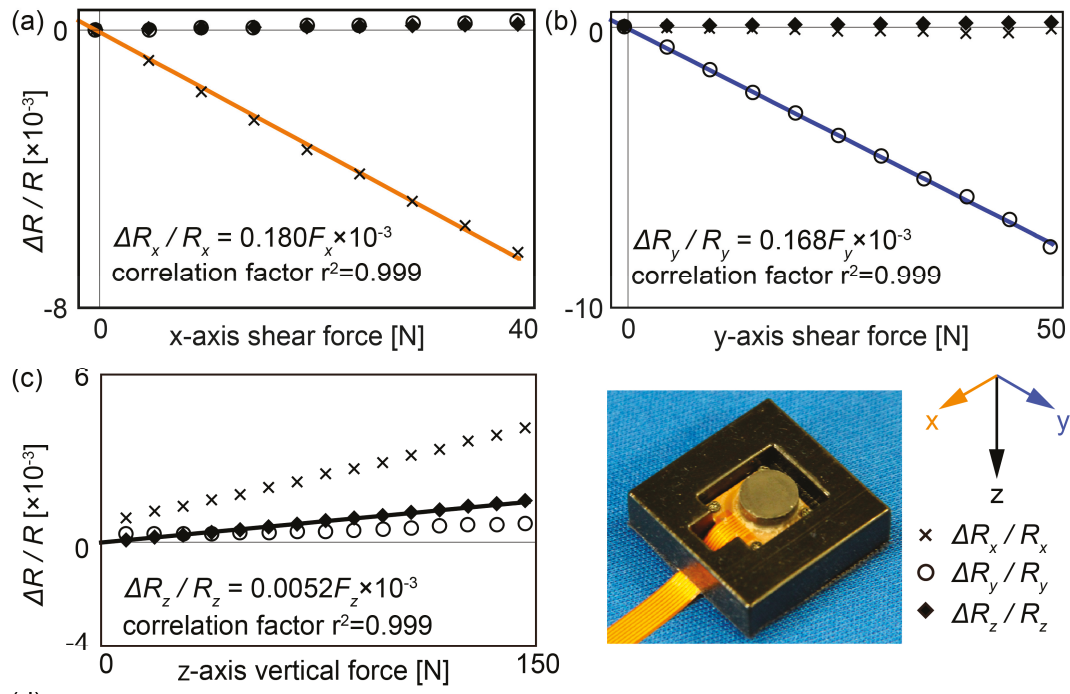

(d) $\left(\begin{array}{l}F_{x} \\ F_{y} \\ F_{z}\end{array}\right)=\left(\begin{array}{rrr}-5.19 & 0.426 & 15.3 \\ -0.131 & -5.90 & 3.36 \\ 2.20 & 1.42 & 88.0\end{array}\right)\left(\begin{array}{l}\Delta R_{x} / R_{x} \\ \Delta R_{y} / R_{y} \\ \Delta R_{z} / R_{z}\end{array}\right) \times 10^{3}$

Figure 2. Outputs of the three-axis force sensor embedded in rubber material used as a sole. (a), (b) and (c) are the results when $\mathrm{x}-, \mathrm{y}$ - and $\mathrm{z}$-axis force were applied to the sensor, respectively. The markers in the graphs are plotted on the measured values. The solid lines in the graphs are fitting values obtained using the least-squares technique. (d) Equation of a force sensor to convert from resistivity changes to forces.

\subsection{Measurement System}

To measure the three-axis GRF distribution during walking, three-axis force sensors were combined with a shoe and circuits.

Commercially available shoes were selected for the measurement experiment (SST8, Dexter, MO, USA). The soles of these shoes could be attached and detached via the hook-and-loop fastener on the bottom surface. This feature was used to attach three-axis force sensors fixed in rubber frames to the left shoe. Rubber with the same thickness was attached to the sole of the right shoe. The shoes used were $26.0 \mathrm{~cm}$ in size. Eleven force sensors were used in the forefoot area, and five force sensors were used in the heel area. We set local coordinates on each sensor. When the subject stands with toes and heels together, the coordinate of the sensors in the heel area and the forefoot area rotates 10 degrees counterclockwise and 7 degrees clockwise with respect to the forward direction, respectively. The cables from the force sensors were fixed using a hook-and-loop fastener on the side surface of the shoes, and were connected with circuits through connectors and wires. The weight of the shoe with sixteen force sensors was $470 \mathrm{~g}$. The circuits were carried on the subject's shoulders with a backpack when the three-axis GRF distribution was measured (Figure 3). 
To correct the force data during walking, a circuit was designed using an IC board (mbed NXP LPC1768, NXP Semiconductors N. V., Eindhoven, The Netherlands). On the circuit board, bridge and amplification circuits, low-pass filters, and A/D converters were mounted for the output channel of each three-axis force sensor. The voltage changes from the three-axis force sensors were measured using the bridge circuits and amplified 247 times, and $25 \mathrm{~Hz}$ were cut off by a low-pass filter. A Bessel filter was used as the low-pass filter, and its group delay was within $3 \%$ error from 0 to $20 \mathrm{~Hz}$. After that, the signals were converted to digital data by 12-bit analog-to-digital (A/D) converters, gathered to the memory on the IC board, and sent to a PC using serial communication. The baud rate of the serial communication was $921.6 \mathrm{kbps}$, and the sampling frequency was $333 \mathrm{~Hz}$. This circuit individually managed four force sensors, and we prepared four sets of circuits for measurement. Lithium batteries were used as the power supply. The dimensions and the weight of the four sets of circuits were $85 \times 106 \times 108 \mathrm{~mm}$ and $660 \mathrm{~g}$, respectively. The total weight of the backpack with four sets of circuits, two batteries, and cables that was carried by the subject was $1100 \mathrm{~g}$.
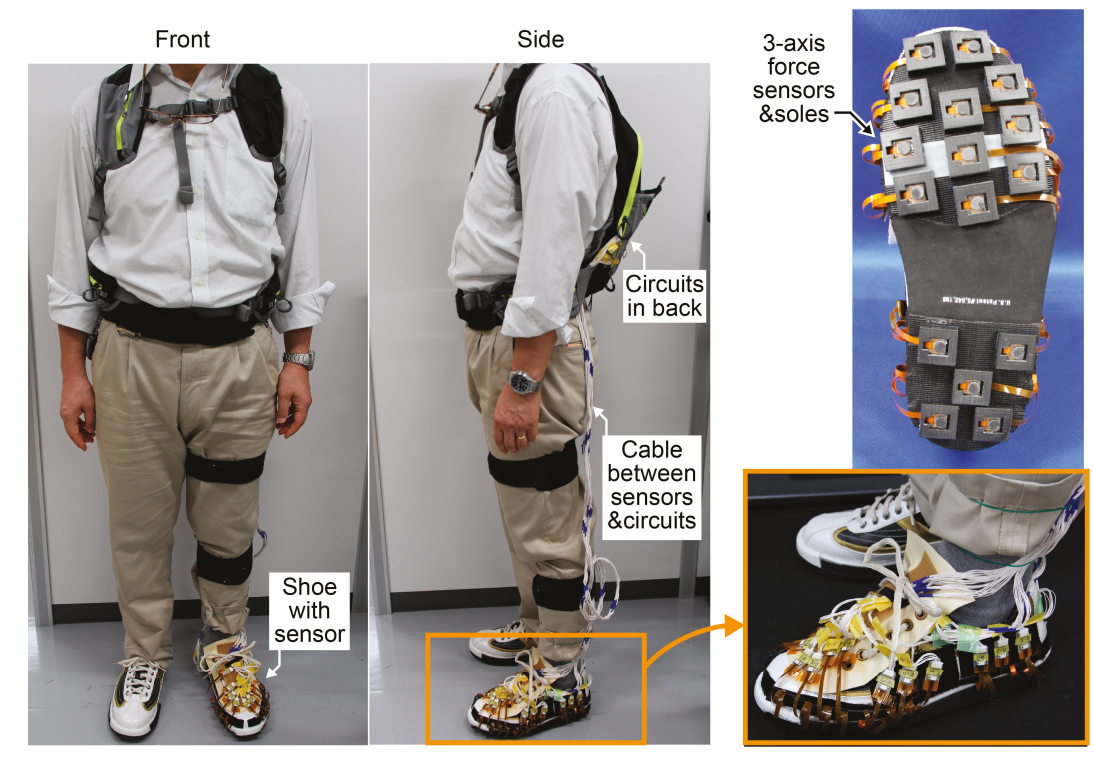

Figure 3. Shoe with three-axis force sensors and appearance of the person who wore the measurement system.

We compared the sum of the sensor read and the body weight when the subject was conducting a static single-leg stand. The result of the former was $528 \mathrm{~N}$, and the latter was $600 \mathrm{~N}$. This indicates that the sensor read has around $10 \%$ error.

\section{Experimental Results and Discussion}

Using the proposed system, the three-axis GRF force distribution was measured when a subject walked on a treadmill. The subject was a healthy man whose height and weight were $168 \mathrm{~cm}$ and $61.2 \mathrm{~kg}$, respectively. The walking velocity was a comfortable speed for the subject, and the analyzed data were averaged over the results from ten steps. The angle of the subject's toe-off was 5 degrees counterclockwise.

Figure 4 shows the change in vertical forces during the foot contact phase. The durations of the single support phase and double support phase were calculated using the foot contact time and walking cycle. As shown in this figure, the contact area under the foot shifted from the heel to the toe. 
At the time of first contact between the left foot and ground, the entire heel area and the bottom of the forefoot area made contact nearly simultaneously. At the beginning of the single support phase, the base area of the toes contacted the ground gradually. Subsequently, the heel area lifted halfway off the ground through the single support phase. At the beginning of the right foot contact, the base area of all the toes were lifting off, and the toe area was in contact with the ground.
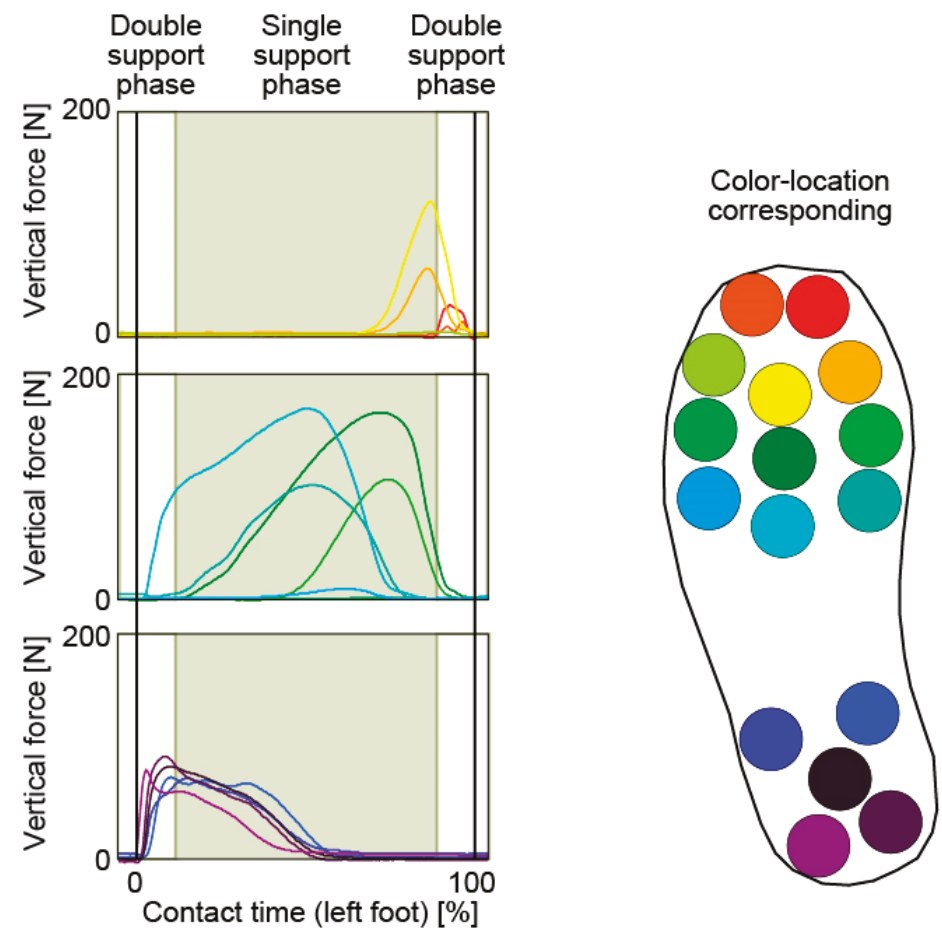

Figure 4. Change of vertical forces during the contact phase of the left foot.

Figure 5 shows the changes in the vertical force distribution over time during the foot contact phase and the center of pressure (COP) trajectory on a schematic of the relation between the location of the force sensors and the foot bone. The vertical forces are represented using a color scale: $0 \mathrm{~N}$ to $5 \mathrm{~N}$ is white, $5 \mathrm{~N}$ to $150 \mathrm{~N}$ gradually changes from purple to red, and over $150 \mathrm{~N}$ is red. The contact phase of the left foot was divided into seven parts, and the time of each data point is shown with the percentage of foot contact time. The time interval is $0.12 \mathrm{~s}$, and the COP trajectory is plotted at $0.06 \mathrm{~s}$ intervals.

At the moment of left foot contact, the vertical force was distributed outside of the heel first and then received by the heel and forefoot (Figure 5i,ii). In this case, the largest vertical force was applied to the back area under the base of the third toe. After the beginning of foot contact, vertical forces at the forefoot were increasingly greater as the subject's total mass moved forward (Figure 5iii,iv). Large vertical forces were applied to a small area under the third metatarsal. During the heel-off phase, vertical forces were primarily applied to the forefoot area of the first, second, and third toes (Figure 5v). During the toe-off phase, the area in which vertical forces were applied changed from the forefoot to the toe, and the forces in these areas became small (Figure 5vi,vii). This trend illustrates that the area under the third metatarsal is an important area during straight walking because of the magnitude of the vertical forces applied. Additionally, this area is likely at high risk of injury when this subject walks an excessively long distance because of the concentrated force. During this foot contact, the COP 
moved from outside of the heel to between the second and third toes. This trajectory is similar to the results of other research [11-13].

Figure 6 shows the change in the shear force distribution over time during the foot contact phase and a schematic of the relation between the force sensor location and foot bone. The shear forces applied from the ground to the shoe are indicated by arrows from the center of each embedded force sensor. The strength of the shear force is represented by the length of the arrow, and the direction of the shear force is represented by the direction of the arrow. The time interval of the presented shear force data is $0.12 \mathrm{~s}$. The contact phase of the left foot is divided into seven parts, and the time of each data point is shown as a percentage of the foot contact time.
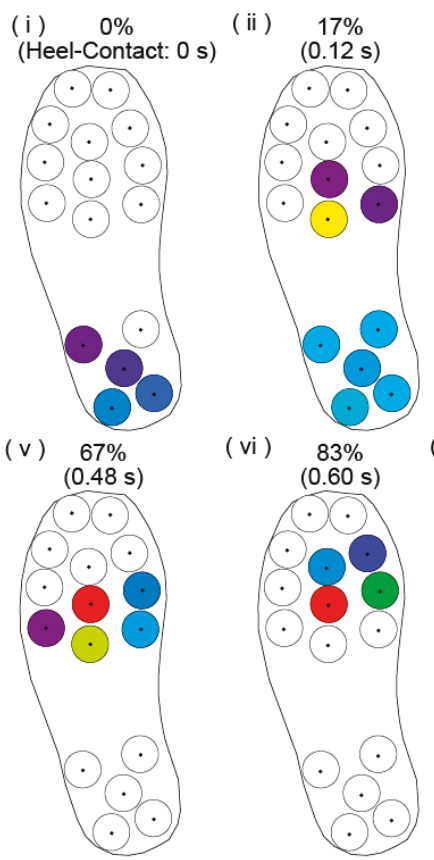

(iii )
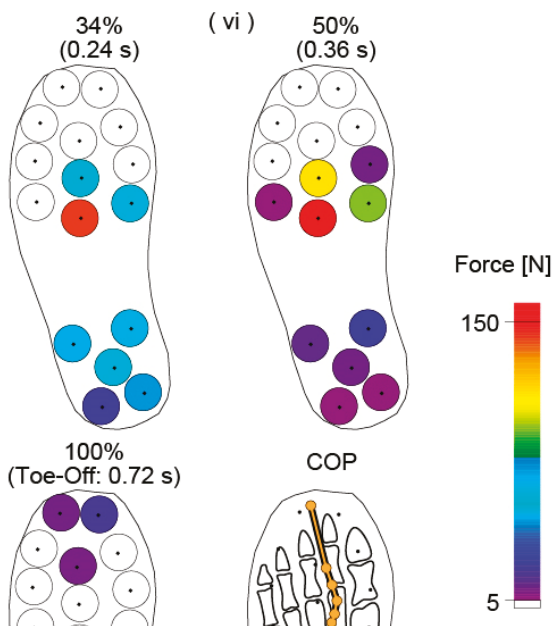

Figure 5. Result of the vertical force distribution measured by the proposed measurement system. COP: center of pressure.

At the moment of left foot contact, a braking force was applied to the heel area and the center back area of the forefoot (Figure 6i,ii). A braking force of approximately $10 \mathrm{~N}$ was evenly applied to those areas. After the beginning of foot contact, the main area where the shear forces were applied moved from the heel to the forefoot. Although the shear forces applied to the heel area decreased in amplitude, the forces applied to the area under the base of the first, second, and third toes increased (Figure 6iii,iv). The shear forces in different areas had different features. The shear forces from the center back area of the first, second, and third toes were in the medial direction, indicating that this area plays a role in translating the center of mass from the left foot to the right foot. The shear force under the base of the third toe was in the anterior direction. Thus, the propulsive force is generated from this area. Additionally, these shear forces generated a torque in the counterclockwise direction along the vertical axis. During the heel-off phase, the area under the base of the big toe received a shear force toward the right foot (Figure 6v). The subject's center of mass also moved toward the right foot due to this shear force and toward the center back area of the first, second, and third toes. During the toe-off phase, propulsive forces were applied under the first, second, and third toes (Figure 6vi,vii). 
Under the big toe, a propulsive force was applied in the medial direction. Conversely, the propulsive force under the third toe was applied in the direction of the long axis of the shoe.
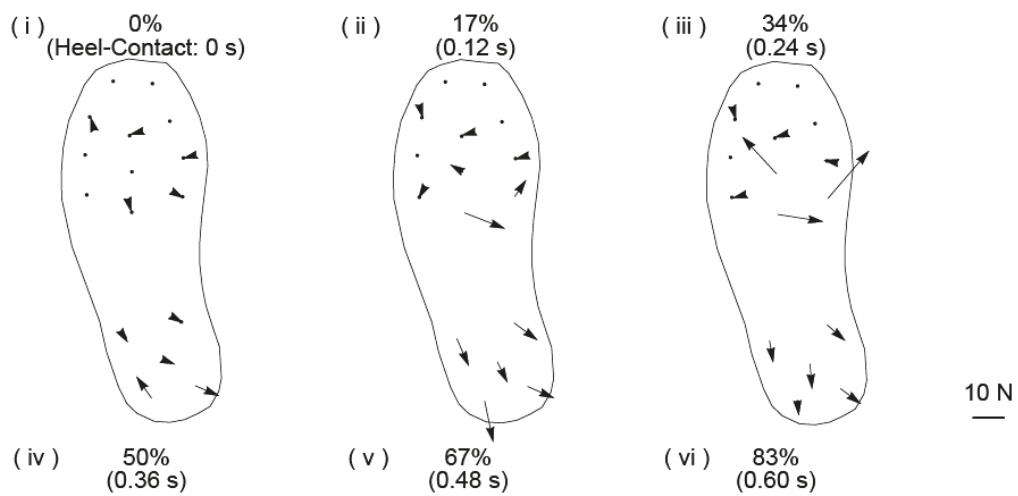

( vi ) $\quad \begin{aligned} & 83 \% \\ & (0.60 \mathrm{~s})\end{aligned}$
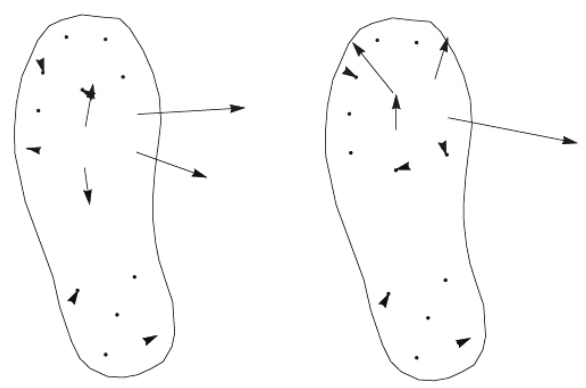

( vii )
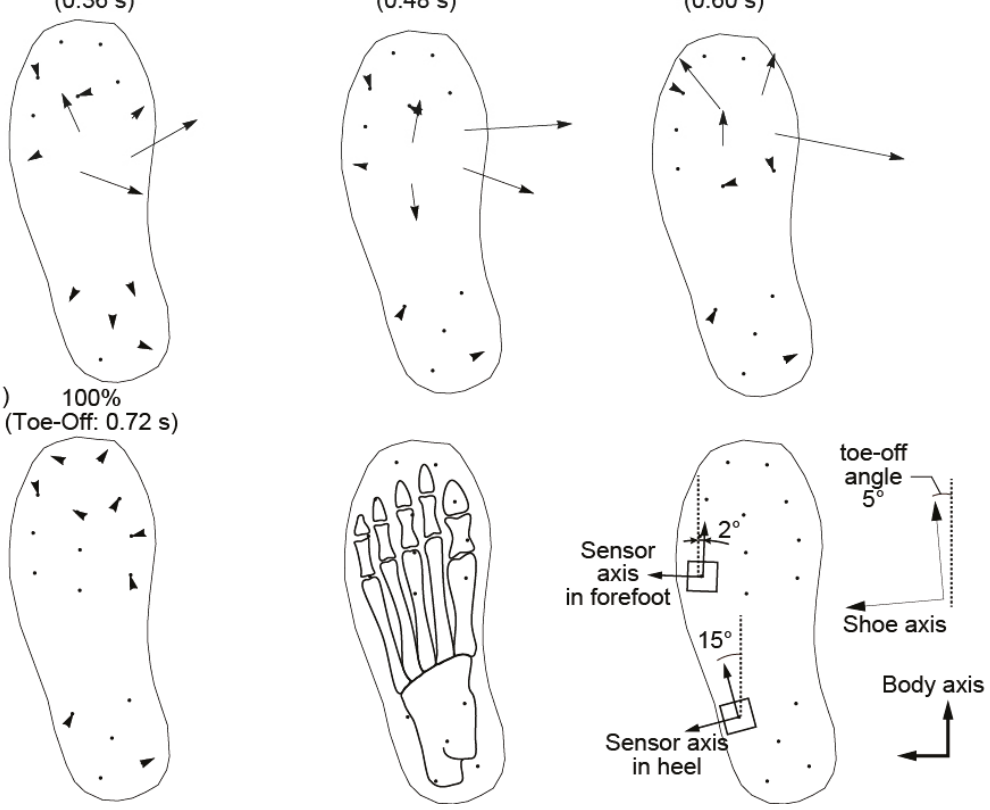

Figure 6. Result of the shear force distribution measured by the proposed measurement system.

These results imply that the features of the shear forces during straight walking differ depending on the location of the sole for this subject. The heel area received braking forces early during straight walking, and the area under the first, second, and third toes generated propulsive forces at the end of walking. Although these features correspond well with previous knowledge of bipedal walking, three features from the forefoot area are observed halfway through straight walking [14]. The first feature was observed under the fourth and fifth toes. In this area, small shear forces were applied during straight walking, indicating that this area has a low degree of importance when the subject is walking straight. The second feature was observed under the base of the big toe. This area helped move the center of mass toward the other foot, and the generated shear force was directed to the right foot while the area was in contact with the ground. This result occurred because the momentum of the center of mass toward the left decreased and changed the momentum toward the right. This result 
indicates that the forefoot is separated into two areas: the area moving the center of mass forward, and the area moving it toward the other foot. The final feature was observed at the base of the third toe. Whereas the front area of the base of the toe generated a propulsive force, the back area of the base of the toe generated a braking force. This feature is assumed to be caused by foot motion in the shoe while walking. When a human is walking, the foot makes a gripping motion in the shoe. During this motion, the toes move as though they are scratching the ground, and the remaining area of the foot holds the ground. The joints between the metatarsal bones and phalanxes support this motion, and the area under these bones becomes the border where the direction of shear force changes. This observation is presumably why the directions of the shear forces are different between the front and back areas under the base of the third toe.

There are several advantages to our method. First, in our method, it is easy to increase the number of force sensors because of their small size. In walking research, several studies have focused on shoes with wearable force sensors [15-18]. In these studies, two-to-six three-axis force sensors were attached to the sole of the shoe, and the researchers attempted to measure the three-axis GRF distribution, even outside the laboratory. Although the results demonstrated the potential usefulness of these proposed measurement systems, the number of force sensors still requires improvement. When using only two-to-six force sensors, the distance between the sensors is rather large. Thus, a large space under the foot is covered by one force sensor, and the spatial resolution of the measured force data is low. This drawback could produce a deficient walking analysis of the three-axis force distribution. To increase the number of force sensors under a foot, where space is limited, the force sensors must be smaller. Our force sensors were fabricated using MEMS and miniaturized. Therefore, we can attach 16 force sensors to a shoe, which is more than twice the number of sensors used in previous studies.

Second, in our method, even when many force sensors are attached to the shoe, the weight of the shoe is approximately maintained because only a small amount of metal material is used. In previous studies attempting to measure three-axis GRF distribution while walking, either the force sensors were made of metal or metal plates were used to fix the force sensors to the soles of commercially available shoes [15-18]. The use of metal could cause subjects discomfort, because such a hard and heavy material is not normally found in shoes. Additionally, if researchers want to attach many force sensors to a shoe, the weight of the shoe will become heavy and may affect the subject's locomotion. To mitigate the drawbacks of using metal as much as possible, our fabricated force sensor was made primarily of rubber, which is in the hardness range of commercially available shoes. By using rubber, our force sensor is lighter than those used in previous studies. Thus, subjects who wear our shoes will experience under-foot sensations that are nearly the same as those before the force sensors were attached, and their locomotion will be largely unaffected.

According to its design, our method can measure a higher three-axis GRF distribution resolution, even outside the laboratory. Our shoes enable data to be collected even in locations where it is difficult to place force plates. The obtained three-axis distribution data cannot be collected using a pressure sheet. Compared to other proposed shoe measurement methods, the shape and weight of the shoe are further preserved, and our proposed method obtains the highest data resolution.

An important observation from this experiment was that the individual features of the area under each subject's sole can be discussed from the measured three-axis force distribution. From the resulting shear force distribution, there are several suggested roles for the forefoot area: a low contribution area, a propulsive force generating area, and a medial force generating area. Additionally, this result implies that the area under the third metatarsal is an area of concentrated applied vertical and shear force. These features observed during our walking experiment cannot be accepted as a common feature of human gait at this time. However, this measurement system can obtain a subject's three-axis force features applied to definite sole areas, and the measurement of the three-axis force is meaningful for walking analysis. In the future, common three-axis force features during walking will be collected using more subjects. 


\section{Conclusions}

A three-axis GRF distribution during straight walking was measured using a shoe with three-axis force sensors. Sixteen small three-axis force sensors were fabricated with rubber and sensor chips. The weight of the three-axis force sensor with a rubber frame was $15 \mathrm{~g}$. The force sensors were attached to the removable sole of the left shoe using a hook-and-loop fastener. The data from these 16 force sensors were collected at a sampling frequency of $333 \mathrm{~Hz}$. Our shoe measurement system was used to measure the three-axis GRF distribution during straight walking on a treadmill. From the experiment, extensive three-axis-GRF-distribution data were obtained for the first time. The local roles under the foot were revealed by analyzing the distribution data: the heel received a braking force during the foot contact phase, the base of the fourth and fifth toes applied a small three-axis force during straight walking, the base of the second and third toes applied a strong vertical force and propulsive force from the mid-standing phase to the take-off phase, and the base of the big toe applied a medial force from the mid-standing phase to the take-off phase. These results indicate that the applied three-axis GRF force differed at different locations under the foot. Therefore, measuring and analyzing the three-axis GRF distribution during walking has enormous significance, and may yield new insights for walking researchers. In the future, we believe that this measurement system and the results of this study will be crucial for further detailed analyses of bipedal locomotion.

Acknowledgments: This work was partially supported by JSPS KAKENHI Grant Number 25000010.

Author Contributions: All authors contributed to the design, data analysis and preparation of the manuscript. Masataka Hori and Akihito Nakai fabricated the sensor, established the shoe measurement system and performed the experiments. Isao Shimoyama planned and supervised the project.

Conflicts of Interest: The authors declare no conflict of interest.

\section{References}

1. Elftman, H. A Cinematic Study of the Distribution of Pressure in the Human Foot. Anat. Rec. 1934, 59, 481-491. [CrossRef]

2. Elftman, H. The Measurement of the External Force in Walking. Science 1938, 88, 152-153. [CrossRef] [PubMed]

3. Rajala, S.; Lekkala, J. Plantar shear stress measurements-A review. Clin. Biomech. 2014, 29, 475-483. [CrossRef] [PubMed]

4. Jung, C.K.; Park, S. Compliant Bipedal Model with the Center of Pressure Excursion Associated with Oscillatory Behavior of the Center of Mass Reproduces the Human Gait Dynamics. J. Biomech. 2014, 47, 223-229. [CrossRef] [PubMed]

5. Stolwijk, N.M.; Duysens, J.; Louwerens, J.W.K.; Keijsers, N.L.W. Plantar Pressure Changes after Long-Distance Walking. Med. Sci. Sports Exerc. 2010, 42, 2264-2272. [CrossRef] [PubMed]

6. Cong, Y.; Lee, W.; Zhang, M. Regional Plantar Foot Pressure Distributions on High-Heeled Shoes-Shank Curve Effects. Acta Mech. Sin. 2011, 27, 1091-1097. [CrossRef]

7. Decker, L.; Houser, J.J.; Noble, J.M.; Karst, G.M.; Stergiou, N. The effects of shoe traction and obstacle height on lower extremity coordination dynamics during walking. Appl. Ergon. 2009, 40, 895-903. [CrossRef] [PubMed]

8. Lieberman, D.E.; Venkadesan, M.; Werbel, W.A.; Daoud, A.I.; D'Andrea, S.; Davis, I.S.; Mang'Eni, R.O.; Pitsiladis, Y. Foot Strike patterns and Collision Forces in Habitually Barefoot versus Shod Runners. Nature 2010, 463, 531-535. [CrossRef] [PubMed]

9. Dxon, S.J.; Collop, A.C.; Batt, M.E. Surface Effects on Ground Reaction Forces and Lower Extremity Kinematics in Running. Med. Sci. Sports Exerc. 2000, 32, 1919-1926. [CrossRef]

10. Takahashi, H.; Nakai, A.; Thanh-Vinh, N.; Matsumoto, K.; Shimoyama, I. A Triaxial Tactile Sensor without Crosstalk Using Pairs of Piezoresistive Beams with Sidewall Doping. Sens. Actuators A: Phys. 2013, 199, 43-48. [CrossRef]

11. Orlin, M.N.; McPoil, T.G. Plantar Pressure Assessment. Phys. Ther. 2000, 80, 399-409. [CrossRef] [PubMed] 
12. Hatala, K.G.; Dingwall, H.L.; Wunderlich, R.E.; Richmond, B.G. The relationship between plantar pressure and footprint shape. J. Hum. Evolut. 2013, 65, 21-28. [CrossRef] [PubMed]

13. Bruening, D.A.; Cooney, K.M.; Buczek, F.L.; Richards, J.G. Measured and estimated ground reaction forces for multi-segment foot models. J. Biomech. 2010, 43, 3222-3226. [CrossRef] [PubMed]

14. Rueterbories, J.; Spaich, E.G.; Larsen, B.; Andersen, O.K. Methods for gait event detection and analysis in ambulatory systems. Med. Eng. Phys. 2010, 32, 545-552. [CrossRef] [PubMed]

15. Veltink, P.H.; Liedtke, C.; Droog, E.; van der Kooij, H. Ambulatory Measurement of Ground Reaction Forces. IEEE Trans. Neural Syst. Rehabil. Eng. 2005, 13, 423-427. [CrossRef] [PubMed]

16. Liedtke, C.; Fokkenrood, S.A.W.; Menger, J.T.; van der Kooij, H.; Veltink, P.H. Evaluation of Instrumented Shoes for Ambulatory Assessment of Ground Reaction Forces. Gait Posture 2007, 26, 39-47. [CrossRef] [PubMed]

17. Liu, T.; Inoue, Y.; Shibata, K. A Wearable Ground Reaction Force Sensor System and Its Application to the Measurement of Extrinsic Gait Variability. Sensors 2010, 10, 10240-10255. [CrossRef] [PubMed]

18. Liu, T.; Inoue, Y.; Shibata, K. A Wearable Force Plate System for the Continuous Measurement of Triaxial Ground Reaction Force in Biomechanical Applications. Meas. Sci. Technol. 2010, 21, 085804. [CrossRef]

(c) 2017 by the authors. Licensee MDPI, Basel, Switzerland. This article is an open access article distributed under the terms and conditions of the Creative Commons Attribution (CC BY) license (http:/ / creativecommons.org/licenses/by/4.0/). 
Article

\title{
An Advanced Hybrid Technique of DCS and JSRC for Telemonitoring of Multi-Sensor Gait Pattern
}

\author{
Jianning $\mathbf{W u}$ *, Jiajing Wang, Yun Ling and Haidong $\mathrm{Xu}$ \\ College of Mathematics and Informatics, Fujian Normal University, Fuzhou 350117, China; \\ jiajingwang_1993@126.com (J.W.); yunling93@163.com (Y.L.); sihaijunmi@fjnu.edu.cn (H.X.) \\ * Correspondence: jianningwu@fjnu.edu.cn
}

Received: 25 October 2017; Accepted: 28 November 2017; Published: 29 November 2017

\begin{abstract}
The jointly quantitative analysis of multi-sensor gait data for the best gait-classification performance has been a challenging endeavor in wireless body area networks (WBANs)-based gait telemonitoring applications. In this study, based on the joint sparsity of data, we proposed an advanced hybrid technique of distributed compressed sensing (DCS) and joint sparse representation classification (JSRC) for multi-sensor gait classification. Firstly, the DCS technique is utilized to simultaneously compress multi-sensor gait data for capturing spatio-temporal correlation information about gait while the energy efficiency of the sensors is available. Then, the jointly compressed gait data are directly used to develop a novel neighboring sample-based JSRC model by defining the sparse representation coefficients-inducing criterion (SRCC), in order to yield the best classification performance as well as a lower computational time cost. The multi-sensor gait data were selected from an open wearable action recognition database (WARD) to validate the feasibility of our proposed method. The results showed that when the comparison ratio and the number of neighboring samples are selected as $70 \%$ and $40 \%$, respectively, the best accuracy (95\%) can be reached while the lowest computational time spends only $60 \mathrm{~ms}$. Moreover, the best accuracy and the computational time can increase by $5 \%$ and decrease by $40 \mathrm{~ms}$, respectively, when compared with the traditional JSRC techniques. Our proposed hybrid technique can take advantage of the joint sparsity of data for jointly processing multi-sensor gait data, which greatly contributes to the best gait-classification performance. This has great potential for energy-efficient telemonitoring of multi-sensor gait.
\end{abstract}

Keywords: multi-sensor gait classification; distributed compressed sensing; joint sparse representation classification; telemonitoring of gait

\section{Introduction}

Recently, wireless body area networks (WBANs), as an emerging wireless sensor networks technology that is applied to the human body [1-3], has been more and more popular in gait telemonitoring applications [4-6]. In such WBANs, each sensor node usually takes the form of a small wearable device equipped with a microcontroller, accelerometer, gyroscope, wireless communication module, and battery. These wearable sensors are co-located on the body for acquiring gait data of people who freely walk in a home or outdoor setting. Moreover, all acquired data can be transmitted, via internet, to a remote terminal such as a hospital for gait monitoring or assessment by further data processing such as gait classification. This greatly contributes to the telemonitoring of gait pattern change, and people do not often visit a hospital for gait function assessment [4-7]. However, in clinical applications, continuous acquisition of the multi-sensor gait data over the long term is usually required for further data processing, in order to accurately evaluate the gait pattern change [8-10]. In such a case, there have been several challenging existing issues such as improving the energy efficiency of the sensor, getting the best classification performance, and getting a lower computation complexity. 
The above existing issues are strangling the WBANs-based gait telemonitoring application, and some advanced techniques for multi-sensor gait data analysis are urgently needed to tackle these existing issues [9-12].

As we know, due to the limited life of the battery, the wearable sensors are usually required to consume the least possible amount of energy for the continuous telemonitoring of gait [10-13]. How to improve the energy efficiency of the sensors has been one challenging endeavor in the WBANs-based telemonitoring applications [10-12]. Many previous studies found that most of the energy of the sensors is consumed by wireless communication, and their works mainly focused on developing the energy-aware MAC protocols for the low energy consumption of sensors. However, these protocols don't provide reliable data transfer because heavy collision is usually generated by some adjacent sensors [10,12]. In recent ten years, compressed sensing (CS)—an emerging data compression methodology that data compression and reconstruction can be perfectly performed based on data sparsity-has attracted wide attention in the study of energy-efficient gait telemonitoring applications [14]. The basic idea is that a larger amount of the acquired gait data is firstly compressed on sensors, in order to significantly reduce the energy consumption during data transmission. Then, all compressed data received in the remote terminal are perfectly reconstructed to perform further data processing $[15,16]$. For example, Wu et al. investigated the application of CS for the energy-efficient telemonitoring of gait using acceleration data [16]. Although significant research efforts are made, many existing works have only showed that the CS technique for energy-efficient gait telemonitoring is feasible only using single-sensor data. Theoretically, the CS technique has no ability to simultaneously compress multi-sensor data for the energy efficiency of sensors. This leads to the possible loss of the highly-correlated information regarding the intrinsic dynamics of human movement. It is essential to search for an advanced technique for jointly processing multi-sensor gait data.

Further data processing such as gait classification is also very important for gait monitoring. How to develop the best gait-classification model has been another challenging issue in gait telemonitoring applications [16-18]. Some studies have showed that the original gait data are directly used to develop the gait-classification models based on machine learning algorithms. In these studies, some commonly used traditional machine learning algorithms include artificial neural networks (ANN) [18], decision tree, $k$-nearest neighbor (KNN) [17], support vector machine (SVM) [16,19], and Hidden Markov Model (HMM) [20-22]. Comparing these methods, the HMM technique has shown higher performance in gait pattern classification using on-body wearable sensors. For instance, Mannini et al. investigated the feasibility of human physical activity classification by using on-body wearable sensors. Especially, Taborri et al. proposed a novel HMM-based distributed classifier for the detection of gait phases by using a wearable inertial sensor network. In their proposed algorithm, a distributed stochastic model and a hierarchical-weighted classification are implemented to detect gait phases by simultaneously processing the data of multi-sensors placed on different body segments of lower limbs. Recently, an emerging sparse representation classification (SRC) algorithm has widely attracted the focus of gait classification. Its basic idea is that all gait training samples are directly used to construct an over-complete dictionary, and a test gait can be sparsely represented as a linear combination of just those training samples with same gait class. The residual error criterion is defined by solving sparse representation coefficients, in order to exactly determine the class of the test gait [23-25]. Zhang et al. investigated the feasibility of a sparse representation-based gait-classification model using wearable sensor gait data [24]. Also, Allen et al. studied the practicality of the sparse representation-based gait-classification model using wearable multi-sensor gait data [25]. Although the SRC algorithm can produce a better accuracy, it has a higher computational time cost. More importantly, all above studies don't take into account the use of the gait data received at a remote terminal to develop a gait-classification model [26,27].

At the same time, other recent studies have focused on using the received gait data to develop gait-classification models. In view of the energy efficiency of sensors, recent relevant studies mainly 
concentrated on the combination of the CS technique with machine learning algorithms for gait classification. Their basic idea is that all compressed gait data received at a remote terminal are reconstructed by the CS technique, and the reconstructed gait data are then employed to develop gait-classification models based on machine learning algorithms [14,16]. In such works, one important step is to reconstruct gait data with a higher quality. However, the traditional CS technique has no ability to perfectly reconstruct the gait data; this is because gait data such as acceleration data are poorly sparse $[14,16]$. This possibly deteriorates the further gait-classification performance. Moreover, these hybrid techniques possibly produce a higher computational complexity. This motivates us to search for advanced approaches to the energy-efficient telemonitoring of multi-sensor gait $[28,29]$.

It is well known that multi-sensor gait data generally interact in a complex fashion, an observation attributable to the intrinsic dynamics of human gait. Theoretically, we can assume multi-sensor gait data as a data ensemble with joint sparsity, in order to jointly process multi-sensors and thus capture the higher-correlation information associated with gait. This can potentially allow the best classification performance and energy efficiency of sensors. Recently, there have emerged some advanced techniques that have a superior ability to jointly process multi-signals based on data joint sparsity. Successful examples include distributed compressed sensing (DCS) [30,31] and the joint sparse representation classification (JSRC) model [32-34]. These two techniques have been widely applied in video coding [35-37], image fusion [38], and multichannel physiological monitoring [39]. So far, no studies have been reported where DCS and JSRC have been employed for jointly processing multi-sensor gait data.

In this study, a novel advanced hybrid technique of DCS and JSRC for the telemonitoring of multi-sensor gait is proposed based on data joint sparsity. Unlike the recent relevant studies, the advantage of our proposed technique is that we directly take advantage of the multi-sensor gait data compressed by DCS to develop the novel neighboring JSRC gait-classification models with better performance. This can not only avoid the complicated step of joint reconstruction of multi-sensor gait data, but can also produce the best classification performance as well as a lower computational time. In our proposed technique, the DCS technique is firstly utilized to simultaneously compress multi-sensor gait data, in order to potentially gain higher-correlation information as well as improved energy efficiency of the sensors. Then, all jointly compressed gait data are directly used to develop a novel neighboring sample-based JSRC model by defining the sparse representation coefficients-inducing criterion (SRCC), not to perform the reconstruction task. The test gait can be sparsely represented by constructing a new over-complete dictionary that includes a few local neighboring training samples containing more valuable information. This possibly produces the best classification performance as well as a lower computational time. Our proposed technique has great potential to enable continuous collection of people's gait information and accurate human gait measurement anywhere and at any time. This possibly provides benefits in telemedicine applications such as the early identification of at-risk gait of elderly in the community, as well as the monitoring of gait rehabilitation progress of people at home. The multi-sensor gait data are selected from an open wearable action recognition database (WARD), in order to validate the feasibility of our proposed method. The results show the superior classification performance of our proposed techniques to the traditional techniques such as SRC and JSRC. Moreover, a lower computational time cost is spent in our proposed technique.

The rest of the paper is organized as follows. In Section 2, we describe the DCS technique for multi-sensor gait data. A novel neighboring JSRC model for multi-sensor gait classification is presented in Section 3. In Section 4, we describe the materials and methods for evaluating our proposed technique. The evaluation results obtained from multi-sensor gait data are given in Section 5. Discussion and conclusions are presented in Sections 6 and 7, respectively. 


\section{Distributed Compressed Sensing for Multi-Sensor Gait Data}

Theoretically, the DCS technique combines distributed source coding theory with compressed sensing theory $[14,30,31]$. It can take advantage of data joint sparsity to capture both inter- and intra-signal correlation among multi-sensor signals. In the DCS technique, three different joint sparsity models (JSM), such as JSM-1, JSM-2, and JSM-3 [30,31], are usually used to perform the simultaneous compression and the joint reconstruction task in the multi-signal case. In this study, since each sensor can acquire the same gait data, such as acceleration data, we assume that each sensor's data has the same sparsity pattern but with different coefficients. Here, JSM-2 is adopted to jointly process the multi-sensor gait data $[30,31]$. That is, all $J$ sensors' gait data in WBANs are assumed as a data ensemble $X=\left[x_{1}, x_{2}, \cdots, x_{J}\right]^{T} \in R^{J N \times 1}$, where $N$ denotes the length of each sensor data $x_{j} \in R^{N \times 1}(j=1,2, \cdots, J)$. Here, we assume that each sensor data $x_{j}$ is sparsely represented as

$$
x_{j}=\Psi \boldsymbol{\theta}_{j}
$$

where $\boldsymbol{\Psi}$ denotes dictionary matrix vectors and $\boldsymbol{\theta}_{j}$ is the corresponding sparse vector that satisfies the same support set $\Omega \subset\{1,2, \cdots, N\}$. Then, the multi-sensor gait data ensemble $X$ can be represented as

$$
X=\Psi \theta
$$

where $\boldsymbol{\theta}=\left[\boldsymbol{\theta}_{1}, \boldsymbol{\theta}_{2}, \cdots, \boldsymbol{\theta}_{j}, \boldsymbol{\theta}_{J}\right]^{T}$ is the joint sparse coefficients vector and $\boldsymbol{\Psi}=\left[\begin{array}{l}\Psi, 0,0, \cdots, 0 \\ 0, \Psi, 0, \cdots, 0 \\ \cdot \\ 0,0,0, \cdots, \Psi\end{array}\right]$ is the corresponding dictionary matrix. Then, the multi-sensor gait data ensemble $\boldsymbol{X}$ can be rewritten as

$$
\left[x_{1}, x_{2}, \cdots, x_{j}, \cdots, x_{J}\right]^{T}=\left[\begin{array}{l}
\Psi, 0,0, \cdots, 0 \\
0, \Psi, 0, \cdots, 0 \\
\cdot \\
0,0,0, \cdots, \Psi
\end{array}\right]\left[\theta_{1}, \theta_{2}, \cdots, \theta_{j}, \cdots, \theta_{J}\right]^{T} .
$$

Next, we define a measurement matrix $\Phi \in \mathrm{R}^{\mathrm{JM} \mathrm{M}_{\mathrm{j}} \times \mathrm{JN}}\left(\boldsymbol{\Phi}_{\mathrm{j}} \in \mathrm{R}^{\mathrm{M}_{\mathrm{j}} \times \mathrm{N}}, \mathrm{M}_{\mathrm{j}} \ll \mathrm{N}\right)$ as

$$
\boldsymbol{\Phi}=\left(\begin{array}{cccccc}
\Phi_{1} & 0 & 0 & 0 & \cdots & 0 \\
0 & \Phi_{2} & 0 & 0 & \cdots & 0 \\
\vdots & \vdots & \vdots & \vdots & \cdots & \vdots \\
0 & 0 & 0 & 0 & \cdots & \Phi_{J}
\end{array}\right)
$$

Thus, the multi-sensor gait data ensemble $X$ can be simultaneously compressed as

$$
\boldsymbol{Y}=\boldsymbol{\Phi} \boldsymbol{X}=\left(\begin{array}{cccccc}
\Phi_{1} & 0 & 0 & 0 & \cdots & 0 \\
0 & \Phi_{2} & 0 & 0 & \cdots & 0 \\
\vdots & \vdots & \vdots & \vdots & \cdots & \vdots \\
0 & 0 & 0 & 0 & \cdots & \Phi_{J}
\end{array}\right)\left[x_{1}, x_{2}, \cdots, x_{j}, \cdots, x_{J}\right]^{T}
$$

where $Y=\left[y_{1}, y_{2}, \cdots, y_{j}, \cdots, y_{L}\right]^{T} \in R^{J M \times 1}$ is the jointly compressed multi-sensor data. Here, it is noted that each $\boldsymbol{y}_{j}=\boldsymbol{\Phi}_{j} \boldsymbol{x}_{j}, j \in\{1,2, \cdots, J\}$ is performed independently of the others. 
In the DCS technique, joint reconstruction is another important task. Its basic idea is to obtain an accurate solution of the joint sparse representation coefficients $\theta=\left[\theta_{1}, \theta_{2}, \cdots, \theta_{j}, \theta_{J}\right]^{T}$ by solving the following $l_{1}$-norm minimization:

$$
\hat{\boldsymbol{\theta}}=\underset{\boldsymbol{\theta}}{\operatorname{argmin}}|| \boldsymbol{\theta} \|_{l_{1}} \text {, s.t. } \mathbf{Y}=\boldsymbol{\Phi} \boldsymbol{X} .
$$

Then, the joint reconstruction of multi-sensor data can be estimated as $\hat{X}=\Psi \hat{\boldsymbol{\theta}}$. The commonly used joint reconstruction algorithms include the One-Step Greedy Algorithm (OSGA) [32] and the Simultaneous Orthogonal Matching Pursuit (SOMP) [31]. However, since multi-sensor gait data such as accelerometer and gyroscope data are poorly sparse, it is very difficult to guarantee the best solution of joint representation coefficients $\hat{\boldsymbol{\theta}}$ by Equation (6). In fact, the jointly compressed multi-sensors data $\boldsymbol{Y}$ by DCS possibly contain enough highly-correlated discriminative information associated with gait. Theoretically, the compressed multi-sensor data $Y$ can be directly used to develop a JSRC-based classification model, but not to perform a joint reconstruction task. This helps to improve multi-sensor gait-classification performance.

\section{A Novel Neighboring JSRC Model for Gait Classification}

Theoretically, the JSRC model is used to generalize the traditional SRC model to the multi-signal case $[33,40-43]$. Its basic idea is to explore the joint sparse representation for the multiple input signals in the classification task, and the joint sparsity can be enforced by imposing a joint sparsity-inducing norm(such as $l_{1,2}$-norm, $l_{1, \infty}$-norm) penalty on representation coefficients [23-25]. However, due to the large-scale gait training samples in this study, more computational time and higher memory cost are needed for the matrix inverse operation in the JSRC algorithm. This possibly yields a high computational complexity as well as a poor classification performance. It is essential to search for a few training samples to develop the best JSRC-based gait-classification models. In this study, a novel neighboring JSRC model is developed based on a few neighboring training samples selected by the sparse representation coefficients-inducing criterion (SRCC). As we know, the same gait class can be theoretically spanned by the training samples (i.e., over-complete dictionary atoms) of the same class in a low-dimensional subspace [33,41-48]. The spanned training sample can be sparsely represented in the high-dimension space. Those samples with high values of sparse representation coefficients are selected as the nearest neighbor samples because they possibly contain the more valuable information associated with gait pattern change. So, these selected neighboring samples are used to reconstruct a new over-complete dictionary for multi-sensor gait classification. The block diagram of the proposed novel neighboring JSRC model is shown in Figure 1. In order to clearly describe the novel neighboring JSRC model, we firstly describe the construction of an over-complete dictionary using all original training samples. 


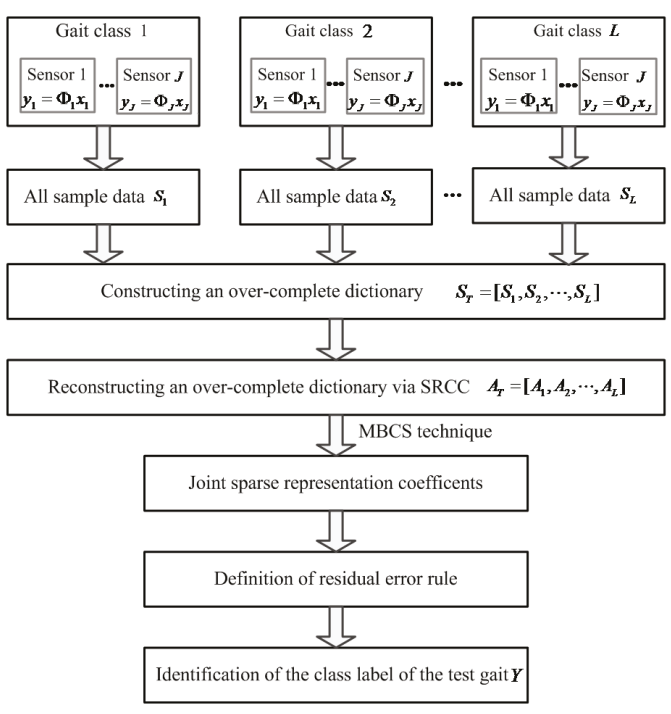

Figure 1. The block diagram of the proposed novel neighboring joint sparse representation classification (JSRC) model.

\subsection{Constructing an Over-Complete Dictionary for JSRC}

In this study, we assume that each sensor node is equipped with a triaxial accelerometer $(x, y, z)$ and a biaxial gyroscope $(\boldsymbol{\theta}, \boldsymbol{\rho})[24,25]$, in order to capture more valuable information associated with gait. Firstly, we define a vector $a_{j}$ to denote the gait data of each sensor $j(j=1,2, \cdots, J)$ at time $t$, i.e.,

$$
a_{j}=\left(x_{j}(\mathrm{t}), y_{j}(\mathrm{t}), z_{j}(\mathrm{t}), \theta_{j}(\mathrm{t}), \rho_{j}(\mathrm{t})\right)^{T} \in \mathrm{R}^{5} .
$$

Then, a vector $\boldsymbol{a}(\boldsymbol{t})$ is defined to represent multi-sensor gait data of all $\boldsymbol{J}$ sensors at time $\boldsymbol{t}$, i.e.,

$$
\boldsymbol{a}(\boldsymbol{t})=\left(\boldsymbol{a}_{1}^{T}(\boldsymbol{t}), \boldsymbol{a}_{2}^{T}(\boldsymbol{t}), \cdots, \boldsymbol{a}_{J}^{T}(\boldsymbol{t})\right)^{T} \in \mathrm{R}^{5 J} .
$$

Next, based on Equation (6), a matrix $S$ is defined to represent the multi-sensor gait data ensemble during the length of time $h$ :

$$
S=[\boldsymbol{a}(1), \boldsymbol{a}(2), \cdots, \boldsymbol{a}(\boldsymbol{h})]^{T}=\left(\boldsymbol{s}^{1}, \boldsymbol{s}^{2}, \cdots, s^{J}\right)^{T} \in \mathrm{R}^{5 J \times \boldsymbol{h}}
$$

where the sub-vector $s^{j}$ denotes the gait data of sensor $j$ with duration $\boldsymbol{h}, s^{j}=\left(\boldsymbol{a}_{j}(1), \boldsymbol{a}_{j}(2), \cdots, \boldsymbol{a}_{j}(\boldsymbol{h})\right)^{T} \in \mathrm{R}^{5 \times \boldsymbol{h}}$.

Here, assuming that there are $L$ different gait classes, we can define a new matrix $S_{T}$ that concatenates the training sample from all $L$ gait classes:

$$
S_{T}=\left[S_{1}, S_{2}, \cdots, S_{i}, S_{L}\right](i=1,2, \cdots, L)
$$

where sub-matrix $S_{i}=\left(s_{i, 1}, s_{i, 2}, \cdots, s_{i, n_{i}}\right)$ represents all training sample data of gait class $i$, and $n_{i}$ denotes the total of training samples of class $i$. In such a case, we can employ the matrix $S_{T}$ to construct an over-complete dictionary that consists of $L$ sub-dictionary $S_{i}$ with respect to all $L$ 
classes $[23,25,33,41,43]$. Thus, based on the constructed over-complete dictionary $\boldsymbol{S}_{T}$, a test gait $\boldsymbol{Y}\left(\boldsymbol{Y}=\left[\mathbf{y}_{1}, \mathbf{y}_{2}, \cdots, \boldsymbol{y}_{j}, \cdots, \mathbf{y}_{J}\right]^{T} \in \boldsymbol{R}^{J M \times 1}\right)$ with class $\boldsymbol{i}$ can be joint-sparsely represented as

$$
\boldsymbol{Y}=S_{T} \boldsymbol{W}
$$

where $W=\left[0,0, \cdots, 0, w_{i, 1}, w_{i, 2}, \cdots, w_{i, n_{i}}, 0, \cdots, 0\right]$ denotes the joint sparse representation coefficients matrix whose entries are zero except those associated with gait class $i$, and the total number of samples $n=\sum_{i}^{L} n_{i}$.

Usually, the joint sparse coefficients matrix $W$ can be estimated by solving the following joint sparse optimization problem $[33,41,43]$ :

$$
\hat{W}=\operatorname{argmin}\left\|\boldsymbol{Y}-S_{T} W\right\|_{F}^{2} \text { s.t. }\|W\|_{l_{0} \backslash l_{2}} \leq k
$$

where $\|\cdot\|_{F}$ denotes the Frobenius norm, $\|W\|_{l_{0} \backslash l_{2}}$ refers to the mixed norm that can be phrased as performing the $\boldsymbol{l}_{2}$-norm cross the column and then the $\boldsymbol{l}_{0}$-norm along the row, i.e., $\|W\|_{l_{0} \backslash l_{2}}=\|[\| W(1$, : )$\left.\left\|_{l_{2}},\right\| W(2,:)\left\|_{l_{2}}, \cdots,\right\| W(L,:) \|_{l_{2}}\right] \|_{l_{0}} . k$ denotes the maximum number of the nonzero coefficients in $W$.

In fact, because the $l_{2} / l_{0}$ regularization norm needs the shared gait pattern across observation, the computation complexity reaches $\boldsymbol{O}\left(\boldsymbol{w}^{2} \boldsymbol{n}\right)$ for the optimization solution of non-zero sparse coefficients $w$. That is, if large-scale training sample data are directly used to construct the over-complete dictionary, then a higher computational complexity is possibly yielded in estimating the optimization solution of $w$. Therefore, it is necessary to find a few training samples to reconstruct an over-complete dictionary for developing the JSRC-based multi-sensor gait-classification model with high quality.

\subsection{Reconstructing a New Over-Complete Dictionary for JSRC}

Here, the sparse representation coefficients-inducing criterion (SRCC) is used to find a few nearest neighboring samples containing the most valuable information, in order to reconstruct a new over-complete dictionary for JSRC. The detailed procedure of selecting the nearest neighbor samples is presented as follows.

Step 1 The test sample $\boldsymbol{y}_{j}$ from sensor $j$ is linearly represented as

$$
y_{\mathrm{j}}=w_{i, 1} s_{1}^{\mathrm{j}}+w_{i, 2} s_{2}^{\mathrm{j}}+\cdots+w_{i, n_{i}} s_{n_{i}}^{\mathrm{j}}
$$

Then, the sparse representation coefficients $w$ are obtained by solving the following $l_{2}$-minimization problem:

$$
\hat{\boldsymbol{w}}=\underset{\hat{w}}{\operatorname{argmin}}\left\|\boldsymbol{y}_{j}-\boldsymbol{s}^{j} \boldsymbol{w}\right\|_{2}+\lambda\|\boldsymbol{w}\|_{2}
$$

where $\lambda$ is a positive regularization, and the $l_{2}$-norm is defined as $\|\boldsymbol{w}\|_{2}=\sum_{i=1}^{n_{i}}\left|\boldsymbol{w}_{i}\right|_{2}$.

Step 2 Based on the solution of $w$, the $m$ training samples with the larger sparse coefficients values are selected as the nearest neighbor training samples. For gait class $i$, a new training sample set is defined as

$$
A_{i}=\left(s_{i, 1}, s_{i, 2}, \cdots, s_{i, m}\right)
$$

Step 3 Based on Equation (15), a new over-completed dictionary is reconstructed as

$$
A_{T}=\left[A_{1}, A_{2}, \cdots, A_{L}\right]^{T}
$$

Therefore, a test gait $Y$ with class $i$ can be joint-sparsely represented as

$$
\boldsymbol{Y}=\boldsymbol{A}_{T} \boldsymbol{W}
$$


Here, the joint sparse coefficients matrix $W$ in Equation (17) can be estimated by solving the following $l_{2} / l_{0}$-minimization problem:

$$
\hat{W}=\operatorname{argmin}\|\boldsymbol{Y}-\mathrm{A} W\|_{F}^{2} \text { s.t. }\|\boldsymbol{W}\|_{l_{0} l_{2}} \leq \boldsymbol{k} .
$$

The detailed procedure of solving joint sparse representation coefficients $\hat{W}$ is presented in Section 3.3.

\subsection{MBCS Technique for Solving Joint Sparse Representation Coefficients}

In this study, the Multitask Bayesian Compressive Sensing (MBCS) technique is applied to solve the joint sparse representation coefficients $W$ in Section 2.2.2 [48], in order to capture more of the temporal-spatial correlation information regarding gait. Due to multi-sensor gait data that are contaminated by noise, the test gait $Y$ can be represented as $Y=A W+E$, i.e.,

$$
\left[\begin{array}{c}
y_{1} \\
y_{2} \\
\vdots \\
y_{J}
\end{array}\right]=\left[\begin{array}{c}
A_{1} \\
A_{2} \\
\vdots \\
A_{J}
\end{array}\right]\left[W_{1}, W_{2}, \cdots, W_{J}\right]+\left[\begin{array}{c}
e_{1} \\
e_{2} \\
\vdots \\
e_{J}
\end{array}\right]
$$

where $E=\left[e_{1}, e_{2}, \cdots, e_{J}\right]^{T}$ denotes the noise. Here, the noise $e_{j}$ satisfies a Gaussian distribution with an unknown parameter $\beta_{0}$, and the prior distribution function of $y_{j}$ is defined as

$$
p\left(\boldsymbol{y}_{j} \mid W_{j}, \beta_{0}\right)=\left(\beta_{0} / 2 \pi\right)^{N / 2} \exp \left(-\frac{\beta_{0}}{2}\left\|\boldsymbol{y}_{j}-\mathbf{A}_{j} W_{j}\right\|_{2}^{2}\right) .
$$

Then, the prior distribution function of the sparse coefficients $\boldsymbol{W}_{j}$ is defined as

$$
p\left(W_{j} \mid \beta\right)=\prod_{i=k}^{M} N\left(W_{j, k} \mid 0, \beta_{k}^{-1}\right)
$$

where $W_{j, k}$ denotes the $k$ th sparse representation coefficient of sensor $j$ and $N\left(\cdot \mid 0, \beta_{k}^{-1}\right)$ refers to the Gaussian distribution function whose mean is equal to zero and variance $\sigma^{2}=1 / \beta_{k}$.

After the optimal parameters $\beta, \beta_{0}$ are estimated by constructing a likelihood function, we can solve the joint sparse representation coefficient $\left\{\boldsymbol{W}_{j}\right\}_{j=1}^{J}$. The production of the solution for $\left\{\boldsymbol{W}_{j}\right\}_{j=1}^{J}$ is presented in the Appendix A, and the detailed procedure for the solution can be found in reference [48].

\subsection{The Definition of Minimal Residual Error Rule for Gait Classification}

Based on the estimated solutions of the joint sparse representation coefficients $\hat{W}$, we firstly define the matrix indication function $\delta_{i}(\cdot)$ whose entries are zero except for those associated with gait class $i$. i.e.,

$$
\delta_{i}(\hat{\boldsymbol{W}})=\left[0^{T}, \cdots, 0^{T}, \boldsymbol{W}_{i}^{T}, 0^{T} \cdots 0^{T}\right]^{T} .
$$

Then, the approximation of test gait sample $Y$ can be defined as

$$
\widetilde{\boldsymbol{Y}}=A \delta_{i}(\hat{\mathbf{W}}) \text {. }
$$

The minimal residual error between $Y$ and $\widetilde{Y}$ can be defined as

$$
\min _{i} r_{i}(\boldsymbol{Y})=\left\|\boldsymbol{Y}-A \delta_{i}(\hat{\mathbf{W}})\right\|_{2}
$$


Thus, the class label of test gait $\boldsymbol{Y}$ can be identified by the following minimal residual error rule $[23,33,41]$ :

$$
\hat{\boldsymbol{i}}=\underset{i}{\operatorname{argmin}}\left\|\boldsymbol{Y}-A \delta_{i}(\hat{\boldsymbol{W}})\right\|_{2}^{2}, i=1,2, \cdots, L .
$$

\section{Materials and Methods}

\subsection{The Selection of Multi-Sensor Gait Data}

In this study, the multi-sensor gait data were selected from an open wearable action recognition database (WARD) database from University of California, in order to evaluate the feasibility of our proposed technique. In the selected WARD database, the multi-sensor gait data with different walking patterns are sampled from a total of 20 subjects ( 7 female subjects and 13 male subjects) with ages ranging from 19 to 75 years. All participants had no known injuries or abnormalities that could affect their walking pattern. The wearable motion sensor networks consist of five wireless motion sensors (two are located on the wrists, one is located on the waist, and two are positioned on the ankles, all using elastic belts). Each sensor node integrates a 3-axis (i.e., $x$-axis, $y$-axis and $z$-axis) accelerometer with a range of $\pm 2 \mathrm{~g}$ and a 2-axis gyroscope with a range of $\pm 500 \mathrm{dps}$, and each axis is reported as a 12 bit value to the sensor node. Each subject is asked to perform 13 activity or gait patterns such as stand, sit, lie down, walk forward, walk left-circle, walk right-circle, turn left, turn right, go upstairs, go downstairs, jog, jump, and push a wheelchair. Each subject is asked to perform each pattern 5 times. The sample ratio is set to $20 \mathrm{~Hz}$. More details about the multi-sensor data collection are available at the website (http:/ / www.eecs.berkeley.edu/ yang/software/WAR/).

\subsection{Data Preprocessing}

In this study, in order to effectively validate the feasibility of our proposed method, we randomly selected nine gait or activity pattern classes from all 13 gait patterns, such as stand, sit, walk forward, turn left, turn right, go upstairs, go downstairs, jog, jump. Firstly, the denoising for the recorded multi-sensor data is performed by using a fifth-order Butterworth with a low-pass filter at $30 \mathrm{~Hz}$. Then, a sliding window technique is employed to segment the acquired time-sequence data into a sequence of fixed-length windows, in order to gain valuable correlation information from the collected multi-sensor gait data [16,24]. Here, the window is set to a length of $N=200$ with $50 \%$ overlap, and 10 windows were randomly extracted from each subject's data. Here, each subject's data consist of the 9 different gait pattern classes data, each class including 10 windows' data (i.e., each subject's data includes 10 stand data, 10 sit data, 10 walk forward data, 10 turn left data, 10 turn right data, 10 go upstairs data, 10 go downstairs data, 10 jog data, 10 jump data). In this study, the 20 subjects' total data are used to train and test, in order to objectively evaluate the classification performance. The total sample data size is 1800 (i.e., 20 subjects $\times 9$ gait pattern classes $\times 10$ window samples), as shown in Table 1. In addition, we perform the classification task of nine gait patterns (walk forward, walk left, walk right, go upstairs, go downstairs, run forward, sitting), in order to further evaluate the feasibility of our proposed model for multi-sensor gait telemonitoring applications.

Table 1. The total amount of available sample data.

\begin{tabular}{ccc}
\hline \multicolumn{3}{c}{ All Samples Data Size } \\
\hline All Subjects & Gait Pattern Classes & Window Samples \\
\hline 20 & 9 & 10 \\
\hline
\end{tabular}

\subsection{Training and Testing the Gait-Classification Model}

In this work, the ten-fold cross validation strategy is adopted to objectively and accurately evaluate gait-classification performance [13-15]. Here, all 20 subjects' data are divided into ten subsets, 
each including 2 subjects' data. To effectively evaluate the generalization performance, the data that are used to test are not included in the training samples data. The detailed training and testing procedure is as follows: Firstly, the training set is constructed to develop a gait-classification model. Here, 18 subjects' data are randomly selected as the training set, containing a sample size of 1620 (i.e., 180 stand data, 180 sit data, 180 walk forward data, 180 turn left data, 180 turn right data, 180 go upstairs data, 180 go downstairs data, 180 jog data, 180 jump data). In the process of training the classification model, a nine-fold cross-validation scheme is employed due to the limited data size. That is, all selected 18 subjects' data are randomly divided into nine subsets, each subset including 2 subjects' data. Eight subsets are arbitrarily selected as the training set while the remaining one is used to test. The above steps are repeated until each subset is employed to test. After the classification model is yielded, the remaining two subjects' data that are not included in training set are used to test the generalization ability. Each subject's data must be used to test the generalization ability. Finally, the whole averaged classification result is obtained for all subjects.

\subsection{Evaluation Criteria for the Proposed Technique}

In addition, some common criteria are adopted to objectively assess the performance of the proposed gait-classification model using multi-sensor gait data, and they are respectively defined as follows.

(1) Compression ratio (CR)

The compression ratio is used to quantitatively evaluate the ability to compress the multi-sensor gait data, and is defined as follows:

$$
C R=\frac{N-M}{N} \times 100 \%
$$

where $N$ and $M$ denote the original data length and the compressed data length, respectively.

(2) Accuracy

Accuracy is employed to objectively evaluate the classification performance, and it is defined as the ratio of the number of gait patterns accurately identified to the total of gait patterns in the tests.

$$
\text { Accuracy }=\frac{N A}{N T} \times 100 \%
$$

where NA and NT denote the number of gait patterns accurately identified and the total number of gait patterns, respectively.

(3) Precision

Precision is defined as a measure of the ability of the classifier to detect gait patterns with class $i$ in the confusion table.

$$
\text { Precision }=\frac{N P}{N P+N F} \times 100 \%
$$

where NP and NF denote the number of gait patterns accurately identified as class $i$ and class $j$, respectively.

(4) Recall

Recall is defined as a measure of the ability of the classifier to specify gait patterns with class $j$ in the confusion table.

$$
\text { Recall }=\frac{N F}{N P+N F} \times 100 \% .
$$

In addition, in this study, statistical analyses to determine the mean and standard deviation (SD) are performed for the outcomes obtained by the different classification algorithms. All indices used are tested by means of a statistical analysis such as ANOVAs, in order to further test the objectivity of 
the outcomes obtained by the different classification algorithms. Here, all different algorithms that are considered as independent variables are performed on all indices.

In this study, all algorithm programs are developed in Matlab 2013a, and they are performed on a computer with an Intel(R) Core(TM) i5-3470 3.20GHz CPU, 4.00GB RAM, and Windows 7 operating system.

\section{Results}

\subsection{The Evaluation of the Effect of DCS on the Developed Neighboring JSRC}

Firstly, we evaluate the effect of the simultaneous compression of multi-sensor gait data by DCS on our developed neighboring JSRC(SRCC-JSRC) model. For comparison, we also perform a JSRC model and a KNN-based JSRC(KNN-JSRC) model. For DCS, the sparse binary matrix was selected as the random projection matrix where only two nonzero entries are included in each column. All 20 subjects' data are used to train and test, and the ten-fold cross validation strategy is adopted to objectively evaluate gait-classification performance. Firstly, we compared three JSRC-based classifications' performances based on multi-sensor gait data which are simultaneously compressed by DCS. The number of neighboring samples are selected as 20,40 and 60 , respectively. All comparative results are shown in Table 2. From Table 2, we can observe that when the number of neighboring samples is selected as 40 or 60 , all neighboring sample-based JSRC models can produce a better accuracy of $94 \%$ as well as a lower computational time cost when compared with the JSRC model. These results suggest that the simultaneous compression of multi-sensor gait data by DCS can provide the valuable high-correlation information regarding gait for the JSRC model.

Table 2. Comparative results from the different JSRC-based gait-classification performance.

\begin{tabular}{ccc}
\hline Classification Algorithms & Accuracy/\% & Computation Time/ms \\
\hline JSRC & 91.33 & 132.8510 \\
KNN-JSRC $(m=20)$ & 94.00 & 77.0239 \\
KNN-JSRC $(m=40)$ & 94.11 & 87.4365 \\
KNN-JSRC $(m=60)$ & 94.44 & 102.5404 \\
SRCC-JSRC $(m=20)$ & 92.67 & 66.1759 \\
SRCC-JSRC $(m=40)$ & 94.44 & 78.0513 \\
SRCC-JSRC $(m=60)$ & 94.89 & 90.7440 \\
\hline
\end{tabular}

It is noted that $\mathrm{KNN}$ is the abbreviation of k-nearest neighbor and SRCC is the abbreviation of sparse representation coefficients-inducing criterion.

\subsection{The Assessment of Gait-Classification Performance Based on the Different Compression Ratios}

Next, we evaluate the classification performance of our proposed method based on the different compression ratios to examine the feasibility of the energy-efficient telemonitoring of gait. The number of neighboring samples are selected as 20 and 40, respectively. The comparative results from all JSRC-based models are shown in Figure 2. The errors of accuracy based on the different compression ratios are also presented in Figure 2. Table 3 shows the mean and SD values of the best accuracy from all selected algorithms. As shown in Figure 2 and Table 3, the SD of all accuracies can obtain lower error. From Figure 2, we obviously see that, based on the different compression ratios, our proposed method (SRCC-JSRC) can produce better accuracy than KNN-JSRC and JSRC, suggesting that the neighboring samples by our defined criteria contain the more valuable information about the test sample. In particular, when the compression ratio increases from 10 to $70 \%$, SRCC-JSRC ( $m=40)$ reaches the best accuracy. These results showed that our proposed method can yield the superior classification performance when the compression ratio is properly selected. That is, our proposed method can potentially produce the best classification performance as well as the best energy efficiency of sensors. In addition, from Figure 2, we also find that all JSRC-based gait classifications produce poor accuracy when the compression ratio is more than $70 \%$. This suggests that the higher compression ratios could lose the more valuable correlation information regarding gait. 


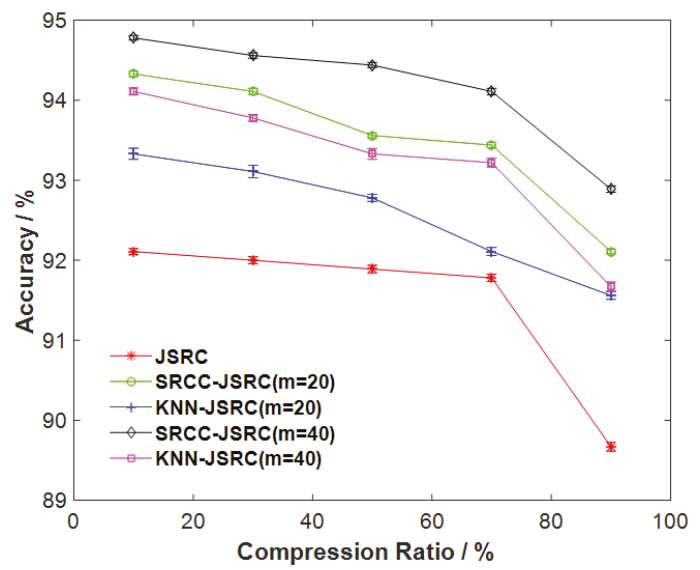

Figure 2. Comparative results of the different gait-classification performances based on the different compression ratios.

In addition, we also compared our proposed model to some tradition classification models such as SRC, naive Bayesian classifier (NBC), and KNN models. Here, the number of nearest neighboring samples are selected as 40 (i.e., $m=40$ ). The comparative results are shown in Figure 3. As shown in Figure 3, our proposed model significantly outcompetes SRC, NBC, and KNN models. In this comparison, the NBC technique is the poorest. These results demonstrate that our proposed model can gain more distinctive temporal-spatial correlation information associated with gait pattern change.

Table 3. The statistical results the best accuracy from all selected algorithms.

\begin{tabular}{|c|c|c|c|c|c|}
\hline Algorithms & $\begin{array}{c}\text { SRCC-JSRC } \\
(m=40)\end{array}$ & $\begin{array}{c}\text { SRCC-JSRC } \\
\quad(m=20)\end{array}$ & $\begin{array}{c}\text { KNN-JSRC } \\
\quad(m=40)\end{array}$ & $\begin{array}{c}\text { KNN-JSRC } \\
(m=20)\end{array}$ & JSRC \\
\hline Mean value (\%) & 94.58 & 94.23 & 93.31 & 93.13 & 92.11 \\
\hline SD value & $(0.043,0.076)$ & $(0.029,0.038)$ & $(0.041,0.066)$ & $(0.042,0.078)$ & $(0.037,0.054)$ \\
\hline
\end{tabular}

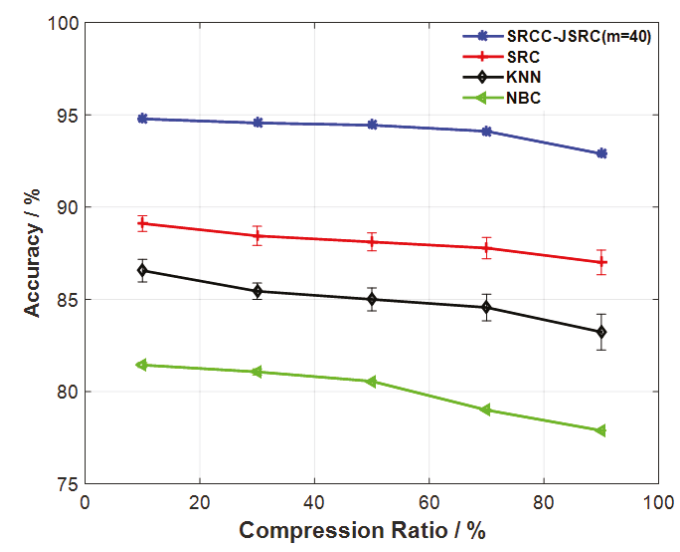

Figure 3. Comparison of accuracy of our proposed technique with KNN, naive Bayesian classifier (NBC), and sparse representation classification (SRC). 


\subsection{The Evaluation of Computation Time Cost Based on the Different Compression Ratios}

Meanwhile, we also evaluate the computational time cost of all JSRC-based models based on the different compression ratios. Here, statistical analyses to determine the mean and standard deviation (SD) are performed for the obtained computational time from the different classification algorithms. All comparative results are shown in Figure 4. The errors of the obtained computational time based on the different compression ratios are also presented in Figure 4. Table 4 shows the mean and SD values of the lowest computation time from all selected algorithms. As shown in Figure 4 and Table 4, the SD of all computation times can obtain lower error. From Figure 4, we can obviously see that the computation time cost of each JSRC-based model is reduced as the compression ratio increases. In comparison, our proposed method (SRCC-JSRC) has the lowest computational time cost. In particular, the lowest computational time cost of SRCC-JSRC $(m=20)$ remains only $67 \mathrm{~ms}$ while the compression ratio increases from 10 to $70 \%$. These results demonstrate that our proposed method could possibly result in a lower computational time cost and better energy efficiency of sensors when the compression ratio is properly selected. Besides this, we also observe that, once the compression ratios are more than 70 , all computation times significantly decline. This is because the higher compression ratios could yield fewer multi-sensor gait data to be processed.

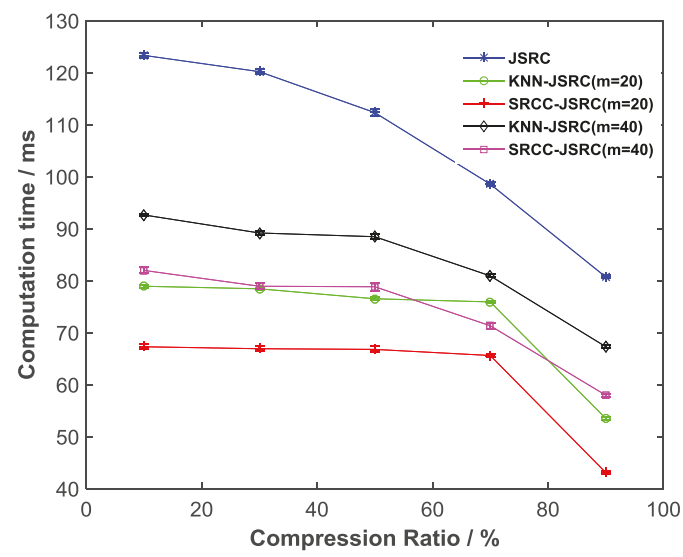

Figure 4. Comparative results of computation time from the different JSRC-based gait-classification algorithms.

Table 4. The statistical results for the lowest computation time from all selected algorithms.

\begin{tabular}{|c|c|c|c|c|c|}
\hline Algorithms & $\begin{array}{c}\text { SRCC-JSRC } \\
(m=40)\end{array}$ & $\begin{array}{c}\text { SRCC-JSRC } \\
(m=20)\end{array}$ & $\begin{array}{c}\text { KNN-JSRC } \\
(m=40)\end{array}$ & $\begin{array}{c}\text { KNN-JSRC } \\
(m=20)\end{array}$ & JSRC \\
\hline Mean value (ms) & 77.65 & 65.98 & 88.03 & 76.45 & 132.06 \\
\hline SD value & $(0.032,0.065)$ & $(0.025,0.034)$ & $(0.036,0.061)$ & $(0.036,0.072)$ & $(0.032,0.049)$ \\
\hline
\end{tabular}

\subsection{Evaluation of Our Proposed Model for Gait Telemonitoring}

We perform the task of classification for nine gait patterns (walk forward, walk left, walk right, go upstairs, go downstairs, run forward, sitting, jog, jump), in order to further evaluate the feasibility of our proposed model for multi-sensor gait telemonitoring applications. Here, the number of nearest neighboring samples is selected as 40 , and the compression ratio is set to $50 \%$. Table 5 shows the confusion table for the classification of the nine gait patterns. In Table 4 , the entry in the $i$ th row and $j$ th column refers to the count of gait patterns belonging to class $i$ that are classified as class $j$. As shown in Table 5, the whole averaged classification accuracy across all nine gait patterns reaches $95 \%$, 
suggesting that our proposed technique can accurately classify these nine gait patterns. In terms of individual patterns, the "turn left" and "jump" patterns obtain the maximal value of $100 \%$ and the "jog" pattern gains the best precision value of $100 \%$. The "downstairs" pattern gains the same maximal precision and recall value of $97 \%$, and the "sitting" pattern obtains poor precision and recall values. For the "jump" pattern, its precision is equal to $100 \%$, whereas its recall is only $90 \%$. The possible reason for this is that some samples of "jump" are easily misclassified as "walk forward" and "downstairs", but samples of "walk forward" are not misclassified as "jump". In conclusion, these results show that our proposed technique can capture the more distinctive and high-correlation information associated with gait for multi-sensor gait classification. Our proposed method has great potential for gait telemonitoring applications.

Table 5. The confusion table for classification of the nine different gait pattern classes.

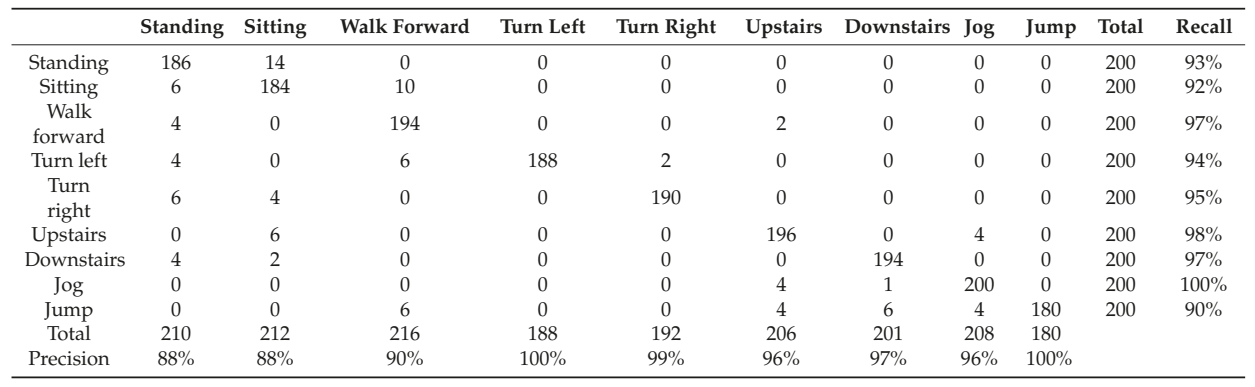

In addition, the results of statistical tests based on ANOVAs are presented in Table 6. As shown in Table 6, all significance values are less than the significance level value of 0.05 , suggesting that the significance of the difference among classification algorithms is at an acceptable level. This further validates the objectivity of the outcomes obtained by the different classification algorithms.

Table 6. The results of statistical tests for all indices based on ANOVAs.

\begin{tabular}{cccccc}
\hline Indices & Compression Ratio & Accuracy & Computation Time & Precision & Recall \\
\hline$F$ values & 70.51 & 101.21 & 146.81 & 224.92 & 182.47 \\
Significance values & 0.0235 & 0.0379 & 0.0342 & 0.0288 & 0.0357 \\
\hline
\end{tabular}

\section{Discussion}

The results of present studies demonstrate that the hybrid technique of DCS and JSRC can take advantage of data joint sparsity to jointly process multi-sensor gait data, which can capture the more valuable high-correlation information hidden in multi-sensor gait data. This greatly contributes to the best classification performance as well as to the energy efficiency of sensors in WBANs-based gait telemonitoring applications. Currently, the discovery of powerful techniques for the quantitative analysis of multi-sensor gait data has become a research focus in the gait telemonitoring field [10-12]. One important issue is how to gain the higher-correlation information regarding gait for the best gait-classification performance as well as for improving the energy efficiency of sensors [13,26-28].

In the present studies, considering that both DCS and JSRC have the best ability to jointly process multi-signals based on data joint sparsity, we investigated the feasibility of the hybrid technique of DCS and JSRC for gait telemonitoring by jointly processing multi-sensor gait data [32-34]. In particular, we try to take advantage of the joint sparsity of multi-sensor gait data to develop a novel neighboring JSRC model for the best classification performance as well as lower computation time. In this study, we firstly evaluate the effect of the simultaneous compression of multi-sensor data by DCS on the JSRC-based classification model. As illustrated in Table 2, all JSRC-based gait-classification models 
can yield better accuracy. This suggests that the DCS technique has the superior ability to capture the higher-correlation information regarding gait by jointly compressing multi-sensor gait data, and all compressed multi-sensor gait data containing the more valuable information significantly helps to produce the better classification performance of the JSRC-based model. These results further suggest that it is feasible that DCS and JSRC can jointly process multi-sensor gait data for gait classification based on data joint sparsity.

Next, we evaluate the feasibility of our proposed method for multi-sensor gait classification based on the different compression ratios. As shown in Figure 2, all selected JSRC models can yield a better gait-classification performance when compression ratios are properly chosen. In the comparison, our proposed model is best. This suggests that our proposed model potentially yields the best performance as well as the best energy efficiency of sensors. In addition, we also compared our proposed method to the traditional SRC, NBC, and KNN models. As shown in Figure 3, our proposed method significantly outcompetes the selected traditional methods. A possible reason for this is that the selected neighboring training samples can contain the more distinctive and high-correlation information associated with gait, which significantly improves multi-sensor gait-classification performance. However, the traditional SRC model does not gain the valuable high-correlation information as it does not jointly processes multi-sensors gait data $[15,16,24,25]$. Similar results in a study on hyperspectral image classification have been found in [45]. Besides this, from Figure 2, we also find that all JSRC-based gait-classification models result in poor accuracy when the compression ratios are more than $70 \%$. This is because the compressed multi-sensor gait data possibly contains more redundant information, thus destroying the classification performance. Similar results in studies on gait telemonitoring have been also reported in [10,15,16,29].

In addition, we also evaluate the computational time cost corresponding to all JSRC-based models based on the different compression ratios. As illustrated in Figure 4, in comparison, our proposed method has the lowest computational cost. The main reasons for this are that the proposed novel neighboring JSRC algorithm has a maximum time complexity of only $T_{1}+\boldsymbol{O}\left(\boldsymbol{w}^{2} m\right)\left(T_{1}\right.$ denotes the time for searching the $m$ nearest neighbor samples) to produce the best classification performance. However, the traditional JSRC algorithm has a computational complexity of $\boldsymbol{O}\left(\boldsymbol{w}^{2} \boldsymbol{n}\right)(n \geq m, n$ is the total number of training sample data points) for multi-sensor gait performance [33,41,43]. In conclusion, all the above results show that our proposed technique has the potential ability to gain the best classification performance, lower computational time cost, and better energy efficiency of sensors in multi-sensor gait classification.

In this study, our proposed model is also employed to classify nine different multi-sensor gait patterns, in order to further examine the practicality of gait telemonitoring applications. As shown in Table 5, our proposed technique shows high-quality gait-classification performance. This suggests that our proposed technique may enforce the robustness of coefficient estimation, which helps to exactly solve the joint sparse representation coefficients. In particular, this is because our hybrid technique can take advantage of the joint sparsity of multi-sensor gait data to gain the more distinctive information associated with the spatial, temporal, and dynamic correlations of gait [13,26-28], which greatly contribute to identifying multi-sensor gait pattern change. Similar findings have been reported in $[9,12,46]$.

\section{Conclusions}

In this study, an advanced hybrid technique of the DCS and JSRC models is proposed for multi-sensor gait telemonitoring. In our technique, both DCS and JSRC feasibly take advantage of data joint sparsity to jointly process multi-sensor gait data. All multi-sensor gait data jointly compressed by DCS contain the more valuable high-correlation information related to gait, and are directly used to develop the JSRC model. This can avoid the multi-sensor gait data reconstruction step and potentially increase the energy efficiency of the sensors. A novel neighboring JRSC model can be developed to gain the best gait-classification performance as well as a lower computational time. It is very hopeful that our proposed technique can serve as a powerful tool for processing multi-sensor gait dat in gait telemonitoring 
applications. Future research paths are to find the effective methods that can significantly improve the classification performance of multi-sensor gait data while compression ratios are higher.

Acknowledgments: We are thankful for the Natural Science project funding from Fujian Province (No. JK2016006, JAT160098 and 2017Y0028) and the Humanities and Social Sciences project funding from Ministry of Education, China (No. 17YJAZH091). In addition, the authors wish to acknowledge the open Wearable Action Recognition Database (WARD) database from University of California, Berkeley.

Author Contributions: Jianning Wu conceived the original idea of the study, along with a corresponding author. He participated in the design of the algorithm and drafted the manuscript. Jiajing Wang, Yun Ling and Haidong $\mathrm{Xu}$ participated in the design of the algorithm and analyzed the data. All authors read and approved the submitted manuscript.

Conflicts of Interest: The authors declare that they have no competing interests.

\section{Appendix A}

In the MBCS technique, the set of unknown parameters $\beta=\left\{\beta_{k}\right\}_{k=1,2, \ldots, N}$ is the hyper-parameter for the prior distribution of $W_{j}$. Here, in order to obtain the best sparsity of coefficients $W_{j}$, the hyper-parameters $\beta_{0}, \beta$ are assumed to satisfy a Gamma distribution, and the prior probabilities of $\beta_{0}, \beta$ are defined, respectively, as

$$
\begin{gathered}
p\left(\beta_{0} \mid a, b\right)=G a\left(\beta_{0} \mid a, b\right), \\
p(\beta \mid c, d)=\prod_{k=1}^{M} G a\left(\beta_{k} \mid c, d\right),
\end{gathered}
$$

where $a, b, c$, and $d$, respectively, denote four different parameters in the Gamma distribution. When $a=b=c=d=0$, the super prior probabilities of $\beta_{0}, \beta$ are available. So, the following maximum likelihood function of $\beta_{0}, \beta$ is defined as

$$
\left\{\beta, \beta_{0}\right\}=\operatorname{argmin} \sum_{j=1}^{J} \log \int d W_{j} p\left(y_{j} \mid W_{j}, \beta_{0}\right) p\left(y_{j} \mid \beta\right) .
$$

Once $\beta_{0}, \beta$ are solved, the posterior probability distribution of $W_{j}$ is defined as

$$
p\left(W_{j} \mid \boldsymbol{y}_{j}, \beta, \beta_{0}\right)=N\left(W_{j} \mid \boldsymbol{\mu}_{j}, \sum_{j}\right)
$$

where $\boldsymbol{\mu}_{j}=\beta_{0} \sum_{j}\left(\boldsymbol{A}_{j}\right)^{T} \boldsymbol{y}_{j}$ is the mean, $\sum_{j}=\left[\beta_{0}\left(\boldsymbol{A}_{j}\right)^{T} \widetilde{\boldsymbol{A}}_{j}+\boldsymbol{\Gamma}\right]^{-1}$ is the covariance, and $\boldsymbol{\Gamma}=\operatorname{diag}\left(\beta_{1}, \beta_{2}, \cdots, \beta_{N}\right)$ is the diagonal matrix.

In order to effectively estimate the parameters $\beta_{0}, \beta$, the likelihood function is constructed as follows:

$$
L\left(\beta, \beta_{0}\right)=\log p\left(y_{j} \mid \beta, \beta_{0}\right)=-\frac{1}{2} \sum_{j=1}^{J}\left(N \log 2 \pi+\log \left|\mathbf{C}_{j}\right|+y_{j} \mathbf{C}_{j}^{-1} y_{j}\right)
$$

where $\mathbf{C}_{j}=\left[\beta_{0}^{-1} \boldsymbol{I}+\boldsymbol{A}_{j} \boldsymbol{\Gamma}^{-1}\left(\boldsymbol{A}_{j}\right)^{T}\right]$. Usually, the derivative of $L\left(\beta, \beta_{0}\right)$ to $\beta, \beta_{0}$ is assumed to be zero, and the iterative rules of $\beta, \beta_{0}$ are respectively defined as follows:

$$
\begin{gathered}
\beta^{\text {new }}=\frac{J-\beta_{k} \sum_{k=1}^{J} \sum_{j,(k, k)}}{\sum_{j=1}^{J} \mu_{j, k}^{2}}, \\
\beta_{0}^{\text {new }}=\frac{\sum_{k=1}^{J}\left(M-N+\sum_{k=1}^{M} \beta_{j} \sum_{j,(k, k)}\right)}{\sum_{j=1}^{J}\left\|y_{j}-A_{j} \mu_{j}\right\|_{2}^{2}} .
\end{gathered}
$$


When the optimal parameters $\beta, \beta_{0}$ are estimated, the solutions of the joint sparse representation coefficients $\left\{W_{j}\right\}_{j=1}^{J}=\left\{\boldsymbol{\mu}_{j}\right\}_{j=1}^{J}$ are available.

\section{References}

1. Ersoy, H.; Alemdar, C. Wireless sensor networks for healthcare: A survey. Comput. Netw. 2010, 54, 2688-2710.

2. Latre, B.; Braem, B.; Moerman, I.; Blondia, C.; Demeester, P. A survey on wireless body area networks. Wirel. Netw. 2011, 17, 1-18. [CrossRef]

3. Chen, M.; Gonzalez, S.; Vasilakos, A.; Cao, H.; Leung, V. Body Area Networks: A Survey. Mob. Netw. Appl. 2011, 16, 171-193. [CrossRef]

4. Wong, W.Y.; Wong, M.S.; Lo, K.H. Clinical applications of sensors for human posture and movement analysis: A review. Prosthet. Orthot. Int. 2007, 31, 62-75. [CrossRef] [PubMed]

5. Hao, Y.; Foster, R. Wireless body sensor networks for health-monitoring applications. Physiol. Meas. 2008, 29, 27-56. [CrossRef] [PubMed]

6. $\quad$ Olivares, A.; Olivares, G.; Mula, F.; Gorriz, J.; Ramirez, J. Wagyromag: Wireless sensor network for monitoring and processing human body movement in healthcare applications. J. Syst. Arch. 2011, 57, 905-915. [CrossRef]

7. Pantelopoulos, A.; Bourbakis, N.G. A survey on wearable sensor-based systems for health monitoring and prognosis. IEEE Trans. Syst. Man Cybern. Part C Appl. Rev. 2010, 40, 1-12. [CrossRef]

8. Taleb, T.; Bottazzi, D.; Guizani, M.; Nait-Charif, H. Angelah: A framework for assisting elders at home. IEEE J. Sel. Areas Commun. 2009, 27, 480-494. [CrossRef]

9. Patel, S.; Park, H.; Bonato, P.; Chan, L.; Rodgers, M. A review of wearable sensors and systems with application in rehabilitation. J. Neuroeng. Rehabil. 2012, 9, 1-17. [CrossRef] [PubMed]

10. Abdelkrim, H.; Marion, S. Wireless sensor networks for rehabilitation applications: Challenges and opportunities. J. Netw. Comput. Appl. 2013, 36, 1-15.

11. Ullah, S.; Higgins, H.; Braem, B.; Latre, B.; Blondia, C.; Moerman, I.; Kwak, K.S. A comprehensive survey of wireless body area networks. J. Med. Syst. 2010, 36, 1065-1094. [CrossRef] [PubMed]

12. Mo, L.F.; Liu, S.P.; Gao, R.X.; John, D.; Staudenmayer, J.W.; Freedson, P.S. Wireless design of a multisensor system for physical activity monitoring. IEEE Trans. Biomed. Eng. 2012, 59, 3230-3237. [PubMed]

13. Marinkovic, S.J.; Popovici, E.M.; Spagnol, C.; Faul, S.; Marnane, W.P. Energy-efficient low duty cycle MAC protocol for wireless body area networks. IEEE Trans. Inf. Technol. Biomed. 2009, 13, 915-925. [CrossRef] [PubMed]

14. Donoho, D. Compressed sensing. IEEE Trans. Inf. Theory 2006, 52, 1289-1306. [CrossRef]

15. Akimura, D.; Kawahara, Y.; Asami, T. Compressed sensing method for human activity sensing using mobile phone accelerometers. In Proceedings of the Ninth International Conference on Networked Sensing Systems (INSS), Antwerp, Belgium, 6 June 2012; pp. 1-4.

16. Wu, J.; Xu, H. An advanced scheme of compressed sensing of acceleration data for telemonintoring of human gait. Biomed. Eng. Online 2016, 15, 1-17.

17. Holmes, C.C.; Adams, N.M. A probabilistic nearest neighbour method for statistical pattern recognition. J. R. Stat. Soc. Ser. B 2002, 64, 295-306. [CrossRef]

18. Hanson, M.A.; Powell, H.C.; Barth, A.T.; Lach, J.; Brandt-Pearce, M. Neural network gait classification for On-Body inertial sensors. In Proceedings of the 6th International Workshop on Wearable and Implantable Body Sensor Networks, Berkeley, CA, USA, 3-5 June 2009; pp. 181-186.

19. Burges, C.J. A Tutorial on Support Vector Machines for Pattern Recognition. Data Min. Knowl. Discov. 1998, 2, 121-167. [CrossRef]

20. Mannini, A.; Sabatini, A.M. Machine Learning methods for classifying human physical activity from on-body accelerometer. Sensors 2010, 10, 1154-1175. [CrossRef] [PubMed]

21. Taborri, J.; Rossi, S.; Palermo, E.; Patanè, F.; Cappa, P. A novel HMM distributed classifier for the detection of gait phases by means of a wearable inertial sensor network. Sensors 2014, 14, 16212-16234. [CrossRef] [PubMed]

22. Mannini, A.; Sabatini, A.M.; Intille, S.S. Human gait detection from wrist-worn accelerometer data. Gait Posture 2013, 37 (Suppl. 1), S26-S27. [CrossRef]

23. Wright, J.; Yang, A.Y.; Ganesh, A.; Sastry, S.S.; Ma, Y. Robust face recognition via sparse representation. IEEE Trans. Pattern Anal. Mach. Intell. 2009, 31, 210-227. [CrossRef] [PubMed] 
24. Zhang, M.; Sawchuk, A.A. Human daily activity recognition with sparse representation using wearable sensors. IEEE J. Biomed. Health Inform. 2013, 17, 553-560.

25. Allen, Y.Y.; Roozbeh, J.; Shankar, S.S.; Ruzena, B. Distributed Recognition of Human Actions Using Wearable Motion Sensor Networks. J. Ambient Intell. Smart Environ. 2009, 1, 103-115.

26. Giannini, S.; Catani, F.; Benedetti, M.G.; Leardini, L. Gait Analysis: Methodologies and Clinical Applications; IOS Press: Amsterdam, The Netherlands, 1994.

27. Chau, T. A review of analytical techniques for gait data. Part 1: Fuzzy, statistical and fractal methods. Gait Posture 2001, 13, 49-66. [PubMed]

28. Chau, T. A review of analytical techniques for gait data. Part 2: Neural network and wavelet methods. Gait Posture 2001, 13, 102-120. [PubMed]

29. Jeevan, K.; Sridhar, K. Compressive sensing of foot gait signals and its application for the estimation of clinical relevant time series. IEEE Trans. Biomed. Eng. 2015, 63, 1401-1415.

30. Baron, D.; Wakin, M.B.; Duarte, M.F.; Sarvotham, S.; Baraniuk, R.G. Distributed Compressed Sensing. Available online: http://www.dsp.rice.edu/ rorb/pdf/DCS112005.pdf (accessed on 1 August 2005).

31. Duarte, M.F.; Sarvotham, S.; Baron, D.; Wakin, M.; Baraniuk, R.G. Distributed compressed sensing of jointly sparse signals. In Proceedings of the 39th Asilomar Conference on Signal, System and Computer, Pacific Grove, CA, USA, 30 October-2 November 2005; pp. 1537-1541.

32. Tropp, J.A.; Gilbert, A.C.; Strauss, M.J. Simultaneous sparse approximation via greedy pursuit. In Proceedings of the IEEE International Conference on Acoustics, Speech, and Signal Processing, Philadelphia, PA, USA, 19-23 March 2005; pp. 721-724.

33. Luo, J.; Yang, B.; Chen, Z. Color image restoration via extended joint sparse model. In Communications in Computer and Information Science, Proceedings of the Pattern Recognition, Beijing, China, 24-26 September 2012; Springer: Berlin/Heidelberg, Germany, 2012; Volume 321, pp. 497-504.

34. Yuan, X.T.; Liu, X.B.; Yan, S.C. Visual classification with multitask joint sparse representation. IEEE Trans. Image Process. 2012, 21, 4349-4360. [CrossRef] [PubMed]

35. Hu, H.; Yang, Z. Spatial correlation-based distributed compressed sensing in wireless sensor networks. In Proceedings of the 2010 6th International Conference on Wireless Communications Networking and Mobile Computing (WiCOM), Chengdu, China, 23-25 September 2010; pp. 1-4.

36. Yang, H.; Huang, L.; Xu, H.; Liu, A. Distributed compressed sensing in wireless local area networks. Int. J. Commun. Syst. 2015, 27, 2723-2743. [CrossRef]

37. Ying, H.; Schlosser, M.; Schnitzer, A.; Schafer, T.; Schlafke, M.E.; Leonhardt, S.; Schiek, M. Distributed intelligent sensor network for the rehabilitation of Parkinson's patients. IEEE Trans. Inf. Technol. Biomed. 2011, 15, 268-276. [CrossRef] [PubMed]

38. Ma, X.; Luong, H.Q.; Philips, W.; Song, H.; Cui, H. Sparse representation and position prior based face hallucination upon classified over-complete dictionaries. Signal Process. 2012, 92, 2066-2074. [CrossRef]

39. Dong, W.; Li, X.; Zhang, D.; Shi, G. Sparsity-based image denoising via dictionary learning and structural clustering. In Proceedings of the 2011 IEEE Conference on Computer Vision and Pattern Recognition (CVPR), Colorado Springs, CO, USA, 20-25 June 2011; pp. 457-464.

40. Sundman, D.; Saikat, C.; Skoglund, M. A greedy pursuit algorithm for distributed compressed sensing. In Proceedings of the 2012 IEEE International Conference on Acoustics, Speech and Signal Processing (ICASSP), Kyoto, Japan, 25-30 March 2012; pp. 2729-2732.

41. Xiao, L.; Li, R.F.; Luo, J.; Duan, M. Activity recognition via distributed random projection and joint sparse representation in body sensor networks. In Proceedings of the 7th China Conference Advances in Wireless Sensor Networks (CWSN2013), Qingdao, China, 17-19 October 2013; pp. 51-60.

42. Zhang, Z.; Jung, T.; Makeig, S.; Pi, Z.; Rao, B.D. Spatiotemporal sparse Bayesian learning with applications to compressed sensing of multichannel physiological signals. IEEE Trans. Neural Syst. Rehabil. Eng. 2014, 22, 1186-1197. [CrossRef] [PubMed]

43. Zhang, H.; Nasrabadi, N.M.; Zhang, Y.; Huang, T.S. Multi-Observation Visual Recognition via Joint Dynamic Sparse Representation. In Proceedings of the IEEE International Conference of Computer Vision, Barcelona, Spain, 6-13 November 2011; pp. 595-602.

44. Guha, T.; Ward, R.K. Learning Sparse Representations for Human Action Recognition. IEEE Trans. Pattern Anal. Mach. Intell. 2012, 34, 1576-1588. [CrossRef] [PubMed] 
45. Chen, C.; Chen, N.; Peng, J. Nearest regularized joint sparse representation for hyperspectral image classification. IEEE Geosci. Remote Sens. Lett. 2016, 13, 424-428. [CrossRef]

46. Shekhar, S.; Patel, V.M.; Nasrabadi, N.M.; Chellappa, R. Joint sparse representation for robust multimodal biometrics recognition. IEEE Trans. Pattern Anal. Mach. Intell. 2013, 36, 113-126. [CrossRef] [PubMed]

47. Sarvotham, S.; Wakin, M.B.; Baron, D.; Duarte, M.F.; Baraniuk, R.G. Analysis of the DCS One-Stage Greedy Algorithm for Common Sparse Supports. Available online: http:/ / www.dsp.rice.edu/sites/dsp.rice.edu/ files/publications/report/2005/analysis-ece-2005.pdf (accessed on 1 October 2005).

48. Ji, S.; Dunson, D.; Carin, L. Multitask compressive sensing. IEEE Trans. Signal Process. 2009, 57, 92-106. [CrossRef]

(C) 2017 by the authors. Licensee MDPI, Basel, Switzerland. This article is an open access article distributed under the terms and conditions of the Creative Commons Attribution (CC BY) license (http://creativecommons.org/licenses/by/4.0/). 
Article

\title{
The Effect of the Accelerometer Operating Range on Biomechanical Parameters: Stride Length, Velocity, and Peak Tibial Acceleration during Running
}

\author{
Christian Mitschke*, Pierre Kiesewetter and Thomas L. Milani \\ Department of Human Locomotion, Chemnitz University of Technology, 09126 Chemnitz, Germany; \\ pierre.kiesewetter@hsw.tu-chemnitz.de (P.K.); thomas.milani@hsw.tu-chemnitz.de (T.L.M.) \\ * Correspondence: christian.mitschke@hsw.tu-chemnitz.de; Tel.: +49-371-531-32196
}

Received: 11 November 2017; Accepted: 4 January 2018; Published: 5 January 2018

\begin{abstract}
Previous studies have used accelerometers with various operating ranges (ORs) when measuring biomechanical parameters. However, it is still unclear whether ORs influence the accuracy of running parameters, and whether the different stiffnesses of footwear midsoles influence this accuracy. The purpose of the present study was to systematically investigate the influence of OR on the accuracy of stride length, running velocity, and on peak tibial acceleration. Twenty-one recreational heel strike runners ran on a 15-m indoor track at self-selected running speeds in three footwear conditions (low to high midsole stiffness). Runners were equipped with an inertial measurement unit (IMU) affixed to the heel cup of the right shoe and with a uniaxial accelerometer at the right tibia. Accelerometers (at the tibia and included in the IMU) with a high OR of $\pm 70 \mathrm{~g}$ were used as the reference and the data were cut at $\pm 32, \pm 16$, and at $\pm 8 \mathrm{~g}$ in post-processing, before calculating parameters. The results show that the OR influenced the outcomes of all investigated parameters, which were not influenced by tested footwear conditions. The lower ORs were associated with an underestimation error for all biomechanical parameters, which increased noticeably with a decreasing OR. It can be concluded that accelerometers with a minimum OR of $\pm 32 \mathrm{~g}$ should be used to avoid inaccurate measurements.
\end{abstract}

Keywords: operating range; accelerometer; stride length; peak tibial acceleration; running velocity; wearable sensors

\section{Introduction}

With the further advancement of sensor technologies and data analyzing techniques, micro-electro-mechanical sensors (MEMS) have become useful tools for biomechanical research and clinical practice [1]. It has been reported that these wearable motion sensors are an inexpensive alternative to optoelectronic systems and force plates, that they are simple to handle, cost-effective, and are suitable for field measurements [2-6]. When using these sensors, spatio-temporal and kinetic parameters during walking and running can be analyzed in clinical as well as in sportive applications. For kinetic measurements, for example, when investigating the impact loads on lower limbs during running under various conditions (e.g., footwear conditions or the influence of fatigue), the peak tibial acceleration (PTA) was examined by some authors using MEMS [7-20]. Thereby, unidirectional accelerations along the longitudinal axis of the tibia, as well as medio-lateral and anterior-posterior accelerations of the tibia were examined. Furthermore, the determination of stride frequency, of walking or running velocity (runVel), and of stride length (strLen) have also been the focus of research that utilizes MEMS [21-25]. To investigate these biomechanical parameters, individually configured sensors or commercially available inertial measurement units (IMUs: e.g., Shimmer, Achillex, or XSens) were used, which combine accelerometers and gyroscopes. In this 
context, Provot et al. [26] compared a calibrated industrial accelerometer (considered as the gold standard) to an IMU accelerometer in two tests: (a) on a shaker, and (b) on the distal anteromedial aspect of the subject's tibia during running at $3.33 \mathrm{~m} / \mathrm{s}$. They concluded that IMUs can be used for valid measurements of tibial acceleration during running.

Besides the different sensor types, sensor locations, and the various sampling rates, sensors with considerably different accelerometer operating ranges (ORs) have also been used. When investigating walking or running, some studies used ORs between \pm 2 and $\pm 70 \mathrm{~g}$, with $\mathrm{g}$ being the acceleration of gravity [27-32]. However, high accelerations act on the sensor in vertical and anterior-posterior directions during the swing phase and in the first $50 \mathrm{~ms}$ after foot strike [11,32]. These accelerations can distinctly exceed the gravitational acceleration of $1 \mathrm{~g}$. For instance, vertical accelerations of $24.62 \pm 4.1 \mathrm{~g}$ were measured with a heel-mounted IMU accelerometer during heel strike, when running at $3.5 \pm 0.1 \mathrm{~m} / \mathrm{s}$ in a neutral running shoe (PUMA FAAS 500) [32]. If the accelerations exceeded the accelerometer OR, a lower accuracy and an underestimated variability of biomechanical parameters derived from accelerometer signals could result. In this context, Ziebart et al. [33] investigated the influence of accelerometer OR and of a sampling rate on peak acceleration during seven jumping tasks. They used a tri-axial accelerometer (Model 7267A, Endevco Corporation, San Juan Capistrano, CA, USA) with a high OR of $\pm 260 \mathrm{~g}$ as the reference and compared the peak accelerations with two commercially available tri-axial accelerometers with an OR of \pm 6 g (device1: ActiGraph GT3X+, ActiGraph LLC, Pensacola, FL, USA and device2: X6-2mini, Gulf Coast Data Concepts, Waveland, MS, USA). They found that the peak impact acceleration was underestimated by up to $35 \%$. Furthermore, the underestimation error was greater for tasks with a greater impact acceleration.

It is currently still unclear whether, and to what extent, high accelerations can influence the accuracy of running parameters when exceeding the accelerometers' OR. However, this information is necessary to determine whether differences in the investigated biomechanical parameters strLen, runVel, and in the PTA between conditions are caused by measurement errors due to an accelerometer OR that is too low, or by the investigated conditions themselves.

In addition, the sensor signal characteristics of the IMU accelerometer, which is affixed to the heel cup of a running shoe, can be influenced by the midsole stiffness of footwear [5]. The authors found that a decreasing midsole stiffness resulted in an increasing delay in specific signal characteristics, when determining the time of foot touchdown. At this time, it is still unclear whether the accuracies of strLen and runVel are also influenced by altering acceleration signal characteristics due to a change in midsole stiffness.

Since acceleration variables are related to running injuries, information about whether the ORs influences running parameters is highly relevant and could assist coaches, researchers, and clinicians in selecting the most appropriate accelerometer specification for their investigations.

Therefore, the aim of the present study was to investigate the influence of the accelerometer OR on running parameters when reducing the OR stepwise from $\pm 70 \mathrm{~g}$ to $\pm 8 \mathrm{~g}$. We hypothesized that the rapid and short spikes at the beginning of ground contact and the high accelerations during the swing phase influenced the accuracy of strLen (H1), runVel (H2), and PTA (H3) significantly, depending on the footwear conditions. The running parameters were determined based on previously published methods, and accelerometers with a high OR of $\pm 70 \mathrm{~g}$ were used as the reference.

\section{Materials and Methods}

\subsection{Participants}

Twenty-one recreational male heel strike runners (age: $24.4 \pm 4.2$ years; height: $178.2 \pm 4.0 \mathrm{~cm}$; weight: $74.1 \pm 6.5 \mathrm{~kg}$; running experience: $8.9 \pm 3.3$ years; training hours: $3.5 \pm 1.7 \mathrm{~h}$ per week), free of injury for the last six months, participated in this study. High force rising rates can be observed after foot touchdown during rearfoot running [34], which results in a higher PTA for rearfoot runners than for forefoot runners [11]. Therefore, to determine the greatest effect of the reduced OR, only 
heel strike runners were investigated. This study was approved by the university's Ethics Committee (V-103-17-HS-CM-Bodenkontakt-25082015), and participants gave written informed consent to their participation in the study.

\subsection{Test Procedure}

During the test, participants wore three different commercially available running shoes, which were provided by our laboratory in men's UK size 8: Adidas AdiStar (ADIDAS); PUMA Speed 600 (PUMA); Asics Gel Nimbus 12 (ASICS). After an individual warm-up and familiarization with the measurement setup, five repeated trials were recorded on a 15-m indoor track in the three footwear conditions. The footwear conditions were investigated in a randomized order. The running speed was individual and self-selected (on average $3.60 \pm 0.4 \mathrm{~m} / \mathrm{s}$ ), however it was constant (range: $\pm 0.1 \mathrm{~m} / \mathrm{s}$ ) for each subject, for all of the 15 trials (three footwear conditions, each having five trials). Running speed was monitored using two light barriers placed 4 meters apart.

\subsection{Experimental Setup}

An individually configured IMU, combining a biaxial accelerometer (ADXL278, Analog Devices, $\mathrm{OR} \pm 70 \mathrm{~g}$ ) and a biaxial gyroscope (IDG-650, InvenSense, $\mathrm{OR} \pm 2000 \mathrm{deg} / \mathrm{s}$ ), was affixed to the heel cup of the right shoe (Figure 1). Wobble-free sensor fixation was achieved using double-sided adhesive tape and an inelastic strap. The sensitive axes of the IMU accelerometer measured the horizontal-forward direction- $($ acc_x $)$ and the vertical (acc_z) acceleration of the shoe. The two sensitive axes of the gyroscope measured the angular velocities in the sagittal $(\omega 1)$, and the frontal planes. Furthermore, to measure accelerations along the longitudinal axis of the tibia (acc_T), double-sided adhesive tape was used to attach a uniaxial lightweight accelerometer (ADXL78, Analog Devices, OR $\pm 70 \mathrm{~g}$ ) to the shaved skin at the medial aspect mid-distance between the malleolus and the plateau of the right tibia $[12,35]$. An elastic strap was used to stabilize the accelerometer and to prevent excessive movements due to its own weight. All sensors operated synchronously, and the data were transmitted by cable to a data logger, which recorded these data. The data logger was secured in a waist belt. To avoid any effects from a sampling rate that was too low, the sensor sampling rate was set to $1000 \mathrm{~Hz}$, which exceeds the minimum requirements to measure these parameters accurately [32].

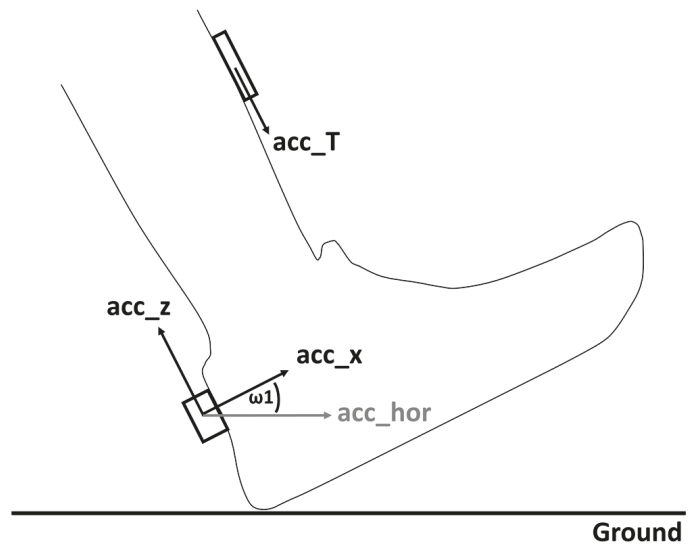

Figure 1. A schematic representation of the sensor setup. Sensitive axes of the accelerometer in the heel cup inertial measurement unit are represented by arrows: horizontal forward direction (acc_x) and vertical acceleration (acc_z). The angular velocity of the shoe was measured in the sagittal ( $\omega 1)$ and frontal plane using a gyroscope. The accelerometer, located at the tibia, measured accelerations along the longitudinal axis of the tibia (acc_T). 


\subsection{Data Analyses}

Data from the sensors were analyzed in post-processing using MATLAB R2016b (MathWorksTM, Natick, MA, USA). Prior to all of the processing steps, the data were filtered using a zero-lag Butterworth low-pass filter (accelerometers: 4th order at $200 \mathrm{~Hz}$; gyroscopes: 4th order at $50 \mathrm{~Hz}$ ) to remove noise. In the next step, accelerations measured with an OR of $\pm 70 \mathrm{~g}$ were cut at $\pm 32, \pm 16$, and at $\pm 8 \mathrm{~g}$ when exceeding their respective thresholds. After the data cutting, running parameters were calculated for all four ORs ( $\pm 70, \pm 32, \pm 16$, and $\pm 8 \mathrm{~g}$ ) separately, as described below.

To separate the strides of the right foot in continuous data, the accelerometer signal of the IMU at the heel cup (vertical axis) was $80 \mathrm{~Hz}$ zero-lag high pass filtered and the first peak in the filtered signal was defined as the foot touchdown $[5,36]$.

For each stride, the orientation angle of the shoe in the sagittal plane $(\theta)$ was calculated using integrated data from $\omega 1$. A nulling algorithm was then applied to the orientation angle of the shoe to eliminate errors resulting from integration offset and from drift error [32]. The time of two consecutive flat shoe phases (flat relative to the ground) was detected by finding the lowest absolute angular velocity in $\omega 1$ for each stride. The shoe orientation angles at these reference times were used in a linear drift model to eliminate the drift error and the integration offset. Based on Sabatini et al. [21] and Mitschke et al. [32], acc_x, acc_z, and the orientation angle $\theta$ were used to calculate the resulting horizontal acceleration (acc_hor) of the right shoe (see Equation (1); Figure 1).

$$
\text { acc_hor }=\text { acc_ } \_\cdot \cos (\theta)-\text { acc } \_z \cdot \sin (\theta)
$$

Subsequently, the horizontal velocity of the shoe $\left(\mathrm{v} \_\mathrm{x}\right)$ was calculated using a numerical integration of acc_hor. To eliminate the drift error and the integration offset of the shoe velocity, the velocity of the shoe during the flat shoe phases was assumed to be temporarily equal to zero [37]. Therefore, velocity was reset to zero as the stride start condition and for error back-propagation with a linear drift model $[32,38]$. The mean running velocity (runVel) was calculated by averaging $v \_x$ over time between two consecutive flat shoe phases. Furthermore, the stride length (strLen) between two consecutive flat shoe phases was calculated using integrated data from $v_{-} \mathrm{x}$.

For kinetics, the positive peak tibial acceleration (PTA) was defined as the maximum acceleration value of the accelerometer at the tibia (Figure 1: acc_T) [12,35].

\subsection{Footwear Conditions}

To quantify the shoe midsole stiffness, shoes were tested in a servo-hydraulic testing device (HC10; Zwick GmbH \& Co. KG; Ulm, Germany) as described in Schwanitz and Odenwald [39]. Each footwear condition underwent a total of ten load tests of 103 load cycles each. Load cycles were applied to the heel of each running shoe by a spherically shaped stamp $(50 \mathrm{~mm})$. The load-time profile was derived from biomechanical measurements of ground reaction force while running at a velocity of $3.5 \pm 0.1 \mathrm{~m} / \mathrm{s}$ [40]. For each footwear condition, midsole deformation at the 101st load cycle was analyzed for each of the ten test sessions. Internal studies have shown that the cycles after the 100th cycle represent reliable results when testing shoes mechanically [41]. The sampling rate was 1000 frames per second, and the data were analyzed using MATLAB R2016b (MathWorksTM, Natick, MA, USA) in post-processing. The midsole stiffness in the rearfoot area between 1000 and $1500 \mathrm{~N}$ was calculated for each footwear condition using Equation (2).

$$
\text { stiffness }=\frac{1500 \mathrm{~N}-1000 \mathrm{~N}}{\text { deformation }_{\mathrm{F}=1500 \mathrm{~N}}-\text { deformation }_{\mathrm{F}=1000 \mathrm{~N}}}
$$

\subsection{Statistical Analyses}

To compare footwear stiffness, the mean and standard deviations (mean $\pm \mathrm{SD}$ ) were calculated. Given that parameters were normally distributed, a one-way analysis of variance (ANOVA) followed 
by Bonferroni post hoc tests were used to determine whether differences existed between mechanical footwear characteristics. The level of significance was set to an alpha of 0.05 . Furthermore, the means of the five trials for each subject and for each footwear condition were used to calculate the group means and the standard deviations (mean $\pm \mathrm{SD}$ ), and the $95 \%$ confidence intervals $(95 \% \mathrm{CI}$ ) for strLen, runVel, PTA, and all ORs. Due to normal distribution, paired sample $t$ tests were used to compare the reference $( \pm 70 \mathrm{~g})$ with the three lower ORs $( \pm 32, \pm 16$, and $\pm 8 \mathrm{~g})$ for strLen and runVel for each of the three footwear conditions. In addition, Wilcoxon tests were used to compare the reference with the lower OR for PTA for each footwear condition. A Bonferroni correction was used to adjust the $p$-values $(p=0.05 / 3 / 3=0.006)$ in relation to the number of comparisons between the investigated OR $(n=3)$ and the investigated biomechanical parameters $(n=3)$. The effect size (Cohen's $d)$ was calculated to quantify the magnitude of differences between the shoe characteristics and the magnitude of differences between the biomechanical parameters examined with different ORs. The coefficients were interpreted as a trivial effect $(d<0.2)$, a small effect $(d<0.5)$, a medium effect $(d<0.8)$, and a large effect $(d \geq 0.8)$ [42]. Additionally, mean differences (MDs), relative mean differences in percent (MD_rel), and root mean square errors (RMSEs) were calculated between the reference OR and each of the lower ORs.

\section{Results}

\subsection{Footwear Characteristics}

All pair-wise comparisons reached statistical significance $(p<0.001)$. Cohen's $d$ showed large effects $(d \geq 0.8)$ in all footwear conditions. The lowest rearfoot stiffness was found for ASICS $(156.9 \pm 0.1 \mathrm{~N} / \mathrm{mm})$ and the highest stiffness was found for ADIDAS $(210.3 \pm 0.4 \mathrm{~N} / \mathrm{mm})($ Table 1$)$.

Table 1. Group mean \pm standard deviation (SD) for rearfoot stiffness of the three footwear conditions; statistical significance $(p<0.05)$ and large effects $(d \geq 0.8)$ were found in all footwear conditions.

\begin{tabular}{ccc}
\hline \multirow{2}{*}{ Footwear Condition } & \multicolumn{2}{c}{ Stiffness $(\mathrm{N} / \mathrm{mm})$} \\
\cline { 2 - 3 } & Mean & SD \\
\hline ADIDAS & 210.3 & 0.4 \\
PUMA & 177.8 & 0.4 \\
ASICS & 156.9 & 0.1 \\
\hline
\end{tabular}

\subsection{Biomechanical Parameters: Stride Length, Running Velocity, and Peak Tibial Acceleration}

For each footwear condition, results of the strLen and the runVel calculations, as well as the PTA values are represented in Figures 2-4 for the reference OR and for the lower ORs.

When comparing the OR of $\pm 70 \mathrm{~g}$ and $\pm 32 \mathrm{~g}$, no significant differences were found for runVel for all three footwear conditions (Table 2). Distinctly greater differences to the reference were found using the ORs of $\pm 16 \mathrm{~g}$ and $\pm 8 \mathrm{~g}$. Setting the OR to $\pm 16 \mathrm{~g}$ resulted in significant differences, up to $3.48 \%$ (ADIDAS: MD: $0.13 \mathrm{~m} / \mathrm{s} ; p<0.001)$, and large effects $(d \geq 0.86)$ for all three footwear conditions. When using an OR of $\pm 8 \mathrm{~g}$, a significantly lower runVel of up to $9.68 \%$ (ADIDAS: MD: $0.36 \mathrm{~m} / \mathrm{s}$; $p<0.001$ ) was found.

When comparing strLen, calculated using ORs of $\pm 70 \mathrm{~g}$ and $\pm 32 \mathrm{~g}$, no significant effects were found for ADIDAS, PUMA, or ASICS (Table 2). When using sensors with a lower OR, strLen calculations resulted in significant differences for all footwear conditions (MD $>2.62 \mathrm{~cm}$; MD_rel $>0.99 \% ; p<0.001$ ), showing large effects for all comparisons $(d>0.84)$. The highest strLen difference between the reduced OR and the reference was found for Adidas. An OR of $\pm 8 \mathrm{~g}$ resulted in a $9.68 \%$ lower stride length in comparison to the reference OR (MD: $26.15 \mathrm{~cm} ; p<0.001$ ).

No significant differences were found for PTA when comparing an OR of $\pm 70 \mathrm{~g}$ and $\pm 32 \mathrm{~g}$, and of $\pm 70 \mathrm{~g}$ and $\pm 16 \mathrm{~g}$ for all footwear conditions (Table 2). The comparison of the $\mathrm{OR} \pm 70 \mathrm{~g}$ 
and \pm 8 g revealed significant differences for all footwear conditions of at least $9.76 \%$ on average (ASICS: MD: $0.61 \mathrm{~g})$, which resulted in a medium effect $(d=0.60)$. The greatest difference between the OR of $\pm 70 \mathrm{~g}$ and $\pm 8 \mathrm{~g}$ was found for ADIDAS (MD: $2.65 \mathrm{~g}$; MD_rel: $28.17 \% ; p=0.002 ; d=0.65$ ). In general, we found that with higher midsole stiffnesses, the differences increased between the reference OR and $\pm 16 \mathrm{~g}$, as well as between the reference OR and $\pm 8 \mathrm{~g}$ (Figure 4 and Table 2).

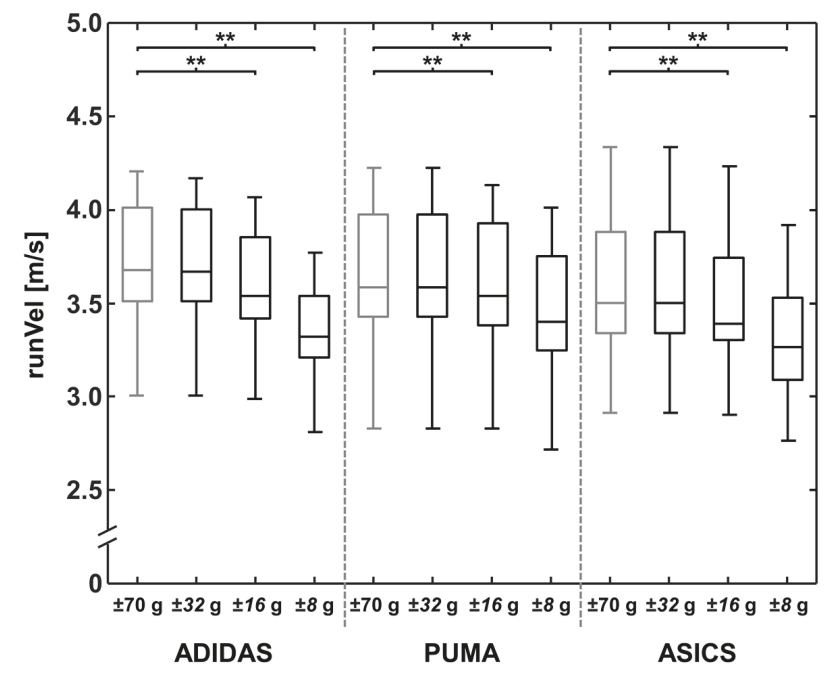

Figure 2. A boxplot of the three footwear conditions for running velocity (runVel), calculated with different accelerometer operating ranges of $\pm 70, \pm 32, \pm 16$, and $\pm 8 \mathrm{~g}$. Significant differences between stride lengths are marked with ${ }^{* *}(p<0.001)$.

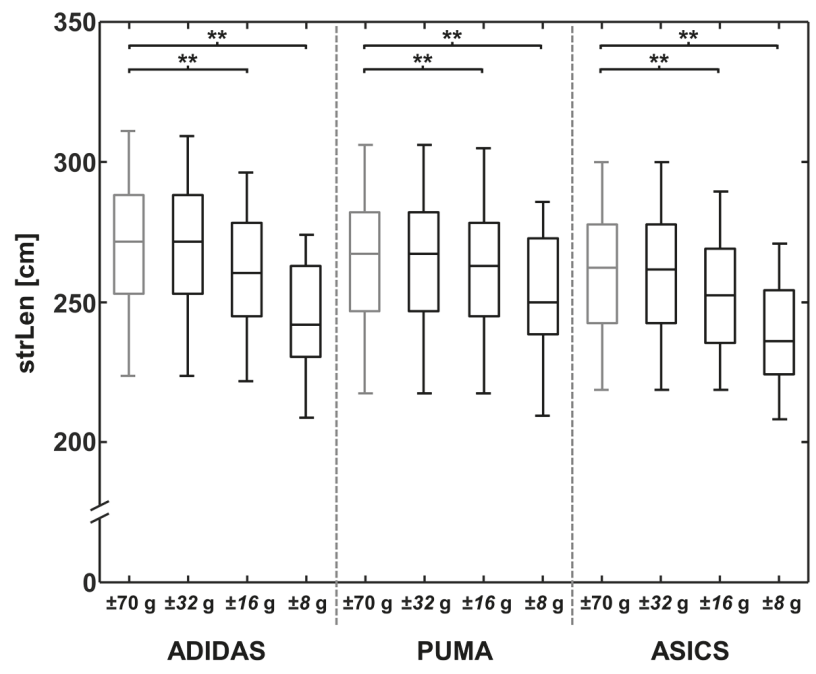

Figure 3. A boxplot of the three footwear conditions for stride length (strLen), calculated with different accelerometer operating ranges of $\pm 70, \pm 32, \pm 16$, and $\pm 8 \mathrm{~g}$. Significant differences between running velocities are marked with ${ }^{* *}(p<0.001)$. 


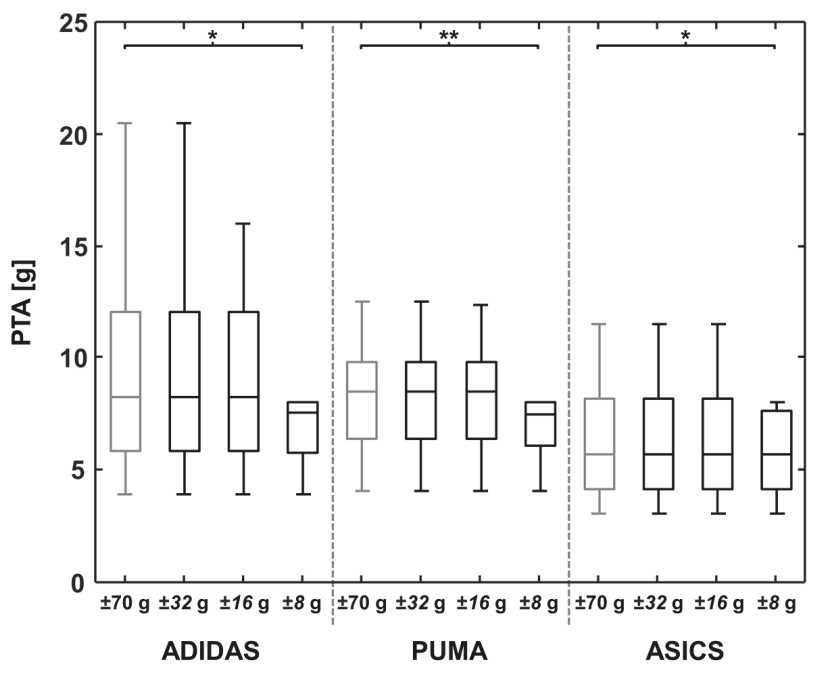

Figure 4. A boxplot of the three footwear conditions for the positive peak tibial acceleration (PTA), determined using different accelerometer operating ranges of $\pm 70, \pm 32, \pm 16$, and $\pm 8 \mathrm{~g}$. Significant differences between peak tibial accelerations are marked with ${ }^{*}(p<0.006)$ and ${ }^{* *}(p<0.001)$.

Table 2. Mean differences (MDs), relative mean differences (MD_rel), $p$-values of pair-wise comparisons, effect size (Cohen's $d$ ), and root mean square errors (RMSEs) between stride lengths (strLen), running velocities (runVel), and PTA, each determined using different accelerometer operating ranges of \pm 70 , $\pm 32, \pm 16$, and $\pm 8 \mathrm{~g}$.

\begin{tabular}{|c|c|c|c|c|c|c|c|c|c|}
\hline $\begin{array}{l}\text { Footwear } \\
\text { Condition }\end{array}$ & & ADIDAS & & & PUMA & & & ASICS & \\
\hline $\begin{array}{c}\text { Operating } \\
\text { ranges }\end{array}$ & $\pm 70- \pm 32 \mathrm{~g}$ & $\pm 70- \pm 16 \mathrm{~g}$ & $\pm 70- \pm 8 \mathrm{~g}$ & $\pm 70- \pm 32 \mathrm{~g}$ & $\pm 70- \pm 16 \mathrm{~g}$ & $\pm 70- \pm 8 \mathrm{~g}$ & $\pm 70- \pm 32 \mathrm{~g}$ & $\pm 70- \pm 16 \mathrm{~g}$ & $\pm 70- \pm 8 \mathrm{~g}$ \\
\hline \multicolumn{10}{|l|}{ runVel } \\
\hline $\mathrm{MD}(\mathrm{cm})$ & 0.01 & 0.13 & 0.36 & 0.00 & 0.04 & 0.18 & 0.00 & 0.09 & 0.29 \\
\hline MD_rel (\%) & 0.14 & 3.48 & 9.68 & 0.00 & 1.00 & 4.88 & 0.05 & 2.64 & 8.18 \\
\hline RMSE (cm) & 0.01 & 0.11 & 0.27 & 0.00 & 0.03 & 0.14 & 0.00 & 0.08 & 0.22 \\
\hline \multicolumn{10}{|l|}{ strLen } \\
\hline $\mathrm{MD}(\mathrm{m} / \mathrm{s})$ & 0.38 & 9.43 & 26.15 & 0.00 & 2.62 & 12.87 & 0.12 & 6.92 & 21.22 \\
\hline MD_rel $(\%)$ & 0.14 & 3.49 & 9.68 & 0.00 & 0.99 & 4.85 & 0.05 & 2.67 & 8.17 \\
\hline$p$ & 0.024 & $<0.001$ & $<0.001$ & 1 & $<0.001$ & $<0.001$ & 0.096 & $<0.001$ & $<0.001$ \\
\hline $\mathrm{MD}(\mathrm{g})$ & 0.00 & 0.59 & 2.65 & 0.00 & 0.31 & 2.08 & 0.00 & 0.00 & 0.61 \\
\hline MD_rel (\%) & 0.00 & 6.25 & 28.17 & 0.00 & 3.48 & 23.02 & 0.00 & 0.04 & 9.76 \\
\hline$p$ & 1 & 0.043 & 0.002 & 1 & 0.109 & $<0.001$ & 1 & 0.317 & 0.005 \\
\hline Cohen's $d$ & - & - & 0.65 & - & - & 0.77 & - & - & 0.60 \\
\hline RMSE (g) & 0.00 & 1.23 & 3.39 & 0.00 & 0.83 & 2.72 & 0.00 & 0.01 & 1.13 \\
\hline
\end{tabular}

\section{Discussion}

The aim of the present study was to investigate the influence of the accelerometer OR on stride length, running velocity, and on peak tibial acceleration when reducing the OR stepwise from $\pm 70 \mathrm{~g}$ to $\pm 32, \pm 16$, and $\pm 8 \mathrm{~g}$. Biomechanical parameters were determined based on previously published methods, and accelerometers (attached at the heel cup and at the tibia) with a high OR of $\pm 70 \mathrm{~g}$ were used as the reference. 
The results of this study revealed that OR influences the outcomes of stride length, running velocity, and peak tibial acceleration, which were not dependent on tested footwear conditions. Lower ORs were associated with an underestimation error for all biomechanical parameters, which increased with decreasing OR. These results confirm hypotheses H1 (strLen), H2 (runVel), and H3 (PTA). Our results show that a sensor OR needs to be carefully considered when interpreting biomechanical parameters of existing investigations and when planning future studies.

Using accelerometers with an OR of $\pm 32 \mathrm{~g}$ resulted in small errors, on average of up to $0.14 \%$ for strLen and $0.14 \%$ for runVel (ADIDAS). However, insignificant differences and low RMSE values were found for all footwear conditions, and therefore, the observed differences can be considered irrelevant. The accelerations during running that were measured in our study did not critically exceed the $\pm 32 \mathrm{~g}$ threshold. However, our results show that when the sensor OR was limited to $\pm 16 \mathrm{~g}$ or $\pm 8 \mathrm{~g}$, distinctly higher differences, when compared to the reference, were found for runVel and strLen. Due to the lower vertical and horizontal forward accelerations (set to $\pm 16 \mathrm{~g}$ and $\pm 8 \mathrm{~g}$ when exceeding the respective thresholds), the resulting horizontal forward velocity of the shoe ( $v_{-} \mathrm{x}$ ) was calculated inaccurately when using the numerical integration of acc_hor. Figure 5 shows v_x for one representative stride. When observing the velocity curve progression, it seems there was a drift error after the numerical integration (frame 120 to 520), which would explain the significantly lower runVel for the OR of $\pm 16 \mathrm{~g}$ and $\pm 8 \mathrm{~g}$ when compared to the reference. However, to eliminate any drift error and integration offset, the shoe velocity during the flat shoe phases was assumed to be temporarily equal to zero [37]. Therefore, the velocity was reset to zero as the stride start condition and for error back-propagation with a linear drift model [32,38]. As shown in Figure 5, the horizontal forward velocity of the foot was reset to zero in both of the flat shoe phases and no additional offset was observed for these phases. We presume, therefore, that the lower velocities were the result of ORs which were too low and were not due to a drift error.

Additionally, the results show that the stiffnesses of the tested footwear conditions do not appear to noticeably influence the accuracy of runVel. Due to the high impact loads during rearfoot running, we expected that the footwear with the highest midsole stiffness in the rearfoot area (ADIDAS) would show distinctly greater differences between $\pm 70 \mathrm{~g}$ and the lower ORs, than the footwear with the lowest stiffness (ASICS). However, whereas ADIDAS revealed the highest differences between the reference OR and the reduced ORs, the ASICS shoe showed higher differences between the reference OR and the reduced ORs than PUMA (medium stiffness) did. Furthermore, similar significant differences $(p<0.001)$ and effect sizes between the reference OR and the OR of $\pm 16 \mathrm{~g}$ and $\pm 8 \mathrm{~g}$ were found for all of the footwear conditions. Therefore, due to the high accelerations which occurred during the heel strike while running in footwear with differing midsole stiffnesses, sensors with an OR greater than $\pm 32 \mathrm{~g}$ should be used to measure these accelerations accurately and to calculate runVel with high accuracy (errors lower than $0.14 \%$ ).

Despite the different acceleration magnitudes which occurred during heel strike for the three footwear conditions, the calculated velocities (OR: $\pm 70 \mathrm{~g}$ ) were comparable using the measurements taken by the light barriers ( $3.60 \pm 0.4 \mathrm{~m} / \mathrm{s}$ ): ADIDAS: $3.70 \pm 0.3 \mathrm{~m} / \mathrm{s}$; PUMA: $3.66 \pm 0.4 \mathrm{~m} / \mathrm{s}$; ASICS: $3.60 \pm 0.3 \mathrm{~m} / \mathrm{s}$. Therefore, the method for calculating runVel can be used when the OR is $> \pm 32 \mathrm{~g}$.

For strLen, effects due to the lower OR were also observed. StrLen was calculated between two consecutive flat shoe phases using the integrated data from $v_{-} x$. As expected, lower velocities for the reduced ORs also resulted in shorter strLen. The values of MD_rel and the significant differences between the reference and the reduced ORs, including effect sizes, were similar for strLen and runVel. This is the result of the numerical integration of $v_{-} x$ between two consecutive flat shoe phases when calculating strLen. Additionally, tested footwear conditions did not influence strLen calculations when using an OR of $\pm 32 \mathrm{~g}$. It seems that the critical accelerations in anterior-posterior and in vertical directions do not critically exceed the OR of $\pm 32 \mathrm{~g}$ during heel strike, regardless of the midsole stiffness. However, using an OR of $\pm 16 \mathrm{~g}$ can lead to strLen differences of $3.49 \%$ (MD: $9.43 \mathrm{~cm}$ ) for each stride when running in the ADIDAS shoe. In contrast, when running in the PUMA shoe, differences of 
only $0.99 \%$ (MD: $2.62 \mathrm{~cm}$ ) were found. With a further decrease in the OR, the differences between the calculated strLen of PUMA and of ADIDAS increased. When investigating mechanical midsole characteristics of footwear in biomechanical tests (e.g., benchmark tests) using an OR that is too low $(< \pm 32 \mathrm{~g})$, errors in the strLen calculation can lead to incorrect conclusions. Therefore, to avoid effects of an OR that is too low, an OR of at least $\pm 32 \mathrm{~g}$ should be used to measure strLen as accurately as possible and independently from the midsole stiffness of footwear.

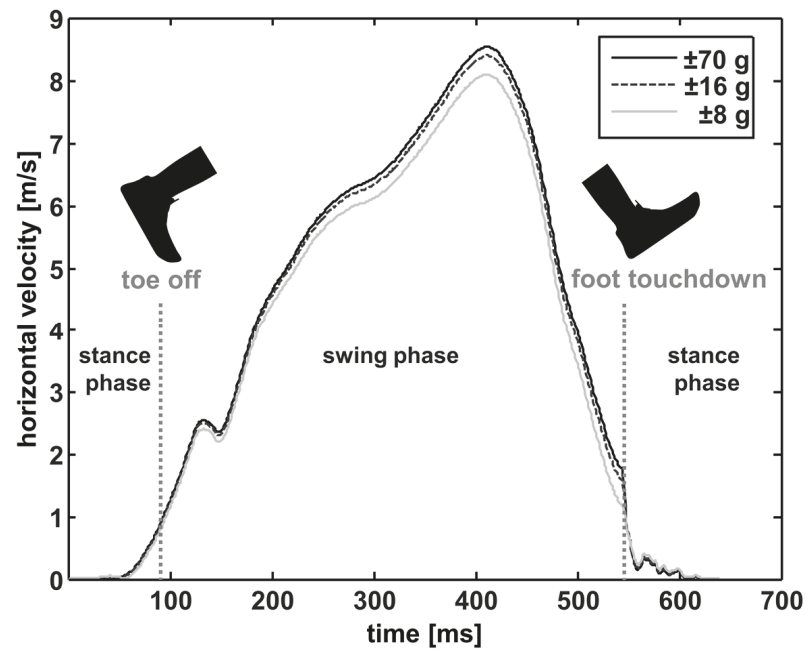

Figure 5. The horizontal velocity (forward direction) of the shoe for one representative stride with an accelerometer operating range of $\pm 70 \mathrm{~g}$ (black line), $\pm 16 \mathrm{~g}$ (grey dotted line), and of $\pm 8 \mathrm{~g}$ (grey line), subdivided in swing phase and foot contact (until toe off, exceeding $2 \mathrm{~g}$ in the horizontal acceleration signal [43] and after the foot touchdown $[5,36])$.

For PTA, none of the subjects in this study generated higher peak tibial accelerations than $\pm 32 \mathrm{~g}$ when running in the three provided footwear conditions (MD_rel: $0.0 \%$; RMSE: $0.0 \mathrm{~g}$ ). However, faster running velocities (e.g., sprint runs), running in shoes with a higher midsole stiffness (e.g., barefoot or minimal shoes), or running barefoot may increase peak tibial acceleration $[10,15,44]$. In some cases, accelerations could then exceed $32 \mathrm{~g}$ and may lead to inaccurate results when measuring with an OR that is too low. Our results for PTA show that accuracy decreased up to $28.17 \%$ (ADIDAS: $\pm 8 \mathrm{~g}$ ) with lower OR. Thereby, we found that a higher midsole stiffness generally decreased the accuracy of PTA. The footwear condition with the stiffest midsole (ADIDAS) showed the greatest PTA for the reference OR (mean \pm SD: $9.41 \pm 5.3 \mathrm{~g}$ ), whereas the footwear with the softest midsole configuration (ASICS) showed the lowest PTA (mean \pm SD: $6.28 \pm 3.0 \mathrm{~g}$ ). When observing the mean values of PTA for each footwear condition, the minimum OR can be derived from these results.

To accurately determine PTA, a distinctly lower OR $(\geq \pm 16 \mathrm{~g})$ can be used in contrast to the strLen and runVel ( $\geq \pm 32 \mathrm{~g}$ ). Due to various influencing factors (e.g., heel fat pad cushioning, foot pronation, shoe midsole deformation, skin displacement, and sensor wobbling), measured accelerations at the tibia were damped and were attenuated in contrast to the wobble-free sensor at the heel cup. Therefore, a distinctly lower OR is necessary for the accelerometer placed at the tibia to determine PTA without a significant loss of information. However, different locations of the accelerometers on the tibia can also influence the temporal and spectral parameters of PTA. Lucas-Cuevas et al. [45] found that a distally placed accelerometer measured a greater PTA and shock attenuation compared to a proximal accelerometer. In our study, the accelerometer was placed at the medial aspect mid-distance between the malleolus and plateau of the right tibia according to Henning et al. [35] and Milani et al. [12]. 
Therefore, a more distal sensor attachment may require a greater OR than was recommended in our study.

\section{Conclusions}

In literature, various accelerometers with ORs between $\pm 2 \mathrm{~g}$ and $\pm 70 \mathrm{~g}$ have been used to determine biomechanical parameters, although some studies did not state the OR used. In the present study, the influence of OR on biomechanical parameters was investigated. Our results are important for coaches, researchers, and clinicians in selecting the best accelerometer specification for their future investigations. Our study shows that an accelerometer OR that is set too low results in an inaccurate determination of strLen, runVel, and PTA. Especially in prolonged runs, when investigating a large number of continuous strides (e.g., a marathon [24]), an OR that is too low leads to an underestimation of strLen for each stride, which results in great differences in the total distance. Furthermore, when investigating strLen or PTA variability during prolonged runs in the context of fatigue, a low PTA or strLen variability can be misinterpreted due to an OR that is too low. Additionally, the midsole stiffnesses of the footwear conditions did not influence the accuracy of biomechanical parameters when the OR was $\geq \pm 32 \mathrm{~g}$. Furthermore, significant differences and effect sizes between the reference OR and the reduced ORs ( $\pm 16 \mathrm{~g}$ and $\pm 8 \mathrm{~g}$ ) were similar for all footwear conditions. However, our results show that when investigating mechanical footwear characteristics in biomechanical tests (e.g., benchmark tests), an OR that is too low (for strLen and runVel $< \pm 32 \mathrm{~g}$, for PTA $< \pm 16 \mathrm{~g}$ ) can lead to an approximation of biomechanical parameters, which leads to incorrect conclusions. Finally, when examining biomechanical parameters during running using accelerometers with an OR lower than $\pm 32 \mathrm{~g}$, these results should be considered carefully.

Acknowledgments: The authors would like to thank Lisa Peterson for proofreading the paper as a native speaker. This research was supported by Puma ${ }^{\circledR}$ Inc., Germany. The publication costs of this article were funded by the German Research Foundation/DFG and the Technische Universität Chemnitz, Chemnitz, Germany, through the funding program Open Access Publishing.

Author Contributions: Christian Mitschke, Pierre Kiesewetter, and Thomas L. Milani conceived and designed the experiments; Christian Mitschke and Pierre Kiesewetter performed the experiments; Christian Mitschke, Pierre Kiesewetter, and Thomas L. Milani analyzed the data; Thomas L. Milani contributed reagents/materials/analysis tools; Christian Mitschke, Pierre Kiesewetter, and Thomas L. Milani wrote the paper.

Conflicts of Interest: The authors declare no conflicts of interest. The funding sponsors had no role in the design of the study, in the collection, analyses, or in the interpretation of data, in the writing of the manuscript, or in the decision to publish the results.

\section{References}

1. Tao, W.; Liu, T.; Zheng, R.; Feng, H. Gait analysis using wearable sensors. Sensors 2012, 12, 2255-2283. [CrossRef] [PubMed]

2. Liu, T.; Inoue, Y.; Shibata, K. Development of a wearable sensor system for quantitative gait analysis. Measurement 2009, 42, 978-988. [CrossRef]

3. Mayagoitia, R.E.; Nene, A.V.; Veltink, P.H. Accelerometer and rate gyroscope measurement of kinematics: An inexpensive alternative to optical motion analysis systems. J. Biomech. 2002, 35, 537-542. [CrossRef]

4. Mitschke, C.; Öhmichen, M.; Milani, T.L. A single gyroscope can be used to accurately determine peak eversion velocity during locomotion at different speeds and in various shoes. Appl. Sci. 2017, 7, 659. [CrossRef]

5. Mitschke, C.; Heß, T.; Milani, T.L. Which method detects foot strike in rearfoot and forefoot runners accurately when using an inertial measurement unit? Appl. Sci. 2017, 7, 959. [CrossRef]

6. Shih, Y.; Ho, C.-S.; Shiang, T.-Y. Measuring kinematic changes of the foot using a gyro sensor during intense running. J. Sports Sci. 2014, 32, 550-566. [CrossRef] [PubMed]

7. Clark, R.A.; Bartold, S.; Bryant, A.L. Tibial acceleration variability during consecutive gait cycles is influenced by the menstrual cycle. Clin. Biomech. 2010, 25, 557-562. [CrossRef] [PubMed] 
8. Chambon, N.; Sevrez, V.; Ly, Q.H.; Guéguen, N.; Berton, E.; Rao, G. Aging of running shoes and its effect on mechanical and biomechanical variables: Implications for runners. J. Sports Sci. 2014, 32, 1013-1022. [CrossRef] [PubMed]

9. Mizrahi, J.; Verbitsky, O.; Isakov, E.; Daily, D. Effect of fatigue on leg kinematics and impact acceleration in long distance running. Hum. Mov. Sci. 2000, 19, 139-151. [CrossRef]

10. Montgomery, G.; Abt, G.; Dobson, C.; Smith, T.; Ditroilo, M. Tibial impacts and muscle activation during walking, jogging and running when performed overground, and on motorised and non-motorised treadmills. Gait Posture 2016, 49, 120-126. [CrossRef] [PubMed]

11. Gruber, A.H.; Boyer, K.A.; Derrick, T.R.; Hamill, J. Impact shock frequency components and attenuation in rearfoot and forefoot running. J. Sport Health Sci. 2014, 3, 113-121. [CrossRef]

12. Milani, T.L.; Hennig, E.M.; Lafortune, M. Perceptual and biomechanical variables for running in identical shoe constructions with varying midsole hardness. Clin. Biomech. 1997, 12, 294-300. [CrossRef]

13. Mercer, J.A.; Vance, J.; Hreljac, A.; Hamill, J. Relationship between shock attenuation and stride length during running at different velocities. Eur. J. Appl. Physiol. 2002, 87, 403-408. [CrossRef] [PubMed]

14. Mercer, J.A.; Bates, B.T.; Dufek, J.S.; Hreljac, A. Characteristics of shock attenuation during fatigued running. J. Sports Sci. 2003, 21, 911-919. [CrossRef] [PubMed]

15. Heidenfelder, J.; Sterzing, T.; Milani, T.L. Systematically modified crash-pad reduces impact shock in running shoes. Footwear Sci. 2010, 2, 85-91. [CrossRef]

16. Meardon, S.; Hamill, J.; Derrick, T.R. Running injury and stride time variability over a prolonged run. Gait Posture 2011, 33, 36-40. [CrossRef] [PubMed]

17. Oriwol, D.; Sterzing, T.; Milani, T.L. The position of medial dual density midsole elements in running shoes does not influence biomechanical variables. Footwear Sci. 2011, 3, 107-116. [CrossRef]

18. Giandolini, M.; Horvais, N.; Farges, Y.; Samozino, P.; Morin, J.B. Impact reduction through long-term intervention in recreational runners: Midfoot strike pattern versus low-drop/low-heel height footwear. Eur. J. Appl. Physiol. 2013, 113, 2077-2090. [CrossRef] [PubMed]

19. Sinclair, J.; Hobbs, S.J.; Protheroe, L.; Edmundson, C.J.; Greenhalgh, A. Determination of gait events using an externally mounted shank accelerometer. J. Appl. Biomech. 2013, 29, 118-122. [CrossRef] [PubMed]

20. García-Pérez, J.A.; Pérez-Soriano, P.; Llana Belloch, S.; Lucas-Cuevas, Á.G.; Sánchez-Zuriaga, D. Effects of treadmill running and fatigue on impact acceleration in distance running. Sports Biomech. 2014, 13, 1-8. [CrossRef] [PubMed]

21. Sabatini, A.M.; Martelloni, C.; Scapellato, S.; Cavallo, F. Assessment of walking features from foot inertial sensing. IEEE Trans. Biomed. Eng. 2005, 52, 486-494. [CrossRef] [PubMed]

22. Mercer, J.A.; Chona, C. Stride length-velocity relationship during running with body weight support. J. Sport Health Sci. 2015, 4, 127-131. [CrossRef]

23. Yang, S.; Mohr, C.; Li, Q. Ambulatory running speed estimation using an inertial sensor. Gait Posture 2011, 34, 462-466. [CrossRef] [PubMed]

24. Reenalda, J.; Maartens, E.; Homan, L.; Buurke, J.H. Continuous three dimensional analysis of running mechanics during a marathon by means of inertial magnetic measurement units to objectify changes in running mechanics. J. Biomech. 2016, 49, 3362-3367. [CrossRef] [PubMed]

25. Watanabe, T.; Saito, H.; Koike, E.; Nitta, K. A preliminary test of measurement of joint angles and stride length with wireless inertial sensors for wearable gait evaluation system. Comput. Intell. Neurosci. 2011, 2011, 6. [CrossRef] [PubMed]

26. Provot, T.; Chiementin, X.; Oudin, E.; Bolaers, F.; Murer, S. Validation of a high sampling rate inertial measurement unit for acceleration during running. Sensors 2017, 17, 1958. [CrossRef] [PubMed]

27. Fong, D.T.P.; Chan, Y.Y. The use of wearable inertial motion sensors in human lower limb biomechanics studies: A systematic review. Sensors 2010, 10, 11556-11565. [CrossRef] [PubMed]

28. Yang, S.; Li, Q. Inertial sensor-based methods in walking speed estimation: A systematic review. Sensors 2012, 12, 6102-6116. [CrossRef] [PubMed]

29. Alvarez, J.C.; González, R.C.; Alvarez, D.; López, A.M.; Rodríguez-Uría, J. Multisensor approach to walking distance estimation with foot inertial sensing. In Proceedings of the Annual International Conference of the IEEE Engineering in Medicine and Biology, Lyon, France, 22-26 August 2007; pp. 5719-5722. 
30. Jasiewicz, J.M.; Allum, J.H.J.; Middleton, J.W.; Barriskill, A.; Condie, P.; Purcell, B.; Li, R.C.T. Gait event detection using linear accelerometers or angular velocity transducers in able-bodied and spinal-cord injured individuals. Gait Posture 2006, 24, 502-509. [CrossRef] [PubMed]

31. Zifchock, R.A.; Davis, I.; Higginson, J.; McCaw, S.; Royer, T. Side-to-side differences in overuse running injury susceptibility: A retrospective study. Hum. Mov. Sci. 2008, 27, 888-902. [CrossRef] [PubMed]

32. Mitschke, C.; Zaumseil, F.; Milani, T.L. The influence of inertial sensor sampling frequency on the accuracy of measurement parameters in rearfoot running. Comput. Methods Biomech. Biomed. Eng. 2017, 20, 1502-1511. [CrossRef] [PubMed]

33. Ziebart, C.; Giangregorio, L.M.; Gibbs, J.C.; Levine, I.C.; Tung, J.; Laing, A.C. Measurement of peak impact loads differ between accelerometers-Effects of system operating range and sampling rate. J. Biomech. 2017, 58, 222-226. [CrossRef] [PubMed]

34. Lieberman, D.E.; Venkadesan, M.; Werbel, W.A.; Daoud, A.I.; D'Andrea, S.; Davis, I.S.; Mang'eni, R.O.; Pitsiladis, Y. Foot strike patterns and collision forces in habitually barefoot versus shod runners. Nature 2010, 463, 531-535. [CrossRef] [PubMed]

35. Hennig, E.M.; Milani, T.L.; Lafortune, M. Use of ground reaction force parameters in predicting peak tibial accelerations in running. J. Appl. Biomech. 1993, 9, 214-306. [CrossRef] [PubMed]

36. Maiwald, C.; Dannemann, A.; Gaudel, J.; Oriwol, D. A simple method to detect stride intervals in continuous acceleration and gyroscope data recorded during treadmill running. Footwear Sci. 2015, 7, 143-144. [CrossRef]

37. Peruzzi, A.; Della Croce, U.; Cereatti, A. Estimation of stride length in level walking using an inertial measurement unit attached to the foot: A validation of the zero velocity assumption during stance. J. Biomech. 2011, 44, 1991-1994. [CrossRef] [PubMed]

38. Hamacher, D.; Hamacher, D.; Taylor, W.R.; Singh, N.B.; Schega, L. Towards clinical application: Repetitive sensor position re-calibration for improved reliability of gait parameters. Gait Posture 2014, 39, 1146-1148. [CrossRef] [PubMed]

39. Schwanitz, S.; Odenwald, S. Long-term cushioning properties of running shoes (P152). In The Engineering of Sport 7; Springer: Paris, France, 2008; pp. 95-100.

40. Brückner, K.; Odenwald, S.; Schwanitz, S.; Heidenfelder, J.; Milani, T. Polyurethane-foam midsoles in running shoes-Impact energy and damping. Procedia Eng. 2010, 2, 2789-2793. [CrossRef]

41. Heidenfelder, J. Entwicklung Eines Dynamischen Tests zur Prüfung der Rückfußdämpfung von Laufschuhen Mittels Biomechanischer Messmethoden; TU Chemnitz: Chemnitz, Germany, 2011.

42. Cohen, J. Quantitative methods in psychology. Psychol. Bull. 1992, 112, 155-159. [CrossRef] [PubMed]

43. Strohrmann, C.; Harms, H.; Kappeler-Setz, C.; Tröster, G. Monitoring kinematic changes with fatigue in running using body-worn sensors. IEEE Trans. Inf. Technol. Biomed. 2012, 16, 983-990. [CrossRef] [PubMed]

44. Mcnair, P.J.; Marshall, R.N. Kinematic and kinetic parameters associated with running in different shoes. Br. J. Sports Med. 1994, 28, 256-261. [CrossRef] [PubMed]

45. Lucas-Cuevas, A.G.; Encarnación-Martínez, A.; Camacho-García, A.; Llana-Belloch, S.; Pérez-Soriano, P. The location of the tibial accelerometer does influence impact acceleration parameters during running. J. Sports Sci. 2017, 35, 1734-1738. [CrossRef] [PubMed]

(C) 2018 by the authors. Licensee MDPI, Basel, Switzerland. This article is an open access article distributed under the terms and conditions of the Creative Commons Attribution (CC BY) license (http:/ / creativecommons.org/licenses/by/4.0/). 
Article

\title{
What is the Best Configuration of Wearable Sensors to Measure Spatiotemporal Gait Parameters in Children with Cerebral Palsy?
}

\author{
Lena Carcreff ${ }^{1,2, *}$, Corinna N. Gerber ${ }^{3}$, Anisoara Paraschiv-Ionescu ${ }^{2}$, Geraldo De Coulon ${ }^{4}$, \\ Christopher J. Newman ${ }^{3}$, Stéphane Armand ${ }^{1}$ and Kamiar Aminian ${ }^{2}$ \\ 1 Laboratory of Kinesiology Willy Taillard, Geneva University Hospitals and University of Geneva, \\ 1205 Geneva, Switzerland; stephane.armand@hcuge.ch \\ 2 Laboratory of Movement Analysis and Measurement, Ecole Polytechnique Fédérale de Lausanne, \\ 1015 Lausanne, Switzerland; anisoara.ionescu@epfl.ch (A.P.-I.), kamiar.aminian@epfl.ch (K.A.) \\ 3 Pediatric Neurology and Neurorehabilitation Unit, Department of Pediatrics, Lausanne University Hospital, \\ 1011 Lausanne, Switzerland; corinna.gerber@chuv.ch (C.N.G.); christopher.newman@chuv.ch (C.J.N.) \\ 4 Pediatric orthopedics, Geneva University Hospitals, 1205 Geneva, Switzerland; geraldo.decoulon@hcuge.ch \\ * Correspondence: lena.carcreff@hcuge.ch; Tel.: +41-(0)22-37-27-872
}

Received: 13 December 2017; Accepted: 25 January 2018; Published: 30 January 2018

\begin{abstract}
Wearable inertial devices have recently been used to evaluate spatiotemporal parameters of gait in daily life situations. Given the heterogeneity of gait patterns in children with cerebral palsy $(\mathrm{CP})$, the sensor placement and analysis algorithm may influence the validity of the results. This study aimed at comparing the spatiotemporal measurement performances of three wearable configurations defined by different sensor positioning on the lower limbs: (1) shanks and thighs, (2) shanks, and (3) feet. The three configurations were selected based on their potential to be used in daily life for children with $\mathrm{CP}$ and typically developing (TD) controls. For each configuration, dedicated gait analysis algorithms were used to detect gait events and compute spatiotemporal parameters. Fifteen children with CP and 11 TD controls were included. Accuracy, precision, and agreement of the three configurations were determined in comparison with an optoelectronic system as a reference. The three configurations were comparable for the evaluation of TD children and children with a low level of disability (CP-GMFCS I) whereas the shank-and-thigh-based configuration was more robust regarding children with a higher level of disability (CP-GMFCS II-III).
\end{abstract}

Keywords: cerebral palsy; gait; inertial sensors; gait events; spatiotemporal parameters

\section{Introduction}

Cerebral palsy (CP) is the most frequent motor disorder in children with a prevalence of 1.8:1000 births in Europe [1]. For the majority of children with $\mathrm{CP}$ who achieve community ambulation, an objective evaluation of their gait is necessary to accurately identify and understand gait impairments, in order to provide adequate and efficient treatment [2]. Such assessments are usually performed in laboratory settings, during standardized clinical gait analysis (CGA). Numerous gait parameters including spatiotemporal parameters (STP), kinematics, kinetics, and muscle activity can be quantified using an optoelectronic (3D motion capture) system, force plates, and an electromyography system [3].

During gait, foot strikes (FS) and foot offs (FO) succeed each other and constitute the base for gait cycle segmentation and the computation of STP. Gait events can be computed from a single force plate, restricting the measure to one step at a time. With the combination of several force plates and an optoelectronic system, it is possible to determine these events over several steps [4]. The advantage of this method is to calculate spatial parameters, such as the stride length, directly 
from the marker positions. Despite its wide use in CGA [5-8], the optoelectronic system has some unavoidable limitations. It suffers from inaccuracies linked to instrumental errors, soft tissue artefacts (the relative displacement between the markers and the underlying bone), and marker misplacement due to anatomical landmark palpation difficulties [8]. Since STP estimation is mainly based on the tracking of markers located on the heels and toes, soft tissue artefacts and marker misplacement are not likely to induce major errors. Therefore, instrumental errors are the main sources of inaccuracy in this situation. In 2005, a review reported mean errors for marker distance estimates between $0.1 \mathrm{~mm}$ and $5.3 \mathrm{~mm}$ depending on the systems [9]. Thanks to the improvement in camera resolution, these errors were expected to decrease. Recently, Di Marco et al. reported that the number of cameras, the calibration volume, and the calibration procedure can induce errors in the marker trajectories reconstruction between $0.2 \mathrm{~mm}$ and $1.7 \mathrm{~mm}[8,10]$. Moreover, the optoelectronic system restrains the measurement volume to the laboratory and thus may hinder the patient's natural gait. As a result, gait parameters obtained through CGA are not fully representative of the usual and daily walking habits (described as 'performance' [11]) of children with CP [12-14]. Performance can currently be estimated through questionnaires and clinical observations which have the inherent drawback of being subjective and evaluator-dependent, and thus associated with potential bias [15,16]. Therefore, there is a need to objectively assess gait performance in daily-life conditions in order to complement CGA, thus enhancing therapeutic choices for children with CP based on real-life data. Wearable inertial sensors can be used to fulfill this need.

Inertial sensors are microelectromechanical systems, including accelerometers and gyroscopes, contained in small casings that can be carried by the patient without restrictions for several hours of measurements. A wearable sensor-based gait analysis system relies on a sensor configuration and an associated algorithm. Many sensor configurations have been tested for STP estimation varying in numbers of sensors (single or multiple), type (single or triaxial), and location on the body [17]. As, in human gait, most body motion comes from the lower limbs, sensors are commonly fixed on the lower limbs [17]. Gait events are detected according to specific features appearing on accelerometer and gyroscope signals in the time and frequency domains [18-20]. Spatial parameters are computed from these signals through methods dependent on the sensor location $[17,20]$. Yang et al. defined three categories of algorithms: the abstraction models where the spatial parameter is estimated from a black box model building the relationship between the sensor measurements and the output (e.g., artificial neural networks, third-order polynomial model, etc.); the human gait models which use the geometric properties of the lower limbs to estimate stride length; and the direct integrations which consist of integrating the acceleration in the global frame between two specific points of the gait cycle in order to calculate stride velocity (simple integration) and stride length (double integration).

The validity of gait event detection and STP computation from wearable devices has been studied in healthy adults, adults with disease, or elderly population, but scarcely in children [21-24]. Lanovaz et al. found that, in healthy children, a system of six inertial sensors was valid for temporal detection but showed consistent bias for spatial parameters estimation with the gait model method [25]. In children with CP, a protocol named "Outwalk" has been developed to measure trunk and lower-limb 3D kinematics using an inertial and magnetic measurement system, but the authors did not assess STP [26,27]. Laudanski et al. observed that gait analysis based on sensors on the feet bring more error than shank-mounted sensors, especially for abnormal gait pattern such as toe-out walking [28]. In children with $\mathrm{CP}$ with low to mild gait impairments, Bregou-Bourgeois et al. used two foot-worn inertial sensors and the direct integration method to estimate STP and showed good accuracy, precision, and agreement against an optoelectronic system for the estimation of stride length, walking speed, and foot angles with regard to the ground [29]. These previous studies pointed out the difficulty of assessing gait in children with CP with inertial sensors since these children cannot achieve the expected movement properly (i.e., full foot contact during gait [29], good alignment in static, or pure flexion/extension of the knee for functional calibration of the sensor axes [26]). Considering these challenges and since gait patterns are very heterogeneous among patients with $\mathrm{CP}$ who are able to 
walk [2], the usability of other sensor set-ups deserves exploration in order to determine which is the most appropriate one. Furthermore, children with a higher level of impairment should be considered as they may benefit even more from the assessment of their gait performance.

The aim of this study was to evaluate and compare the measurement performance of three wearable configurations and to identify the most appropriate set-up for gait assessment of children with CP. For this purpose, a standard optoelectronic system was used as a reference to evaluate event detection and STP computation.

\section{Materials and Methods}

This study was an observational case-control validation study with a single center setup.

\subsection{Participants}

Fifteen children and adolescents with $\mathrm{CP}$ and eleven age- and sex-matched typically developing (TD) controls were evaluated in the laboratory of kinesiology within Geneva University Hospitals (HUG). Participants of the CP group were recruited from the patients followed at the HUG pediatric orthopedics unit if they met the following inclusion criteria: (a) aged between 8 and 20 years; (b) diagnosis of $\mathrm{CP}$; (c) ability to walk in the community with or without mechanical walking aids; and (d) with a level of Gross Motor Function Classification System (GMFCS) [30] between I and III. For the control group, children were recruited among collaborators' or patients' acquaintances. The exclusion criteria for both groups were those that precluded adequate participation in the measurement sessions (mental age $<8$ years, severe visual disorder, attention deficit, and other significant behavioral issues). All participants provided written consent, and the protocol was approved by the hospital's institutional ethical committee (CCER-15-176).

\subsection{Protocol}

A trained experimenter measured the following anthropometric data: leg, thigh, shank, and foot lengths, as well as knee, ankle, and pelvis widths which were required inputs for the computation of gait parameters (by the optoelectronic and the wearable systems). Participants were subsequently equipped with the equipment described below, as illustrated in Figure 1 (all sensor configurations were worn at the same time), and asked to walk barefoot on a 10-m walkway. They walked at their self-selected speed for six to eight trials, in order to record a sufficient quantity of gait cycles per participant (minimum of 60 cycles). Participants with a high level of disability (GMFCS III) were allowed to perform the evaluation with their mechanical aid (e.g., walker).

\subsection{Reference Spatiotemporal Parameters of Gait Using Laboratory Setting}

\subsubsection{Equipment}

A set of 35 reflective markers ( $14 \mathrm{~mm}$ diameter) was placed on specific anatomical landmarks of the participant's head, trunk, pelvis, arms, thighs, shanks, and feet according to the full-body Plug-In-Gait model (Davis, 1991) (Figure 1). Leg lengths, pelvis, knee, and ankle widths previously measured were used as inputs in the Plug-In-Gait model. Marker trajectories were recorded by a twelve-camera optoelectronic system (Qualisys Motion capture systems, Göteborg, Sweden) set at a sampling frequency of $100 \mathrm{~Hz}$. Two force plates (AMTI Accugait, Watertown, NY, USA) embedded in the middle of the walkway recorded ground reaction forces at $1000 \mathrm{~Hz}$. 


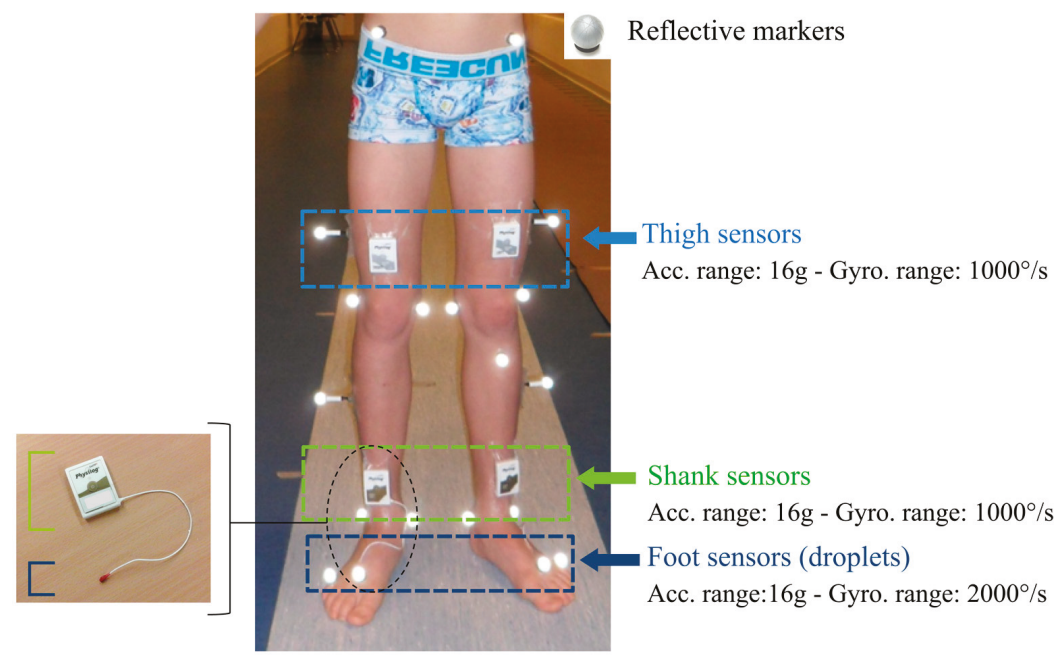

Figure 1. Equipment on the participant's lower limbs: reflective markers for the optoelectronic system and inertial Physilog sensors for the wearable configurations on the thighs, shanks, and feet. Feet sensors are embedded in a droplet (red PCB resin on the picture on the left), corresponding to deported $6 \mathrm{D}$ inertial units connected to the shank sensors.

\subsubsection{Spatiotemporal Parameters Computation}

As it is the clinical standard for in-laboratory gait analysis, the force plates and optoelectronic systems were set as reference to compare the three wearable configurations [31,32]. When an entire foot contact on the force plate was available, the gait events (FS and FO) were determined from the vertical ground reaction force based on a threshold sets to $20 \mathrm{~N}$. The subsequent occurrences of the same events were detected on the marker trajectories by the kinematic-based model [33]. All rated events were manually checked frame by frame on the sagittal view of the heel and toe markers in the MOKKA software [34]. The stride time was calculated as the time difference between two successive FS, the stride length was computed as the distance separating the heel markers at two successive FS times, and the stride velocity was calculated as the stride length divided by the stride time for each gait cycle [35].

\subsection{Spatiotemporal Parameters of Gait Using Wearable Setting}

\subsubsection{Equipment}

Six synchronized inertial sensors (Physilog $4^{\circledR}$, Gait Up, Renens, Switzerland) were used in this study. Physilog $4^{\circledR}$ is a standalone device (dimensions: $50 \mathrm{~mm} \times 37 \mathrm{~mm} \times 9.2 \mathrm{~mm}$, weight: $19 \mathrm{~g}$ ) comprising a triaxial accelerometer, triaxial gyroscope, triaxial magnetometer, and barometer with adjustable ranges, battery, a memory unit, and microcontroller. The magnetometer and barometer were disabled. The sampling frequency was set at $100 \mathrm{~Hz}$. Sensors were fixed on the participant's thighs, shanks, and feet bilaterally using a hypoallergenic adhesive film (Opsite Flexigrid, Smith \& Nephew Medical, Hull, UK), as shown in Figure 1. Sensors on the feet were embedded in a droplet (a small deported 6D sensor unit containing the 3D accelerometer and 3D gyroscope) in order to be appropriate for measuring activities in daily life settings: either barefoot or with shoes. Shank and thigh sensors were always oriented in the same way relating to the thigh and the shank, as shown in Figure 1. 
The optoelectronic and wearable systems were synchronized using an additional Physilog $4^{\circledR}$ receiving a trigger signal from one camera for every trial start and stop. The synchronization was performed in post-treatment according to the pulse train recorded by the additional sensor.

\subsubsection{Sensor Configuration and Associated Algorithm}

Three configurations were chosen, which all have the following in common: (1) algorithms described in research studies with high number of subject $(n>800)$ [36-38], (2) commercially available sensors, (3) easy to setup sensors and possibility of long-term measurements in daily-life, and (4) validation against laboratory system in adult populations (healthy or with disease). The three configurations use different sensor locations: first, the 'Shanks and Thighs' (ShTh) with four sensors fixed on both thighs and shanks; second, the 'Shanks' (Sh) with only one sensor on each shank; and finally, the 'Feet' with one sensor on each foot. The algorithms associated to the three above-mentioned configurations were used in this study: the double pendulum gait model in ShTh [39] and Sh, with a reduced number of sensors in Sh [40], and the direct integration method in Feet [41,42]. The ShTh and Sh methods have the same algorithm for gait event detection. Table 1 provides details about the three configurations regarding sensor location, algorithms, event detection, output gait parameters, and information about validation of the algorithms.

\subsection{Data Analysis}

Two gait events, FS and FO, as well as three STP, stride time, stride length, and stride velocity, were extracted from the three wearable configurations for each participant and each side, and compared cycle by cycle with the same outputs extracted from the reference (optoelectronic system). These parameters were selected as they are fundamental descriptors of gait and the computation of other STP parameters is based on them.

For TD participants, the parameters extracted from left and right sides were congregated since there was no difference between limbs. For children with CP-GMFCS I, the parameters were separated into paretic and non-paretic limbs according to their clinical profiles. Children with CP-GMFCS II and III were all bilaterally affected and, therefore, left and right sides were congregated and reported as paretic limbs.

The number of non-detected gait cycles, resulting from irregularities in the signal pattern (mostly due to pathological gait), was reported for each method. In cases where gait events were detected but no associated STP was computed, the cycle was not considered and reported as non-detected.

\subsection{Statistical Analysis}

We conducted descriptive analyses to evaluate the consistency of the three configurations with regard to the reference system. To that purpose, error (mean and standard deviation of the difference with the reference) was determined for each configuration. Regarding gait event detection, positive/negative values stand for late/early gait event detection, respectively, and over/underestimation of STP. The analyses were performed for the subgroups of TD, CP-GMFCS I (subdivided into paretic and non-paretic sides), and CP-GMFCS II and III in order to observe the influence of the impairment level on the error.

Spearman's correlation coefficients $(r)$ were used to evaluate the linear association between each configuration and the reference for the three STP in each subgroup. Furthermore, to quantify the agreements between each configuration and the reference system and visually represent the distribution of the errors, a graphical analysis through Bland-Altman plots was performed for each configuration for the three STP for each group. 


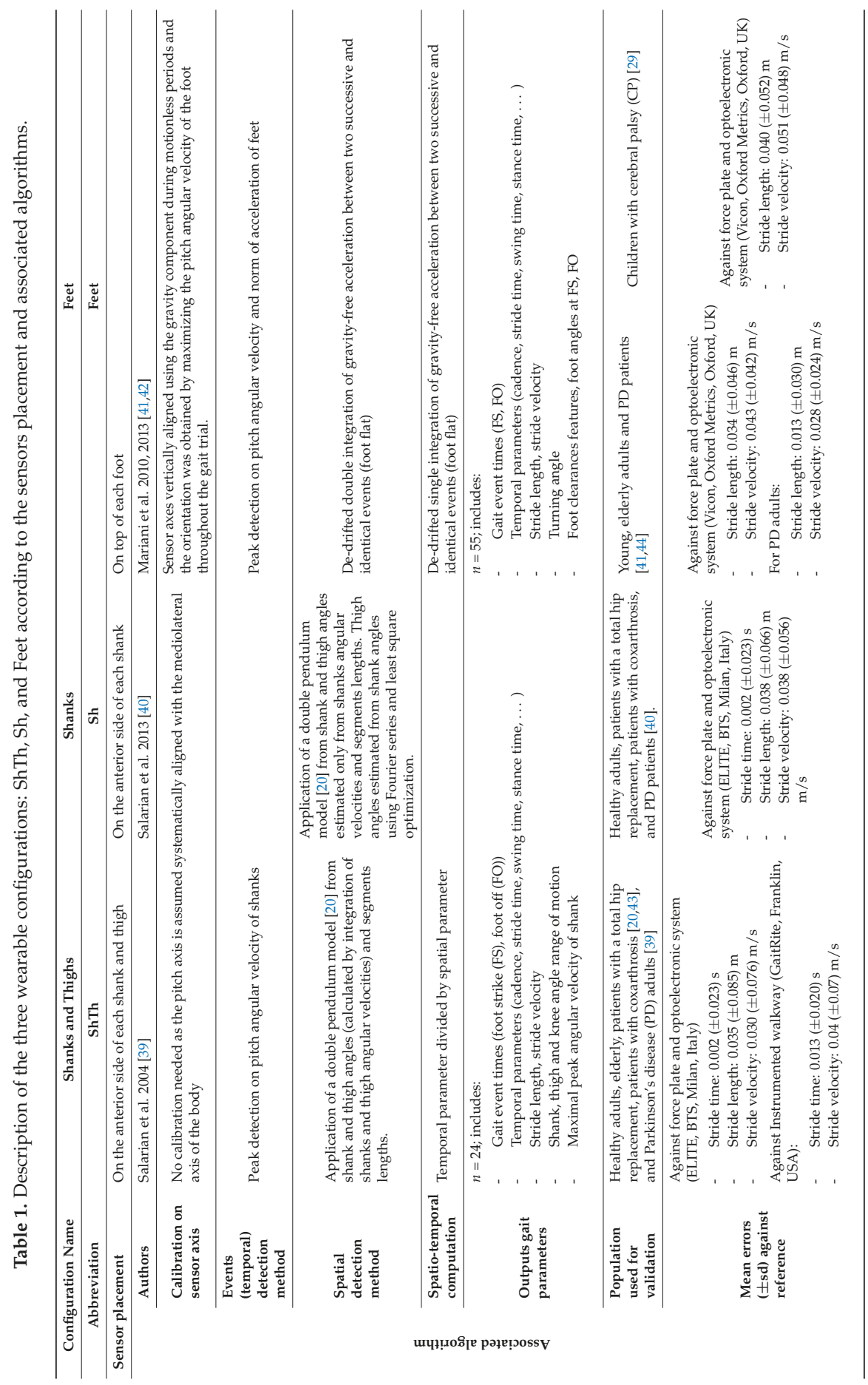




\section{Results}

Characteristics of the study population are summarized in Table 2. In total, 2395 cycles (TD: 998, CP-I: 747, CP-II-III: 650) were analyzed with ShTh and Sh, and 2099 cycles (TD: 934, CP-I: 681, CP-II-III: 484) with Feet. The corresponding cycles were selected for the reference system. No false positive gait events were detected. The number of non-detected cycles is shown in Table 3. Feet missed five cycles and ShTh/Sh missed three cycles for the TD group. Feet missed 197 cycles and ShTh/Sh missed 58 cycles for the CP group. Feet did not detect any events for one participant who walked exclusively on his toes.

Table 2. General characteristics of the study population.

\begin{tabular}{|c|c|c|c|}
\hline & TD & \multicolumn{2}{|c|}{$\mathrm{CP}$} \\
\hline \multirow{4}{*}{$\mathbf{N}$} & 11 & \multicolumn{2}{|c|}{15} \\
\hline & & GMFCS I & GMFCS II-III \\
\hline & & 7 & 8 \\
\hline & & $\begin{array}{l}\text { Unilateral } \\
5\end{array}$ & $\begin{array}{l}\text { Bilateral } \\
10\end{array}$ \\
\hline $\begin{array}{l}\text { Sex } \\
\text { Number of girls in the group (\%) }\end{array}$ & $5(45)$ & \multicolumn{2}{|c|}{$8(55)$} \\
\hline $\begin{array}{l}\text { Age in years } \\
\text { mean } \pm \text { std }\end{array}$ & $13.5 \pm 2.9$ & \multicolumn{2}{|c|}{$12.8 \pm 3.1$} \\
\hline
\end{tabular}

Table 3 summarizes the errors in estimating FS and FO times from the three configurations against the reference. Figure 2 illustrates an example for each group of FS and FO detection with ShTh/Sh and Feet configurations as compared to the reference. The three configurations showed similar errors for FS detection within the TD and the non-paretic groups (errors $<0.042 \pm 0.030 \mathrm{~s}$ ). Feet showed a higher error in detecting FS for the paretic groups $(0.051 \pm 0.053 \mathrm{~s}$ for GMFCS I and $0.077 \pm 0.299 \mathrm{~s}$ for GMFCS II-III) compared to ShTh/Sh $(0.037 \pm 0.051 \mathrm{~s}$ for GMFCS I and $0.053 \pm 0.048 \mathrm{~s}$ for GMFCS II-III). The error of FO detection was lower compared to FS detection, for the three configurations and the whole population ( $\mid$ errors $\mid<0.029 \pm 0.058 \mathrm{~s}$ for FO and $\mid$ errors $\mid<0.077 \pm 0.299 \mathrm{~s}$ for FS).

Table 3 also reports the errors for STP computations (stride time, stride length, and stride velocity). The error of all configurations was inferior to $0.003 \pm 0.072 \mathrm{~s}$ for stride time estimation within each group, except for the CP-GMFCS II-III group with Feet (0.012 $\pm 0.129 \mathrm{~s})$. Feet showed lower errors compared to the two other configurations for stride length estimation, for all the groups except the CP-GMFCS I paretic group where the errors were equivalent between ShTh and Feet $(0.019 \pm 0.066 \mathrm{~m}$ and $0.018 \pm 0.069 \mathrm{~m})$. The highest errors for stride length estimation were found with Sh in each group. For stride velocity, ShTh showed lower errors for the CP groups (e.g., $0.024 \pm 0.118 \mathrm{~m} / \mathrm{s}$ (ShTh) against $-0.127 \pm 0.133 \mathrm{~m} / \mathrm{s}$ (Sh) and $0.073 \pm 0.123 \mathrm{~m} / \mathrm{s}$ (Feet) in the GMFCS II-III group), whereas lower errors were found with Feet for the TD group $(0.030 \pm 0.045 \mathrm{~m} / \mathrm{s}$ (Feet) against $0.073 \pm 0.067 \mathrm{~m} / \mathrm{s}$ (ShTh) and $0.133 \pm 0.091 \mathrm{~m} / \mathrm{s}(\mathrm{Sh})$ ).

Figure 3 presents the cycle-by-cycle comparison between each configuration and the reference for the three STP and the different groups. All correlations were significant $(p<0.05)$. Correlation coefficients were high to very high $(r>0.7)$ according to previous recommendations [45] for the three configurations except for stride length estimation in the CP-GMFCS II-III group with ShTh and Sh where the correlation coefficients were moderate ( $r=0.545$ and $r=0.576$ respectively).

The agreement assessed by Bland-Altman plots is shown in Figure 4. Higher levels of agreement were found for ShTh/Sh compared to Feet for stride time detection in CP-GMFCS I (0.095 s against $0.053 \mathrm{~s}$ ) and CP-GMFCS II-III (0.252 s against $0.142 \mathrm{~s}$ ). However, a higher agreement was reported with Feet for stride length in all groups as compared to the two other configurations. For the stride velocity, a better agreement was found with Feet for the TD and the CP-GMFCS I groups $(0.088 \mathrm{~m}$ and $0.121 \mathrm{~m} / \mathrm{s})$ as compared to ShTh $(0.134 \mathrm{~m} 0.0 .133 \mathrm{~m} / \mathrm{s})$ and Sh $(0.179 \mathrm{~m}$ and $0.207 \mathrm{~m} / \mathrm{s})$; however, ShTh showed a better agreement for CP-GMFCS II-III $(0.232 \mathrm{~m} / \mathrm{s})$ as compared to Feet $(0.242 \mathrm{~m} / \mathrm{s})$ and Sh $(0.260 \mathrm{~m} / \mathrm{s})$. 


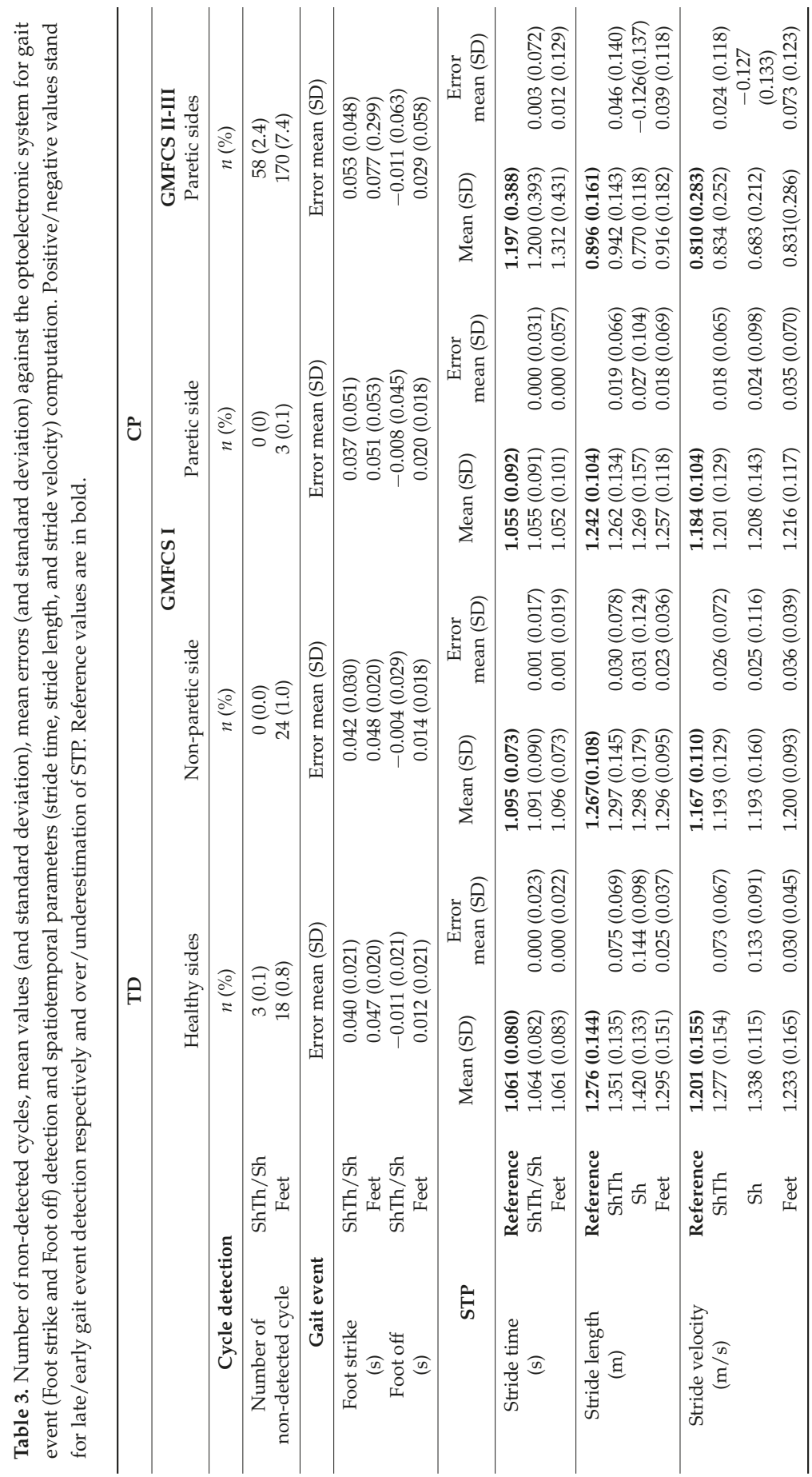



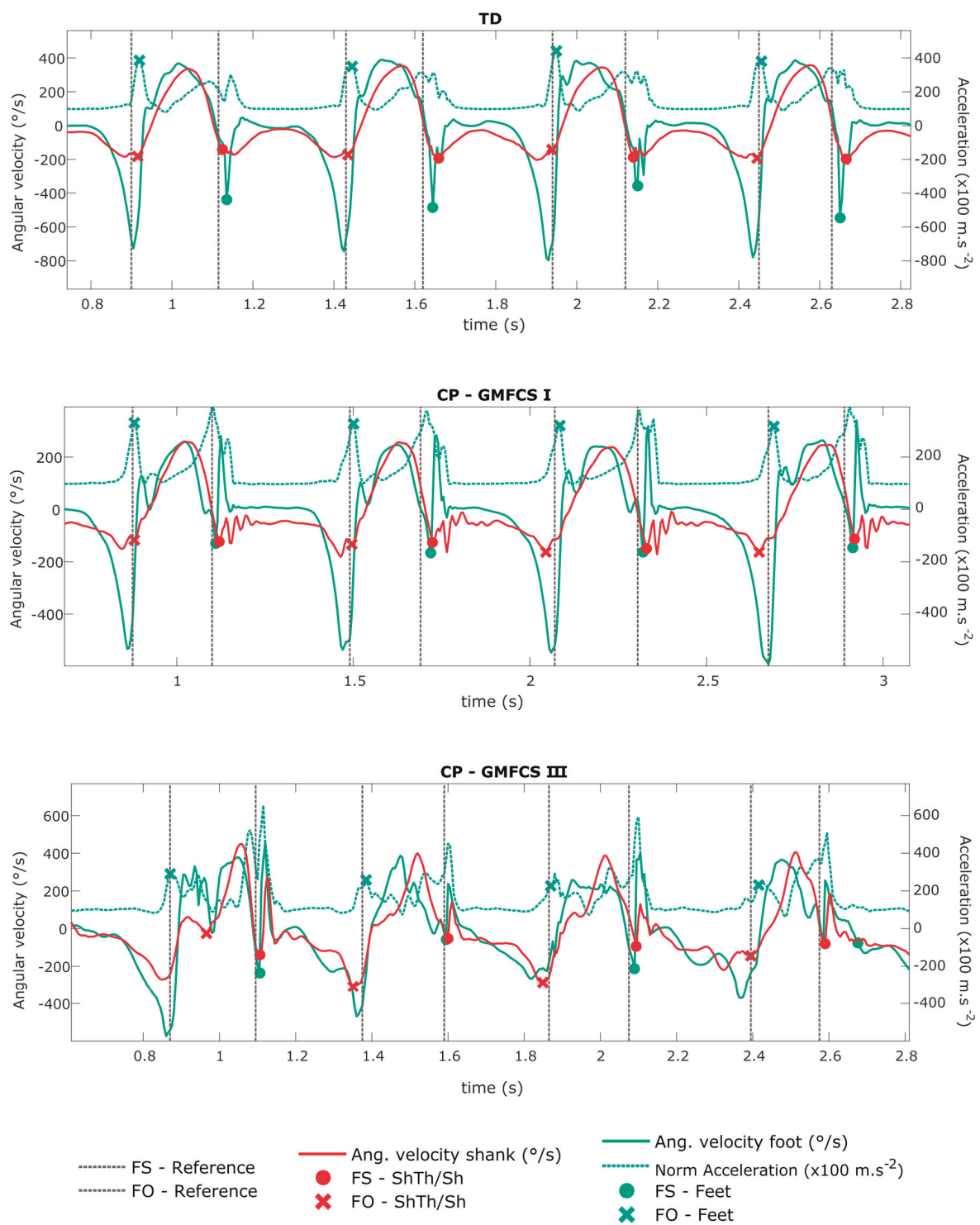

Figure 2. Shank angular velocity and foot angular velocity and norm of acceleration signals (measured by the wearable configurations ShTh/Sh and Feet). The gait events (FS and FO) detected by the wearable configurations are shown by the symbols on the corresponding signals. The vertical lines define FS and FO detected by the reference. Four strides are represented for each example, corresponding to a typically developing (TD) child, a child with CP-GMFCS I, and a child with CP-GMFCS III. 
Stride time (s)

ShTh
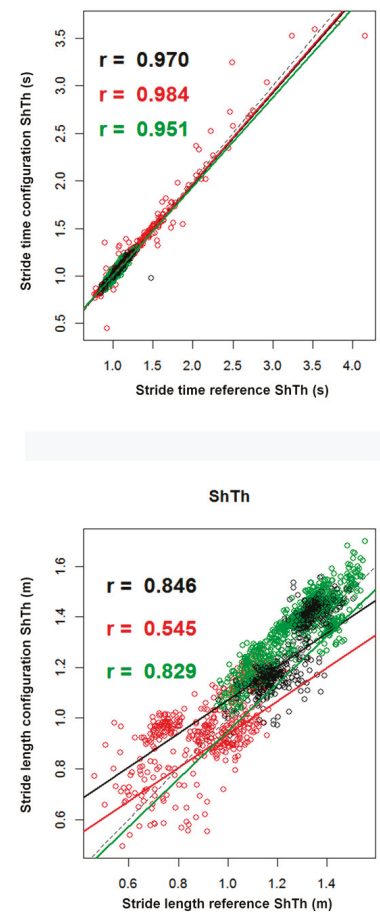

ShTh

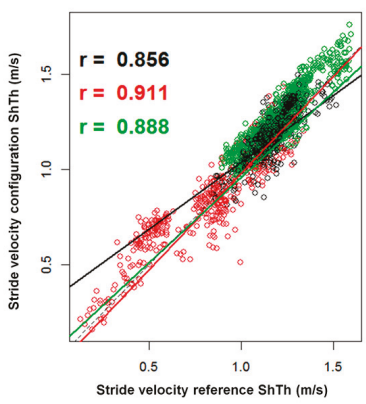

Sh

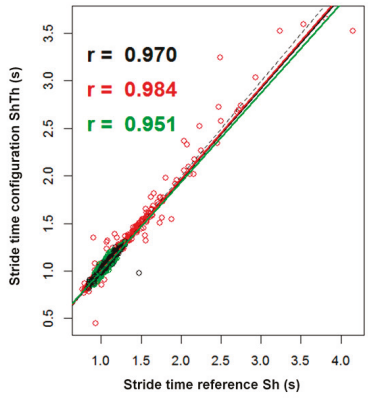

Stride length $(\mathrm{m})$

Sh

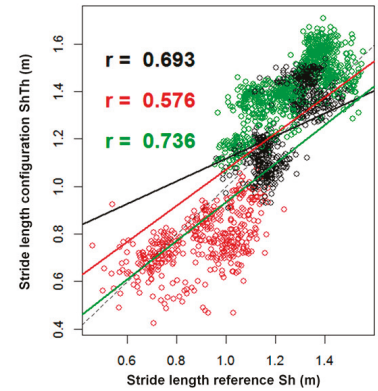

Stride velocity $(\mathrm{m} / \mathrm{s})$

Sh

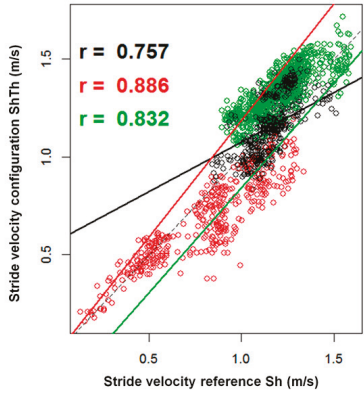

CP GMFCS

CP-GMFCS $\|-\mid$
Feet
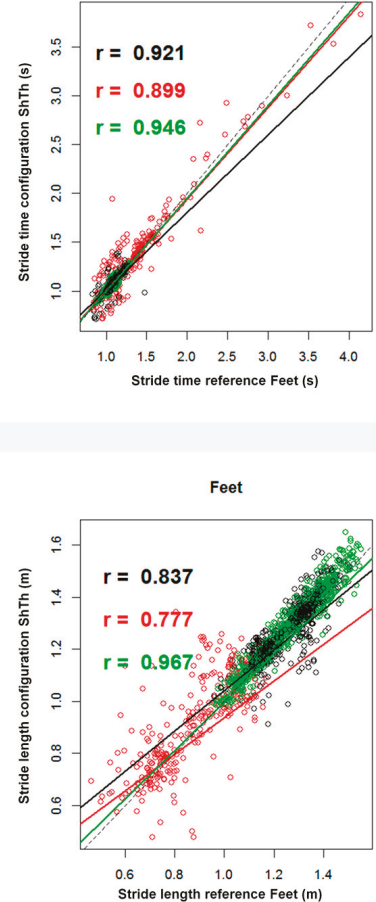

Feet

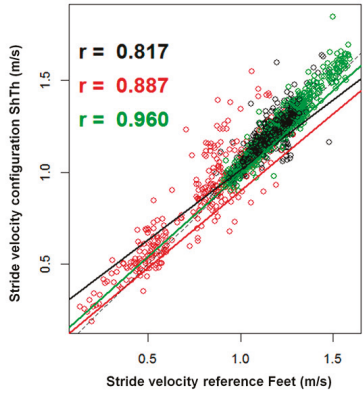

Figure 3. Correlations between the wearable configurations (ShTh, Sh, and Feet) and the optoelectronic system for the three spatiotemporal parameters (stride time, stride length, and stride velocity). Spearman's correlation coefficients are reported. Dashed lines represent $y=x$. Green: TD; Black: CP-GMFCS I level; and Red: CP-GMFCS II-III levels. 

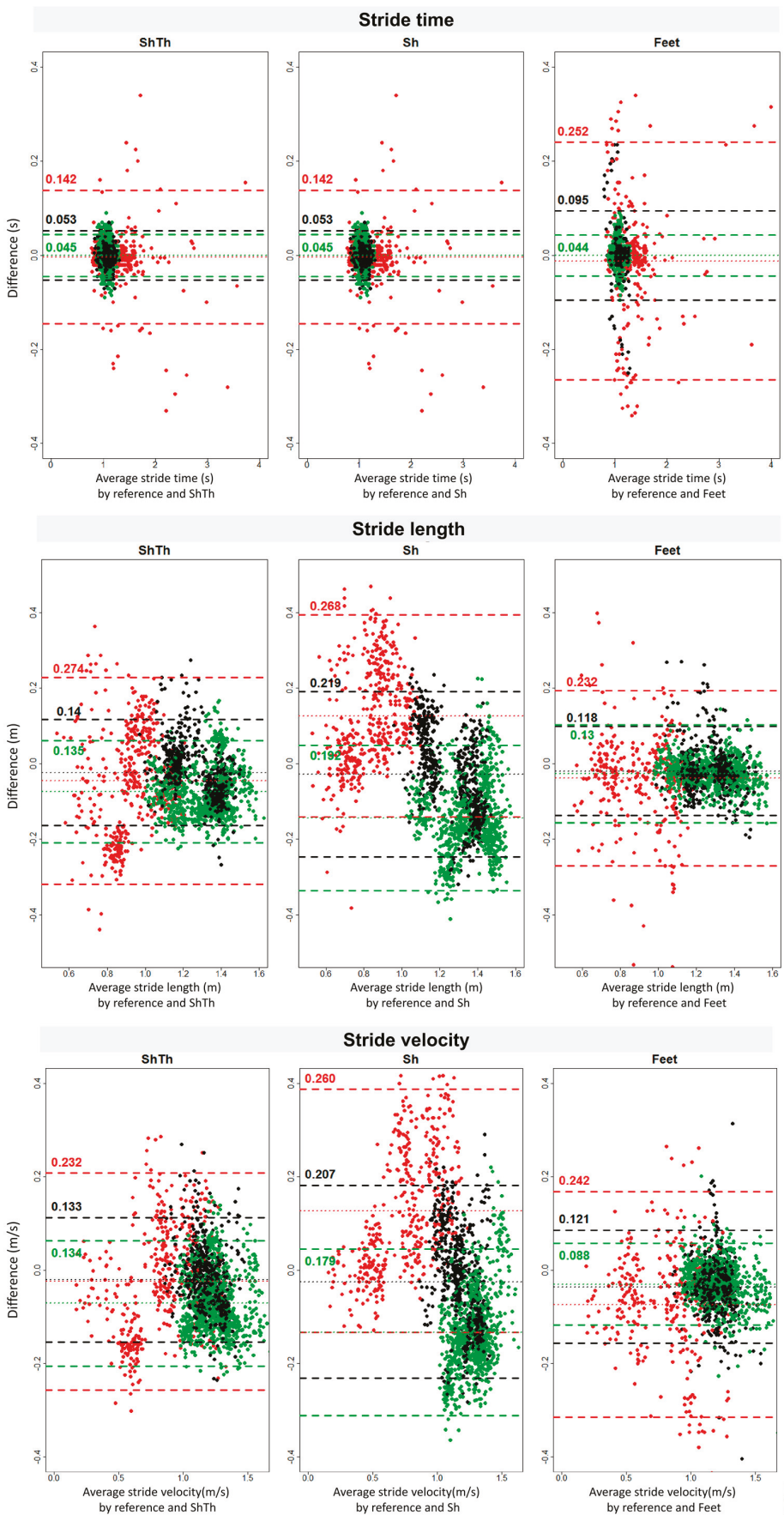

Figure 4. Bland-Altman plots of the three wearable configurations (ShTh, Sh, and Feet) against the optoelectronic system for the three spatiotemporal parameters (stride time, stride length, and stride velocity). Mean error and level of agreement ( \pm 1.96 standard deviation) are represented by horizontal lines. Green: TD; Black: CP-GMFCS I level; and Red: CP-GMFCS II-III levels. 


\section{Discussion}

In this study, we aimed to compare three wearable configurations defined by different sensor positioning and associated algorithms in order to choose the most appropriate one for gait performance evaluation in children with CP. The main findings were that the configuration using sensors only on the shanks was not appropriate for the spatial and spatio-temporal estimation for the whole study population, and ShTh and Feet provided advantages depending on the estimated parameter and the level of severity of the child. Indeed, temporal parameters were better estimated by the ShTh configuration in the pathological groups, whereas spatial parameters were better estimated by the Feet configuration. For velocity estimation, the Feet configuration was better in TD children and children with a mild gait impairment (GMFCS I), but the ShTh configuration was better in children with more impaired gait (GMFCS II and III).

\subsection{Temporal Detection}

The key starting point of gait analysis is the segmentation of gait episodes into gait cycles using specific temporal events: FS and FO. For the whole study population, the three configurations were comparable regarding the detection of these events, with ShTh and Sh being more robust than Feet to detect events in challenging gait patterns (less non-detected cycles and better FS detection in the CP group compared to Feet).

When discussing the temporal results, one has to consider that the resolution frame of both systems was $0.01 \mathrm{~s}$ (sample frequency: $100 \mathrm{~Hz}$ ), that the reference system error can reach $0.02 \mathrm{~s}$ [33], and that the systems' synchronization precision was up to $0.01 \mathrm{~s}$ due to the delay between the trigger signal recorded by the additional Physilog and the actual start of the cameras. Considering these statements, FO detection with wearable systems was very close to FO detection of the reference ( 1 to 3 frames difference). For FS detection, the difference between the systems was larger (superior to 3 frames) especially for Feet for the most severely affected patients (up to 7 frames difference), indicating that ShTh/Sh was more accurate to segment gait trials and therefore appropriate to estimate the stride time for these patients. The errors of FS time related to the ShTh method in TD children was five times higher than those of the original method found by Salarian et al. including Parkinson's disease and healthy adults [39]. This could be due to the difference in reference event detection or sampling frequency of the inertial sensors twice higher than in the present study.

The difficulty to detect events with inertial sensors is directly linked to, first, the pattern of the signal and, second, its smoothness. As illustrated in Figure 2, in abnormal gait patterns, expected features of the signal (such as specific thresholds, time windows, or peaks) can be missing, and non-expected features (such as extra-peaks) can appear which decrease the algorithm performance for event detection. In CP, these abnormal patterns are found especially at the distal parts of the limbs (at feet level) since movements of the distal limbs are more affected by the pathology than the proximal segments [46]. This explains why ShTh seems preferable for children with a high level of disability for gait events detection.

\subsection{Spatial Detection}

The Sh configuration was not suitable for children. Sh errors were between 3.5 and 3.8 times higher in our study compared to previously published results for healthy adults [40]. Indeed, with Sh, we found a systematic error for TD (11\% of mean stride length) and CP-GMFCS II-III groups (14\% of mean stride length). The Sh method uses a thigh predictor to estimate the thigh angles for stride length computation which has been trained on adults data (healthy and Parkinson's disease patients). Even if the method aimed to suit any target population [40], it proved unfit for the children population, even the TD children. Furthermore, as the thigh angle is predicted from the shank sensor, misalignment of the sensor axis with the mediolateral axis of the shank has a greater impact on Sh results compared to the results where both thigh and shank angles are estimated from the thigh- and shank-fixed sensors. 
A calibration procedure that aligns the sensor frame with the anatomical frame of the shank would probably increase the performance of the system [40]. Such a calibration was not performed in this study as it was not straightforward for the heterogeneous and highly affected children we evaluated. The focus of this paper was the evaluation of errors before any calibration.

Previous studies found that STP computation with inertial sensors in healthy populations was improved when the sensors were closer to the ground [47,48] (i.e., closer to the measure). The results of this study partially confirmed this since Feet was found more accurate in average to estimate stride length compared to ShTh. On the other hand, Feet configuration was not able to analyze one toe-walking participant. Indeed, the Feet method is based on the assumption that the participant achieves 'foot-flat' contact at mid-stance to set the initial orientation of the sensor relative to the fixed frame [41]. This condition was not fulfilled for all children with CP included in this study, especially in the GMFCS II-III group, which caused the decreased performance of the algorithm. Overall, Feet configuration might be more accurate for STP parameters but is limited for highly abnormal gait patterns, which are more accurately estimated with sensors on shanks and thighs.

Finally, both ShTh and Feet configurations reported errors lower than the in-laboratory intra-subject variability of gait in children [49], so they can both be considered as valid and suitable for gait assessments.

\subsection{Choice for Daily Life Assessment}

The main perspective of this study is to determine the optimal configuration of sensors for the assessment of gait in the daily-life of children with CP. While measurement errors were the main criteria of comparison between the three systems, other aspects require careful consideration when choosing the best set-up for daily-life application.

On one hand, it is useful to keep the number of sensor units as low as possible to increase comfort for the patient, decrease the set-up time, and thus, make the system acceptable for long measurements [50]. Feet sensors were embedded in a droplet fixed directly on the skin to be independent of shoe wearing in order to allow the monitoring of all gait episodes within a day, including various environments. However, this system sometimes provided discomfort when tested outside the laboratory, because the shoe pressuring on the sensor droplet caused pain. Even if the shank and thigh sensors were larger, they did not hinder the children's gait and were more convenient. However, the positioning of sensors on the shanks might need to be adapted for the children while wearing orthoses. On the other hand, more sensors can detect a higher variety of gait parameters and activities which confer a substantial advantage for daily life assessment [51,52]. ShTh configuration allows the recognition of the sitting, lying, and standing posture whereas Feet configuration cannot distinguish sitting from standing posture. Computation of complementary parameters, valuable for the assessment of children with $\mathrm{CP}$, must also be considered. For example, foot clearance and foot/floor angles can be computed only with the Feet configuration, and knee angle only with the ShTh method.

Combining these practical aspects with the accuracy of the measurements, we selected the configuration using shank and thigh sensors for further testing of its usability for long-term assessments in laboratory-free conditions.

\subsection{Limitations and Perspectives}

The results of this study were obtained in a controlled environment which is not representative of daily-life conditions. Consequently, in daily-life settings, environmental disruptions, incorrect set-up, unintentional switch-off, or sensor fall might downgrade the results. Our laboratory setting reference, even though considered a 'gold standard', can reach $0.02 \mathrm{~s}$ in timing error [33] and a mean accuracy of $5.3 \mathrm{~mm}$ for marker trajectory estimates [9]. The errors reported can, therefore, be a combination of errors of both systems. Furthermore, only configurations using sensors on the lower limbs have been tested in this study. Other configurations including those using sensors on the wrists, the chest, or the pelvis exist [21,53] but were not tested as we wished to analyze the paretic and non-paretic limbs separately. Finally, we evaluated a limited number of subjects and, since CP gait patterns are very heterogeneous, 
a larger number of participants, especially in the $\mathrm{CP}$ group with a high level of disability (GMFCS II-III), would permit to strengthen the conclusions. Besides this, an increased number of participants would allow subgroup analysis within the GMFCS II-III group according to what algorithms could then be refined to be more versatile and flexible enough to fulfill the requirements of every individual clinical picture.

\section{Conclusions}

We compared three configurations of wearable sensors able to compute STP in children with $\mathrm{CP}$ and TD children on the base of the comparison with an optoelectronic system in the laboratory. The results showed that the configuration using sensors on both feet was more accurate for typical and regular gait patterns (i.e., for of TD children), while sensors located on the shanks and thighs performed better for moderate to severely impaired gait patterns (CP with GMFCS levels II and III). Overall, the results of this study indicate that inertial sensors proved promising for an objective evaluation of gait in daily-life. Such evaluations have the potential to shed light on patients' daily difficulties.

Acknowledgments: This work was supported by the Leenaards Foundation 2015 award for translational research. We thank Rebekka Anker and Stéphane Lovejoy from GaitUp Company for their technical support, Benedikt Fasel and Christopher Moufawad El Achkar for their assistance in data processing, Anne Tabard-Fougère for her assistance in data analysis and insightful discussions, and Alice Bonnefoy-Mazure for proofreading. The authors wish to give special thanks to the participants and their families without whom this study would not have been possible.

Author Contributions: Stéphane Armand, Anisoara Paraschiv-Ionescu, and Christopher J. Newman conceived the study and found the financial support. Lena Carcreff, Corinna N. Gerber, Stéphane Armand, Anisoara Paraschiv-Ionescu, and Christopher J. Newman designed the experiments. Lena Carcreff, Geraldo De Coulon, Corinna N. Gerber, and Stéphane Armand recruited the participants. Lena Carcreff and Corinna N. Gerber performed the experiments. Kamiar Aminian provided the algorithms. Lena Carcreff processed and analyzed the data and wrote the paper. Lena Carcreff, Corinna N. Gerber, Stéphane Armand, Anisoara Paraschiv-Ionescu, Geraldo De Coulon, Christopher J. Newman, and Kamiar Aminian discussed the results. Stéphane Armand and Kamiar Aminian substantially revised the work. All authors have approved the submitted version of the paper.

Conflicts of Interest: Christopher J. Newman and Kamiar Aminian are shareholders of GaitUp SA.

\section{References}

1. Sellier, E.; Platt, M.J.; Andersen, G.L.; Krägeloh-Mann, I.; De La Cruz, J.; Cans, C.; Cans, C.; Van Bakel, M.; Arnaud, C.; Delobel, M.; et al. Decreasing prevalence in cerebral palsy: A multi-site European population-based study, 1980 to 2003. Dev. Med. Child Neurol. 2016, 58, 85-92. [CrossRef] [PubMed]

2. Armand, S.; De Coulon, G.; Bonnefoy-mazure, A. Gait analysis in children with cerebral palsy. EFORT Open Rev. 2016, 1, 448-460. [CrossRef] [PubMed]

3. Carcreff, L.; Bonnefoy-mazure, A.; De Coulon, G.; Armand, S. Analyse quantifiée de la marche. Mov. Sport Sci. 2016, 3, 7-21. [CrossRef]

4. Sutherland, D.H. The evolution of clinical gait analysis Part II Kinematics. Gait Posture 2002, 16, 159-179. [CrossRef]

5. Desailly, E.; Daniel, Y.; Sardain, P.; Lacouture, P. Foot contact event detection using kinematic data in cerebral palsy children and normal adults gait. Gait Posture 2009, 29, 76-80. [CrossRef] [PubMed]

6. Ghoussayni, S.; Stevens, C.; Durham, S.; Ewins, D. Assessment and validation of a simple automated method for the detection of gait events and intervals. Gait Posture 2004, 20, 266-272. [CrossRef] [PubMed]

7. Bruening, D.A.; Ridge, S.T. Automated event detection algorithms in pathological gait. Gait Posture 2014, 39, 472-477. [CrossRef] [PubMed]

8. Di Marco, R.; Rossi, S.; Castelli, E.; Patanè, F.; Mazzà, C.; Cappa, P. Effects of the calibration procedure on the metrological performances of stereophotogrammetric systems for human movement analysis. Meas. J. Int. Meas. Confed. 2017, 101, 265-271. [CrossRef]

9. Chiari, L.; Della Croce, U.; Leardini, A.; Cappozzo, A. Human movement analysis using stereophotogrammetry. Part 2: Instrumental errors. Gait Posture 2005, 21, 197-211. [CrossRef] [PubMed] 
10. Di Marco, R.; Rossi, S.; Patanè, F.; Cappa, P. Technical quality assessment of an optoelectronic system for movement analysis. J. Phys. Conf. Ser. 2015, 588. [CrossRef]

11. World Health Organization. Towards a Common Language for Functioning, Disability and Health: ICF The International Classification of Functioning, Disability and Health; World Health Organization: Geneva, Switzerland, 2002; Volume 1149.

12. Holsbeeke, L.; Ketelaar, M.; Schoemaker, M.M.; Gorter, J.W. Capacity, Capability, and Performance: Different Constructs or Three of a Kind? Arch. Phys. Med. Rehabil. 2009, 849-855. [CrossRef] [PubMed]

13. Van Eck, M.; Dallmeijer, A.J.; Voorman, J.M.; Becher, J.G. Longitudinal study of motor performance and its relation to motor capacity in children with cerebral palsy. Dev. Med. Child Neurol. 2009, 51, 303-310. [CrossRef] [PubMed]

14. Smits, D.-W.; Gorter, J.W.; Van Schie, P.E.; Dallmeijer, A.J.; Ketelaar, M. How Do Changes in Motor Capacity, Motor Capability, and Motor Performance Relate in Children and Adolescents With Cerebral Palsy? Arch. Phys. Med. Rehabil. 2014, 95, 1577-1584. [CrossRef] [PubMed]

15. Keawutan, P.; Bell, K.; Davies, P.S.W.; Boyd, R.N. Systematic review of the relationship between habitual physical activity and motor capacity in children with cerebral palsy. Res. Dev. Disabil. 2014, 35, 1301-1309. [CrossRef] [PubMed]

16. Olivier, I.; Baker, C.; Cordier, J.; Thomann, G.; Nougier, V. Cognitive and motor aspects of a coincidence-timing task in Cerebral Palsy children. Neurosci. Lett. 2015, 602, 33-37. [CrossRef] [PubMed]

17. Yang, S.; Li, Q. Inertial sensor-based methods in walking speed estimation: A systematic review. Sensors 2012, 12, 6102-6116. [CrossRef] [PubMed]

18. Boutaayamou, M.; Denoël, V.; Brüls, O.; Demonceau, M.; Maquet, D.; Forthomme, B.; Croisier, J.-L.; Schwartz, C.; Verly, J.; Garraux, G. Algorithm for Temporal Gait Analysis Using Wireless Foot-Mounted Accelerometers; Springer: Cham, Switzerland, 2017; ISBN 978-3-319-54716-9.

19. Khandelwal, S.; Wickström, N. Novel methodology for estimating Initial Contact events from accelerometers positioned at different body locations. Gait Posture 2017. [CrossRef] [PubMed]

20. Aminian, K.; Najafi, B.; Büla, C.; Leyvraz, P.F.; Robert, P. Spatio-temporal parameters of gait measured by an ambulatory system using miniature gyroscopes. J. Biomech. 2002, 35, 689-699. [CrossRef]

21. Zijlstra, W.; Hof, A.L. Assessment of spatio-temporal gait parameters from trunk accelerations during human walking. Gait Posture 2003, 18, 1-10. [CrossRef]

22. Esser, P.; Dawes, H.; Collett, J.; Feltham, M.G.; Howells, K. Assessment of spatio-temporal gait parameters using inertial measurement units in neurological populations. Gait Posture 2011, 34, 558-560. [CrossRef] [PubMed]

23. Trojaniello, D.; Cereatti, A.; Della Croce, U. Accuracy, sensitivity and robustness of five different methods for the estimation of gait temporal parameters using a single inertial sensor mounted on the lower trunk. Gait Posture 2014, 40, 487-492. [CrossRef] [PubMed]

24. Mansour, K.B.; Rezzoug, N.; Gorce, P. Analysis of several methods and inertial sensors locations to assess gait parameters in able-bodied subjects. Gait Posture 2015, 42, 409-414. [CrossRef] [PubMed]

25. Lanovaz, J.L.; Oates, A.R.; Treen, T.T.; Unger, J.; Musselman, K.E. Validation of a commercial inertial sensor system for spatiotemporal gait measurements in children. Gait Posture 2017, 51, 14-19. [CrossRef] [PubMed]

26. Van Den Noort, J.C.; Ferrari, A.; Cutti, A.G.; Becher, J.G.; Harlaar, J. Gait analysis in children with cerebral palsy via inertial and magnetic sensors. Med. Biol. Eng. Comput. 2013, 51, 377-386. [CrossRef] [PubMed]

27. Cutti, A.G.; Ferrari, A.; Garofalo, P.; Raggi, M.; Cappello, A.; Ferrari, A. “Outwalk”: A protocol for clinical gait analysis based on inertial and magnetic sensors. Med. Biol. Eng. Comput. 2010, 48, 17-25. [CrossRef] [PubMed]

28. Laudanski, A.; Yang, S.; Li, Q. A concurrent comparison of inertia sensor-based walking speed estimation methods. In Proceedings of the Annual International Conference of the IEEE Engineering in Medicine and Biology Society, EMBC, Boston, MA, USA, 30 August-3 September 2011; pp. 3484-3487. [CrossRef]

29. Brégou Bourgeois, A.; Mariani, B.; Aminian, K.; Zambelli, P.Y.; Newman, C.J.; Bre, A. Spatio-temporal gait analysis in children with cerebral palsy using, foot-worn inertial sensors. Gait Posture 2014, 39, 436-442. [CrossRef] [PubMed]

30. Palisano, R.; Rosenbaum, P.; Walter, S.; Russell, D.; Wood, E.; Galuppi, B. Development and reliability of a system to classify gross motor function in children with cerebral palsy. Dev. Med. Child Neurol. 1997, 39, 214-223. [CrossRef] [PubMed] 
31. Veilleux, L.-N.; Raison, M.; Rauch, F.; Robert, M.; Ballaz, L. Agreement of spatio-temporal gait parameters between a vertical ground reaction force decomposition algorithm and a motion capture system. Gait Posture 2015, 43, 257-264. [CrossRef] [PubMed]

32. Caldas, R.; Mundt, M.; Potthast, W.; Buarque, F.; Neto, D.L. A systematic review of gait analysis methods based on inertial sensors and adaptive algorithms. Gait Posture 2017, 57, 204-210. [CrossRef] [PubMed]

33. Stanhope, S.J.; Kepple, T.M.; McGuire, D.A.; Roman, N.L. Kinematic-based technique for event time determination during gait. Med. Biol. Eng. Comput. 1990, 28, 355-360. [CrossRef] [PubMed]

34. Barre, A.; Armand, S. Biomechanical ToolKit: Open-source framework to visualize and process biomechanical data. Comput. Methods Programs Biomed. 2014, 114, 80-87. [CrossRef] [PubMed]

35. Hollman, J.H.; McDade, E.M.; Pertersen, R.C. Normative Spatiotemporal Gait Parameters in Older Adults. Gait Posture 2011, 34, 111-118. [CrossRef] [PubMed]

36. Dadashi, F.; Mariani, B.; Rochat, S.; Büla, C.J.; Santos-Eggimann, B.; Aminian, K. Gait and foot clearance parameters obtained using shoe-worn inertial sensors in a large-population sample of older adults. Sensors 2014, 14, 443-457. [CrossRef] [PubMed]

37. Rochat, S.; Büla, C.J.; Martin, E.; Seematter-Bagnoud, L.; Karmaniola, A.; Aminian, K.; Piot-Ziegler, C.; Santos-Eggimann, B. What is the Relationship between Fear of Falling and Gait in Well-Functioning Older Persons Aged 65 to 70 Years? Arch. Phys. Med. Rehabil. 2010, 91, 879-884. [CrossRef] [PubMed]

38. Seematter-Bagnoud, L.; Santos-Eggimann, B.; Rochat, S.; Martin, E.; Karmaniola, A.; Aminian, K.; Piot-Ziegler, C.; Büla, C.J. Vulnerability in high-functioning persons aged 65 to 70 years: The importance of the fear factor. Aging Clin. Exp. Res. 2011, 22, 485-486. [CrossRef]

39. Salarian, A.; Russmann, H.; Vingerhoets, F.J.G.; Dehollain, C.; Blanc, Y.; Burkhard, P.R.; Aminian, K. Gait assessment in Parkinson's disease: Toward an ambulatory system for long-term monitoring. IEEE Trans. Biomed. Eng. 2004, 51, 1434-1443. [CrossRef] [PubMed]

40. Salarian, A.; Burkhard, P.R.; Vingerhoets, F.J.G.; Jolles, B.M.; Aminian, K. A novel approach to reducing number of sensing units for wearable gait analysis systems. IEEE Trans. Biomed. Eng. 2013, 60, 72-77. [CrossRef] [PubMed]

41. Mariani, B.; Hoskovec, C.; Rochat, S.; Büla, C.; Penders, J.; Aminian, K. 3D gait assessment in young and elderly subjects using foot-worn inertial sensors. J. Biomech. 2010, 43, 2999-3006. [CrossRef] [PubMed]

42. Mariani, B.; Rouhani, H.; Crevoisier, X.; Aminian, K. Quantitative estimation of foot-flat and stance phase of gait using foot-worn inertial sensors. Gait Posture 2013, 37, 229-234. [CrossRef] [PubMed]

43. Najafi, B.; Helbostad, J.L.; Moe-Nilssen, R.; Zijlstra, W.; Aminian, K. Does walking strategy in older people change as a function of walking distance? Gait Posture 2009, 29, 261-266. [CrossRef] [PubMed]

44. Mariani, B.; Jiménez, M.C.; Vingerhoets, F.J.G.; Aminian, K. On-shoe wearable sensors for gait and turning assessment of patients with parkinson's disease. IEEE Trans. Biomed. Eng. 2013, 60, 155-158. [CrossRef] [PubMed]

45. Mukaka, M.M. Statistics corner: A guide to appropriate use of correlation coefficient in medical research. Malawi Med. J. 2012, 24, 69-71. [CrossRef] [PubMed]

46. Berker, A.N.; Yalçin, M.S. Cerebral Palsy: Orthopedic Aspects and Rehabilitation. Pediatr. Clin. N. Am. 2008, 55, 1209-1225. [CrossRef] [PubMed]

47. Iosa, M.; Picerno, P.; Paolucci, S.; Morone, G. Wearable inertial sensors for human movement analysis. Expert Rev. Med. Devices 2016, 13, 641-659. [CrossRef] [PubMed]

48. Washabaugh, E.P.; Kalyanaraman, T.; Adamczyk, P.G.; Claflin, E.S.; Krishnan, C. Validity and repeatability of inertial measurement units for measuring gait parameters. Gait Posture 2017, 55, 87-93. [CrossRef] [PubMed]

49. Steinwender, G.; Saraph, V.; Scheiber, S.; Zwick, E.B.; Uitz, C.; Hackl, K. Intrasubject repeatability of gait analysis data in normal and spastic children. Clin. Biomech. 2000, 15, 134-139. [CrossRef]

50. Hegde, N.; Bries, M.; Sazonov, E. A Comparative Review of Footwear-Based Wearable Systems. Electronics 2016, 5, 48. [CrossRef]

51. Paraschiv-Ionescu, A.; Perruchoud, C.; Buchser, E.; Aminian, K. Barcoding human physical activity to assess chronic pain conditions. PLoS ONE 2012. [CrossRef] [PubMed] 
52. Attal, F.; Mohammed, S.; Dedabrishvili, M.; Chamroukhi, F.; Oukhellou, L.; Amirat, Y. Physical Human Activity Recognition Using Wearable Sensors. Sensors 2015, 15, 31314-31338. [CrossRef] [PubMed]

53. Fasel, B.; Duc, C.; Dadashi, F.; Bardyn, F.; Savary, M.; Farine, P.A.; Aminian, K. A wrist sensor and algorithm to determine instantaneous walking cadence and speed in daily life walking. Med. Biol. Eng. Comput. 2017, 55, 1773-1785. [CrossRef] [PubMed]

(C) 2018 by the authors. Licensee MDPI, Basel, Switzerland. This article is an open access article distributed under the terms and conditions of the Creative Commons Attribution (CC BY) license (http:/ / creativecommons.org/licenses/by/4.0/). 
Article

\title{
Estimation of Foot Plantar Center of Pressure Trajectories with Low-Cost Instrumented Insoles Using an Individual-Specific Nonlinear Model
}

\author{
Xinyao $\mathrm{Hu}^{1}$, Jun Zhao ${ }^{1}$, Dongsheng Peng ${ }^{1}$, Zhenglong Sun ${ }^{2}$ and Xingda Qu ${ }^{1, *}$ \\ 1 Institute of Human Factors and Ergonomics, Shenzhen University, Shenzhen 518060, China; \\ huxinyao@szu.edu.cn (X.H.); zhaojun2016@email.szu.edu.cn (J.Z.); 2013110350@email.szu.edu.cn (D.P.) \\ 2 Institute of Robotics and Intelligent Manufacturing, the Chinese University of Hong Kong, \\ Shenzhen 518172, China; sunzhenglong@cuhk.edu.cn \\ * Correspondence: quxd@szu.edu.cn; Tel.: +86-755-86965716
}

Received: 29 November 2017; Accepted: 30 January 2018; Published: 1 February 2018

\begin{abstract}
Postural control is a complex skill based on the interaction of dynamic sensorimotor processes, and can be challenging for people with deficits in sensory functions. The foot plantar center of pressure (COP) has often been used for quantitative assessment of postural control. Previously, the foot plantar COP was mainly measured by force plates or complicated and expensive insole-based measurement systems. Although some low-cost instrumented insoles have been developed, their ability to accurately estimate the foot plantar COP trajectory was not robust. In this study, a novel individual-specific nonlinear model was proposed to estimate the foot plantar COP trajectories with an instrumented insole based on low-cost force sensitive resistors (FSRs). The model coefficients were determined by a least square error approximation algorithm. Model validation was carried out by comparing the estimated COP data with the reference data in a variety of postural control assessment tasks. We also compared our data with the COP trajectories estimated by the previously well accepted weighted mean approach. Comparing with the reference measurements, the average root mean square errors of the COP trajectories of both feet were $2.23 \mathrm{~mm}( \pm 0.64)$ (left foot) and $2.72 \mathrm{~mm}( \pm 0.83)$ (right foot) along the medial-lateral direction, and $9.17 \mathrm{~mm}( \pm 1.98)$ (left foot) and $11.19 \mathrm{~mm}( \pm 2.98)$ (right foot) along the anterior-posterior direction. The results are superior to those reported in previous relevant studies, and demonstrate that our proposed approach can be used for accurate foot plantar COP trajectory estimation. This study could provide an inexpensive solution to fall risk assessment in home settings or community healthcare center for the elderly. It has the potential to help prevent future falls in the elderly.
\end{abstract}

Keywords: postural control; falls in the elderly; fall risk assessment; low-cost instrumented insoles; foot plantar center of pressure

\section{Introduction}

Postural control refers to the control and maintenance of body's center of mass (COM) within the base of support during static or dynamic activities [1]. It has drawn much attention from research community in recent decades. The main functional goals of postural control include postural orientation and postural equilibrium, both of which require the integration of sensory information from visual, vestibular and somatosensory systems to stabilize the body and coordinate the movement strategies [2]. Therefore, postural control is a complex skill based on the interaction of dynamic sensorimotor processes [3], and can be challenging for people with deficits in sensory functions. For example, elderly who suffered age-related degeneration in sensory systems $[4,5]$ and people with pathological conditions (such as cerebral palsy [6], stroke [7] and Parkinson's disease [8,9]) were found to be associated with impaired postural control. 
The foot plantar center of pressure (COP) has often been used for quantitative assessment of postural control. For example, Rocchi et al. examined 14 COP measures by using the principle component analysis and found that five of these measures, including root mean square distance, mean velocity, principal sway direction, centroidal frequency of the power spectrum, and frequency dispersion, can effectively reflect the postural control mechanism among patients with Parkinson's disease [9]. Liu et al. used the velocity of COP trajectory during quiet upright standing to quantify the intensity of postural sway among young adults, healthy old adults and fall prone old adults [10]. Lafond et al. suggested that the velocity of COP trajectory was the most reliable measure for assessing postural stiffness among the elderly [11]. Biswas et al. have shown that the characteristics of COP can enhance the predictive power to a constructed index for dynamic stability [12]. In other studies, COP measures were also used to assess postural stability among stroke patients [13], patients with post-stroke hemiparesis [14] and patients with rheumatic disease [15]. In a recent study, Johansson et al. investigated how the COP sway can be used to predict future falls. They found that fall risk increased by $75 \%$ for participants with the COP sway lengths $\geq 400 \mathrm{~mm}$ during quiet standing with eyes open. They also suggested that fall risk could almost be doubled if the sway lengths $\geq 920 \mathrm{~mm}$ during quiet standing with eyes closed [16].

Conventionally, the COP trajectory is measured by force plates or force mapping systems [17]. However, such systems are restricted in laboratory settings. As such, they cannot be used to assess postural control in daily activities. To address this, a variety of insole-based plantar pressure measurement systems have been developed. Some of them are commercially available, such as the F-scan measurement system (Tekscan, Inc., Boston, MA, USA) and Novel Pedar system (Novel Inc., Kirkland, WA, USA). These systems allow the COP trajectory to be captured in a more extended space compared to force plates and force mapping systems. However, they have to rely on cables for data acquisition and power supply, which makes them obstructive and compromise their wearability. More importantly, these systems are too expensive for personal or daily use in home settings.

Providing inexpensive and wearable solutions for postural control assessment will provide vital information of fall risks, and thus could be useful for preventing future falls especially among elderly [18]. Recent advancement in microelectronics technology makes such wearable solutions feasible. For instance, Balaga et al. proposed a method that used a single body-fixed inertial sensor to quantitatively describe the postural control strategy during the lying-to-sit-to-stand-to-walk transfer tasks [19]. Similarly, wearable sensors (including those integrated in smart phones) were reported to be useful for fall risk assessment [20-23]. Many low-cost instrumented insole systems have also been developed [24-33]. Among the existing low-cost insole systems, different sensor technologies were implemented, such as force sensitive resistors (FSRs) [25,33], fabric or textile pressure sensing arrays [28], or piezoelectric sensors [32], etc. The numbers of pressure sensors or sensing arrays also varied among different studies, ranging from four FSRs [31] to 48 pressure sensor arrays [32].

Regardless of the sensor types and the number of sensors, the "weighted-mean approach" was most commonly used to estimate COP trajectories. In the weighted-mean approach, the force/pressure detected by each sensor was weighted by its corresponding coordinate (i.e., sensor location) and then summed. The COP trajectory was calculated by dividing the sum of weighted force/pressure by the overall force/pressure [26-30,32,33]. The accuracy of the weighted-mean COP trajectory estimation approach is not robust. In particular, when implementing the weighted-mean approach, the accuracy of COP trajectory estimation is dependent on the areas covered by the pressure sensors, which will be a potential source for errors. In other words, the weighted-mean approach depends on the locations and numbers of the pressure sensors. Theoretically, more sensors or sensor arrays implemented in the insole will result in higher accuracy. However, more sensors or sensor arrays always means high cost and system complexity, which makes it practically infeasible today.

In the present study, we aimed to develop and evaluate low-cost instrumented insoles for the estimation of foot plantar COP trajectories. In order to address the limitations of the weighted-mean approach, we proposed a novel individual-specific nonlinear model. This model was expected to 
accurately estimate foot plantar COP trajectories using the data from a small number of low-cost FSR sensors. An experiment involving a variety of postural control assessment tasks was carried out to provide data for least square error approximation and model coefficients specification. The accuracy of the foot plantar COP trajectories estimation by this model was examined by comparing the estimated COP data with the reference data from a commercial insole-based plantar pressure measurement system. Accuracy comparisons were also carried out between the proposed nonlinear model and the weighted-mean approach.

\section{Materials and Methods}

\subsection{Hardware Design}

The block diagram of the proposed instrumented insole is shown in Figure 1. It mainly consists of three parts: a 12 integrated FSR (FSR402, Interlink Electronics, Los Angeles, CA, USA) based insole, a lower-shank mounter block, and a PC end graphic user interface (GUI). Each FSR402 sensor has a $12.7 \mathrm{~mm}$ diameter sensing area made of fiberglass resin that is attached to a base $18.1 \mathrm{~mm}$ in diameter. The lower-shank mounter block consisted of a Micro-Computer Unit (MCU, STM32 32-bit ARM Cortex, ARM, Ltd., Cambridge, UK), a Bluetooth module (HC-06, Wavesen Co. Ltd. Guangzhou, China), a customized A2D module and a battery. Twelve FSRs were strategically adhered onto a silicone made insole, the size of which was US 9. The layout of the FSRs is depicted in Figure 2. This layout is similar to what has been suggested by Howell et al. [34], where the 12 sensors can cover the important foot plantar pressure distribution areas such as great toe, metatarsophalangeal joint, arch of the foot, and heel. An insole coordinate system was used to help identify the actual location of each sensor. As illustrated in Figure 2, the $x$-axis is the tangent line to the bottom edge of the insole, which defines the medial-lateral direction (ML); and the $y$-axis is the tangent line to the left edge of the insole, which defines the anterior-posterior (AP) direction. The origin is the intersection between the $x$-axis and the $y$-axis.

Prior to usage, each FSR was calibrated and conditioned following the techniques suggested by Hall et al. [35]. This was to eliminate the creep effect (i.e., the change of FSR resistance over prolonged time) and minimize the hysteresis effect. After that, each FSR sensor was connected to a Voltage-to-Current (V2C) converting circuit, as recommended by the manufacturer [36]. The thickness of the connecting cable is only $0.4 \mathrm{~mm}$ so that it will not lead to uneven surface of the insole. The V2C converting circuit converts the FSR resistance value to an inverse voltage output, which were subsequently converted into readable voltage output through a 10-bit analog-2-digital (A2D) module. The force measured by each sensor was obtained based on its voltage output following a fourth order polynomial equation, as suggested by Hall et al. [35]. The data were transmitted wirelessly by a Bluetooth module to a PC end, where the GUI was designed by a customized MATLAB script to visualize the pressure output in real time. 


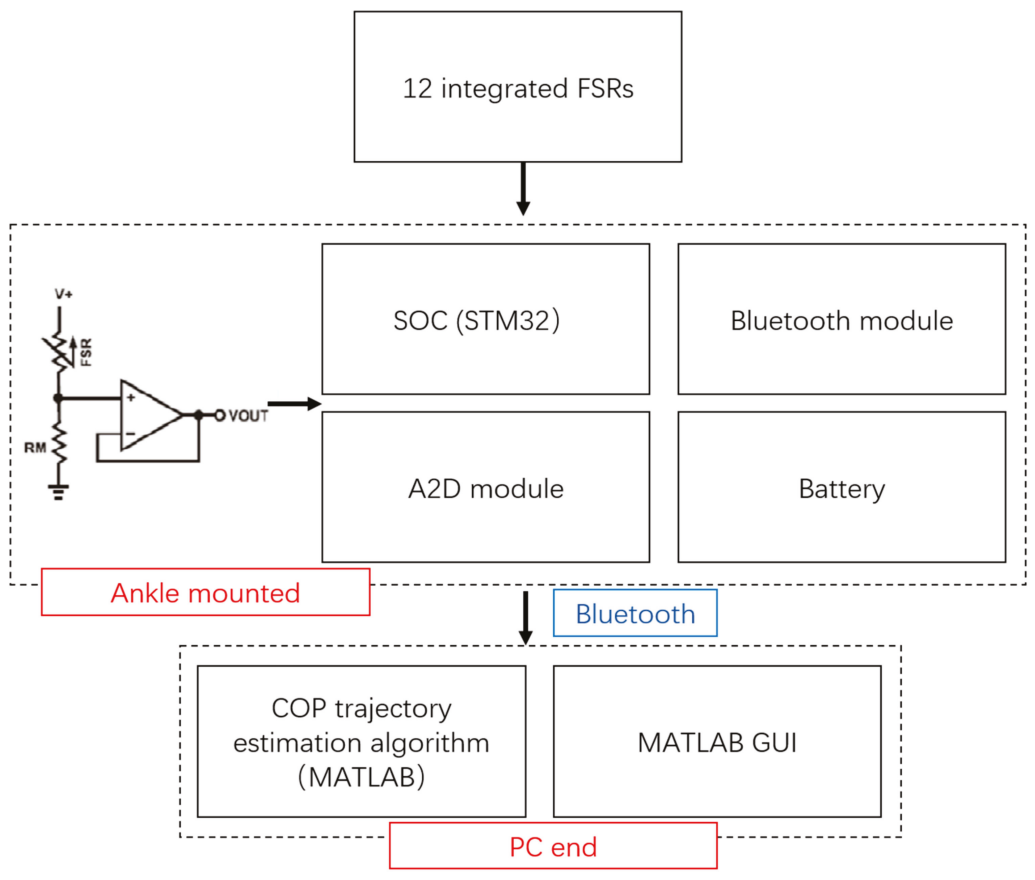

Figure 1. The block diagram of the instrumented insole.

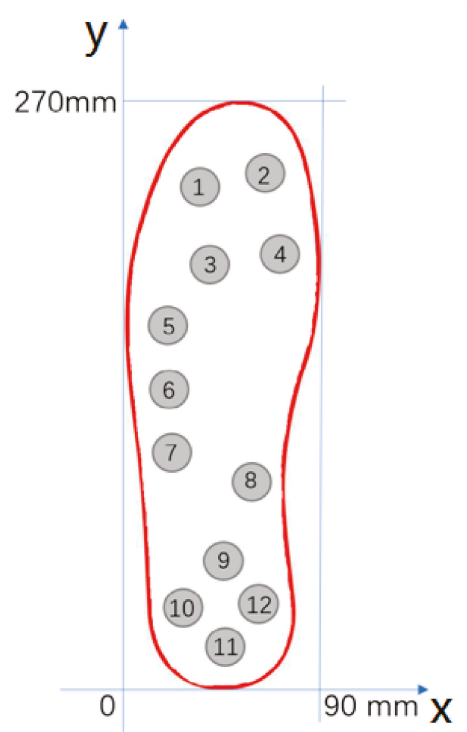

\begin{tabular}{|c|c|c|}
\hline FSR No. & $x-\operatorname{coor}$ & y-coor \\
\hline 1 & 30 & 236 \\
\hline 2 & 60 & 239 \\
\hline 3 & 37 & 194 \\
\hline 4 & 62 & 197 \\
\hline 5 & 10 & 176 \\
\hline 6 & 9 & 148 \\
\hline 7 & 9 & 122 \\
\hline 8 & 49 & 109 \\
\hline 9 & 42 & 69 \\
\hline 10 & 14 & 40 \\
\hline 11 & 37 & 31 \\
\hline 12 & 52 & 45 \\
\hline
\end{tabular}

Figure 2. The layout of the 12 Force Sensitive Resistor (FSR) sensors and the corresponding coordinates. 


\subsection{The Experiment}

The experiment was carried out for model development and validation. A convenience sample of 10 young (age $=22.6 \pm 1.5$ years, height $=173.8 \pm 4.4 \mathrm{~cm}$, weight $=64.9 \pm 4.4 \mathrm{~kg}$ ) and 10 older adults (age $=65.7 \pm 3.4$ years, height $=167.5 \pm 3.9 \mathrm{~cm}$, weight $=65.2 \pm 2.9 \mathrm{~kg}$ ) were recruited from the local community. These participants all had the shoe size of US 9. The young participants were between 21 and 24 years old. The elderly participants were all above 60 years old and living independently, and could perform the activities of daily living (ADLs) without external assistance. Both young and elderly participants reported no injuries, illness, or medical surgery history. All participants signed the informed consent form approved by the Shenzhen University ethics committee.

Previous research has demonstrated that the F-scan system can measure the COP trajectory accurately [37]. Therefore, the foot COP trajectories obtained from the F-scan system were considered as the reference measurement (i.e., the response data). Prior to the experiment, the F-scan sensor sheets were tailored to make sure it had the same shape and size as the instrumented insoles. They were then pasted underneath the instrumented insoles (Figure 3a). Cautious actions were taken before data collection to make sure that the instrumented insoles and F-scan sensor sheet were adhered evenly and firmly. The insoles were inserted into a pair of sports shoes (size US 9), as depicted in Figure 3b.

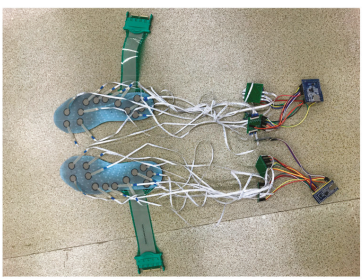

(a)

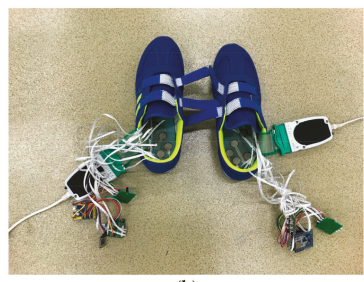

(b)

Figure 3. (a) the F-scan sensor sheets (green) were tailored and adhered underneath the instrumented insole (blue); (b) the insoles were inserted into a pair of sports shoes.

Participants were asked to wear the shoes with the instrumented insoles. Both the lower-shank mounted block of the instrumented insoles and the signal box of the F-scan system were attached to the lower shank (Figure 4). Then, participants were instructed to perform a variety of postural control assessment tasks including (1) quiet standing with open eyes, (2) quiet standing with closed eyes, (3) standing up from a chair with armrests (wooden chair, seat height $435 \mathrm{~mm}$, armrests height $250 \mathrm{~mm}$ ), (4) sitting down to a chair with armrests, (5) standing up from a chair without armrests (wooden chair, seat height $435 \mathrm{~mm}$ ), and (6) sitting down to a chair without armrests. During quiet standing trials, participants were asked to stand quietly with a self-chosen comfortable posture for $10 \mathrm{~s}$. During standing up and sitting down trials, participants were asked to keep the feet on the ground when they started standing up or sitting down. When performing standing up and sitting down tasks with the chair with armrests, participants were instructed to use the armrests to help the body ascending and descending. These tasks were performed in a random order. Three trials were collected for each task. Each of these trials was separated to form three blocks of trials, which would be used for cross validation purposes. Prior to data collection, participants were given approximately 5 min practice to get familiar with these tasks. The activation of the F-Scan system and our proposed instrumented insoles is initiated with the application of external pressure. Thus, these two insole systems were synchronized by manually applying external pressure onto them at the same time. The sampling frequency was set at $50 \mathrm{~Hz}$. 


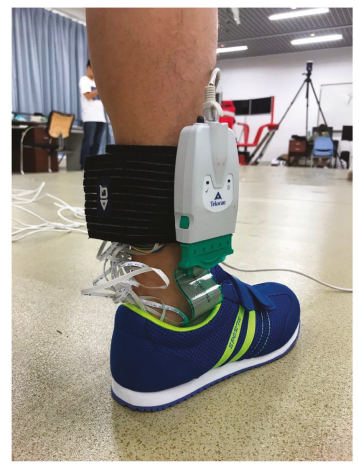

Figure 4. Both the lower-shank mounted block of the instrumented insole and the signal box of the F-scan system were attached to the lower-shank.

We noted that the instrumented insole was placed in between the foot and F-scan sensor sheet. The overlapping between the instrumented insole and F-scan sensor sheet might modify the foot plantar pressure distribution and induce errors in F-scan measurement. Such errors might compromise the accuracy of the reference data. A test was carried out to examine the possible errors caused by the overlap. One male volunteer (age: 31, height: $180 \mathrm{~cm}$, weight: $65 \mathrm{~kg}$, shoe size: US 9) participated. Firstly, the participant was asked to wear the experimental shoes that had both the proposed instrumented insole and F-scan sensor sheet (overlapping condition), and perform the above-mentioned postural control assessment tasks. Each task was performed 20 times in a random order. Then, the participant wore the same shoes with F-scan sensor sheet only (no overlapping condition) and performed each postural control assessment task 20 times in a random order. Two $\mathrm{COP}$ parameters including COP range and mean COP were compared between the 'overlapping' and 'no overlapping' conditions by using $t$-tests. As shown in Table 1, when the level of significance was set at 0.05 , no significant differences existed in the COP parameters between the 'overlapping' and 'no overlapping' conditions. This suggests that the possible errors due to the overlapping did not significantly affect the COP estimation results. Thus, we used the F-scan measurement data in the overlapping condition as the reference data in the following analysis.

Table 1. Comparisons of COP parameters between the 'overlapping' and 'no overlapping' conditions.

\begin{tabular}{|c|c|c|c|c|c|c|c|}
\hline & & \multicolumn{3}{|c|}{ Medial-Lateral (ML) COP } & \multicolumn{3}{|c|}{ Anterior-Posterior (AP) COP } \\
\hline & & Overlapping & No Overlapping & $p$-Value & Overlapping & No Overlapping & $p$-Value \\
\hline \multirow[t]{2}{*}{ Task 1} & COP range $(\mathrm{mm})$ & $2.5 \pm 0.3$ & $3.5 \pm 0.2$ & 0.83 & $6.9 \pm 2.5$ & $5.4 \pm 2.1$ & 0.54 \\
\hline & Mean COP (mm) & $42.4 \pm 1.4$ & $46.3 \pm 3.2$ & 0.19 & $189.8 \pm 2.6$ & $192.3 \pm 2.3$ & 0.73 \\
\hline \multirow[t]{2}{*}{ Task 2} & COP range $(\mathrm{mm})$ & $8.7 \pm 1.2$ & $7.7 \pm 1.3$ & 0.55 & $16.4 \pm 3.0$ & $13.0 \pm 3.5$ & 0.55 \\
\hline & Mean COP (mm) & $45.2 \pm 7.2$ & $47.9 \pm 6.4$ & 0.18 & $193.9 \pm 1.6$ & $196.7 \pm 2.4$ & 0.18 \\
\hline \multirow[t]{2}{*}{ Task 3} & COP range $(\mathrm{mm})$ & $15.4 \pm 1.7$ & $12.5 \pm 1.9$ & 0.89 & $67.0 \pm 18.3$ & $68.6 \pm 22.1$ & 0.81 \\
\hline & Mean COP (mm) & $45.7 \pm 0.7$ & $50.1 \pm 1.5$ & 0.30 & $187.3 \pm 18.8$ & $186.9 \pm 14.3$ & 0.91 \\
\hline \multirow[t]{2}{*}{ Task 4} & COP range $(\mathrm{mm})$ & $16.3 \pm 1.9$ & $14.5 \pm 2.2$ & 0.74 & $70.4 \pm 15.3$ & $74.6 \pm 19.2$ & 0.66 \\
\hline & Mean COP (mm) & $48.4 \pm 3.5$ & $49.0 \pm 4.7$ & 0.25 & $186.9 \pm 13.8$ & $183.7 \pm 15.5$ & 0.96 \\
\hline \multirow[t]{2}{*}{ Task 5} & COP range $(\mathrm{mm})$ & $13.4 \pm 3.3$ & $14.9 \pm 4.5$ & 0.12 & $73.2 \pm 11.5$ & $70.0 \pm 14.7$ & 0.81 \\
\hline & Mean COP (mm) & $45.1 \pm 5.7$ & $48.7 \pm 3.1$ & 0.06 & $208.7 \pm 29.2$ & $200.7 \pm 22.1$ & 0.78 \\
\hline \multirow[t]{2}{*}{ Task 6} & COP range $(\mathrm{mm})$ & $12.8 \pm 1.5$ & $15.1 \pm 2.0$ & 0.83 & $70.6 \pm 10.9$ & $74.3 \pm 11.8$ & 0.86 \\
\hline & Mean COP (mm) & $47.5 \pm 0.8$ & $43.9 \pm 0.9$ & 0.13 & $204.8 \pm 9.5$ & $213.2 \pm 9.8$ & 0.62 \\
\hline
\end{tabular}

\subsection{The Individual-Specific Nonlinear Model for COP Estimation}

The foot plantar COP is practically defined as the centroid of all the external forces acting on the plantar surface of the foot [38]. Therefore, in the previous weighted-mean approach, the COP 
trajectories were estimated by a spatial average of all the forces measured by pressure sensors. However, with a reduced number of pressure sensors, the external forces acting on the foot plantar might not be registered completely. Thus, errors might be induced.

To address the limitation of the weighted-mean approach, a multivariate nonlinear regression model was proposed where the FSR sensor outputs were considered as the predictor data. Similar to the weighted-mean approach, in the proposed model, the output of FSR sensor was weighted and summed to determine COP locations (Equations (1) and (2)). However, instead of using the sensor locations, the model coefficients (i.e., weighting parameters) were determined by a least square error approximation process. The sensor locations (i.e., the coordination of each sensor defined in Figure 2) were only used to set the initial values of the model coefficients. The least square error approximation process would tune the model coefficients to achieve improved COP trajectory estimation capability. This model is as follows:

$$
\begin{aligned}
& X_{\mathrm{COP}}=\frac{\sum_{i}^{12} C_{i}^{x} F_{i}}{C_{t o t}^{x} \boldsymbol{F}_{\text {tot }}}, \\
& \boldsymbol{Y}_{\mathrm{COP}}=\frac{\sum_{i}^{12} C_{i}^{y} F_{i}}{C_{t o t}^{y} \boldsymbol{F}_{\text {tot }}},
\end{aligned}
$$

where $\boldsymbol{X}_{\mathrm{COP}}$ and $\boldsymbol{Y}_{\mathrm{COP}}$ are the COP coordinates defined in the coordinate system (Section 2.1). $\boldsymbol{X}_{\mathrm{COP}}$ represents the COP location along the medial-lateral direction, $Y_{\mathrm{COP}}$ represents the COP location along the anterior-posterior direction. $C_{i}^{x}$, and $C_{i}^{y}(i=1,2,3 \ldots 12)$ are the model coefficients that weight each sensor output $\left(F_{i}\right) . C_{t o t}^{x}$ and $C_{t o t}^{y}$ are the model coefficients that weights the sum of all forces registered by FSRs. $\boldsymbol{F}_{\text {tot }}$ is the sum of all forces registered by FSRs $\left(\boldsymbol{F}_{t o t}=\sum_{i}^{12} \boldsymbol{F}_{i}\right) . \boldsymbol{C}_{i}^{x}, \boldsymbol{C}_{i}^{y}, \boldsymbol{C}_{\text {tot }}^{x}$ and $C_{\text {tot }}^{y}$ are determined through the least square error approximation process.

An iterative least square error approach was carried out to determine the model coefficients. The model coefficients were written in the vector form as:

$$
\begin{aligned}
& \widehat{C^{x}}=\left(C_{i}^{x} ; C_{t o t}^{x}\right), \\
& \widehat{C^{y}}=\left(C_{i}^{y} ; C_{t o t}^{y}\right),
\end{aligned}
$$

The reference measurement (i.e., the foot COP trajectories obtained from the F-scan system) in the vector form was as follows:

$$
\begin{aligned}
& X_{\mathrm{COP}}=\left(X_{\mathrm{COP}, 1} ; X_{\mathrm{COP}, 2} ; \ldots X_{\mathrm{COP}, m}\right), \\
& Y_{\mathrm{COP}}=\left(Y_{\mathrm{COP}, 1} ; Y_{\mathrm{COP}, 2} ; \ldots Y_{\mathrm{COP}, m}\right),
\end{aligned}
$$

where $m$ is the number of observations in the training data (which related to the time taken in each postural control assessment task). Similarly, the predictor data $(\boldsymbol{F})$ can be written as a $m \times i$ matrix ( $i$ is the number of FSR sensors) as follows:

$$
\boldsymbol{F}=\left(\begin{array}{ccccc}
\boldsymbol{F}_{11} & \boldsymbol{F}_{12} & \cdots & \boldsymbol{F}_{1 i} & \sum_{i}^{12} F_{1 i} \\
\boldsymbol{F}_{21} & \boldsymbol{F}_{22} & \cdots & \boldsymbol{F}_{2 i} & \sum_{i}^{12} F_{2 i} \\
\vdots & \vdots & \ddots & \vdots & \vdots \\
\boldsymbol{F}_{m 1} & \boldsymbol{F}_{m 2} & \cdots & \boldsymbol{F}_{m i} & \sum_{i}^{12} F_{m i}
\end{array}\right)=\left(\begin{array}{cc}
\boldsymbol{F}_{1}^{T} & \sum_{i}^{12} \boldsymbol{F}_{1} \\
\boldsymbol{F}_{2}^{T} & \sum_{i}^{12} \boldsymbol{F}_{2} \\
\vdots & \vdots \\
\boldsymbol{F}_{m}^{T} & \sum_{i}^{12} \boldsymbol{F}_{m}
\end{array}\right) .
$$


The goal of the approximation process is to find $\widehat{C^{x}}$ and $\widehat{C^{y}}$ that can minimize the square error between the reference measurements and the model predicted values. Let $E_{\widehat{C}^{x}}=$

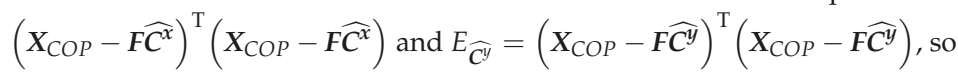

$$
\begin{aligned}
& {\widehat{C^{x}}}^{*}=\underset{\widehat{C^{x}}}{\arg \min } E_{\widehat{C}^{x}} \\
& {\widehat{C^{y}}}^{*}=\underset{\widehat{C^{y}}}{\arg \min } E_{\widehat{C}^{y}} \text {. }
\end{aligned}
$$

In multivariable calculus, to find the minimum value of $E_{\widehat{C^{x}}}$ and $E_{\widehat{C^{y}}}$, it requires solving the following partial derivative functions [39]. This process went iteratively until the closed form optimal solution for $\widehat{C^{x}}$ and $\widehat{C^{y}}$ were found:

$$
\begin{aligned}
& \frac{\partial E_{\widehat{C^{x}}}}{\partial{\widehat{C^{x}}}^{x}}=2 \boldsymbol{F}^{T}\left(\boldsymbol{F} \widehat{C^{x}}-X_{C O P}\right)=0, \\
& \frac{\partial E_{\widehat{C^{y}}}}{\partial \widehat{C^{y}}}=2 \boldsymbol{F}^{T}\left(\boldsymbol{F} \widehat{C^{y}}-Y_{C O P}\right)=0 .
\end{aligned}
$$

Three-fold cross validation was implemented for model evaluation. The data from the postural control assessment tasks were equally assigned into three groups, and each group contained data from tasks (1)-(6) (as described in Section 2.2). For each evaluation procedure, two groups were selected as the training set while the other group as the evaluation set. This repeated three times until all the group combinations were tested. Then, the mean value of root mean square errors (RMSE), the correlation coefficients (CC), the maximum error (MaxE) and minimum error (MinE) between the estimated COP trajectories and the reference measurements were calculated. In addition, in order to determine the statistical significance of the CC, the $p$-values were examined for CC. In addition, the COP trajectory was also estimated by the weighted mean approach. Similarly, three-fold cross validation was implemented to evaluate the weighted mean approach. Comparisons were carried out between the nonlinear model and weighted mean approach in RMSE, CCs, MaxE and MinE.

\subsection{Graphic User Interface (GUI)}

The GUI is shown in Figure 5. The left-hand side panel shows the pair of instrumented insole and the locations of FSR sensors. Each red dot presents the instant sensor pressure, the size of which corresponds to the pressure magnitude. The green dot indicates the estimated location of the COP. The right-hand side panel shows the COP trajectories along the time. It also shows the pressure output of each FSR sensor and instant $\boldsymbol{F}_{\text {tot }}$ normalized by body weight (BW). 


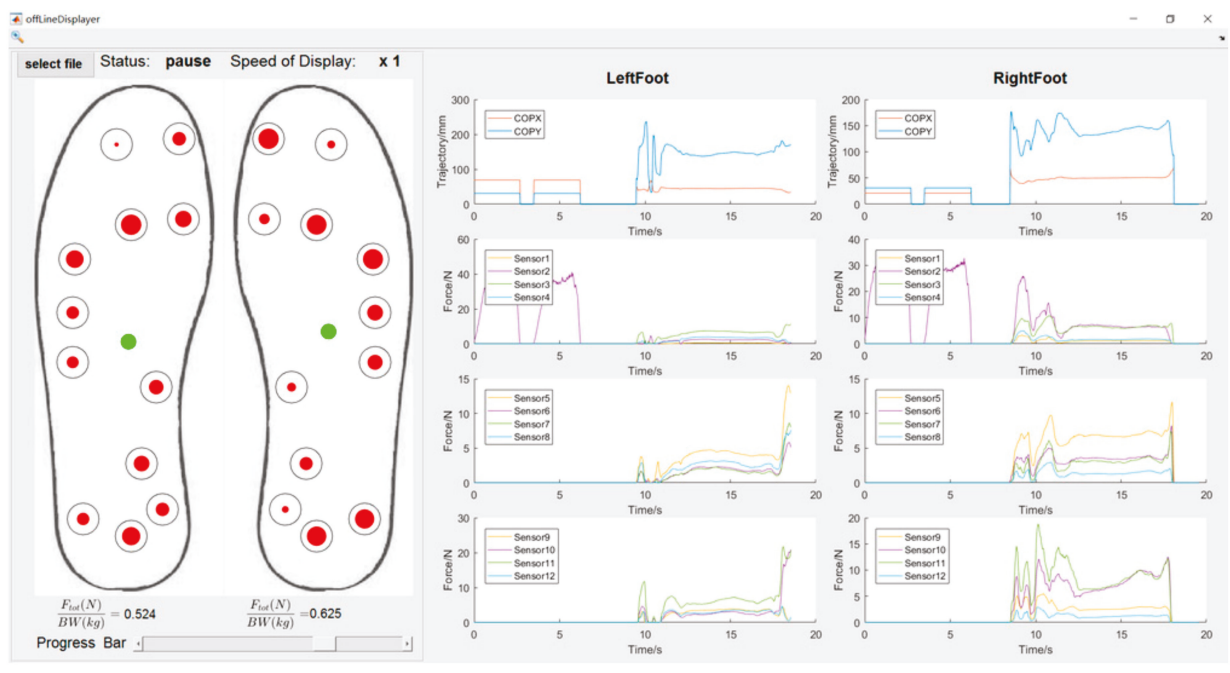

Figure 5. The GUI developed in the MATLAB to show the output of each sensor, $\boldsymbol{F}_{\text {tot }}$ normalized by body weight (BW), and the estimated foot plantar COP trajectory.

\section{Results}

Figure 6 shows an example of COP trajectory estimation by the proposed nonlinear model. The COP trajectories along the ML direction and AP direction were plotted against the reference measurements. This example shows that the estimated COP trajectories were trending closely with the reference, suggesting an accurate estimation.

Table 2 shows the RMSE, CC, MaxE and MinE results of the COP trajectories calculated by the proposed nonlinear model compared with the reference measurements. The overall mean and standard deviation were also summarized. The RMSE reflects estimation accuracy. For the ML COP, the mean RMSEs were $2.23( \pm 0.64)$ and $2.72( \pm 0.83)$ for the left and right foot, respectively. For the AP COP, the mean RMSEs were $9.17( \pm 1.98)$ and $11.19( \pm 2.98)$, respectively. The RMSEs along the AP were larger than that along the ML direction because the COP trajectories typically have a larger moving range along the AP direction. The small RMSEs suggested that the proposed COP trajectory estimation model had high COP trajectory estimation accuracy.

The CC accounts for the similarity of the estimated COP trajectory time series, compared to the reference measurements. The mean value of CCs was $0.91( \pm 0.05)$ to $0.93( \pm 0.02)$ along the ML and AP direction, respectively. In addition, the $p$-values of each CC were all smaller than 0.0001 , indicating that the correlation is highly significant.

The MaxE and MinE indicate the least and best estimation each model can achieve. The MaxE for ML COP was $26.119 \mathrm{~mm}$ and $25.254 \mathrm{~mm}$ for the left and right foot, respectively; for AP COP, it was $124.86 \mathrm{~mm}$ and $116.18 \mathrm{~mm}$ for the left and right foot, respectively. As for the minimum error, both the weighted mean approach and nonlinear model can achieve a high accuracy with the minimum error less than $1 \mathrm{~mm}$.

Figure 7 shows the comparison of the RMSE between the nonlinear model and weighted mean approach. For all participants, the RMSE between the estimated COP by the nonlinear model and the reference measurements are smaller compared to their counterparts estimated by the weighted mean approach. Figure 7 also shows the CCs. Overall, the weighted mean approach yielded a fairly good CC, which was approximately $0.77-0.85$ along the ML and AP direction, respectively. However, the 
nonlinear model yielded a higher CC. These results indicate that the COP trajectories estimated by the nonlinear model have a more similar trending with the reference COP.

The least square error approximations were completed by a customized MATLAB script running on a laptop computer (Intel ${ }^{\circledR}$ Core (TM) i7-7500U 2.7GHz, 8.00GB RAM, 256GB HD, Windows x64 operation system, HP Inc., Palo Alto, CA, USA). The number of iterations in least square error approximation was between 5-8 (Mean $\pm \mathrm{SD}=6.5 \pm 1.0$ iterations) across different trials. It took $0.053-0.124 \mathrm{~s}$ (Mean $\pm \mathrm{SD}=0.112 \pm 0.005 \mathrm{~s}$ ) to complete the approximation procedure.
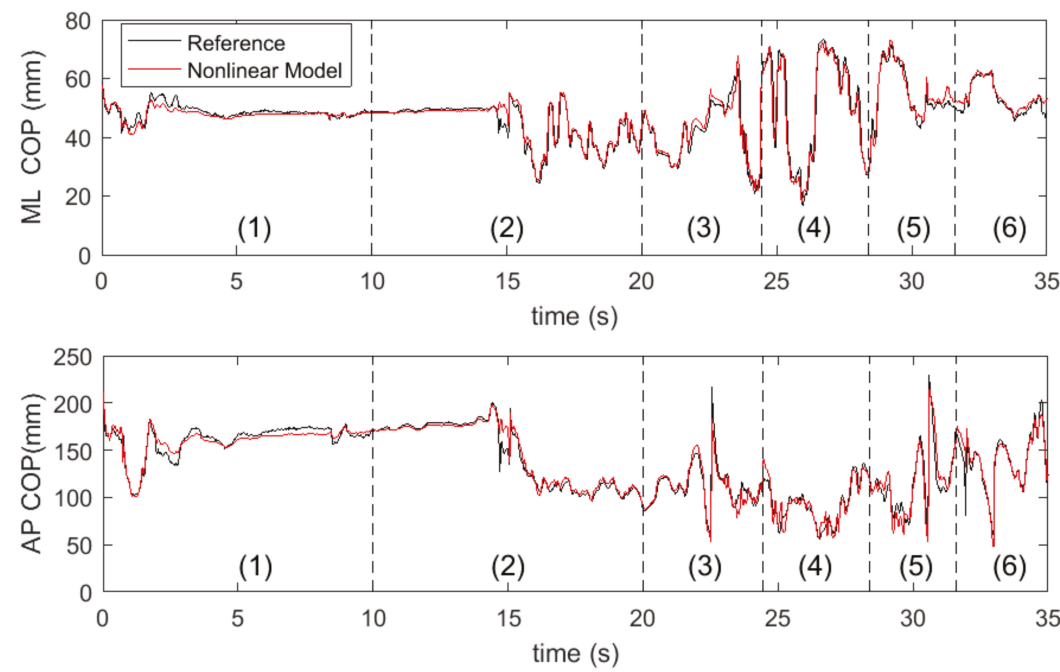

Figure 6. Representative plot of the comparison of COP trajectory estimation by the nonlinear model and the reference data. The data were obtained from the left foot and for the different tasks: (1) quiet standing with open eyes, (2) quiet standing with closed eyes, (3) standing up from a chair with armrests, (4) sitting down to a chair with armrests (task 3 and 4 have multi-contacts phases where participant's hands are in contact with armrests or the seat) (5) standing up from a chair without armrests, and (6) sitting down to a chair without armrests. 


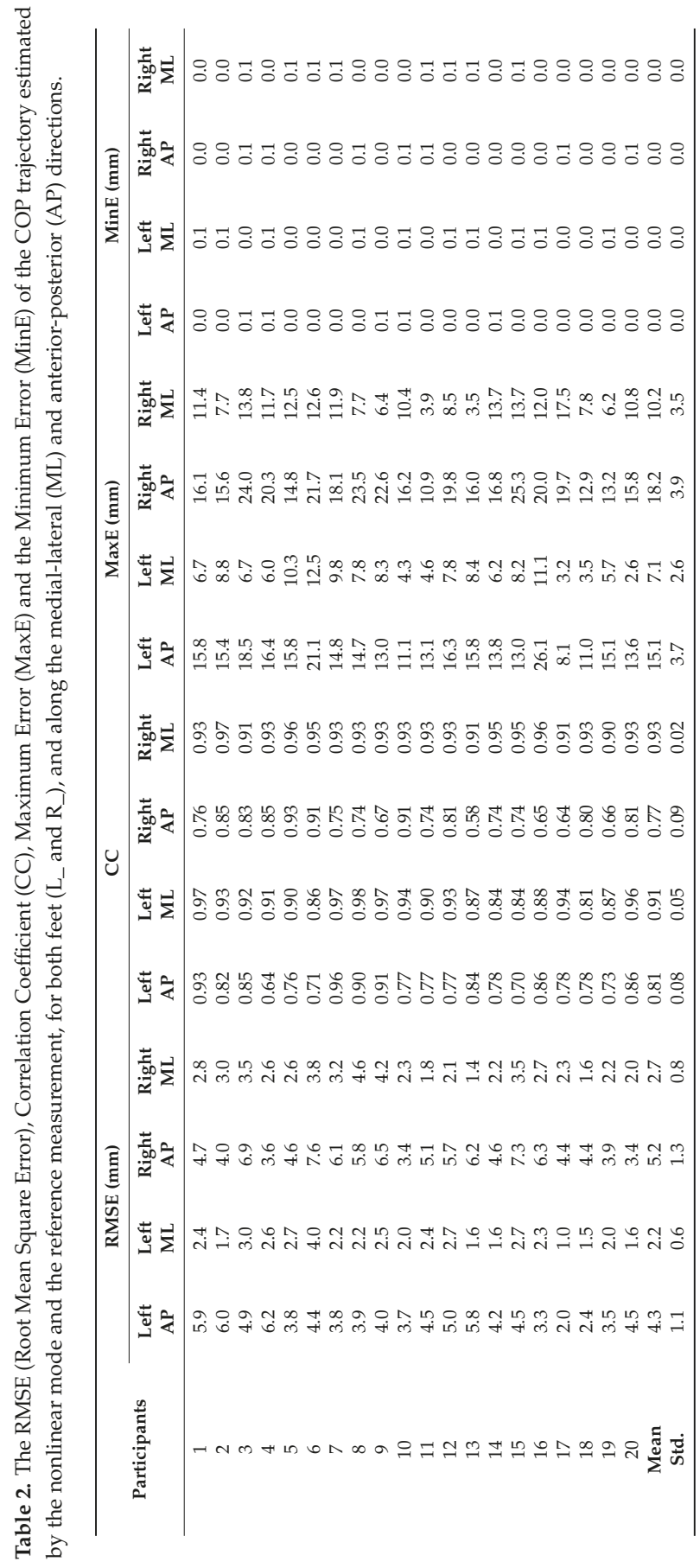



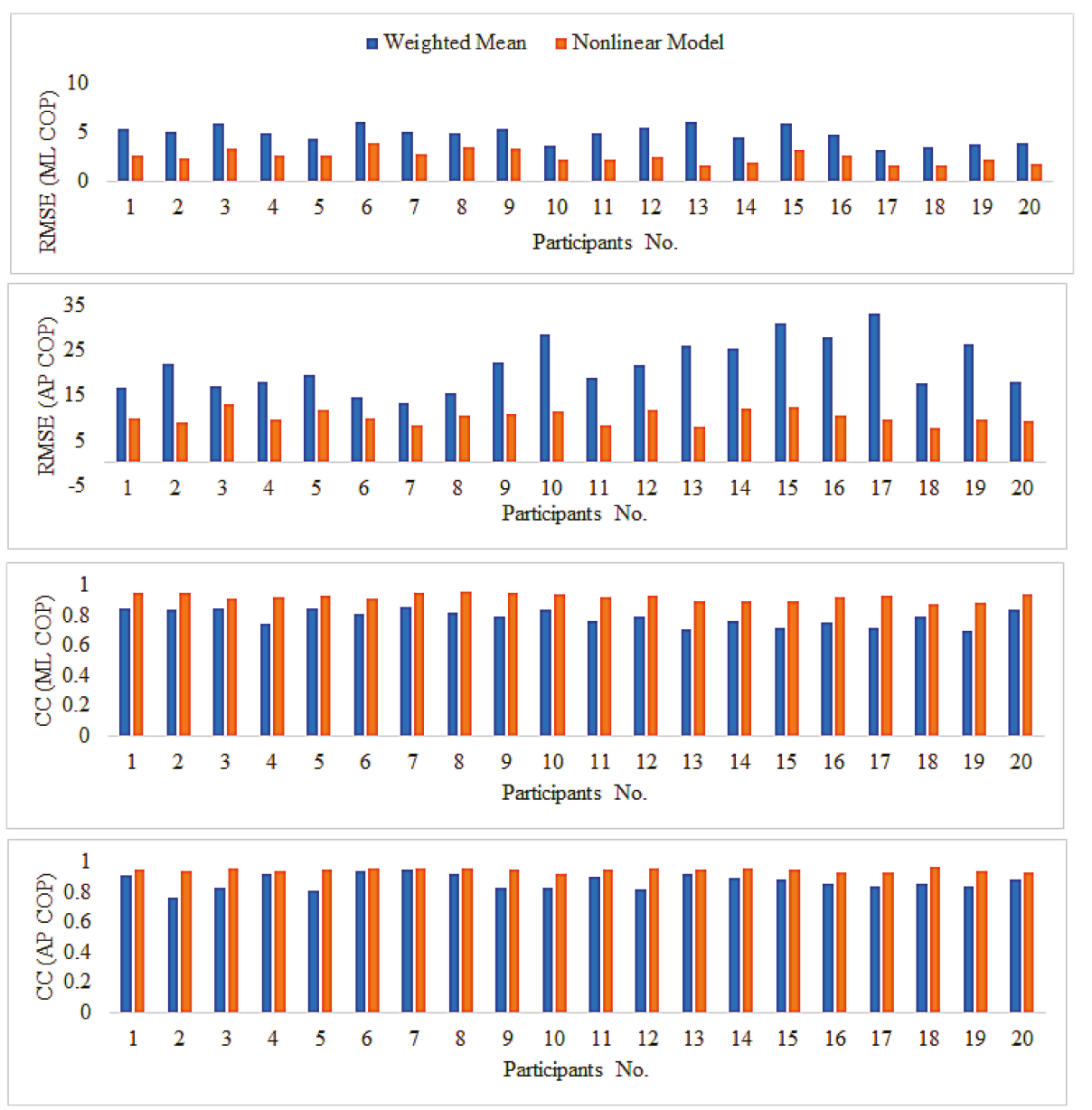

Figure 7. Comparisons of the RMSE and CC between the nonlinear model and the weighted mean approach.

\section{Discussion}

This study presents an individual-specific nonlinear model that can help estimate the foot plantar COP trajectories with an instrumented insole. Among the 20 participants involved in this study, the average RMSE between the estimated COP trajectories by the proposed model and the reference measurements was less than $3 \mathrm{~mm}$ along the medial-lateral direction of the foot, and less than $12 \mathrm{~mm}$ along the anterior-posterior direction. In addition, the estimated COP trajectories by this nonlinear model established high correlation coefficients with the reference measurements (0.91-0.93 with $p$-values all less than 0.0001). To our knowledge, the results are the smallest errors and highest correlation coefficients reported in relevant studies where the foot plantar COP trajectories were estimated by a small number of low-cost pressure sensors [28,29]. For instance, Shu [28] reported a mean relative difference of 7.6 and $9.9 \mathrm{~mm}$ between the estimated COP trajectories and the reference measurements during normal standing and standing on one leg. Dyer et al. [29] reported the RMSE ranging from $7 \mathrm{~mm}$ to $24 \mathrm{~mm}$ during locomotion. Overall, the results suggested that the proposed nonlinear model had excellent COP trajectory estimation capability. 
Previously, the weighted mean approach was the most commonly used method to estimate the foot plantar COP trajectory by instrumented insoles [40]. However, as mentioned earlier, this approach may not lead to desirable estimation, especially when the number of low-cost pressure sensors is small. To address the limitation of the weighted mean approach, we proposed the nonlinear model here and used this model to estimate COP trajectories during a variety of postural control assessment tasks. Meanwhile, to identify the improvement of the proposed nonlinear model, the weighted mean approach was also used for COP trajectory estimation by using the same set of data. The results show that the mean RMSE between the estimated COP trajectories by the weighted mean approach and the reference measurements were $\sim 4-6 \mathrm{~mm}$ along the ML direction, and $\sim 18-20 \mathrm{~mm}$ along the AP direction. These nearly doubled the RMSEs obtained by the nonlinear model. The correlation coefficients were ranging from 0.80 to 0.86 , which were also lower than those calculated by the nonlinear model approach. These results suggested that the proposed nonlinear model can lead to improved COP trajectory estimation compared to the weighted mean approach.

There might be two reasons that can help explain why the nonlinear model works better than the weighted mean approach with a small number of sensors. First, in the weighted mean approach, the accuracy depends largely on the numbers of sensors and their predefined locations. As we discussed earlier, the small number of sensors might induce errors. Different sensor placement strategy will also influence the accuracy of COP trajectory estimation. However, in the nonlinear model, least square error approximation will help rectify the errors due to the small number of sensors and their placement strategy. Another reason is that the weighted mean approach cannot address individual differences, which may induce additional intra-subject errors. In addition, the least square error approximation process in the nonlinear model is dependent on individual data from the sensors. In other words, the model coefficients were determined by each participant's own experimental data. Thus, the proposed nonlinear model is individual-specific and is able to address individual differences.

This study demonstrated that the foot plantar COP can be accurately estimated by a small number of low-cost pressure sensors. Many instrumented insole systems have been developed and are even commercially available. Clinically, more accurate COP estimation help better postural control assessment. However, there is always a trade-off between the number of sensors and estimation accuracy. The increased number of sensors will complicate the instrumented insole system and possibly compromise the reliability of the whole system. In addition, a larger number of sensors or sensor array will increase the cost substantially and make it unaffordable for the home-dwelling elderly. Therefore, an instrumented insole with a small number of low-cost sensors like what we proposed in the present study has its own merits.

From a practical point of view, this study can benefit the home-based postural control assessment for elderly and patients with pathological conditions. The impaired postural control is considered as an important risk factor of falls [41,42]. Though consensus is still lacking, postural control parameters have been widely suggested as indicators of fall risks [43-46]. Based on these, the authors believe that this can benefit the fall prevention research.

There are still some limitations in this study. First, this study mainly focused on postural control assessment during static stance and sit-to-stand transitions. Although previous research suggested that increased postural sway during stance can be a risk factor for prospective falls in community-dwelling elderly individuals [10], future work needs to be carried out to test the validity of this nonlinear approach during other activities of daily living, such as walking and stair negotiation. Second, we did not examine insole size other than US 9 just for convenience. However, as the proposed COP estimation model in the present study was individual-specific, we believe that this model would be applicable for other insole sizes as well.

\section{Conclusions}

Falls are still a major safety and health problem among aged population. Fall risk assessment is an effective approach to reduce fall accidents among the elderly. A substantial number of falls in the elderly 
result from loss of balance. Thus, the plantar COP, as an indicator of postural control performance, is an important fall risk assessment parameter. This study presented a low-cost instrumented insole system that uses a nonlinear model for COP trajectory estimation. Results show that this system is able to provide accurate COP trajectory data. Compared to traditional COP trajectory estimation approaches (i.e., weighted mean approach), the proposed nonlinear model performed better in terms of estimation accuracy. Based on this, we suggest that the proposed instrumented insole system could serve as an inexpensive solution to fall risk assessment in home settings or community healthcare centers for the elderly. It has the potential to help prevent future falls in the elderly.

Acknowledgments: This work was supported in part by the Natural Science Foundation of China (11702175; 31570944), the Natural Science Foundation of Guangdong Province (2015A030313553; 2016A030310068), and the Science, Technology and Innovation Committee of Shenzhen City (JCYJ20160422145322758; JCYJ20150525092940994). The authors would like to thank Wenzhen Chen and Jialun Cai for their help during data collection.

Author Contributions: X.H. and X.Q. conceived and designed the experiments; X.H., D.P. and J.Z. performed the experiments; D.P., Z.S. and X.H. analyzed the data; and X.H., Z.S. and X.Q. wrote the paper.

Conflicts of Interest: The authors declare no conflict of interest.

\section{References}

1. Horak, F.B. Postural orientation and equilibrium: What do we need to know about neural control of balance to prevent falls? Age Ageing 2006, 35, ii7-ii11. [CrossRef] [PubMed]

2. Massion, J. Postural control system. Curr. Opin. Neurobiol. 1994, 4, 877-887. [CrossRef]

3. Tresch, M.C. A balanced view of motor control. Nat. Neurosci. 2007, 10, 1227-1228. [CrossRef] [PubMed]

4. Lord, S.R.; Sambrook, P.N.; Gilbert, C.; Kelly, P.J.; Nguyen, T.; Webster, I.W.; Eisman, J.A. Postural stability, falls and fractures in the elderly: Results from the Dubbo Osteoporosis Epidemiology Study. Med. J. Aust. 1994, 160, 684-685. [CrossRef]

5. Melzer, I.; Benjuya, N.; Kaplanski, J. Postural stability in the elderly: A comparison between fallers and non-fallers. Age Ageing 2004, 33, 602-607. [CrossRef] [PubMed]

6. Nashner, L.M.; Shumway-Cook, A.; Marin, O. Stance posture control in select groups of children with cerebral palsy: Deficits in sensory organization and muscular coordination. Exp. Brain Res. 1983, 49, 393-409. [CrossRef] [PubMed]

7. Benaim, C.; Pérennou, D.A.; Villy, J. Validation of a standardized assessment of postural control in stroke patients. Stroke 1999, 30, 862-1868. [CrossRef]

8. Horak, F.B.; Nutt, J.G.; Nashner, L.M. Postural inflexibility in parkinsonian subjects. J. Neurol. Sci. 1992, 111, 46-58. [CrossRef]

9. Rocchi, L.; Chiari, L.; Cappello, A.; Horak, H.B. Identification of distinct characteristics of postural sway in Parkinson's disease: A feature selection procedure based on principal component analysis. Neurosci. Lett. 2006, 394, 140-145. [CrossRef] [PubMed]

10. Liu, J.; Zhang, X.; Lockhart, T.E. Fall risk assessments based on postural and dynamic stability using inertial measurement unit. Saf. Health Work 2012, 3, 192-198. [CrossRef] [PubMed]

11. Lafond, D.; Corriveau, H.; Hébert, R.; Prince, F. Intrasession reliability of center of pressure measures of postural steadiness in healthy elderly people. Arch. Phys. Med. Rehabil. 2004, 85, 896-901. [CrossRef] [PubMed]

12. Biswas, A.; Lemaire, E.D.; Kofman, J. Dynamic gait stability index based on plantar pressures and fuzzy logic. J. Biomech. 2008, 41, 1574-1581. [CrossRef] [PubMed]

13. Van Dijk, M.M.; Meyer, S.; Sandstad, S.; Wiskerke, E.; Thuwis, R.; Vandekerckhove, C.; Myny, C.; Ghosh, N.; Beyens, H.; Dejaeger, E.; et al. A cross-sectional study comparing lateral and diagonal maximum weight shift in people with stroke and healthy controls and the correlation with balance, gait and fear of falling. PLoS ONE 2017, 12, e0183020. [CrossRef] [PubMed]

14. Sawacha, Z.; Carraro, E.; Contessa, P.; Guiotto, A.; Masiero, S.; Cobelli, C. Relationship between clinical and instrumental balance assessments in chronic post-stroke hemiparesis subjects. J. Neuroeng. Rehabil. 2013, 10, 95. [CrossRef] [PubMed] 
15. Sawacha, Z.; Carraro, E.; Din, S.D.; Guiotto, A.; Bonaldo, L.; Punzi, L.; Cobelli, C.; Masiero, S. Biomechanical assessment of balance and posture in subjects with ankylosing spondylitis. J. Neuroeng. Rehabil. 2012, 9, 63. [CrossRef] [PubMed]

16. Johansson, J.; Nordstrom, A.; Gustafson, Y.; Westling, G.; Nordstrom, P. Increased postural sway during quiet stance as a risk factor for prospective falls in community-dwelling elderly individuals. Age Aging 2017, 46, 946-970. [CrossRef] [PubMed]

17. Robertson, G.; Caldwell, G.; Hamill, J.; Kamen, G.; Whittlesey, S. Research Methods in Biomechanics, 2nd ed.; Human Kinetics: Champaign, IL, USA, 2013.

18. Klenk, J.; Schwickert, L.; Palmerini, L.; Mellone, S.; Bourke, A.; Ihlen, E.A.F.; Kerse, N.; Hauer, K.; Pijnappels, M.; Synofzik, M.; et al. The FARSEEING real-world fall repository: A large-scale collaborative database to collect and share sensor signals from real-world falls. Eur. Rev. Aging Phys. Act. 2016, 13, 8. [CrossRef] [PubMed]

19. Bagala, F.; Klenk, J.; Cappello, A.; Chiari, L.; Becker, C.; Lindemann, U. Quantitative description of the lie-to-sit-to-stand-to-walk transfer by a single body-fixed sensor. IEEE Trans. Neural Syst. Rehabil. Eng. 2013, 21, 624-633. [CrossRef] [PubMed]

20. Klenk, J.; Becker, C.; Lieken, F.; Nicolai, S.; Maetzler, W.; Alt, W.; Zijlstra, W.; Hausdorff, J.M.; van Lummel, R.C.; Chiari, L.; et al. Comparison of acceleration signals of simulated and real-world backward falls. Med. Eng. Phys. 2011, 33, 368-373. [CrossRef] [PubMed]

21. Becker, C.; Schwickert, L.; Mellone, S.; Bagala, F.; Chiari, L.; Helbostad, J.L.; Zijlstra, W.; Aminian, K.; Bourke, A.; Todd, C.; et al. Proposal for a multiphase fall model based on real-world fall recordings with body-fixed sensors. Z. Gerontol. Geriatr. 2012, 45, 707-715. [CrossRef] [PubMed]

22. Bagala, F.; Becker, C.; Cappello, A.; Chiari, L.; Aminian, K.; Hausdorff, J.M.; Zijlstra, W.; Klenk, J. Evaluation of accelerometer-based fall detection algorithms on real-world falls. PLoS ONE 2012, 7, e37062. [CrossRef] [PubMed]

23. Mellone, S.; Tacconi, C.; Schwickert, L.; Klenk, J.; Becker, C.; Chiari, L. Smartphone-based solutions for fall detection and prevention: The FARSEEING approach. Z. Gerontol. Geriatr. 2012, 45, 722-727. [CrossRef] [PubMed]

24. Chesnin, K.J.; Selby-Silverstein, L.; Besser, M.P. Comparison of an in-shoe pressure measurement device to a force plate: Concurrent validity of center of pressure measurements. Gait Posture 2000, 12, 128-133. [CrossRef]

25. Forner-Cordero, A.; Koopman, H.J.F.M.; van der Helm, F.C.T. Use of pressure insoles to calculate the complete ground reaction forces. J. Biomech. 2004, 37, 1427-1432. [CrossRef] [PubMed]

26. Forner-Cordero, A.; Koopman, H.J.F.M.; van der Helm, F.C.T. Inverse dynamics calculations during gait with restricted ground reaction force information from pressure insoles. Gait Posture 2006, 23, 189-199. [CrossRef] [PubMed]

27. Liedtke, C.; Fokkenrood, W.; Menger, T.; van der Kooij, H.; Veltink, H. Evaluation of instrumented shoes for ambulatory assessment of ground reaction forces. Gait Posture 2007, 26, 39-47. [CrossRef] [PubMed]

28. Shu, L.; Hua, T.; Wang, Y.; Li, Q.; Feng, D.D.; Tao, X. In-shoe plantar pressure measurement and analysis system based on fabric pressure sensing array. IEEE Trans. Inf. Technol. Biomed. 2010, 14, 767-775. [PubMed]

29. Dyer, P.S.; Bamberg, S.J.M. Instrumented insole vs. force plate: A comparison of center of plantar pressure. In Proceedings of the 2011 IEEE Annual International Conference of Engineering in Medicine and Biology Society, Boston, MA, USA, 30 August-3 September 2011; pp. 6805-6809.

30. Crea, S.; Donati, M.; De Rossi, S.M.M.; Oddo, C.M.; Vitiello, N. A wireless flexible sensorized insole for gait analysis. Sensors 2014, 14, 1073-1093. [CrossRef] [PubMed]

31. Ayena, J.C.; Otis, M.J.; Menelas, B.A. An efficient home-based risk of falling assessment test based on Smartphone and instrumented insole. In Proceedings of the IEEE International Symposium on Medical Measurements and Applications (MeMeA), Turin, Italy, 7-9 May 2015; pp. 416-421.

32. Lin, F.; Wang, A.; Zhuang, Y.; Tomita, M.R.; Xu, W. Smart Insole: A wearable sensor device for unobtrusive gait monitoring in daily life. IEEE Trans. Ind. Inform. 2016, 12, 2281-2291. [CrossRef]

33. Claverie, L.; Ille, A.; Moretto, P. Discrete sensors distribution for accurate plantar pressure analyses. Med. Eng. Phys. 2016, 38, 1489-1494. [CrossRef] [PubMed]

34. Howell, A.M.; Kobayashi, T.; Hayes, H.A.; Foreman, K.B.; Bamberg, S.J.M. Kinetic gait analysis using a low-cost insole. IEEE Trans. Biomed. Eng. 2013, 60, 3284-3290. [CrossRef] [PubMed] 
35. Hall, R.S.; Desmoulin, G.T.; Milner, T.E. A technique for conditioning and calibrating force-sensing resistors for repeatable and reliable measurement of compressive force. J. Biomech. 2008, 41, 3492-3495. [CrossRef] [PubMed]

36. Interlink. Available online: https://www.interlinkelectronics.com/datasheets/Datasheet_FSR.pdf (assessed on 10 August 2017).

37. Chen, B.; Bates, B.T. Comparison of F-Scan in-sole and AMTI forceplate system in measuring vertical ground reaction force during gait. Physiother. Theory Pract. 2000, 16, 43-53. [CrossRef]

38. Cavanagh, P.R.; Ae, M. A technique for the display of pressure distributions beneath the foot. J. Biomech. 1980, 13, 69-75. [CrossRef]

39. Bickel, P.J.; Doksum, K.A. Mathematical Statistics: Basic Ideas and Selected Topics; Prentice Hall: Upper Saddle River, NJ, USA, 1977.

40. Ma, C.Z.H.; Wong, D.W.C.; Lam, W.K.; Wan, A.H.P.; Lee, W.C.C. Balance improvement effects of biofeedback systems with state-of-the-art wearable sensors: A systematic review. Sensors 2016, 16, 434. [CrossRef] [PubMed]

41. Prudham, D.; Evans, J. Factors associated with falls in the elderly: A community study. Age Ageing 1981, 10, 141-146. [CrossRef] [PubMed]

42. Tinetti, M.; Speechley, M.; Cinter, S. Risk factors for falls among elderly persons living in the community. N. Engl. J. Med. 1988, 319, 1701-1707. [CrossRef] [PubMed]

43. Palumbo, P.; Klenk, J.; Cattelani, L.; Bandinelli, S.; Ferrucci, L.; Rapp, K.; Chiari, L.; Rothenbacher, D. Predictive performance of a fall risk assessment tool for community-dwelling older people(FRAT-up) in 4 European cohorts. J. Am. Med. Dir. Assoc. 2016, 17, 1106-1113. [CrossRef] [PubMed]

44. Palumbo, P.; Palmerini, L.; Bandinelli, S.; Chiari, L. Fall risk assessment tools for elderly living in the community: Can we do better? PLoS ONE 2015, 10, e0146247. [CrossRef] [PubMed]

45. Cattelani, L.; Palumbo, P.; Palmerini, L.; Bandinelli, S.; Becker, C.; Chesani, F.; Chiari, L. FRAT-up, a web-based fall-risk assessment tool for elderly people living in the community. J. Med. Internet Res. 2015, 17, e41. [CrossRef] [PubMed]

46. Palumbo, P.; Palmerini, L.; Chiari, L. A probabilistic model to investigate the properties of prognostic tools for fall. Methods Inf. Med. 2015, 54, 189-197. [CrossRef] [PubMed] 


\title{
Review \\ Shoe-Insole Technology for Injury Prevention in Walking
}

\author{
Hanatsu Nagano and Rezaul K. Begg * \\ Institute for Health and Sport (IHES), Victoria University, Melbourne, VIC 3032, Australia; \\ hanatsu.nagano@vu.edu.au \\ * Correspondence: rezaul.begg@vu.edu.au; Tel.: +61-3-9919-1116; Fax: +61-3-9919-1242
}

Received: 29 March 2018; Accepted: 29 April 2018; Published: 8 May 2018

\begin{abstract}
Impaired walking increases injury risk during locomotion, including falls-related acute injuries and overuse damage to lower limb joints. Gait impairments seriously restrict voluntary, habitual engagement in injury prevention activities, such as recreational walking and exercise. There is, therefore, an urgent need for technology-based interventions for gait disorders that are cost effective, willingly taken-up, and provide immediate positive effects on walking. Gait control using shoe-insoles has potential as an effective population-based intervention, and new sensor technologies will enhance the effectiveness of these devices. Shoe-insole modifications include: (i) ankle joint support for falls prevention; (ii) shock absorption by utilising lower-resilience materials at the heel; (iii) improving reaction speed by stimulating cutaneous receptors; and (iv) preserving dynamic balance via foot centre of pressure control. Using sensor technology, such as in-shoe pressure measurement and motion capture systems, gait can be precisely monitored, allowing us to visualise how shoe-insoles change walking patterns. In addition, in-shoe systems, such as pressure monitoring and inertial sensors, can be incorporated into the insole to monitor gait in real-time. Inertial sensors coupled with in-shoe foot pressure sensors and global positioning systems (GPS) could be used to monitor spatiotemporal parameters in real-time. Real-time, online data management will enable 'big-data' applications to everyday gait control characteristics.
\end{abstract}

Keywords: gait; insole; injury prevention

\section{Introduction}

Walking is a fundamental locomotor task essential to healthy, active living, but it is accompanied by injury risk, particularly among the senior population. Walking is a continuum of gait cycles repeated thousands of times daily, and suboptimal features of the gait cycle can increase the probability of injury. Older adults (particularly females), for example, are prone to falls-related acute injuries due to gait impairments [1,2], and impaired foot pressure control during the loading response can cause foot problems [3]. The knee joint can be also affected over time due to functionally undesirable weight bearing, possibly resulting in osteoarthritis (OA) [4]. While walking is critically important both from a functional perspective and to ensure adequate exercise, injury risks must be minimised by optimising some essential biomechanical features of the gait pattern. Biomechanical interventions for injury prevention should, however, fulfil certain practical requirements, including low cost, easy engagement, immediate effects, and little physical effort; otherwise, interventions are unlikely to be adopted voluntarily and maintained in the longer term [5]. Footwear interventions have the potential to satisfy these requirements.

Typically, shoes are constructed with a number of components, all of which can influence gait mechanics [6]. An elevated heel is a factor in lateral instability and may result in caution-related adaptations reflected in spatio-temporal parameters [6]. Compared to standard soles, hard soles can 
more effectively provide tactile sensation for quicker reactions to maintain balance. Footwear-collars improve balance due to increased tactile sensation around the ankle while reducing swing foot clearance [6]. The outsole provides the interface with the walking surface and affects the frictional demands of walking and associated risk of slipping [7]. In contrast, the insole has direct contact with the sole of the foot and directly controls foot pressure and ankle joint motion that, in turn, influences the individual's gait pattern [8]. While some features of 'safe shoes' tend to be avoided, such as firm shoe-lace fixation, particularly in individuals with impaired activities of daily living (ADL) [9], insole interventions have the potential to be more readily accepted due to their practicality when applied to various footwear types.

As summarised in Table 1, three main types of insole modification can be identified as having the potential to support safer walking. Until recently, most high-grade insoles were produced using custom-moulding, which was designed to accommodate the individual's foot shape and influence foot pressure distribution. While this approach has provided a springboard, sensor technologies are now available that can provide a highly detailed biomechanical analysis of foot pressure and gait patterns to considerably advance shoe-insole development. Such progress could revolutionise injury prevention. For example, three-dimensional (3D) motion capture systems (e.g., Optotrack, Vicon, Optitrack) can accurately model gait motions, which is useful for identifying suboptimal gait features. By utilising this sensor technology, insole development can be undertaken to optimise gait control.

Foot pressure mapping, another very useful in-shoe sensor technology (e.g., F-scan, Pedar), is often synchronised with 3D motion capture to reveal the foot's pressure distribution and centre of pressure (CoP) in real-time [10]. The advantages of insole sensor systems are portability and wireless communication. Foot pressure measurement can not only be utilised in developing new insoles but also has the potential to acquire and store data to record gait patterns. Body-mounted inertial sensors are a related technology with similar potential to sample gait parameters in the natural environment. The portability of sensor-based systems will be their essential advantage in future gait assessments and will gradually overcome the limitations of laboratory-based 3D motion capture systems.

Table 1. Shoe-insole modification and biomechanical effects [11-18].

\begin{tabular}{cc}
\hline Modification Type & Potential Biomechanical Effects \\
\hline Material & Shock absorption \\
& Pressure distribution \\
& Energy efficiency \\
\hline Geometry (ankle control) & Shock absorption \\
& CoP \\
& Balance \\
Extra features (texture/heel cup etc.) & Energy efficiency \\
& Reaction speed \\
& CoP \\
& Balance \\
\hline
\end{tabular}

$\mathrm{CoP}=$ centre of pressure

In the current review, typical locomotor injuries are first explained and then insole developments and their significance are thoroughly discussed. Finally, the concept of a wireless gait measurement insole will be introduced and future directions in gait-related sensor technology outlined.

\section{Biomechanics of Locomotive Injuries Based on Gait Analysis}

In daily locomotion, both acute and overuse injuries should be considered. The primary cause of acute injury during locomotion is falls, particularly in the older population [1]. In contrast, certain types of ankle and knee pathology can be classified as overuse injuries due to the accumulation of 
negative gait features over time rather than a single traumatic event. This section introduces the biomechanics of falls and lower limb injuries primarily at the ankle and knee joints.

\subsection{Falls}

Approximately $33 \%$ of senior adults fall annually and up to $20 \%$ of cases lead to serious injuries [1,2,19]. Falls in this context can be defined as "an unintentional coming to the ground or the lower level due to balance loss" [20]. Biomechanically, falls occur when balance is disturbed and not able to be restored, with the consequence that the individual makes forceful contact with either the walking surface or surrounding objects. This twofold process comprises, therefore, (1) an event that disturbs balance and (2) failure in balance recovery.

Of the many balance-disturbing events, tripping has been identified as the leading cause of falls, accounting for up to 53\% of all falls [21]. Tripping is due to physical contact of the swing foot with the walking surface or an object on it, which creates the momentum to significantly destabilise balance. To prevent tripping, therefore, swing foot clearance should provide a sufficient vertical margin, particularly at the mid-swing event-Minimum Foot Clearance (MFC)—illustrated in Figure 1. At MFC, the vertical margin of the swing foot from the walking surface is low (i.e., $1-2 \mathrm{~cm}$ ) while moving at maximum speed and, as a consequence, causes high impact in the case of tripping [22]. Previous research by Moosabhoy and Gard suggests that, to prevent tripping, ankle dorsiflexion should be the most effective lower limb joint control strategy, whereby one degree of ankle dorsiflexion at MFC can be predicted to elevate the toe by $.3 \mathrm{~cm}$ [23], a response that can significantly reduce the probability of tripping [24].
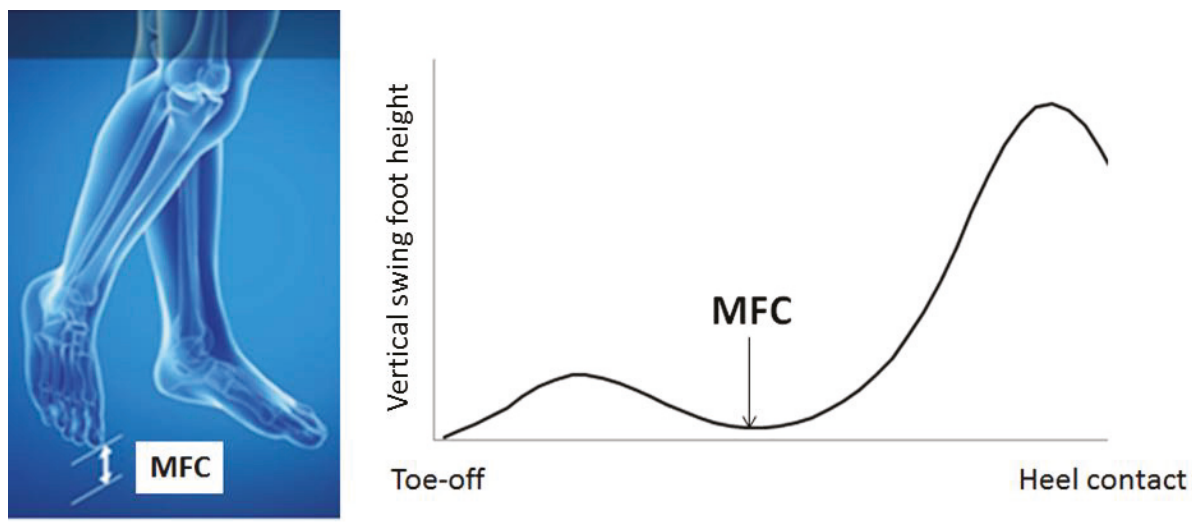

Swing Phase

Figure 1. Minimum Foot Clearance (MFC).

Biomechanically, dynamic balance is defined by the relationship between body centre of mass $(\mathrm{CoM})$ and base of support (BoS) [25]. In gait analysis, sensors, such as infrared light-emitting diodes (IREDs), light-emitting diodes (LEDs), and passive reflective markers, are usually attached to anatomical landmarks to model the subject's motion relative to a pre-registered laboratory coordinate system $(x, y, z)$. In static conditions, when the CoM is preserved within BoS, balance is considered to be secure, whereas in dynamic conditions, including walking, extrapolated CoM (XCoM) is used rather than CoM, as in the equation below [26].

$$
\mathrm{XCoM}=\operatorname{CoM} \text { position }+\frac{\text { CoM velocity }}{\sqrt{\frac{\text { gravity }}{l}}}
$$


In the above equation, $l$ indicates the distance between the ankle and the end of invertedpendulum movement, $\mathrm{CoM}$ [27]. XCoM has been considered to more accurately represent the positional threshold within BoS because CoM position by itself does not differentiate static CoM from fast moving CoM. The distance between XCoM and either the lateral or anterior-posterior boundary of the BoS, depending on CoM movement direction, is defined as the margin of stability (MoS) (Figure 2). A greater MoS indicates that balance is secure while a negative $(<0)$ MoS indicates balance loss and that the body is falling. From an injury prevention perspective, the most important feature of these relationships is that CoM motion is highly dependent on foot CoP control [28], which, as described above, can be influenced by footwear manipulations.

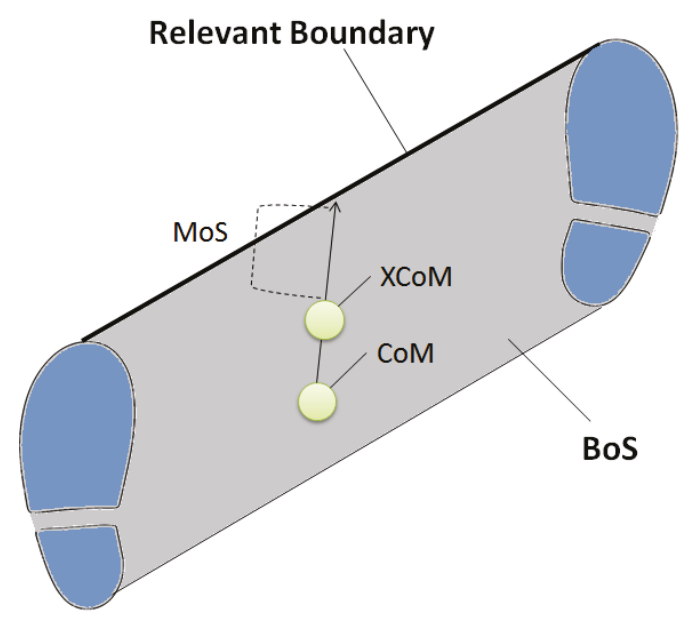

Figure 2. Description of balance in the transverse plane. MoS = margin of stability; BoS = base of support; $\mathrm{CoM}=$ centre of mass; $\mathrm{XCoM}=$ extrapolated centre of pass; Relevant boundary depending on the direction of CoM movement.

\subsection{Foot-Related Conditions}

Older adults' footwear often has inappropriate features, perhaps due to prioritising comfort over safety [9]. This is reflected in the tendency to avoid shoes with fixation or heel counter, and sandals are commonly worn $(22 \%)$ when they experience falls [9]. Improper footwear structures that cause undesirable foot pressure distribution and ineffective impact distribution can lead to chronic pain over time, which discourages active walking [29]. Footwear, including the insole, should be designed to promote walking mechanics that do not impose excessive stress on the foot and lower limb joints [3]. Foot problems often arise from inadequate weight bearing, which is reflected in undesirable ankle motion and foot pressure distribution [30,31]. Pressure distribution is the important element of gait control in preventing conditions, such as ulcer development, around the plantar areas, particularly among the diabetic population [32]. Fifteen million people in the U.S. have diabetes and $15-20 \%$ of those experience hospitalisation [3]. Another consequence of inadequate foot pressure distribution is Hallux valgus, which is especially common among high-heel wearers [14,33]. Hallux deformities are one of the major causes of foot pain [3]. Control of foot pressure is reflected in CoP movement, which is not only responsible for foot disorders but also controls dynamic balance. Inversion ankle sprain, for example, is a common acute injury caused by excessively lateral CoP [34], while Hallux valgus is a result of overpronation during mid to late stance [35]. As reported in previous studies [13,17] and as shown in Table 1, insole modifications can alter $\mathrm{CoP}$ control and foot pressure distribution, 
possibly reducing the incidence of debilitating foot conditions caused by inadequate $\mathrm{CoP}$ and foot pressure control.

Foot segment orientation is controlled by ankle motion, including dorsiflexion/plantarflexion and eversion/inversion. Pronation is the combination of dorsiflexion and eversion while inversion and plantarflexion form supination [36]. For adequate loading, the foot should be pronated in the early stance phase to absorb impact while quickly accommodating the walking surface environment [36,37]. Foot contact sometimes occurs with a supinated ankle but immediately starts to pronate, the essential countermovement to maximise the range of pronation, with accompanying tibial internal rotation [36,38]. Towards toe-off via foot-flat, the foot supinates, controlled first by eccentric work until foot-flat followed by the concentric work of supinators approaching toe-off [36,39]. This series of functional ankle motions most efficiently oscillates foot contact impact through the stance phase and into forward progression. These ankle motions are responsible for efficient weight transfer but also affect CoP movement, which can be measured using either force plates or in-shoe foot pressure monitoring systems.

Ankle kinematics can be monitored using 3D motion capture systems. For modelling the foot, a typical marker setup includes the toe (the distal and superior surface of the foot), second metatarsal head, fifth metatarsal head, lateral and medial malleolus, and heel. To visualise ankle motion, the tibia should also be modelled by incorporating lateral and medial epicondyles in addition to the lateral and medial malleolus at the ankle (Figure 3).

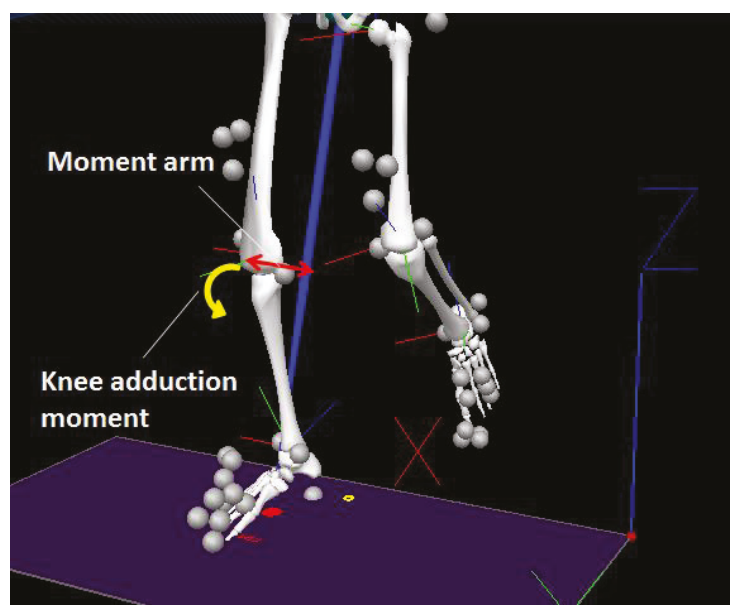

Figure 3. Illustration of marker setup for gait analysis; knee adduction moment due to shank external rotation.

\subsection{Knee Osteoarthritis}

Knee osteoarthritis $(\mathrm{OA})$ is a painful condition that discourages people from walking and increases the risk of falling [39]. OA is frequently associated with pain caused by micro-fractures due to bone-to-bone collision with reduced articular cartilage. Knee adduction moment is the kinetic marker most reliably related to the progression of $\mathrm{OA}$, especially at the medial compartment $[40,41]$. A comprehensive review suggested that approximately $10-15 \%$ of the senior population show clinical evidence of OA [42], but it is possible that gait modification interventions using insoles slow the progression of $\mathrm{OA}$ if knee adduction moment can be reduced [43].

There are two peaks in knee adduction moment during a gait cycle. The first peak is associated with OA and timing approximates the first Ground Reaction Force (GRF) peak and maximum knee flexion [44]. Reducing knee adduction moment is, therefore, the challenge in OA prevention. 
Biomechanically, this can be achieved by (1) reducing GRF and (2) creating a shorter moment arm by reducing external shank rotation in the frontal plane after foot contact $[45,46]$. Shock absorption at the heel could reduce peak GRF, while tibial realignment in early stance is associated with reduced moment arm and knee adduction moment. Biomechanical gait data obtained using a combination of 3D motion capture systems and force plates (i.e., kinematic and kinetic analysis) can compute knee joint kinetics via an analysis technique known as inverse dynamics. As introduced later, a lateral wedge insole may be able to control $\mathrm{CoP}$ and realign the tibia to reduce knee adduction moment.

In summary, safe walking is essential to maintaining mobility without injury and, as shown in Table 1 , shoe insoles can control gait functions to optimise walking mechanics [8,47]. In the current review, the primary focus is insole modifications to improve gait function.

\section{Shoe Insole Technology for Gait Control and Injury Prevention}

Shoe-insoles provide the interface between the foot and the footwear. Potential modifications include (1) modifying insole geometry (i.e., changing ankle angle) [18]; (2) increasing contact area (i.e., custom-moulding) [11]; (3) adjusting resilience (i.e., lower for shock absorption and greater for reusing mechanical energy) [12]; (4) treating insole surface (e.g., texture installation) [48]; (5) providing assistive support (e.g., heel counter) [49]; and (6) incorporating portable sensor systems, such as pressure sensors or inertial sensors [50]. Fundamental functions relating to reducing the above injury risks are as follows.

\subsection{Shock Absorption}

Impact energy at heel contact can be dissipated to minimise soft tissue damage, and softer materials are likely to reduce shock more effectively [51]. Ethylene vinyl acetate (EVA) foam is one of the more common shoe-insole materials, is usually used with a density range between 300 and $400 \mathrm{~m} / \mathrm{s}^{3}$, and is ideal for semi-customised shoe-insole moulding in commercial production [52]. The choice of insole material characteristics, such as density, can be used to control elasticity and resilience.

Technology for shock absorption at the heel has been marketed commercially using materials such as gels. One biomechanical explanation for the effectiveness of shock absorption products is that they prolong the time from initial foot-ground contact until complete compression of the footwear. Although GRF and foot pressure distribution can be modified by construction materials [53], some studies have reported that shock absorbers may not reduce injury risks [54]. In addition to shock absorbing materials, insole structure and hardness of the mid-sole are important for reducing load [51,55].

Shock absorption utilising either a material's elastic properties or a spring mechanism may be effective in storing mechanical energy at impact and then recovering the energy later in the stance phase toward toe-off as demonstrated by Zhang et al. using spring-loaded Axillary clutches [56]. Energy recovery can therefore engender more efficient walking by minimising the energy required at push-off. As detailed further in the following Section 3.2. Ankle, foot-ground impact can be viewed as an unexploited source of external energy because in human walking only $60-70 \%$ of impact energy can be oscillated through the loading response while the remainder is lost as vibration, sound, or heat $[57,58]$.

\subsection{Ankle}

Ankle motions responsible for shock absorption during early loading are a combination of dorsiflexion and eversion (i.e., pronation) [36,59-61]. Flat-foot contact is not considered desirable for shock absorption because the interval from initial foot contact to foot flat determines impact distribution over time due to the eccentric work of dorsiflexors [37,38,61]. Ageing appears to be a factor in reduced dorsiflexion at foot contact [62].

A pronated ankle during early loading rotates the shank internally and triggers knee flexion [63-65], but both these kinematic adaptations have the potential to reduce knee adduction moment [51]. 
An over-pronation problem arises following mid-stance when the ankle should begin to supinate [36]. Given the anatomical constraints on foot kinematics during the stance phase, during early-loading foot orientation for shock absorption is important and after mid-loading over-pronation should be avoided.

Stored mechanical energy should be transferred throughout the loading response from the pronated ankle up to supinated orientation at mid-stance towards toe-off [36]. Quantification of mechanical energy transfer is possible by calculating the recovery rate (see below), the percentage of mechanical energy transferred to oscillate the loading response from heel contact to toe-off [64-66].

$$
\text { Recovery Rate }(\%)=100 \times[\Delta \mathrm{KE}+\Delta \mathrm{PE}-\Delta(\mathrm{KE}+\mathrm{PE})] /(\Delta \mathrm{KE}+\Delta \mathrm{PE})
$$

where $\Delta \mathrm{KE}=$ increase in kinetic energy; $\Delta \mathrm{PE}=$ increase in potential energy; $\Delta(\mathrm{KE}+\mathrm{PE})=$ increase in the sum of kinetic energy and potential energy, all measured in the double support phase of the gait cycle based on CoM kinematics.

Step-to-step CoM transition during double support requires mechanical energy to produce GRF to continue walking [67]. More efficient loading is possible by utilising the impact at heel contact to initiate toe-off without dispersing the mechanical energy transferred to other lower limb joints (i.e., the knee). A higher recovery rate is, therefore, considered advantageous in reducing the heel contact forces transferred to other lower limb joints. For efficient loading, insole geometry can be re-designed to support pronation of the subtalar joint at heel contact while ensuring no disturbance to later ankle supination $[36,59-61,68]$. In the presence of foot deformity or other pathological conditions, however, careful consideration is required prior to any insole modification. For example, a custom-moulding insole modification may be necessary for seriously deformed feet, such as deformation due to neuropathy in diabetic patients [69]. Levinger et al. [45,46] suggested that a lateral wedge insole may be effective in reducing knee adduction moment by supporting pronation during early stance. They have, however, also stated that a lateral wedge insole may not be suitable for an already pronated foot.

\subsection{Foot Pressure Control}

The most common method for foot pressure distribution by shoe-insoles is to increase the contact area between the foot and the insole surface [70]. This can be achieved by custom-moulding the shoe insole to accommodate differences in foot shape [11]. Semi-customisation is also possible using an insole surface material that gradually adapts to the foot's shape. Foot CoP trajectory in a normal gait starts at the heel and ends at the toe, with a lateral curvature [71]. This lateral excursion seems to be a reflection of ankle supination during mid-stance.

As indicated above, balance control is determined by the relationship between CoM and BoS. A specially designed insole can control the $\mathrm{CoP}$ which changes $\mathrm{CoM}$ motion and it is particularly important to regulate excessive lateral CoP excursion to stabilise sideways balance [59]. A potential solution is an insole incorporating enhanced texture to consistently guide the optimal CoP path (Figure 4).

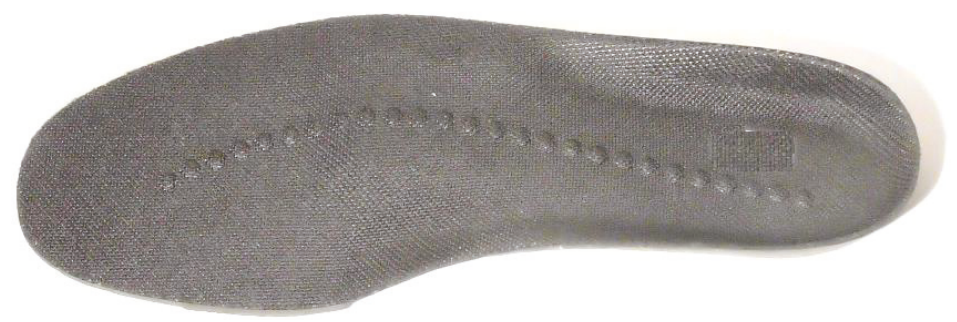

Figure 4. Example of texture installation to guide the CoP (WO2016015091A1-Injury Reduction Insole). 
If the $\mathrm{CoP}$ is variable over multiple gait cycles, associated $\mathrm{CoM}$ motions could also become variable and dynamic balance control could become unstable. Ensuring adequate cutaneous stimulation to assist the optimal CoP path is, therefore, a possible approach to improving balance. As described below, texture installation may also assist balance control by improving the reaction speed to balance perturbations.

\subsection{Reaction Speed}

Stimulation of cutaneous receptors increases afferent feedback and may, therefore, decrease reaction time. Ideally, the $\mathrm{CoP}$ should travel through (i.e., stimulate) high sensitivity cutaneous receptors without excessive pressure [72] and if balance is lost, recovery can be faster. Stimulation of the plantar surface is possible either by incorporating enhanced texture or using vibration devices [15,73]. The findings from these previous studies of this manipulation, however, leave some doubt as to how effectively such augmented stimulation can enhance afferent feedback and proprioception $[15,73,74]$.

Priplata et al. [15] tested the effect of vibrating insoles on balance control and found that older adults had improved dynamic balance due to plantar stimulation, but the study methodology was questioned by Lafond et al. [74]. One of their primary concerns was the methods employed for estimating balance, which is a more general problem arising from the lack of standardised biomechanical analysis methods to evaluate footwear effects on gait and balance. Similarly, some studies have reported enhanced proprioceptive reaction effects of textured insoles $[73,75,76]$, while others have not [77], and these discrepancies may, again, have been due to methodology but also to the fact that the textured or vibration insoles tested were not the same [78]. If cutaneous receptors are stimulated more systematically rather than stimulated by randomly installing textures over the entire insole surface, gait and balance could be controlled more effectively [78]. A further methodological limitation of previous insole research has been sample size; however, an ongoing study by Hatton et al. [79] with a large sample is expected to reveal the effects of texture stimulation on gait patterns with greater confidence.

There are a few reports of texture installation that is not applied over the entire surface. For example, SoleSensor is a commercially available shoe-insole that takes advantage of tactile stimulation to enhance reaction speed [16] using only a narrow tube peripheral to the insole surface. When foot pressure shifts peripherally and balance is disturbed, stimulation is provided by the tube that increases reaction speed. Similarly, Ritchile et al. applied textures only to the medial portion of the insole and reported that it supported functionally important mid-foot supination [68]. Due to the variety of texture installations with respect to area, size, shape, and hardness, further research is necessary to confirm the most effective stimulation properties. Research evidence suggests, however, that stimulation of cutaneous receptors has considerable promise for enhancing gait and balance [78].

\subsection{Lateral Wedge Insole}

The lateral wedge insole is designed to stabilize the ankle in a more everted position, helping to more internally align the tibia [46]. This adaptation reduces the tibial moment arm from the GRF vector and consequently decreases knee adduction moment [40,41]. For the Varus deformity, an everted ankle reduces knee adduction moment, softening the compression of the medial knee structure between the femur and tibia. The degrees of eversion support are usually between $5^{\circ}$ and $15^{\circ}$ [80].

Despite these biomechanical theories, the lateral wedge insole effects on knee OA are controversial. Weinhandl et al. [41], for example, reported no apparent effects in a young group who wore a lateral wedge insole for one week. As with the stimulation insoles, the conflicting results may be attributable to differences between the lateral wedge insoles in each study. Paradoxically, if the optimum structure can be identified, lateral wedges appear to have potential as a knee OA treatment [81]. Sawada et al. [82] reported that an individual's foot alignment also determines the effectiveness of a lateral wedge insole in reducing peak knee adduction moment. Walking speed was also suggested to affect experimental results in terms of knee adduction moment, but this hypothesis has been rejected by one study [83]. 
An additional modification that may enhance the lateral wedge insole is arch-support [84]. Based on data from 90 participants [81], soft, rather than hard, lateral wedge insoles have also been found to be more effective.

An additional advantage of lateral wedge insoles is CoP control [45], although this feature has not been examined thoroughly in previous reports. As discussed, CoP excursion through the stance phase demonstrates a characteristic lateral curvature. This response is functional in leading the pronated ankle into a supinated position to efficiently oscillate mechanical energy from heel contact to toe-off. This deviation may, however, be associated with lateral balance disturbance and ankle inversion sprain [85]. Thus, eversion support has potential for injury prevention by regulating excessive lateral $\mathrm{CoP}$ excursion. Careful consideration is required when incorporating this feature into shoe-insoles to correct excessive Valgus knee so as not to disturb functional supination.

\section{Concerns for Custom-Moulding}

In populations with significant foot deformities, it may be difficult to wear conventional shoes and customised insoles may be required. Custom-made insoles can accommodate individual-specific foot shapes and maximise the contact area between the foot and insole [11,30]. This promotes foot pressure distribution, and for those with significantly deformed feet, customisation appears to be essential [86,87].

Caution is, however, required for custom-moulding for two reasons. First, every part of the foot does not contribute equally to weight bearing; for example, the mid-foot has been reported to have little responsibility [14]. It is, therefore, possible that some parts of the foot may be more vulnerable to foot pressure than others. The second caution is that customisation could reflect and accentuate negative foot control. If a misaligned foot is scanned and moulded without correction, progression of the deformity may advance due to inadequate foot posture and additional foot problems may arise as a consequence [29]. Lateral CoP excursion is associated with the risk of inversion sprain [34]. If a foot is susceptible to inversion sprain, for example, custom-moulding without CoP modification may not help or could even further increase the risk of injury. A lateral wedge assists foot pronation and reduces knee adduction moment, but the lateral wedge may not adequately assist the foot with excessive Valgus [45]. It can, therefore, be implied that a foot with excessive Valgus may benefit from a medial wedge rather than a lateral wedge. Furthermore, custom-moulding was found not to treat Hallux valgus by regulating hyperpronation of the subtalar joint during the later stance phase when weight is concentrated on the metatarsal area [35]. The results suggest, therefore, that custom-moulding alone may not correct inadequate ankle joint kinematics.

Although custom-moulding is important, the insole's fundamental geometry should support optimum foot pressure control and energy efficient loading by providing adequate pronation-supination coupling. Ideally, the thin insole surface layer should be individual-specific, while the layers below can be rigidly constructed to assist optimum foot control. Telfer et al. [31] described the potential of 3D foot scanning and 3D printing for custom-moulding. This is a more finely-tuned approach to insole design, and future customisation of insoles and footwear is likely to take advantage of this technology. Other recent studies have also reported that 3D foot scanning could be an effective method for custom-made footwear despite further research being required to more comprehensively test the precision of this technique, including an increased number of tested subjects and wider measurement parameters to define the foot segment $[88,89]$.

\section{Gait Analysis for Insole Development}

While shoe-insoles assist walking, detailed gait analysis has not been widely utilized to test their effects on gait patterns. While pressure data are instructive, as mentioned above, gait analysis can record human walking precisely to identify various problems, including the risk of falling and balance control and lower limb joint (ankle and knee) biomechanics problems. The utility of 3D gait analysis in identifying the causes of foot and knee pain has been discussed by Rao et al. [29], and Menant et al. [6] 
used these techniques to determine how footwear features influenced gait biomechanics. Heel collars, for example, also improve balance by providing increased tactile sensation around the ankle via the extended contact area provided by the collar, while a high collar was found to increase the risk of tripping by reducing swing foot height at MFC. In addition to identifying condition-specific biomechanical parameters, such as those associated with knee pain described above, spatio-temporal gait parameters also reflect walking fundamentals (Figure 5). For example, gait impairments due to injury, ageing, psychological conditions, or medications show similar gait patterns, including slower gait velocity (due to shorter step length), increased step width, and prolonged double support time [90]. Utilising 3D motion capture systems, the fundamental spatio-temporal gait parameters can be easily measured by markers attached only to the heel and toe. Alternatively, other gait assessment tools, such as the GaitRite mat, can also record stride cycle parameters. Motion capture systems have also been used for testing commercial products, such as footwear and anti-slip strips [91,92]. Commercially available 3D motion capture systems are now relatively affordable (e.g., Optitrack, NaturalPoint), and the complex programming for 3D gait analysis can be overcome using low-cost commercial software that automatically extracts a range of gait parameters given a pre-specified standardized marker setup at data collection.

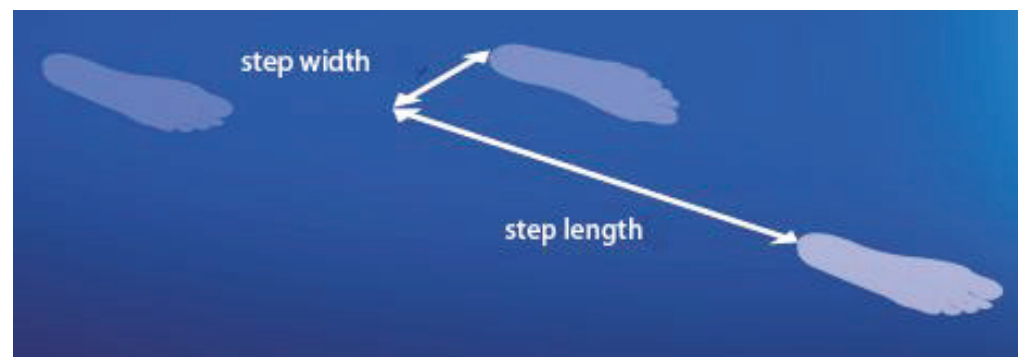

Figure 5. Spatio-temporal gait parameters, step length, and step width.

\section{Potential of a Wearable, Sensor-Integrated Insole for Real-Time Gait Monitoring}

Digital gait analysis began using multiple standard video cameras to monitor human movement from various angles to estimate 3D motion. Later, 3D motion capture systems utilising either infrared light emitting diodes (active systems) or reflective markers (passive systems) provided highly accurate position-time data and are now widely used in experimental gait research. While these systems are used as the gold standard for gait assessment, limitations in this technology include a complicated setup, high time demands, a lack of portability, and a requirement for specialized skills for system operation and data analysis. In Section 5, potential directions to overcome these problems have been proposed, and one approach is wearable sensor technology, which, despite its limitations, receives considerable ongoing research attention due to its immensely practical application in gait measurement.

Small, inexpensive body-mounted inertial measurement units (IMU) can measure angular velocity and linear acceleration [93]. IMU data can also be transmitted to Android devices using Bluetooth [94]. These innovations create the potential for online management, such as mass data storage and automatic recording of personal gait data. Difficulties in utilising IMUs for gait monitoring remain in deriving position-time data due to complex noise filtering [93]. To date, a number of techniques have been proposed to obtain greater accuracy in estimating positional data but further efforts may be required until sufficiently reliable kinematic data estimation is achieved.

Incorporation of IMUs into an insole is a promising approach to gait management because IMU attachment to the foot has the advantage of concurrently recognising gait cycle events and, using them, estimating walking speed [95]. It is, however, still difficult to precisely estimate walking speed and other associated parameters, such as stride length, using IMUs alone. It is, accordingly, fruitful 
to consider adding other technology, such as foot pressure sensors (Figure 6) and global positioning systems (GPS) within the insole. In-shoe foot pressure sensors can more precisely detect foot contact than IMU data, while the GPS can track the walking path and, therefore, total distance travelled. In-shoe pressure monitoring insoles are available [10,96], and real-time monitoring can alert the user to inadequate pressure distribution, such as excessive plantar pressure, to prevent foot ulcers or identify a lateral CoP excursion warning of potential balance loss.

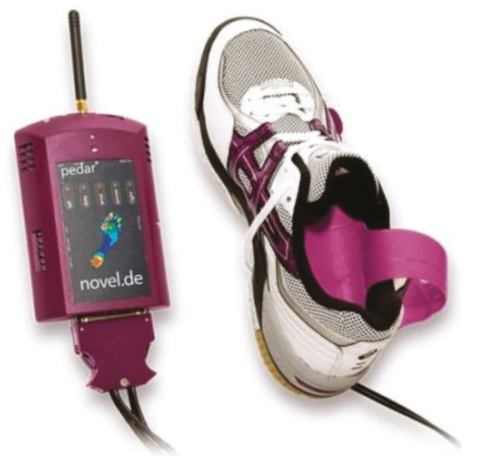

Figure 6. Foot-pressure monitoring system, Pedar (Novel, Munich, Germany, www.novel.de).

The VitaliSHOE project utilises wearable IMUs and pressure sensors integrated into the shoe-insole to detect the risk of falling in the senior population [97]. In an early paper, they showed the successful detection of transition between stance and swing based on both IMU and insole pressure data [97]. They identified, however, limitations due to gait measurement at a high walking speed and fragility of the IMU associated with mechanical stress [97]. In a later project [98], temporal variables were measured with high reliability but they also acknowledged the difficulties in acquiring reliable spatial data (e.g., step length) from IMUs [98]. It could also be interesting to utilise GPS technology to obtain spatial data in studies conducted in real-world settings, and smart shoes incorporating a mobile GPS have already been piloted as a fruitful direction for gait analysis in everyday settings [99].

\section{Long-Term Effects}

When developing shoe-insoles, the long-term effects of wearing them should be carefully evaluated to prevent overuse injuries, restriction of natural lower limb motion, and potential anatomical deformity [100]. Long-term use of a lateral wedge insole was found to reduce adductor moment semi-permanently, a positive adaptation; but if structured inadequately, adverse effects are possible [101]. As discussed earlier, foot control modifications can cause biomechanical changes to potentially all lower limb joint actions. Even very small negative features of the gait cycle can be accumulated by the thousands of steps taken every day and can eventually cause injury. Laboratory-based gait testing is useful in inspecting whether there are hazards in gait control. If gait kinematics and kinetics are maintained, it can be speculated that no negative long-term effects may arise. It is, however, still important to conduct human trials for a prolonged period to ensure that insoles are unlikely to cause orthopaedic problems.

\section{Conclusions}

Shoe-insoles have potential as an effective intervention to encourage safe walking. Biomechanical gait analysis is available for monitoring insole effects on walking performance. Insole modifications could support more adaptive ankle angles, improve foot pressure distribution, absorb shock, and reduce proprioceptive reaction time. Long-term effects of shoe-insoles should be tested to support 
and confirm experimental biomechanical evidence for their safety and effectiveness. Integration of wearable sensors into shoe-insoles will be a very important future direction for real-time gait measurement. Taking advantage of online data management, it will be possible to achieve the goal of detailed gait analysis available to everyone in performing the gait activities of everyday life.

Author Contributions: Conceptualization, H.N. and R.B.; Investigation, H.N.; Writing-Original Draft Preparation, H.N.; Writing-Review \& Editing, R.B.; Supervision, R.B.

Acknowledgments: The authors thank W.A. Sparrow for proof-reading the manuscript, Caitac for providing the insole image and Shinichi Tajima for illustrations.

Conflicts of Interest: The authors declare no conflict of interest.

\section{References}

1. Hill, K.; Schwarz, J.; Flicker, L.; Carroll, S. Falls among healthy, community-dwelling, older women: A prospective study of frequency, circumstances, consequences and prediction accuracy. Aust. N. Z. J. Public Health 1999, 23, 41-48. [CrossRef] [PubMed]

2. Keskin, D.; Borman, P.; Ersöz, M.; Kurtaran, A.; Bodur, H.; Akyüz, M. The risk factors related to falling in elderly females. Geriatr. Nurs. 2008, 29, 58-63. [CrossRef] [PubMed]

3. Bálint, G.P.; Korda, J.; Hangody, L.; Bálint, P.V. Foot and ankle disorders. Best Pract. Res. Clin. Rheumatol. 2003, 27, 87-111. [CrossRef]

4. Favre, J.; Jolles, B.M. Gait analysis of patients with knee osteoarthritis highlights a pathological mechanical pathway and provides a basis for therapeutic interventions. EFFORT Open Rev. 2017, 1, 368-374. [CrossRef] [PubMed]

5. Yardley, L.; Kirby, S.; Ben-Shlomo, Y.; Gilbert, R.; Whitehead, S.; Todd, C. How likely are older people to take up different falls prevention activities? Prev. Med. 2008, 47, 554-558. [CrossRef] [PubMed]

6. Menant, J.C.; Steele, J.R.; Menz, H.B.; Munro, B.J.; Lord, S.R. Effects of footwear features on balance and stepping in older people. Gerontology 2008, 54, 18-23. [CrossRef] [PubMed]

7. Yamaguchi, T.; Hokkirigawa, K. Development of a high slip-resistant footwear outsole using a hybrid rubber surface pattern. Ind. Health 2014, 52, 414-423. [CrossRef] [PubMed]

8. Kim, S.H.; Ahn, S.H.; Jung, G.S.; Kim, J.H.; Cho, Y.W. The effects of biomechanical foot orthoses on the gait patterns of patients with malaignment syndrome as determined by three dimensional gait analysis. J. Phys. Ther. Sci. 2016, 28, 1188-1193. [CrossRef] [PubMed]

9. Sherrington, C.; Menz, H.B. An evaluation of footwear worn at the time of fall-related hip fracture. Age Ageing 2003, 32, 310-314. [CrossRef] [PubMed]

10. Razak, A.H.A.; Zayegh, A.; Begg, R.K.; Wahab, Y. Foot plantar pressure measurement system: A review. Sensors 2012, 12, 9884-9912. [CrossRef] [PubMed]

11. Hellstrand Tang, U.; Zügner, R.; Lisovskaja, V.; Karlsson, J.; Hagberg, K.; Tranberg, R. Comparison of plantar pressure in three types of insole given to patients with diabetes at risk of developing foot ulcers a two-year, randomized trial. J. Clin. Transl. Endocrinol. 2014, 1, 121-132. [CrossRef] [PubMed]

12. Pratt, D.J.; Rees, P.H.; Rodgers, C. Assessment of some shock absorbing insoles: Technical note. Prosthet. Orthot. Int. 1986, 10, 43-45. [PubMed]

13. Jamshidi, N.; Rostami, M.; Najarian, S.; Menhaj, M.B.; Saadatnia, M.; Salami, F. Differences in centre of pressure trajectory between normal and steppage gait. J. Res. Med. Sci. 2010, 15, 33-40. [PubMed]

14. Donatelli, R. Normal biomechanics of the foot and ankle. J. Orthop. Sports Phys. Ther. 1985, 7, 91-95. [CrossRef] [PubMed]

15. Priplata, A.A.; Niemi, J.B.; Harry, J.D.; Lipsitz, L.A.; Collins, J.J. Vibrating insoles and balance control in elderly people. Lancet 2003, 362, 1123-1124. [CrossRef]

16. Maki, B.E.; Perry, S.D.; Scovil, C.Y.; Peters, A.L.; McKay, S.M.; Lee, T.A.; Corbeil, P.; Fernie, G.R.; Mcilroy, W.E. Interventions to promote more effective balance-recovery reaction in industrial settings: New perspectives on footwear and handrails. Ind. Health 2008, 46, 40-50. [CrossRef] [PubMed]

17. Aboutorabi, A.; Saeedi, H.; Kamali, M.; Farahmand, B.; Eshraghi, A.; Dolagh, R.S. Immediate effect of orthopedic shoe and functional foot orthosis on centre of pressure displacement and gait parameters in juvenile flexible flat foot. Prosthet. Orthot. Int. 2014, 38, 218-223. [CrossRef] [PubMed] 
18. Seo, K.; Park, S.; Park, K. Impact of wearing a functional foot orthotic on the ankle joint angle of frontal surface of young adults with flatfoot. J. Phys. Ther. Sci. 2017, 29, 819-821. [CrossRef] [PubMed]

19. Stevens, J.A.; Corso, P.S.; Finkelstein, E.A.; Miller, T.R. The costs of fatal and nonfatal falls among older adults. Inj. Prev. 2006, 12, 290-295. [CrossRef] [PubMed]

20. Zecevic, A.A.; Salmoni, A.W.; Speechley, M.; Vandervoort, A.A. Defining a fall and reasons for falling: Comparisons among the views of seniors, health care providers, and the research literature. Gerontologist 2006, 46, 367-376. [CrossRef] [PubMed]

21. Blake, A.J.; Morgan, K.; Bendall, M.J.; Dallosso, H.; Ebrahim, S.B.J.; Arie, T.H.D.; Fentem, P.H.; Bassey, E.J. Falls by elderly people at home prevalence and associated factors. Age Ageing 1988, 17, 365-372. [CrossRef] [PubMed]

22. Begg, R.; Best, R.; Dell'Oro, L.; Taylor, S. Minimum foot clearance during walking: Strategies for the minimisation of tri-related falls. Gait Posture 2007, 25, 191-198. [CrossRef] [PubMed]

23. Moosabhoy, M.A.; Gard, S.A. Methodology for determining the sensitivity of swing leg toe clearance and leg length to swing leg joint angles during gait. Gait Posture 2006, 24, 493-501. [CrossRef] [PubMed]

24. Best, R.; Begg, R. A method for calculating the probability of tripping while walking. J. Biomech. 2008, 41, 1147-1151. [CrossRef] [PubMed]

25. Lugade, V.; Lin, V.; Chou, L.S. Centre of mass and base of support interaction during gait. Gait Posture 2011, 33, 406-411. [CrossRef] [PubMed]

26. Hof, A.L.; Gazendam, M.G.J.; Sinke, W.E. The condition for dynamic stability. J. Biomech. 2005, 38, 1-8. [CrossRef] [PubMed]

27. Lafond, D.; Duarte, M.; Prince, F. Comparison of three methods to estimate the centre of mass during balance assessment. J. Biomech. 2004, 37, 1421-1426. [CrossRef]

28. Kao, P.; Ferris, D.P. Motor adaptation during dorsiflexion-assisted walking with a powered orthosis. Gait Posture 2009, 29, 230-236. [CrossRef] [PubMed]

29. Rao, S.; Riskowski, J.; Hannan, M.T. Musculoskeletal Conditions of the Foot and Ankle: Assessments and Treatment Options. Best Pract. Res. Clin. Rheumatol. 2012, 26, 345-368. [CrossRef] [PubMed]

30. Bus, S.A.; van Deursen, R.W.M.; Kanade, R.V.; Wissink, M.; Manning, E.A.; van Baal, J.G.; Harding, K.G. Plantar pressure relief in the diabetic foot using forefoot offloading shoes. Gait Posture 2009, 4, 618-622. [CrossRef] [PubMed]

31. Telfer, S.; Woodburn, J.; Collier, A.; Cavanagh, P.R. Virtually optimised insoles for offloading the diabetic foot: A randomised crossover study. J. Biomech. 2017, 60, 157-161. [CrossRef] [PubMed]

32. Nyska, M.; Liberson, A.; McCabe, C.; Linge, K.; Klenrman, L. Plantar foot pressure distribution in patients with Hallux valgus treated by distal soft tissue procedure and proximal metatarsal osteotomy. Foot Ankle Surg. 1998, 4, 35-41. [CrossRef]

33. Koller, U.; Willegger, M.; Windhager, R.; Wanivenhaus, A.; Tmka, H.; Schuh, R. Plantar pressure characteristics in Hallux valgus feet. J. Orthop. Res. 2014, 32, 1688-1693. [CrossRef] [PubMed]

34. Morrison, K.E.; Kaminski, T.W. Foot characteristics in association with inversion ankle injury. J. Athlet. Train. 2007, 42, 135-142.

35. Reina, M.; Lafuente, G.; Munuera, P.V. Effect of custom-made foot orthoses in female hallux valgus after one-year follow up. Prosthet. Orthot. Int. 2013, 37, 113-119. [CrossRef] [PubMed]

36. De la Cruz, B.; Garcia, C.; Sanchez, M.D.; Albornoz, M.; Espejo, L.; Dominguez-Maldonado, G. Therapeutic physical exercise for lower limb overpronation in young athletes. Eur. J. Integr. Med. 2014, 7, 211-217. [CrossRef]

37. Stergiou, N.; Bates, B.T. The relationship between subtalar and knee joint function as a possible mechanism for running injuries. Gait Posture 1997, 6, 177-185. [CrossRef]

38. Usherwood, J.R.; Channon, A.J.; Myatt, J.P.; Rankin, J.W.; Hubel, T.Y. The human foot and heel-sole-toe walking strategy: A mechanism enabling an inverted pendular gait with low isometric muscle force? J. R. Soc. 2012, 9, 2396-2402. [CrossRef] [PubMed]

39. Tasci, B.G.; Sendur, O.F.; Aydemir, A.H. Primary knee osteoarthritis increases the risk of falling. J. Back Musculoskelet. Rehabil. 2017, 30, 785-789. [CrossRef] [PubMed]

40. Jones, R.K.; Nester, C.J.; Richards, J.D.; Kim, W.Y.; Johnson, D.S.; Jari, S.; Laxton, P.; Tyson, S.F. A comparison of the biomechanical effects of valgus knee braces and lateral wedged insoles in patients with knee osteoarthritis. Gait Posture 2013, 37, 368-372. [CrossRef] [PubMed] 
41. Weinhandl, J.T.; Sudheimer, S.E.; Van Lunen, B.L.; Stewart, K.; Hock, M.C. Immediate and 1 week effects of laterally wedge insoles on gait biomechanics in healthy females. Gait Posture 2016, 45, 164-169. [CrossRef] [PubMed]

42. Dillon, C.F.; Rasch, E.G.; Gu, Q.; Hirsch, R. Prevalence of knee osteoarthritis in the United States: Arthritis data from the third national health and nutrition examination survey 1991-1994. J. Rheumatol. 2006, 33, 2271-2279. [PubMed]

43. Jones, R.K.; Chapman, G.J.; Parkes, M.J.; Forsythe, L.; Felson, D.T. The effect of different types of insoles or shoe modifications on medial loading of the knee in persons with medial knee osteoarthritis: A randomised trial. J. Orthop. Res. 2015, 33, 1646-1654. [CrossRef] [PubMed]

44. Nagano, H.; Tatsumi, I.; Sarashina, E.; Sparrow, W.A.; Begg, R.K. Modelling knee flexion effects on joint power absorption and adduction moment. Knee 2015, 22, 490-493. [CrossRef] [PubMed]

45. Levinger, P.; Menz, H.B.; Fotoohabadi, M.R.; Feller, J.A.; Bartlett, J.R.; Bergman, N.R. Foot posture in people with medial compartment knee osteoarthritis. J. Foot Ankle Res. 2010, 3, 29. [CrossRef] [PubMed]

46. Levinger, P.; Menz, H.B.; Morrow, A.D.; Bartlett, J.R.; Feller, J.A.; Bergman, N.R. Relationship between foot function and medial knee joint loading in people with medial compartment knee osteoarthritis. J. Foot Ankle Res. 2013, 6, 33. [CrossRef] [PubMed]

47. Chen, Y.C.; Lou, S.Z.; Huang, C.Y.; Su, F.C. Effects of foot orthoses on gait patterns of flat feet patients. Clin. Biomech. 2010, 25, 265-270. [CrossRef] [PubMed]

48. Aruin, A.S.; Kanekar, N. Effect of a textured insole on balance and gait symmetry. Exp. Brain Res. 2013, 231, 201-208. [CrossRef] [PubMed]

49. Yung-Hui, L.; Wei-Hsien, H. Effects of shoe inserts and heel height on foot pressure, impact force, and perceived comfort during walking. Appl. Ergon. 2005, 36, 355-362. [CrossRef] [PubMed]

50. Benocci, M.; Rocchi, L.; Farella, E.; Chiari, L.; Benini, L. A wireless system for gait and posture analysis based on pressure insoles and inertial measurement units. In Proceeding of the 3rd IEEE International Conference on Pervasive Computing Technologies for Healthcare-Pervasive Health (PCTHealth '09), London, UK, 1-3 April 2009.

51. Creaby, M.W.; May, K.; Bennell, K.L. Insole effects on impact loading during walking. Ergonomics 2011, 54, 665-671. [CrossRef] [PubMed]

52. Crabtree, P.; Dhokia, V.G.; Newman, S.T.; Answell, M.M. Manufacturing methodology for personalised symptom-specific sports insoles. Robot. Comput.-Integr. Manuf. 2009, 25, 972-979. [CrossRef]

53. De Castro, M.P.; Abreu, S.; Pinto, V.; Santos, R.; Machado, L.; Vaz, M.; Vilas-Boas, J.P. Influence of pressure-relief insoles developed for loaded gait (backpackers and obese people) on plantar pressure distribution and ground reaction forces. Appl. Ergon. 2014, 45, 1028-1034. [CrossRef] [PubMed]

54. Malisoux, L.; Delattre, N.; Urhausen, A.; Theisen, D. Shoe cushioning, body mass and running biomechanics as risk factors for running injury: As study protocol for a randomised controlled trial. BMJ Open 2017, 7, e017379. [CrossRef] [PubMed]

55. Baltich, J.; Maurer, C.; Nigg, BM. Increased vertical impact forces and altered running mechanics with softer midsole shoes. PLoS ONE 2015, 10, e0125196. [CrossRef] [PubMed]

56. Zhang, Y.; Beaven, M.; Liu, G.; Xie, S. Mechanical efficiency of walking with spring-loaded axillary crutches. Assist. Technol. 2013, 25, 111-116. [CrossRef] [PubMed]

57. Dipaola, M.; Pavan, E.E.; Cattaneo, A.; Frazzitta, G.; Pezzoli, G.; Cavallari, P.; Frigo, C.A.; Isaias, I.U. Mechanical Energy Recovery during Walking in Patients with Parkinson Disease. PLoS ONE 2016, 11, e0156420. [CrossRef] [PubMed]

58. Neptune, R.R.; Zajac, F.E.; Kautz, S.A. Muscle mechanical work requirements during normal walking: The energetic cost of raising the body's center-of-mass is significant. J. Biomech. 2004, 37, 817-825. [CrossRef] [PubMed]

59. Silver-Thorn, B.; Herrmann, A.; Current, T.; McGuire, J. Effect of ankle orientation on heel loading and knee stability for post-stroke individuals wearing ankle-foot orthoses. Prosthet. Orthot. Int. 2011, 35, 150-162. [CrossRef] [PubMed]

60. Fitzgerald, R.H.; Vedpathak, A. Plantar pressure distribution in a hyperpronated foot before and after intervention with an extraosseous talotarsal stabilisation device-A retrospective study. J. Foot Ankle Surg. 2013, 52, 432-443. [CrossRef] [PubMed] 
61. Brunner, R.; Rutz, E. Biomechanics and muscle function during gait. J. Child. Orthop. 2013, 7, 367-371. [CrossRef] [PubMed]

62. Nagano, H.; Begg, R.K.; Sparrow, W.A.; Taylor, S. Ageing and limb dominance effects on foot-ground clearance during treadmill and overground walking. Clin. Biomech. 2011, 26, 962-968. [CrossRef] [PubMed]

63. Fong, C.; Blackburn, J.T.; Norcros, M.F. Ankle-dorsiflexion range of motion and landing biomechanics. J. Athlet. Train. 2011, 46, 5-10. [CrossRef] [PubMed]

64. Cavagna, G.A.; Zamboni, A. The sources of external work in level walking and running. J. Physiol. 1976, 262, 639-657. [CrossRef] [PubMed]

65. Collett, J.; Dawes, H.; Howells, K.; Elswowrth, C.; Izadi, H.; Sackley, C. Anomalous centre of mass energy fluctuations during treadmill walking in healthy individuals. Gait Posture 2007, 26, 400-406. [CrossRef] [PubMed]

66. Schepens, B.; Bastien, J.; Heglund, C.; Williems, P.A. Mechanical work and muscular efficiency in walking children. J. Exp. Biol. 2004, 207, 587-596. [CrossRef] [PubMed]

67. Kuo, A. The six determinants of gait and the inverted pendulum analogy: A dynamic walking perspective. Hum. Mov. Sci. 2007, 26, 617-656. [CrossRef] [PubMed]

68. Ritchie, C.; Paterson, K.; Bryant, A.L.; Bartold, S.; Clark, R.A. The effects of enhanced plantar sensory feedback and foot orthoses on midfoot kinematics and lower leg neuromuscular activation. Gait Posture 2011, 33, 576-581. [CrossRef] [PubMed]

69. Bus, S.A.; Ulbrecht, J.S.; Cavanagh, P.R. Pressure relief and load redistribution by custom-made insoles in diabetic patients with neuropathy and foot deformity. Clin. Biomech. 2004, 19, 629-638. [CrossRef] [PubMed]

70. Bonanno, D.R.; Landorf, K.B.; Menz, H.B. Pressure-relieving properties of various shoe inserts in older people with plantar heel pain. Gait Posture 2011, 33, 385-389. [CrossRef] [PubMed]

71. Shankthikumar, S.; Low, Z.; Falvey, E.; McCrory, P.; Franklyn-Miler, A. The effect of gait velocity on calcaneal balance at heel strike; implications for orthotic prescription in injury prevention. Gait Posture 2010, 31, 9-12. [CrossRef] [PubMed]

72. Nurse, M.A.; Nigg, B.M. The effect of changes in foot sensation on plantar pressure and muscle activity. Clin. Biomech. 2001, 16, 719-727. [CrossRef]

73. Nurse, M.A.; Hulliger, M.; Wakeling, J.M.; Nigg, B.M.; Stefanyshyn, D.J. Changing the texture of footwear can alter gait patterns. J. Electromyogr. Kinesiol. 2005, 15, 496-506. [CrossRef] [PubMed]

74. Lafond, D.; Mouchnino, L.; Prince, F. Tactile stimulation of insoles and balance control in elderly people. Lancet 2004, 363, 84-86. [CrossRef]

75. Ma, C.C.; Lee, Y.; Chen, B.; Aruin, A.S. Immediate and short-term effects of wearing a single textured insole on symmetry of stance and gait in healthy adults. Gait Posture 2016, 49, 190-195. [CrossRef] [PubMed]

76. Bancroft, R.J.; McDonough, T.; Shakespeare, J.; Lynas, K. Orthotics. Eur. Geriatr. Med. 2011, 2, 122-125. [CrossRef]

77. Alfuth, M. Textured and stimulating insoles for balance and gait impairments in patients with multiple sclerosis and Parkinson's disease: A systematic review and meta-analysis. Gait Posture 2017, 51, 132-141. [CrossRef] [PubMed]

78. Orth, D.; Davids, K.; Wheat, J.; Seifert, L.; Liukkonen, J.; Jaakkola, T.; Ashford, D.; Kerr, G. The role of textured material in supporting perceptual-motor functions. PLoS ONE 2013, 8, e60349. [CrossRef] [PubMed]

79. Hatton, A.L.; Dixon, J.; Rome, K.; Brauer, S.G.; Williams, K.; Kerr, G. The effects of prolonged wear of textured shoe insoles on gait, foot sensation and proprioception in people with multiple sclerosis: Study protocol for a randomised controlled trial. Trials 2016, 17, 208. [CrossRef] [PubMed]

80. Xing, F.; Lu, B.; Kuang, M.; Wang, Y.; Zhao, Y.; Zhao, J.; Sun, L.; Wang, Y.; Ma, J.; Ma, X. A systematic review and meta-analysis into the effect of lateral wedge arch support insoles for reducing knee joing load in patients with medial knee osteoarthritis. Medicine 2017, 96, e7168. [CrossRef] [PubMed]

81. Hsieh, R.; Lee, W. Clinical effects of lateral wedge arch support insoles in knee osteoarthritis. Medicine 2016, 95, 27. [CrossRef] [PubMed]

82. Sawada, T.; Tokuda, K.; Tanimoto, K.; Iwamoto, Y.; Ogata, Y.; Anan, M.; Takahashi, M.; Kito, N.; Shinkoda, K. Foot alignments influence the effect of knee adduction moment with lateral wedge insoles during gait. Gait Posture 2016, 49, 451-456. [CrossRef] [PubMed]

83. Kluge, F.; Krinner, S.; Lochmann, M.; Eskofier, B.M. Speed dependent effects of laterally wedged insoles on gait biomechanics in healthy subjects. Gait Posture 2017, 55, 145-149. [CrossRef] [PubMed] 
84. Nakajima, K.; Kakihana, W.; Nakagawa, T.; Mitomi, H.; Hikita, A.; Suzuki, R.; Akai, M.; Iwaya, T.; Fukui, N. Addition of an arch support improves the biomechanical effect of a laterally wedged insole. Gait Posture 2009, 29, 208-213. [CrossRef] [PubMed]

85. Willems, T.; Witvrouw, E.; Delbaere, K.; De Cock, A.; De Clercq, D. Relationship between gait biomechanics and inversion sprains: A prospective study of risk factors. Gait Posture 2005, 21, 379-387. [CrossRef] [PubMed]

86. Enna, C.D.; Brand, P.W.; Reed, J.K.; Welch, D. The orthotic care of the denervated foot in Hansen's disease. Orthot. Prosthet. 1976, 30, 33-39.

87. Verity, S.; Sochocki, M.; Embil, J.M.; Trepman, E. Treatment of Charcot foot and ankle with a prefabricated removable walker brace and custom insole. Foot Ankle Surg. 2008, 14, 26-31. [CrossRef] [PubMed]

88. Dombroski, C.E.; Balsdon, M.E.; Froats, A. The use of a low cost 3D scanning and printing tool in the manufacture of custom-made foot orthoses: A preliminary study. BMC Res. Notes 2014, 7, 443. [CrossRef] [PubMed]

89. Menz, H.B.; Auhl, M.; Ristevski, S.; Frescos, N.; Munteanu, S.E. Evaluation of the accuracy of shoe fitting in older people using three-dimensional foot scanning. J. Foot Ankle Res. 2014, 7, 3. [CrossRef] [PubMed]

90. Whittle, M. Gait Analysis: An Introduction, 4th ed.; Butterworth-Heinemann Elsevier: Amsterdam, The Netherland, 2007; ISBN 9780750688833.

91. Nagano, H.; Sparrow, W.A.; Begg, R.K. Biomechanical characteristics of slipping during unconstrained walking, turning, gait initiation and termination. Ergonomics 2013, 56, 1038-1048. [CrossRef] [PubMed]

92. Nagano, H.; Sparrow, W.; Bowman, R.; Begg, R. A Biomechanical Model of the Spacing and Width Effects of Anti-Slip Strips. In Proceeding of the International Conference on Fall Prevention and Protection, Tokyo, Japan, 23-25 October 2013; pp. 105-111.

93. Santhiranayagam, B.K.; Lai, D.T.H.; Sparrow, W.A.; Begg, R.K. A machine learning approach to estimate minimum toe clearance using inertial measurement units. J. Biomech. 2015, 48, 4309-4316. [CrossRef] [PubMed]

94. Aqueveque, P.; Sobarzo, S.; Saavedra, F.; Maldonado, C.; Gomez, B. Android platform for realtime gait tracking using inertial measurement units. Eur. J. Transl. Myol. 2016, 26, 262-267. [CrossRef] [PubMed]

95. Yang, S.; Li, Q. Inertial sensor-based methods in walking speed estimation: A systematic review. Sensors 2012, 12, 6102-6116. [CrossRef] [PubMed]

96. Stassi, S.; Canavese, G.; Cauda, V.; Fallauto, C.; Corbellini, S.; Motto, P.; Demarchi, D.; Pirri, C.F. Wearable and flexible pedobarographic insole for continuous pressure monitoring. In Proceeding of the IEEE Sensors, Baltimore, MD, USA, 3-6 November 2013; pp. 1-4.

97. Jagos, H.; Oberzaucher, J.; Reichel, M.; Zagler, W.L.; Hlauschek, W. A multimodal approach for insole motion measurement and analysis. Procedia Eng. 2010, 2, 3103-3108. [CrossRef]

98. Jagos, H.; Pils, K.; Haller, M.; Wassermann, C.; Chhatwal, C.; Rafolt, D.; Rattay, F. Mobile gait analysis via eSHOEs instrumented shoe insoles: A pilot study for validation against the gold standard GAITRite®. J. Med. Eng. Technol. 2017, 41, 375-386. [CrossRef] [PubMed]

99. Zhang, Z.; Poslad, S. Improved use of foot force sensors and mobile phone GPS for mobility activity recognition. Proc. IEEE Sens. 2014, 14, 4340-4347. [CrossRef]

100. Franklin, S.; Grey, M.J.; Heneghan, N.; Bowen, L.; Li, F.X. Barefoot vs common footwear: A systematic review of the kinematic, kinetic and muscle activity differences during walking. Gait Posture 2015, 42, 230-239. [CrossRef] [PubMed]

101. Riskowski, J.; Dufour, A.B.; Hannan, M.T. Arthritis, foot pain and shoe wear: Current musculoskeletal research on feet. Curr. Opin. Rheumatol. 2011, 23, 148-155. [CrossRef] [PubMed]

(C) 2018 by the authors. Licensee MDPI, Basel, Switzerland. This article is an open access article distributed under the terms and conditions of the Creative Commons Attribution (CC BY) license (http:/ / creativecommons.org/licenses/by/4.0/). 


\title{
Article \\ Self-Tuning Threshold Method for Real-Time Gait Phase Detection Based on Ground Contact Forces Using FSRs
}

\author{
Jing Tang ${ }^{1,2}$, Jianbin Zheng ${ }^{1,2}$, Yang Wang ${ }^{1,2, *}$, Lie Yu ${ }^{3}$, Enqi Zhan ${ }^{1,2}$ and Qiuzhi Song ${ }^{4}$ \\ 1 School of Information Engineering, Wuhan University of Technology, Wuhan 430070, China; \\ mimitang85119@163.com (J.T.); jbzheng@whut.edu.cn (J.Z.); eqzhan@whut.edu.cn (E.Z.) \\ 2 Key Laboratory of Fiber Optic Sensing Technology and Information Processing, Ministry of Education, \\ Wuhan University of Technology, Wuhan 430070, China \\ 3 School of Electronic and Electrical Engineering, Wuhan Textile University, Hongshan District, \\ Wuhan 430070, China; lyu@wtu.edu.cn \\ 4 School of Electromechanical, Beijing Institute of Technology, Beijing 100081, China; qzhsong@bit.edu.cn \\ * Correspondence: powerflow@whut.edu.cn; Tel.: +86-1334-996-6226
}

Received: 8 December 2017; Accepted: 31 January 2018; Published: 6 February 2018

\begin{abstract}
This paper presents a novel methodology for detecting the gait phase of human walking on level ground. The previous threshold method (TM) sets a threshold to divide the ground contact forces (GCFs) into on-ground and off-ground states. However, the previous methods for gait phase detection demonstrate no adaptability to different people and different walking speeds. Therefore, this paper presents a self-tuning triple threshold algorithm (STTTA) that calculates adjustable thresholds to adapt to human walking. Two force sensitive resistors (FSRs) were placed on the ball and heel to measure GCFs. Three thresholds (i.e., high-threshold, middle-threshold andlow-threshold) were used to search out the maximum and minimum GCFs for the self-adjustments of thresholds. The high-threshold was the main threshold used to divide the GCFs into on-ground and off-ground statuses. Then, the gait phases were obtained through the gait phase detection algorithm (GPDA), which provides the rules that determine calculations for STTTA. Finally, the STTTA reliability is determined by comparing the results between STTTA and Mariani method referenced as the timing analysis module (TAM) and Lopez-Meyer methods. Experimental results show that the proposed method can be used to detect gait phases in real time and obtain high reliability when compared with the previous methods in the literature. In addition, the proposed method exhibits strong adaptability to different wearers walking at different walking speeds.
\end{abstract}

Keywords: adaptability; force sensitive resistors; self-tuning triple threshold algorithm

\section{Introduction}

From a medical and industrial perspective, wearable devices have evolved and continue to develop in terms of providing assistance to humans [1]. Gait analysis through wearable devices is an extensive area in the field of biomechanics that provides knowledge in terms of identifying pathologies, evaluating athletes' performance, design of sports products and rehabilitation engineering [2]. Generally, the wearable devices used for gait analysis are designed by equipping various sensors on it.

Thus far, different kinds of sensor types, including force sensitive resistors (FSRs) [3-5], air pressure sensors [6], inertial sensors [7-14], inclinometers [15], foot switches [16], and electromyography (EMG) sensors [17], are readily available in the industry and can be applied to gait analysis. Yan et al. [18] estimated the gait events through a sensory apparatus, which combine three subsystems such as a primary phase estimator, a desired gait event detector and a phase error compensator. This method 
is sensitive to the tuning of the adaptive oscillators parameters. However, different parameters may change the results. In the literature, several works $[11,12]$ have detected the gait phase using the inertial sensors that could measure the body segment orientations and joint angles. Bejarano et al. [13] proposed an adaptive algorithm based on the inertial and magnetic sensors to detect the gait events. However, the inertial sensor is sensitive to temperature, shock and magnetic disturbances, which would result in the misdetections of gait phases. In order to overcome these limitations, Muller et al. [14] proposed a novel online gait phase detection algorithm, which can be used indoor and in the presence of magnetic disturbances. However, the delays of the heel off and initial contact are considerably high. Nevertheless, an alternative solution to inertial sensors is FSRs [16]. As described by Catalfamo et al. [19], force platforms, such as FSRs, represent the gold standard method for gait analysis. Yu et al. [20] used proportion an adaptive method, which calculates the sums and proportions of ground contact forces (GCFs). However, one parameter is affected by the attachment of the shoe to the foot, which would affect the reliability of the whole system. Additionally, force platforms measure the GCFs to detect gait phases, and each gait phase has a unique GCF pattern [7].

As reported by Smith et al. [21], 80\% of errors in gait phase detection using FSRs was due to the setting of threshold value. In order to set appropriate thresholds for gait phase detection, a number of researchers $[4,5,19]$ had presented their methods. Mariani et al. [4] defined $5 \%$ body weight as a threshold. However, different subjects had different body weights such that different thresholds should be set for different subjects. In addition, no matter how slowly or fast the subject walked, only one threshold was set for the same subject in all experiments. As a result, this method of using body weight percentage as a threshold was not adaptable to different subjects and different walking speeds (i.e., slow and fast). Lopez-Meyer et al. [5] and Catalfamo et al. [19] used the maximum and minimum GCFs of gait cycles to compute the threshold. However, the use of the maximum and minimum GCFs for the threshold computation could not detect gait phases in real time because the maximum and minimum GCFs were obtained in data post-processing.

The main purpose of this study is to develop a gait phase detection method, which can detect gait phases in real time during different walking trials. Therefore, we propose a self-tuning triple-threshold algorithm (STTTA), which obtains the maximum and minimum GCFs of the present gait cycle to calculate adjustable thresholds. In addition, the acquirement of the maximum and minimum GCFs, and the calculations of adjustable thresholds are completed in the walking process. This paper also proposes a gait phase detection algorithm (GPDA), which provides the rules that determine calculations for STTTA. In order to evaluate the reliability of STTTA, three previous methods in the literature are introduced to obtain comparative results.

We hypothesize that the proposed STTTA can gain high and stable reliabilities for different subjects walking at different speeds. To better test the adaptability, the same initial threshold values are set for all subjects in all experiments.

\section{Methods}

\subsection{Subjects}

This study included fourteen males and ten females (age $=24.5 \pm 2.0$ years, weight $=67.3 \pm 8.8 \mathrm{~kg}$; mean \pm SD) with no history of foot diseases. All of them volunteered to participate in our experiments. The participants walked on the treadmill for $30 \mathrm{~s}$ duration at designated constant speeds of $2 \mathrm{~km} / \mathrm{h}$, $3 \mathrm{~km} / \mathrm{h}, 4 \mathrm{~km} / \mathrm{h}, 5 \mathrm{~km} / \mathrm{h}$ and $6 \mathrm{~km} / \mathrm{h}$, respectively.

\subsection{Instrumentation and Data Processing}

As shown in Figure 1, the sensor units comprised two FSRs (LOSON LSH-10, LOSON Instrumetation, Nankin, China), which were located in the sole of the ball and the sole of the heel. The FSRs collected GCF data at a frequency of $2000 \mathrm{~Hz}$ with a high resolution of 16 bits AD converter using an ARM11 computer (S3C6410). The measuring range of each FSR was 0-200 kg. The accuracy 
(including linearity and repeatability) of each FSR was $\pm 0.5 \%$ full scale (FS). Standard load cells $(5 \mathrm{~kg}, 10 \mathrm{~kg}, 20 \mathrm{~kg}, 25 \mathrm{~kg}, 50 \mathrm{~kg}, 100 \mathrm{~kg}$ and $200 \mathrm{~kg}$ ) were used to calibrate the FSRs. As the FSRs output a weak micro-voltage signal, an amplification circuit was equipped. Because of this circuit, the output signal of each FSR was amplified to 0-5 V, which correlated with the measured mass of 0-200 kg.

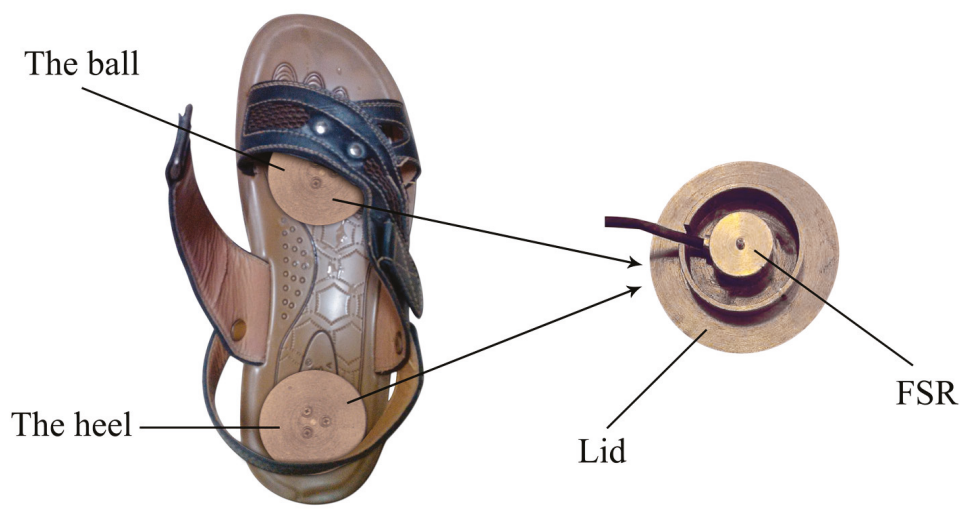

Figure 1. FSRs placed inside one shoe with one in the ball and the other in the heel. A lid is made to enlarge the press area.

After data acquisition, the GCF data from FSRs were filtered by second order Butterworth low pass filter with a cut-off frequency of $10 \mathrm{~Hz}$. The results of the gait phase detection were processed in Matlab (version2012, MathWorks, MA, USA) using the proposed method.

\subsection{Previous Methods}

In previous, the GCFs measured by an FSR can be divided into on-ground and off-ground statuses through setting a threshold $T$ :

$$
G=\left\{\begin{array}{l}
\text { on-ground, } F \geq T \\
\text { off }- \text { ground, } F<T
\end{array},\right.
$$

where $F$ is the GCF from the ball or heel, and Tis the threshold. $G$ is the result that "on-ground" status means "FSR pressed" and "off-ground" status means "FSR not pressed".

In our experiments, three previous ways were applied to the threshold computations. To be specific, one way was to define body weight percentage as a threshold, which had been affirmed to detect gait phase in real time. The other two ways were to use the maximum and minimum GCFs of gait cycles for threshold calculation. Meanwhile, the two ways carried out data post-processing to calculate an appropriate threshold, and were finally chosen as reference methods.

\subsubsection{Mariani Method}

Mariani et al. [4] defined 5\% of body weight to compute the threshold T:

$$
T=0.05 \cdot \mathrm{mg}
$$

where $\mathrm{m}$ is the subject's mass and $\mathrm{g}$ is the acceleration of gravity.

\subsubsection{Timing analysis module Method}

Catalfamo et al. [19] employed a Timing analysis module (TAM) software (version5.24, Tekscan, South Boston, MA, USA) as their reference method. After data acquisition of each experiment, 
the maximum and minimum GCFs (i.e., $T_{\max }$ and $T_{\min }$, respectively) were searched out for threshold calculation:

$$
T=T_{\min }+\left(T_{\max }-T_{\min }\right) \times \frac{10}{100} .
$$

Additionally, $T$ needed to be calculated severally for each set of FSR in each experiment.

\subsubsection{Lopez-Meyer Method}

Lopez-Meyer et al. [5] used the average value of the maximum GCFs and the average value of the minimum GCFs to compute the threshold T. For each experiment, the threshold computation needed all the local maximum GCFs and local minimum GCFs (i.e., $T_{\max }(i)$ and $T_{\min }(j)$, respectively) of gait cycles. In a complete gait cycle, there were only one $T_{\max }$ and only one $T_{\min }$ for each set of FSR. In one experiment, there were many gait cycles. For one set of FSR, there were $k T_{\max }$ and $l T_{\min }$ in one experiment. However, $k$ did not equal $l$ as there might exist an incomplete gait cycle in the experiment:

$$
\begin{gathered}
T_{M A X}=\frac{1}{k} \sum_{i=1}^{k} T_{\max }(i), \\
T_{M I N}=\frac{1}{l} \sum_{j=1}^{l} T_{\min }(j), \\
T=T_{M I N}+\alpha\left(T_{M A X}-T_{M I N}\right),
\end{gathered}
$$

where $\alpha$ was a proportional factor for the threshold adjustment to compensate for interindividual variability in pressure levels. In our experiments, the selection of proportional factor was made that $\alpha$ was set to 0.084 .

\subsection{Self-Tuning Triple-Threshold Algorithm}

With respect to the Mariani method, different thresholds should be set for different subjects, and the same threshold is set for one subject in five experiments with different walking speed each time. The Mariani method is not adaptable to different subjects and different walking speeds. Meanwhile, the TAM and Lopez-Meyer methods both carry out data post-processing to calculate the thresholds and are incapable of real-time application. In order to seek a method to detect gait phase in real time and be adaptable to different walking conditions, we propose a self-tuning triple-threshold algorithm (STTTA) that uses three thresholds to obtain the maximum and minimum GCFs for threshold computations.

\subsubsection{Setting of Three Thresholds}

In this section, the magnitudes of three thresholds are amplified. Figure 2 demonstrates the GCFs processing through three thresholds, including high-threshold, middle-threshold and low-threshold (i.e., $T_{H}, T_{M}$ and $T_{L}$, respectively). Specifically, $T_{H}$ is utilized to search the maximum GCF (i.e., $T_{\max }(j)$ ) of the present gait cycle, and chosen as the main threshold that divides the GCFs into on-ground and off-ground statuses. $T_{M}$ is used to search time points (points $b$, e and h shown in Figure 2) for the adjustments of $T_{H}$ and $T_{L}$. Meanwhile, $T_{L}$ is employed to search the minimum GCF (i.e., $T_{\min }(j)$ ) of the present gait cycle, and the time point (point $\mathrm{f}$ ) for the adjustments of $T_{M}$.

At the present gait cycle, the three thresholds are newly calculated for the next gait cycle. The three thresholds (i.e., $T_{H}(i-1), T_{M}(i-1)$ and $T_{L}(i-1)$ ), by which the GCFs are processed at the present gait cycle, have been calculated at the last gait cycle. 


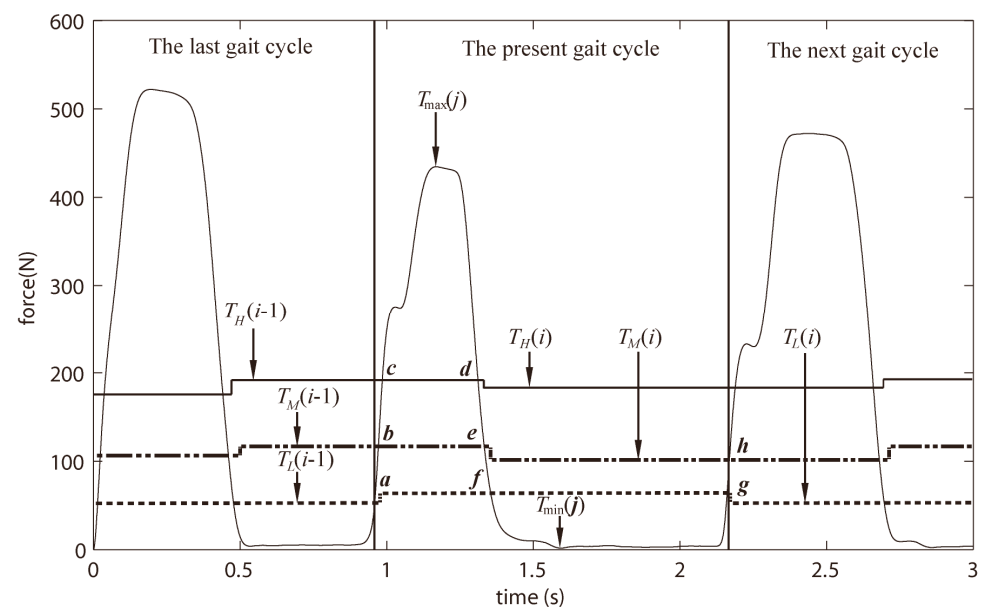

Figure 2. Three thresholds used for the GCFs processing.

As Figure 2 shows, the present gait cycle starts at point a and ends at point $\mathrm{g}$. Point a is the end point of the last gait cycle, and also the start point of the present gait cycle. Point $b$ is used to calculate the $T_{L}(i-1)$. Points $\mathrm{c}$ and $\mathrm{d}$ make a region $[\mathrm{c}, \mathrm{d}]$. When the GCF $(F(k))$ is larger than $T_{H}(i-1)$, it goes to region $[c, d]$. Then, the $T_{\max }(j)$ can be searched out as follows:

$$
T_{\max }(j)=M A X=\left\{\begin{array}{l}
F(k), F(k) \geq T_{H}(i-1) \& F(k-1)<T_{H}(i-1) \\
F(k), F(k)>M A X \& F(k)>T_{H}(i-1) \& F(k-1) \geq T_{H}(i-1) \\
M A X, F(k)<M A X \& F(k)>T_{H}(i-1) \& F(k-1)>T_{H}(i-1)
\end{array},\right.
$$

where $k$ is the total number of the collected GCFs from one FSR in each experiment. The numerical range of $k$ is $1 \sim 60,000$ because each experiment lasts $30 \mathrm{~s}$ with a sampling frequency of $2000 \mathrm{~Hz}$.

At point $\mathrm{e}, T_{H}(i)$ is calculated as a new threshold for the next gait cycle:

$$
T_{H}(i)=\beta \cdot\left(T_{\max }(j)-T_{L}(i-1)\right)+T_{L}(i-1),
$$

where $\beta$ is a proportion factor and chosen to be a constant. $i$ means that the present gait cycle is the $i$-th step of one subject walking in one experiment, while $j$ means that the $T_{\max }(j)$ is the $j$-th maximum GCF of the total gait cycles. However, $i$ was not necessarily equal to $j$ as incomplete gait cycles may have occurred.

At point $\mathrm{f}, T_{M}(i)$ is newly calculated for the next gait cycle:

$$
T_{M}(i)=\gamma \cdot\left(T_{\max }(j)-T_{L}(i-1)\right)+T_{L}(i-1),
$$

where $\gamma$ is a proportion factor and chosen to be a constant.

In common with points $\mathrm{c}$ and $\mathrm{d}$, the points $\mathrm{f}$ and $\mathrm{g}$ also make a region $[\mathrm{f}, \mathrm{g}]$. The calculation of $T_{\min }(j)$ is made in the regions $[\mathrm{f}, \mathrm{g}]$, which means that the $F(k)$ is smaller than $T_{L}(i-1)$ :

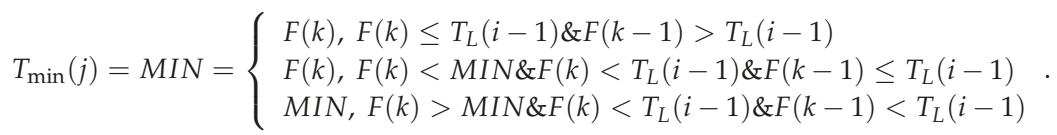

Additionally, point $\mathrm{g}$ is the end point of the present gait cycle, and also the start point of the next gait cycle. 
However, $T_{L}(i)$ is newly set at point $h$, as the same to $T_{L}(i-1)$ calculated at point $b$ :

$$
T_{L}(j)=\lambda \cdot T_{\min }(j)+(1-\lambda) \cdot T_{M}(i),
$$

where $\lambda$ is a proportion factor and chosen to be a constant.

\subsubsection{Setting of Initial Threshold Values}

Before the experiments, it is necessary to set initial values for the three thresholds. In this paper, $T_{H}(1), T_{M}(1)$ and $T_{L}(1)$ are the initial values of $T_{H}, T_{M}$ and $T_{L}$. However, the setting should follow that $T_{L}(1)<T_{M}(1)<T_{H}(1)$.

\subsection{Gait Phase Detection Algorithm}

The gait cycle can be divided into stance-phase and swing-phase. The transitions between gait phases are gait events. As proposed by Pappas et al. [3,22], swing-phase, stance-phase, heel-strike and heel-off are the most common gait phases and gait events for a single foot. For the proposed STTTA, $T_{H}$ is the main threshold, which divided the GCFs into on-ground and off-ground statuses:

$$
G=\left\{\begin{array}{l}
\text { on }- \text { ground, } F \geq T_{H} \\
\text { off }- \text { ground, } F<T_{H}
\end{array} .\right.
$$

As is the same for Equation (1), $F$ is the GCF from the ball or heel, and $T_{H}$ is the threshold. $G$ is the result that "on-ground" status means "FSR pressed" and "off-ground" status means "FSR not pressed".

When the GCFs from the ball and heel are processed from Equations (1) and (12), the gait phases and gait events can be distinguished by following the rules in Table 1.

Table 1. Rules of gait phase detection algorithm.

\begin{tabular}{cccc}
\hline Heel & Ball & \multicolumn{2}{c}{ Gait Types } \\
\hline on-ground & on-ground & Gait phase & Stance \\
off-ground & off-ground & Gait phase & Swing \\
on-ground & off-ground & Gait event & Heel-strike \\
off-ground & on-ground & Gait event & Heel-off \\
\hline
\end{tabular}

\subsection{Reference Methods}

In order to obtain the reliability of the proposed STTTA, reference methods should be determined. The TAM and Lopez-Meyer methods carried out data post-processing to calculate an appropriate threshold for each FSR at each walking speed. In addition, the TAM method had been chosen as a reference method to test the reliability of gait events detection. On the other hand, the Lopez-Meyer method had been tested on a shoe-based wearable sensor system, which had been compared with the "GAITRite system". The Lopez-Meyer method acquired 95\% confidence to compare detection results with the GAITRite system. Therefore, in this paper, the TAM and

Lopez-Meyer methods were both selected as reference methods. Finally, both the STTTA and Mariani methods were compared with the reference methods to obtain their reliabilities.

\section{Experimental Results}

\subsection{Selection of Coefficients}

Before the experiments, the values of $T_{H}(1), T_{M}(1)$ and $T_{L}(1)$ were determined. The value selections of $T_{H}(1), T_{M}(1)$ and $T_{L}(1)$ followed the rules that $T_{\min }<T_{L}(1)<T_{M}(1)<T_{H}(1)<T_{\max }$. Based on data analysis, the value range of $T_{\min }$ was $0-5 \mathrm{~N}$ and the value range of $T_{\max }$ was $300-1300 \mathrm{~N}$. Therefore, the values of $T_{H}(1), T_{M}(1)$ and $T_{L}(1)$ could be selected within a wide range. To evaluate this in our 
experiments, we randomly chose a set of initial threshold values where $T_{L}(1)=15 \mathrm{~N}, T_{M}(1)=20 \mathrm{~N}$ and $T_{H}(1)=25 \mathrm{~N}$.

After the selection of $T_{H}(1), T_{M}(1)$ and $T_{L}(1)$, the proportion factor $\beta, \gamma$, and $\lambda$ in Equations (8), (9) and (11) were optimized to determine the highest reliability of STTTA. From Equations (9) and (11), the values of $T_{M}$ and $T_{L}$ were affected by each other. As a result, one of $\gamma$ and $\lambda$ should be firstly determined such that the value of $\lambda$ was chosen to be 0.5 . Then, using the data from nine subjects as training data, the reliabilities of the proposed STTTA were calculated by using various values of $\beta$ and $\gamma$, which is shown in Figure 3. The selections for $\beta$ and $\gamma$ to acquire the highest reliability was made such that $\beta=0.071$ and $\gamma=0.042$.

Finally, the same $T_{H}(1), T_{M}(1)$ and $T_{L}(1)$ were used for all 24 subjects in all experiments. In addition, data from the remaining subjects were processed using the selected $\beta, \gamma$, and $\lambda$.

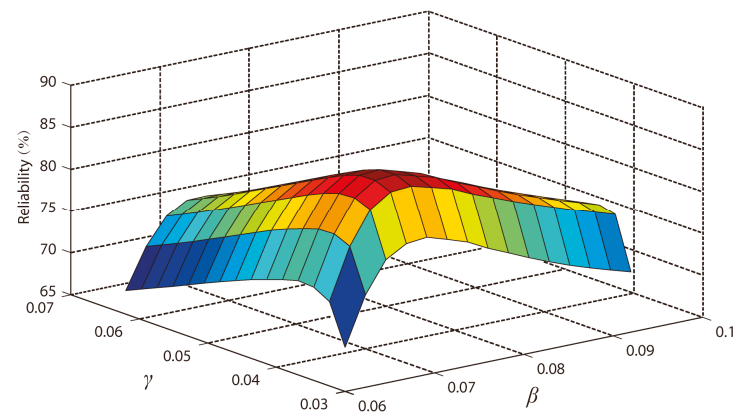

Figure 3. The reliabilities of the proposed STTTA in various values of $\beta$ and $\gamma$.

\subsection{The Results of Gait Phase Detection and Experimental Comparisons}

Figure 4 showed the detection results of the proposed STTTA. In Figure $4 \mathrm{a}$, the GCFs from the ball and heel were displayed. The self-adjustments of three thresholds for the GCFs from the ball and heel is demonstrated in Figure 4 b,c. It was qualitatively illustrated in Figure $4 d$ where the gait phases and gait events were detected through the proposed GPDA. In order to clarify the self-adjustment effect, the main threshold $\mathrm{T}_{\mathrm{H}}$ of the proposed STTTA was compared with the thresholds calculated by the previous methods. As pictured in Figure $4 \mathrm{e}, \mathrm{f}$, the amplitudes of the threshold $\mathrm{T}_{\mathrm{H}}$ were adjustable and adaptable to walking conditions, while constant thresholds were figured out for the previous methods.

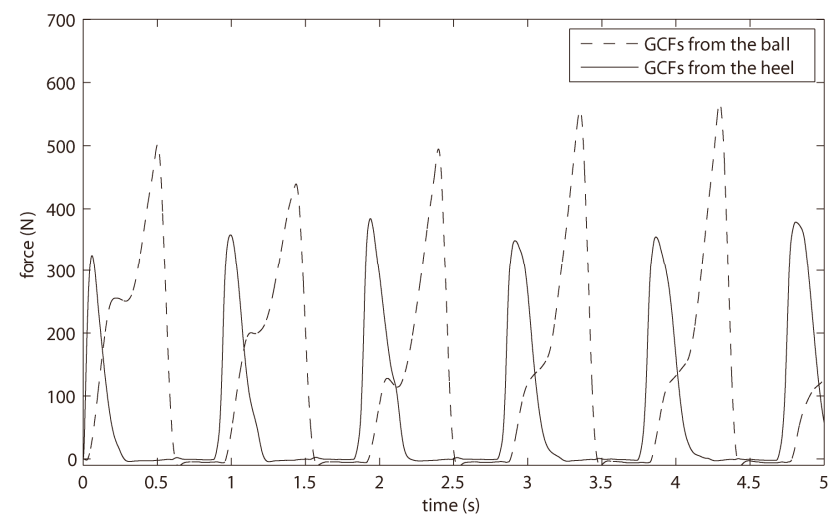

(a)

Figure 4. Cont. 


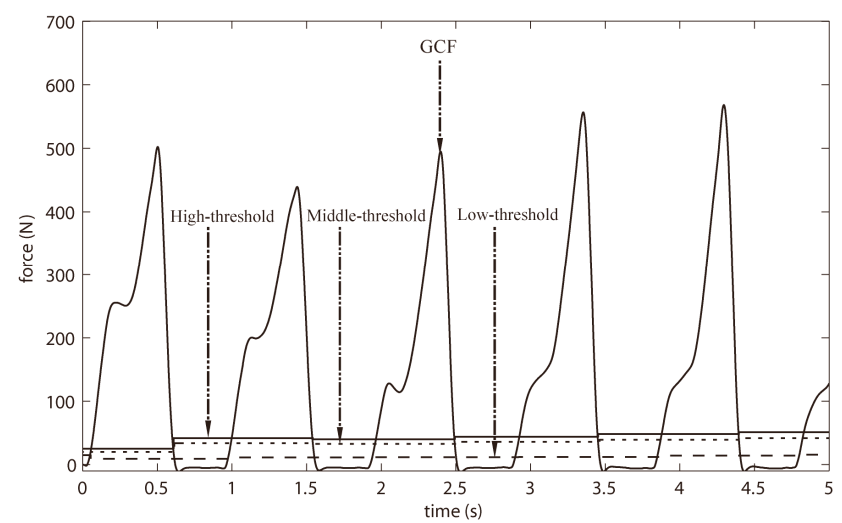

(b)

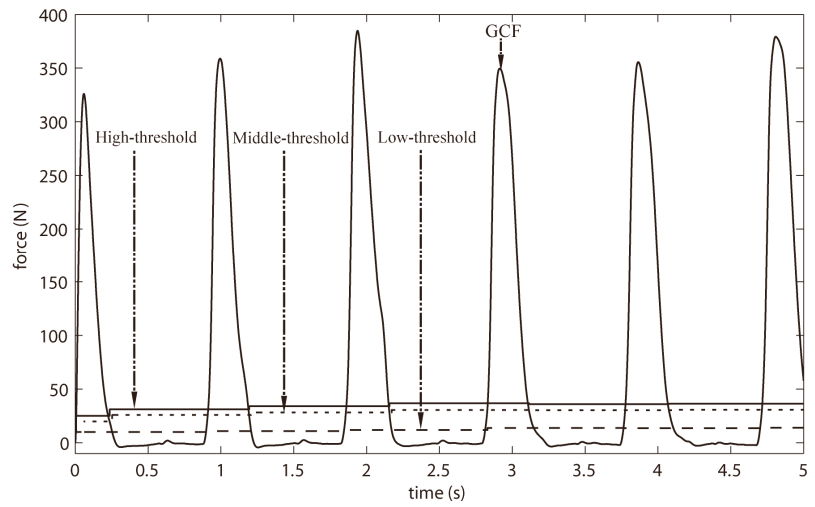

(c)

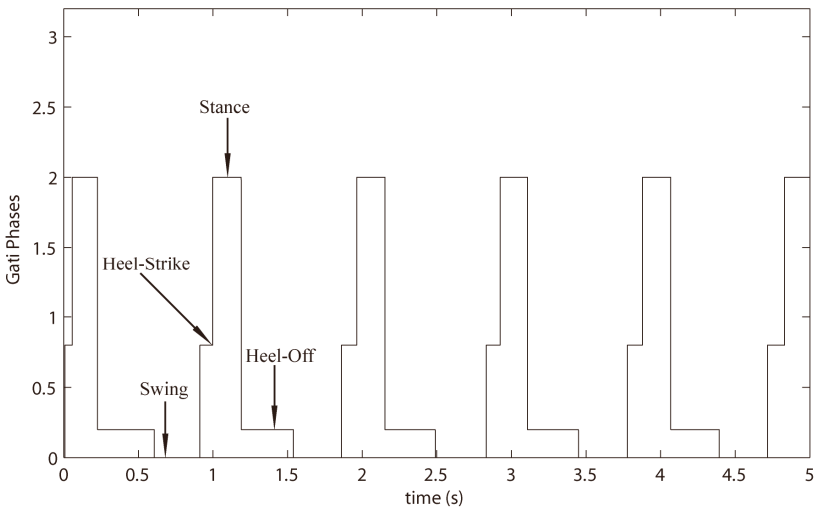

(d)

Figure 4. Cont. 


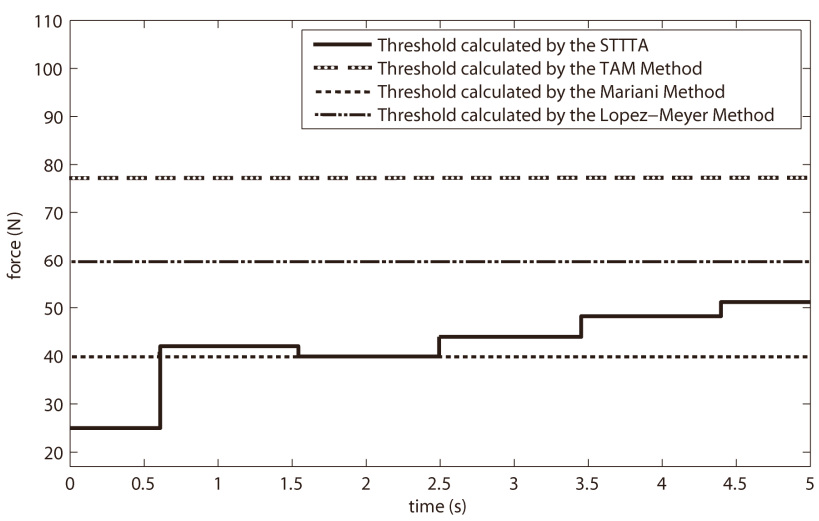

(e)

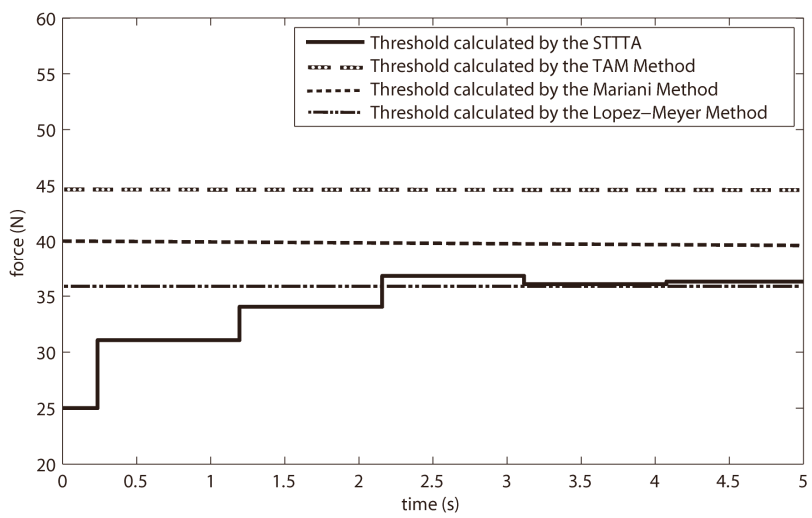

(f)

Figure 4. (a) the GCFs of heel and ball measured by FSRs; (b) three self-tuning thresholds for the processing of GCF from the ball; (c) three self-tuning threshold for the processing of GCF from the heel; (d) the result of gait phase detection through GPDA; (e) thresholds calculated for the ball by four kinds of methods; and (f) thresholds calculated for the heel by four kinds of methods.

\subsection{Real-Time Application for Gait Phase Detection}

After data acquisitions, the data processing included the initial threshold setting, acquirement of the maximum and minimum GCFs, and threshold computations, was made prior to the GPDA. When the threshold computations were achieved, the gait phase detection could be implemented through the GPDA.

In this gait phase detection system, the sampling frequency was set to $2000 \mathrm{~Hz}$. Data acquisitions, data processing and gait phase detection could be all accomplished within one sampling period. The time delay between data acquisition and gait phase detection was less than $0.5 \mathrm{~ms}$. Therefore, the proposed STTTA could be used to detect gait phases in real time.

\subsection{Adaptability to Different Walking Conditions}

Needless to obtain the body weights of all subjects, the proposed STTTA acquired higher average reliabilities than the Mariani method as shown in Table 2. In addition, irrespective of the reference method applied (TAM method or the Lopez-Meyer method), higher reliability was gained for the 
STTTA compared to the Mariani method. The comparative results affirmed that the proposed STTTA was more reliable than the Mariani method in real-time gait phases detection.

Table 2. Comparative reliability results for the proposed method.

\begin{tabular}{|c|c|c|c|c|c|}
\hline \multirow[b]{2}{*}{ Subjects } & \multirow[b]{2}{*}{ Gender } & \multicolumn{2}{|c|}{ Mariani Method } & \multicolumn{2}{|c|}{ The Proposed STTTA } \\
\hline & & $\begin{array}{c}\text { Compared } \\
\text { with TAM } \\
\text { Method }\end{array}$ & $\begin{array}{l}\text { Compared with } \\
\text { Lopez-Meyer } \\
\text { Method }\end{array}$ & $\begin{array}{c}\text { Compared } \\
\text { with TAM } \\
\text { Method }\end{array}$ & $\begin{array}{l}\text { Compared with } \\
\text { Lopez-Meyer } \\
\text { Method }\end{array}$ \\
\hline 1 & Male & $91.66 \%$ & $80.70 \%$ & $95.01 \%$ & $87.50 \%$ \\
\hline 2 & Male & $86.80 \%$ & $83.96 \%$ & $93.07 \%$ & $92.90 \%$ \\
\hline 3 & Male & $93.42 \%$ & $82.55 \%$ & $92.82 \%$ & $89.58 \%$ \\
\hline 4 & Male & $83.72 \%$ & $78.14 \%$ & $94.14 \%$ & $90.96 \%$ \\
\hline 5 & Male & $88.29 \%$ & $82.28 \%$ & $89.97 \%$ & $80.00 \%$ \\
\hline 6 & Male & $91.16 \%$ & $81.75 \%$ & $96.49 \%$ & $89.95 \%$ \\
\hline 7 & Male & $91.77 \%$ & $84.40 \%$ & $96.57 \%$ & $91.07 \%$ \\
\hline 8 & Male & $93.93 \%$ & $85.32 \%$ & $92.68 \%$ & $88.88 \%$ \\
\hline 9 & Male & $87.41 \%$ & $77.79 \%$ & $91.87 \%$ & $94.58 \%$ \\
\hline 10 & Male & $88.33 \%$ & $85.18 \%$ & $88.49 \%$ & $82.76 \%$ \\
\hline 11 & Male & $94.64 \%$ & $84.12 \%$ & $92.62 \%$ & $88.00 \%$ \\
\hline 12 & Male & $93.00 \%$ & $87.83 \%$ & $96.54 \%$ & $92.42 \%$ \\
\hline 13 & Male & $93.63 \%$ & $84.47 \%$ & $95.14 \%$ & $87.45 \%$ \\
\hline 14 & Male & $94.03 \%$ & $84.64 \%$ & $96.03 \%$ & $89.46 \%$ \\
\hline 15 & Female & $92.59 \%$ & $86.21 \%$ & $96.86 \%$ & $92.58 \%$ \\
\hline 16 & Female & $95.92 \%$ & $88.27 \%$ & $95.5 \%$ & $91.34 \%$ \\
\hline 17 & Female & $91.81 \%$ & $88.42 \%$ & $93.10 \%$ & $91.58 \%$ \\
\hline 18 & Female & $89.81 \%$ & $84.18 \%$ & $86.00 \%$ & $83.03 \%$ \\
\hline 19 & Female & $88.847 \%$ & $79.80 \%$ & $94.42 \%$ & $88.92 \%$ \\
\hline 20 & Female & $84.47 \%$ & $69.96 \%$ & $94.02 \%$ & $83.53 \%$ \\
\hline 21 & Female & $91.68 \%$ & $90.84 \%$ & $80.08 \%$ & $93.95 \%$ \\
\hline 22 & Female & $81.07 \%$ & $85.68 \%$ & $85.09 \%$ & $89.64 \%$ \\
\hline 23 & Female & $85.75 \%$ & $90.54 \%$ & $89.75 \%$ & $94.39 \%$ \\
\hline 24 & Female & $80.56 \%$ & $84.43 \%$ & $88.86 \%$ & $92.30 \%$ \\
\hline Average & -- & $89.62 \%$ & $83.81 \%$ & $92.29 \%$ & $89.45 \%$ \\
\hline
\end{tabular}

To demonstrate the adaptability of the proposed STTTA, the experimental results of one male subject and one female subject were taken as examples. As shown in Figure $5 \mathrm{a}-\mathrm{d}$, the Mariani method could achieve high reliability at one walking speed, and then reduce significant reliability at other walking speeds. However, the proposed STTTA gained stable reliabilities at five walking speeds. The stability to speeds proved that the proposed STTTA was adaptable to different walking speeds.

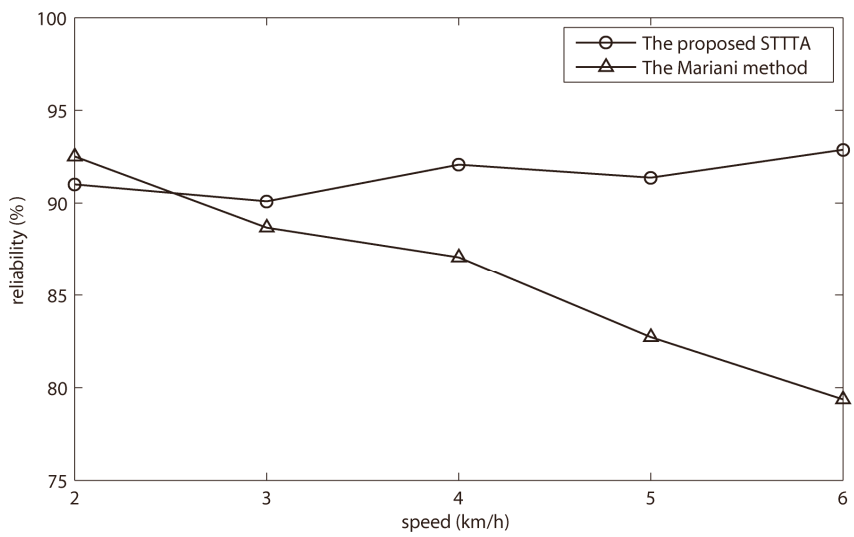

(a)

Figure 5. Cont. 


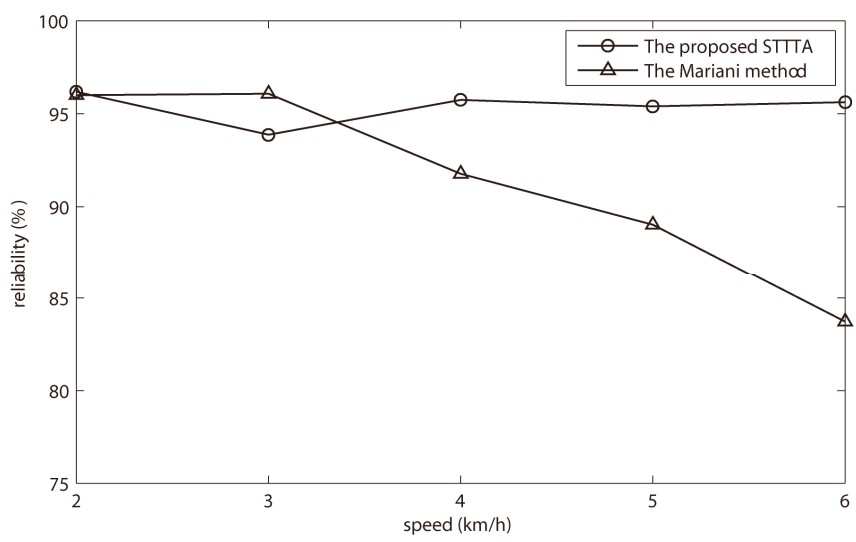

(b)

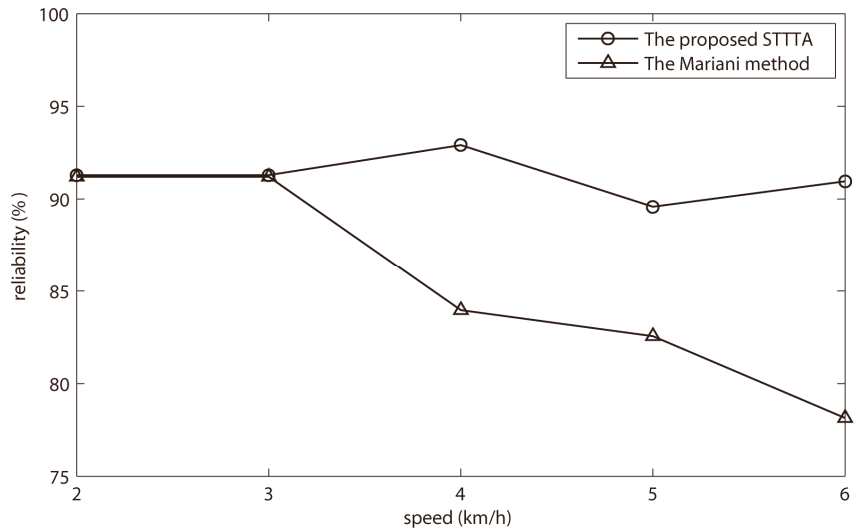

(c)

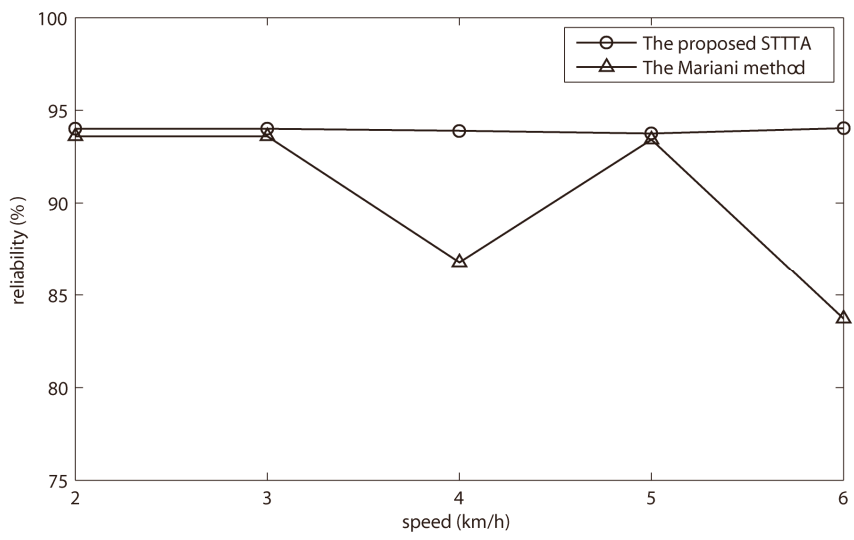

(d)

Figure 5. Reliabilities of the Mariani and STTTA method for one male subject at five walking speeds: (a) TAM method as reference method; (b) Lopez-Meyer method as reference method. Reliabilities of the Mariani and STTTA method for one female subject at five walking speeds; (c) TAM method as reference method; (d) Lopez-Meyer method as reference method. 


\section{Discussion}

\subsection{The Reason of Using Three Thresholds for GCFs Processing}

At the beginning, one threshold was expected to obtain the maximum and minimum GCFs of the present gait cycle, and the time point of a new threshold calculation for the next gait cycle. As shown in Figure 6a, the region (c,d) was used to search the maximum GCF of the present gait cycle, and the point $d$ was used as the time point for new threshold calculation. However, the new threshold would be calculated twice (i.e., it should be only once) occasionally at one gait cycle as shown in Figure $6 \mathrm{~b}$. The threshold $T(i)$, which was the correct threshold calculated for the next gait cycle at time point $d$. Nonetheless, it possibly existed that the GCFs after time point $d$ were larger than the newly computed threshold $T(i)$ in a new region $\left(c^{\prime}, \mathrm{d}^{\prime}\right)$. In this situation, the algorithm would search the maximum GCFs in region $\left(\mathrm{c}^{\prime}, \mathrm{d}^{\prime}\right)$, and figured out an unexpected new threshold $T^{\prime}(i)$, which replaced the $T(i)$ for threshold processing of the next gait cycle, at time point $d^{\prime}$. Additionally, the secondly calculated threshold $T^{\prime}(i)$ was a false threshold for the next gait cycle. Similarly, using two thresholds also resulted in the same problem.

However, using three thresholds avoided the above-mentioned problem. As Figure 2 showed, the maximum and minimum GCFs were severally obtained in region (c, d) and (f, g); meanwhile, the new thresholds (i.e., $T_{H}$ and $T_{L}$ ) were severally computed at time point $\mathrm{e}$ and $\mathrm{h}$. The middle threshold $\mathrm{T}_{\mathrm{M}}$ was to search the time point $\mathrm{e}$ and $\mathrm{h}$, which avoided the new threshold computation at time point $\mathrm{d}$ or g. To sum up, the use of three thresholds avoided the problem of twice threshold computations.

\subsection{Adaptability to Variable Speed Walking}

Generally, when the subjects walk faster, their feet hit the ground harder, i.e., the magnitude of GCFs gets bigger. As a result, the magnitude of GCFs changes with the variation of walking speeds; meanwhile, the maximum GCF increases (or decreases) as the walking speed increases (or decreases). According to the formula in Equation (8), when the subjects walk slowly, the maximum GCF possesses small magnitude such that a small threshold is calculated. Similarly, when the subjects walk fast, the formula calculated a big threshold. As to variable speed walking, one computed threshold should be set for each walking speed to conduct correct gait phase detection. If the subject suddenly changes walking speed, the ground contact force changes accordingly. The subject will take one step, and the thresholds will be adjusted to the new speed because three thresholds of the next gait cycle are calculated by the GCFs of the present gait cycle. The proposed STTTA uses the maximum and minimum GCFs from the last gait cycle to calculate a threshold (i.e., $T_{H}$ ) for the present gait cycle. The maximum and minimum GCFs of the last gait cycle are the closest to those of the present cycle. As a result, appropriate thresholds are calculated for each gait cycle, which correlates with speed variation. To sum up, the proposed STTTA is adaptable to variable speed walking.

\subsection{Limitation of the Research}

In our study, only healthy subjects have been studied on level ground, not taking the pathological subjects into account. The experiments were done on a treadmill because the method is not suitable for irregular terrain and stairs walking. Uneven road conditions and obstacles on the ground may lead to misdetections.

In the experiments, some rules of GPDA were not observed. However, the specific walking habits of individuals may employ the unused rules. The GPDA rules could be used to detect abnormal gaits for podiatric diagnoses. 


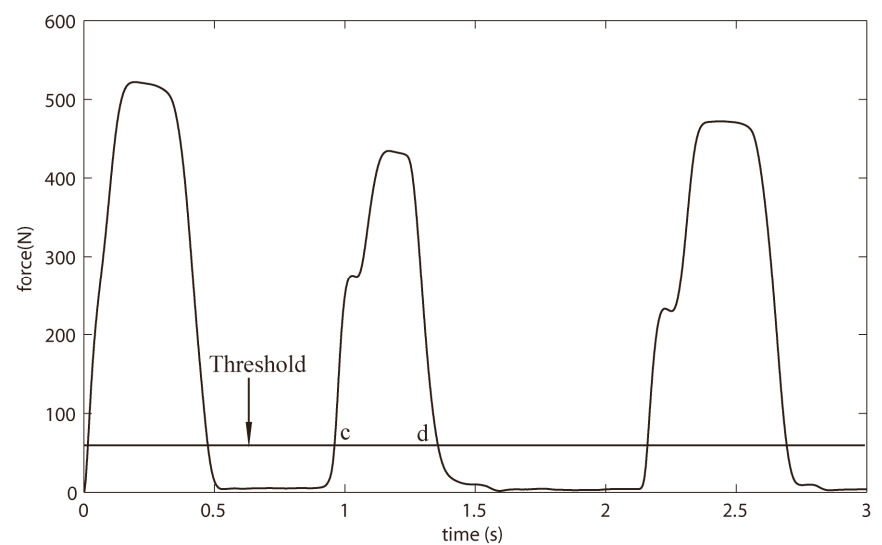

(a)
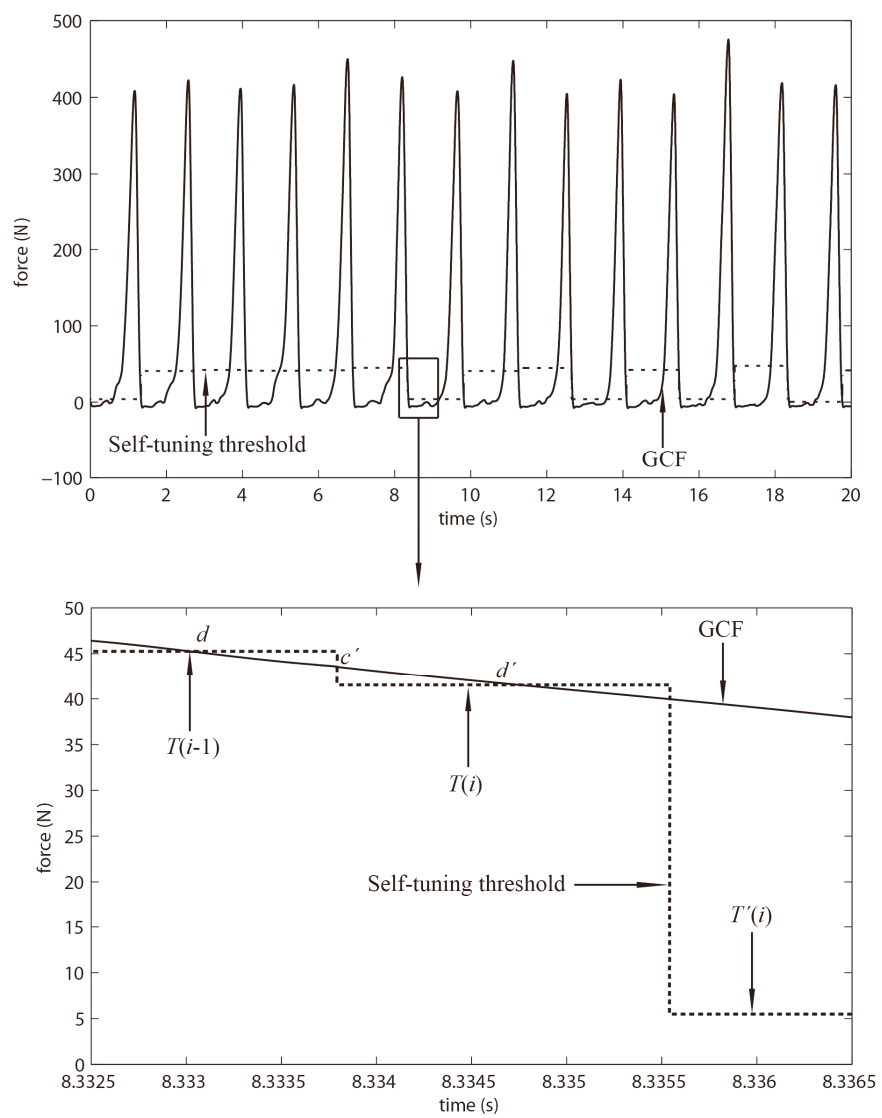

(b)

Figure 6. (a) the description of using one threshold for threshold adjustment; (b) demonstration of threshold adjustment using one threshold and analysis of twice threshold computations. 


\section{Conclusions}

This paper proposes a self-tuning triple-threshold algorithm that calculates adjustable thresholds to adapt to human walking. In addition, the adjustable thresholds are calculated in the walking process, and the same initial threshold values are set for all subjects in all experiments. For all subjects, high average reliabilities are gained when the proposed method is severally compared with two reference methods. For each subject, the proposed method acquires stable reliabilities in five experiments with different walking speeds each time. It comes to a conclusion that the proposed STTTA can be used to detect gait phases in real time, and shows adaptability to different walking conditions on level ground. In the future work, the method of gait phase detection would be studied on irregular terrains or when climbing stairs. Furthermore, it needs more types of sensors to detect the sub-phases of swing.

Acknowledgments: We would like to thank all participants for helping us with data acquisitions. This work was supported by the National Key R\&D Program of China "The study on Load-bearing and Moving Support Exoskeleton Robot Key Technology and Typical Application" (2017YFB1300500).

Author Contributions: Jing Tang, Jianbin Zheng and Yang Wang proposed the conception and design of this research, acquired the data and drafted this article. Lie Yu and Enqi Zhan helped with the analysis and interpretation of data. Qiuzhi Song revised for important intellectual content. All authors revised and approved the final paper version to be published.

Conflicts of Interest: None of these authors has conflict of interest in this research.

\section{References}

1. Novak, D.; Riener, R. A survey of sensor fusion methods in wearable robotics. Robot. Auton. Syst. 2015, 73, 155-170. [CrossRef]

2. Senanayake, C.; Member, S.; Senanayake, S.M.N.A.; Member, S. Fuzzy Logic based Implementation of a Real-Time Gait Phase Detection Algorithm using Kinematical Parameters for Walking. In Proceedings of the International Conference of Soft Computing and Pattern Recognition, Malacca, Malaysia, 4-7 December 2009; pp. 592-597.

3. Pappas, I.P.I.; Popovic, M.R.; Keller, T.; Dietz, V.; Morari, M. A Reliable Gait Phase Detection System. IEEE Trans. Neural Syst. Rehabil. Eng. 2001, 9, 113-125. [CrossRef] [PubMed]

4. Mariani, B.; Rouhani, H.; Crevoisier, X.; Aminian, K. Quantitative estimation of foot-flat and stance phase of gait using foot-worn inertial sensors. Gait Posture 2013, 37, 229-234. [CrossRef] [PubMed]

5. Lopez-meyer, P.; Fulk, G.D.; Sazonov, E.S. Automatic Detection of Temporal Gait Parameters in Poststroke Individuals. IEEE Trans. Inf. Technol. Biomed. 2011, 15, 594-601. [CrossRef] [PubMed]

6. Kong, K.; Member, S.; Tomizuka, M. A Gait Monitoring System Based on Air Pressure Sensors Embedded in a Shoe. IEEE/ASME Trans. Mechatron. 2009, 14, 358-370. [CrossRef]

7. Rueterbories, J.; Spaich, E.G.; Andersen, O.K. Characterization of gait pattern by 3D angular accelerations in hemiparetic and healthy gait. Gait Posture 2013, 37, 183-189. [CrossRef] [PubMed]

8. Williamson, R.; Andrews, B.J. Gait Event Detection for FES Using Accelerometers and Supervised Machine Learning. IEEE Trans. Rehabil. Eng. 2000, 8, 312-319. [CrossRef] [PubMed]

9. Lee, J.K.; Park, E.J. Quasi real-time gait event detection using shank-attached gyroscopes. Med. Biol. Eng. Comput. 2011, 49, 707-712. [CrossRef] [PubMed]

10. Greene, B.R.; Mcgrath, D.; Burns, A.; Caulfield, B. An adaptive gyroscope-based algorithm for temporal gait analysis. Med. Biol. Eng. Comput. 2010, 48, 1251-1260. [CrossRef] [PubMed]

11. Gouwanda, D.; Gopalai, A.A. A robust real-time gait event detection using wireless gyroscope and its application on normal and altered gaits. Med. Eng. Phys. 2015, 37, 219-225. [CrossRef] [PubMed]

12. Seel, T.; Landgraf, L.; Escobar, V.C.; Schauer, T. Online gait detection with automatic to gait velocity changes using accelerometers and gyroscopes. Biomed. Tech. 2014, 59. [CrossRef]

13. Bejarano, N.C.; Ambrosini, E.; Pedrocchi, A.; Ferrigno, G.; Monticone, M.; Ferrante, S. A Novel Adaptive, Real-Time Algorithm to Detect Gait Events From Wearable Sensors. IEEE Trans. Neural Syst. Rehibil. Eng. 2015, 23, 413-422. [CrossRef] [PubMed] 
14. Müller, P.; Seel, T.; Schauer, T. Experimental Evaluation of a Novel Inertial Sensor Based Realtime Gait Phase Detection Algorithm. In Proceedings of the European Conference on Technically Assisted Rehabilitation, Berlin, Germany, 12-13 March 2015.

15. Dai, R.; Stein, R.B.; Andrews, B.J.; James, K.B.; Wieler, M. Application of Tilt Sensors in Functional Electrical 1s ti minlation. IEEE Trans. Rehabil. Eng. 1996, 4, 63-72. [PubMed]

16. Blanc, Y.; Balmer, C.; Landis, T. Temporal parameters and patterns of the foot roll over during walking: Normative data for healthy adults. Gait Posture 1999, 10, 97-108. [CrossRef]

17. Sousa, A.S.P.; Silva, A.; Macedo, R.; Santos, R.; Tavares, R.S. Influence of long-term wearing of unstable shoes on compensatory control of posture: An electromyography-based analysis. Gait Posture 2014, 39, 98-104. [CrossRef] [PubMed]

18. Yan, T.; Parri, A.; Garate, V.R.; Cempini, M. An oscillator-based smooth real-time estimate of gait phase for wearable robotics. Auton. Robot. 2017, 41, 759-774. [CrossRef]

19. Catalfamo, P.; Moser, D.; Ghoussayni, S.; Ewins, D. Detection of gait events using an F-Scan in-shoe pressure measurement system. Gait Posture 2008, 28, 420-426. [CrossRef] [PubMed]

20. Yu, L.; Zheng, J.; Wang, Y.; Song, Z.; Zhan, E. Adaptive method for real-time gait phase detection based on ground contract forces. Gait Posture 2015, 41, 269-275. [CrossRef] [PubMed]

21. Smith, B.T.; Coiro, D.J.; Finson, R.; Betz, R.R.; Mccarthy, J. Evaluation of Force-Sensing Resistors for Gait Event Detection to Trigger Electrical Stimulation to Improve Walking in the Child With Cerebral Palsy. IEEE Trans. Neural Syst. Rehabi. Eng. 2002, 10, 22-29. [CrossRef] [PubMed]

22. Pappas, I.P.I.; Keller, T.; Mangold, S.; Popovic, M.R.; Dietz, V.; Morari, M. A Reliable Gyroscope-Based Gait-Phase Detection Sensor Embedded in a Shoe Insole. IEEE Sens. J. 2004, 4, 268-274. [CrossRef]

(C) 2018 by the authors. Licensee MDPI, Basel, Switzerland. This article is an open access article distributed under the terms and conditions of the Creative Commons Attribution (CC BY) license (http:/ / creativecommons.org/licenses/by/4.0/). 
Article

\title{
A Real-Time Wireless Sweat Rate Measurement System for Physical Activity Monitoring
}

\author{
Andrew Brueck ${ }^{1}$, Tashfin Iftekhar ${ }^{1}$, Alicja B. Stannard ${ }^{2}$, Kumar Yelamarthi ${ }^{1}$ and Tolga Kaya ${ }^{3, *}$ \\ 1 School of Engineering and Technology, Central Michigan University, Mt Pleasant, MI 48859, USA; \\ bruec1ap@cmich.edu (A.B.); iftek1a@cmich.edu (T.I.); yelam1k@cmich.edu (K.Y.) \\ 2 Department of Physical Therapy and Human Movement Science, Sacred Heart University, Fairfield, \\ CT 06825, USA; stannarda@sacredheart.edu \\ 3 School of Computing, Sacred Heart University, Fairfield, CT 06825, USA \\ * Correspondence: kayat@sacredheart.edu; Tel.: +1-203-396-6589
}

Received: 20 October 2017; Accepted: 8 February 2018; Published: 10 February 2018

\begin{abstract}
There has been significant research on the physiology of sweat in the past decade, with one of the main interests being the development of a real-time hydration monitor that utilizes sweat. The contents of sweat have been known for decades; sweat provides significant information on the physiological condition of the human body. However, it is important to know the sweat rate as well, as sweat rate alters the concentration of the sweat constituents, and ultimately affects the accuracy of hydration detection. Towards this goal, a calorimetric based flow-rate detection system was built and tested to determine sweat rate in real time. The proposed sweat rate monitoring system has been validated through both controlled lab experiments (syringe pump) and human trials. An Internet of Things (IoT) platform was embedded, with the sensor using a Simblee board and Raspberry Pi. The overall prototype is capable of sending sweat rate information in real time to either a smartphone or directly to the cloud. Based on a proven theoretical concept, our overall system implementation features a pioneer device that can truly measure the rate of sweat in real time, which was tested and validated on human subjects. Our realization of the real-time sweat rate watch is capable of detecting sweat rates as low as $0.15 \mu \mathrm{L} / \mathrm{min} / \mathrm{cm}^{2}$, with an average error in accuracy of $18 \%$ compared to manual sweat rate readings.
\end{abstract}

Keywords: sweat sensor; sweat rate; dehydration; IoT; PDMS

\section{Introduction}

With the popularity of smartwatches, a new lifestyle is growing, particularly in urban areas, where people are more conscious of how they treat their own bodies [1]. Numerous devices are available for professionals and exercise/technology enthusiasts that monitor body vitals such as heart rate monitors [2], temperature sensors [3], and even optical glucose measurement devices [4]. One of the growing exercise activities to stay fit and healthy is endurance sports such as marathons, biking, and triathlons [5]. Not only is there a significant increase in the number of professional athletes competing in these events, but also there has been growing interest among non-professional athletes every year in the last decade [6]. There are several smartwatches on the market that can help monitor exercise intensity and performance. Even though heart rate monitoring is considered the most effective way of monitoring the effort level, athletes are well aware that fluid intake before, during, and after a hard training is quite important $[7,8]$. Fluid intake of less than what is required by the body would lead to dehydration (or hypohydration), and cause both physical and mental performance degradation [8-10]. On the other hand, too much fluid intake, more than required (hyperhydration), can be fatal [7].

It is imperative that most of the fluid losses during a race or training occur through sweating in order to regulate the core body temperature [11]. Physical exercise leads to an increase in core body 
temperature, and sweating through skin cools down the body via thermoregulation. Sweat glands that are located under the skin are responsible for sweating action. Eccrine sweat glands are the main sweat glands located in human skin, and are responsible for regulating core body temperature [12]. On the other hand, apocrine sweat glands are located at armpits and groin areas that are activated during emotional and sexual arousement [13]. Although the density of eccrine sweat glands varies significantly throughout the body, some averages can be given, such as 104 glands $/ \mathrm{cm}^{2}$ for forearms, 155 glands $/ \mathrm{cm}^{2}$ for the forehead, etc. [14].

Research on the physiology of sweat is more than a century old, when contents of sweat were first analyzed [13]. What is in sweat is well understood; it is mostly water, with electrolytes such as sodium, potassium, urea, lactate, and other trace minerals [12,14]. Several sweat-based hydration detection monitoring systems were proposed that included optical [15,16], electrochemical [17,18], or amperometric $[17,19]$ techniques. It is known that the amounts of individual constituents of sweat not only vary from person to person, but also show significant variations in different regions of the same individual $[14,20,21]$. Without establishing a base level of sweat on a particular individual's particular body region, comparisons cannot be made. Furthermore, the concentration of sweat also changes with the rate of sweat, which is directly related to the intensity of the exercise and environmental conditions $[11,22,23]$. Therefore, sweat rate plays a significant role in physical conditioning, and is a useful variable for determining the performance of an athlete, particularly in endurance sports. A real-time measurement of sweat rate can ultimately provide more deterministic results on how to interpret sweat concentration data that could be used to gather physiological information about an athlete.

Sweat-rate sensing was first performed by utilizing traditional sweat collection methods such as whole-body wash [24,25] or absorbent pads [26]. Although accurate, these approaches could only provide a limited number of data points, and cannot be considered in real time. Toward sweat rate sensing, flow rate sensors were considered as an alternative. It was widely accepted that microflow sensing (flow rates around $\mu \mathrm{L} / \mathrm{min}$ ) is implemented with thermal flow rate sensors, particularly calorimetric or thermal pulse flow sensors $[27,28]$. Matzeu et al. demonstrated the detection of sweat rate using image analysis where they took images of the sweat filling a commercial sweat collector (Megaduct, a larger volume than a Macroduct) [29]. This approach was arguably the first real-time sweat rate detection system. However, it requires cameras and post-processing, altering the use of the system as a wearable device. Coyle et al. also proposed a preliminary sweat rate sensing textile patch employed by discrete humidity sensors [30]. Although promising, this device was only capable of measuring the evaporated sweat rather than the total secreted sweat from a region, which is called transepidermal water loss, and is not the same as the sweat rate. Wei et al. used a cellulose-based sweat absorber to measure sweat levels [31]. Real-time sweat rates were obtained, but these devices were not validated through manual measurements. Furthermore, their system relied on the weight of the absorbed sweat, which would cause hidromeiosis, i.e., the local dysfunction of sweat glands due to the blockage of sweat ducts.

Building on our years-long sweat concentration research [16,32-34], we have recently proposed a concept that could be used for sweat rate sensing applications, and stated that it could potentially be utilized for sweat-rate sensing applications [35]. Building upon our expertise on the Internet of Things (IoT) [36-39], we are bringing our concept into life by proposing a wearable, real-time sweat rate device. Although we have shown the feasibility of the concept in our previous work [30], this paper provides an innovative implementation of the concept, and expands the scope with human subject validation (compared with manual data collection). A unique modification in building a microfluidic calorimetric sweat rate sensor allowed us to feed the sensor with sweat via a commercial sweat collector (Macroduct). Sweat rate data was obtained, processed, analyzed, and sent to the cloud for instantaneous and longitudinal monitoring and analysis. A smartphone app was developed that displayed the sweat rate information obtained wirelessly from a LilyPad Simblee board [40]. A custom 
printed circuit board (PCB) was designed with surface mount components. Human trials were also conducted to validate the proposed IoT-based sweat rate monitoring system.

\section{Materials and Methods}

The flow rate sensor was built using a polydimethylsiloxane (PDMS) silicone elastomer kit (Dow Corning) by using two pieces of PDMS. A silicon wafer with a long rectangle channel mold was used. PDMS was poured into the silicon mold to create the inverse channel, which was $26 \mathrm{~mm} \times 3 \mathrm{~mm} \times 1 \mathrm{~mm}$. Inlet and outlet holes were punched through the PDMS channel. The upper PDMS block had a 10-ohm resistor embedded in it that acted as a heater, and holes were also punched through to allow two copper-constantan T-type thermocouples (Omega, with 30 gauge wiring) to insert. Upper and lower PDMS pieces were attached together by using the plasma cleaner (Harrick PDC-32G) to form the closed channel. Thermocouples were then slid into place through the already punched holes. Loctite was used to ensure a tight seal around the thermocouples and the heating resistor.

A PCB was designed and fabricated to minimize the footprint of the electronics. A power transistor (NPN MJD200T4G) was used to supply enough current to the heating resistor. An instrumentation amplifier (AD8237ARMZ) was utilized to convert the differential temperature readings into voltage. An entire PCB was powered via the Simblee BLE's (Bluetooth Low Energy) regulated 3.3-V output. The voltage output of the amplifier was connected to the Simblee board for wireless communication.

The characterization of two copper-constantan T-type thermocouples was done by using a Revlon turbo/lightweight/1875 W hairdryer. Thermocouples were physically kept farther apart, and one of them was heated. The process was reversed for thermocouples in order to obtain a symmetric temperature difference. The temperature reading of the thermocouples was obtained from two Omega HHM9007R multimeters (OMEGA, Norwalk, CT, USA). The voltage output of the differential temperature readings of the thermocouples was obtained from the instrumentation amplifier. A non-zero reference voltage was used for the amplifier. The temperature value and corresponding output voltage were then recorded.

The characterization of the sweat rate sensor device was performed by pumping deionized (DI) water through the channel with a syringe pump (KDS Scientific 100) at flow rates from $3 \mu \mathrm{L} / \mathrm{min}$ to $100 \mu \mathrm{L} / \mathrm{min}$, which were equally spaced logarithmically. The temperatures and the output voltage of the amplifier were recorded once the flow rate reached a steady state value (about $10 \mathrm{~min}$ ).

The Macroduct sweat collector (ELITechGroup Biomedical Systems, South Logan, UT, USA) was used to guide the sweat to the PDMS channel. A 3.7-V $500 \mathrm{mAh}$ rechargeable LiPo PKCell battery was used to power the Simblee board, which then powered the PCB. The entire device was housed in a box that was custom designed using TinkerCAD (Version 3.9, Autodesk, CA, USA), and three-dimensional (3D) printed by Makerbot Replicator + (New York City, NY, USA).

Human trials were conducted using an exercise bike. In order to record the sweat rate sensor manually, a Macroduct was placed on the left upper forearm of the sweat rate sensor prototype, and the volume of sweat in the Macroduct tubing was recorded over time. The manual rate of sweat was then calculated by taking into account the dimensions of the Macroduct tubing. The sweat rate sensing device was put on the right upper forearm. Output voltages of the device were wirelessly transmitted to a smartphone (HTC One M7 WLS and iPhone 6s) via the LilyPad Simblee BLE board, and a mobile app was utilized to read the data every $15 \mathrm{~s}$ or $30 \mathrm{~s}$. A cloud interface was built around ThingSpeak. A Raspberry Pi 3 was used as the receiver and connection to the cloud. An online stopwatch (Free-stopwatch.com) was used to keep time accurately for the sweat rate testing. Five subjects were tested after they gave informed consent. Institutional Review Board (IRB) approval was obtained at Sacred Heart University (IRB \#170922A). Subjects were asked to bike on the exercise bike at $80 \%$ workload of their maximum heart rate, which was calculated as $208-(0.7 \times$ age $)$. Room temperature was $24{ }^{\circ} \mathrm{C}$, and the relative humidity was recorded as $40 \%$. Subjects were allowed to drink water before, during, and after ad libitum. 


\section{Device Architecture}

The device was built on the commercial Macroduct sweat collector with some modifications. The coiled tubing of the Macroduct was shortened to $1 \mathrm{~cm}$, and the tubing was connected to the inlet of the calorimetric sweat rate sensor to guide the sweat into the sweat rate sensing channel. Temperature information readings, as well as heating element control circuitry, were designed and custom built on a PCB. Information gathered from the sensor was processed at the PCB electronics, and the voltage output of the sweat rate information was sent to the Simblee board for wireless transmission. The circuitry was powered by the Simblee board, which had a LiPo battery as its own power supply. The individual components of the entire system and the assembled device photos are shown in Figure 1a,b, respectively.
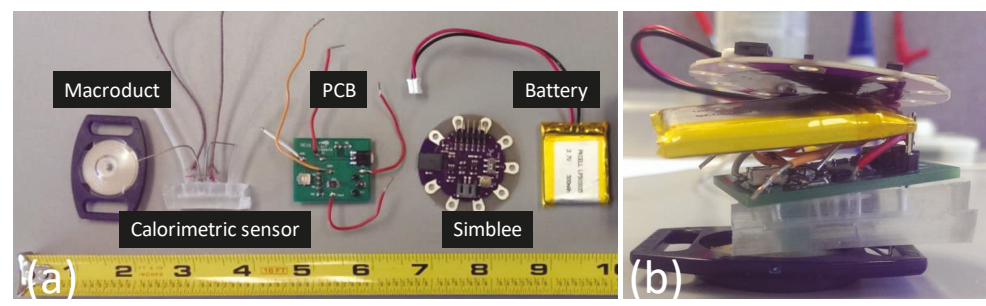

Figure 1. Components of the sweat rate sensing device: (a) Individual parts that were used to build the prototype; (b) overall device view after the assembly and connections were made.

Two system architectures have been developed to test the sweat rate sensor's IoT functionality, as illustrated in Figure 2. Both architectures are compatible with the current system, and no hardware modifications needed to be made on the device. The first architecture purely focuses on monitoring the instantaneous sweat rate of just one user, and provides a warning to the user so that appropriate actions can be taken when the level goes beyond a threshold value. This monitoring is accomplished through a cell phone application with which the LilyPad Simblee can directly communicate. The second architecture is designed such that the sweat rate from multiple users could be obtained simultaneously, longitudinal data analytics could be performed, and data could be stored for further analysis by a clinical expert. This architecture requires an internet gateway, which was implemented by a Raspberry Pi 3 on the receiver side. A ThingSpeak IoT platform was utilized for data analytics, storage, and visualization.

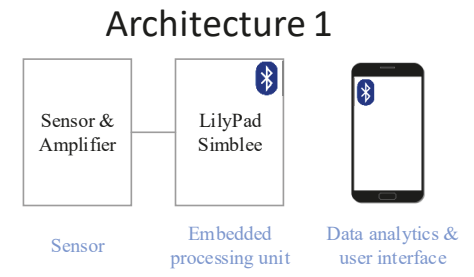

Architecture 2

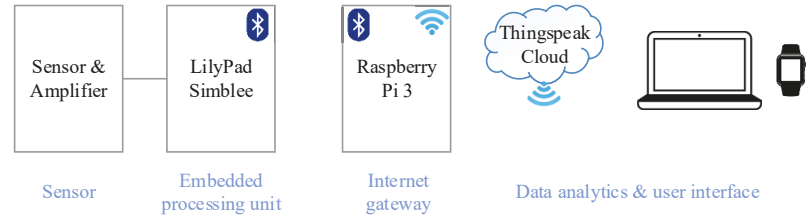

Figure 2. Architectural components of an Internet of Things (IoT) framework for instantaneous (architecture 1) and longitudinal (architecture 2) sweat rate analysis. 


\section{Results}

Figure 3a shows the initial sweat rate sensing concept where the initial theoretical operation was introduced by the authors [30]. A heating element is placed in a flow channel. Two thermocouples (upstream and downstream) are placed on each side of the heater (2-mm spacing) to sense the temperature. Once there is a flow, temperature around the upstream sensor $\left(T_{u}\right)$ cools off because of the fluid flow. Temperature around the downstream sensor $\left(T_{d}\right)$, on the other hand, increases with the flow as the temperature profile is pushed further with the flow. Once a critical flow rate value is reached (detailed theoretical analysis can be found in [31]), $T_{d}$ starts to cool off as well (due to high flow rates). The temperature difference, $\Delta T=T_{d}-T_{u}$, reaches a maximum at this critical flow rate, as illustrated in Figure 1b. In practice, this threshold occurs at high flow rates that are well beyond the human sweat rate. Therefore, a more realistic region of $\Delta T$ was represented with a solid line, and the theoretical (but not practical) extrapolation of $\Delta T$ is shown as the dashed line in Figure $2 b$.
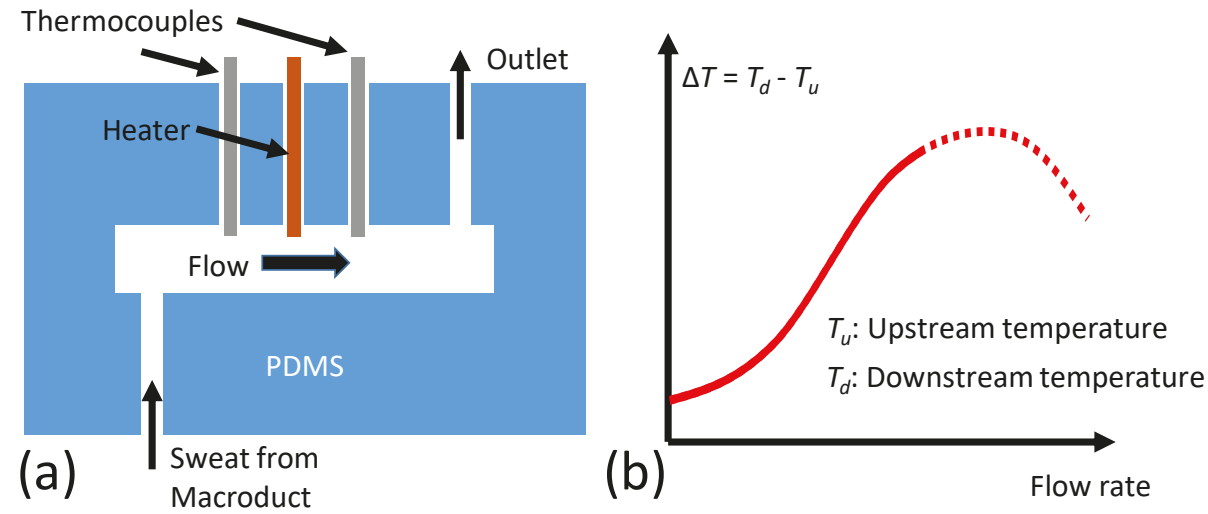

Figure 3. Theoretical principles of the calorimetric flow rate device. (a) Two thermocouples (upstream and downstream) were placed along the heating element. Sweat entered the channel from the bottom, and exited from the top as an outlet; (b) The temperature difference between thermocouple readings increased with the flow and reached a maximum. Realistic flow rates were well below this maximum. The temperature difference is a monotonously increasing function of the flow rate.

The overall circuit diagram of the sensor and its electronics was provided in Figure 4 . The base voltage of a transistor (NPN MJD200T4G) is biased using a simple voltage divider. The current that the transistor draws flows through a 10-ohm resistor connected between the emitter and the ground. This resistor acts as a heater, and is embedded in the PDMS device. The temperature upstream and downstream of the heater are both sensed by copper-constantan T-type thermocouples (Omega). The thermocouples produce a voltage depending on the temperature with a slope of $40 \mu \mathrm{V} /{ }^{\circ} \mathrm{C}$. Both of the thermocouples are inputs to a linear instrumentation amplifier (AD8237ARMZ), which takes the difference between the two and amplifies the result to obtain voltage values that can be detected by the microcontroller. External resistors set the gain of the amplifier, and the reference voltage is set by a $10-\mathrm{k} \Omega$ potentiometer. The output voltage of the amplifier is then sent to the Simblee board for data conversion and wireless transfer. The Simblee board has a regulated 3.3-V power supply that powers the transistor network, as well as the amplifier. An external LiPo 3.7 V 500 mAh PKCell battery powers the Simblee board. 


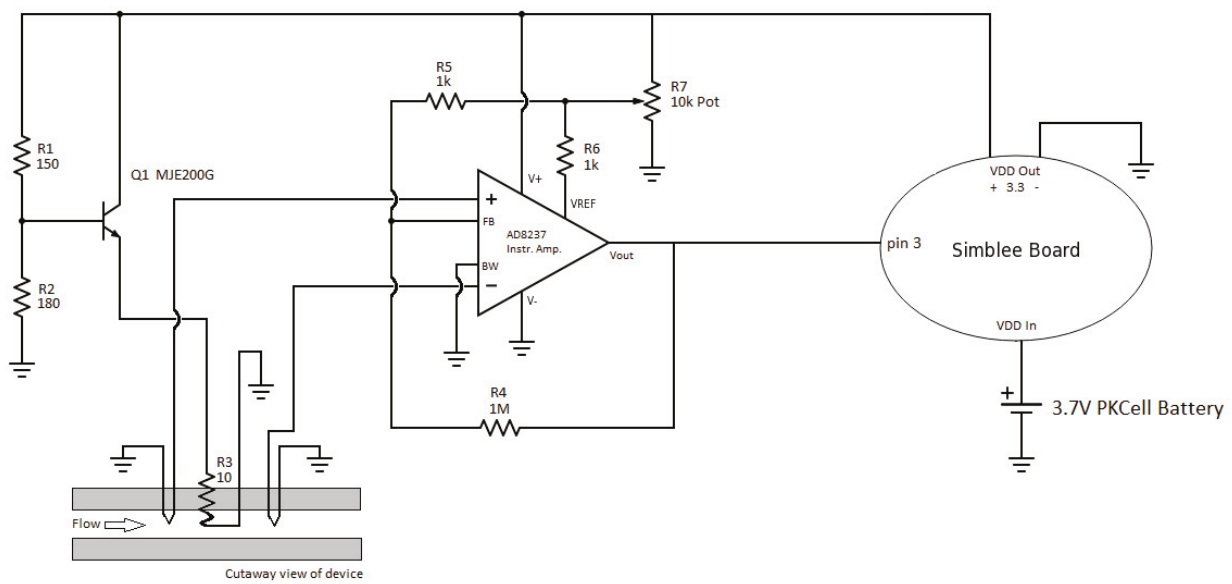

Figure 4. Schematic diagram of the IoT platform. A power transistor was used to set the current to the heating resistor. The temperature difference was converted into voltage via the instrumentation amplifier, and fed to the Simblee board for wireless communication.

The circuitry was first tested by changing the temperature around the thermocouples using a hairdryer to characterize the temperature to voltage conversion. It can be seen from the Figure 5 inset that a linear voltage increase occurs with temperature changes. In this experiment, $\Delta T$ was changed on a range of approximately $4{ }^{\circ} \mathrm{C}$ to show the trend. By setting the instrumentation amplifier gain to 1000 , and knowing that the thermocouple voltage conversion is $40 \mu \mathrm{V} /{ }^{\circ} \mathrm{C}$, an ideal function can be derived as $V_{\text {out }}=40 \mu \mathrm{V} /{ }^{\circ} \mathrm{C} \times 1000 \times \Delta T+V_{\text {ref }}$, where $V_{\text {ref }}$ represents the reference voltage for the amplifier. The reference voltage in Figure 5 inset was subtracted for visualization purposes.

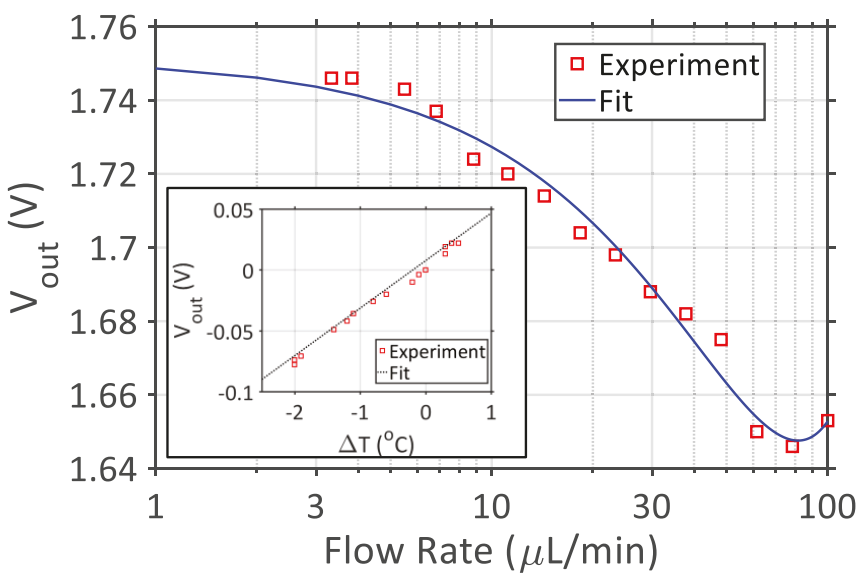

Figure 5. Characterization of the IoT device. The inset shows the linear relationship between the output voltage and the temperature difference. The main figure shows the output voltages that were obtained from different flow rates (generated with a syringe pump). A quadratic polynomial was fit to the data so that the output voltages can be converted into flow rate in real human trials.

Once the circuitry operation has been validated, the sweat rate device was characterized by using a syringe pump for various volumetric flow rates (in $\mu \mathrm{L} / \mathrm{min}$ ). Figure 5 shows the output voltage 
values with respect to varying flow rates, which were aligned with the theoretical illustration in Figure $2 \mathrm{~b}$. It can be seen that $V_{\text {out }}$ starts to increase back from the minimum point at around $80 \mu \mathrm{L} / \mathrm{min}$, which would make detection difficult (two flow rate data points would correspond to the same voltage value). On the other hand, flow rates below $1 \mu \mathrm{L} / \mathrm{min}$ do not result in a significant change in output voltage, reducing the resolution of the measurement. Therefore, the volumetric flow rate detection limit is determined to be between $1-80 \mu \mathrm{L} / \mathrm{min}$. It is important here to note that the sweat rate is given in flow rates per unit area $\left(\mathrm{cm}^{2}\right)$. Although these experiments were conducted by pushing the liquid to the PDMS channel directly from a syringe pump, corresponding sweat rate values could be calculated by using the area of the Macroduct, which would feed the sweat to the sensing area. As the diameter of the Macroduct is $2.7 \mathrm{~cm}$, the minimum sweat rate that can be detected with the current system would be $0.15 \mu \mathrm{L} / \mathrm{min} / \mathrm{cm}^{2}$. Although the upper limit of detection for the device is calculated to be around $13 \mu \mathrm{L} / \mathrm{min} / \mathrm{cm}^{2}$, this limit would never be reached, because it is known that the sweat rate for humans does not exceed $5 \mu \mathrm{L} / \mathrm{min} / \mathrm{cm}^{2}$. Hence, the range of the device fits well to the application. A second order polynomial was fit to the experimental data, which can be used to obtain the flow rate (FR) from the output voltage $\left(V_{\text {out }}\right)$.

In order to validate the sweat rate device, human subject trials were conducted. A commercial Macroduct was used as the control. The Macroduct collects the sweat in the spiral tubing as the collector makes contact with the skin, and the small pinhole at the bottom allows the sweat to go through the tube. Spiral Macroduct tubing was marked every $45^{\circ}$ turn, which corresponds to a $5-\mathrm{mm}$ distance between each mark. The exact locations of the marks were determined by uncoiling the spiral tube, as shown in Figure 6a. The volume of the sweat between each mark was calculated using the inner diameter of the tubing $(0.64 \mathrm{~mm})$. The time it took the sweat to go from one mark to another was noted, and the volumetric flow rate was calculated accordingly. This method allowed us to record sweat rate values approximately every $2 \mu \mathrm{L}$ in the tube. The Macroduct tube has a dye in it that made it easier to visualize the location of the sweat. An actual Macroduct usage on a subject is shown in Figure $6 b$.
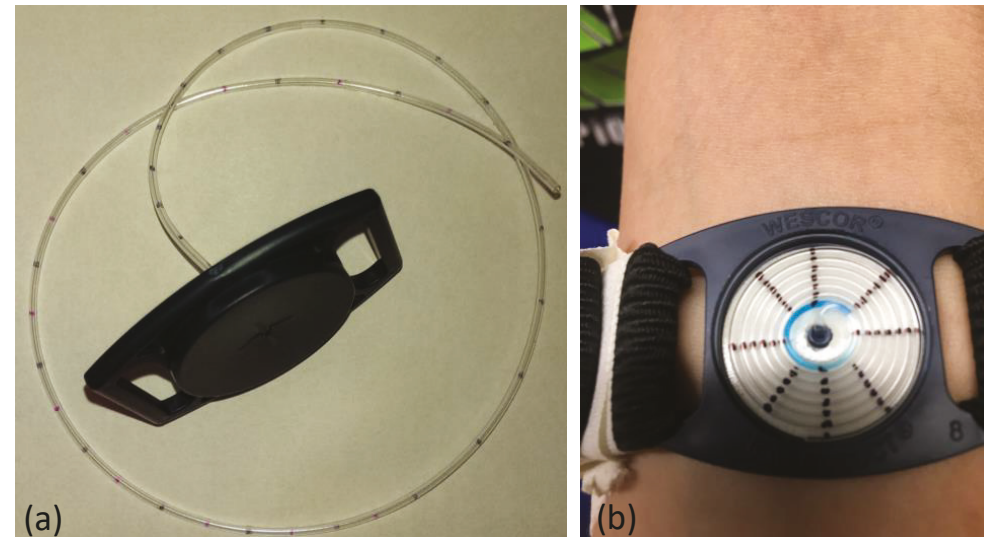

Figure 6. Manual determination of the sweat rate was achieved with a Macroduct sweat collector. (a) Uncoiled Macroduct tube to measure the distance between each mark; (b) Photo of the Macroduct being filled with the sweat during an actual subject trial.

Two male and three female participants completed the exercise protocol (Table 1). Exercise duration ranged from $18 \mathrm{~min}$ to $30 \mathrm{~min}$, while the average sweat rate was $0.76 \pm 0.41 \mu \mathrm{L} / \mathrm{min} / \mathrm{cm}^{2}$. Pre and post-weights were measured with clothes. Therefore, weight loss during the exercise only provides a qualitative measure, and can be assumed that the overall weight loss was greater. No fluids 
were ingested during the trial. In order to provide more quality information regarding the validation of the device, error values in accuracy were devised from the data by calculating the percent deviation of the sweat rate $(S R)$ from the device data $\left(S R_{\text {device }}\right)$ to the manual Macroduct collection $\left(S R_{\text {control }}\right)$, and are presented in the Table 1 as the percentage error (calculated as $\left.100 x\left(S R_{\text {device }}-S R_{\text {control }}\right) / S R_{\text {control }}\right)$. The percent error in accuracy for all of the subjects ranged from 4-30\% (Table 1).

Table 1. Results from the validation of the sweat rate sensor device.

\begin{tabular}{ccccccc}
\hline Subject \# & Gender & Pre Test Weight $\mathbf{( k g )}$ & Weight Loss $\mathbf{( k g )}$ & Duration $(\mathbf{m i n})$ & Sweat Rate $\left(\mu \mathrm{L} / \mathbf{m i n} / \mathbf{c m}^{2}\right)$ & Error $(\%)$ \\
\hline 1 & Female & 62.4 & $>0.2$ & 18 & 0.91 & 1.21 \\
2 & Male & 93.9 & $>0.3$ & 20 & 0.98 & 16 \\
3 & Male & 75.6 & $>0.3$ & 20 & 0.17 & 15 \\
4 & Female & 52.7 & $>0.2$ & 30 & 0.54 & 27 \\
5 & Female & 76.0 & $>0.2$ & 30 & \\
\hline
\end{tabular}

The sweat rate device was worn on the opposite arm to the manual data collection, and data was collected simultaneously. Sweat rate readings were gathered every $30 \mathrm{~s}$ from the phone app. Figure 7 shows four of the five subject tests. It is apparent that the manual readings from the Macroduct collection started 7-10 min after the exercise started, as it takes time for the Macroduct reservoir to begin filling. Similarly, device data for subjects 1 and 2 were also delayed. Subject 4 and 5 data from the device started from the very beginning, due to the residual DI water left from rinsing the device. This residual water was left in the tube intentionally to lower the latency of the device. As expected, subjects' sweat rate patterns varied during the exercise protocol, with most of the patterns being relatively constant after $12 \mathrm{~min}$ of exercise.
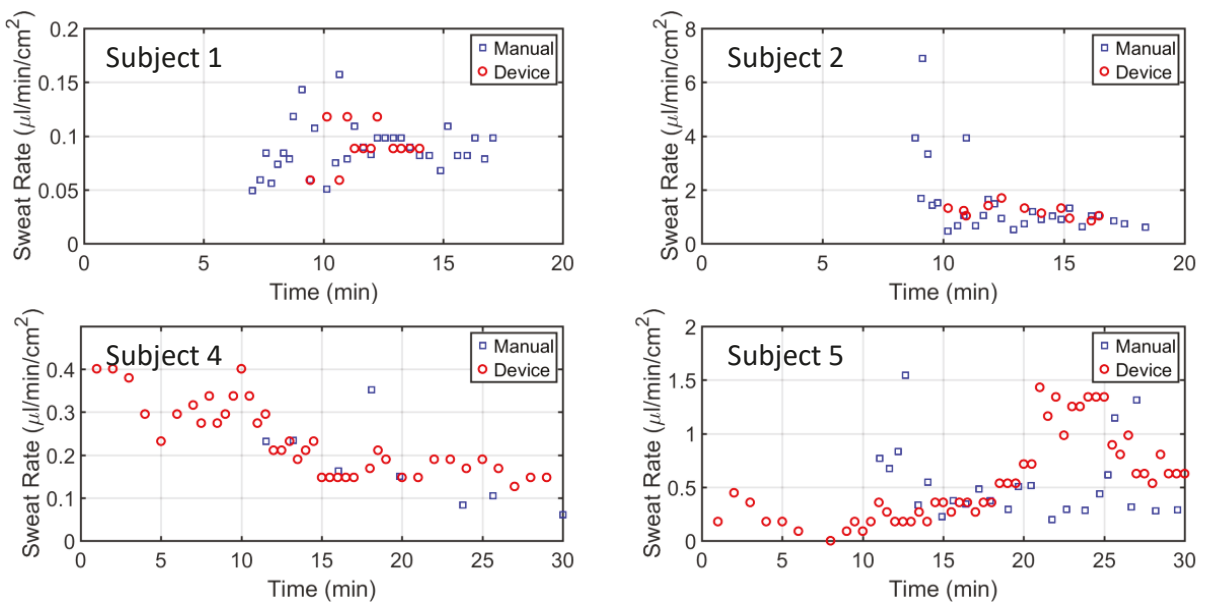

Figure 7. Human trials were conducted to validate the sweat rate sensing device. Manual data collection from the Macroduct was recorded by visually observing the sweat going through the spiral tube. Sweat rate from the device was recorded from the Smartphone.

The IoT framework was tested with the actual sweat rate sensor platform. Figure 8a shows the real-time data of the sweat rate sensor, where different flow rates were introduced to the device. The flow rate was calculated from the obtained voltage values using the second-degree polynomial obtained earlier. ThingSpeak offers a great way of post-processing the data using embedded MATLAB functions (as seen in green buttons). The mobile app, as shown in Figure $8 b, c$, was designed to give instantaneous feedback to the user if the sweat rate values reached excessively high levels. Currently, 
this threshold was set to $1.5 \mu \mathrm{L} / \mathrm{min} / \mathrm{cm}^{2}$, which can easily be adjusted depending on the situation. If the threshold is exceeded, the app gives a warning (shown in Figure 8c) on the screen.
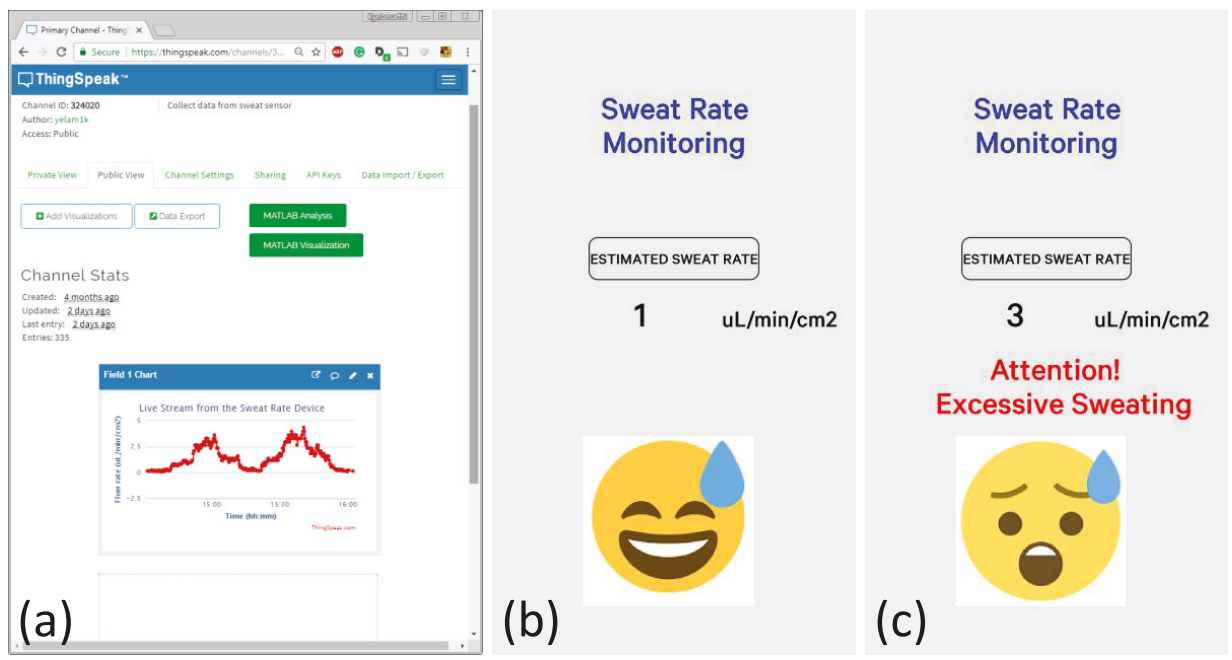

Figure 8. The IoT framework with a sweat rate sensor prototype. (a) The ThingSpeak web interface was used for real-time monitoring of the data; $(\mathbf{b}, \mathbf{c})$ The smartphone app was developed based on the Simblee's interface to give instantaneous feedback to the user.

\section{Discussion}

The current device has a size of $6 \mathrm{~cm} \times 6 \mathrm{~cm} \times 5 \mathrm{~cm}$, which equates to a form factor of $180 \mathrm{~cm}^{3}$. Considering that the form factor of a sports watch is around $25 \mathrm{~cm}^{3}$, the size of the current sweat rate sensing device is big. From the end product point of view, the PDMS device can be made much thinner, and a smaller battery could be used. Lastly, a PCB can be embedded into the PDMS. These efforts could potentially reduce the form factor to below $100 \mathrm{~cm}^{3}$, which would be acceptable for commercialization.

The overall current consumption of the device is around $80 \mathrm{~mA}$. Most of the current is consumed at the heating element. The Simblee board is capable of providing enough current to the device. The current consumption of the instrumentation amplifier is $0.1 \mathrm{~mA}$, which can be considered negligible. During the data transmission, the Simblee board can consume as high as $10 \mathrm{~mA}$. The relatively large current consumption of the entire device results in the need for a high-capacity battery. If the current is lowered on the heating element, the temperature differences, and hence the output voltage swings, get significantly smaller, limiting the sensitivity of the conversion. One way to reduce the current consumption is to implement the heater using thin films, i.e., microfabrication techniques. This approach would significantly lower the form factor and allow the device to become more sensitive at below $0.15 \mu \mathrm{L} / \mathrm{min} / \mathrm{cm}^{2}$. The authors had theoretically proved that the effective range of the device can be shifted to lower flow rates by downscaling the device dimensions, particularly the distance between the heater and the respective thermocouples [35].

In order for the Macroduct sweat collector to start filling up the coiled tube, the interface between the skin and the Macroduct needs to fill first. However, because the Macroduct conforms to the skin, we assumed this volume is less than $1 \mu \mathrm{L}$. There is about $1 \mathrm{~cm}$ of coiled tube before the sweat enters the sweat-sensing channel, resulting about $4 \mu \mathrm{L}$ of sweat that is not analyzed. Furthermore, both of the thermocouples that are in the sweat channel need to be immersed in sweat. Considering this distance is around $1 \mathrm{~cm}$ (from the location where the sweat enters the channel to the downstream thermocouple), and the cross-section of the channel is $3 \mathrm{~mm} \times 1 \mathrm{~mm}$, potentially $30 \mu \mathrm{L}$ of sweat would be required. 
Overall, almost $35 \mu \mathrm{L}$ of sweat would be needed to start receiving sweat rate data. An average sweat volume rate of $3 \mu \mathrm{L} / \mathrm{min}$ (approximately $0.5 \mu \mathrm{L} / \mathrm{min} / \mathrm{cm}^{2}$ ) would result in a 10-min latency, which is consistent with our observations for subjects 1 and 2 (Figure 7). However, the sweat channel and Macroduct tubing can be filled with DI water from PDMS outlet in order to reduce the latency. It can be seen from subjects 4 and 5 (Figure 7) that the latency was completely removed.

It is apparent that the local sweat rate cannot be a good representative of the whole body sweat rate. However, a rough estimate can be performed. Taylor et al. provided a detailed study on regional sweat rate variations [14]. In their seminal work, morphologically normalized and referenced adult values (weight: $70 \mathrm{~kg}$, height, $1.7 \mathrm{~m}$, body surface area, BSA: $1.8 \mathrm{~m}^{2}$ ) were defined, and the active sweat gland density for the forearm was given as 104 glands $/ \mathrm{cm}^{2}$ [14]. The total number of active eccrine sweat glands on the entire body was estimated to be around two million [14]. An average of $1 \mu \mathrm{L} / \mathrm{min} / \mathrm{cm}^{2}$ of local sweat on the forearm then would result in a $0.4 \mathrm{~L}(\sim 0.4 \mathrm{~kg})$ of sweat loss in $20 \mathrm{~min}$. Our preliminary weight loss measurements revealed that subjects lost at least $0.2-0.3 \mathrm{~kg}$ during the exercise. Although these numbers are approximations, it is evident that the values are within the same scale.

The average error in the accuracy of the sweat rate device was found to be $18 \%$. However, it must be noted here that manual data collection with the Macroduct also presents inherent challenges in terms of its own accuracy. The time it takes the sweat to travel from one marked location on the tube to the next was visually observed, which could easily yield data-recording errors. Also, each Macroduct was attached to forearms using stretchable bands. The amount of pressure that is applied to the skin can also affect the functionality of both data collection methods.

All of the subsystems that were used in the IoT framework were off-the-shelf components with open-source software libraries available, and minimal effort would be required if the system were to be modified for a different analysis.

\section{Conclusions}

We have presented a wireless real-time sweat rate sensing platform for athlete hydration detection applications. A calorimetric flow rate sensor was utilized in combination with a Macroduct sweat collector to detect the rate of sweat. Data collection was carried out by establishing an IoT framework through either: (1) displaying the sweat rate information on a smartphone app, or (2) displaying on the cloud via ThingSpeak. Controlled flow rate characterization was performed using a syringe pump to calibrate the device. Human subject trials were conducted to validate the device.

The lower limit of sweat rate detection for the current device is around $0.15 \mu \mathrm{L} / \mathrm{min} / \mathrm{cm}^{2}$. Considering that the device was developed for athletes who would sweat more due to heavy exercise, this limit could potentially not be a practical limit in field tests. For example, one of our subjects (subject 4) was a very light sweater, but we were still able to gather her sweat rate information via our device.

The theoretical concept of the calorimetric flow rate system was investigated by our group [36]. Our current work significantly expanded on this knowledge, and we built a standalone real-time sweat rate device. Our implementation of combining the Macroduct sweat collector with the calorimetric sweat rate sensor (inlet from the bottom, and outlet from the top by using a double PDMS mold) is novel. Furthermore, the device was validated with five subjects, resulting in an average error in accuracy of $18 \%$, which is considered to be very promising. Our device is unique in approach and validation, which separates us from camera-recorded sweat rate measurements [29] or the absorbent patch approach $[30,31]$ that was discussed earlier.

We believe the current developments on IoT interfaces and physiological sensor devices will lead to cloud-based health awareness and tracking tools for unique exercise routines. Our current approach will help pave the way for wireless, real-time sweat-based hydration systems. 
Acknowledgments: This work was funded in part by the National Science Foundation under Grant \#1542368. Zach Nelson helped with $3 \mathrm{~d}$ designs and prints.

Author Contributions: Andrew Brueck performed the experiments and contributed to the writing. Tashfin Iftekhar helped with PDMS fabrication. Alicja B. Stannard helped with subject tests and contributed to writing. Kumar Yelamarthi contributed to writing and building and testing the IoT interface. Tolga Kaya wrote the article, performed the data analysis, and supervised the project.

Conflicts of Interest: The authors declare no conflict of interest.

\section{References}

1. How Smart Watches Can Help Improve Your Health. Available online: https://intermountainhealthcare. org/blogs/topics/live-well/2015/03/how-smart-watches-can-help-improve-your-health (accessed on 24 January 2018).

2. Achten, J.; Jeukendrup, A.E. Heart rate monitoring. Sports Med. 2003, 33, 517-538. [CrossRef] [PubMed]

3. Vaz, A.; Ubarretxena, A.; Zalbide, I. Full Passive UHF Tag with a Temperature Sensor Suitable for Human Body Temperature Monitoring. IEEE Trans. Circuits Syst. II 2010, 57, 95-99. [CrossRef]

4. Heinemann, L.; Schmelzeisen-Redeker, G. Non-invasive Continuous Glucose Monitoring in Type I Diabetic Patients with Optical Glucose Sensors. Diabetologia 1998, 41, 848-854. [CrossRef] [PubMed]

5. Is the Marathon Running Trend Taking over the Fitness World? Available online: https:// fashionmagazine. com/lifestyle/health/marathon-running-trend/ (accessed on 24 January 2018).

6. Rogers, M.M.; Pai, P.M.; Conroy, R.S. Recent Advances in Wearable Sensors for Health Monitoring. IEEE Sens. J. 2015, 15, 3119-3129. [CrossRef]

7. Casa, D.J.; Armstrong, L.E.; Montain, S.J.; Rich, B.S.E.; Stone, J.A. National Athletic Trainers' Association position statement: Fluid replacement for athletes. J. Athl. Train. 2000, 35, 212-224. [PubMed]

8. Sawka, M.N. Physiological consequences of hypohydration: Exercise performance and thermoregulation. Med. Sci. Sports Exerc. 1992, 24, 657-670. [CrossRef] [PubMed]

9. Murray, B. Hydration and physical performance. J. Am. Coll. Nutr. 2007, 26, 542S-548S. [CrossRef] [PubMed]

10. Grandjea, A.C.; Grandjean, N.R. Dehydration and Cognitive Performance. J. Am. Coll. Nutr. 2007, 26, 549S-554S. [CrossRef]

11. Gagnon, D.; Kenny, G.P. Sex differences in thermoeffector responses during exercise at fixed requirements for heat loss. J. Appl. Physiol. 2012, 113, 746-757. [CrossRef] [PubMed]

12. Sato, K.; Kang, W.H.; Saga, K.; Sato, K.T. Biology of sweat glands and their disorders. I. Normal sweat gland function. J. Am. Acad. Dermatol. 1989, 20, 537-563. [CrossRef]

13. Kuno, Y. The Physiology of Human Perspiration; J. \& A. Churchill: London, UK, 1934.

14. Taylor, N.A.; Machado-Moreira, C.A. Regional variations in transepidermal water loss, eccrine sweat gland density, sweat secretion rates and electrolyte composition in resting and exercising humans. Extreme Physiol. Med. 2013, 2, 4. [CrossRef] [PubMed]

15. Morris, D.; Colye, S.; Wu, Y. Bio-sensing textile based patch with integrated optical detection system for sweat monitoring. Sens. Actuators B-Chem. 2009, 139, 231-236. [CrossRef]

16. Alomari, M.; Liu, G.; Mueller, A.; Mock, A.; Ghosh, R.; Smith, K.; Kaya, T. A Portable Optical Human Sweat Sensor. J. Appl. Phys. 2014, 116, 183102. [CrossRef]

17. Bandodkar, A.J.; Wang, J. Non-invasive wearable electrochemical sensors: a review. Trends Biotechnol. 2014, 32, 363-371. [CrossRef] [PubMed]

18. Dam, V.A.T.; Zevenbergen, M.A.G.; van Schaijk, R. Toward wearable patch for sweat analysis. Sens. Actuators B-Chem. 2016, 236, 834-838. [CrossRef]

19. Gao, W.; Emaminejad, S.; Nyein, H.; Challa, S.; Chen, K. Fully integrated wearable sensor arrays for multiplexed in situ perspiration analysis. Nature 2016, 509, 509-526. [CrossRef] [PubMed]

20. Bain, A.R.; Deren, T.M.; Jay, O. Describing individual variation in local sweating during exercise in a temperate environment. Eur. J. Appl. Physiol. 2010, 111, 1599-1607. [CrossRef] [PubMed]

21. Patterson, M.J.; Galloway, S.D.R.; Nimmo, M.A. Variations in regional sweat composition in normal human males. Exp. Physiol. 2000, 85, 869-875. [CrossRef] [PubMed] 
22. Tanaka, H.; Osaka, Y.; Obara, S.; Yamaguchi, H.; Miyamoto, H. Changes in the concentrations of $\mathrm{Na}^{+}, \mathrm{K}^{+}$ and $\mathrm{Cl}-$ in secretion from the skin during progressive increase in exercise intensity. Eur. J. Appl. Physiol. Occup. Physiol. 1992, 64, 557-561. [CrossRef] [PubMed]

23. Buono, M.J.; Ball, K.D.; Kolkhorst, F.W. Sodium ion concentration vs. sweat rate relationship in humans. J. Appl. Physiol. 2007, 103, 990-994. [CrossRef] [PubMed]

24. Shirreffs, S.M.; Maughan, R.J. Whole body sweat collection in humans: an improved method with preliminary data on electrolyte content. J. Appl. Physiol. 1997, 82, 336-341. [CrossRef] [PubMed]

25. Baker, L.B.; Stofan, J.R.; Hamilton, A.A.; Horswill, C.A. Comparison of regional patch collection vs. whole body washdown for measuring sweat sodium and potassium loss during exercise. J. Appl. Physiol. 2009, 107, 887-895. [CrossRef] [PubMed]

26. Morris, N.B.; Cramer, M.N.; Hodder, S.G.; Havenith, G.; Jay, O. A comparison between the technical absorbent and ventilated capsule methods for measuring local sweat rate. J. Appl. Physiol. 2013, 114, 816-823. [CrossRef] [PubMed]

27. Kuo, J.; Yu, L.; Meng, E. Micromachined Thermal Flow Sensors-A Review. Micromachines 2012, 3, 550-573. [CrossRef]

28. Elwenspoek, M. Thermal Flow Micro Sensors. In Proceedings of the IEEE International Semiconductor Conference, Sinaia, Romania, 5-9 October 1999; pp. 423-435.

29. Matzeu, G.; Fay, C.; Caillant, A.; Coyle, S.; Diamond, D. A wearable device for monitoring sweat rates via image analysis. IEEE Trans. Biomed. Eng. 2016, 63, 1672-1680. [CrossRef] [PubMed]

30. Coyle, S.; Lau, K.; Moyna, N.; O'Gorman, D.; Diamond, D.; Francesco, F.; Costanzo, D.; Salvo, P.; Trivella, M.; De Rossi, D.; et al. BIOTEX-biosensing Textiles for Personalized Healthcare Management. IEEE Trans. Inf. Technol. Biomed. 2010, 14, 364-370. [CrossRef] [PubMed]

31. Wei, P.; Morey, B.; Dyson, T.; McMahon, N.; Hsu, Y.-Y.; Gazman, S.; Klinker, L.; Ives, B.; Dowling, K.; Rafferty, C. A Conformal Sensor for Wireless Sweat Level Monitoring. In Proceedings of the IEEE Sensors, Baltimore, MD, USA, 3-6 November 2013.

32. Liu, G.; Ho, C.; Slappey, N.; Zhou, Z.; Snelgrove, S.E.; Brown, M.; Grabinski, A.; Guo, X.; Chen, Y.; Miller, K.; et al. A wearable conductivity sensor for wireless real-time sweat monitoring. Sens. Actuators B-Chem. 2016, 227, 35-42. [CrossRef]

33. Liu, G.; Fuentes, R.; Koser, H.; Kaya, T. A self-powered power conditioning circuit for battery-free energy scavenging applications. Analog Integr. Circuits Signal Process. 2015, 83, 203-207. [CrossRef]

34. Alomari, M.; Mueller, A.; Sel, K.; Edwards, J.; Kaya, T. Detection of Relative $\left[\mathrm{Na}^{+}\right]$and $\left[\mathrm{K}^{+}\right]$Levels in Sweat with Optical Measurements. J. Appl. Phys. 2014, 115, 203107. [CrossRef]

35. Iftekhar, A.T.; Ho, J.C.T.; Mellinger, A.; Kaya, T. 3D Modeling and Characterization of a Calorimetric Flow Rate Sensor for Sweat Rate Sensing Applications. J. Appl. Phys. 2017, 121, 094505. [CrossRef]

36. Yelamarthi, K.; Aman, M.S.; Abdelgawad, A. An Application-Driven Modular IoT Architecture. Wirel. Commun. Mob. Comput. 2017, 2017, 1350929. [CrossRef]

37. Laubhan, K.; Talaat, K.; Riehl, S.; Aman, M.-S.; Abdelgawad, A.; Yelamarthi, K. A low-power IoT framework: From sensors to the cloud. In Proceedings of the IEEE International Conference on Electro Information Technology (EIT), Grand Forks, ND, USA, 19-21 May 2016.

38. Turner, J.; Zellner, C.; Khan, T.; Yelamarthi, K. A Smartphone based Continuous Heart Rate Monitoring System. In Proceedings of the IEEE International Conference on Electro Information Technology, Lincoln, NE, USA, 14-17 May 2017.

39. Abdelgawad, A.; Yelamarthi, K.; Khattab, A. IoT-Based Health Monitoring system for Active and Assisted Living. In Proceedings of the 2nd EAI International Conference on Smart Objects and Technologies for Social Good, Venice, Italy, 30 November-1 December 2016.

40. LilyPad Simblee BLE Board, Sparkfun. Available online: https://www.sparkfun.com/products/13633 (accessed on 24 January 2018).

(C) 2018 by the authors. Licensee MDPI, Basel, Switzerland. This article is an open access article distributed under the terms and conditions of the Creative Commons Attribution (CC BY) license (http:/ / creativecommons.org/licenses/by/4.0/). 
Article

\title{
Estimation of Handgrip Force from SEMG Based on Wavelet Scale Selection
}

\author{
Kai Wang ${ }^{1}$, Xianmin Zhang ${ }^{1}$, Jun Ota ${ }^{2}$ and Yanjiang Huang ${ }^{1,2, *}$ \\ 1 Guangdong Provincial Key Laboratory of Precision Equipment and Manufacturing Technology, \\ South China University of Technology, Guangzhou 510640, China; \\ 201410100131@mail.scut.edu.cn (K.W.); zhangxm@scut.edu.cn (X.Z.) \\ 2 Research into Artifacts, Center for Engineering, University of Tokyo, Chiba 113-8654, Japan; \\ ota@race.u-tokyo.ac.jp \\ * Correspondence: mehuangyj@scut.edu.cn
}

Received: 15 January 2018; Accepted: 22 February 2018; Published: 24 February 2018

\begin{abstract}
This paper proposes a nonlinear correlation-based wavelet scale selection technology to select the effective wavelet scales for the estimation of handgrip force from surface electromyograms (SEMG). The SEMG signal corresponding to gripping force was collected from extensor and flexor forearm muscles during the force-varying analysis task. We performed a computational sensitivity analysis on the initial nonlinear SEMG-handgrip force model. To explore the nonlinear correlation between ten wavelet scales and handgrip force, a large-scale iteration based on the Monte Carlo simulation was conducted. To choose a suitable combination of scales, we proposed a rule to combine wavelet scales based on the sensitivity of each scale and selected the appropriate combination of wavelet scales based on sequence combination analysis (SCA). The results of SCA indicated that the scale combination VI is suitable for estimating force from the extensors and the combination $\mathrm{V}$ is suitable for the flexors. The proposed method was compared to two former methods through prolonged static and force-varying contraction tasks. The experiment results showed that the root mean square errors derived by the proposed method for both static and force-varying contraction tasks were less than $20 \%$. The accuracy and robustness of the handgrip force derived by the proposed method is better than that obtained by the former methods.
\end{abstract}

Keywords: surface electromyography; handgrip force; force-varying muscle contraction; nonlinear analysis; wavelet scale selection

\section{Introduction}

Various dynamic handgrip tasks are required in a number of applications, including manual fabrication and machining, handling tools, monitoring for surgery, human-robot interactions and so on [1-3]. Usually, these tasks require long duration muscle contractions. With respect to the characteristics of usage, a high level of repetition and force is required in the handgrip tasks, which can be considered a risk factor for work-related upper extremity muscular disorder (WUED) [4]. Therefore, accurately monitoring the handling force in real time is important for preventing WUED $[5,6]$. To obtain real-time handgrip force measurements, one indirect measurement method is based on surface electromyography (SEMG), which is a common, non-invasive technique for analyzing muscle contractions for real-world applications $[4,7,8]$. However, SEMG signals are affected by physiological factors and non-physiological factors during prolonged muscle contractions, which affects force estimation from SEMG $[1,9,10]$. These influence factors are complex and varied under a prolonged muscle contraction environment. Therefore, it is necessary to obtain valid signals from the SEMG to realize accurate prediction of handgrip force in a long duration gripping motion. 
In previous studies, a number of correlations between SEMG of a target muscle and force output of a target task have been discussed. In muscle contraction, the activation of motor units within a target muscle follows a mechanism (motor unit rotation and substitution) to maintain a given force. However, muscle fatigue resulting from prolonged muscle contractions can reduce the force generation performance of the target muscles. To overcome the decline in performance, more fresh motor units are recruited to replace the burden of fatigued motor units and compensate for a lack of performance $[7,9,11,12]$. Many researchers have reported that the increase in amplitude of SEMGs collected from long duration muscle contractions is caused by complex electrophysiological events (i.e., motor unit rotation or substitution), motion artifact, and so on, which, as noises, affect the SEMG signals at different frequencies and reduce the force estimation performance [8,13-17]. According to this phenomenon, many time-frequency and time-scale techniques have been used to eliminate the influence of these noises on force estimation from SEMG.

Many researchers used Fast Fourier Transform (FFT) to observe that the power spectrum of SEMG shifts to a lower frequency range when complex noises (electrophysiological effect of fatigue, random noises) develops [18-21]. The frequency banding technique based on FFT showed that the low frequency band amplitude is increased linearly during endurance isometric contraction tasks [22]. Kumar et al. (2003) found that the amplitude of the SEMG signal in the high frequency band does not change significantly with the development of complex physiological noise, through wavelet analysis [23]. To analyze the changes in SEMG in varying muscle contractions, time-frequency techniques have typically been adopted. A comparison of different time-frequency techniques indicated that continuous wavelet transformation (CWT) produces precise results with good representation of time and frequency localization [24]. The frequency-band analysis technique based on wavelet transformation has been generally used to explore the valid signals from SEMG for accurately estimating force. Sparto et al. (1999) used different wavelet scales of the Daubechies (db) wavelet function of order 6 to report that a decrease in high frequency wavelet coefficients and an increase in low frequency wavelet coefficients may be correlated with fatiguing muscle contractions [25]. Soo et al. (2010) performed a comparison of 55 types of wavelet scale combinations (WSC) and reported that a correlation between two scales (scale 2 and scale $3 \mathrm{of} \mathrm{db2)} \mathrm{and} \mathrm{handgrip} \mathrm{force} \mathrm{provides} \mathrm{a} \mathrm{stable}$ estimate of handgrip force from SEMG during endurance isometric contractions [6].

Many CWT-based methods, such as frequency band analysis, have been utilized to obtain a suitable wavelet scale for estimating accurate force from SEMG. These methods have used a single scale or particular WSC to analyze and filter the original signal [14,22]. An original signal can be decomposed into signals under different wavelet scales (i.e., different frequency band ranges) and retains the time domain features through CWT. In practical grasping tasks, each wavelet scale signal contains a variety of complex physiological and non-physiological factors. It is difficult to identify which factors are present at different scales. Currently, the nonlinear differences in quantification between each wavelet scale and the measured force are not clear. Thus, it is necessary to analyze the nonlinear correlations (i.e., nonlinear degree) between each wavelet scale and force in the estimation of handgrip force from SEMG.

The purpose of this paper is to find an appropriate WSC for accurate evaluation of handgrip force from SEMG during force-varying muscle contractions of long duration. The suitable WSC was obtained by using the sequence combination analysis (SCA) method based on Monte Carlo sensitivity analysis. As a simple and reliable sensitivity analysis method, Monte Carlo simulation can calculate the degree of nonlinearity between variables and the actual target output, regardless of how many factors the variable contains [26,27]. We first obtained the nonlinear correlations between wavelet scales and handgrip force during prolonged force-varying muscle contraction in the Monte Carlo sensitivity analysis, and then selected the appropriate WSC by analyzing the nonlinearity degree of each correlation. Finally, we evaluated the proposed method by comparing it to former methods. The root mean square error (RMSE) was used to evaluate the difference between the estimated force and the measured force during the WSC selection procedure, as well as comparisons between the 
proposed method and former methods. The contribution of this study is that we have investigated the nonlinear correlations between the wavelet scale and handgrip force. These correlations can enhance the selection of an appropriate WSC to accurately estimate handgrip force during force-varying muscle contractions of long duration.

\section{Experimentation and Methodology}

\subsection{Experimental Setup}

Twenty healthy participants (16 men and 4 women), whose ages ranged from 22 to 31 years, participated in the experiment. At this stage, we did not consider the differences between males and females. Ten subjects (group A: 8 men and 2 women) were used for determining the suitable WSC for estimating handgrip force in the force-varying contractions as a training group. The other ten subjects (group B: 8 men and 2 women) were used as a validation group, to evaluate the performance of the suitable WSC. Before the experiment, all participants were informed of the experiment process and the risk of muscle fatigue, and agreed to perform the experiment.

In the gripping tasks, SEMG data was recorded from the flexor digitorum superficialis (FDS) muscle and extensor carpi radialis (ECR) muscle of the forearm, as shown in Figure 1. These are the dominant forearm muscles that contribute to handgrip force [28,29]. The SEMG was measured using reusable integral bipolar dry surface electrodes (Green Sensor Electrodes SX230, Biometrics Ltd., Newport, UK) with a skin contact area of $10 \mathrm{~mm}^{2}$ for each electrode and a center-to-center recording distance of $20 \mathrm{~mm}$. Electrode locations were determined in accordance with a previous study [6,29]. EMG signals were amplified 20 times and bandpass prefiltered $(15-450 \mathrm{~Hz})$, together with the force signals that were obtained at a sampling rate of $1000 \mathrm{~Hz}$ (14 bits), using a portable electrophysiological amplifier system (Biometrics Ltd., DataLOG-MWX8-Bluetooth, UK, input resistance > 1015 ohms, CMRR-60 Hz $>96 \mathrm{~dB}$ ) and stored on a computer. Handgrip forces $(\mathrm{kg})$ were measured using an adjustable handgrip dynamometer. The sampling rate was set to $1000 \mathrm{~Hz}$, and the data was digitized at a 14-bit resolution. To reduce the influence of grip width, a grip width of $65 \mathrm{~mm}$ was selected [5]. The force signal was visualized online, on a computer screen, to provide feedback about the exerted force by subjects during the data collection procedure, as shown in Figure 1.

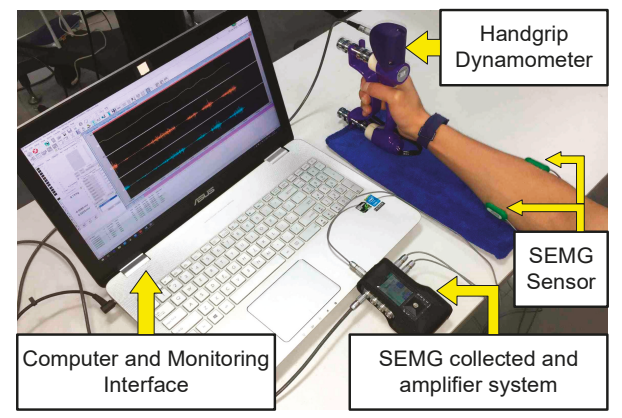

Figure 1. Experimental setup. SEMG: surface electromyogram.

\subsection{Experimental Procedure}

At the beginning of the experiment, each participant was asked to perform a maximal voluntary contraction task (MVC) three times, and from this, the average value of maximum grip strength $(\mathrm{kg})$ and maximum amplitude $(\mathrm{mV})$ of the SEMG were calculated. In the experiment, the participants in Group A first performed the force-varying analysis task to obtain the suitable WSC using the proposed method. Then, all participants in Groups A and B performed the static and force-varying validation 
tasks to evaluate the performance of the proposed method. The force-varying analysis task, static validation task, and force-varying validation task are defined as follows.

\subsubsection{Force-Varying Analysis Task (Force-Varying Contraction)}

Participants in Group A were required to perform a force-varying fluctuating contraction. In the force-varying analysis task, a series of varying handgrip forces, occurring within ten minutes, was increased or decreased respectively by squeezing and releasing the dynamometer. Participants were asked to increase their handgrip force gradually to provide an adequate correlation between handgrip force and SEMG for each minute, as shown in Figure 2.

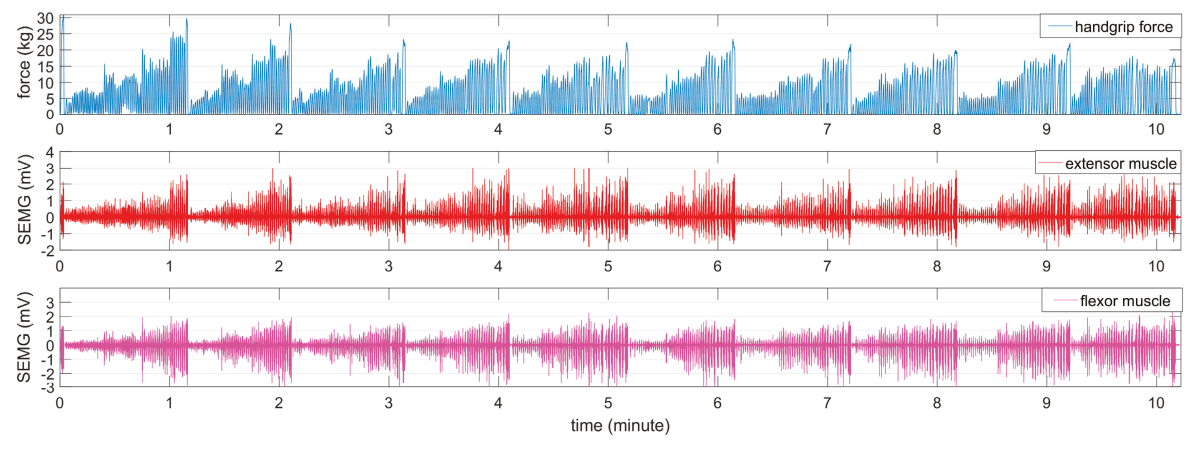

Figure 2. The process of the force-varying analysis task.

\subsubsection{Static Validation Task (Isometric Muscle Contraction)}

Static tasks were performed at four force levels, which included $20 \%, 30 \%, 50 \%$, and $70 \%$ of the MVC in a random order during the isometric contraction [6,29]. The participants sustained these contractions until the generated force dropped below $5 \%$ from the target force level for $5 \mathrm{~s}$. The experiment with each force level was executed only once. To avoid the effect of muscle fatigue, participants were allowed to rest for $15 \mathrm{~min}$ before performing the next force level experiment [6].

\subsubsection{Force-Varying Validation Task (Voluntary Contraction)}

In the force-varying validation task, a force level that varied between $0 \%$ and $80 \%$ of the MVC was performed over a 10-min period. Before the force-varying validation task, the participant rested for $60 \mathrm{~min}$. A sample of the recorded handgrip force and SEMG for both muscles is illustrated in Figure 3.
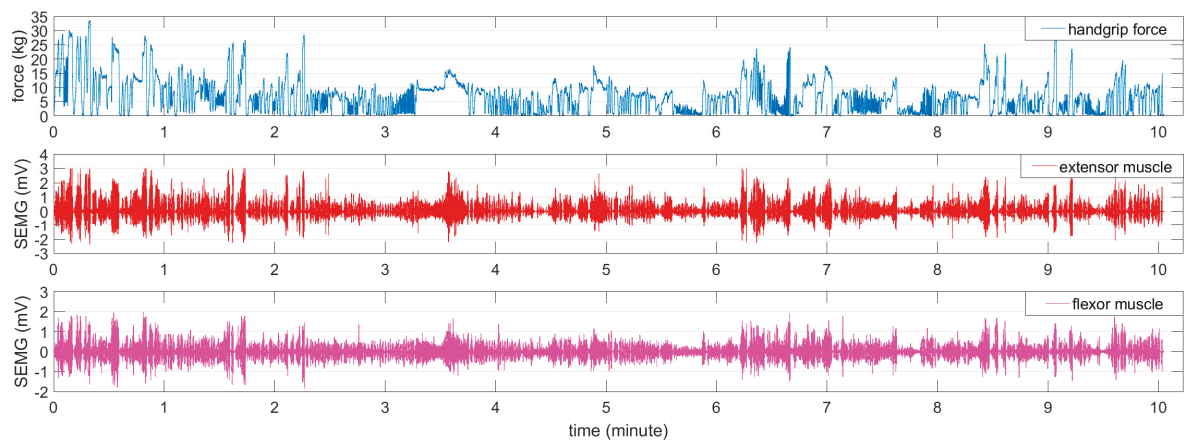

Figure 3. The process of the force-varying validation task. 


\subsection{Proposed Method}

To accurately estimate the handgrip force from the SEMG signals during the long duration force-varying grip motion, this paper utilized the Monte Carlo sensitivity analysis to determine the nonlinear degree of the relationship between each wavelet scale and handgrip force from the initial nonlinear SEMG-handgrip force relationship, and used a nonlinear correlation-based scale-selection method (SCA) to obtain the suitable WSC of the scale.

\subsubsection{Initial Nonlinear SEMG-Handgrip Force Relationship}

The initial nonlinear SEMG-handgrip force relationship was built using the ten wavelet (db2) scales (scale number: $12,3,4,5,6,7,8,9,10)$ from the SEMG signals and handgrip force collected from the force-varying analysis tasks of group A. These wavelet scales are most relevant to handgrip force [23]. This model is based on the second-order polynomial [29,30]. Given the predictors $e(1), \ldots$, $e(P)$, the response of handgrip force $F($ estimation $)$ is predicted by:

$$
F(\text { estimation })=\sum_{p=1}^{P}\left(\theta_{1 p} e(p)+\theta_{2 p} e^{2}(p)\right)
$$

where $1 \leq p \leq P=10, P$ is the total number of wavelet scales, and the vectors of coefficients $\theta_{1}=\left(\theta_{11}, \ldots, \theta_{1 p}, \ldots, \theta_{1 P}\right)$ and $\theta_{2}=\left(\theta_{21}, \ldots, \theta_{2 p}, \ldots, \theta_{2 P}\right)$ are produced by ordinary least squares. In this paper, the predictors, $e(p)$, are the wavelet coefficients' intensities of the wavelet scale of $p$, which are collected from the original SEMG $x_{n}$, with data size, $N$, through CWT and root mean square (RMS)

$$
c_{n}(p)=\sum_{n=0}^{N-1} x_{n} \psi^{*}\left[\frac{\left(n^{\prime}-n\right) \delta t}{p}\right]
$$

where $0 \leq n \leq N-1, c_{n}(p)$ is the wavelet coefficient, $\delta t$ is the sampling time, $\psi$ is the mother wavelet, and $\left(^{*}\right)$ indicates the complex conjugate [31]. Then, the valid intensity of wavelet coefficients can be calculated after pre-processing of the RMS with the window frame size of $M$

$$
e_{n}(p)=\sqrt{\frac{1}{M} \sum_{n=k M}^{(k+1) M-1} c_{n}(p)^{2}}
$$

where $0 \leq k \leq \frac{N}{M}-1$. A moving average filter (200 ms) [32] was used to smooth the $e_{n}(p)$.

\subsubsection{Sensitivity Analysis}

For a nonlinear index, the sensitivity analysis is important [26]. In contrast to other sensitivity analysis methods, Monte Carlo simulation, as a variance-based global sensitivity analysis method, can obtain the sensitivity of interactions between the parameters [27]. This paper used the multivariate Monte Carlo simulation to obtain the sensitivity value of each wavelet scale related to force, predicted from the original nonlinear model (Equation (1)). The Monte Carlo simulation is often used to calculate the expected estimation of parameters in known mathematical models with random variables $[26,27]$. The main steps of Monte Carlo simulation can be categorized as follows:

(1) CWT was used to convert the original SEMG signals to wavelet coefficients of the ten scales in the force-varying analysis task.

(2) Wavelet coefficients of the ten scales normalized by MVC data were used to build the initial nonlinear SEMG-handgrip force relationship.

(3) A specific number of random variables were used to conduct a simulation of the handgrip force in the initial nonlinear relationship. 
(4) Mean square deviation was used to calculate the simulated sensitivity value corresponding to each correlation between ten scales and handgrip force.

(5) The sensitivity value of each correlation was normalized by the sum of the sensitivity values of the ten scales.

(6) Steps 1-5 were repeated for every minute in the force-varying analysis task.

In this paper, the random variable was distributed evenly in a specific range (0-100) as a basic step to realize the Monte Carlo simulation. In each iteration of the simulation, each model parameter used a given number of random variables to obtain the expected sensitivity value. The given number of random variables was obtained by a finite difference-based convergence experiment for all participants in Group A [27]. This experiment showed a relationship between the number of random variables and the expected sensitivity estimator, as shown in Figure 4. This experiment was performed at a specific variable number range, $r$, that was between 100 to 140,000, with an interval of 100 . By using this range, $r$, the sensitivity of each wavelet scale was obtained from the Monte Carlo simulation at each given variable quantity and these sensitivities were calculated by first order differential calculation.

$$
\begin{gathered}
d_{i}(p)=\left|y_{i+1}(p)-y_{i}(p)\right| \\
i=\frac{r}{100}
\end{gathered}
$$

where $p=1,2, \ldots, 10$, is the ordinal number of the wavelet scale, $r=100,200 \ldots, 140,000$, and $y_{i}(p)$ is the sensitivity value of the wavelet scale of $p$ with a specific random variable number $(r=i \times 100)$. Then, the sum, $d_{i}$ (integration), of the sensitivity differences under a variable number for Group A can be calculated as follows:

$$
d_{i}(\text { integration })=\sum_{p=1}^{10} d_{i}(p)
$$
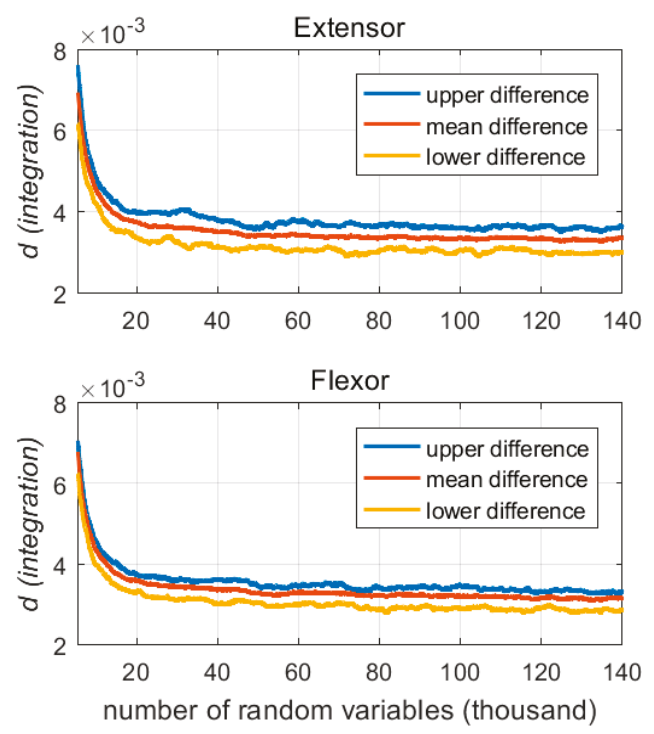

Figure 4 . The results of the convergence experiment for determining the number of random variables. 
Figure 4 depicts the influence of the number of random variables on the sensitivity distribution. The mean difference (red line) decreased as the number of random variables increased, and tended to stabilize from 60,000. The upper difference (blue line) and the lower bound difference (yellow line) became stable when the number of random parameters exceeded 100,000. Therefore, this paper used 100,000 random parameters, which were uniformly distributed between $0-100$, to perform the Monte Carlo simulation for analyzing the sensitivity of each wavelet scale to handgrip force.

\subsubsection{Sequence Combination Analysis (SCA)}

Based on the Monte Carlo simulation, we obtained the nonlinear degree of each scale-handgrip force correlation through the force-varying analysis tasks. To choose the suitable WSC, we designed a set of scale permutations and combination rules based on the mean value of sensitivity of each scale. The mean sensitivity value was calculated from all participants in Group A. The distribution and mean sensitivity value of each scale is shown in Figure 5. Through frequency band analysis, Soo et al. (2010) observed that wavelet scales 2 and 3 have large effects on fatigue resistance during muscle isometric contractions [6]. By using the Monte Carlo sensitivity analysis, it was shown that these two scales have lower sensitivity to handgrip force than other scales. Therefore, this paper determined a specified sequence and combination rule of the wavelet scale. The rule is that the scale with low sensitivity is preferred for retention and the scale with high sensitivity is eliminated, in turn. By considering the wavelet scales described in Section 2.3.1, we can obtain ten WSCs (combination serial numbers I, II, III, IV, V, VI, VII, VIII, IX, X) based on the proposed sequence combination analysis. The details of the WSCs are illustrated in Figure 6. The coefficient tern structure and parameters of the original nonlinear SEMG-handgrip force relationship were adjusted to match the different WSCs. This paper uses RMSE as the evaluation index to determine which WSC has the appropriate estimation accuracy for handgrip force.

\subsection{Performance Comparison}

\subsubsection{Former Method}

In this paper, two former methods were utilized to be compared with the proposed method. Formerly, force estimation was realized by calculating the amplitude of the SEMG signal, for example, using RMS and the pre-defined SEMG-force relationship. Former method A builds a linear SEMG-force relationship by using the normalized SEMG signal, which is bandpass-filtered between 15 and $450 \mathrm{~Hz}$ [5]. Former method B builds a SEMG-force model based on the frequency-band technique, which determines a suitable WSC (wavelet scales 2 and 3 ) to estimate the handgrip force from SEMG signals during an isometric muscle contraction task [6].

\subsubsection{Performance Index}

To obtain the estimated error, a comparison between the estimated and actual handgrip force was conducted using the RMSE:

$$
\text { RMSE }=\sqrt{\frac{1}{T} \sum_{t=1}^{T}\left(F(\text { actual })_{t}-F(\text { estimation })_{t}\right)^{2}}
$$

where $F($ actual) is the sample data of the observed griping force from the grip dynamometer and F(estimation) is the predicted force from SEMG. The sampling number of handgrip force is $1 \leq t \leq T$. A value of RMSE closer to zero indicates that the model has a smaller random error component. 


\section{Results and Discussion}

\subsection{Nonlinear Correlation between Wavelet Scale and Handgrip Force}

Figure 5 describes the distribution between the wavelet scale and its sensitivity to handgrip force for Group A. These sensitivity values can be used to evaluate the degree of nonlinear correlation between the wavelet and handgrip force. According to the observation of the sensitivity distribution of the two muscles, scales 1, 2, 3, 4 have lower degree nonlinear correlations with handgrip force, the degree of nonlinear correlation between scales $6,7,8,9,10$ and handgrip force are generally higher, and scale 5 is in the middle sensitivity position. According to Soo et al. (2010), the four wavelet scales with a low degree of nonlinear correlation to handgrip force are in the 181.82-727.27 Hz central frequency range, the five scales with a higher degree of nonlinear correlation to handgrip force are between $72.72 \mathrm{~Hz}$ and $121.21 \mathrm{~Hz}$ of central frequency and the center frequency of scale 5 is in the middle at $145.45 \mathrm{~Hz}$ [6]. Wakeling et al. (2002) reported that the frequency ranges for recruitment of slow and fast twitch muscle fibers are approximately 143 and $397 \mathrm{~Hz}$ of SEMG, respectively [33]. Thus, it can be approximated that slow fiber signal actions at scale 5, and fast fibers, are located at low sensitivity scales. Begg et al. (2007) reported that the SEMG of low frequency signals contain motion artifacts [34], which may result in high sensitivity. Many researchers reported that the power spectrum of SEMG shifts to a lower frequency range when muscle fatigue develops $[11,18,19]$. According to the center frequency of the wavelet scale, the wavelet scales 6-10 belong to the low frequency range of the SEMG signal. This is the reason why the wavelet scales of low frequency signals (i.e., scale 6-10 in Figure 5) have a high degree of sensitivity.

From the results shown in Figure 5, we can observe that each scale has a different sensitivity distribution. To simplify the distribution, we calculated the mean sensitivity of each scale to express the relationship between the ten wavelet scales and their sensitivity to handgrip force. Then, wavelet scales were arranged, in ascending order, in regard to mean sensitivity value, as shown in Figure 6.
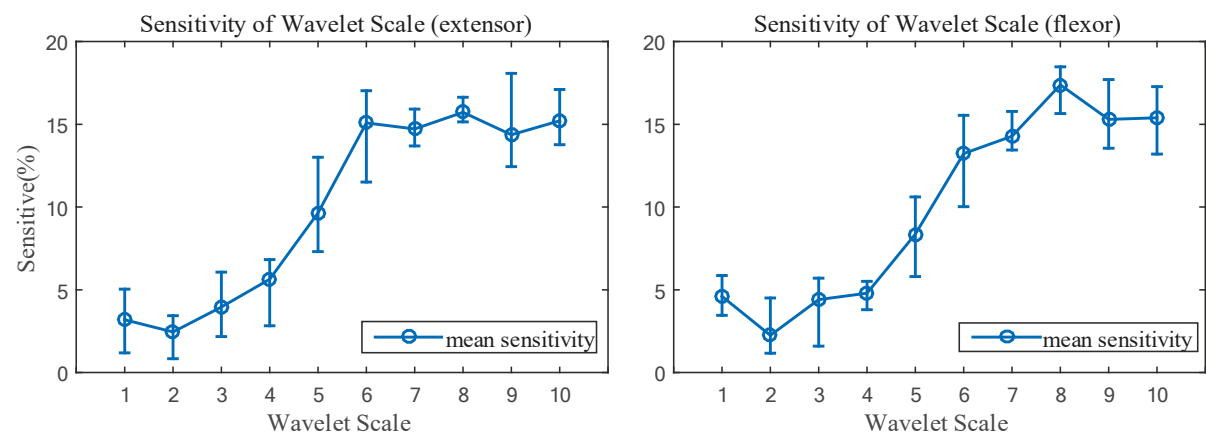

Figure 5. Sensitivity distribution of the ten wavelet scales to handgrip force.

\subsection{Selection of Wavelet Scale Combination}

Figure 6 describes a layout of the SCA. The layout shows the sequence and combination of the ten wavelet scales. For instance, the WSC II covered all scales, except scale 8, and the WSC X only had scale 2. The performances of force estimation under the ten WSCs were evaluated by RMSE during the force-varying validation tasks. The RMSE was calculated based on the difference between the handgrip force estimated from SEMG and the handgrip force measured from the grip dynamometer. 


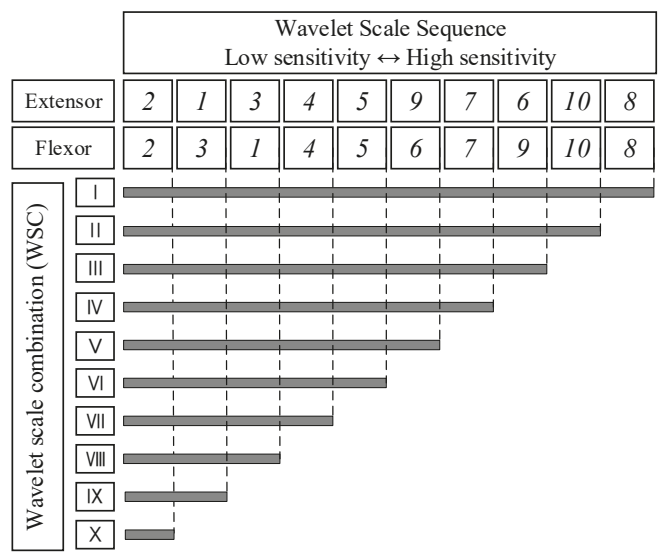

Figure 6. Sequence combination of wavelet scales based on the sensitivity value.

The performance evaluation consisted of a varying RMSE process of the ten WSCs during the force-varying validation task. Figure 7 illustrates that the performance evaluation of the ten WSCs is a time-varying RMSE process. Each WSC has a different error curve during the force-varying validation task. Figure 7 shows that WSC I, II, III, IV, and V obtained from the extensor muscles have unsatisfactory estimated accuracies, and WSC VI to X have similar estimation accuracies in the amplificatory graph. In comparison to the five WSCs (I, II, III, IV, and V), the removal of highly sensitive wavelet scales can help to improve the estimation accuracy of handgrip force over a long duration griping motion. WSC I, II, III and IV obtained from the flexor muscle have unsatisfactory accuracies of estimated force, and WSC V to X have similar estimated accuracies. To choose the suitable WSC, a statistical method was used to evaluate the differences between estimated accuracy for ten WSCs, as shown in Figure 8.
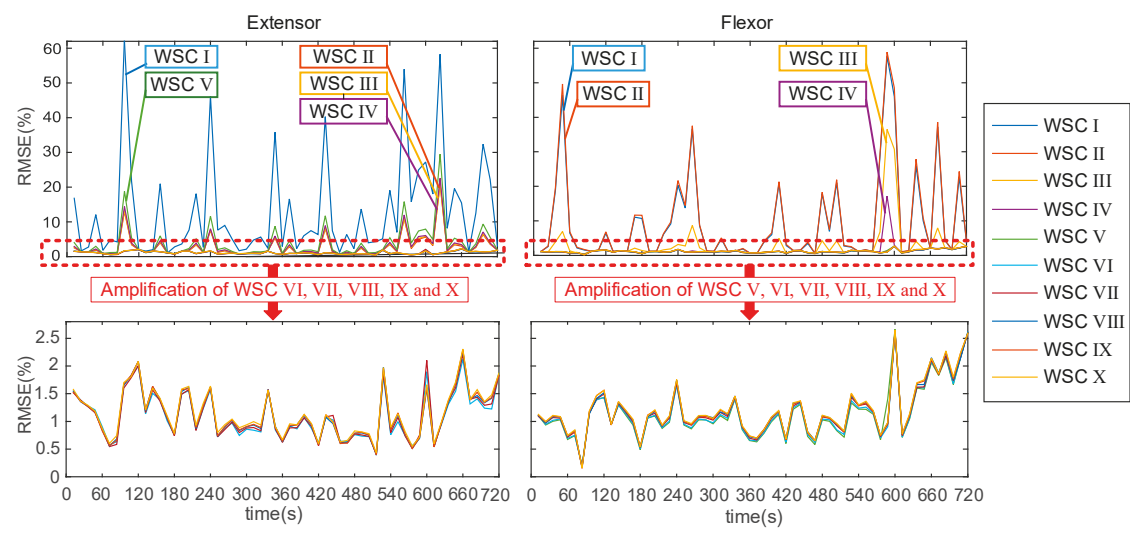

Figure 7. Varying RMSE process of each wavelet scale combination (WSC) in the force-varying validation task. 
Extensor

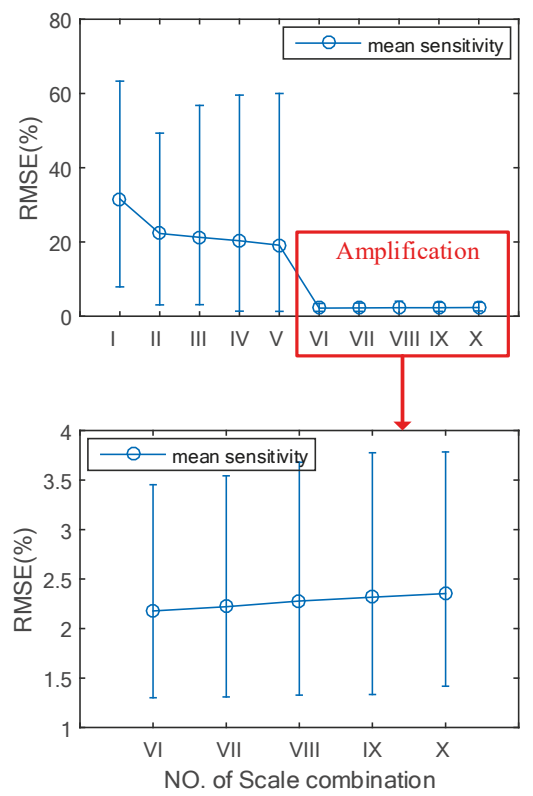

Flexor

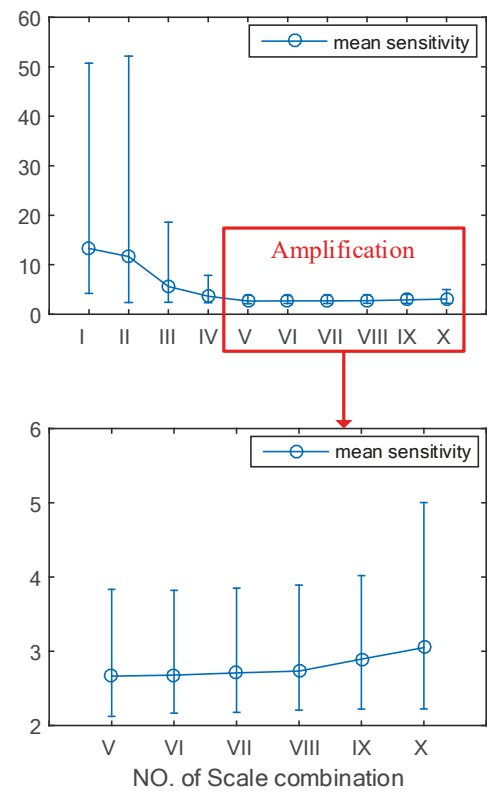

Figure 8. Comparison of the ten WSCs.

Figure 8 shows the handgrip force estimation error that was derived from Group A under different WSCs. The WSCs of I, II, III, IV, and V in the extensor muscle have larger predicted errors of handgrip force than the others. However, after amplification of local errors, it was found that the minimum force estimation error occurred in WSC VI. The suitable WSC selection for the flexor muscle is similar to that for the extensor muscle. The WSC V of the flexor muscle is considered to have the minimum RMSE range and mean in estimation of the long duration handgrip force from SEMG.

\subsection{Performance Comparison}

Figures 9 and 10 show the comparisons between RMSE of handgrip force, estimated by the proposed method and former methods, in both force-varying and static validation tasks. Figure 9A,C present the RMSE obtained by the participants in Groups A and B within the first minute of the force-varying validation task. In the first minute of the task, the SEMG is affected by a low degree of physiological effect of fatigue and motion artifact. Figure 9B,D shows the performance comparisons in the last minute of the validation task. In the last minute of task, the complex noise (electrophysiological effect of fatigue, motion artifact, and so on) seriously affects SEMG amplitude and frequency, and reduces the correlation between SEMG and handgrip force. This is the reason why the RMSE in the first minute is smaller than that in the last minute. Figure 10 shows the RMSE derived by the proposed method and former methods during the static task. Since the static task is more likely to produce fatigue than the force-varying task, we compared the estimated accuracy of these three methods in the first $10 \mathrm{~s}$ and the last $10 \mathrm{~s}$ of the isometric contraction task, at four force levels. The results show that the RMSE in the first $10 \mathrm{~s}$ is smaller than that in the last $10 \mathrm{~s}$. 

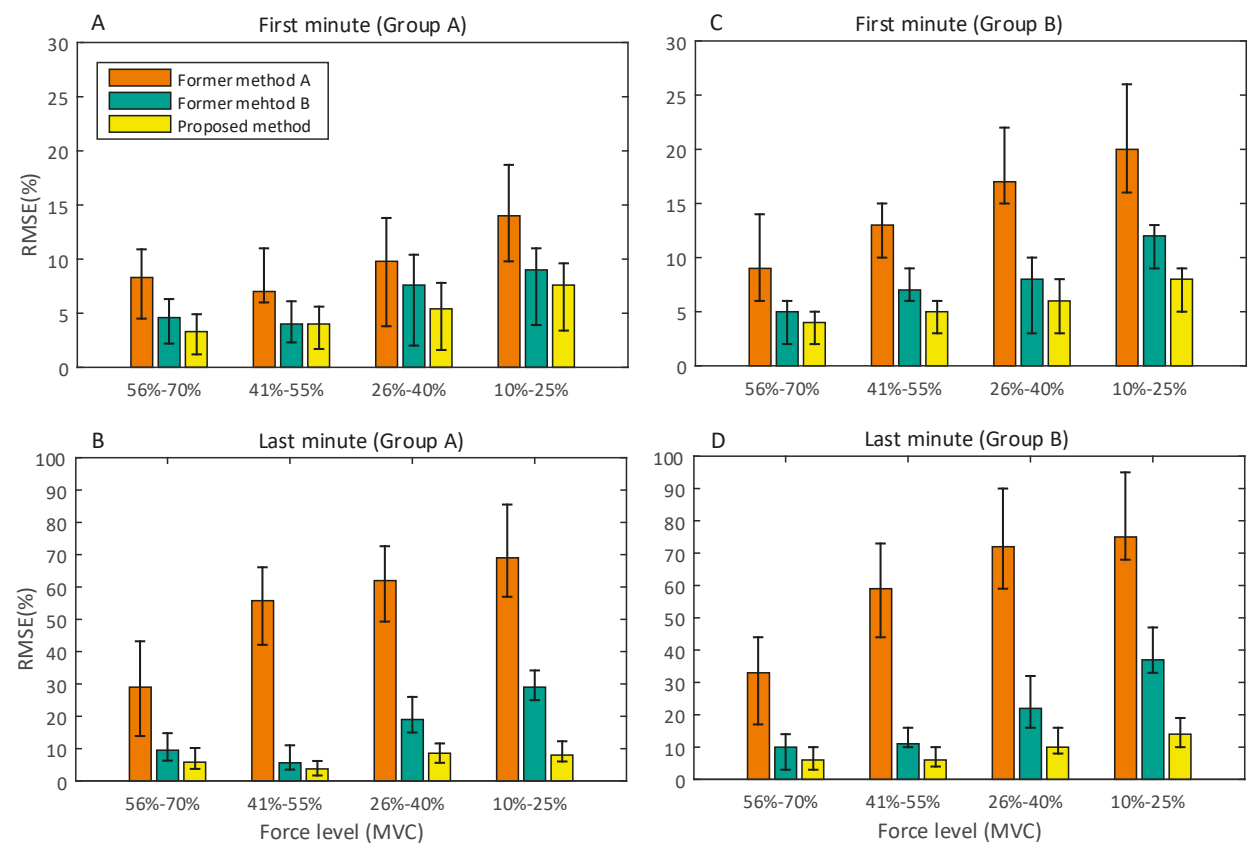

Figure 9. Results of the force-varying validation tasks.
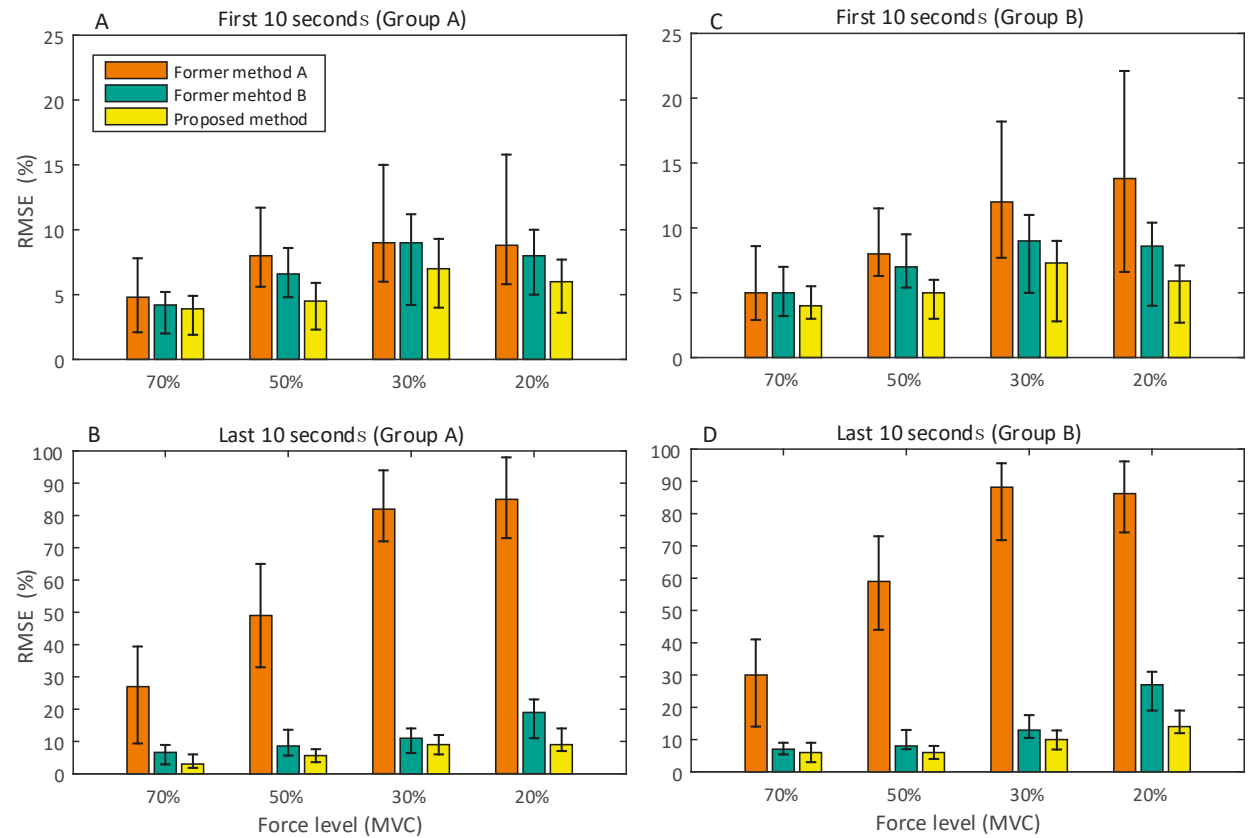

Figure 10. Results of the static validation tasks. 
In the force varying validation task, the experimental results (Figure 9) show that the proposed method has a better performance than the others in terms of force estimation, especially in the force range of $10-25 \%$. This is because the proposed method can obtain a better pre-defined SEMG-handgrip force relationship than the former methods. In contrast with the high degree of force (greater than $41 \%$ MVC), the performances of these three methods are slightly different. Because high force muscle contraction requires a large number of motor units to maintain the particular level of force, the high force muscle contraction is different from the low force contraction. Based on the principle of motor unit recruitment [1,9], the three methods can obtain a similar pre-defined SEMG-handgrip force relationship at a high force level. Since former method A contains all wavelet scales, except for scale 1, its pre-defined SEMG-handgrip force relationship is based on the scales with considerable noise (motion artifacts and random noise); thus, the performance of this method is weak compared to the other methods. The similar results can be obtained in the static validation task, as shown in Figure 10.

The proposed method has a significant performance improvement for fatigued muscle contractions with long durations (see Figures 9B,D and 10B,D). The proposed method reduces the force-varying validation RMSEs by $27 \%, 53 \%, 54 \%$, and $61 \%$, compared to former method $\mathrm{A}$, in the handgrip force ranges of $56-70 \%, 41-55 \%, 26-40 \%$ and $10-25 \%$ of MVC, respectively. The RMSE of the proposed method is reduced by $4 \%, 2 \%, 11 \%$ and $21 \%$, respectively, in these four ranges of force, compared to former method B. In the static validation tasks, the proposed method can significantly improve the performance of force estimation at the low force level. A large amount of noise caused by the electrophysiological effect of fatigue and motion artifacts affects the amplitude and frequency of SEMG, which causes the pre-defined SEMG-handgrip force relationship built by former methods to no longer be accurate.

Compared to the first minute, the estimated accuracy of former method B in the last minute decreased by an average of $11 \%$ in the force range of $10-40 \%$ of MVC. From the perspective of sensitivity of each scale to handgrip force, this is because the wavelet scales 2 and 3 have lower sensitivities to gripping force prediction than the other scales and thus, cannot characterize flexibles change during force-varying contractions for low forces. The signal noise induced by the intramuscular electrophysiological effect and motion artifacts has consequences for the correlation between the two scales and handgrip force and further reduces the estimated performance of former method B. Previous studies have shown that low strength muscle contractions are mainly controlled by slow-twitch muscle fibers (approximately $143 \mathrm{~Hz}$ of SEMG), and high strength muscle contractions are mainly controlled by fast-twitch muscle fibers (approximately $397 \mathrm{~Hz}$ ) [33,35]. In former method B, the SEMG signal of $143 \mathrm{~Hz}$ is missing due to the limitation of scale pre-layout in frequency band analysis technology [6]. This is the reason why the performance of former method B is worse than our proposed method.

In contrast to the former methods, the proposed method can effectively inhibit the influence of complex electrophysiological noises on the predicted accuracy of the handgrip force from SEMG during long duration fatiguing muscle contractions. However, the prediction errors for low-level forces are greater than those for high level forces. The reason for this is that the intramuscular electrophysiological effect and motion artifacts can reduce the degree of correlation between different levels of force and SEMG to varying degrees. During the long duration muscle contractions, all participants overcame fatigue to produce a specified handgrip force with extra shaking of the forearm. Motion artifact noise may be caused by the shaking of these forearm muscles. When the fatigue reduces the force generated from the muscle, the rotation and substitution between fatigued motor units and fresh motor units is performed to generate and maintain the requisite force level. In this situation, the complex electrophysiology within muscles affects the changes in the SEMG signal. Thus, the intramuscular electrophysiological effect and motion artifacts ultimately change the amplitude and frequency of the SEMG signal. 


\section{Conclusions}

In this paper, we proposed a nonlinear correlation-based method to select appropriate wavelet scales to accurately estimate handgrip force from SEMG during a force-varying muscle contraction of long duration. The sensitivity analysis of the Monte Carlo simulation was used to explore the nonlinear correlation between ten wavelet scales and handgrip force. A nonlinear, correlation-based SCA method was proposed to determine the suitable WSC. The results derived via SCA indicated that the suitable scales for the extensor muscles is WSC VI, consisting of scales 1 to 5 , and WSC V including scales 1 to 6 is suitable for flexor muscles. The appropriate WSC, obtained by the proposed method, was compared to two former methods through a set of static and force-varying contraction tasks. The experimental results showed that the proposed method has a better estimation performance than the former methods, especially for low level contractions (0-30\% maximal voluntary contraction).

The proposed method enhances the performance of grip strength estimation from SEMG. However, the actual working muscles not only provide force output but also drive limb posture changes. Further research using the proposed nonlinear correlation-based scale selection method is needed to explore the correlation between wavelet scales of SEMG and muscle loads or limb posture in various work tasks, such as human-robot interactions, and gait analysis based on SEMG of lower limb muscles. An accurate force estimation based only on electromyography data can be used for gait analysis instead of using inverse dynamics or similar methods [36,37]. In addition, the participants in this study were limited in age, health, and sex ratio (i.e., 16 healthy male students and four healthy female students); thus, further research should take into account the gender differences in the results of the proposed method.

Acknowledgments: This work was supported by the [Natural Science Foundation of China] under Grant [51505151,91748111]; [Natural Science Foundation of Guangdong Province] under Grant [2015A030310239].

Author Contributions: Kai Wang, Xianmin Zhang, Jun Ota and Yanjiang Huang conceived and designed the experiments; Kai Wang and Yanjiang Huang performed the experiments and analyzed the data; Kai Wang and Yanjiang Huang wrote the paper; Xianmin Zhang, Jun Ota and Yanjiang Huang revised the paper. All of the authors have read and approved the final manuscript.

Conflicts of Interest: The authors declare no conflict of interest.

\section{References}

1. Staudenmann, D.; Roeleveld, K.; Stegeman, D.F.; van Dieën, J.H. Methodological aspects of SEMG recordings for force estimation-A tutorial and review. J. Electromyogr. Kinesiol. 2010, 20, 375-387. [CrossRef] [PubMed]

2. Scheme, E.; Englehart, K. Electromyogram pattern recognition for control of powered upper-limb prostheses: State of the art and challenges for clinical use. J. Rehabil. Res. Dev. 2011, 48, 643-659. [CrossRef] [PubMed]

3. Daley, H.; Englehart, K.; Hargrove, L.; Kuruganti, U. High density electromyography data of normally limbed and transradial amputee subjects for multifunction prosthetic control. J. Electromyogr. Kinesiol. 2012, 22, 478-484. [CrossRef] [PubMed]

4. Hermens, H.; Freriks, B.; Disselhorst-Klug, C.; Rau, G. Development of recommendations for SEMG sensors and sensor placement procedures. J. Electromyogr. Kinesiol. 2000, 10, 361-374. [CrossRef]

5. Hoozemans, M.J.M.; van Dieen, J.H. Prediction of handgrip forces using surface EMG of forearm muscles. J. Electromyogr. Kinesiol. 2005, 15, 358-366. [CrossRef] [PubMed]

6. Soo, Y.; Sugi, M.; Yokoi, H.; Arai, T.; Nishino, M.; Kato, R.; Nakamura, T.; Ota, J. Estimation of handgrip force using frequency-band technique during fatiguing muscle contraction. J. Electromyogr. Kinesiol. 2010, 20, 888-895. [CrossRef] [PubMed]

7. Liu, J.; Brown, R.; Yue, G. A dynamical model of muscle activation, fatigue, and recovery. Biophys. J. 2002, 82, 2344-2359. [CrossRef]

8. Edwards, R.H. Human muscle function and fatigue. Ciba Found. Symp. 1981, 82, 1-18. [CrossRef] [PubMed]

9. Marco, G.; Alberto, B.; Taian, V. Surface EMG and muscle fatigue: Multi-Channel approaches to the study of myoelectric manifestations of muscle fatigue. Physiol. Meas. 2017, 38, 27-60. [CrossRef] [PubMed] 
10. Vieira, T.M.; Bisi, M.C.; Stagni, R.; Botter, A. Changes in tibialis anterior architecture affect the amplitude of surface elexctromyograms. J. Neuroeng. Rehabil. 2017, 14, 14. [CrossRef] [PubMed]

11. Klaver-krol, E.G.; Rasker, J.J.; Henriquez, N.R.; Verheijen, W.G.; Zwarts, M.J. Muscle fiber velocity and electromyographic signs of fatigue in fibromyalgia. Muscle Nerve 2012, 46, 738-745. [CrossRef] [PubMed]

12. Krou-Lund, C.; Jørgensen, K. Myo-electric fatigue manifestations revieited: Power spectrum, conduction velocity, and amplitude of human elbow flexor muscles during isolated and repetitive endurance contractions at 30\% maximal voluntary contraction. Eur. J. Appl. Physiol. 1993, 66, 161-173. [CrossRef]

13. Basmajian, J.V.; De Luca, C.J. Muscles Alive: Their Functions Revealed by Electromyography; Williams and Wilkins: Baltimore, MD, USA, 1985; pp. 201-222.

14. Bonato, P.; Roy, S.H.; Knaflitz, M.; De Luca, C.J. Time-frequency parameters of the surface myoelectric signal for assessing muscle fatigue during cyclic dynamic contractions. IEEE Trans. Biomed. Eng. 2001, 48, 745-753. [CrossRef] [PubMed]

15. Molinari, F.; Knaflitz, M.; Bonato, P.; Actis, M.V. Electrical manifestations of muscle fatigue during concentric and eccentric isokinetic knee flexion-extension movements. IEEE Trans. Biomed. Eng. 2006, 53, 1309-1316. [CrossRef] [PubMed]

16. Farina, D. Interpretation of the surface electromyogram in dynamic contractions. Exerc. Sport Sci. Rev. 2006, 34, 121-127. [CrossRef] [PubMed]

17. González-Izal, M.; Rodríguez-Carreño, I.; Malanda, A.; Mallor-Giménez, F.; Navarro-Amézqueta, I.; Gorostiaga, E.M.; Izquierdo, M. sEMG wavelet-based indices predicts muscle power loss during dynamic contractions. J. Electromyogr. Kinesiol. 2010, 20, 1097-1106. [CrossRef] [PubMed]

18. Sadoyama, T.; Miyano, H. Frequency analysis of surface EMG to evaluation of muscle fatigue. Eur. J. Appl. Physiol. 1981, 47, 239-246. [CrossRef]

19. Merletti, R.; Lo, C.L.R.; Orizio, C. Indices of muscle fatigue. J. Electromyogr. Kinesiol. 1991, 1, 20-33. [CrossRef]

20. Gallina, A.; Merletti, R.; Vieira, T.M.M. Are the myoelectric manifestations of fatigue distributed regionally in the human medial gastrocnemius muscle? J. Electromyogr. Kinesiol. 2011, 21, 929-938. [CrossRef] [PubMed]

21. Farina, D.; Leclerc, F.; Arendt-Nielsen, L.; Buttelli, O.; Madeleine, P. The change in spatial distribution of upper trapezius muscle activity is correlated to contraction duration. J. Electromyogr. Kinesiol. 2008, 18, 16-25. [CrossRef] [PubMed]

22. Allison, G.; Fujiwara, T. The relationship between EMG median frequency and low frequency band amplitude changes at different levels of muscle capacity. Clin. Biomech. 2002, 17, 464-469. [CrossRef]

23. Kumar, D.K.; Pah, N.D.; Bradley, A. Wavelet analysis of surface electromyography to determine muscle fatigue. IEEE Trans. Neural Syst. Rehabilit. 2003, 11, 400-406. [CrossRef] [PubMed]

24. Karlsson, S.; Yu, J.; Akay, M. Time-frequency analysis of myoelectric signals during dynamic contractions: A comparative study. IEEE Trans. Biomed. Eng. 2000, 47, 228-238. [CrossRef] [PubMed]

25. Sparto, P.J.; Parnianpour, M.; Jagadeesh, J.M. Wavelet analysis of electromyography for back muscle fatigue detection during dynamic constant-torque exertions. Spine 1999, 24, 1791-1798. [CrossRef] [PubMed]

26. Keenan, K.G.; Valero-Cuevas, F.J. Experimentally Valid Predictions of Muscle Force and EMG in Models of Motro-Unit Function Are Most Sensitive to Neural Properties. J. Neurophysiol. 2007, 98, 1581-1590. [CrossRef] [PubMed]

27. Chen, W.; Zhang, X.; Li, H.; Wei, J.; Fatikow, S. Nonlinear analysis and optimal design of a novel piezoelectric-driven compliant microgripper. Mech. Mach. Theory 2017, 118, 32-78. [CrossRef]

28. Mogk, J.; Keir, P. The effects of posture on forearm muscle loading during gripping. Ergonomics 2003, 46, 956-975. [CrossRef] [PubMed]

29. Wang, K.; Huang, Y.; Zhang, X. Estimation of Handgrip Force from Nonlinear SEMG-Force Relationship during Dynamic Contraction Tasks. In Proceedings of the IEEE International Conference on Robotics and Biomimetics, Macau, China, 5-8 December 2017; pp. 412-417.

30. Lawrence, J.H.; De Luca, C.J. Myoelectric signal versus force relationship in different human muscle. J. Appl. Physiol. 1983, 54, 1653-1659. [CrossRef] [PubMed]

31. Christopher, T.; Gilbert, P.A. Practical guide to wavelet analysis. Bull. Am. Meteorol. Soc. 1998, 79, 61-78. [CrossRef]

32. Asefi, M.; Moghimi, S.; Kalani, H.; Moghimi, A. Dynamic modeling of SEMG-force relation in the presence of muscle fatigue during isometric contractions. Biomed. Signal Process. 2016, 28, 41-49. [CrossRef] 
33. Wakeling, J.M.; Kaya, M.; Temple, G.K.; Johnston, I.A.; Herzog, W. Determining patterns of motor recruitment during locomotion. J. Exp. Biol. 2002, 205, 359-369. [PubMed]

34. Begg, R.; Lai, D.T.H.; Palaniswami, M. Computational Intelligence in Biomedical Engineering; CRC: New York, NY, USA, 2007.

35. Henneman, E.; Clamann, H.P.; Gillies, J.D.; Skinner, R.D. Rank order of motoneurons within a pool: Law of combination. J. Neurophysiol. 1974, 37, 1338-1349. [CrossRef] [PubMed]

36. Hortobágyi, T.; Solnik, S.; Gruder, A.; Rider, P.; Steinweg, K.; Helseth, J.; DeVita, P. Interaction between age and gait velocity in the amplitude and timing of antagonist muscle coactivation. Gait Posture 2009, 29, 558-564. [CrossRef] [PubMed]

37. Nardo, F.D.; Maranesi, E.; Mengarelli, A.; Ghetti, G.; Burattini, L.; Fioretti, S. Assessment of the variability of vastii myoelectric activity in young healthy females during walking: A statistical gait analysis. J. Electromyogr. Kinesiol. 2015, 25, 800-807. [CrossRef] [PubMed]

(C) 2018 by the authors. Licensee MDPI, Basel, Switzerland. This article is an open access article distributed under the terms and conditions of the Creative Commons Attribution (CC BY) license (http:/ / creativecommons.org/licenses/by/4.0/). 
Article

\title{
An Automatic Gait Feature Extraction Method for Identifying Gait Asymmetry Using Wearable Sensors
}

\author{
Arif Reza Anwary ${ }^{1}$, Hongnian $\mathrm{Yu}^{1, *}$ and Michael Vassallo ${ }^{2}$ \\ 1 Faculty of Science and Technology, Bournemouth University, Fern Barrow, Poole BH12 5BB, UK; \\ manwary@bournemouth.ac.uk \\ 2 Royal Bournemouth Hospital, UK, CoPMRE Bournemouth University, Fern Barrow, Poole BH12 5BB, UK; \\ Michael.vassallo@rbch.nhs.uk \\ * Correspondence: yuh@bournemouth.ac.uk; Tel.: +44-01202-961150
}

Received: 6 January 2018; Accepted: 20 February 2018; Published: 24 February 2018

\begin{abstract}
This paper aims to assess the use of Inertial Measurement Unit (IMU) sensors to identify gait asymmetry by extracting automatic gait features. We design and develop an android app to collect real time synchronous IMU data from legs. The results from our method are validated using a Qualisys Motion Capture System. The data are collected from 10 young and 10 older subjects. Each performed a trial in a straight corridor comprising 15 strides of normal walking, a turn around and another 15 strides. We analyse the data for total distance, total time, total velocity, stride, step, cadence, step ratio, stance, and swing. The accuracy of detecting the stride number using the proposed method is $100 \%$ for young and $92.67 \%$ for older subjects. The accuracy of estimating travelled distance using the proposed method for young subjects is $97.73 \%$ and $98.82 \%$ for right and left legs; and for the older, is $88.71 \%$ and $89.88 \%$ for right and left legs. The average travelled distance is $37.77(95 \% \mathrm{CI} \pm 3.57)$ meters for young subjects and is $22.50(95 \% \mathrm{CI} \pm 2.34)$ meters for older subjects. The average travelled time for young subjects is $51.85(95 \% \mathrm{CI} \pm 3.08)$ seconds and for older subjects is $84.02(95 \% \mathrm{CI} \pm 9.98)$ seconds. The results show that wearable sensors can be used for identifying gait asymmetry without the requirement and expense of an elaborate laboratory setup. This can serve as a tool in diagnosing gait abnormalities in individuals and opens the possibilities for home based self-gait asymmetry assessment.
\end{abstract}

Keywords: inertial measurement unit; accelerometer; gyroscope; asymmetry; feature extraction; wearable sensors; gait analysis

\section{Introduction}

Gait asymmetry (GA) is an indicator of different diseases and disease progression. It results in reduced gait efficiency and activity levels. Objective assessment of GA is important in the treatment and rehabilitation of patients with various conditions such as falls or after orthopaedic surgery. Gait is the result of a series of rhythmic alternating movement of arms, legs, and trunk which create forward movement of the body [1]. It relies on complex mechanisms depending upon the closely integrated actions of musculoskeletal, nervous system (central and peripheral), visual, vestibular, auditory systems; joint mobility and the smooth propulsive movement of the center of gravity. Every individual's gait pattern should be symmetrical with right and left sides performing identical movements. This is not the case since every individual has a unique gait pattern and the limb movement of one side is not exactly repeated on the other side, which results in GA. GA analysis provides bilateral locomotive information of gait parameters (e.g., length and period of stride, step, stance and swing), kinematic and kinetic measurements (e.g., angular joint trajectories, angular joint velocities, joint forces, and reaction forces), muscular measurements (e.g., muscle contraction, muscle force) and energy expenditure (e.g., oxygen consumption, heart rate) [2]. This is employed in different 
domains such health, sports and rehabilitation. GA can be a determinant of recovery in patients with different diseases such as Parkinson's disease [3] and stroke [4]. It can be used to monitor and improve an athlete's performance [5] as well as a patient's progress in orthopedics and rehabilitation [6]. In biometrics and biomedical engineering areas, gait analysis is used as an assistive tool to characterize human locomotion [7]. GA is important in elderly patient fall risk assessment [8], and is a predictor of functional and cognitive decline [9].

The tools and methodologies used to assess GA are often arbitrary [10] and often studied in non-natural controlled conditions. Currently available methods include reflective skin markers [11], ear-worn sensors [12] and camera based motion analysis systems [13]. Other methods for measuring GA include ground reaction forces [14], dynamic electromyography [15], and instrumented walkways [16]. These methods are laboratory based, complicated, costly, and often carried out by technical or clinical staff, which may be difficult for patients to use at their homes.

Clinical scales used to analyse gait parameters are subjective or semi-subjective and a poor replacement to laboratory based methods for identifying changes in GA. Different assessment tools such as the Gait Abnormality Rating Scale [17], Figure of 8 Walk Test [18], Four Square Step Test [19], The Functional Gait Assessment [20], Groningen Meander Walking Test [21] and Berg Balance Scale [22], are used to observe a patient's gait and balance. Their use has a high chance of intra and inters observer variation and human error. This may affect the accuracy of diagnosis, follow-up and treatment [2]. Therefore, a more objective way of assessing GA is required.

A variety of wearable sensors including accelerometer, gyroscope, magnetometer, foot pressure sensor, inclinometer, and goniometer $[23,24]$ are generally used to measure various characteristics of human gait. Inertial Measurement Unit (IMU) sensors are used in different situations such as monitoring of post-operative gait abnormalities [25], stride variability [24], measurement of GA [26], fall-related gait characteristics measured on a treadmill in daily life [27], nature of the Parkinsonian gait [28] and human waking foot trajectory [29]. Accelerometer based gait parameters such as times of stance, swing, single support and double [30]; stride and stance phase [31]; gait velocity, cadence and step length [32]; step number, moving distances, every step instant speed and average speed [33]; step counting [34,35]; times of heel strike, toe strike, heel-off, and toe-off [36]; stride length and duration [37]; walking distance, time and speed [38] were investigated.

Although, IMU based gait analysis methods are available, specific GA was actually found in few studies [30]. Some studied a single gait parameter [24], single sensor [12] or applied simple statistical methods for comparisons [38]. Force-sensitive resistors placed in insoles to detect ground contact were used to estimate the stance time for GA [39]. Microsoft Kinect-based GA [13], IMU and pressure sensitive shoe insole was used to detect gait onset and toe-off detection [40] and IMU-based knee flexion/extension angle measurements [41] and GA using gyroscopes [42] were also used. Systems developed for the detection of GA require a fully automatic system for data collection, feature extraction and quantitative measurements where both limbs are evaluated.

In order to broaden the use of accurate quantitative GA monitoring in clinical screening and research, an affordable GA tool is required which can be used in clinic or home. This study aims to design and implement an automatic lower limb gait features extraction method based on accelerometer and gyroscope data to increase the reliability and validity of monitoring GA. We set out to develop an affordable multi-sensor based synchronous data collection system for a comprehensive physical gait analysis extracting 24 commonly reported GA features. We develop a novel android app for collecting synchronous accelerometer and gyroscope data from both legs. Features include total distance, total time, total velocity, stride, step, cadence, step ratio, stance, and swing. We also estimate the mean, standard deviation, variance, minimum and maximum values. The paper has the following contributions: (1) designing and developing a novel android app to collect real time synchronous IMU data from legs; (2) proposing a gait asymmetry feature extraction method (a novel stride detection technique, a stance and swing detection technique, and a method for estimating travelled distance); and (3) creating a data set using our developed app and designed MetaWear casing, Velcro elastic belt, 
and buckles for validating the designed app and the proposed method. The paper is organized in the following sections: Section 2 presents the design and the methods of the proposed system. Section 3 delivers the experimental results and discussion. The conclusion is given in Section 4 .

\section{Design and Methods}

\subsection{Subjects Selection}

We recruited a convenience sample of 20 subjects with 10 healthy young subjects (nine male, mean age 25.3 years, standard deviation 4.64, range 19-35 years), and 10 older subjects (nine male, mean age 69.4 years, standard deviation 7.28, range 62-86 years). Older subjects 1, 3, 5, 6, 9 and 10 did not have any health problems. Subject 2 has a right foot drop and drags the foot and toes. Subject 4 has pain in the right leg lower muscle and walks without any support. Subject 7 has pain in the lower part of his left leg and uses a crutch during walking. Subject 8 has pain in both ankles and walks with support of a walker. According to World Health Organization (WHO), the life expectancy at birth is 71 years in Bangladesh [43]. Therefore, 65+ is considered old age in Bangladesh although would be viewed as young old in the Western countries. The subjects are purposefully chosen for this study to provide a variety of gaits for evaluation.

\subsection{Experimental Protocol and Calibration}

The experiment is performed in two different locations for young and older. The older subjects are residents in a care home. All subjects perform a walk in a straight corridor comprising of 15 strides of normal forward walking, a turn-around and another 15 strides. Accelerometer and gyroscope data from sensors attached on two foot locations are recorded in a database synchronously using our Android app. The distance carried out by walking on the corridor is measured by a tape. The several older subjects perform less than 15 strides. Calibration was performed individually where the distance travelled is measured manually and the result compared to the output from the sensor. The Qualisys motion capture system (Qualisys AB, Kvarnbergsgatan 2, Gothenburg, Sweden) is used for calibration in the validation stage as well.

\subsection{Sensor Placing Location}

From our investigation [44], it is found that placing a sensor in different foot locations gives quite different signal patterns. It is also observed that the orientation of the sensor has a significant effect on output data. In order to increase the sensor accuracy and reliability, and reduce the variability, all sensors are fitted tightly to the barefoot. We choose the barefoot rather than sensors attached to a shoe because wear and tear in the shoe can affect the position of the sensor and accuracy of the data output. The plantar aspect is not covered as this is the part of the foot in contact with the floor and is not practical for the subject to walk on the sensor. In this study, the sensors are placed at metatarsal foot locations of both legs (Figure 1) for collecting data since the best performance [44] can be achieved.
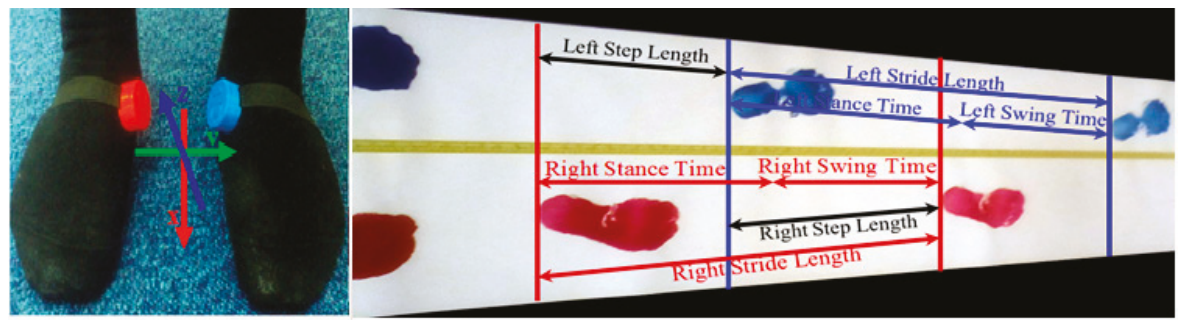

Figure 1. IMU sensors placement in right and left metatarsal foot locations of the barefoot. 


\subsection{Sensor Selection}

There are wearable wireless IMU sensors commercially used for health rehabilitation and activity monitoring [45]. An affordable wearable Bluetooth, long autonomy, minimum consumption, multiple synchronized data transmission supported IMU sensor is important for our investigation. With all these considerations the recently introduced sensor MetaWear CPro (MBIENTLAB Inc., San Francisco, CA, USA) [46] (price: \$30.00) was chosen. The power consumption of our sensor is low during sleep mode. The sensor (Figure 2) is in an active state when connected by Bluetooth to our android device and only goes to sleep mode once it is disconnected. It is sensitive to acceleration and rotatory movements that occur during normal human locomotion.

The IMU sensors are sampled at a frequency range of $20 \mathrm{~Hz}$ to $200 \mathrm{~Hz}$ [47]. In practice, a low sampling rate for the accelerometer possibly produces excellent recognition and accuracy in posture and activity classification [48]. For this study, the accelerometer range is $\pm 8 \mathrm{~m} / \mathrm{s}^{2}$ and gyroscope range is \pm 500 degrees / s with the sampling rate of $50 \mathrm{~Hz}$. A casing for the sensor is designed using SolidWorks [49] and printed using a 3D printer. A Velcro elastic belt and buckles are used to adjust and attach the sensor (Figure 2).

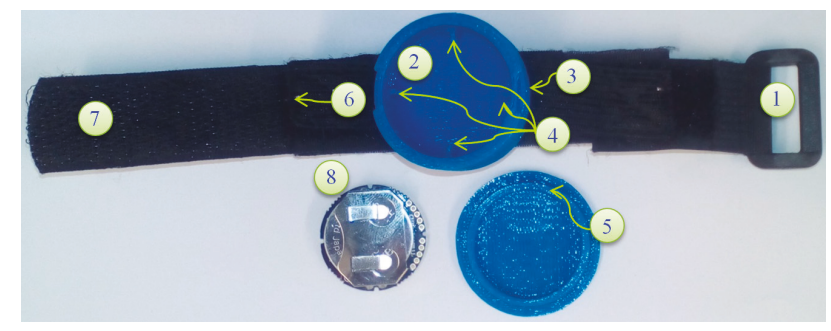

Figure 2. Proposed MetaWear casing, Velcro elastic belt, buckles and IMU sensor: (1) Buckle and Elastic Belt: the buckle is sewn onto an elastic belt for fastening to Velcro; (2) Bottom case which keeps the sensor safe from pressure, temperature and water; (3) Lock Open Edge which helps to open the cover from bottom case; (4) Sensor Lock Mechanism: The four locks keep the sensor sideways movement and orientation; (5) Cover Lock Mechanism which tightly locks with the case; (6) Velcro-Elastic Joint: The elastic belt is sewed with Velcro; (7) Velcro which adjusts and tighten when the sensor is attached; and (8) IMU sensor and battery.

\subsection{Android App Design, Development and Data Collection}

The sensor provides an Android API library for interacting with the MetaWear board on an Android mobile phone. A minimum of Android 4.3 (SDK 18) is required to use this library, however some features will not properly function due to the underlying Bluetooth LE (BLE) implementation. For getting the best results, it is recommended to use an Android 4.4 (SDK 19) or higher and BLE 4.0 or higher. Based on these criteria, an Android app is developed using Android Studio 2.2 to collect real time accelerometer and gyroscope data, and store data on an external SD card in a csv file. The data soring format in a csv file is date (dd/mm/yyyy), time (HH:MM:SS.ss), system clock (milliseconds), accelerometer (X, Y, Z) and gyroscope (X, Y, Z), shown in Figure 3.

The app is designed through the following steps: (1) requirement analysis through the literature review and interview with the expert and users; (2) reviewing the market available software development platform; (3) initial design of the mobile app using Android Studio 2.2; (4) testing and debugging with the user feedback for improvement; (5) documentation for development. We develop the proposed app shown in Figure 3. The details of the design are beyond the scope of this paper and will be reported in the future.

Pressing the SCAN button the availability of all the devices for data collection is checked. The order of data collection is selected by Slot number. Each sensor then automatically connects 
with the corresponding mac address by showing CONNECTED. Pressing the DATA RECORDING switch makes a dialog box to get the file name for storing data. Pressing the OK button starts data collection. Pressing the STOP button stores the collected data.

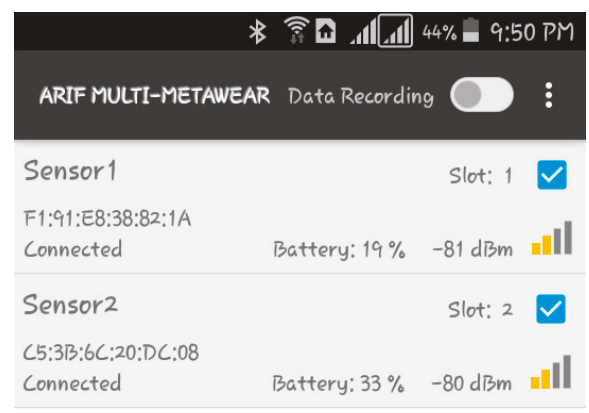

Figure 3. Proposed android app to collect data from MetaWear CPro.

A stride is a whole gait cycle with stance and swing events. This horizontal movement produces high acceleration during walking and this movement is the subject of investigation in this study for GA monitoring. The data with the horizontal movement information from the feet are analysed using our method to find GA information. Figure 4 shows the raw data.
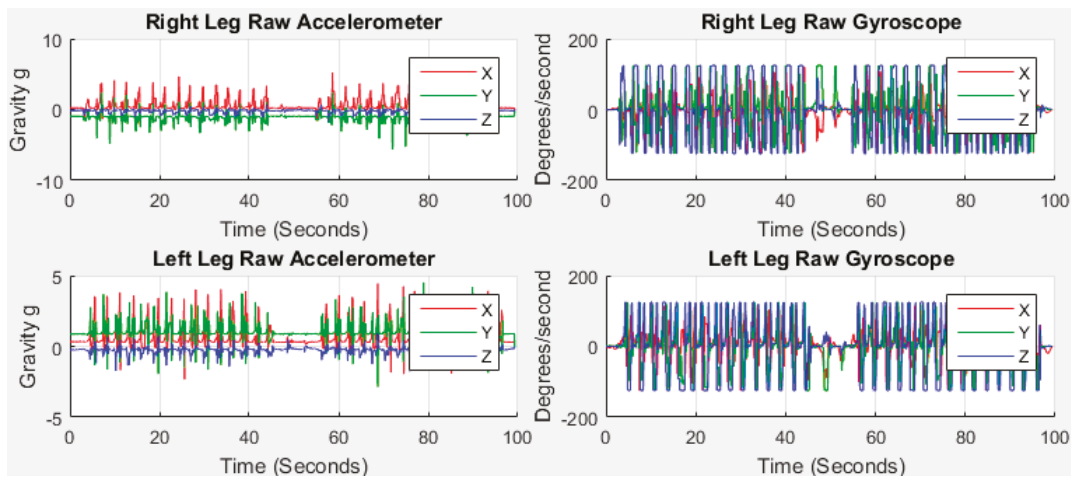

Figure 4. Raw accelerometer and gyroscope data from right and left feet of older subject 1.

The red, green and blue lines in Figure 4 stand for accelerometer readings on $x, y$ and $z$ axis, respectively, with $g$ units $\left(9.81 \mathrm{~m} / \mathrm{s}^{2}\right)$ in the sensor frame. We can observe from the raw data that the accelerometer reading on $x$ is the highest and $z$ is the lowest before the commencement of walking for right leg indicative of the initial gravitational force. Similarly, the accelerometer reading on $y$ is the highest and $z$ is the lowest before the commencement of walking for left leg. The initial data is not aligned to zero means that the sensors are not placed perfectly upright position with the earth frame in the foot locations due to the initial gravity part of $y$ and $z$. For this study, the sensors do not need to be perfectly upright which in any case is not user-friendly and impossible. The discrepancy between the sensor frame, the foot frame and the earth frame are compensated for in this study.

\subsection{Gait Asymmetry Feature Extraction Method}

\subsubsection{Raw Data Processing}

It is noted that accelerometers are sensitive to altitude and impact forces, while gyroscopes are sensitive to temperature changes and suffer from a low-changing bias. Consequently accelerometers 
have poor dynamic features and gyroscopes have poor static features [50]. To provide a robust absolute orientation vector in the form of quaternion or Euler angles, the sensor combines the measurements from 3-axis accelerometer and 3-axis gyroscope sensors. The algorithm in [46] fuses the raw data in an intelligent way to improve each sensors output. This includes offset calibration of each sensor, monitoring of the calibration status and Kalman filter fusion to provide distortion-free and refined orientation vectors [46]. The IMU sensor provides accelerometer $A\left(a_{x}, a_{y}, a_{z}\right)$ and gyroscope $G\left(g_{x}, g_{y}, g_{z}\right)$ with respect to time $t$. As the accelerometer is sensitive to acceleration due to movement and the local gravitational force, the input data consists of the user acceleration and gravitational acceleration.

\subsubsection{Coordinate Systems}

In this study, there are three coordinate systems, the foot frame describing the foot rotation, the sensor frame describing the motion of the sensor and the global or Earth frame. Since the sensor is attached to the foot tightly using an elastic Velcro belt, we assume that the sensor does not slip or move during walking time. Therefore we consider that the foot frame and sensor frame are the same. Our approach is to transfer the sensor frame to the Earth frame and then to remove the gravitational component. The high gravitational force of the Earth frame is downward towards Earth. The $A_{x}$ axis is aligned along the foot axis of the IMU sensor, $A_{z}$ points downwards so that it is aligned with gravity so that the three axes from a right handed coordinate system shown in Figure 5a.

\subsubsection{Quaternion}

Quaternion is a concept related to the foundations of algebra and number theory. While the accelerometer and gyroscope sensors enable the tracking of translational and rotational movements, the accurate measurement of the sensor orientation is important to interpret sensor information. Quaternions are a mathematical construct that consist of four individual numeric complex number components that can be used to represent the orientation of a ridged body or coordinate frame in a three dimensional space.

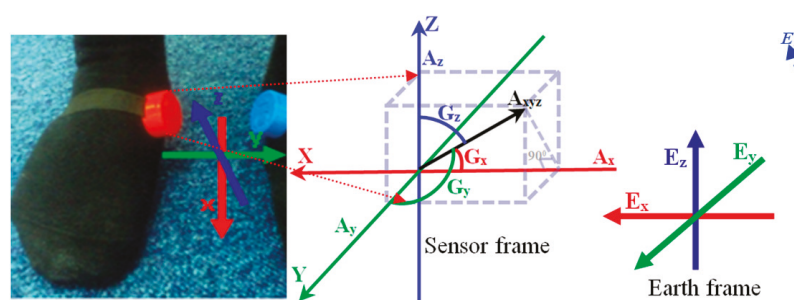

a)

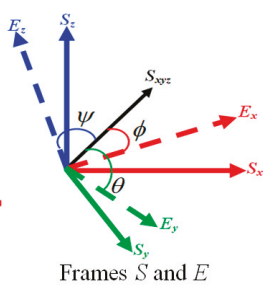

b)

Figure 5. (a) Sensor frame and earth frame of accelerometer and gyroscope axes; (b) The orientation of frame $E$ is achieved by a rotation, from alignment with frame $S$, of angle of $\phi, \theta$, and $\psi$ around the axis $S_{x y z}$.

Many quaternions are available to estimate the orientation from accelerometer, gyroscope and magnetometer data. We use the Madgwick technique [51] which fuses accelerometer, gyroscope and magnetometer for estimating quaternion. An arbitrary orientation of frame $S$ relative to frame $E$ can be achieved through a rotation of angle $\theta$ around an axis of $S_{x y z}$ defined in frame $E$ shown in Figure $5 \mathrm{~b}$ where the mutually orthogonal unit vectors $S_{x}, S_{y}, S_{z}$ and $E_{x}, E_{y}, E_{z}$ define the principle axis of coordinate frames $S$ and $E$, respectively.

$S_{x}, S_{y}$ and $S_{z}$ define the components of the unit vector $S_{x y z}$ in the $x, y$ and $z$ axes of the frame $S$. To denote the relative frames of orientations and vectors, $S_{E} \hat{q}$ in Equation (1) represents the orientation of frame $E$ relative to $S$ and $S_{x y z}$ is a vector described in $S$ [51].

$$
{ }_{E}^{S} \hat{q}=\left[\begin{array}{llll}
q_{1} & q_{2} & q_{3} & q_{4}
\end{array}\right]=\left[\begin{array}{llll}
\cos \frac{\theta}{2} & -S_{x} \sin \frac{\theta}{2} & -S_{y} \sin \frac{\theta}{2} & -S_{z} \sin \frac{\theta}{2}
\end{array}\right]
$$


The compensated gyroscope measurement ${ }^{S} \omega_{c}$ then is used in place of the gyroscope measurements ${ }^{S} \omega$, where the magnitude of the angular error in each axis ${ }^{S} \omega_{\in}$ is equal to a quaternion derivative of unit length and then the integral gain $\varsigma$ directly defines the rate of convergence of the estimated gyroscope bias ${ }^{S} \omega_{b}$ expressed as the magnitude of a quaternion derivative [51]. The complete orientation of ${ }_{E}^{S} \hat{q}_{e s t, t}$ is achieved and Figure 6 shows a block diagram representation of the complete orientation filter implemented for an IMU. The details derivation is described in the Supporting Information.

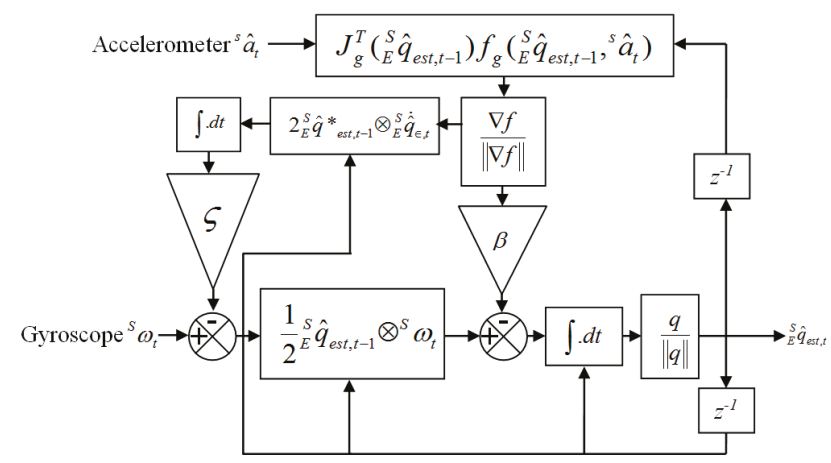

Figure 6. The process diagram of the complete orientation filter for an IMU.

We apply the technique shown in Figure 6 to our collected data for body acceleration to the Earth frame with a sampling frequency of $50 \mathrm{~Hz}, \beta$ gain of 0.1 and $\varsigma$ gain of 0.5 . The gravity components are removed and the conversion of the accelerometer from gravitational force $g$ to user acceleration of movement $\left(A M_{x y z}\right) \mathrm{m} / \mathrm{s}^{2}$ is achieved by multiplying 9.81 . The three axis data are transformed due to the fact that looking at specific axes is sensitive to the sensor orientation [52]. Figure 7 shows the acceleration due to user movement $A M_{x y z}=\left[a m_{x}, a m_{y}, a m_{z}\right]$ for both feet of older subject 1 .

Figure 8 shows the acceleration of total $A T_{x y z}$ and gyroscope $G T_{x y z}$ towards $x, y$ and $z$ directions estimated using Equation (2):

$$
\left|A T_{x y z_{i}}\right|=\sqrt{a m_{x i}{ }^{2}+a m_{y i}{ }^{2}+a m_{z i}{ }^{2}} \text { and }\left|G T_{x y z i}\right|=\sqrt{g_{x i}{ }^{2}+g_{y i}{ }^{2}+g_{z i}{ }^{2}}
$$
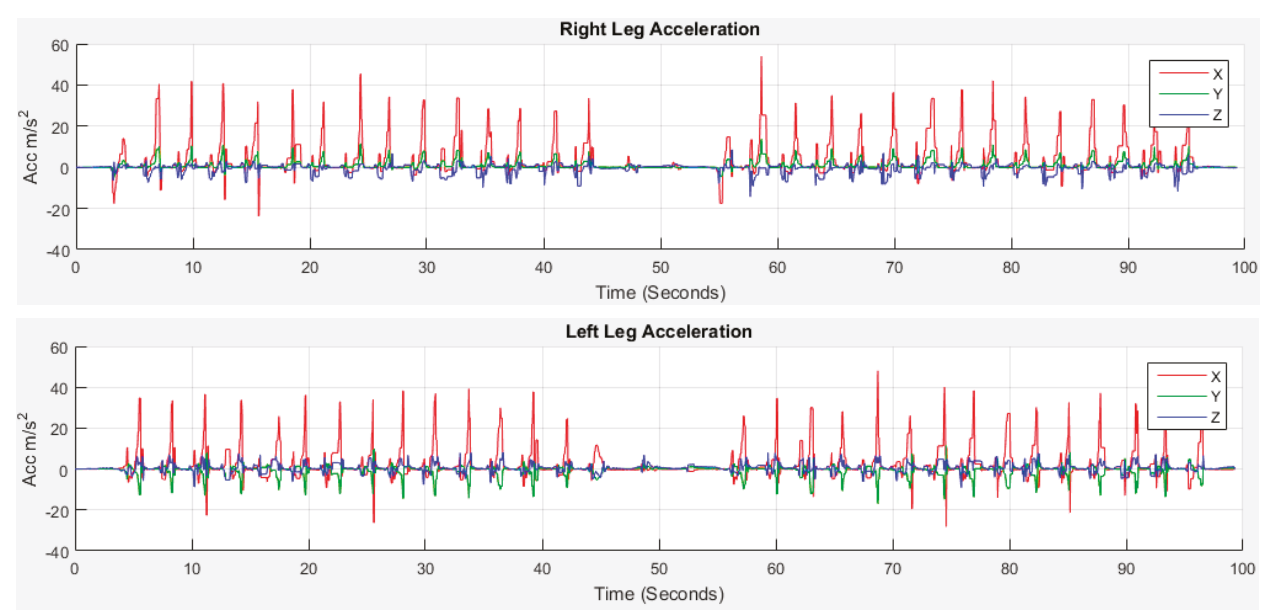

Figure 7. Acceleration due to user movement $A M_{x y z}$ after removing gravity component. 

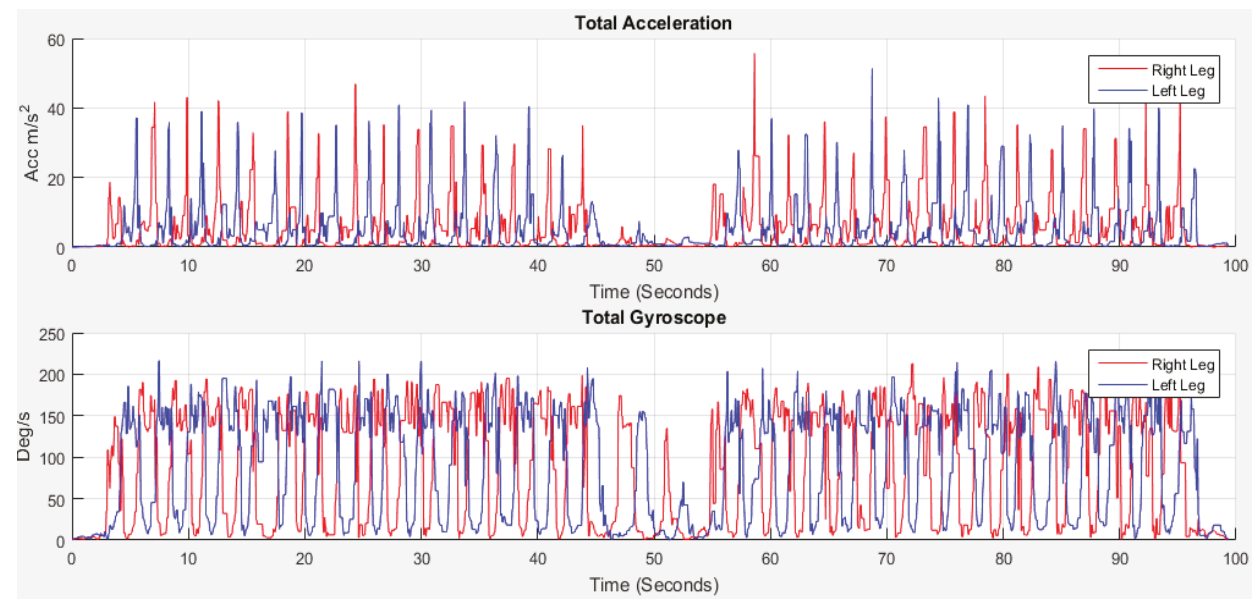

Figure 8. The total acceleration $A T_{x y z}$ and gyroscope $G T_{x y z}$.

\subsubsection{Stride, Stance, Swing and Step Events Detection}

Human walking can be described and characterized in the context of a gait cycle. A stride is the distance between a point on one foot at the first foot contact and the same point on that foot at the next foot contact. It is the equivalent of a gait cycle made up of two steps. Each stride contains stance and swing relevant phases. Stance and swing phases of a gait cycle consists eight relevant phases shown in Figure 9.

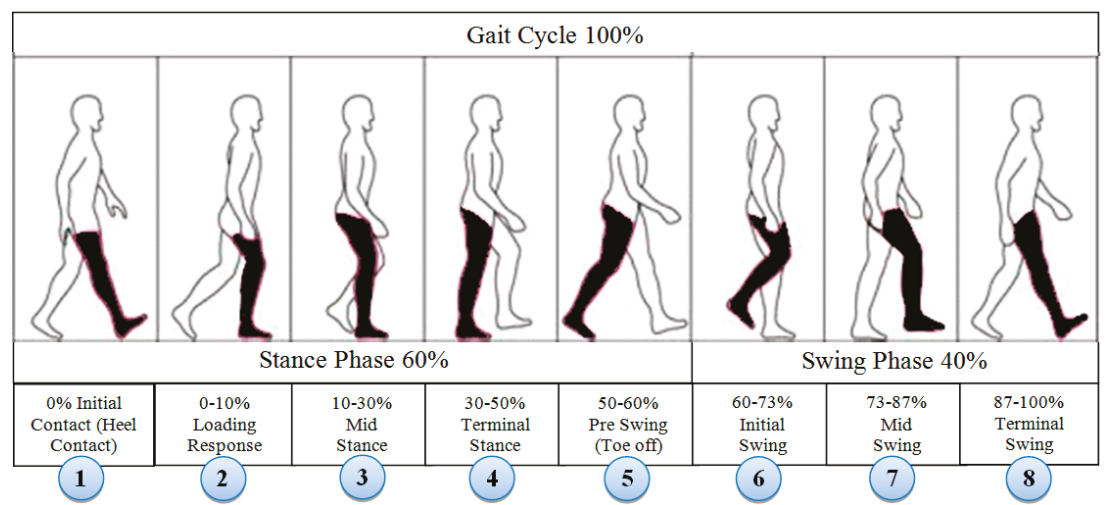

Figure 9. Normal human gait phases [53].

The first phase starts when the heel contacts the ground and the waist is in its lowest position during the entire step. There is deceleration of the leg towards the horizontal axis as the velocity moves to zero. The zero velocity remains until the terminal stance phase where the foot is flat on the ground. The next phase is pre-swing where the toe is off the ground and starts forward movement demonstrating initial acceleration towards horizontal axis. The swing phase is when the heel moves off the ground. The acceleration interval corresponds to the change from the heel lift to the swing at the height point at mid-swing phase. Deceleration starts during the terminal swing phase from the highest point to the foot back flat on the ground. There is zero velocity again in the interval corresponding to the change from a flat foot to a heel lift. These different phases of gait cycle presented 
in Figure 10 are identifiable from the IMU acceleration signal. The same phenomenon of human limb kinematic with accelerometer signal output during a typical walking cycle has been identified in the literature. Our gait cycle accelerometer signal $A T_{x y z}$ (Figure 10) is agreed with the signal pattern in $[53,54]$. The different phases of the gait cycle (Figure 9) with corresponding accelerometer signal are shown in Figure 10.

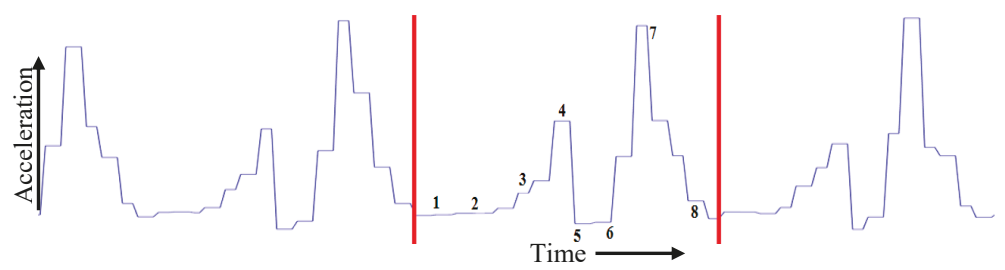

Figure 10. Eight different phases of a gait cycle from accelerometer data.

From Figure 10, we can observe that at the start and end of each stride, the walker's feet are stationary on the ground. As the IMU sensor is attached to the foot, the stance phase is stationary and swing phase is non-stationary. Due to the walker's movement towards the $x$ axis, the acceleration shows its highest value in the swing phase. The output of the accelerometer signal will be different, if the sensor is placed on foot [54,55], waist [56] or different body locations [34,57].

Many algorithms [34] are available for stride event detection from IMU sensors. During human walking, a consistent sequence of motions is performed at each stride that results in a maximum peak value that lies in the mid-swing phase. This mid-swing phase appears when a user pushes off this foot and shortens the limb to clear ground thus releasing the foot from the ground until it again contacts with ground as shown in Figures 9 and 10. A particular threshold value is set to detect these characteristics for detecting stride [35,58]. One disadvantage of these algorithms is that any motion with a similar periodicity of walking will trigger for a false stride event. In addition, difficulty arises in finding the automatic selection of the threshold value which can vary between users, surfaces and shoes [59]. The variation in the peak magnitude gets larger for faster human waking velocities [57] and a window based threshold calculation [58] was used to obtain an acceptable level of accuracy for a larger window size. However increasing the window size may degrade the step detection accuracy during the translation of step mode because the threshold calculated from a larger window may not be able to effectively handle the variation in the recent statistics [57]. Due to peak magnitude variation, the threshold value also varies based on individuals walking style and even differs from left to right leg as shown in Figure 11.

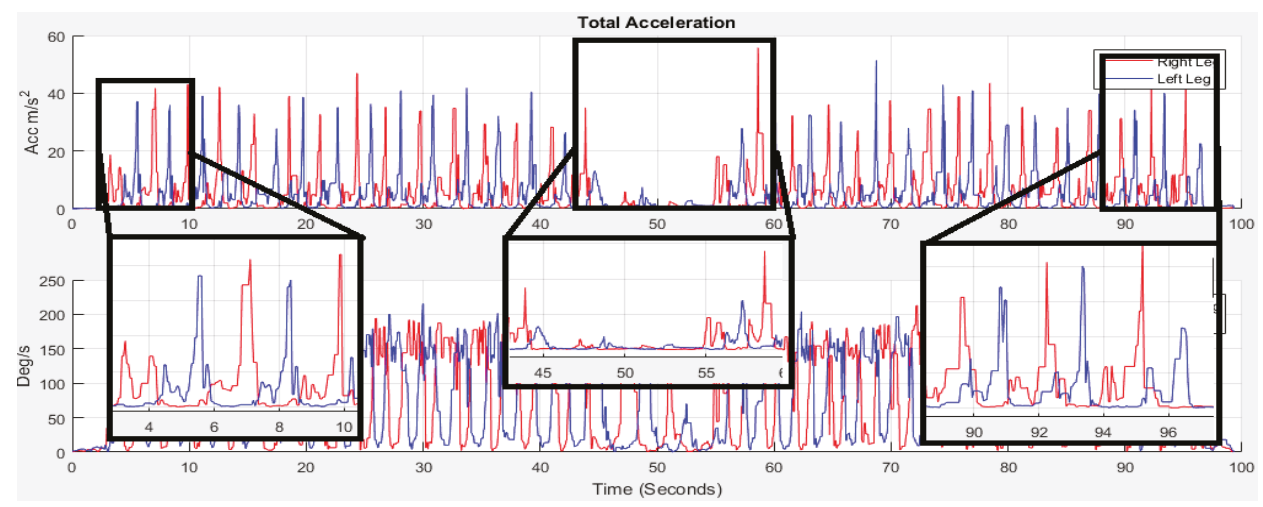

Figure 11. Peaks magnitude variation from Figure 8. 
The different threshold may result in a different output of detecting steps. Another important point is that when a subject begins walking from a standing state, stops walking for a turnaround or stops, there is poor acceleration and it is crucial to detect the gait cycle in these situations. For this reason the 1st stride is not considered for gait analysis by researchers [60]. We take this in consideration to address this point in this study. As the mid-swing phase in accelerometer data is a good indicator for performing a complete gait cycle, thus for counting the number of strides, the number of mid-swing phase in accelerometer data is analysed as walking strides are equal to the number of mid-swing phases. The highest peak is occurred at the push off phase starting from the terminal stance at the 4 th to pre-swing at 5th phases shown in Figure 9 for gyroscope data. We apply threshold based algorithms obtaining low accuracy to detect the stride number for our collected accelerometer and gyroscope dataset. As the peaks at terminal stance phase are more prominent than the mid-swing phase, the threshold based algorithm detects two strides instead of single stride. To avoid this, a novel stride detection technique is proposed based on the local minimal prominence characteristics of strides associated with the time-varying magnitude of acceleration shown in Figure 12. The technique consists of designing a high-pass filter, computing the absolute value, designing a low-pass filter, shifting data to centroid and finding the strides using findpeaks [61] function.

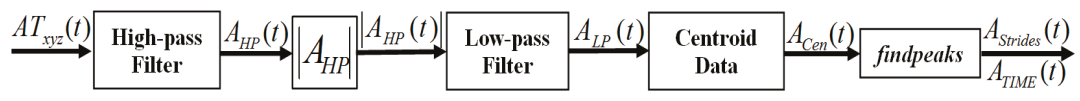

Figure 12. The proposed step detection technique.

The accelerometer converts acceleration to an electrical signal and in the process, unwanted constant bias in acceleration becomes a linear error called drift. Thus the 2nd order Butterworth digital high-pass filter with a sampling rate $f_{S}=50 \mathrm{~Hz}$ and cut off frequency $f_{\mathcal{C}}=1000 \mathrm{~Hz}$ is applied to $A T_{x y z}$ to remove the corrupted data and the DC component of the acceleration signal. The smoothness is achieved at the price of decreased roll off steepness. The output from the filter is then passed to the zero phase filtfilt delay filter. The filtfilt corrects for phase distortion introduced by a one-pass filter [62]. The output of filtfilt filter then is passed through a low-pass filter with $f_{\mathcal{C}}=5 \mathrm{~Hz}$ to obtain $A_{L P}$ which is shifted to centroid using Equation (3).

$$
A_{\text {Cen }}=A_{L P}-\operatorname{mean}\left(A_{L P}\right)
$$

To find the local minima prominences, $A_{C e n}$ is passed through a findpeaks function which finds local peaks in the data vector and ignores small peaks that occur in the neighbourhood of a larger peak. A local peak is a data sample that is either greater than its two neighbouring samples or is equal to Inf. If a peak is flat, the function returns only the point with the lowest index. The findpeaks function detects the stationary periods when the foot touches the ground the point of minimal prominence during walking. The function returns two vectors containing the minimal local peaks $A_{\text {Strides }}$ and the locations $A_{\text {TIME }}$ at which the peaks occur. The number of strides is the same as the length of $A_{\text {Strides }}$ vector. Again, as each stride consists of stance and swing events, thus the initial contact and the transition between pre-swing and initial swing (4th and 5th phases in Figure 9) are detected using steps in Figure 13 to get stance and swing information.

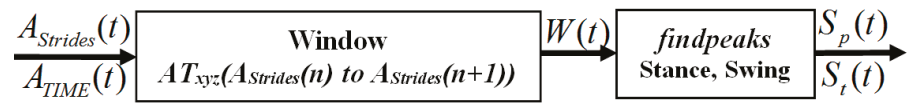

Figure 13. Proposed stance and swing detection technique. 
A window is prepared whose size is the difference between a pairwise consecutive strides from $A_{\text {Strides. }}$. Each window is then passed through findpeaks function as there is only one local maximum in each stride located between 4th and 5th phases in Figure 9. A loop from 1 to total detected strides number is used to find the stance and swing event for all strides. The detected Start (purple circle), SS (cyan triangle) and End (black rectangle) information of each stride are shown in Figure 14 for right and legs where the stance phase information is provided by the difference between Start and SS; and the swing information is the difference between SS and End.

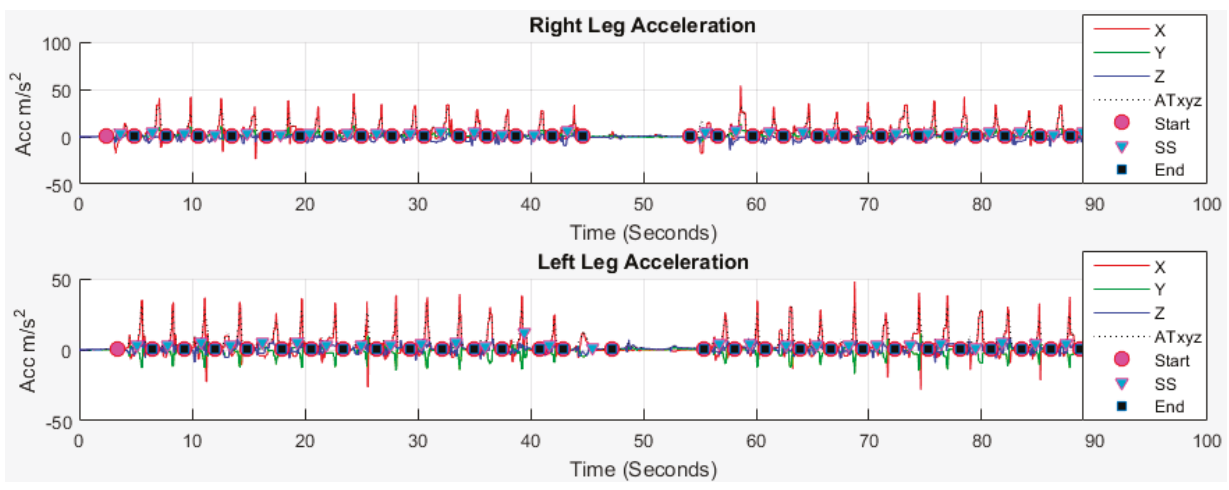

Figure 14. Result of stride, stance and swing event detection using proposed method.

A step is the sequence of events between the contact of one foot and the next contact of the opposite foot. At the beginning of the stance phase, the initial contact of the foot contacts with ground of the one leg. The loading response begins at the initial contact and ends when the toe of opposite leg leaves the ground, midstance then begins and finishes when the center of gravity is over the same foot. The terminal stance begins when the center of gravity is over the supporting foot and ends when the opposite leg contacts the ground. The strides, stance and swing event are detected from right and left legs. The step event is then detected between the heel of two subsequent feet shown in Figure 15.

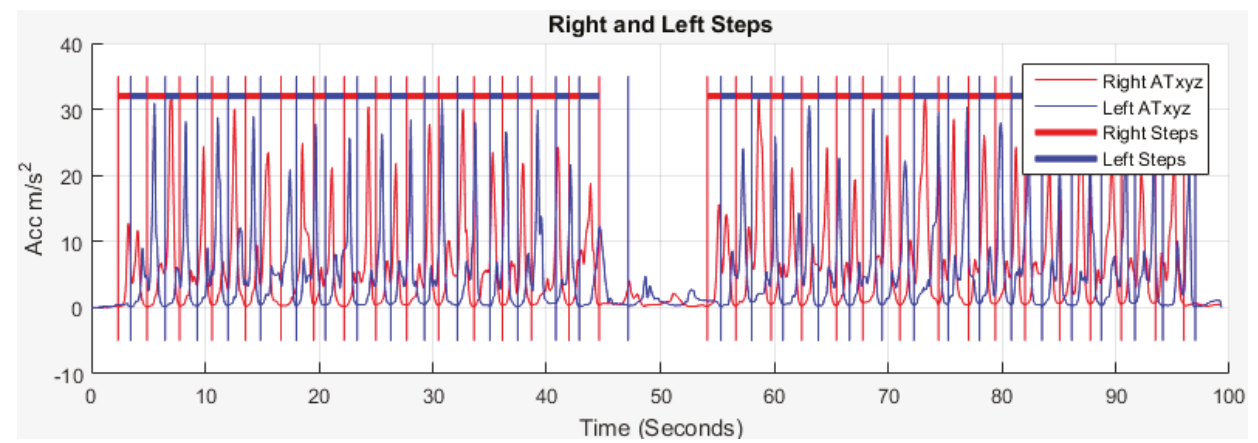

Figure 15. Result of step event detection using proposed method.

\subsubsection{Velocity and Distance Estimation}

A pedestrian navigation system (PNS) has diverse applications in airports, theatres, underground parking, other indoor and outdoor places and modern cities. In order to estimate PNS in a virtual environment, a number of navigation methods [63] are available to derive pose estimates from 
electrical measurements of mechanical, inertial, acoustic, magnetic, optical, and radio frequency sensors. There are popular conventional approaches of IMU-based PNS known as Pedestrian Dead-Reckoning (PDR) solution and inertial navigation system (INS) [64]. In PDR, a constant step length is assumed on a relatively smooth surface, often usable in office-like environment and the average step length is integrated along with the orientation estimations obtained from IMU at each detected step to track the position. IMU based foot mounted PNS methods and systems have been proposed in $[55,65]$. The foot mounted method uses a double integral on horizontal acceleration to estimate distance and a gyroscope or magnetometer to measure the heading distance [64]. The disadvantage of PDR is that it assumed on relative smooth surfaces and a constant step length that needs tuning for individual users. Again, if the walking pattern is different from the predefined step length model, this may adversely affect the distance estimation [64]. The INS is adapted from the aerospace community in which IMU is used for tracking position, velocity and attitude $[55,66,67]$. Sensor drift is a well-known problem in INS system which depends on the precision of the sensor used and high end inertial sensors are very costly [66]. The IMU sensors have some small errors when estimating the distance and direction and signal noise can further exacerbate this problem described details in $[64,66]$.

In this paper, we consider the walking constrains of a user with an IMU fitted on the both right and left legs. We apply appropriate methods to detect the movement of the leg, changes in position and compute its velocity and travelled distance from the initial location by means of the data collected from the accelerometers. The basic approach lies on the double integral of the accelerometer data where the first applying integration retrieves the current velocity and then the second applying integration computed on the velocity provides the distance travelled. Distance travelled is obtained principally from trapezoidal double integration [68] of the user movement signal on each stride detected in the direction of travel as mentioned in Section 2.6.4. However, there are two main problems for performing a double integration of the acceleration signal, unknown initial condition and drift. The unknown initial condition problem means integration requires a known initial condition. Drift means IMU sensors are subject to errors in acceleration that when integrated in to velocity and distance, leads to drastic integration error. This can be unbound over time if the acceleration signal is integrated without filtering $[55,64-66,68]$. The integration works properly with known initial conditions. Thus, to calculate the actual displacement, integration errors must be minimized. A method known as zero-velocity update (ZUPT) $[64,66,67]$ is often used to correct for drift and is often used to aid in autonomous inertial pedestrian navigation. ZUPT uses the fact that during human walking time, one foot is always stationary on the ground. When a stationary period of the acceleration is detected the assumption is made that the foot is on the ground and the velocity at that time is set to 0 . In this way, the drift is greatly reduced. However, ZUPT assumption implies that the angular rate is 0 as well and consequently if the accelerometer is moving at a constant velocity, the algorithm would misjudge the motion as stationary. ZUPT therefore cannot reduce all errors $[50,66]$. Based on our experience, an accelerometer is very sensitive to movement and walking is a complex course of acceleration and deceleration. The detection of zero velocity does not fail due to misjudgement, but adjusting the threshold value for motion detection plays an important role in that misjudgement when motion detection is not properly set (discussed and showed in Section 2.6.4).

In addition, this issue may not relevant to this study as the "foot stationary event" is already detected based on local minimal prominence as described in Section 2.6.4. The stationary period remains in the stance phase and the movement period remain in the swing phase shown in Figure 9. As IMU sensors are mounted on each foot, the acceleration is high in the swing phase due to the movement of the leg during walking. The zero-velocity in non-stationary period of stance phase is used in the ZUPT scheme to reduce the drift. The ZUPT based on local minimal prominence to detect the swing phase is shown in Figure 16. 

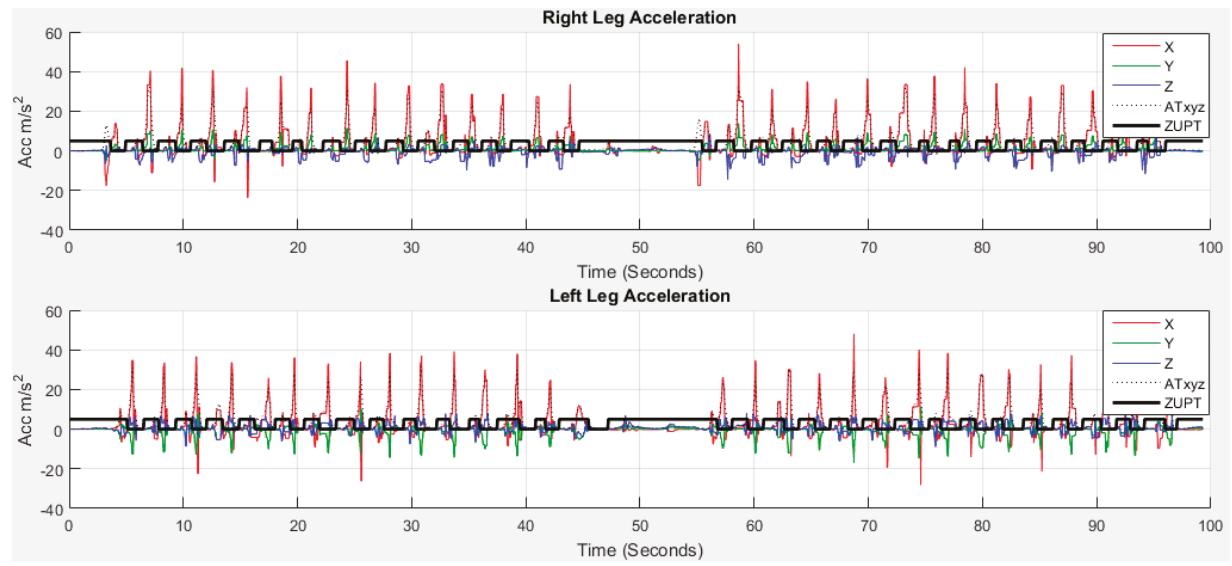

Figure 16. Zero-velocity update (ZUPT) from $\left|A T_{x y z_{i}}\right|$.

Another concern regarding the double integration is that the displacement signal emphasizes the low frequency data more than the acceleration signal, a low-pass filter effect of the integrator. Therefore, the input data are passed through a high-pass filter to remove the direct component of the acceleration signal. Considering these issues, a double integral method shown in Figure 17 is proposed for calculating travelled distance.

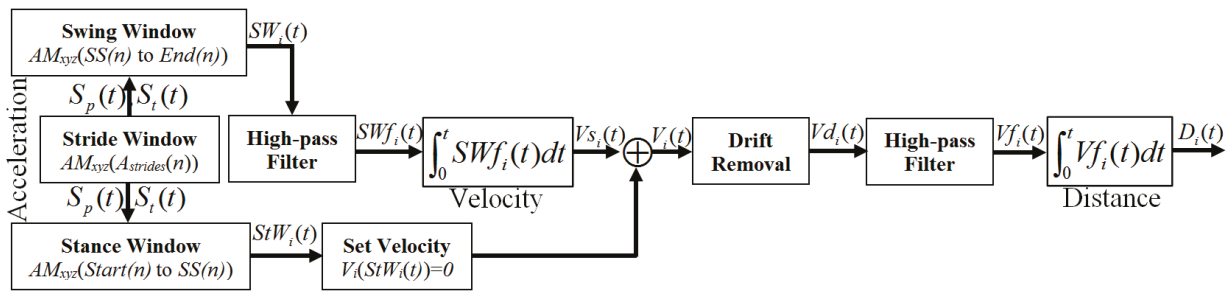

Figure 17. Proposed method for estimating travelled distance.

In order to obtain the velocity and distance in time series, two stages of integration and two stages of high-pass filtering are applied. A stride window is prepared from $A_{\text {Strides }}$ and $A M_{x y z}$. The swing and stance windows are brought out from the corresponding stride window using Start, SS and End mentioned in Section 2.6.4. A 1st order Butterworth high-pass filer is designed with $f_{S}=50 \mathrm{~Hz}$ and $f_{C}=1000 \mathrm{~Hz}$. The 1st integral operation cumtrapz in Matlab is applied on $S W f_{i}$ with respect to time $t$ that gives the $V s_{i}(t)$ velocity for the 1st swing phase. The ZUPT is applied on stance phase to set the stationary velocity to 0 . The non-stationary period of swing velocity and stationary period of stance zero velocity are then combined to obtain $V_{i}(t)$ shown in Figure 18.

As the stationary period in stance phase velocity is set to zero, the integral constant from non-stationary period in swing phase exists in $V_{i}(t)$. Therefore, it is important to remove the drift caused by integration from $V_{i}(t)$. To remove the integral drift [50], the velocity difference between the initial and end of a non-stationary period is estimated. The velocity difference is then divided by the number of samples during this non-stationary period to get the drift rate. The drift rate is multiplied with the corresponding data index to estimate the drift value at that certain point. The drift value is then subtracted from the calculated velocity $V_{i}(t)$ to obtain the error free velocity $V d_{i}(t) . V d_{i}(t)$ is then passed through the high-pass filter for the 2 nd time and the distance $D_{i}(t)$ is estimated after 
2nd integral operation. $D_{i}(t)$ consists of the distance towards $x, y$ and $z$ coordinates. Repeat the same procedure for all strides to calculate velocity and distance. Then estimate the travelled distance using Equation (4):

$$
T D_{x y z_{i}}=\sqrt{D_{x_{i}}^{2}+D_{y_{i}}^{2}+D_{z_{i}}^{2}}
$$

Figure 19 shows the estimated distance $D_{i}(t)$ towards $x, y$ and $z$ and travelled distance $T D_{x y z}$.

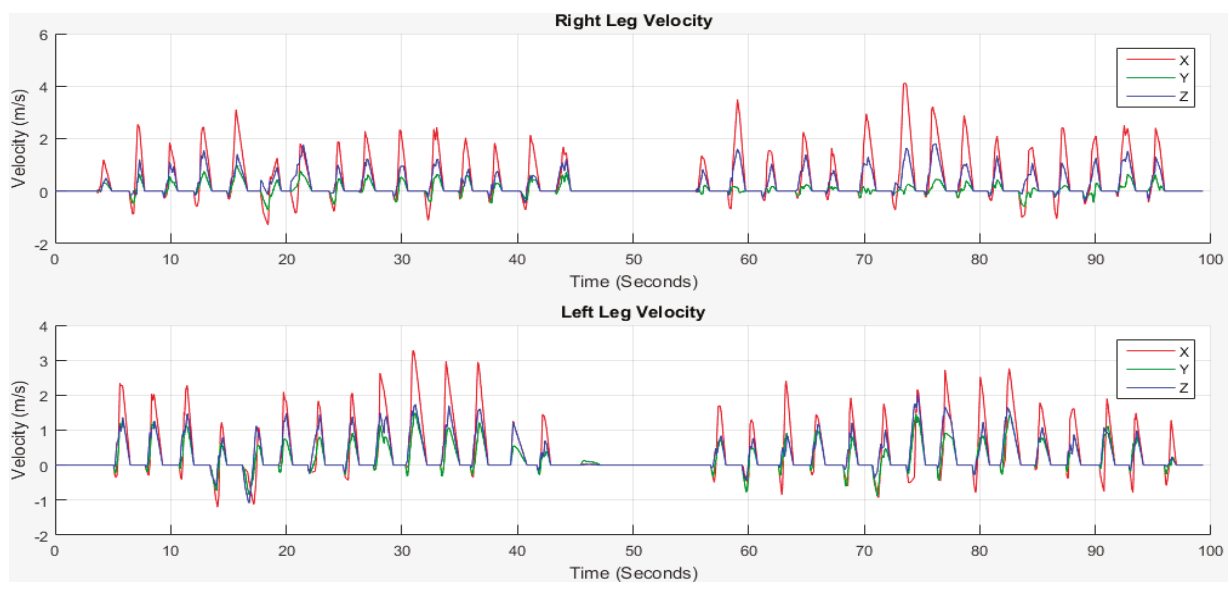

Figure 18. First integral operation to get velocity $V_{i}(t)$.

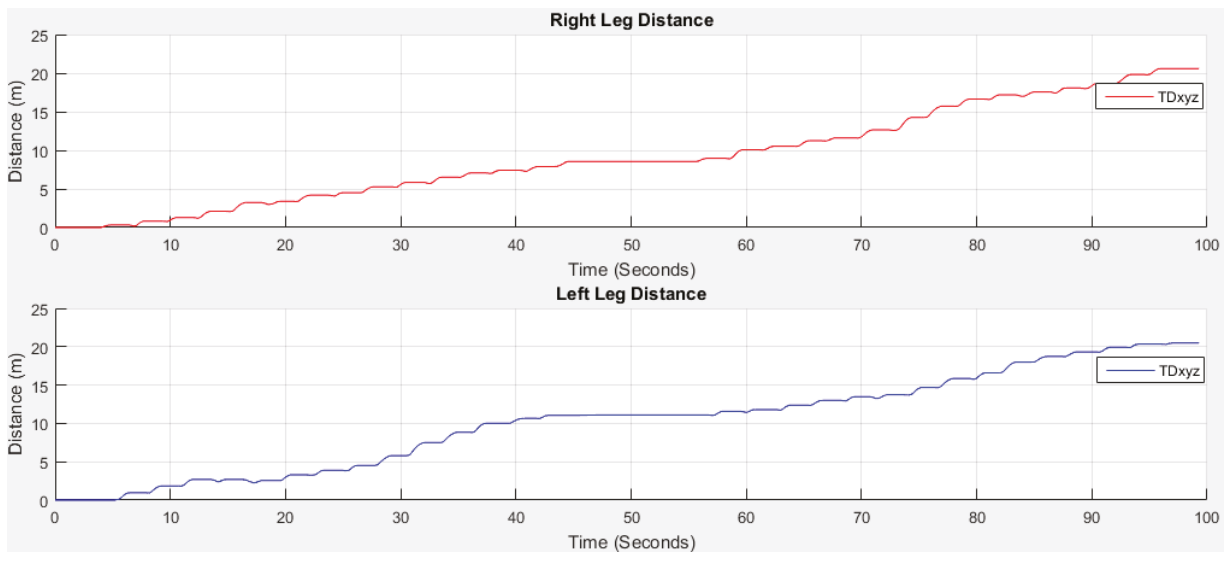

Figure 19. 2nd integral operation to get distance $D_{i}(t)$.

\subsubsection{Selection of Gait Asymmetry Variables}

GA in this study is considered as an indicator to show the difference between right and left leg walking which may serve as a diagnostic tool for clinicians. There is no commonly accepted superior guideline, preferred methodology or protocol for GA evaluation. The European GAITRite Network Group has developed Guidelines for Clinical Applications of Gait Analysis [69] to provide guidance to clinicians who implement spatiotemporal gait analysis to the clinic. Two issues are addressed in [69]: (1) Environmental measurement conditions and safety issues describe lighting, noise, visual distraction, clothing, footwear and safety; (2) Measurement procedures describe steady-state gait at 
different velocities, standardized walking instructions, assistive devices, stride-to-stride variability, gait analysis in association with simultaneous cognitive tasks and description of study population. To evaluate stride-to-stride variability, they recommend the highest possible number of gait cycles from a practical standpoint, with a minimum of three consecutive gait cycles for both left and right sides (i.e., a total of six gait cycles) [69]. Many issues relevant in GA assessment is reported in [69]. However there is no recommended systematic procedure for developing a GA assessment. General measurement of gait variability includes cadence, stride length and gait velocity [70], alone or in combination with other outcome measures such as stride to stride variability assessed by an accelerometer, gyroscope and magnetometer [24,36,39,40]. Researchers presented a set of 31 gait variables in [71] and 16 variables were investigated. Stride-to-stride variability [72] is commonly used to quantify walking consistency which is strongly associated with motor ability [73], mild cognitive impairment [74], dementia [75] and stroke [76]. In our study, a set of 24 commonly reported physical gait variables are initially considered for this analysis from both right and left legs. Figure 20 shows the GA variables.

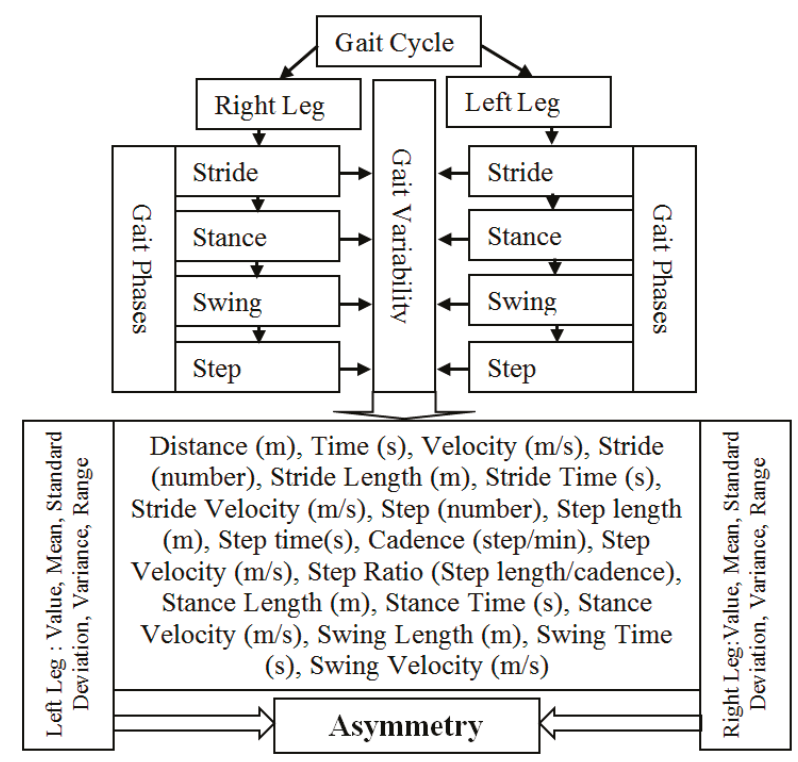

Figure 20. Proposed variability monitoring for GA.

A stride length is the distance between the heel contacts of the same foot and a stride time is the interval between sequential initial heel contacts by the same limb. A step length is the distance and a step time is the interval from one foot strike to the other foot strike. The number of full steps taken within a minute is known as cadence. A stance length is the distance between the heel contact and pre-swing phases and a stance time is the interval of stance length. As the IMU sensor is placed at the foot location, the stance length is stationary. During the rest gait cycle, the foot is off the ground as the limb is swung forward to begin the next stride referred as swing phase. A swing length is the distance between the initial swing and terminal swing phases and a swing time is the interval of swing length. Figure 21 shows the stride, stance and swing information of older subject 1. 

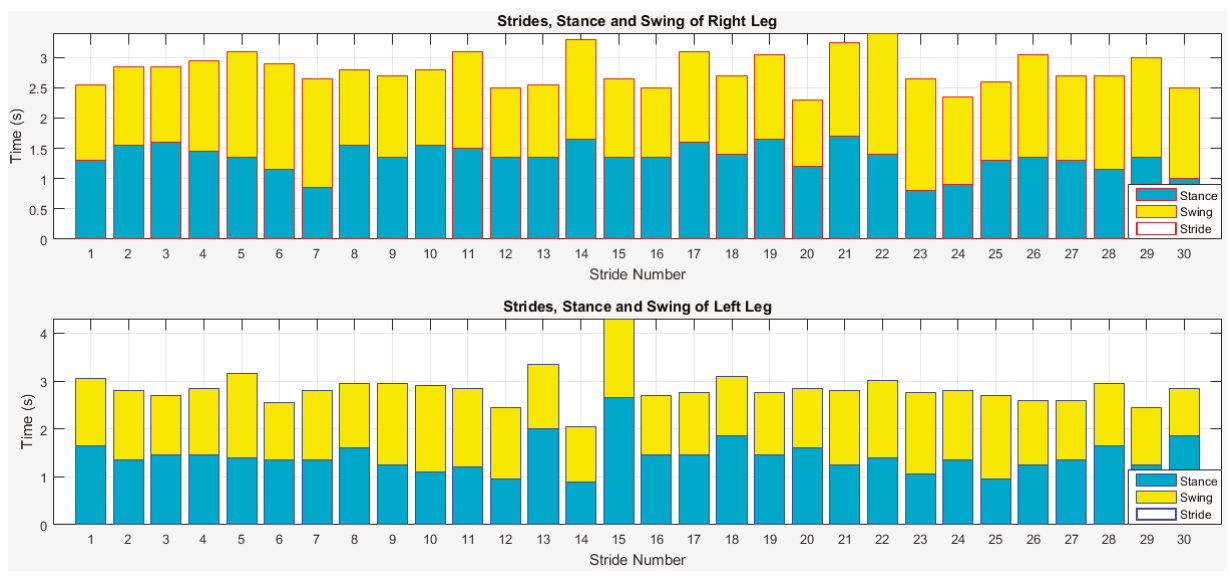

Figure 21. Stride, stance and swing information of right and left legs.

In the normal gait and the gait in patients after disease or injury, a certain GA level should be considered as normal [77]. Structural GA in human movement in limb length without underlying disease or injury is present in $90 \%$ of the population with an average magnitude of $5.2 \mathrm{~mm}$ and limb inequality of $20 \mathrm{~mm}$ must be present before considering clinically significant [78].

To quantify the temporal and spatial asymmetry of gait pattern, symmetry deviations (unaffected side - affected side, expressed as a fraction of the stride duration) [79], symmetric index (dividing the absolute difference of unaffected and affected by their average) [80], asymmetry ratios (1 (affected/unaffected) $)$ [81], Robinson symmetry index $(2 \times(($ unaffected - affected $) /($ unaffected + affected $)) \times 100)$ [82], a log-transformed symmetry ratio $(\mid 100 \times(\ln ($ affected/unaffected $)) \mid)[83]$, and symmetry angles $\left(\left(\left[45^{\circ}-\arctan (\right.\right.\right.$ affected/unaffected $\left.\left.) \times 100\right] / 90\right)$ [84] were used. However quantifying indices have known limitations as described in [77]. A certain amount of GA is usually present in able-bodied individuals [77], but no agreement exists regarding the clinical criteria for quantifying of GA. In visual observation or self-reports of physical function, GA is frequently reported as present or not present which may not satisfy scientific criteria of reliability and validity [85]. Thus, an arbitrary cut-off value of $10 \%$ deviation from perfect symmetry was previously used as a criterion of asymmetry in gait assessment $[76,82]$. This was subsequently criticized due to its non-parameter specific nature [86]. Other previously used criteria to describe the presence or absence of GA included sensitivity and specificity [87], the use of 95\% confidence intervals (GA within the limits of a $95 \%$ confidence interval (CI) obtained in a healthy population would define able-bodied gait, while GA outside the $95 \%$ CI would define pathologic gait) [86], and significant limbs difference [77]. In this study, we present gait variability quantities and validate the results with the ground truth for temporal GA monitoring. However, a parameter-specific criterion with optimal cut-off value that best discriminate GA from normal GA for each individual will be investigated in future study. The stride and step asymmetry information from older subject 1 is presented in Figures 22 and 23. 

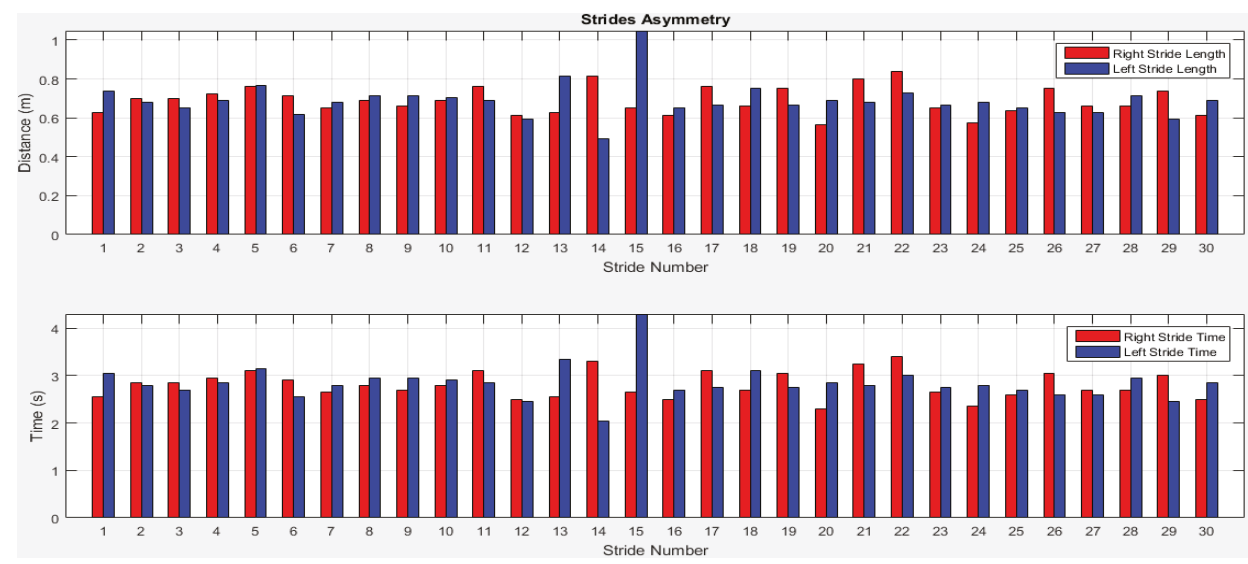

Figure 22. Stride asymmetry information of right and left legs.
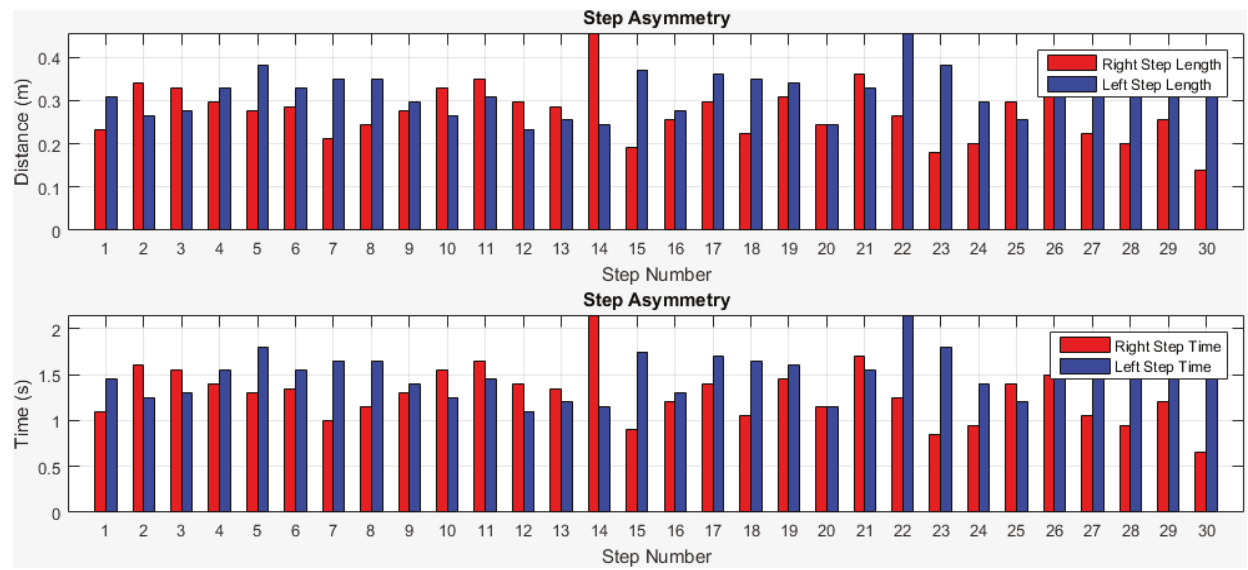

Figure 23. Step asymmetry estimation of right and left legs.

\subsubsection{Statistical Analysis}

Our estimated travelled distance and Qualisys results are tested for normality using Shapiro-Wilk [88]. The data is found to be normally distributed and a comparison of means is performed using the t-test for significance assuming equal variance. The estimated period and Qualisys results are found not normally distributed and a comparison of means were performed using the Wilcoxon Signed Ranks Test [89] for significance. The $p$ values of $<0.05$ are considered to be significant for both analysis. Statistics are performed using SPSS Version 24 [90].

\section{Experimental Results}

Initial experimental results from older subject 1 (male, age 67 , height $1.52 \mathrm{~m}$ and weight $68 \mathrm{~kg}$ ) are presented. We extract automatic GA features based on the data collected from both feet.

\subsection{GA Results of Older Subject 1}

Table 1 shows the accuracy of the distance travelled and estimated, detecting stride and step number from both legs. 
Table 1. Velocity, distance, stride and step information.

\begin{tabular}{|c|c|c|c|c|c|}
\hline Older subject 1 & Age & Height (m) & Weight (Kg) & Gender & \\
\hline & 67 & 1.57 & 68 & Male & \\
\hline \multirow[t]{2}{*}{ Total Time (s) } & & & 99.3 & & \\
\hline & Actual * & Right Leg ** & Accuracy $(\%)$ & Left Leg ** & Accuracy (\%) \\
\hline Total Distance (m) & 21.03 & 20.59 & 97.91 & 20.47 & 97.34 \\
\hline Estimated Velocity (m/s) & 0.21 & 0.21 & 97.91 & 0.21 & 97.34 \\
\hline Detected Stride Number & 30 & 30 & 100 & 30 & 100 \\
\hline Detected Step Number & 30 & 30 & 100 & 30 & 100 \\
\hline
\end{tabular}

$$
\text { Accuracy }=\left(100-\left|\frac{\text { ActualValue }- \text { EstimatedValue }}{\text { ActualVale }}\right| \times 100\right) \%
$$

The accuracy is estimated using Equation (5). The actual distance travelled is $21.03 \mathrm{~m}$ measured using manual tape with $99.3 \mathrm{~s}$ walking time. The estimated both legs travelled distances are $20.59 \mathrm{~m}$ and $20.47 \mathrm{~m}$. The actual and estimated distances are very close. Normal human walking velocity may vary from 1.5 to $2.5 \mathrm{~m} / \mathrm{s}$ [91] and the walking velocity for this subject is $0.21 \mathrm{~m} / \mathrm{s}$ which is slow. The accuracy of stride and step event detection are $100 \%$. Table 2 shows the summery of average gait variability.

We can observe from Table 2 that the mean stride lengths of both legs are the same. Although, the standard deviations are low and the right leg's value is lower indicating that the left stride length has more variation compared to the right stride length. The highest stride length is found at the 15th stride (last stride) on the left leg which is before turning. The mean stride times are close for both legs. Although, the right and left leg stride length, time and velocity difference is low, Figure 24 shows that a little stride asymmetry is noticeable in right and left strides time and distance. The difference of other parameters between the legs is also low. However, it is noted from Figure 25 that step asymmetry is more prominent than stride asymmetry which may result in an inconsistent gait.

Table 2. Gait Asymmetry Variability.

\begin{tabular}{cccccccccccc}
\hline & \multicolumn{4}{c}{ Right Leg } & \multicolumn{5}{c}{ Left Leg } \\
\hline Gait Features & Mean & Std & Var & Min & Max & Mean & Std & Var & Min & Max \\
\hline Stride Length (m) & 0.69 & 0.07 & 0.00 & 0.56 & 0.84 & 0.69 & 0.09 & 0.01 & 0.49 & 1.05 \\
Stride Time (s) & 2.80 & 0.28 & 0.08 & 2.30 & 3.40 & 2.85 & 0.37 & 0.13 & 2.05 & 4.30 \\
Stride Velocity (m/s) & 0.25 & & & & & 0.24 & & & & \\
Step length (m) & 0.27 & 0.06 & 0.00 & 0.14 & 0.46 & 0.32 & 0.05 & 0.00 & 0.23 & 0.46 \\
Step time(s) & 1.28 & 0.30 & 0.09 & 0.65 & 2.15 & 1.52 & 0.26 & 0.07 & 1.10 & 2.15 \\
Step Velocity (m/s) & 0.25 & & & & & 0.24 & & & & \\
Cadence (step/min) & 18.13 & & & & & 18.13 & & & & \\
Step Ratio (Step length/cadence) & 0.02 & & & & & 0.02 & & & & \\
Stance Time (s) & 1.65 & 0.23 & 0.05 & & & 1.67 & 0.35 & 0.12 & \\
Swing Length (m) & 0.69 & 0.35 & 0.12 & & & 0.69 & 0.44 & 0.19 & & \\
Swing Time (s) & 1.16 & 0.23 & 0.05 & & & 1.18 & 0.21 & 0.04 & & \\
Swing Velocity (m/s) & 0.59 & & & & & 0.58 & & & &
\end{tabular}

Std = Standard Deviation, Var = Variance, Min = Minimum and Max = Maximum.

We validate our results using 10 young subjects (age average $27.55 \pm 3.54$ ) by conducting trials using the Qualisys Motion Capture System (Qualisys Medical AB, Gothenburg, Sweden) [92] and our IMU sensor concurrently. Applying our method to the collected data leads to the result in Table 3. The average accuracy of the result is $97.57 \%$ with $95 \%$ confidence interval 1.327 for the estimated distance and $99.01 \%$ with $95 \%$ confidence interval 0.266 for the Period. 
Table 3. Validation our results with Qualisys.

\begin{tabular}{|c|c|c|c|c|c|c|c|}
\hline \multirow{2}{*}{ Participants } & \multirow[t]{2}{*}{ Leg } & Qualisys & Estimated & \multirow[b]{2}{*}{ Accuracy (\%) } & Qualisys & Period & \multirow[b]{2}{*}{ Accuracy (\%) } \\
\hline & & \multicolumn{2}{|c|}{ Distance (m) } & & Tir & & \\
\hline \multirow[b]{2}{*}{1} & Right & 7.650 & 7.607 & 99.435 & 12.670 & 12.510 & 98.740 \\
\hline & Left & 7.522 & 7.459 & 99.159 & 12.330 & 12.330 & 100.000 \\
\hline \multirow{2}{*}{2} & Right & 7.402 & 7.155 & 96.664 & 12.670 & 12.830 & 98.740 \\
\hline & Left & 7.456 & 7.327 & 98.270 & 12.330 & 12.280 & 99.590 \\
\hline \multirow[b]{2}{*}{3} & Right & 8.181 & 8.126 & 99.330 & 8.720 & 8.740 & 99.770 \\
\hline & Left & 7.984 & 7.747 & 97.034 & 8.280 & 8.150 & 98.430 \\
\hline \multirow{2}{*}{4} & Right & 7.978 & 7.806 & 97.848 & 8.720 & 8.880 & 98.170 \\
\hline & Left & 8.121 & 8.061 & 99.259 & 8.280 & 8.180 & 98.790 \\
\hline \multirow[b]{2}{*}{5} & Right & 7.735 & 7.699 & 99.531 & 9.780 & 9.750 & 99.690 \\
\hline & Left & 7.842 & 7.791 & 99.345 & 9.720 & 9.640 & 99.180 \\
\hline \multirow[b]{2}{*}{6} & Right & 7.564 & 7.493 & 99.066 & 9.780 & 9.710 & 99.280 \\
\hline & Left & 7.481 & 7.518 & 99.505 & 9.720 & 9.830 & 98.870 \\
\hline \multirow[b]{2}{*}{7} & Right & 7.693 & 6.784 & 88.181 & 7.380 & 7.250 & 98.240 \\
\hline & Left & 7.626 & 7.197 & 94.377 & 7.030 & 7.130 & 98.580 \\
\hline \multirow[b]{2}{*}{8} & Right & 7.422 & 6.939 & 93.497 & 7.380 & 7.310 & 99.050 \\
\hline & Left & 7.144 & 6.669 & 93.344 & 7.030 & 7.140 & 98.440 \\
\hline \multirow{2}{*}{9} & Right & 7.769 & 7.744 & 99.678 & 7.940 & 7.910 & 99.620 \\
\hline & Left & 7.755 & 7.626 & 98.331 & 7.870 & 8.000 & 98.350 \\
\hline \multirow{2}{*}{10} & Right & 7.508 & 7.485 & 99.698 & 7.940 & 7.960 & 99.750 \\
\hline & Left & 7.623 & 7.613 & 99.870 & 7.870 & 7.800 & 99.110 \\
\hline
\end{tabular}

Estimated $=$ Estimated distance using our method; Period = Total time of travelling the distance.

Although the sample size is small, the significance of the test of normality for Qualisys and Estimated Distance are 0.83 and 0.37 using Shapiro-Wilk. The $t$-test shows that there is no difference in means $(p=0.094)$ between Qualisys $\left(\mu_{1}=7.67, \sigma_{1}=0.26\right)$ and Estimated Distance $\left(\mu_{2}=7.49, \sigma_{2}=0.39\right)$. There is a strong correlation $(r=0.81)$ present. The Wilcoxon Signed Ranks Test for Qualisys and Estimated Period shows no mean difference $(p=0.83)$.

\subsection{GA Results of Young and Older Subjects}

Table 4 shows the gait data from the 10 young subjects. Table 4 shows that the accuracy of estimating the total distance compared with the actual distance is also high for both legs. The detected stride and step number using the proposed method is excellent. For all young subjects, the accuracy of detecting stride number using proposed method is $100 \%$. The accuracy of estimating travelled distance using proposed method is $97.73 \%$ for right and $98.82 \%$ for left legs.

Table 4. Velocity, distance, stride and step results for young subjects.

\begin{tabular}{|c|c|c|c|c|c|}
\hline AVERAGE & Age & Height (m) & Weight (Kg) & Gender & \\
\hline & 25.30 & 1.61 & 61.90 & $9 \mathrm{M}, 1 \mathrm{~F}$ & \\
\hline \multirow[t]{2}{*}{ Total Time (s) } & & & 51.85 & & \\
\hline & Actual * & Right Leg ** & Accuracy & Left Leg ** & Accuracy \\
\hline Total Distance (m) & 37.77 & 37.19 & 97.73 & 37.81 & 98.82 \\
\hline Estimated Velocity $(\mathrm{m} / \mathrm{s})$ & 0.73 & 0.72 & 97.73 & 0.73 & 98.82 \\
\hline Detected Stride Number & 30.00 & 30.00 & 100.00 & 30.00 & 100.00 \\
\hline Detected Step Number & 30.00 & 30.00 & 100.00 & 30.00 & 100.00 \\
\hline
\end{tabular}

Table 5 shows the details of both legs asymmetry variables information. The stride lengths of legs are the same for young subjects. The overall difference between legs is low for young subjects. In natural walking, the foot is on the ground for about $60 \%$ of the total gait cycle during stance phase 
and $40 \%$ during swing phase shown in Figure 9. The ratio of stance and swing is found closest to the 60:40\% split for average stride, stance and swing information (Table 5) for young subjects.

Table 5. Right and left legs asymmetry of young subjects.

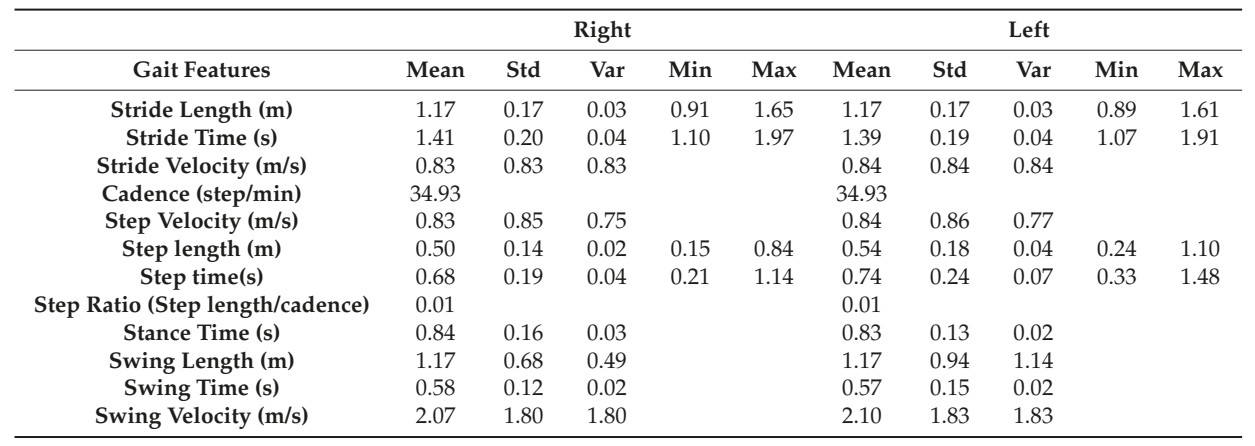

Table 6 shows that the accuracy of estimating the total distance compared with the actual distance is high for both legs. For all older subjects the accuracy of detecting stride number using the proposed method is $92.67 \%$. The accuracy of estimating the travelled distance using the proposed method is $88.71 \%$ for the right and $89.88 \%$ for the left legs. The detected stride and step number using the proposed method is also high. However, comparing to results in young subjects (Table 4), the accuracy is lower for older subjects. This is likely to be due to older people walking slowly resulting in a poorer signal output. Table 7 shows the details of both legs asymmetry variables for older subjects. Overall the stride lengths of both legs are similar. The overall difference between legs is very low.

Table 6. Velocity, distance, stride and step results for older subjects.

\begin{tabular}{cccccc}
\hline Average & Age & Height $(\mathbf{m})$ & Weight $(\mathrm{Kg})$ & \multicolumn{2}{c}{ Gender } \\
\hline & $\mathbf{6 9 . 4 0}$ & $\mathbf{1 . 5 2}$ & $\mathbf{6 3 . 4 0}$ & $\mathbf{9 ~ M , ~ 1 F}$ \\
\hline Total Time (s) & & & $\mathbf{8 0 . 2 6}$ & & \\
\hline Total Distance (m) & 22.49 & 22.21 & 88.71 & 21.19 & 89.88 \\
Estimated Velocity (m/s) & 0.31 & 0.29 & 88.71 & 0.27 & 89.88 \\
Detected Stride Number & 30 & 27.80 & 92.67 & 27.80 & 92.67 \\
Detected Step Number & 30 & 27.80 & 92.67 & 27.80 & 92.67 \\
\hline
\end{tabular}

${ }^{*}$ ActualValue, ${ }^{* *}$ EstimatedValue.

Table 7. Right and left legs asymmetry of older subjects.

\begin{tabular}{|c|c|c|c|c|c|c|c|c|c|c|}
\hline \multirow[b]{2}{*}{ Gait Features } & \multicolumn{5}{|c|}{ Right } & \multicolumn{5}{|c|}{ Left } \\
\hline & Mean & Std & Var & Min & Max & Mean & Std & Var & Min & Max \\
\hline Stride Length (m) & 0.74 & 0.14 & 0.02 & 0.54 & 1.16 & 0.74 & 0.13 & 0.02 & 0.55 & 1.09 \\
\hline Stride Time (s) & 2.47 & 0.46 & 0.24 & 1.84 & 3.88 & 2.44 & 0.39 & 0.16 & 1.80 & 3.47 \\
\hline Stride Velocity $(\mathrm{m} / \mathrm{s})$ & 0.32 & 0.32 & 0.32 & & & 0.33 & 0.32 & 0.32 & & \\
\hline Cadence (step/min) & 24.34 & & & & & 24.34 & & & & \\
\hline Step Velocity $(\mathrm{m} / \mathrm{s})$ & 0.32 & 0.33 & 0.13 & & & 0.33 & 0.33 & 0.13 & & \\
\hline Step length (m) & 0.22 & 0.23 & 0.06 & 0.22 & 0.63 & 0.35 & 0.18 & 0.04 & 0.04 & 0.57 \\
\hline Step time(s) & 0.54 & 0.86 & 1.02 & 1.02 & 2.07 & 1.13 & 0.73 & 0.81 & 0.18 & 2.20 \\
\hline Step Ratio (Step length/cadence) & 0.007 & & & & & 0.014 & & & & \\
\hline Stance Time (s) & 1.39 & 0.30 & 0.10 & & & 1.37 & 0.28 & 0.08 & & \\
\hline Swing Length (m) & 0.74 & 0.78 & 0.73 & & & 0.74 & 0.76 & 0.66 & & \\
\hline Swing Time (s) & 1.09 & 0.30 & 0.10 & & & 1.07 & 0.23 & 0.05 & & \\
\hline Swing Velocity $(\mathrm{m} / \mathrm{s})$ & 0.78 & 0.68 & 0.68 & & & 0.78 & 0.65 & 0.65 & & \\
\hline
\end{tabular}


We check the data for statistical errors and assessed whether the estimated values are reasonable. Figure 24 shows the boxplot of travelled distance from young and older subjects. It is noted that the observations identified by the boxplots are not especially extreme. The young subjects' travelled distance for 30 strides has a wider range and is significantly different than older ones. On average young subjects travelled distance is $37.77(95 \% \mathrm{CI} \pm 3.57) \mathrm{m}$ and in older ones is $22.50(95 \% \mathrm{CI} \pm 2.34)$ $\mathrm{m}$. Similarly the legs stride and step variation is low for older ones than young ones. Older ones gait is slow and results in a low variation in walking comparing with young ones. The step length has more variation then stride length. Based on the total travelled distance, stride and step information, it can be seen that young and older subjects are distinguishable.

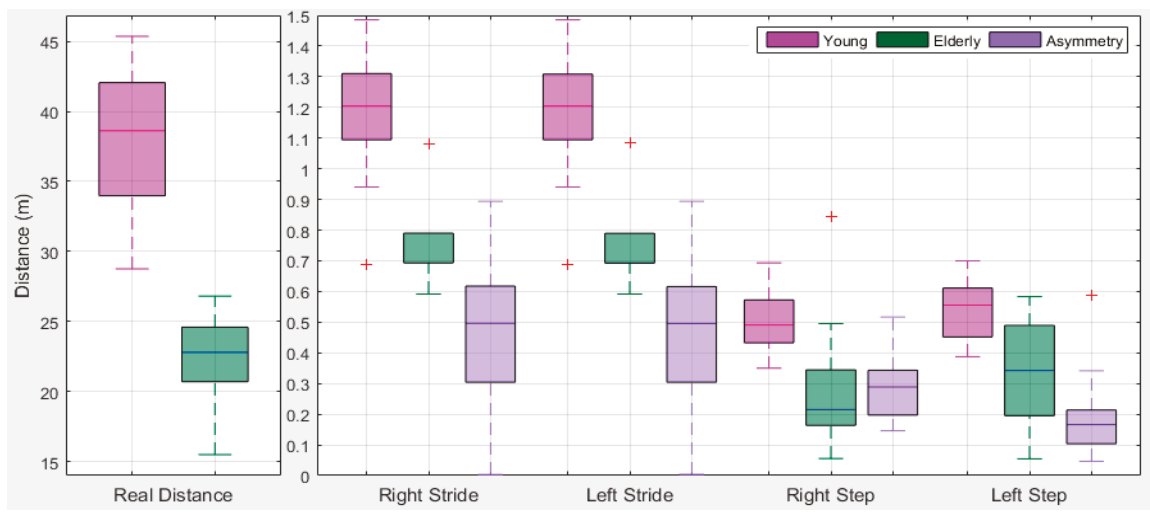

Figure 24. BoxPlot of stride and step asymmetry in distances from right and left legs.

Figure 25 shows a boxplot of total time from young and older subjects with their difference. The total time for performing a total of 30 strides is lower for young subjects than older ones. On average the young subjects travelled time is 51.85 (95\% CI \pm 3.08$) \mathrm{s}$ and older ones is 84.02 $(95 \% \mathrm{CI} \pm 9.98) \mathrm{s}$. Young subjects show low leg variation with a lower range than older ones. Based on the total time, stride and step timing information, it can be seen that young subjects and older ones are distinguishable.

The detailed results of the 20 subjects are presented in the Supporting Information.

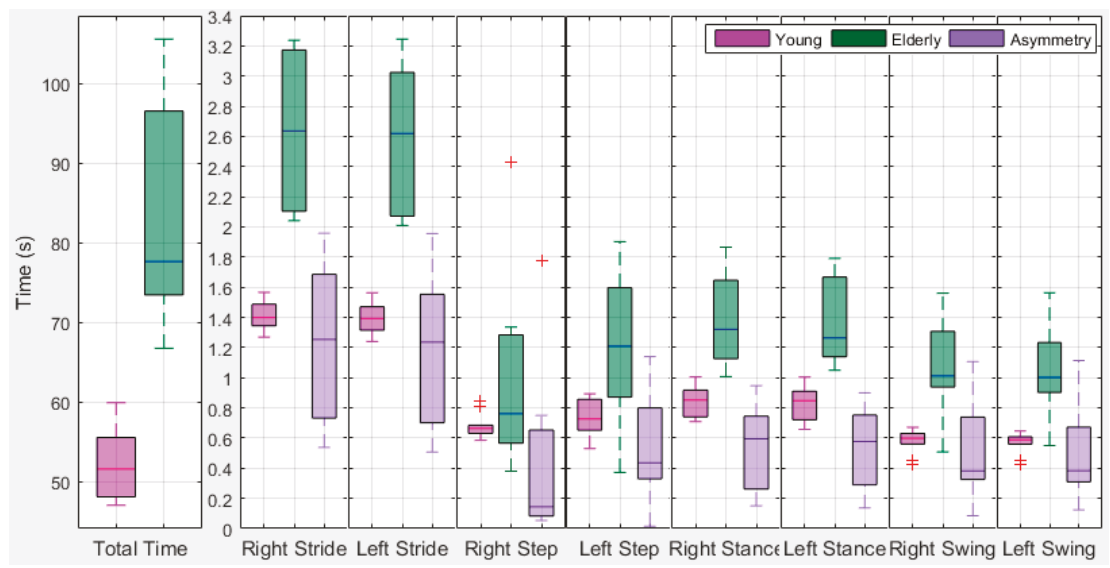

Figure 25. Box Plot of stride and step asymmetry in times from right and left legs. 


\subsection{Discussion}

In this research we show that in a clinical setting outside of a gait laboratory it is possible to collect information about GA using IMU sensors. From Figure 10, we can see that our gait cycle accelerometer signal $A T_{x y z}$ is agreed with the signal pattern in $[53,54]$. We demonstrate the systematic steps of an automatic gait features extraction method that we deployed. Our research enriches the current literature in GA assessment. It is possible to evaluate walking distance using a multisensor approach. Current methods however rely on the threshold based detection of the spike $[35,36,58,60]$. Our method uses minimal prominence characteristics for detecting gait phases. The former relies on generating a movement of sufficient magnitude to generate the spike and therefore has limited utility in people with slow gait. Our method therefore has the potential for broader use as it can be used in people with slower gaits such as older adults. We demonstrate that our method can deliver accurate results of stride detection and distance travelled similar to accuracy levels demonstrated by other authors $[36,60]$. We believe that there are advantages to using the minimal prominence approach as it can be used in a wider population people with different gait patterns.

There are however a number of limitations. The number of subjects is still relatively small (20). There is the potential of a Type 1 error (false positive) in detecting an effect that is not there. IMU calibration is an essential part for distance estimation. Although in our methods we try to minimize errors, as gait features are intrinsically variable from person to person, any such algorithm should involve a degree of calibration and error in the measurements. Individual quirks, heel strike, significant body up-down movement and other factors can affect the results. However this can be considered a proof of concept study that has established our method for extracting automatic GA features. There are several other possible sources of errors [93] that may arise from the use of IMU sensors including errors of repeatability, stability and drift. Although IMU sensors performance has been ramped up dramatically, the errors in measurement are unavoidable, especially for miniature micro-electro-mechanical (MEMS) sensors. Future developments should focus on MEMS sensor error modelling and accommodation to further improve parameter estimation accuracy [94]. Other possible areas of error may arise from frictional noise and the relative movement of clothing and shoes to the sensor. However we compared the output from our sensor to a gold standard Qualisys motion capture system which shows good accuracy making the effect of such errors minimal.

To achieve our goal, data are collected from two sensors placed on the barefoot at the medial aspect of foot over the bony prominence of the first metatarsal. It is noted that the orientation of the sensor has a significant effect on output and placing the sensor in different locations gives a different pattern to the data. The position and orientation of the sensor are crucial as changes in position through human error may give different data patterns which might be difficult to interpret. This highlights the importance of properly fixing the sensor to the optimal location to avoid inaccuracies. The placing of sensors on foot locations requires other generic considerations such as battery life and android device that is BLE enabled to pick up sensor data.

To estimate the orientation of the IMU sensors, we apply the Madgwick technique [51] for our collected data but not the magnetic field parameter. The technique is developed assuming that the acceleration would only measure gravity. In practice, accelerations due to motion will result in an erroneous observed direction of gravity and the distortion will present for only short periods of time. Therefore, the magnitude of the filter gain $\beta$ (Section 2.6.3) is chosen low enough that the divergence caused by the erroneous gravitational observations is reduced to an acceptance level over the period. In future, an investigation of dynamic values of gains $\beta$ and $\varsigma$ will be conducted to reduce errors.

A threshold is used for detecting steps $[36,60]$ and different value may result in a different output. It is crucial to detect the 1st and last strides of gait cycle when a person starts and stop walking. Thus, the 1st stride is not considered by researchers [60]. Our proposed method for detecting the stride information is based on the local minimal prominence which starts when the heel contacts the ground resulting in the stationary period (Section 2.6.4) and estimated the total number of strides. We also confirm these results obtained by counting the highest peak in the mid-swing phase as it also 
is a good indicator for a complete gait cycle. From each stride, the local minimal prominence which is the transition between pre-swing and initial swing (4th and 5th phases in Figure 10) is detected. We find that that when turning or when stopping there is a poor acceleration signal. As gait of older subjects is much slower, it is crucial to detect strides, stance and swing phases from the gait cycle. However, the stationary stance phase is prominent for both young and older subjects. For this reason, we use the local minimal prominence characteristics to detect different events to avoid these crucial phases. We have shown that it is possible to detect, stride, stance and swing events but further analysis of the eight events including single and double support phases in a gait cycle is necessary to provide more accurate information for GA analysis.

In order to track the position in a virtual environment, several navigation methods [63] are available to derive pose estimates from electrical measurements of mechanical, inertial, acoustic, magnetic, optical, and radio frequency sensors. Each approach has advantages and limitations including modality-specific limitations related to the physical medium, measurement-specific limitations imposed by the devices, associated signal-processing electronics, and circumstantial limitations that arise in a specific application [95]. Our velocity and distance estimation is based on results of a double integral with ZUPT. We apply the high pass filter on acceleration data that removes linear trend from the signal and then remove drift to estimate distance. We use the simplest technique of trapezoidal rule for estimating distance for our collected data and our estimated distance results are close to the actual distance. There are many other types of numerical integration schemes available which are much more involved and with the potential for more accuracy. However, the trapezoidal rule is the simplest technique of an entire class of numerical integration schemes which are known as the Newton-Cotes formulas [96] and which we have adopted. Our future plan is to investigate other methods with our collected data.

The results show that our method is capable of extracting automatic gait features and has the potential to be used in GA assessment and gait change monitoring for home and clinical use. Gait with slow velocity is common in older adults [97] and an automatic system sensitive enough to detect gait features in these circumstances is required. Our low cost portable personalized proposed solution could bring out automatic GA features for monitoring longitudinal gait changes or abnormalities. In future work, we plan to use our automatic extracted GA features information to classify gait changes over time to identify abnormal gait patterns for the assessment of elderly fall risk, rehabitation and sports applications.

\section{Conclusions}

In the present work, two IMU sensors were placed at right and left metatarsal barefoot locations to collect accelerometer and gyroscope data. We designed and developed an android app to collect real time synchronous data from both sensors. We proposed a systematic method to extract automatic gait features for the GA assessment. We first applied the quaternion technique to raw data for estimating actual sensor orientation. We applied our proposed stride, stance, swing and step event detection technique and analysed for stride, step, cadence, step ratio, stance, and swing. We then estimated distance using double integration with drift removing from acceleration and analyzed for total velocity, distance and time. Our method was validated with the Qualisys motion capture system. We applied our method for 10 young and 10 older subjects. Our results show that it is possible to extract GA features automatically in a clinical setting outside of a gait laboratory. This has the potential to make the evaluation of GA widely available in clinical practice rather than being limited to gait laboratories.

Supplementary Materials: The supporting information is available online at http:/ /www.mdpi.com/1424-8220/ 18/2/676/s1.

Acknowledgments: This work was supported by European Commission funding (ERASMUS MUNDUS FUSION project). The authors would like to thank all participants that participated in the study. The authors would like to thank Andrew Callaway for his help to collect data from Qualisys and all participants that participated in the study.

Conflicts of Interest: The authors declare no conflict of interest. 


\section{References}

1. Murray, M.P. Gait as a total pattern of movement: Including a bibliography on gait. Am. J. Phys. Med. Rehabilit. 1967, 46, 290-333.

2. Muro-de-la-Herran, A.; Garcia-Zapirain, B.; Mendez-Zorrilla, A. Gait analysis methods: An overview of wearable and non-wearable systems, highlighting clinical applications. Sensors 2014, 14, 3362-3394. [CrossRef] [PubMed]

3. Plotnik, M.; Giladi, N.; Balash, Y.; Peretz, C.; Hausdorff, J.M. Is freezing of gait in parkinson's disease related to asymmetric motor function? Ann. Neurol. 2005, 57, 656-663. [CrossRef] [PubMed]

4. Lewek, M.D.; Bradley, C.E.; Wutzke, C.J.; Zinder, S.M. The relationship between spatiotemporal gait asymmetry and balance in individuals with chronic stroke. J. Appl. Biomech. 2014, 30, 31-36. [CrossRef] [PubMed]

5. Wahab, Y.; Bakar, N.A. Gait Analysis Measurement for Sport Application Based on Ultrasonic System. In Proceedings of the 2011 IEEE 15th International Symposium on Consumer Electronics (ISCE), Singapore, 14-17 June 2011; pp. 20-24.

6. Steultjens, M.P.M.; Dekker, J.; Baar, M.E.V.; Oostendorp, R.A.B.; Bijlsma, J.W.J. Range of joint motion and disability in patients with osteoarthritis of the knee or hip. Rheumatology 2000, 39, 955-961. [CrossRef] [PubMed]

7. Bora, N.M.; Molke, G.V.; Munot, H.R. Understanding human gait: A survey of traits for biometrics and biomedical applications. In Proceedings of the 2015 International Conference on Energy Systems and Applications, Pune, India, 30 October-1 November 2015; pp. 723-728.

8. Yogev, G.; Plotnik, M.; Peretz, C.; Giladi, N.; Hausdorff, J.M. Gait asymmetry in patients with parkinson's disease and elderly fallers: When does the bilateral coordination of gait require attention? Exp. Brain Res. 2007, 177, 336-346. [CrossRef] [PubMed]

9. Plotnik, M.; Dagan, Y.; Gurevich, T.; Giladi, N.; Hausdorff, J.M. Effects of cognitive function on gait and dual tasking abilities in patients with parkinson's disease suffering from motor response fluctuations. Exp. Brain Res. 2011, 208, 169-179. [CrossRef] [PubMed]

10. Caldas, R.; Mundt, M.; Potthast, W.; Buarque, F.; Markert, B. A systematic review of gait analysis methods based on inertial sensors and adaptive algorithms. Gait Posture 2017, 57, 204-210. [CrossRef] [PubMed]

11. Kirtley, C. Clinical Gait Analysis: Theory and Practice; Elsevier Health Sciences: Philadelphia, PA, USA, 2006.

12. Jarchi, D.; Lo, B.; Wong, C.; Ieong, E.; Nathwani, D.; Yang, G.-Z. Gait analysis from a single ear-worn sensor: Reliability and clinical evaluation for orthopaedic patients. IEEE Trans. Neural Syst. Rehabilit. Eng. 2016, 24, 882-892. [CrossRef] [PubMed]

13. Auvinet, E.; Multon, F.; Manning, V.; Meunier, J.; Cobb, J.P. Validity and sensitivity of the longitudinal asymmetry index to detect gait asymmetry using microsoft kinect data. Gait Posture 2017, 51, 162-168. [CrossRef] [PubMed]

14. Su, B.L.; Song, R.; Guo, L.Y.; Yen, C.W. Characterizing gait asymmetry via frequency sub-band components of the ground reaction force. Biomed. Signal Process. Control 2015, 18, 56-60. [CrossRef]

15. Bervet, K.; Bessette, M.; Godet, L.; Crétual, A. Ker-egi, a new index of gait quantification based on electromyography. J. Electromyogr. Kinesiol. 2013, 23, 930-937. [CrossRef] [PubMed]

16. Williams, A.J.; Peterson, D.S.; Earhart, G.M. Gait coordination in Parkinson disease: Effects of step length and cadence manipulations. Gait Posture 2013, 38, 340-344. [CrossRef] [PubMed]

17. Brach, J.S.; VanSwearingen, J.M. Physical impairment and disability: Relationship to performance of activities of daily living in community-dwelling older men. Phys. Ther. 2002, 82, 752-761. [PubMed]

18. Hess, R.J.; Brach, J.S.; Piva, S.R.; VanSwearingen, J.M. Walking skill can be assessed in older adults: Validity of the figure-of-8 walk test. Phys. Ther. 2010, 90, 89-99. [CrossRef] [PubMed]

19. Duncan, R.P.; Earhart, G.M. Four square step test performance in people with Parkinson disease. J. Neurol. Phys. Ther. 2013, 37, 2-8. [CrossRef] [PubMed]

20. Wrisley, D.M.; Kumar, N.A. Functional gait assessment: Concurrent, discriminative, and predictive validity in community-dwelling older adults. Phys. Ther. 2010, 90, 761-773. [CrossRef] [PubMed]

21. Bossers, W.J.R.; van der Woude, L.H.V.; Boersma, F.; Scherder, E.J.A.; van Heuvelen, M.J.G. The groningen meander walking test: A dynamic walking test for older adults with dementia. Phys. Ther. 2014, 94, 262-272. [CrossRef] [PubMed] 
22. Berg, K.O.; Wood-Dauphinee, S.L.; Williams, J.I.; Maki, B. Measuring balance in the elderly: Validation of an instrument. Can. J. Public Health 1992, 83, 7-11.

23. Agostini, V.; Knaflitz, M.; Antenucci, L.; Lisco, G.; Gastaldi, L.; Tadano, S. Wearable sensors for gait analysis. In Proceedings of the 2015 IEEE International Symposium on Medical Measurements and Applications (MeMeA), Torino, Italy, 7-9 May 2015; pp. 146-150.

24. Urbanek, J.K.; Harezlak, J.; Glynn, N.W.; Harris, T.; Crainiceanu, C.; Zipunnikov, V. Stride variability measures derived from wrist- and hip-worn accelerometers. Gait Posture 2017, 52, 217-223. [CrossRef] [PubMed]

25. Hanly, R.; Doyle, F.; Whitehouse, S.; Timperley, A. Outpatient 3-d gait analysis one year after THA using a portable IMU system. Orthop. Proceed. 2016, 98-B, 1.

26. Esser, P.; Howells, K.; Dawes, H.; Collet, J. Gait Asymmetry Measurement. U.S. Patent 14/122,344, 24 April 2014.

27. Rispens, S.M.; Van Dieën, J.H.; Van Schooten, K.S.; Cofré Lizama, L.E.; Daffertshofer, A.; Beek, P.J.; Pijnappels, M. Fall-related gait characteristics on the treadmill and in daily life. J. NeuroEng. Rehabilit. 2016, 13, 1-9. [CrossRef] [PubMed]

28. Okuda, S.; Takano, S.; Ueno, M.; Hara, Y.; Chida, Y.; Ikkaku, T.; Kanda, F.; Toda, T. Gait analysis of patients with parkinson's disease using a portable triaxial accelerometer. Neurol. Clin. Neurosci. 2016, 4, 93-97. [CrossRef]

29. Kitagawa, N.; Ogihara, N. Estimation of foot trajectory during human walking by a wearable inertial measurement unit mounted to the foot. Gait Posture 2016, 45, 110-114. [CrossRef] [PubMed]

30. Lee, J.A.; Cho, S.H.; Lee, J.W.; Lee, K.H.; Yang, H.K. Wearable accelerometer system for measuring the temporal parameters of gait. In Proceedings of the 2007 29th Annual International Conference of the IEEE Engineering in Medicine and Biology Society, Lyon, France, 22-26 August 2007; pp. 483-486.

31. Chung, P.C.; Hsu, Y.L.; Wang, C.Y.; Lin, C.W.; Wang, J.S.; Pai, M.C. Gait analysis for patients with alzheimer's disease using a triaxial accelerometer. In Proceedings of the 2012 IEEE International Symposium on Circuits and Systems, Seoul, Korea, 20-23 May 2012; pp. 1323-1326.

32. Kavanagh, J.J. Lower trunk motion and speed-dependence during walking. J. Neuroeng. Rehabilit. 2009 , 6, 9. [CrossRef] [PubMed]

33. Song, Y.; Shin, S.; Kim, S.; Lee, D.; Lee, K.H. Speed estimation from a tri-axial accelerometer using neural networks. In Proceedings of the 2007 29th Annual International Conference of the IEEE Engineering in Medicine and Biology Society, Lyon, France, 22-26 August 2007; pp. 3224-3227.

34. Brajdic, A.; Harle, R. Walk detection and step counting on unconstrained smartphones. In Proceedings of the 2013 ACM International Joint Conference on Pervasive and Ubiquitous Computing, Zurich, Switzerland, 8-12 September 2013; pp. 225-234.

35. Mladenov, M.; Mock, M. A step counter service for java-enabled devices using a built-in accelerometer. In Proceedings of the 1st International Workshop on Context-Aware Middleware and Services: Affiliated with the 4th International Conference on Communication System Software and Middleware (COMSWARE 2009), Dublin, Ireland, 6-16 June 2009; pp. 1-5.

36. Boutaayamou, M.; Schwartz, C.; Stamatakis, J.; Denoël, V.; Maquet, D.; Forthomme, B.; Croisier, J.-L.; Macq, B.; Verly, J.G.; Garraux, G.; et al. Development and validation of an accelerometer-based method for quantifying gait events. Med. Eng. Phys. 2015, 37, 226-232. [CrossRef] [PubMed]

37. Rebula, J.R.; Ojeda, L.V.; Adamczyk, P.G.; Kuo, A.D. Measurement of foot placement and its variability with inertial sensors. Gait Posture 2013, 38, 974-980. [CrossRef] [PubMed]

38. Brandes, M.; Zijlstra, W.; Heikens, S.; van Lummel, R.; Rosenbaum, D. Accelerometry based assessment of gait parameters in children. Gait Posture 2006, 24, 482-486. [CrossRef] [PubMed]

39. Afzal, M.R.; Oh, M.-K.; Lee, C.-H.; Park, Y.S.; Yoon, J. A portable gait asymmetry rehabilitation system for individuals with stroke using a vibrotactile feedback. BioMed Res. Int. 2015, 2015, 16. [CrossRef] [PubMed]

40. Novak, D.; Reberšek, P.; De Rossi, S.M.M.; Donati, M.; Podobnik, J.; Beravs, T.; Lenzi, T.; Vitiello, N.; Carrozza, M.C.; Munih, M. Automated detection of gait initiation and termination using wearable sensors. Med. Eng. Phys. 2013, 35, 1713-1720. [CrossRef] [PubMed]

41. Seel, T.; Raisch, J.; Schauer, T. Imu-based joint angle measurement for gait analysis. Sensors 2014, 14, 6891-6909. [CrossRef] [PubMed] 
42. Gouwanda, D.; Arosha Senanayake, S.M.N. Identifying gait asymmetry using gyroscopes-A cross-correlation and normalized symmetry index approach. J. Biomech. 2011, 44, 972-978. [CrossRef] [PubMed]

43. Paranietharan, D.N. Who I Bangladesh. In WHO; World Health Organization: Geneva, Switzerland, 2017.

44. Anwary, A.R.; Yu, H.; Vassallo, M. Optimal foot location for placing wearable IMU sensors and automatic feature extraction for gait analysis. IEEE Sens. J. 2017. [CrossRef]

45. Cornacchia, M.; Ozcan, K.; Zheng, Y.; Velipasalar, S. A survey on activity detection and classification using wearable sensors. IEEE Sens. J. 2017, 17, 386-403. [CrossRef]

46. MbientLab. Available online: https:// mbientlab.com/ (accessed on 3 March 2016).

47. Sazonov, E.S.; Fulk, G.; Hill, J.; Schutz, Y.; Browning, R. Monitoring of posture allocations and activities by a shoe-based wearable sensor. IEEE Trans. Biomed. Eng. 2011, 58, 983-990. [CrossRef] [PubMed]

48. SolidWorks, I. Solidworks Corporation; Solidworks Corporation: Concord, MA, USA, 2002.

49. Zhi, R. A Drift Eliminated Attitude \& Position Estimation Algorithm in 3D. Master's Thesis, University of Vermont, Burlington, VA, USA, 2016.

50. MbientLab. Available online: https://mbientlab.Com/docs/metawearcpsv0.5.Pdf (accessed on 25 May 2015).

51. Madgwick, S.O. An efficient orientation filter for inertial and inertial/magnetic sensor arrays. Citado 2010, 5, 9-19.

52. Starlino, B. A Guide to Using IMU (Accelerometer and Gyroscope Devices) in Embedded Applications. Available online: http:/ /www.starlino.com/imu_guide.html (accessed on 29 December 2017).

53. Liu, D.-X.; Wu, X.; Du, W.; Wang, C.; Xu, T. Gait phase recognition for lower-limb exoskeleton with only joint angular sensors. Sensors 2016, 16, 1579. [CrossRef] [PubMed]

54. Patterson, M.; Delahunt, E.; Sweeney, K.; Caulfield, B. An ambulatory method of identifying anterior cruciate ligament reconstructed gait patterns. Sensors 2014, 14, 887-899. [CrossRef] [PubMed]

55. Foxlin, E. Pedestrian tracking with shoe-mounted inertial sensors. IEEE Comput. Graph. Appl. 2005, 25, 38-46. [CrossRef] [PubMed]

56. Alvarez, D.; González, R.C.; López, A.; Alvarez, J.C. Comparison of step length estimators from weareable accelerometer devices. In Proceedings of the 28th Annual International Conference of the IEEE Engineering in Medicine and Biology Society (EMBS'06), New York, NY, USA, 30 August-3 September 2006; pp. 5964-5967.

57. Lee, H.-H.; Choi, S.; Lee, M.-J. Step detection robust against the dynamics of smartphones. Sensors 2015, 15, 27230-27250. [CrossRef] [PubMed]

58. Chon, Y.; Talipov, E.; Cha, H. Autonomous management of everyday places for a personalized location provider. IEEE Trans. Syst. Man Cybern. Part C 2012, 42, 518-531. [CrossRef]

59. Gafurov, D.; Snekkenes, E. Towards understanding the uniqueness of gait biometric. In Proceedings of the 8th IEEE International Conference on Automatic Face \& Gesture Recognition (FG'08), Amsterdam, The Netherlands, 17-19 September 2008; pp. 1-8.

60. Truong, P.; Lee, J.; Kwon, A.-R.; Jeong, G.-M. Stride counting in human walking and walking distance estimation using insole sensors. Sensors 2016, 16, 823. [CrossRef] [PubMed]

61. The MathWorks Inc. Find Local Maxima-Matlab Findpeaks, Mathworks United Kingdom. Available online: https:/ / uk.mathworks.com/help/signal/ref/findpeaks.html (accessed on 14 December 2016).

62. Oppenheim, A.V.; Schafer, R.W. Discrete-Time Signal Processing; Prentice Hall: Englewood Cliffs, NJ, USA, 1989.

63. Hasan, A.M.; Samsudin, K.; Ramli, A.R.; Azmir, R.S.; Ismaeel, S.A. A review of navigation systems (integration and algorithms). Aust. J. Basic Appl. Sci. 2009, 3, 943-959.

64. Lan, K.-C.; Shih, W.-Y. On calibrating the sensor errors of a PDR-based indoor localization system. Sensors 2013, 13, 4781-4810. [CrossRef] [PubMed]

65. Sukumar, C.F.P.; Hazas, M. Tutorial: Implementation of a pedestrian tracker using foot-mounted inertial sensors. IEEE Pervasive Comput. 2012, 12, 17-27.

66. Ilyas, M.; Cho, K.; Baeg, S.-H.; Park, S. Drift reduction in pedestrian navigation system by exploiting motion constraints and magnetic field. Sensors 2016, 16, 1455. [CrossRef] [PubMed]

67. Fang, J.; Gu, Q.-T.; Ding, T.-H. Dynamic zero velocity update for vehicle inertial navigation system. J. Chin. Inert. Technol. 2008, 3, 004.

68. Thong, Y.K.; Woolfson, M.S.; Crowe, J.A.; Hayes-Gill, B.R.; Jones, D.A. Numerical double integration of acceleration measurements in noise. Measurement 2004, 36, 73-92. [CrossRef] 
69. Kressig, R.W.; Beauchet, O. Guidelines for clinical applications of spatio-temporal gait analysis in older adults. Aging Clin. Exp. Res. 2006, 18, 174-176. [CrossRef] [PubMed]

70. Whittle, M. Gait Analysis: An Introduction; Butterworth Heinmann: Oxford, UK, 2002.

71. Thingstad, P.; Egerton, T.; Ihlen, E.F.; Taraldsen, K.; Moe-Nilssen, R.; Helbostad, J.L. Identification of gait domains and key gait variables following hip fracture. BMC Geriatr. 2015, 15, 150. [CrossRef] [PubMed]

72. Hausdorff, J.M.; Cudkowicz, M.E.; Firtion, R.; Wei, J.Y.; Goldberger, A.L. Gait variability and basal ganglia disorders: Stride-to-stride variations of gait cycle timing in Parkinson's disease and Huntington's disease. Mov. Disord. 1998, 13, 428-437. [CrossRef] [PubMed]

73. Zeni, J.A.; Higginson, J.S. Gait parameters and stride-to-stride variability during familiarization to walking on a split-belt treadmill. Clin. Biomech. 2010, 25, 383-386. [CrossRef] [PubMed]

74. Beauchet, O.; Allali, G.; Launay, C.; Herrmann, F.; Annweiler, C. Gait variability at fast-pace walking speed: A biomarker of mild cognitive impairment? J. Nutr., Health Aging 2013, 17, 235-239. [CrossRef] [PubMed]

75. IJmker, T.; Lamoth, C.J. Gait and cognition: The relationship between gait stability and variability with executive function in persons with and without dementia. Gait Posture 2012, 35, 126-130. [CrossRef] [PubMed]

76. Balasubramanian, C.K.; Neptune, R.R.; Kautz, S.A. Variability in spatiotemporal step characteristics and its relationship to walking performance post-stroke. Gait Posture 2009, 29, 408-414. [CrossRef] [PubMed]

77. Sadeghi, H.; Allard, P.; Prince, F.; Labelle, H. Symmetry and limb dominance in able-bodied gait: A review. Gait Posture 2000, 12, 34-45. [CrossRef]

78. Knutson, G.A. Anatomic and functional leg-length inequality: A review and recommendation for clinical decision-making. Part I, anatomic leg-length inequality: Prevalence, magnitude, effects and clinical significance. Chiropr. Osteopat. 2005, 13, 11. [CrossRef] [PubMed]

79. Schauer, M.; Mauritz, K.-H. Musical motor feedback (MMF) in walking hemiparetic stroke patients: Randomized trials of gait improvement. Clin. Rehabilit. 2003, 17, 713-722. [CrossRef] [PubMed]

80. Chou, S.-W.; Wong, A.M.; Leong, C.-P.; Hong, W.-S.; Tang, F.-T.; Lin, T.-H. Postural control during sit-to stand and gait in stroke patients. Am. J. Phys. Med. Rehabilit. 2003, 82, 42-47. [CrossRef]

81. Lin, P.-Y.; Yang, Y.-R.; Cheng, S.-J.; Wang, R.-Y. The relation between ankle impairments and gait velocity and symmetry in people with stroke. Arch. Phys. Med. Rehabilit. 2006, 87, 562-568. [CrossRef] [PubMed]

82. Robinson, R.; Herzog, W.; Nigg, B. Use of force platform variables to quantify the effects of chiropractic manipulation on gait symmetry. J. Manip. Physiol. Ther. 1987, 10, 172-176.

83. Plotnik, M.; Giladi, N.; Hausdorff, J.M. A new measure for quantifying the bilateral coordination of human gait: Effects of aging and Parkinson's disease. Exp. Brain Res. 2007, 181, 561-570. [CrossRef] [PubMed]

84. Zifchock, R.A.; Davis, I.; Higginson, J.; Royer, T. The symmetry angle: A novel, robust method of quantifying asymmetry. Gait Posture 2008, 27, 622-627. [CrossRef] [PubMed]

85. Archer, K.R.; Castillo, R.C.; MacKenzie, E.J.; Bosse, M.J. Gait symmetry and walking speed analysis following lower-extremity trauma. Phys. Ther. 2006, 86, 1630. [CrossRef] [PubMed]

86. Herzog, W.; Nigg, B.M.; Read, L.J.; Olsson, E. Asymmetries in ground reaction force patterns in normal human gait. Med. Sci. Sports Exerc. 1989, 21, 110-114. [CrossRef] [PubMed]

87. Leddy, A.L.; Crowner, B.E.; Earhart, G.M. Functional gait assessment and balance evaluation system test: Reliability, validity, sensitivity, and specificity for identifying individuals with Parkinson disease who fall. Phys. Ther. 2011, 91, 102. [CrossRef] [PubMed]

88. Shapiro, S.S.; Wilk, M.B. An analysis of variance test for normality (complete samples). Biometrika 1965, 52, 591-611. [CrossRef]

89. Woolson, R.F. Wilcoxon signed-rank test. In Wiley Encyclopedia of Clinical Trials; John Wiley \& Sons, Inc.: Hoboken, NJ, USA, 2007.

90. Corp, I. IBM Spss Statistics for Windows, 24; IBM Corp: Armonk, NY, USA, 2013

91. Mohler, B.J.; Thompson, W.B.; Creem-Regehr, S.H.; Pick, H.L.; Warren, W.H. Visual flow influences gait transition speed and preferred walking speed. Exp. Brain Res. 2007, 181, 221-228. [CrossRef] [PubMed]

92. Qualisys Motion Capture. Available online: https://www.Qualisys.Com/ (accessed on 4 August 2017).

93. Warren, S.F.; Wall, J.H.; Bevly, D.M. Characterization of Various Imu Error Sources and the Effect on Navigation Performance. In Proceedings of the 18th International Technical Meeting of the Satellite Division of The Institute of Navigation (ION GNSS 2005), Long Beach Convention Center, Long Beach, CA, USA, 13-16 September 2005. 
94. Yang, S.; Laudanski, A.; Li, Q. Inertial sensors in estimating walking speed and inclination: An evaluation of sensor error models. Med. Biol. Eng. Comput. 2012, 50, 383-393. [CrossRef] [PubMed]

95. Welch, G.; Foxlin, E. Motion tracking: No silver bullet, but a respectable arsenal. IEEE Comput. Graph. Appl. 2002, 22, 24-38. [CrossRef]

96. Weisstein, E.W. Newton-cotes formulas. MathWorld. Available online: http://mathworld.wolfram.com/ Newton-CotesFormulas.html2004 (accessed on 8 April 2017).

97. Brach, J.S.; VanSwearingen, J.M. Interventions to improve walking in older adults. Curr. Transl. Geriatr. Exp. Gerontol. Rep. 2013, 2. [CrossRef] [PubMed]

(C) 2018 by the authors. Licensee MDPI, Basel, Switzerland. This article is an open access article distributed under the terms and conditions of the Creative Commons Attribution (CC BY) license (http:/ / creativecommons.org/licenses/by/4.0/). 
Article

\title{
An Affordable Insole-Sensor-Based Trans-Femoral Prosthesis for Normal Gait
}

\author{
Srinivas Pandit ${ }^{1}$, Anoop Kant Godiyal ${ }^{1}$, Amit Kumar Vimal ${ }^{1}$, Upinderpal Singh ${ }^{2}$, \\ Deepak Joshi ${ }^{1,3, *}$ and Dinesh Kalyanasundaram ${ }^{1,3}$ \\ 1 Centre for Biomedical Engineering, Indian Institute of Technology Delhi, New Delhi 110016, India; \\ ird11831@cbme.iitd.ac.in (S.P.); bmz138038@cbme.iitd.ac.in (A.K.G.); bmz128466@cbme.iitd.ac.in (A.K.V.); \\ dineshk.iitdelhi@gmail.com (D.K.) \\ 2 Department of Physical Medicine and Rehabilitation, All India Institute of Medical Sciences, \\ New Delhi 110029, India; usingh@aiims.ac.in \\ 3 Department of Biomedical Engineering, All India Institute of Medical Sciences, New Delhi 110029, India \\ * Correspondence: joshideepak2004@gmail.com; Tel.: +91-11-2659-1155
}

Received: 18 January 2018; Accepted: 19 February 2018; Published: 27 February 2018

\begin{abstract}
This paper proposes a novel and an affordable lower limb prosthesis to enable normal gait kinematics for trans-femoral amputees. The paper details the design of a passive prosthesis with magneto-rheological (MR) damping system and electronic control. A new control approach based on plantar insole feedback was employed here. Strategically placed sensors on the plantar insole provide required information about gait cycle to a finite state controller for suitable action. A proportional integral (PI) based current controller controls the required current for necessary damping during gait. The prosthesis was designed and developed locally in India keeping in view the cost, functionality, socio-economic, and aesthetic requirements. The prototype was experimentally tested on a trans-femoral amputee and the results are presented in this work. The implementation of the proposed design and control scheme in the prototype successfully realizes the notion that normal gait kinematics can be achieved at a low cost comparable to passive prostheses. The incurring cost and power expenditure of the proposed prosthesis are evaluated against passive and active prostheses, respectively. The commercial implications for the prosthesis were explored on the basis of recommendations of ISPO Consensus Conference on Appropriate Prosthetic Technology in Developing Countries. The key objective of this work is to enable lucid design for development of an affordable prosthesis in a low-resource setting.
\end{abstract}

Keywords: lower limb prosthesis; trans-femoral amputee; MR damper; knee damping control

\section{Introduction}

About 30 million amputees are residing in low income countries [1,2]. Among those, only $5 \%$ to $15 \%$ are capable to receive a much-needed prosthesis [3]. Although the popular state-of-the art prostheses used in developed countries offer several advanced features, its application is hindered in low income developing countries primarily due to high cost, different functional demands, cultural issues, and unavailability of components locally $[4,5]$.

Gait parameters of trans-femoral amputees including step length, inter-leg symmetry, hip exertion, and involvement of upper body are highly affected [6]. Over the years, there have been considerable efforts to develop affordable prostheses for trans-femoral amputees in developing countries [6-9]. These efforts have resulted in a variety of lower limb prosthetic designs at reduced cost. However, these prosthetic designs include low-end technologies like manual locking knee joints, the weight activated braking mechanism, and polycentric knee joint mechanisms [2,6,10-12]. In manual-locking-knee joints, knee stability is improved by providing more stiffness which renders lower speed and increased 
energy expenditure during gait [10]. Problems associated with stiff-legged gait were overcome by incorporating weight activated brakes with a knee joint. A typical weight activated arrangement suffers from limitations of abnormal gait and delayed initiation of swing phase [10]. Polycentric knee joint mechanisms were quite useful in developed countries and now, with some modifications, are being developed to suit the needs of low-income countries. The polycentric knee joint linkages can be configured to improve the stability in the stance phase with fair swing phase initiation. It does impose a limitation. As the extent of stability is increased in the stance phase, the swing phase initiation gets delayed proportionally $[8,13]$. In this regard, some recent prostheses such as Le Torneau polycentric knee (Limbs International, El Paso, TX, USA), Stanford-Jaipur leg (Stanford University, Stanford, CA, USA), Remotion Knee (D-Rev), SASPL knee joint LCKnee (Andrysek et al.) and a three axes knee (Arelekatti et al.) at MIT [2,10-12] have been developed.

Le Torneau knee and Stanford-Jaipur knee joints have a similar polycentric design of four-bar linkage with little to offer other than stance phase stability. On the other hand, Remotion Knee is the advanced version of Stanford-Jaipur knee with curvature mimicking to address late stance phase initiation $[2,10]$. Studies have revealed that such prosthetic designs though provide a low-cost solution to a grieving population, it does not take into account the need for an early stance flexion-extension and properly timed late stance flexion $[11,12]$. The basic biomechanical aspects, when overlooked, can result in many psychological, physiological, and socio-economic problems [14]. Abnormal and sub-normative gait puts immediate and extended costs in terms of psychological stigma and socio-economic distress, whereas in the longer term it results in musculoskeletal impairments and adversely affects the physiological equilibrium [15-18]. It suggests that the excessive focus on cost reduction alone cannot serve the desired purpose and low-cost prostheses must also have the ability to provide a gait kinematics closer to that of a healthy person.

Also, Andrysek et al. and Arelekatti et al. addressed the problem of abnormal gait in low-cost passive prostheses. Andrysek et al. SASPL engages or disengages depending on the loading of the prosthetic limb during weight bearing but is not able to provide early stance flexion-extension and optimal swing phase damping [11]. Arelekatti et al. incorporated able-bodied gait kinematics using an automatic early stance lock for stability, a linear spring for early stance flexion-extension and swing control. The design suffers from few limitations such as the necessity of full knee extension at the end of swing phase and failure to lock the knee before stance phase that can lead to instability and risk of fall. Further, due to the lack of any clinical gait analysis, the study does not establish the extent of improvement in amputee kinematics towards normative gait [12]. It offers sufficient motivation to look for a new prosthesis design and control scheme that can enable able-bodied gait kinematics in trans-femoral amputees at an affordable cost in developing countries. So far, all developed prostheses designed for developing countries are primarily passive in nature, the authors believe that a variable damping can provide the subject with comfortable walking, thereby yielding the desired results at a low cost.

In this paper, a novel low cost lower limb prosthesis was developed and its clinical testing and analysis are presented. A new foot plantar insole feedback-based control strategy was developed and implemented to provide necessary damping using an MR damper. The prosthesis enables the amputee to realize the able-bodied normal gait kinematics and meets the functional, socio-economic, and aesthetic needs of a trans-femoral amputee and is affordable even in low-income economies. The paper also focuses on essential design features and requirements of a prosthesis to assess its commercial viability in these countries such as low cost, light-weight, functionality, biomechanically appropriate, durable, using locally available materials etc., based on the recommendations of ISPO Consensus Conference on Appropriate Prosthetic Technology in Developing Countries and elsewhere [19-22]. The details are discussed later in Section 5.7. The key objective of this work is to enable lucid design for development of an affordable prosthesis in low-income economies. 


\section{Prosthesis Design}

This rationale of the prosthesis design is presented in this section. The trans-femoral prosthesis shall serve the major function of providing stability during stance phase and controlled flex-extension during the swing phase of a gait cycle. In the stance phase, the stability was ensured by providing suitable resistance to knee joint flexion while the user transfers body weight from the sound/healthy limb to the prosthetic limb. Similarly, the swing phase flex-extension was controlled by regulating walking speed [22]. Both the functions were achieved by including springs and dampers in the design [3]. Either a variable damping system or a system with multiple dampers is required for a variable damping system incorporated in state of art active prostheses, whereas multiple dampers are used in recent advanced passive prostheses. The multiple-damper approach inherently puts the design at disadvantage in terms of serviceability $[10,11]$.

A study conducted by Herr and Wilkenfeld [23] serves as the theoretical background for our design to realize able-bodied gait kinematics. The authors used an MR knee prosthesis to adapt knee damping using local sensing of knee force, torque, and position. A few recent studies conducted by $\mathrm{Xu}$ et al. [24], Park et al. [25], and Fu et al. [26] have also attempted to design a lower limb prosthesis based on an MR damper. Complex sensing, data-driven AI control and lack of local climatic adaptations as well as increased cost of the prosthesis arising from various factors ranging from non-inclusion of off-the-shelf components or locally manufactured components increased the inherent cost, making it unsuitable for applications in low-income economies. Further, the control architecture based on stiff tracking of the knee angle should be avoided in order to enable the amputee to use the prosthesis more interactively, rather than reacting to it [24]. Therefore, the potential of prostheses based on MR damper remain untapped in such low-income economies due to the unavailability, complexity, and incurring cost of design and manufacturing [20,23,27].

It is proposed that a single damper with a controllable damping capacity, such as an $\mathrm{MR}$ damper, if used with suitably designed sensing modality, driving circuitry, and control architecture, can provide able-bodied gait kinematics yet at a cost below par with advanced passive prostheses. Here, strategically placed sensors on the plantar insole provide sufficient information for normal gait kinematics, thereby enabling the control of the current in the MR damper during the different phases of a gait cycle. This forms the basis for the design of a simple MR damper-based knee joint mechanism and its control with plantar insole feedback.

\subsection{Mechanical Design}

The design of the prosthesis' mechanical structure is shown in Figure 1. The design includes an MR damper, a hinge-type knee joint, and braces to support the leg assembly. In the MR damper, modulation of magnetic field strength can offer variable resistance to the flow of MR fluid. Placement of electromagnets around the piston rod and MR fluid as a working medium inside a hydraulic cylinder can result in a variable damping $[20,23,27]$. Damping can be modulated by changing the current flowing in the coils of the electromagnet. A variety of MR dampers are available to suit lower limb prostheses. In this study, a commercially available MR damper (Make: Lord Corporation, Cary, NC, USA; Model RD-8041 with a stroke length of $74 \mathrm{~mm}$, and extended length of $248 \mathrm{~mm}$ ) was employed in the prototype.

The piston rod and outer cylinder of the MR damper were fixed to the knee joint and the shank individually with swing link and lower-link rods. The upper-link and lower-link rods are attached to the braces. The upper link rod and swing-link rod form the knee axis and the control axis of the prosthesis, respectively; the upper link rod along with knee body articulates the knee braces via the knee axis. The control axis primarily responds to the ground reaction forces that cause relative motion between the knee and the braces. The swing-link rod transfers the forces generated by the MR damper during locomotion. The biomechanical mechanism of the control axis has been extensively reported elsewhere [26]. The control of the prosthesis in both the stance and swing phases was achieved by the same mechanical assembly and no separate mechanical component was used to lock the knee. 
The upper part of the knee joint (knee body) was connected to a socket connector to fasten the socket properly. The braces along with MR damper form the shank of the leg. It was connected to a Sach foot with a shank connector. The socket and shank connectors are connected to the knee and ankle adapters used by prosthetists for proper alignment of the prosthesis to the individual amputee. Knee Movement has been restricted to 90 degrees with protective bars (stoppers) placed in the forward direction of the damper. Custom designed components were used for link rods, plates, braces, and knee joint to meet the design specifications. Proper motion of the knee joint was ensured by simulating the solid model design in Solid Works ${ }^{\circledR}$. During the simulation along the entire knee range, there was no interference between the joint links, bars, plates, and braces. With proper machining of all the custom components, the mechanism was assembled by bolting the braces to the knee joint. The MR damper was shoulder-bolted with the links of the assembly. Bearings were employed for smooth motion of joint between knee body plates and link rods. Off-the-shelf components such as connectors, adaptors, and the Sach foot were employed to extend the design with existing prosthetic components.

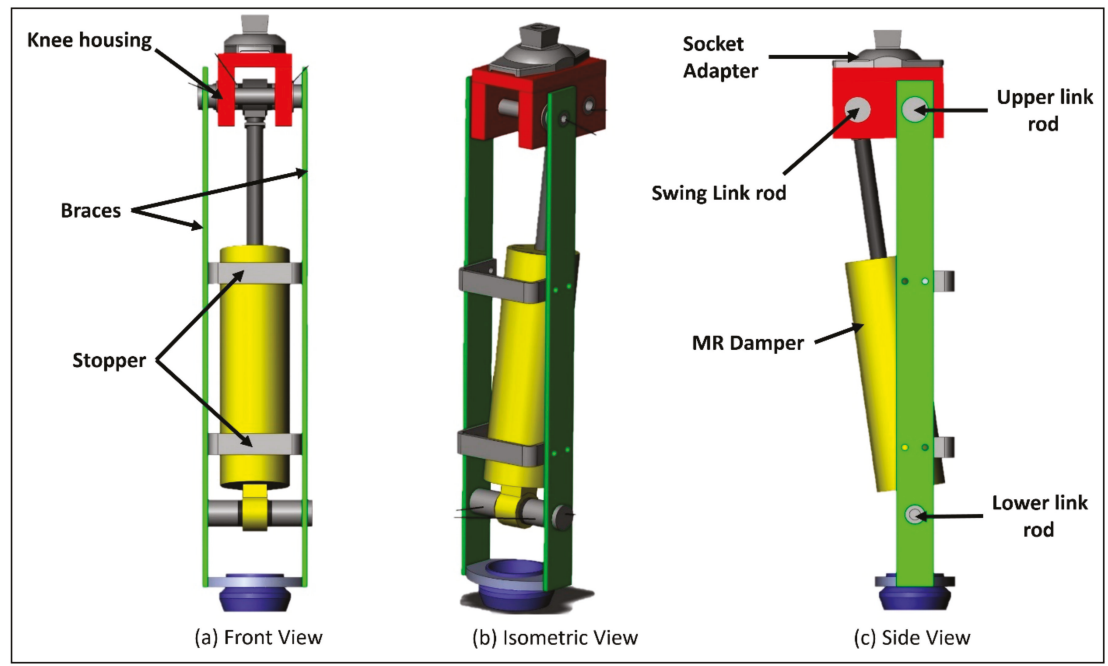

Figure 1. Three-dimensional structure and mechanical design of the proposed prosthesis.

\subsection{Sensing Mechanism}

Plantar insoles are extensively used for gait analysis of many locomotion impairments [28,29]. Although plantar insoles provide information during only the stance phase, it is sufficient for most of the rehabilitation support systems, posture and balance control [28]. Most of the plantar insoles employ a discrete number of sensors to obtain the required information; the minimum number of sensors vary from 2 to 16, or more depending upon the need [28]. Taking into account the advantages of foot plantar insoles, a novel sensing method was developed to control the prosthesis both in the stance and swing phases. It is composed of 24 switches strategically placed on the heel (S1), mid-foot (S2), metatarsal (S3), and toe (S4) of the plantar insole as shown in Figure 2. The sensor placement was determined by considering dominant pressure points of the plantar surface during walking as reported by Razak et al. [28]. The rationale for considering sensors on plantar insole lies in the wheel-like-behavior of plantar foot and its subsequently resulting roll-over shapes (ROS) [30,31]. Such ROSs are formed for various biomechanical events corresponding to different phases of a gait cycle of human locomotion i.e., loading response, mid-stance, terminal stance, pre-swing, and swing [32]. The progression of a typical gait cycle of a healthy individual along with subsequent phases and events are depicted in Figure 3. As the five segments are sequential in nature, the phases are distinctly noticed 
due to the formation of ROSs based on the combination of states of four groups or sensors placed on the foot plantar insole. The grouping of the 24 sensors into 4 groups is shown in Figure $2 \mathrm{~b}$. The stance phase events can be related to different combinations of the states of four sensor groups as tabulated in Table 1. If the state of any of the sensors in a group is high, then the state of the same group is assigned the value of 1 .

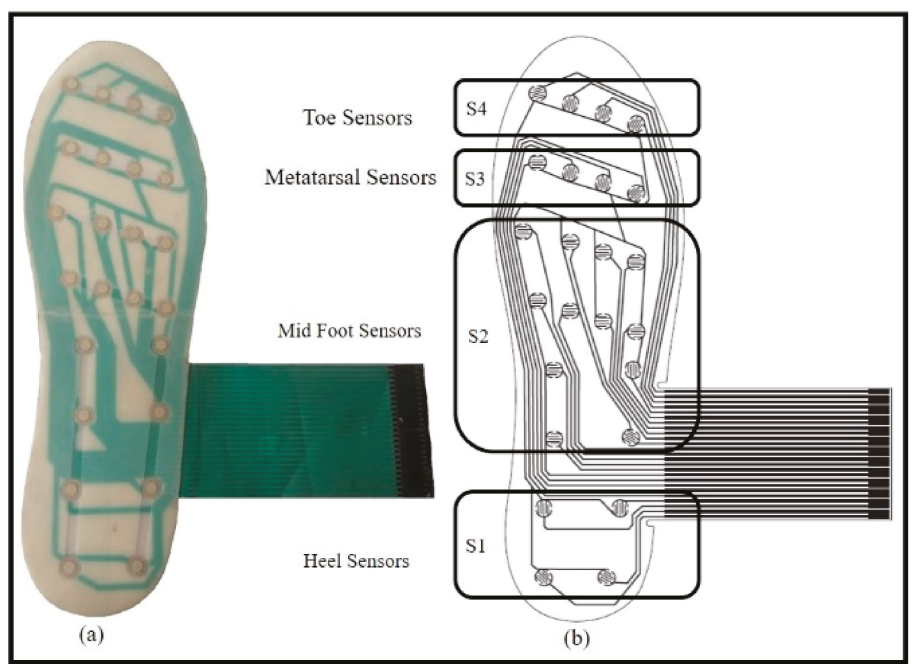

Figure 2. (a) Photograph of plantar insole and (b) sensor grouping.

The circuitry and mechanical dimensions of the insole were designed using Coral Draw ${ }^{\circledR}$. The insole was printed using the silk screen printing process (Keywell Industrial Co., Noida, UP, India). In this process, silver-conducting ink and dielectric was used to print the circuit. While designing the insole, it was important to determine the minimum thickness of the dielectric layer and the amount of pressure that the insole may undergo during walking. It enables the designer to use an optimum thickness of the dielectric layer that is sufficient to trigger the events. For the initial prototype, this trade-off between minimum dielectric layer thickness and maximum loading was made using the ground reaction force (GRF) profile of the amputee on a force plate.

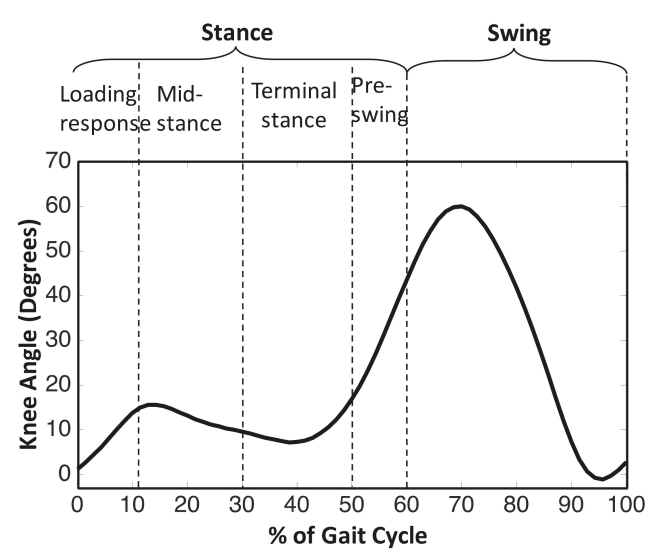

Figure 3. Typical gait cycle of a healthy person [33] and its classification [30]. 
Table 1. Sensor states during different phases of gait.

\begin{tabular}{ccccc}
\hline Gait Segment & S1 & S2 & S3 & S4 \\
\hline Loading Response & 1 & 0 & 0 & 0 \\
\hline Mid-Stance & 1 & 1 & 0 & 0 \\
\hline \multirow{3}{*}{ Terminal-Stance } & 0 & 1 & 0 & 0 \\
& 0 & 1 & 1 & 0 \\
Pre-Swing & 0 & 0 & 1 & 1 \\
\hline Swing & 0 & 0 & 0 & 1 \\
\hline
\end{tabular}

\subsection{Embedded System}

To feed the desired current to the MR damper at the given time instant, an electronic circuit that uses sensor readings was designed to regulate the current. The MR damper requires variable current to provide variable damping; the MR damper current can be varied by varying the input DC voltage across the load. It was achieved by a step-down DC/DC converter, controlled by a pulse width modulation (PWM) scheme. The duty cycle of the PWM signal was varied to reduce the error in the reference and actual feedback current. Error in reference and feedback current can be reduced to a permissible extent in desirable time by employing a proportional integral (PI) controller. The actual feedback current was measured with a Hall current sensor ACS712. The power circuit includes a battery bank rated at $9 \mathrm{~V}$ and $2.4 \mathrm{Ah}$, a power MOSFET, freewheeling diode, low pass LC filter, and MR damper.

The power and control circuits were isolated with an optocoupler and gate-driving circuit to ensure smooth operation. The control circuit includes the sensing mechanism along with a control unit as shown in Figure 4. A microcontroller is the central component of the control unit, that in-turn is the brain of the total signal processing system. The primary objective of the microcontroller is to control the power driving unit when required to change the damping. After careful analysis of the requirements, the Atmel mega (ATmega) 329P microcontroller was chosen for this work for its low cost, versatile characteristics, and more advanced features. The necessary processing of sensor signals and implementation of control algorithm was achieved with the firmware. The details of the components of the embedded system are given in Table 2 .

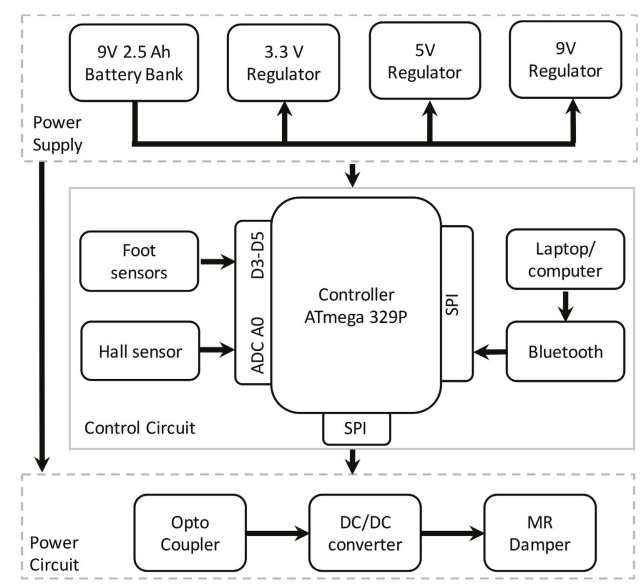

Figure 4. Embedded system layout. 
Table 2. Details of the embedded system components.

\begin{tabular}{cc}
\hline Components & Rating \\
\hline MOSFET IRF 540N & $100 \mathrm{~V}, 33 \mathrm{~A}$ \\
Diode SR 360 & $60 \mathrm{~V}, 3 \mathrm{~A}$ \\
Hall current sensor ACS 712 & $230 \mathrm{~V}, 5 \mathrm{~A}$ \\
Opto-coupler MCT2E 4050 & $3 \mathrm{~V}, 50 \mathrm{~mA}$ \\
MR damper RD 8041 & $12 \mathrm{~V}, 1 \mathrm{~A}$ \\
Filter capacitor & $50 \mathrm{~V}, 100 \mu \mathrm{F}$ \\
Filter inductor & $1 \mathrm{~A}, 33 \mu \mathrm{H}$ \\
\hline
\end{tabular}

\subsection{Control Architecture}

A two-level control scheme was employed to achieve able-bodied gait kinematics as depicted in Figure 5. The control approach involves a finite state controller as a secondary controller and a conventional PI controller as a primary controller. The secondary controller generates the required current references for the MR damper using a finite state machine that ultimately modulates the impedance of the gait, depending on the gait event segment. The primary controller is a closed loop PI controller for MR damper current, which compensates for the load transfer dynamics, thus enabling the faithful tracking of current references with a higher bandwidth and accuracy as compared to its open loop counterparts. Prosthetic control is different from robotic control where the oscillatory response was avoided and the system was tasked to follow a stiff trajectory. PD, P, PID, sliding mode, and hysteresis controllers are inherently suitable for stiff tracking, whereas a PI controller offers a more suitable response. Furthermore, a PI controller is the most widely used current controller in industrial applications, and its incorporation would attract more commercial acceptability.

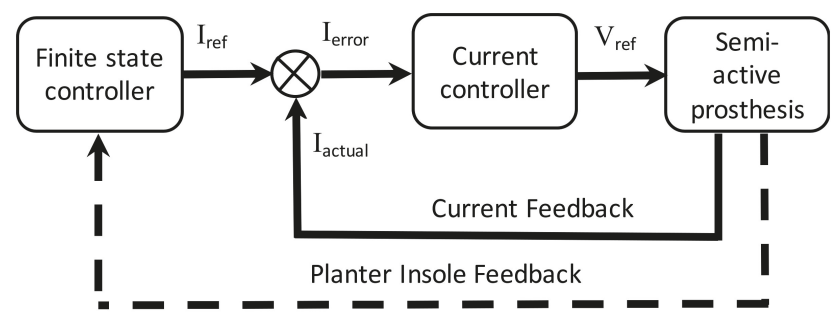

Figure 5. Block diagram of the control scheme.

This ordered behavior of state machines are utilized in neuro-prostheses with event triggered control [34,35]. The states of the finite state machine were determined by considering various demanded requirements [36]; here we intend to achieve the flexion-extension of the knee during the stance phase with a high degree of stability and a suitably damped swing phase to adjust the walking speed. In order to meet the stated requirements, a gait cycle with five segments/phases as described earlier were considered i.e., loading response, mid-stance, terminal stance, pre-swing, and swing. The above states are illustrated in Figure 6. Though there could be any number of states that can be used to form a state machine, as previous studies have reported, the introduction of a foot plantar insole with four sensor groups provides a total of 16 states, out of which 15 belong to stance phase and one to swing phase. Due to the ROS trajectory during level walking, only 7 states of stance can be assigned to four phases as tabulated in Table 1, whereas the remaining 8 states of stance do not represent any of the ROS segments. The available literature suggests that consideration of 4 states have successfully resulted in desired kinematics in the stance phase [27], whereas treatment of swing phase as a single state does not deviate much from the same [37]. 
In state 0 , the knee flexes near to the maximum stance flexion. A relatively high damping was applied during this state to prevent buckling at the knee due to the user's weight. During state 1, the knee begins to extend after maximum flexion. The rate of extension was lower than that of flexion during state 0 , thereby requiring a high damping. The level of damping shall account for slow extension in order to avoid potential slamming of rotating parts to the knee housing and stoppers. State 2 involves the flexion-extension while the heel is off the ground and the sound limb is sharing the body weight, a moderate damping shall serve the purpose here. State 3 encounters the extension with a high rate of change and low body weight bearing; a low damping will provide the desired profile. The final state (state 4) represents a large knee flexion and associated extension after flexing to its maximum. The rate of change of flexion-extension suggests a very small damping for the same. All states can be tuned to suitable current references which can be accurately followed by a well-designed PI controller. The response of the PI controller must be fast and accurate as it lies in the inner loop of the control architecture.

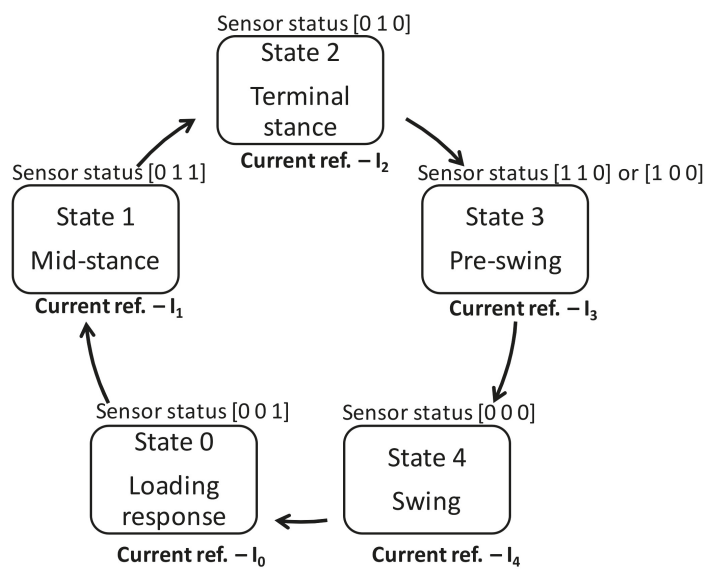

Figure 6. Finite state machine diagram.

\section{Methods}

The developed prosthesis was tested on a 24-year-old male $(1.63 \mathrm{~m}, 60 \mathrm{~kg})$ unilateral trans-femoral amputee in India as part of the target population. The subject was using a commercially available passive prosthesis (single axis extension assisting prosthetic knee joint) for the last three years post traumatic amputation. The amputee mobility predictor (AMP) score of the amputee was more than 35 , i.e., above the $\mathrm{K} 3$ activity level. Table 3 shows the measurement of amputee and component used in the proposed prosthesis. A custom made Ischial containment socket was designed for the amputee by a licensed professional prosthetist. The prosthesis was assembled according to the measurements of the amputee.

Table 3. Details of the amputation and prosthesis.

\begin{tabular}{cc}
\hline Stump Length & $22 \mathrm{~cm}$ \\
\hline $\begin{array}{c}\text { knee axis w.r.t. } \\
\text { Ischial bone }\end{array}$ & $34 \mathrm{~cm}$ \\
\hline Knee axis to floor & $47 \mathrm{~cm}$ \\
\hline Socket design & Ischial containment socket \\
\hline Prosthetic foot & SACH foot \\
\hline
\end{tabular}


The dynamic alignment of the prosthesis was performed by the trained prosthetist who is one of the co-authors of the current work. The participant did not have any musculoskeletal injuries, sensory or neurological impairment or related disabilities other than trans-femoral amputation. The study was approved by the All India Institute of Medical Sciences ethics committee (Ref. No. IEC-35/09.02.17). The participant was explained the experiment protocols before starting the experiment and written consent was obtained.

\subsection{Parameter Tuning and Training}

The controller parameters were tuned sequentially considering one controller at a time. Firstly, the innermost current controller was designed for the electrical time constant of the MR damper, so that a maximum current of $1 \mathrm{~A}$ could be attained with a desired speed and accuracy. The gain parameters were tuned separately, and the speed was optimized after attaining a fair accuracy. It was achieved by the Ziegler-Nicholas method of tuning the PI controller. After tuning of the PI controller, i.e., the current controller, the prosthesis was fitted on the subject for familiarization. When the subject was able to walk properly with certain level of comfort, the prosthesis was then considered ready to be tuned for the secondary level of control. As stated in Section 2.2, a gait cycle was segmented into 5 states (shown in Figure 7), and for each state one current reference was to be set, thereby a total of 5 parameters of the finite state controller needed to be tuned. Figure 7e,f depict the same damping as shown in Figure 8, however, for the sake of visualization, they are shown separately. It was usually straight-forward to make an initial guess of only 5 parameters. However, to avoid unnecessary discomfort to the subject, the parameters were tuned sequentially in each state depending upon the feedback from the subject and the prosthetist.

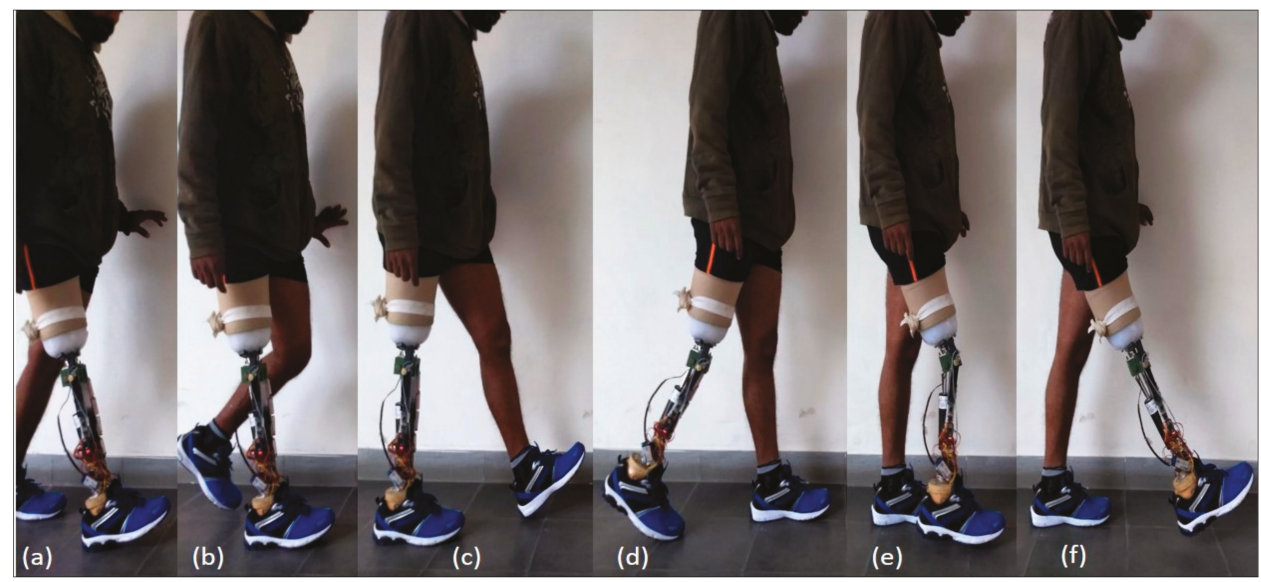

Figure 7. Photograph of subject with developed prosthesis during training in segment (a) loading response, (b) mid-stance, (c) terminal-stance, (d) pre-swing, (e) extended knee in swing, and (f) fully flexed knee in swing.

After obtaining an initial set of parameters, all the values were iteratively fine-tuned to attain a gait characteristic closer to that of a healthy individual. The knee angle was used as an essential quantitative measure to observe the performance. Subject feedback along with that of the trained prosthetist was considered primary qualitative criteria to tune the parameters. This type of tuning avoids unnecessary discomfort to the subject. The final set of current references are depicted in Figure 8. 


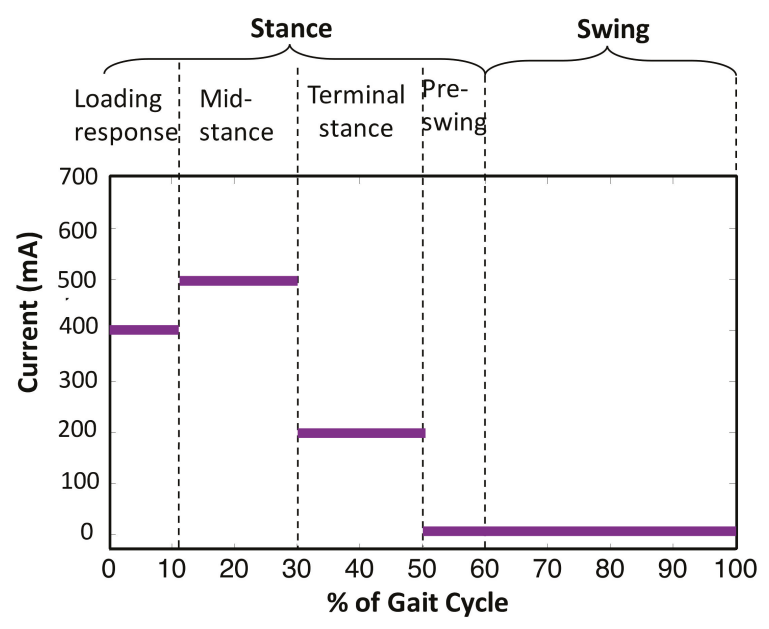

Figure 8. Final set of current references after tuning.

Once the prosthesis was tuned for level walking, the subject was given sufficient training time to get proper acclimatization to it. A number of training sessions were conducted for the subject in accordance with the training methods reported in several studies [38-41]. The training was usually specific to each subject, thus the sessions were planned by a trained prosthetist keeping in view that the subject had been using a passive prosthesis for a long duration. When the subject actively started to walk on level ground, the experimental data was collected for analysis.

\subsection{Experimental Setup}

The subject was asked to walk on a $24.38 \mathrm{~m}$ ( 80 feet)-long corridor at a uniform subject comfortable speed. The initial walk of $3.05 \mathrm{~m}$ (10 feet) and $3.05 \mathrm{~m}$ of walk towards the end were excluded to avoid any deviation in biomechanical data that may arise due to acceleration and deceleration during beginning and ending, respectively. Hence the walking variables were measured over an active length of corridor of $18.29 \mathrm{~m}$ (60 feet) which was assumed to be uniform.

For the subject (fitted with the prosthesis as shown in Figure 9), a total of 10 trials were conducted; one trial included initial standing of $10 \mathrm{~s}$ and then level walking of $24.38 \mathrm{~m}$ ( 80 feet) followed by standing for $10 \mathrm{~s}$. The starting, stopping, and other necessary instructions were provided verbally to the subject. After each trial, the subject was asked to take a minimum of 2 min rest which could be increased based on subject's requirement. In order to evaluate the performance of the developed prosthesis, the data obtained from the knee goniometer, foot plantar insole, on-board clock, and the voltage and current sensors were transmitted wirelessly with a Bluetooth module to a remote desktop.

A freeware serial port terminal application (CoolTerm, version 1.4.7) was used to record the data with a sampling frequency of $100 \mathrm{~Hz}$. The data was filtered in MATLAB ${ }^{\circledR} \mathrm{R} 2015 \mathrm{~b}$ using a low pass Butterworth filter of 4 th order with a cut-off frequency of $10 \mathrm{~Hz}$. The cut-off frequency of $10 \mathrm{~Hz}$ was preferred to filter the data as the frequency of human motion was typically less than $5 \mathrm{~Hz}$. The signal was further smoothened using a 5-point moving average filter. 


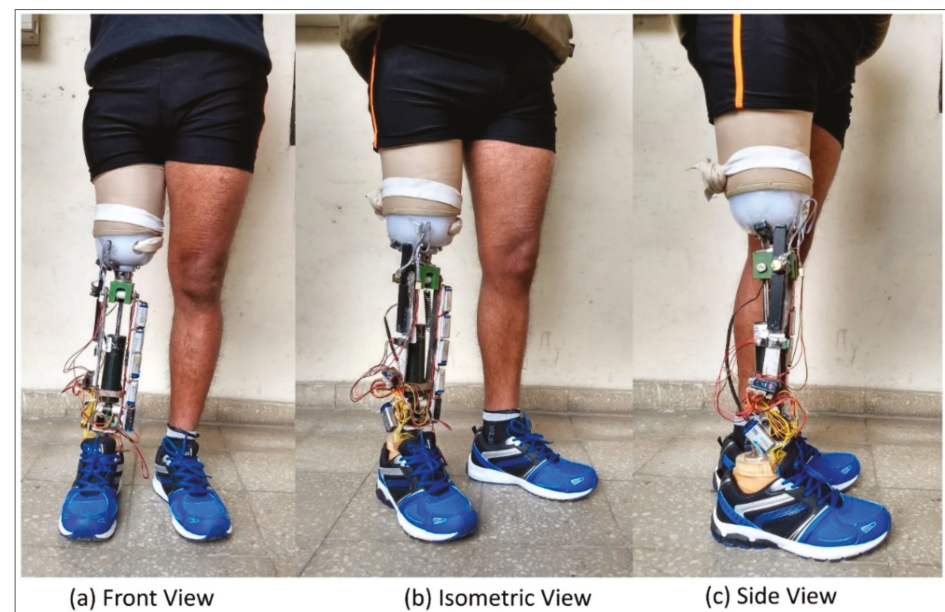

Figure 9. Photograph of experimental setup and subject during testing of prosthesis.

\section{Results}

\subsection{Gait Kinematics}

Knee joint angle is the most established parameter to evaluate the kinematic performance of a prosthesis [37]. The measured knee angle from the on-board goniometer during level walk is shown in Figure 10. The prosthesis was able to demonstrate an early stance phase knee flexion-extension; at a self-selected walking speed of a gait cycle, the knee joint in early stance flexed up to $15 \pm 5$ degrees. The swing phase flexion was constrained up to $45 \pm 7$ degrees, which is below the biologically acceptable limit of 70 degrees. Combining both, the prosthesis realizes a knee angle trajectory that is closer to that of able-bodied or normal gait kinematics.

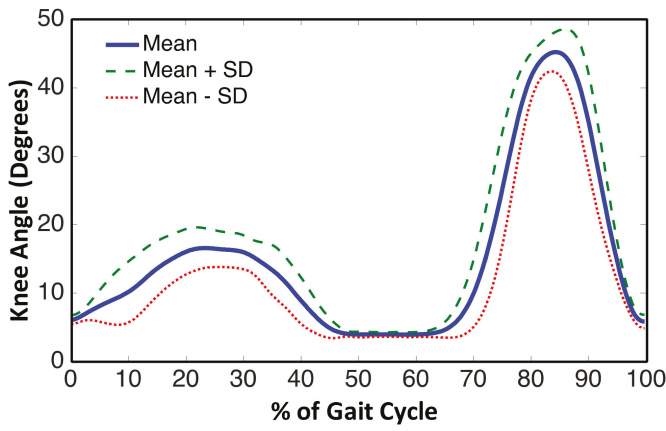

Figure 10. Gait kinematics measured during experiment.

\subsection{Power Consumption}

External power requirement is the major concern for both active or semi-active prostheses. To evaluate the performance of the prosthesis, the assessment of power consumption was carried out by measuring the average current through the battery. In this study, the average electrical power provided by battery bank and the consumption by MR dampers were measured by Hall Effect current sensors (ACS712). The input electrical power consumed in a gait cycle can be evaluated from Figure 11 
by considering supply voltage of $9 \mathrm{~V}$. In-line with the expectation, the peak power was perceived during mid-stance as the prosthesis supports entire body weight at this time. The power consumption during loading response was less than the peak power due to double support. In terminal stance, the body transfers the load to the sound limb. Hence, a moderate power was consumed during this phase. During the swing and pre-swing phases, the prosthesis consumes very little or almost no power due to very little load of prosthesis itself. An average power of $2.25 \mathrm{~W}$ was consumed by the MR damper during a typical gait cycle of over-ground level walking.

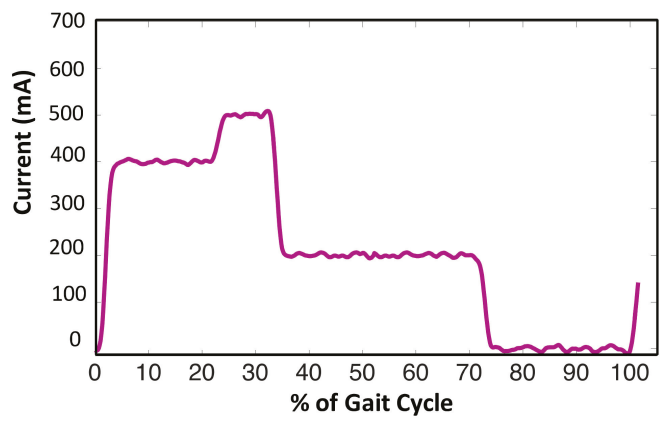

Figure 11. Actual current flowing in the MR damper during a typical gait cycle.

\section{Discussion}

\subsection{Gait Biomechanics}

The response of developed prosthesis is seen in Figure 10. It can be noticed that the gait kinematics are more similar to that of a healthy person performing a level walk. On comparison of measured knee angle to a typical gait data [29] (as shown in Figure 3), the response is quite similar to that of normal gait. Contrary to its passive counterparts, it enables the amputee to achieve knee flexion-extension during heel strike and mid stance with the required degree of stability. Such an extent of stance flexion and extension is absent in the typical gait cycle of an amputee using any passive prosthesis [38-40]. Controlled swing phase knee flexion-extension is also achieved; thus meeting the two necessary requirements of a prosthesis to provide able-bodied gait kinematics [35]. Though the kinematic data of healthy individuals and amputees can be compared, one should avoid trying to find a precise match between the two. The healthy gait data serves as a reference for essential characteristics only. The results presented in this study can best compared with that in the study conducted by Herr and Wilkenfeld on four unilateral transfemoral amputees using MR damper knee joints [23]. The knee joint angle of the proposed prosthesis exhibits all the characteristics similar to that of the knee angle trajectory obtained in [23].

The lack of smooth stance-to-swing transition is a major problem witnessed in many of the previous prostheses [11,37]. This problem is usually associated with hyper-stabilizing stance phase knee joints, such as polycentric knee joints, causing delayed initiation of swing phase. The developed prosthesis, due to its single axis design and control strategy, effectively overcomes the stance to swing transition problems. The design and control of the prosthesis also eliminates the necessity of full knee extension at the end of the swing phase as observed by Arelekatti et al. [16].

\subsection{Control with Plantar Insole}

The control of a semi-active or active prosthesis is usually achieved by highly sophisticated methods including complex sensing, data driven advanced AI, and state of the art embedded systems $[23,26]$. All these methods require computationally efficient software and hardware platforms associated with delicate peripherals and sophisticated electronic circuits, thereby making it a costly 
affair for low-income economies despite its huge potential to realize normal gait kinematics. The novel control method introduced in this study provides a low cost, effective, and efficient alternative to existing prostheses. The simple form of digital information requires almost no processing and does away with the unnecessary delay caused by Analog to Digital Convertors. Hence, it enables the designer to select a simple, low cost and low power microcontroller, a major component contributing to higher cost.

The study establishes that a foot plantar insole possesses significant information required to control the prosthesis. The basic principle of extraction of information from a plantar insole lies in ROS created by the wheel-like mechanism of the foot during locomotion, as also reported earlier [30,31]. Different ROSs are caused by different kinematic patterns. However, the kinematic data is also subject dependent but has well defined characteristic trajectory. The patterns usually vary with age, type of locomotion, and kind of locomotion impairment. This gives the opportunity to extend to other fields, such as orthoses for lower limbs. The electronically controlled prostheses are either joint angle controlled or torque controlled. The angle control approach is inherently not suitable for prostheses due to biomechanical reasons, as reported by Sup et al. [37]. On the other hand, the torque control or tracking with state impedance control schemes, have already established its suitability in active prostheses [42], but its application in semi-active prostheses [43] may not be cautious enough as semi-active prostheses reported in [24-26] using an MR damper do not contribute to net propulsive power during walking. Its function still remains as a passive device with impedance or damping modulation despite torque control. Hence, torque as a kinetic variable representing work and energy interaction is not suitable and is an over-applied idea for semi-active prostheses. Damping modulation can be achieved by simpler yet efficient control methods based on variables representing sufficient information of a gait cycle.

\subsection{Cost and Weight}

The developed prosthesis was designed in-house, fabricated, and assembled in the lab in India. Other than the MR damper, all the components were customized and locally manufactured with locally available materials. In an initial attempt to test the feasibility of the control scheme in less time, the fabrication of the MR damper was avoided and the most widely used MR damper (Lord Corporation Model RD-8041) was imported. The total cost of developed prosthesis is 17,000 INR excluding the damper; a suitably customized, locally designed, and manufactured MR damper is estimated to incur 5000 INR more, amounting the total cost to 22,000 INR. This cost is comparable to commonly available low cost passive prostheses which typically cost ranging from 6000 to 150,000 INR [13]. The total weight of developed prosthesis is 1.77 kilograms $(\mathrm{kg})$ excluding damper and is expected to add $0.5 \mathrm{~kg}$ after attachment of customized locally manufactured damper. A total weight of $2.27 \mathrm{~kg}$ is quite lower than that of reported active and semi-active prostheses [12,23]. The component-wise manufacturing/production cost and weight is summarized in Table 4.

Table 4. Manufacturing cost and weight of various components.

\begin{tabular}{ccc}
\hline Component & Production Cost (INR) & Weight (kg) \\
\hline Knee joint \& mechanical structure & 7000 & 0.810 \\
Sensorized plantar insole & 1000 & 0.015 \\
Sach Foot & 900 & 0.710 \\
Embedded electronics & 2000 & 0.085 \\
Rechargeable batteries & 5000 & 0.150 \\
Total & 17,000 & 1.770 \\
\hline
\end{tabular}

${ }^{a}$ Excluding MR damper. 


\subsection{Battery Life and Power Consumption}

While importing external power to the prosthesis, the power consumption assessment is vital to its commercial acceptance. The average power consumption during a gait cycle was measured to be less than $2.5 \mathrm{~W}$ during over-ground level walking, whereas that of a typical active prostheses is in the range of $60-100 \mathrm{~W}$ for the same [37,42]. Hence, (i) the power consumption of the developed prosthesis is almost 30 times less than its active commercial counterparts, and (ii) a reduction in power requirement can enable further reduction in weight and cost. For a battery bank of $9 \mathrm{~V}$ and $2.4 \mathrm{Ahr}$, the battery can deliver up to $8 \mathrm{~h}$ of continuous supply to the developed prosthesis on a level walk. With this power arrangement, considering stride time of $2 \mathrm{~s}$ and stride length of $70 \mathrm{~cm}$, the amputee will still be able walk 14,400 steps or a distance of around $10 \mathrm{~km}$. As suggested by references, an adult walks 4000 to 18,000 steps per day [44,45]. Hence, the prosthesis is capable of operating an entire day with one time charging in a day. This is a significant advantage over active prostheses that requires $\sim 115 \mathrm{Wh}$ (Equivalent to a $9 \mathrm{~V}, 12 \mathrm{Ahr}$ battery) to work all day [37]. In low-income/developing countries, where access to usual electric charging may not be available at all times, a customized provision of charging with renewable sources such as PV panel, wind etc. can be made at a minimal additional cost.

\subsection{Prosthesis Utilization}

The level of utilization is one of the parameters to evaluate the usability of any system. A system usability score (SUS) [46] has been calculated based on the questionnaire response of the subject after using the developed prosthesis. The same questionnaire has been used in the past on several rehabilitation studies [47]. The SUS score of 85 shows a promising scope for high usability of the prosthesis in Indian scenario (Table 5).

Table 5. System usability scale (SUS) score ${ }^{\mathrm{a}}$.

\begin{tabular}{lc}
\hline \multicolumn{1}{c}{ Questionnaire Item } & Weight \\
\hline I think that I would like to use this prosthesis frequently. & 5 \\
I found the system unnecessarily complex. & 1 \\
I thought the system was easy to use. & 5 \\
I think that I would need the support of a technical person to be able to use this system. & 1 \\
I found the various functions in this system were well integrated. & 4 \\
I thought there was too much inconsistency in this system. & 2 \\
I would imagine that most people would learn to use this system very quickly. & 3 \\
I found the system very cumbersome to use. & 2 \\
I felt very confident using the system. & 5 \\
I needed to learn a lot of things before I could get going with this system. & 2 \\
SUS Score & 85 \\
\hline
\end{tabular}

a The SUS scores range from 1 ("strongly disagree") to 5 ("strongly agree"). The SUS score was calculated based on the responses following Brooke [46].

\subsection{Implications for Commercial Applications}

There are a variety of factors that affect the applicability, commercial viability, and social acceptability of a prosthesis in many low-income economies. The prosthetic needs in these countries differ based on functional demand, local availability of materials, cost, and cultural barriers [4]. As suggested, some points of consideration of appropriate technologies for prosthetics in developing countries like India primarily include low cost, local availability, consideration of local climate, suitability for local manufacturing, and durability [5,21,45,48,49]. Similar recommendations were made at the ISPO Consensus Conference on Appropriate Prosthetic Technology in developing Countries [21]. The developed prosthesis complies with most of the recommendations, and these suggested features, along with description, are tabulated in Table 6. The cost of the passive prosthesis ranges from US $\$ 125$ to 1875 as per Sam et al. [9]. 
Table 6. Technical features and descriptions based on recommendations of ISPO consensus conference on appropriate prosthetic technology in developing countries $[5,20,21]$.

\begin{tabular}{|c|c|}
\hline Technical Feature & Description \\
\hline Low cost & $\begin{array}{l}\text { An expected cost of 22,000 INR is very low keeping in view the performance and } \\
\text { features offered. The mass production of prosthesis would further cut the cost. } \\
\text { Those already using modern passive prostheses can afford such a cost and others } \\
\text { can be supported by governmental schemes as well as non-profit organizations } \\
\text { already working in this field. }\end{array}$ \\
\hline Technical functionality & $\begin{array}{l}\text { It serves the basic functions of providing stability during stance phase and } \\
\text { controlled flexion during the swing phase of a gait cycle. It successfully prevents } \\
\text { knee buckling on heel strike and delayed transition from stance to swing phase. }\end{array}$ \\
\hline $\begin{array}{l}\text { Biomechanical } \\
\text { appropriateness }\end{array}$ & $\begin{array}{l}\text { The feasibility of early stance flexion-extension of knee joint makes it } \\
\text { biomechanically appropriate; incorporation of suitably damped swing phase can } \\
\text { enable the amputee to match its speed with that of the sound limb. }\end{array}$ \\
\hline Light weight & $\begin{array}{l}\text { An expected total weight of } 2.270 \mathrm{~kg} \text { of prosthetic leg is comparable to } \\
\text { commercially available modern passive prostheses in India. The usual weight of } \\
\text { a passive prosthetic leg varies between } 2 \text { to } 3 \mathrm{~kg} \text { in India due to physiological } \\
\text { parameters of Indian population. }\end{array}$ \\
\hline $\begin{array}{c}\text { Use of locally } \\
\text { available materials }\end{array}$ & $\begin{array}{l}\text { The prosthesis is made up of SS and Metalon which are widely available in most } \\
\text { parts of India. Both the materials are affordable, high strength, and durable for a } \\
\text { long period. }\end{array}$ \\
\hline $\begin{array}{l}\text { Consideration } \\
\text { for local climate }\end{array}$ & $\begin{array}{l}\text { The prosthesis is designed keeping in view the hot and humid conditions of the } \\
\text { Indian subcontinent. The material used in the prosthesis can bear several times } \\
\text { the excessive weather conditions including water, heat, and dirt. }\end{array}$ \\
\hline Durable & $\begin{array}{l}\text { The simple design and suitable material selection can make a prosthesis durable } \\
\text { in developing countries. ISO- } 10328 \text { specifies that a prosthetic leg/knee must } \\
\text { endure } 3 \text { million cycles which is equivalent to approximately } 3 \text { years of use. }\end{array}$ \\
\hline $\begin{array}{l}\text { Simple to process } \\
\text { and repair }\end{array}$ & $\begin{array}{l}\text { The design of the prosthesis is modular and each part can be replaced or repaired } \\
\text { separately. The components are reproducible by local personnel and can be } \\
\text { manually fabricated. The prosthesis is simple to process and repair using local } \\
\text { production capability. }\end{array}$ \\
\hline
\end{tabular}

\subsection{Parameter Tuning}

It may appear that there are seven parameters to tune, but usually in a scenario where the prosthesis is manufactured at a facility and the patient is trained by prosthetist, the current controller parameters can be tuned by the manufacturers and the remaining five can be tuned by the prosthetist. It is justified as the prosthetist is trained and experienced at analyzing gait patterns, and therefore it is easier for he/she to judge the suitable value of the parameters. The highlight of the proposed design is the ease of fabrication in low-resource settings, and thereby can help amputees in locomotion.

\subsection{Limitations and Future Work}

Though the prosthesis achieves its objective in terms of kinematic performance and other parameters explained earlier, there is ample scope to take it to the next level, as this work is a pilot study. Factors such as heat dissipation, the reliability of the insole sensors, and security of control to prevent falls need further attention. The developed prosthesis design is in its initial stages and further modifications need to be made to bring the design out of the lab setting. A locally manufactured MR damper needs to be fixed to the prosthesis in order to extensively evaluate the performance of the developed prosthesis on a large number of the targeted population as the prosthesis has been tested on single amputee only. A more aesthetic make over with suitable casing and fitting could unveil its humanoid appearance. The present design and control method addresses level walking with a single speed only, a variable speed adaptation would enhance its maneuverability and user 
friendliness. The future work would include the implementation of the above left-over points with design, development of control scheme for variable speed adaptation, and thorough testing of the modified design on more subjects.

\section{Conclusions}

A novel low-cost lower limb prosthesis based on sensors placed in a plantar insole was presented in this work. The hardware with suitable control architecture enabled the trans-femoral amputee to attain able-bodied gait kinematics. The results are encouraging and provide a new dimension to the control philosophy of proposed prostheses. The study presents the initial prototype and hence may need thorough testing and related modifications before commercialization. The developed prosthesis is an optimum choice for lower limb amputees in low-income or low-resource settings as well as in developing countries. The attractive features of low cost, durability, and able-bodied gait performance are expected to expand its commercial reach beyond India to other low and medium income developing countries.

Acknowledgments: The authors gratefully acknowledge the active participation of Vipin Kumar as a subject in this study. His patient support during training and testing of prosthesis is highly appreciable. We thank Indian Council of Medical Research (ICMR) New Delhi, Govt. of India for funding this research via research grant No.5/20/13/Bio/2011-NCD-I.

Author Contributions: D.J. conceived and suggested the development; S.P., A.K.G., D.J., and A.K.V. contributed in the development and experiment; A.K.V. and U.S. provided training; D.K. contributed in mechanical structure development; D.J., U.S., and D.K. wrote the paper.

Conflicts of Interest: The authors declare no conflict of interest. The founding sponsors had no role in the design of the study; in the collection, analyses, or interpretation of data; in the writing of the manuscript, and in the decision to publish the results.

\section{References}

1. WHO (World Health Organization). World report on disability 2011. Am. J. Phys. Med. Rehabil. Assoc. Acad. Physiatr. 2011, 91, 549. [CrossRef]

2. Andrysek, J. Lower-Limb Prosthetic Technologies in the Developing World: A Review of Literature from 1994-2010. Prosthet. Orthot. Int. 2010, 34, 378-398. [CrossRef] [PubMed]

3. Pearlman, J.; Cooper, R.; Krizack, M.; Lindsley, A.; Wu, Y.; Reisinger, K.; Armstrong, W.; Casanova, H.; Chhabra, H.; Noon, J. Lower-limb prostheses and wheelchairs in low-income countries: An Overview. IEEE Eng. Med. Biol. Mag. 2008, 27, 12-22. [CrossRef] [PubMed]

4. Craig, J. Prosthetic Feet for Low-Income Countries. J. Prosthet. Orthot. 2005, 17, S47-S49. [CrossRef]

5. Cummings, D. Prosthetics in the developing world: A review of the literature. Prosthet. Orthot. Int. 1996, 20, 51-60. [CrossRef] [PubMed]

6. Williams, M.R.; D'Andrea, S.; Herr, H.M. Impact on gait biomechanics of using an active variable impedance prosthetic knee. J. Neuroeng. Rehabil. 2016, 13, 1-11. [CrossRef] [PubMed]

7. Ingraham, K.M.; Fey, N.P.; Simon, A.M.; Hargrove, L.J. Assessing the relative contributions of active ankle and knee assistance to the walking mechanics of transfemoral amputees using a powered prosthesis. PLOS ONE 2016, 11, 1-19. [CrossRef] [PubMed]

8. Gregg, R.D.; Lenzi, T.; Hargrove, L.J.; Sensinger, J.W. Virtual Constraint Control of a Powered Prosthetic Leg: From Simulation to Experiments with Transfemoral Amputees. IEEE Trans. Robot. 2013, 30, 1455-1471. [CrossRef] [PubMed]

9. Sam, M.; Childress, D.; Hansen, A.; Meier, M.; Lambla, S.; Grahn, E.; Rolock, J. The "Shape\&Roll" Prosthetic Foot: I. Design and Development of Appropriate Technology for Low-Income Countries. Med. Confl. Surviv. 2004, 20, 294-306. [CrossRef] [PubMed]

10. Gibson, D.A. Atlas of Limb Prosthetics. Can. Med. Assoc. J. 1982, 127, 324.

11. Radcliffe, C.W. Four-bar linkage prosthetic knee mechanisms: Kinematics, alignment and prescription criteria. Prosthet. Orthot. Int. 1994, 18, 159-173. [CrossRef] [PubMed]

12. Jensen, J.S.; Raab, W. Clinical field testing of trans-femoral prosthetic technologies: Resin-wood and ICRC-polypropylene. Prosthet. Orthot. Int. 2004, 28, 141-151. [CrossRef] [PubMed] 
13. Meier, M.R.; Sam, M.; Hansen, A.H.; Childress, D.S. The "shape\&roll" prosthetic foot: II. Field testing in El Salvador. Med. Confl. Surviv. 2010, 20, 307-325. [CrossRef]

14. Hamner, S.R.; Narayan, V.G.; Donaldson, K.M. Designing for scale: Development of the remotion knee for global emerging markets. Ann. Biomed. Eng. 2013, 41, 1851-1859. [CrossRef] [PubMed]

15. Andrysek, J.; Klejman, S.; Torres-Moreno, R.; Heim, W.; Steinnagel, B.; Glasford, S. Mobility function of a prosthetic knee joint with an automatic stance phase lock. Prosthet. Orthot. Int. 2011, 35, 163-170. [CrossRef] [PubMed]

16. Arelekatti, V.N.M.; Winter, A.G. Design of fully passive prosthetic knee for persons with transfemoral amputation in India. In Proceedings of the 2015 IEEE International Conference on Rehabilitation Robotics (ICORR), Nanyang Technological University, Singapore, 11-14 August 2015; pp. 350-356.

17. Gailey, R. Review of secondary physical conditions associated with lower-limb amputation and long-term prosthesis use. J. Rehabil. Res. Dev. 2008, 45, 15-30. [CrossRef] [PubMed]

18. Pohjolainen, T.; Alaranta, H.; Kärkkäinen, M. Prosthetic use and functional and social outcome following major lower limb amputation. Prosthet. Orthot. Int. 1990, 14, 75-79. [CrossRef] [PubMed]

19. Wyss, D.; Lindsay, S.; Cleghorn, W.L.; Andrysek, J. Priorities in lower limb prosthetic service delivery based on an international survey of prosthetists in low- and high-income countries. Prosthet. Orthot. Int. 2015, 39, 102-111. [CrossRef] [PubMed]

20. Day, H.J. A review of the consensus conference on appropriate prosthetic technology in developing countries. Prosthet. Orthot. Int. 1996, 20, 15-23. [PubMed]

21. ISPO. ISPO consensus conference on appropriate orthopaedic technology for low-income countries: Conclusions and recommendations. Prosthet. Orthot. Int. 2001, 25, 168-170. [CrossRef]

22. Steen, J.; Dmsc, J.M.; Sexton, S. Appropriate Prosthetic and Orthotic Technologies in Low Income Countries (2000-2010); International Society for Prosthetics and Orthotics (ISPO): Brussels, Belgium, 2010.

23. Herr, H.; Wilkenfeld, A. User-adaptive control of a magnetorheological prosthetic knee. Ind. Robot Int. J. 2003, 30, 42-55. [CrossRef]

24. Xu, L.; Wang, D.-H.; Fu, Q.; Yuan, G.; Hu, L.-Z. A novel four-bar linkage prosthetic knee based on magnetorheological effect: Principle, structure, simulation and control. Smart Mater. Struct. 2016, 25, 115007. [CrossRef]

25. Park, J.; Yoon, G.-H.; Kang, J.-W.; Choi, S.-B. Design and control of a prosthetic leg for above-knee amputees operated in semi-active and active modes. Smart Mater. Struct. 2016, 25, 85009. [CrossRef]

26. Fu, Q.; Wang, D.H.; Xu, L.; Yuan, G. A magnetorheological damper-based prosthetic knee (MRPK) and sliding mode tracking control method for an MRPK-based lower limb prosthesis. Smart Mater. Struct. 2017, 26. [CrossRef]

27. Martinez-Villalpando, E. C.; Herr, H. Agonist-antagonist active knee prosthesis: A preliminary study in level-ground walking. J. Rehabil. Res. Dev. 2009, 46, 361-373. [CrossRef] [PubMed]

28. Abdul Razak, A.H.; Zayegh, A.; Begg, R.K.; Wahab, Y. Foot plantar pressure measurement system: A review. Sensors 2012, 12, 9884-9912. [CrossRef] [PubMed]

29. Lou, C.; Wang, S.; Liang, T.; Pang, C.; Huang, L.; Run, M.; Liu, X. A graphene-based flexible pressure sensor with applications to plantar pressure measurement and gait analysis. Materials 2017, 10, 1068. [CrossRef] [PubMed]

30. Torricelli, D.; Gonzalez, J.; Weckx, M.; Jiménez-Fabián, R.; Vanderborght, B.; Sartori, M.; Dosen, S.; Farina, D.; Lefeber, D.; Pons, J.L. Human-like compliant locomotion: State of the art of robotic implementations. Bioinspir. Biomim. 2016, 11, 51002. [CrossRef] [PubMed]

31. Hansen, A.H.; Childress, D.S.; Knox, E.H. Roll-over shapes of human locomotor systems: Effects of walking speed. Clin. Biomech. 2004, 19, 407-414. [CrossRef] [PubMed]

32. Shorter, K.A.; Kogler, G.F.; Loth, E.; Durfee, W.K.; Hsiao-Wecksler, E.T. A portable powered ankle-foot orthosis for rehabilitation. J. Rehabil. Res. Dev. 2011, 48, 459. [CrossRef] [PubMed]

33. Winter, D. Biomechanics as an Interdiscipline; John Wiley \& Sons, Inc.: Hoboken, NJ, USA, 2009; ISBN 9780470398180.

34. Wang, Q.; Yuan, K.; Zhu, J.; Wang, L. Finite-state control of a robotic transtibial prosthesis with motor-driven nonlinear damping behaviors for level ground walking. In Proceedings of the 2014 IEEE 13th International Workshop on Advanced Motion Control (AMC), Yokohama, Japan, 14-16 March 2014; pp. 155-160. [CrossRef] 
35. Sweeney, P.C.; Lyons, G.M.; Veltink, P.H. Finite state control of functional electrical stimulation for the rehabilitation of gait. Med. Biol. Eng Comput. 2000, 38, 121-126. [CrossRef] [PubMed]

36. Zlatnik, D.; Steiner, B.; Schweitzer, G. Finite-state control of a trans-femoral (TF) prosthesis. IEEE Trans. Control Syst. Technol. 2002, 10, 408-420. [CrossRef]

37. Sup, F.; Varol, H.A.; Mitchell, J.; Withrow, T.J.; Goldfarb, M. Preliminary evaluations of a self-contained anthropomorphic transfemoral prosthesis. IEEE/ASME Trans. Mechatron. 2009, 14, 667-676. [CrossRef] [PubMed]

38. Chin, T.; Sawamura, S.; Fujita, H.; Nakajima, S.; Ojima, I.; Oyabu, H.; Nagakura, Y.; Otsuka, H.; Nakagawa, A. Effect of endurance training program based on anaerobic threshold (AT) for lower limb amputees. J. Rehabil. Res. Dev. 2001, 38, 7-11. [PubMed]

39. Esquenazi, A. Amputation rehabilitation and prosthetic restoration. From surgery to community reintegration. Disabil. Rehabil. 2004, 26, 831-836. [CrossRef] [PubMed]

40. Majumdar, D.; Pal, M.S.; Majumdar, D. Effects of military load carriage on kinematics of gait. Ergonomics 2010, 53, 782-791. [CrossRef] [PubMed]

41. Franchignoni, F.; Orlandini, D.; Ferriero, G.; Moscato, T.A. Reliability, validity, and responsiveness of the locomotor capabilities index in adults with lower-limb amputation undergoing prosthetic training. Arch. Phys. Med. Rehabil. 2004, 85, 743-748. [CrossRef] [PubMed]

42. Shultz, A.H.; Lawson, B.E.; Goldfarb, M. Running with a powered knee and ankle prosthesis. IEEE Trans. Neural Syst. Rehabil. Eng. 2015, 23, 403-412. [CrossRef] [PubMed]

43. Fey, N.P.; Simon, A.N.N.M.; Young, A.J.; Member, S.; Hargrove, L.J. Controlling Knee Swing Initiation and Ankle Plantarflexion with an Active Prosthesis on Level and Inclined Surfaces at Variable Walking Speeds. IEEE J. Transl. Eng. Health Med. 2014, 2. [CrossRef] [PubMed]

44. Tudor-Locke, C.; Craig, C.L.; Brown, W.J.; Clemes, S.A.; De Cocker, K.; Giles-Corti, B.; Hatano, Y.; Inoue, S.; Matsudo, S.M.; Mutrie, N.; et al. How many steps/day are enough? For adults. Int. J. Behav. Nutr. Phys. Act. 2011, 8, 79. [CrossRef] [PubMed]

45. Tudor-Locke, C.; Craig, C.L.; Beets, M.W.; Belton, S.; Cardon, G.M.; Duncan, S.; Hatano, Y.; Lubans, D.R.; Olds, T.S.; Raustorp, A.; et al. How many steps/day are enough? for children and adolescents. Int. J. Behav. Nutr. Phys. Act. 2011, 8, 78. [CrossRef] [PubMed]

46. Brooke, J. SUS-A quick and dirty usability scale. Usability Eval. Ind. 1996, 189, 4-7. [CrossRef]

47. Crea, S.; Edin, B.B.; Knaepen, K.; Meeusen, R.; Vitiello, N. Time-discrete vibrotactile feedback contributes to improved gait symmetry in patients with lower limb amputations: Case series. Phys. Ther. 2017, 97, 198-207. [CrossRef] [PubMed]

48. Flor, H. Phantom-limb pain: Characteristics, causes, and treatment. Lancet Neurol. 2002, 1, 182-189. [CrossRef]

49. Cutson, T.M.; Bongiorni, D.R. Rehabilitation of the older lower limb amputee: A brief review. J. Am. Geriatr. Soc. 1996, 44, 1388-1393. [CrossRef] [PubMed]

(C) 2018 by the authors. Licensee MDPI, Basel, Switzerland. This article is an open access article distributed under the terms and conditions of the Creative Commons Attribution (CC BY) license (http:/ / creativecommons.org/licenses/by/4.0/). 
Article

\title{
Inertial Measurement Units for Clinical Movement Analysis: Reliability and Concurrent Validity
}

\author{
Mohammad Al-Amri ${ }^{1,2, *}$, Kevin Nicholas ${ }^{1,2,3}$, Kate Button ${ }^{1,2,3}$, Valerie Sparkes ${ }^{1,2}$, \\ Liba Sheeran ${ }^{1,2}$ and Jennifer L Davies ${ }^{1,2}$ \\ 1 School of Healthcare Sciences, College of Biomedical and Life Sciences, Cardiff University, Cardiff CF24 0AB, \\ Wales, UK; NicholasKA@cardiff.ac.uk (K.N.); ButtonK@cardiff.ac.uk (K.B.); SparkesV@cardiff.ac.uk (V.S.); \\ SheeranL@cardiff.ac.uk (L.S.); DaviesJ@cardiff.ac.uk (J.L.D.) \\ 2 Arthritis Research UK Biomechanics and Bioengineering Centre, Cardiff University, Cardiff CF10 3AXB, \\ Wales, UK \\ 3 Cardiff and Vale University Health Board, Heath Park, Cardiff CF14 4XW, Wales, UK \\ * Correspondence: Al-AmriM@cardiff.ac.uk; Tel.: +44-292-068-7115
}

Received: 15 January 2018; Accepted: 26 February 2018; Published: 28 February 2018

\begin{abstract}
The aim of this study was to investigate the reliability and concurrent validity of a commercially available Xsens MVN BIOMECH inertial-sensor-based motion capture system during clinically relevant functional activities. A clinician with no prior experience of motion capture technologies and an experienced clinical movement scientist each assessed 26 healthy participants within each of two sessions using a camera-based motion capture system and the MVN BIOMECH system. Participants performed overground walking, squatting, and jumping. Sessions were separated by $4 \pm 3$ days. Reliability was evaluated using intraclass correlation coefficient and standard error of measurement, and validity was evaluated using the coefficient of multiple correlation and the linear fit method. Day-to-day reliability was generally fair-to-excellent in all three planes for hip, knee, and ankle joint angles in all three tasks. Within-day (between-rater) reliability was fair-to-excellent in all three planes during walking and squatting, and poor-to-high during jumping. Validity was excellent in the sagittal plane for hip, knee, and ankle joint angles in all three tasks and acceptable in frontal and transverse planes in squat and jump activity across joints. Our results suggest that the MVN BIOMECH system can be used by a clinician to quantify lower-limb joint angles in clinically relevant movements.
\end{abstract}

Keywords: inertial measurement units; motion analysis; kinematics; gait; functional activity; repeatability; reliability; biomechanics

\section{Introduction}

Assessment of movement patterns during functional activities such as walking and squatting, and during sporting manoeuvres such as jumping, is a cornerstone of musculoskeletal physiotherapy. Commonly, the physical examination involves observation by the clinician and completion of clinicianor patient-rated scales [1]. However, it is challenging to accurately evaluate multiple joints of both legs in multiple planes of movement when an individual is performing a dynamic functional activity, which often occurs at speed. Three-dimensional optoelectronic (camera-based) motion systems can be used to provide comprehensive, objective measurements [2], but this typically requires the patient to attend a specialised movement analysis laboratory. The equipment within these laboratories is expensive, non-portable, and requires a high level of technical expertise and a lengthy calibration process. The use of these systems is therefore not widespread in clinical practice, and clinicians typically do not have access to objective biomechanical information for assessing patient performance. A more rigorous approach to quantifying joint movement in the clinic is required. 
A potential solution to this problem is inertial measurement units (IMUs), which could be used in clinical settings to objectively measure movement patterns during functional activities. An IMU is comprised of accelerometers, gyroscopes, and magnetic sensors combined with a fusion algorithm, for example a Kalman filter [3-5]. It can be attached to a body segment to estimate the movement of that segment in space. When combined with other IMUs on adjacent body segments, the kinematics of movements can be calculated [6-12]. IMU motion-capture systems are portable and less expensive than traditional camera-based motion-capture systems. The validity of joint kinematics calculated with IMU systems has been confirmed with respect to optoelectronic systems $[10,11,13]$.

Commercial IMU systems are increasingly available, from companies such as Xsens Technologies B.V., (Enschede, The Netherlands), Shimmer Sensing (Dublin, Ireland), BioSyn Systems (Surrey, BC, Canada), I Measure U (Auckland, New Zealand), and APDM Wearable Technologies (Portland, OR, USA). The Xsens MVN BIOMECH system is composed of wireless motion IMUs (called MTw2 sensors) and native biomechanical protocols, and estimates three-dimensional joint kinematics [14]. The accuracy of the orientation information provided by an individual IMU [15-17] and the joint angles provided by MVN BIOMECH software $[18,19]$ have been reported. Robert-Lachaine et al. [18] and Zhang et al. [19] compared joint angles obtained from MVN BIOMECH software against those obtained from a biomechanical model associated with the Optotrak camera-based motion analysis system. During walking and stair climbing, the mean differences in lower-limb joint angles between the two systems were between $1.4^{\circ}$ and $6.7^{\circ}$, and the coefficient of multiple correlation (CMC) was between 0.39 and 0.99 [19]. In more complex manual handling tasks, errors due to technology were $<5^{\circ}$ and $\mathrm{CMC}$ was 0.79 to 0.97 [18]. This suggests that the MVN BIOMECH system is able to quantify joint kinematics during complex functional movements. In these studies, the motion analysis systems were operated by individuals who were experienced in performing movement analysis experiments, and not by clinicians. However, if an IMU system is to be used in a clinical setting, it is most likely to be used by a clinician with little or no experience of collecting biomechanical data. At present it is not clear if undergoing brief training using only the guides provided by the manufacturer is sufficient to enable a clinician to collect accurate and reliable biomechanical information using a commercially available IMU system. Additionally, the tasks evaluated in previous studies were limited to walking and stair climbing [19], and manual handling tasks [18]. To facilitate transfer of this technology to the clinic it is necessary to quantify the validity and reliability of the Xsens MVN BIOMECH system when used by a clinician, and for clinically relevant functional movements that may involve a greater range of motion or dynamic movements, such as squat and jump.

The primary aim of this study was to determine the reliability of joint angular kinematics provided by the Xsens MVN BIOMECH system when used by a clinically experienced musculoskeletal physiotherapist (MSKP) with no experience of using IMUs across different sessions (i.e., within-rater, between-session repeatability). Secondary aims were (1) to determine the reliability of joint angular kinematics provided the Xsens MVN BIOMECH system when used by an experienced MSKP and an experienced clinical movement scientist (i.e., between-rater, within-session repeatability); and (2) to determine the concurrent validity (agreement) of joint angular kinematics provided the Xsens MVN BIOMECH system when used by an experienced MSKP against data collected using a gold-standard camera-based motion capture system (VICON motion analysis system, Oxford Metrics Group Ltd., Oxford, UK). Validity and reliability were evaluated during walking, squatting and jumping to cover a larger range of motion (squat, jump) and a more dynamic movement (jump) than previously investigated. 


\section{Materials and Methods}

\subsection{Research Participants and Setting}

The study was carried out in the Research Centre for Clinical Kinaesiology at Cardiff University. The study was conducted as part of the Arthritis Research UK Biomechanics and Bioengineering Centre at Cardiff University, and was approved by the Wales Research Ethics Committee 3 (10/MRE09/28). The required sample size $(n=16)$ was determined according to the recommendation of Walter et al. (Table II, [20]), using $\alpha=0.05, \beta=0.2, \rho_{0}=0.2$ and $\rho_{1}=0.7$. To account for an anticipated attrition of $40 \%$ over the repeated sessions, the target sample size was increased to 27 . A convenience sample of healthy participants was recruited using the following criteria: age between 18 and 60 years; healthy with no known neurological, cardiovascular, or musculoskeletal condition. Written informed consent was obtained prior to participation.

\subsection{Raters}

The Xsens MVN BIOMECH system was used by two raters. Rater 1 (MSKP) had 13 years experience as a qualified physiotherapist and 5 years experience as a specialist knee physiotherapist, but no prior experience of biomechanical motion capture. Rater 2 (clinical movement scientist) had 10 years experience performing biomechanical motion capture studies in laboratory settings. The two raters received the same information and instructions for use of the Xsens MVN BIOMECH system. This consisted of self-training using the MVN user manual [21] and online tutorials (https: / tutorial. xsens.com/) and a half-day training session provided by Xsens Technologies at Cardiff University.

\subsection{Experimental Protocol}

Each participant underwent two movement analysis sessions, approximately 1 week apart (mean \pm standard deviation, $4 \pm 3$ days). In the first session, anthropometric measurements were taken by the MSKP from the right lower limb with the participant in a standing posture. Body mass was recorded using SECA scales (Birmingham, UK). These measurements were only taken in the first session. Apart from this, the two sessions were identical and proceeded as follows:

At the start of each session, 16 retroreflective markers (14-mm diameter) were placed on the participant according to the Plug-in-Gait lower-body model (Vicon Motion Systems, Oxford Metrics Group Ltd.). In every session, retroreflective markers were placed by the MSKP using standardised palpation methods on anatomical landmarks [22]. Retroreflective markers were always attached prior to placement of any IMU, and were held in position with medical grade double-sided adhesive tape.

Seven MTw2 trackers (Xsens Technologies) were then placed in accordance with Xsens instructions [21]. MTw2 trackers were placed by either rater 1 (MSKP) or rater 2 (clinical movement scientist). The order of the raters was randomised across participants, but consistent within participants across sessions. MTw2 trackers were secured using elasticated Velcro straps on each upper thigh (centrally and halfway between the greater trochanter and lateral epicondyle of the knee), each lower leg (proximal medial surface of the tibia), the dorsum of each foot and one centrally over the sacrum. Each lower-limb tracker was placed between the two outermost layers of the strap and attached to the Velcro of the inner layer to secure its position and minimise any movement. The sacral tracker was placed directly over the sacrum with the upper border of the sensor aligned centrally between the two posterior superior iliac spines. The sacral sensor was held in position with medical grade double-sided adhesive tape. Care was taken not to interfere with the reflective markers.

Each participant performed eight repetitions of each of the following three activities: over-ground walking, squatting, and vertical jumping. Prior to performing each activity the participant was provided a demonstration by the MSKP, and was allowed to ask any questions. All activities were performed at a self-selected speed to best mimic performance of these activities in a clinical setting. The order of the activities was randomised across participants, but consistent within participants and across sessions. Each walking trial consisted of a walk in a straight line across the laboratory 
(approximately $8 \mathrm{~m}$ ). Squat depth was standardised to prevent occlusion of the reflective markers on the anterior superior iliac spines at low squat depths. This was done using a plinth placed behind the participant at the height of the knee joint line plus $5 \mathrm{~cm}$, which prevented the participant from squatting past this depth. Once all activities were completed, the MTw2 trackers were removed by the same rater who had placed them. The participant rested for at least $10 \mathrm{~min}$ to allow any strap marks on the skin to disappear, and then the MTw2 trackers were placed by the other rater. The retroreflective markers were not moved during this rest period. The participant then repeated the activities in the same order as in the first half of the session.

\subsection{Data Collection}

Kinematic data were collected at $120 \mathrm{~Hz}$ using a ten-camera VICON MX3+ motion analysis system (Oxford Metrics Group Ltd.) and $60 \mathrm{~Hz}$ using the Xsens MVN BIOMECH system (Xsens Technologies). The systems were synchronised using a trigger sent from the MVN BIOMECH studio software (Version 4.4) to VICON Nexus software (Version 1.8). Prior to beginning the tasks, the participant was asked to stand in a static N-pose, as per the instructions in the MVN BIOMECH user manual [21]. This was maintained for $\sim 30 \mathrm{~s}$. At the start of this period of quiet stance, the MTw2 trackers were calibrated within the MVN BIOMECH software. During this process the software establishes the relation between segment and tracker orientations [23,24]. Once this was complete ( 10 s), the remaining duration of the quiet stance was synchronously recorded by the two systems. These data were used offline in VICON Nexus software as the static anatomical calibration trial for the Plug-In-Gait model.

\subsection{Data Processing}

Data collected using the MVN BIOMECH system were exported as an mvnx file. For data collected using the VICON system, reconstruction and auto-labelling of marker trajectories was first performed in VICON Nexus software. Each trial was then visually inspected and unmarked trajectories were manually labelled. Gaps in trajectories of up to 10 samples were joined with linear interpolation filtered with a quintic spline filter (Woltring; mean square error of 15). The Plug-in Gait pipeline was then implemented in VICON Nexus software, and resulting kinematic data were exported as a c3d file. Plug-in Gait is the commercial name for the implementation of what is commonly called the conventional gait model [22,25-27].

Custom analysis scripts were created in Matlab software (version 2015a; The MathWorks Inc., Natick, MA, USA) and used to perform subsequent analyses. Hip, knee and ankle joint angles calculated by MVN BIOMECH software and VICON Nexus software were extracted from the mvnx and c3d files, respectively. In VICON, positive joint values indicate flexion, adduction and internal rotation in the sagittal, frontal and transverse planes, respectively. In MVN BIOMECH, positive joint angles indicate flexion, abduction and internal rotation, respectively. Frontal plane joint angles from MVN BIOMECH were therefore inverted before any further analysis. Data were filtered with a 6- $\mathrm{Hz}$ low-pass fourth-order Butterworth filter. Vicon data were resampled to $60 \mathrm{~Hz}$ using the resample function in Matlab. No other post-processing was performed on the joint angles provided by the two systems. Although the systems were synchronised, we observed discrepancies in the duration of the files recorded by the two systems. Due to the uncertainty as to whether this failure of synchronisation occurred at the start or the end of the recording, the mvnx and c3d files were aligned using the 'aligndata' function within Matlab performed on the sagittal plane knee angle.

Movement cycles were defined using data from VICON, as described in Table 1. For each movement cycle, the following variables were quantified for hip, knee and ankle joints on both sides of the body in the sagittal, frontal and transverse planes: minimum angle, maximum angle, range of motion. For walking trials, the joint angle at heel strike was also identified. The minimum and maximum angle, the range of motion and the angle at heel strike represent discrete, clinically relevant 
events in the movement cycles [28-31]. If the MVN BIOMECH system is to be transferred into the clinic it is important that it can accurately and reliably measure these angles at these time points.

Table 1. Definition of start and end of movement cycles for each activity.

\begin{tabular}{ccc}
\hline & Start & End \\
\hline Walk & Heel strike * & Subsequent heel strike * on the same side \\
\hline Jump & $\begin{array}{c}\text { Local maxima in left knee flexion angle } \\
\text { that preceded each local peak in vertical } \\
\text { heel position }\end{array}$ & $\begin{array}{c}\text { Local maxima in left knee flexion angle that } \\
\text { succeeded each local peak in vertical heel position }\end{array}$ \\
\hline $\begin{array}{l}\text { Squat } \\
\text { * Heel strike was determined as described by Zeni et al. [32], as the local minima in the anterior-posterior position of } \\
\text { the heel relative to the sacrum. The sacrum was defined as the average position of left and right posterior superior } \\
\text { iliac spines. }\end{array}$
\end{tabular}

\subsection{Data Analysis}

Data analysis was carried out in Matlab software for each activity (walk, jump, squat), each joint (hip, knee, ankle), each plane of movement (sagittal, frontal, transverse) and each side of the body (left, right). For each walk trial, a single stride from the middle of the trial was used for analysis. This gave eight strides for analysis, alongside eight repetitions of squat and jump. The descriptive analysis included mean, standard deviation, difference, and $95 \%$ confidence interval of difference of each outcome measure. These were calculated for each participant as an average of all repetitions, before being averaged across all participants. Reliability and validity were quantified for each activity, each joint, and each plane according to the COnsensus-based Standards for the selection of health Measurement INstrument (COSMIN) standards [33,34] as detailed below.

\subsubsection{Reliability}

Within-rater, between-session reliability was evaluated for rater 1 (MSKP) by comparing data from session 1 and session 2. Between-rater (within-session) reliability was evaluated for session 1 by comparing data from rater 1 (MSKP) and rater 2 (clinical movement scientist). Reliability was quantified using single ICC. For this calculation, the two-way random effects model was used, with a confidence of $95 \%$. ICC was interpreted according to Shrout and Fleiss [35] where ICC $\geq 0.75$ indicates excellent repeatability, ICC $0.4-0.74$ indicates fair-to-high repeatability, and ICC $\leq 0.39$ indicates poor repeatability. Standard error of measurement (SEM) was also calculated using Equation (1) [36]:

$$
\mathrm{SEM}=\mathrm{SD} \times \sqrt{1-\mathrm{ICC}}
$$

where SD refers to the standard deviation. SEM was used to evaluate absolute reliability and provide information on variability over repeated measurements [37]. Absolute reliability indicates the reliability of scores within individual participants on different occasions, and was considered excellent if SEM $<3.0^{\circ}$ and acceptable if SEM $<5^{\circ}[38]$.

\subsubsection{Concurrent Validity}

Concurrent validity was evaluated for rater 1 (MSKP) using data from session 1 . For each activity (walk, jump, squat), each joint (hip, knee, ankle), each plane of movement (sagittal, frontal, transverse) and each side of the body (left, right), MVN BIOMECH and VICON data were compared using the difference in minimum angle, maximum angle, and range of motion $(\Delta \min \theta, \Delta \max \theta$, and $\Delta \mathrm{ROM}$, respectively), the coefficient of multiple correlation (CMC $[10,25,39,40])$, and the linear fit method (LFM [41]). In addition, for walking trials, MVN BIOMECH and VICON data were compared using the difference in the angle at heel strike $(\Delta @ H S)$. 
Delta values indicate the similarity of the two signals at clinically relevant points in the movement cycle. The CMC and LFM indicate the similarity of the two signals across the full movement cycle. The CMC was calculated using the formula provided in Ferarri et al. [10]. When the range of motion of the two waveforms is comparable to the offset between them, the CMC is not a real number. Following Ferarri et al. [10,39], CMC was calculated before and after the offset was removed from the two signals. For each signal (MVN BIOMECH and VICON), offset was calculated as the mean of the signal over the entire movement cycle. This value was then subtracted from the signal, giving a signal with zero offset. The $\mathrm{CMC}$ before and after offset removal is referred to as $\mathrm{CMC}_{1}$ and $\mathrm{CMC}_{2}$ respectively. $\mathrm{CMC}$ is reported only for joints and planes where all values are real numbers [10]. The LFM gives three parameters: $\alpha 1$ indicates the scaling factor, $\alpha$ indicates the scalar addition (i.e., shift or offset) and $R^{2}$ indicates the strength of the linear relation between the two signals [41]. Concurrent validity was considered excellent if CMC and $R^{2}>0.75$, fair-to-high if CMC and $R^{2} 0.4-0.74$, and poor if CMC and $R^{2}<0.39$.

\section{Results}

\subsection{Demographics and Descriptive}

Twenty-six participants (mean \pm standard deviation age: $35.2 \pm 8.4$ years; height: $162.0 \pm 32.9 \mathrm{~cm}$; body mass: $71.6 \pm 12.8 \mathrm{~kg}$; body mass index: $25.3 \pm 3.8 \mathrm{~kg} / \mathrm{m}^{2}$ ) were enrolled in the study and completed the first session. These individuals were all recruited in the first wave of recruitment. Although this was one below our target recruitment of 27, at the end of the first wave of our recruitment it was clear that our attrition of $4 \%$ was well below the allowed $40 \%$, and so a 27 th participant was not sought. One participant ( $4 \%$ ) did not return for the second session. For the remaining 25 participants, the duration between sessions was $4 \pm 3$ days. For a second participant, data were not processed for session 1 and for a third participant, data were not processed for the jump activity for session 1, rater 1 . In both cases this was because clothing obscured the right thigh marker. Between-session reliability of MVN BIOMECH data was therefore evaluated for 24 participants, apart from the jump where it was evaluated for 23 participants. Similarity of MVN BIOMECH and VICON data was evaluated using data from session 1, and was therefore evaluated for 25 participants. Results were similar for left and right sides of the body, and only results for the left side of the body are presented. The mean \pm SD speed of walking was $1.40 \pm 0.14 \mathrm{~m} / \mathrm{s}$ in session 1 and $1.39 \pm 0.13 \mathrm{~m} / \mathrm{s}$ in session 2 . The mean $\pm \mathrm{SD}$ duration of squat was $2.25 \pm 0.60 \mathrm{~s}$ in session 1 and $2.18 \pm 0.62 \mathrm{~s}$ in session 2 . The mean $\pm \mathrm{SD}$ duration of jump was $1.21 \pm 0.55 \mathrm{~s}$ in session 1 and $1.13 \pm 0.38 \mathrm{~s}$ in session 2 .

\subsection{Reliability}

The mean and standard deviation of each measure, and the mean difference of each measure between days and raters are presented in Appendix A. For all activities and all joints, the between-session (within-rater) reliability of sagittal plane joint angles provided by MVN BIOMECH was high, with ICC between 0.6 and 0.95 (Figure 1). The between-session (within-rater) reliability of the frontal plane knee angle and transverse plane ankle angle at the heel strike was poor (Figure 1). The absolute between-session (within-rater) reliability of the MVN BIOMECH data was generally acceptable (SEM $<5^{\circ}$; Figure 2$)$. 
ICC: MVN BIOMECH data. Rater 1. Session 1 vs. Session 2.
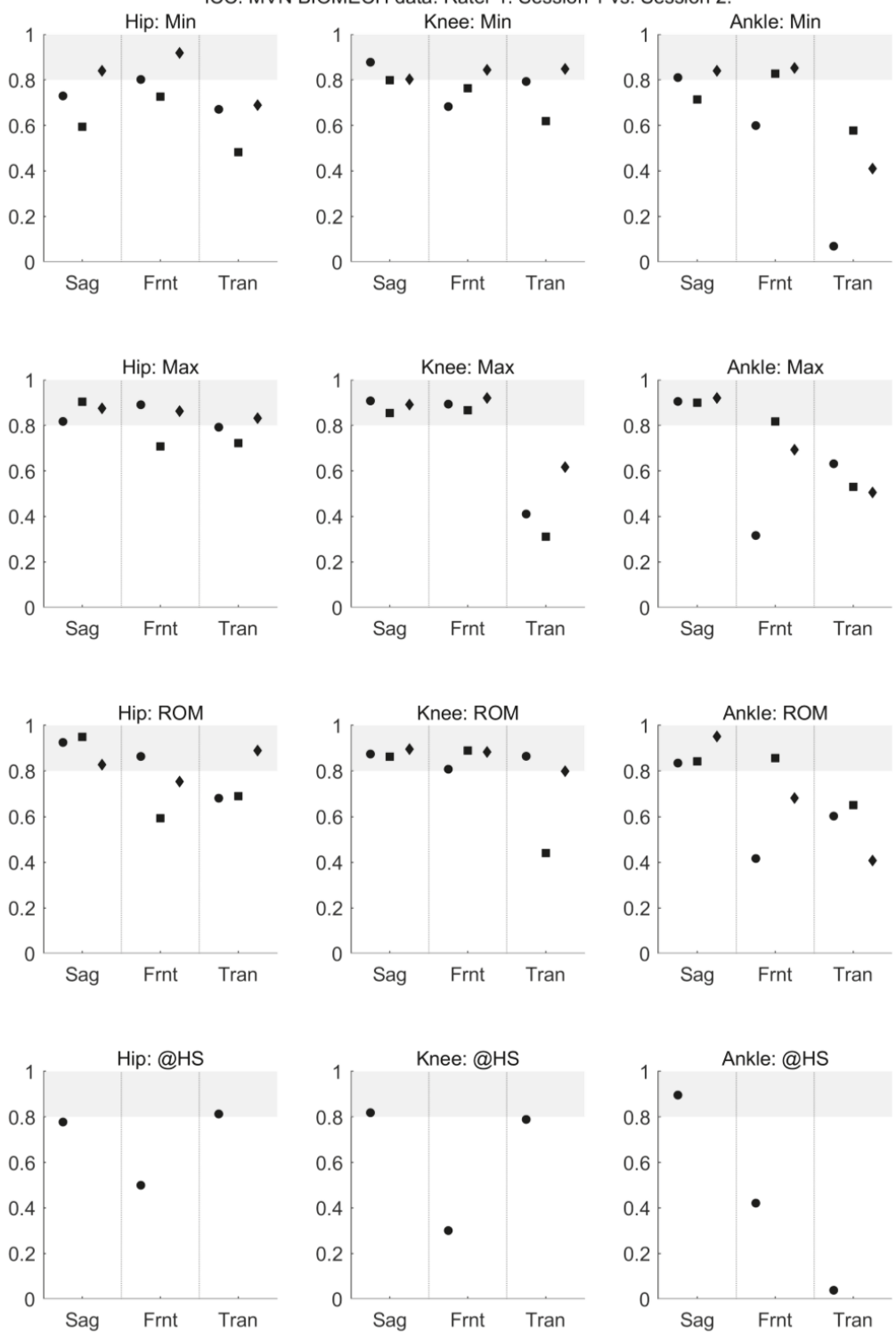

Figure 1. Between-session (within-rater) intraclass correlation coefficient of MVN data collected by rater 1 (musculoskeletal physiotherapist) in sessions 1 and 2. Intraclass correlation coefficient is shown for the minimum angle (min; top row), maximum angle (max; second row), range of motion (ROM; third row) and angle at heel strike (@HS; bottom row) for the hip (left-most column), knee (centre column) and ankle (right-most column) joints in the sagittal (Sag), frontal (Frnt) and transverse (Tran) planes of movement. The three data points in each plane correspond to the walk (left-most point; circle), squat (centre point; square) and jump (right-most point; diamond) tasks. Grey shading indicates values considered to indicate excellent reliability. 

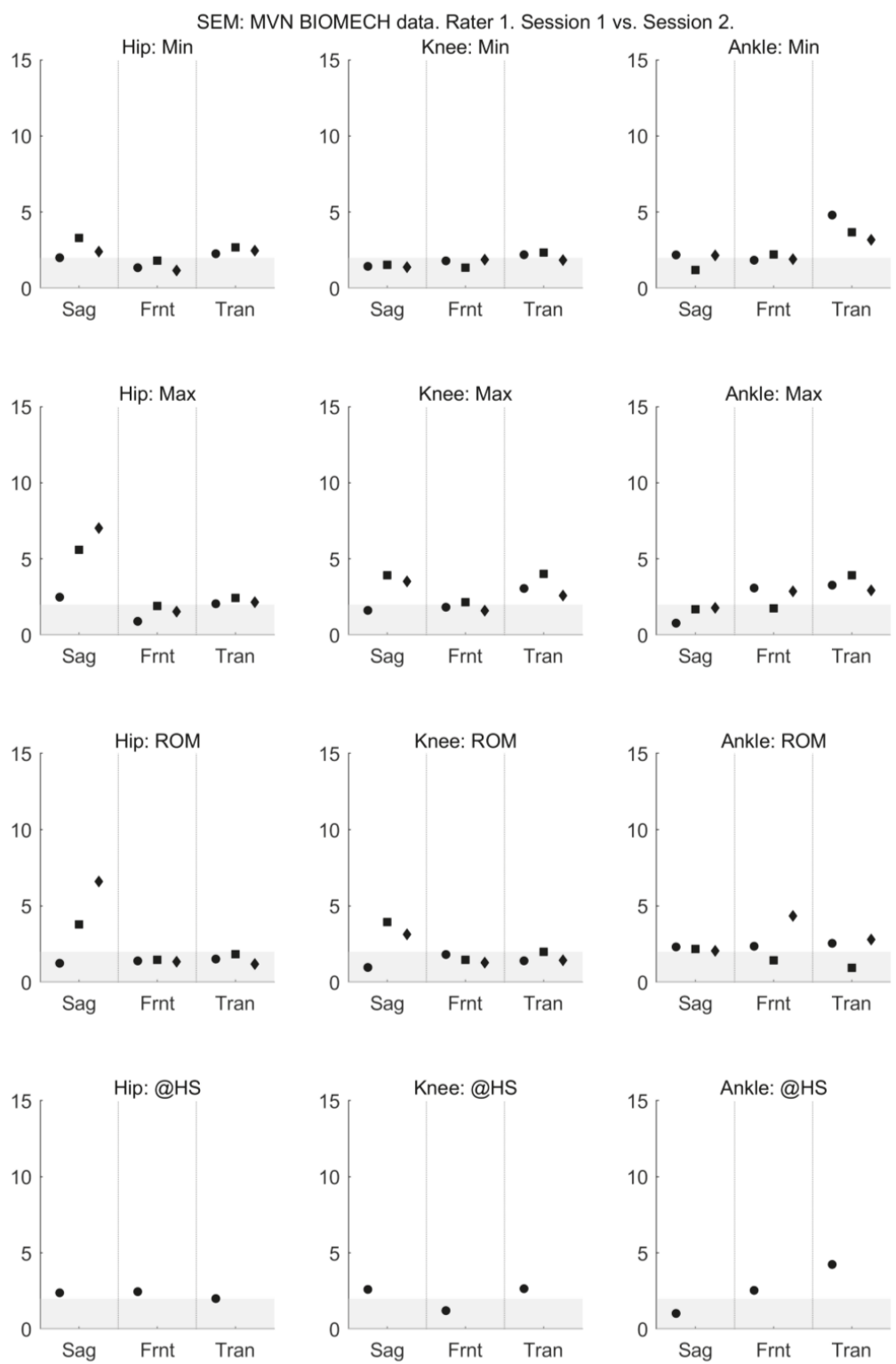

Figure 2. Between-session (within-rater) standard error of measurement of MVN data collected by rater 1 (musculoskeletal physiotherapist) in sessions 1 and 2. Standard error of measurement is shown for the minimum angle (min; top row), maximum angle (max; second row), range of motion (ROM; third row) and angle at heel strike (@HS; bottom row) for the hip (left-most column), knee (centre column) and ankle (right-most column) joints in the sagittal (Sag), frontal (Frnt) and transverse (Tran) planes of movement. The three data points in each plane correspond to the walk (left-most point; circle), squat (centre point; square) and jump (right-most point; diamond) tasks. Grey shading indicates values considered to indicate excellent reliability. 
For walking and squatting, the between-rater (within-session) reliability of all joint angles provided by MVN BIOMECH was acceptable, with ICC > 0.6 (Figure 3) and SEM < $5^{\circ}$ (Figure 4) across all planes. For jumping, the between-rater (within-session) reliability of joint angles ranged from poor to excellent (Figures 3 and 4).
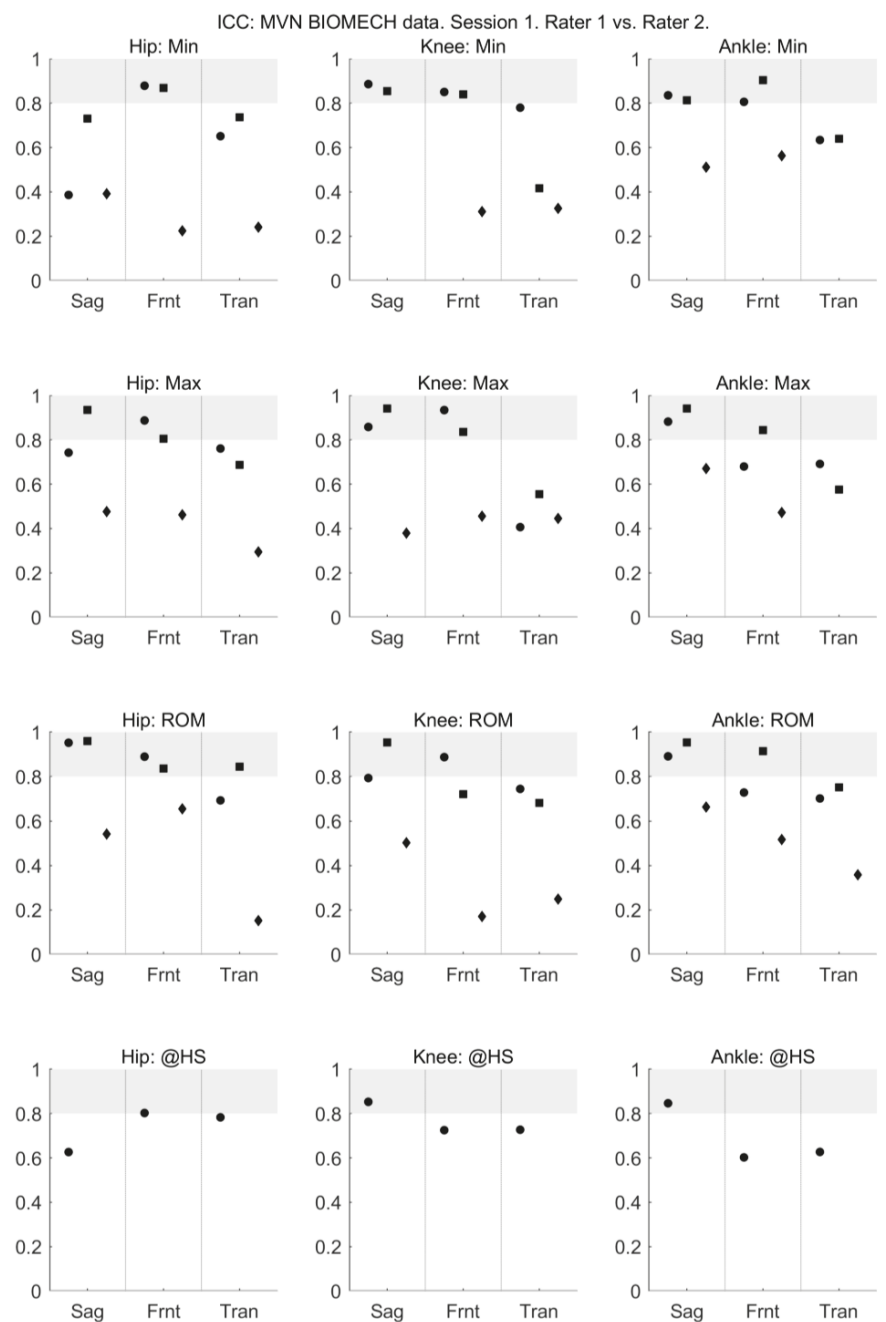

Figure 3. Between-rater intraclass correlation coefficient of MVN data collected by rater 1 (musculoskeletal physiotherapist) and rater 2 (clinical movement scientist) in session 1. Intraclass correlation coefficient is shown for the minimum angle (min; top row), maximum angle (max; second row), range of motion (ROM; third row) and angle at heel strike (@HS; bottom row) for the hip (left-most column), knee (centre column) and ankle (right-most column) joints in the sagittal (Sag), frontal (Frnt) and transverse (Tran) planes of movement. The three data points in each plane correspond to the walk (left-most point; circle), squat (centre point; square) and jump (right-most point; diamond) tasks. Grey shading indicates values considered to indicate excellent reliability. 

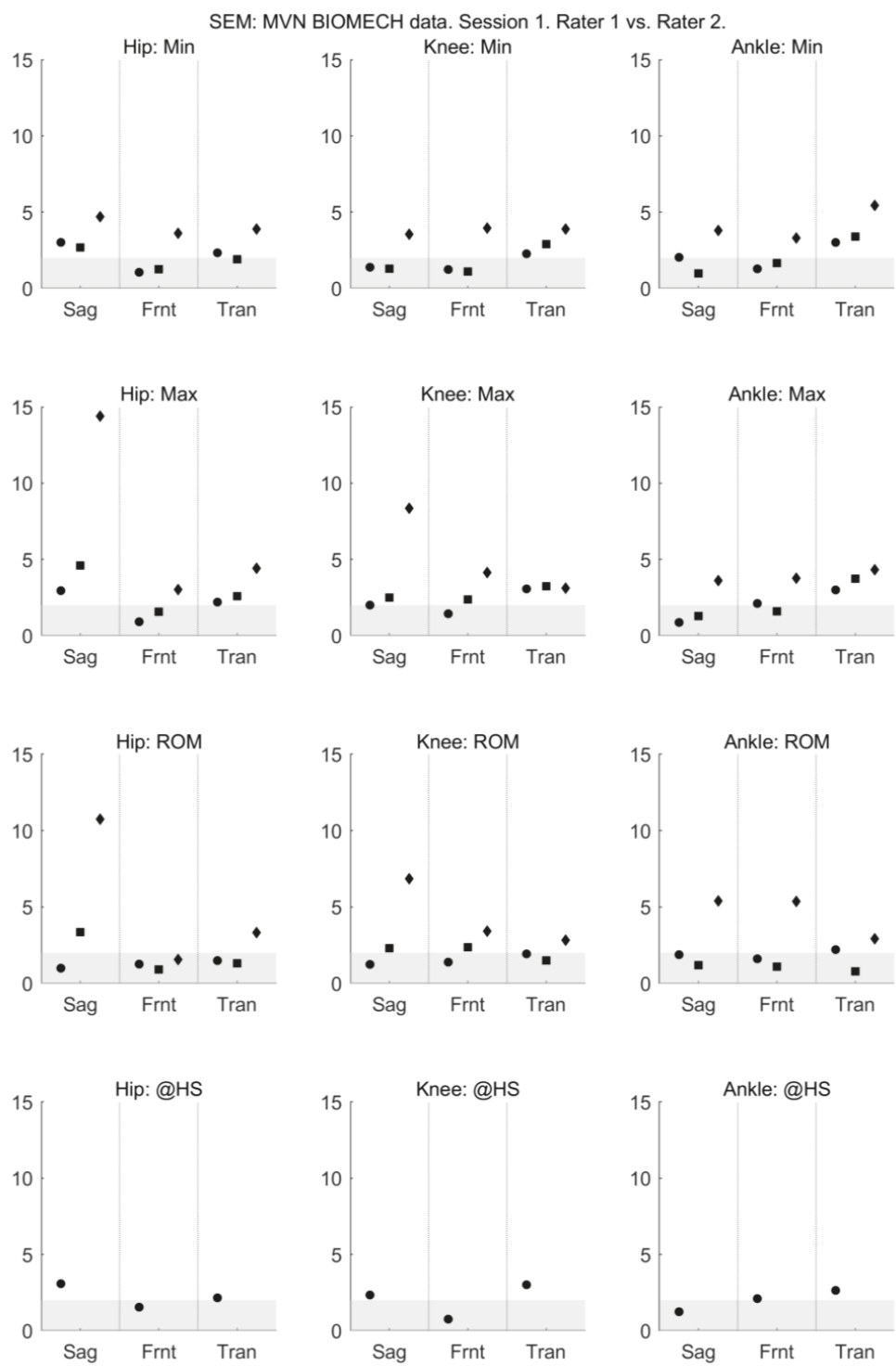

Figure 4. Between-rater standard error of measurement of MVN data collected by rater 1 (musculoskeletal physiotherapist) and rater 2 (clinical movement scientist) in session 1 . Standard error of measurement is shown for the minimum angle (min; top row), maximum angle (max; second row), range of motion (ROM; third row) and angle at heel strike (@HS; bottom row) for the hip (left-most column), knee (centre column) and ankle (right-most column) joints in the sagittal (Sag), frontal (Frnt) and transverse (Tran) planes of movement. The three data points in each plane correspond to the walk (left-most point; circle), squat (centre point; square) and jump (right-most point; diamond) tasks. Grey shading indicates values considered to indicate excellent reliability. 


\subsection{Concurrent Validity}

Figures 5-7 present the joint angle time series' obtained from MVN BIOMECH and VICON systems for each participant during walk, squat, and jump. Across all three activities there appears to be good similarity between the pattern of sagittal and frontal joint angles provided by the two systems. However, an offset in the absolute values provided by the two systems is evident, especially for the hip in the sagittal plane (Figure 5) and the knee and ankle in the transverse plane (Figure 7).
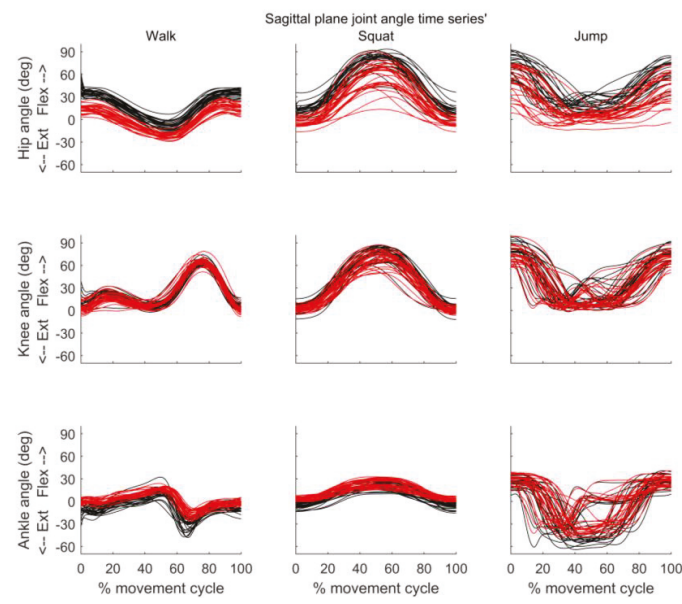

Figure 5. Sagittal plane joint angles throughout the movement cycle for each participant. Time series of hip (top row), knee (middle row) and ankle (bottom row) joint angles obtained from VICON (black) and MVN BIOMECH (red) systems for each participant during walk (left), squat (centre), and jump (right). Y-axis represents joint angles in degrees and $\mathrm{X}$-axis represent the movement cycle in percentage.

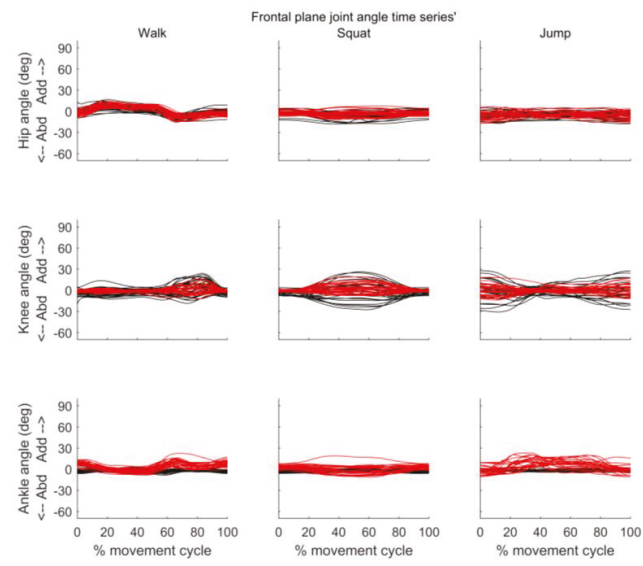

Figure 6. Frontal plane joint angles throughout the movement cycle for each participant. Time series of hip (top row), knee (middle row) and ankle (bottom row) joint angles obtained from VICON (black) and MVN BIOMECH (red) systems for each participant during walk (left), squat (centre), and jump (right). Y-axis represents joint angles in degrees and $\mathrm{X}$-axis represent the movement cycle in percentage. $\mathrm{Y}$-axis scale is the same as in Figure 5 to allow comparison. 

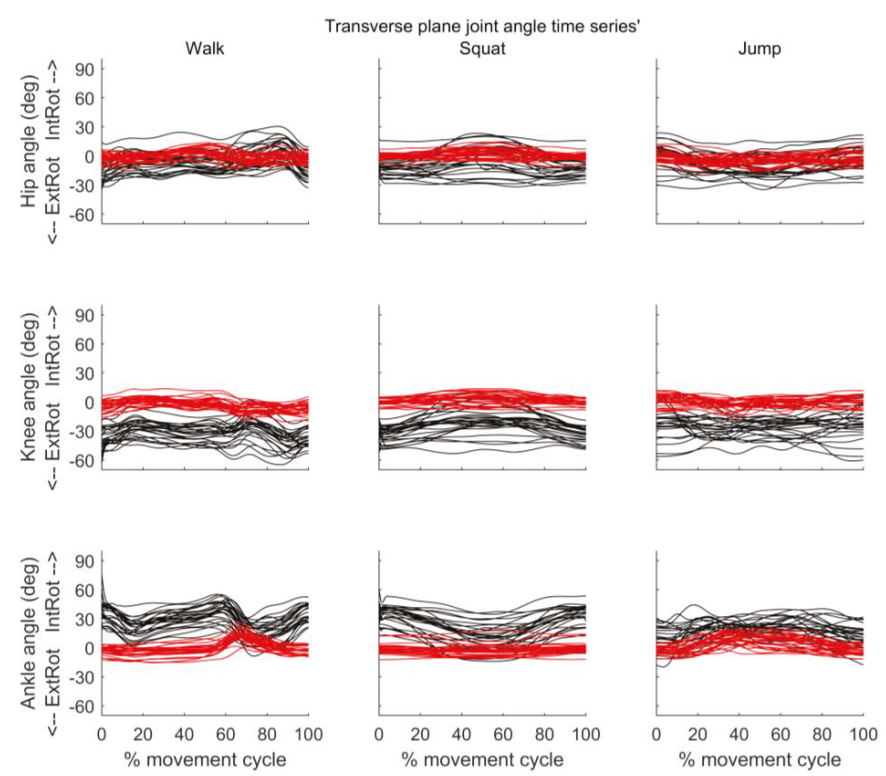

Figure 7. Transverse plane joint angles throughout the movement cycle for each participant. Time series of hip (top row), knee (middle row) and ankle (bottom row) joint angles obtained from VICON (black) and MVN BIOMECH (red) systems for each participant during walk (left), squat (centre), and jump (right). $Y$-axis represents joint angles in degrees and $X$-axis represent the movement cycle in percentage. $Y$-axis scale is the same as in Figures 5 and 6 to allow comparison.

The difference in joint angle provided by the MVN BIOMECH and VICON systems at discrete, time points is shown in Figure 8.
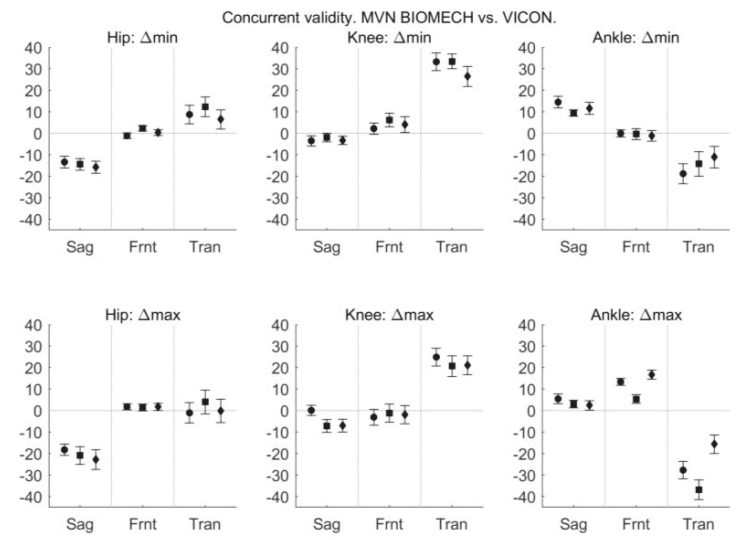

Figure 8. Cont. 

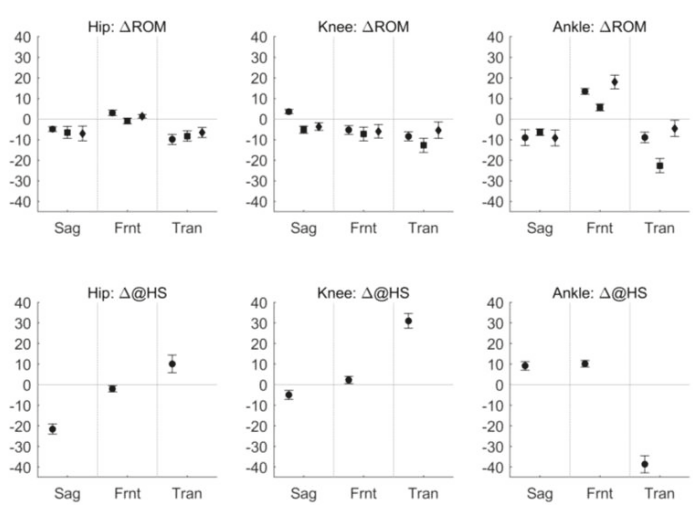

Figure 8. Difference between MVN BIOMECH and VICON data at discrete, clinically relevant events. The difference in minimum angle ( $\Delta$ min; top row), maximum angle ( $\Delta$ max; second row), range of motion ( $\triangle \mathrm{ROM}$; third row) and angle at heel strike ( $\triangle @ \mathrm{HS}$; bottom row) between the MVN BIOMECH and VICON systems for the hip (left), knee (centre) and ankle (right) joints in the sagittal (Sag), frontal (Frnt) and transverse (Tran) planes of movement. The three data points in each plane correspond to the walk (left-most point; circle), squat (centre point; square) and jump (right-most point; diamond) tasks. Positive values indicate that MVN BIOMECH angle was larger than VICON angle. Error bars indicate $95 \%$ confidence interval of the difference.

The similarity between the joint angle waveforms obtained from the MVN BIOMECH and VICON systems during walk, squat, and jump was evaluated using CMC and $R^{2}$ provided by the LFM. CMC indicated excellent similarity between MVN BIOMECH and VICON systems $\left(\mathrm{CMC}_{2}>0.9\right)$ for all three joints in the sagittal plane in all functional activities, and excellent similarity for the hip joint in the frontal plane during walking (Figure 9). CMC is not reported for other planes and activities because of the presence of non-real numbers (see Section 2.6.2).

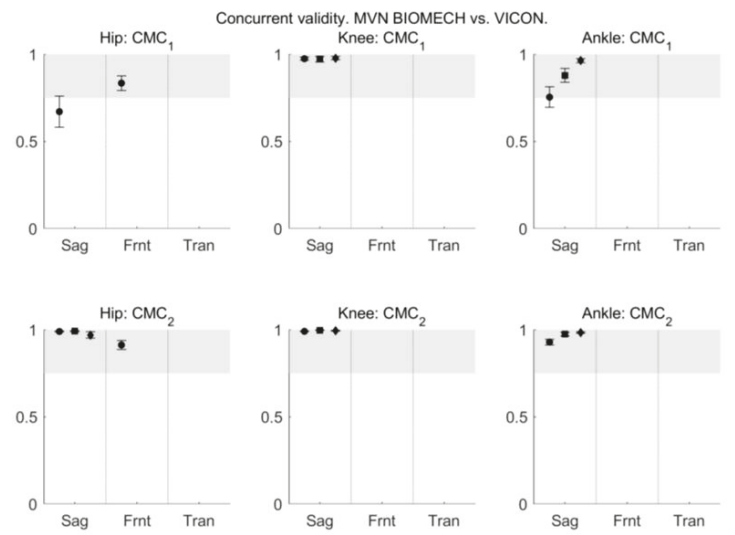

Figure 9. The coefficient of multiple correlation (CMC) between MVN BIOMECH and VICON data before $\left(\mathrm{CMC}_{1}\right.$; top row) and after $\left(\mathrm{CMC}_{2}\right.$; bottom row) offset removal for the hip (left), knee (centre) and ankle (right) joints in the sagittal (Sag), frontal (Frnt) and transverse (Tran) planes of movement. $\mathrm{CMC}$ is reported only for joints and planes where all values are real numbers [39]. Where there are three data points in each plane they correspond to the walk (left-most point; circle), squat (centre point; square) and jump (right-most point; diamond) tasks. Where there is only one data point it corresponds to the walk task. Error bars indicate $95 \%$ confidence intervals. 
$R^{2}$ provided by the LFM indicated excellent similarity between MVN BIOMECH and VICON systems $\left(R^{2}>0.8\right)$ for sagittal plane angles for all joints across all activities, and fair-to-good similarity $\left(R^{2} 0.4-0.8\right)$ for transverse and frontal plane angles for all joints during squat and jump and knee joint during walking (Figure 10). The similarity between the two systems was poor for transverse plane hip and ankle joint angles during walking and frontal plane ankle angle during walking.
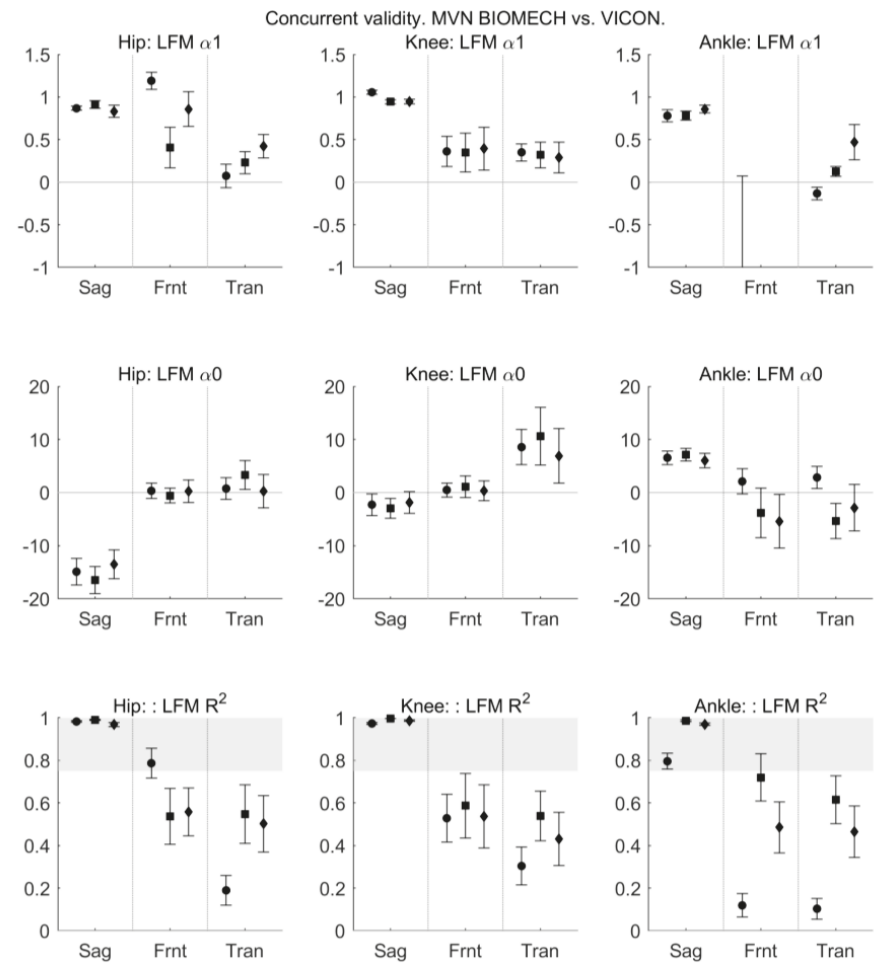

Figure 10. The outcome parameters of linear fit method comparing MVN BIOMECH and VICON data. Outcome parameters are $\alpha 1$ (scaling factor; top row), $\alpha 0$ (scalar addition; middle row) and $R^{2}$ (strength of the linear relation between the two signals; bottom row) for the hip (left), knee (centre) and ankle (right) joints in the sagittal (Sag), frontal (Frnt) and transverse (Tran) planes of movement. The three data points in each plane correspond to the walk (left-most point; circle), squat (centre point; square) and jump (right-most point; diamond) tasks. Error bars indicate $95 \%$ confidence intervals.

\section{Discussion}

This study aimed to quantify the within- and between-rater reliability and the concurrent validity of joint angles provided by the commercially available Xsens MVN BIOMECH system during three clinically relevant functional activities. Within-rater reliability was fair-to-excellent in all three planes for hip, knee, and ankle joint angles in all three tasks when comparing the kinematics obtained on two different testing days, i.e., between sessions. Between-rater reliability (between a MSKP and a clinical movement scientist) was fair-to-excellent in all three planes during walking and squatting, and poor-to-high during jumping. Concurrent validity (agreement between the Xsens MVN BIOMECH system and VICON Plug-in Gait, a camera-based motion capture system) was excellent in the sagittal plane for hip, knee, and ankle joint angles in all three tasks and acceptable in frontal and transverse planes in squat and jump. These results indicate that, in the present study, the MVN BIOMECH 
system was reliably used by an experienced clinician with no prior experience of biomechanical motion capture to quantify lower-limb joint angles in clinically relevant movements. The implications of this for transition of this technology to the clinic are discussed below.

\subsection{Reliability}

For walking, the between- and within-rater reliability of discrete kinematic parameters provided by the MVN BIOMECH system was fair to excellent. The ICC and SEM values are similar to published values for camera-based systems [38,42-45]. Most reports of camera-based systems have not reported the reliability of ankle angles in the transverse and frontal planes; however, our results for ankle ROM and maximum ankle angle in the transverse plane during walking are comparable to those of Meldrum et al. [46]. According to a systematic review [38], between-rater ICC values for kinematic variables obtained from camera-based systems across joints in sagittal and frontal planes ranged from 0.5 to 0.99 . We report ICC values for the MVN BIOMECH system of between 0.65 and 0.99 , with a relatively small $\operatorname{SEM}\left(<3.0^{\circ}\right)$ for all joints, planes and tasks, with the exception of minimum hip angle in the sagittal plane (ICC 0.39 and SEM $3.0^{\circ}$ ). This suggests that between- and within-rater reliability of kinematic variables obtained from the MVN BIOMECH system across joints and planes is comparable to or better than those obtained from optoelectronic motion capture systems [38,42-46].

For squatting, the between-rater reliability of kinematic parameters was fair-to-excellent for all parameters across planes. The within-rater reliability was good-to-excellent for all parameters except maximum hip angle in the sagittal plane, where SEM reached $5.5^{\circ}$. For sagittal plane angles, this within-rater reliability compares well to published values for a camera-based system [47]. Between- and within-rater reliability of joint angles in the frontal and transverse planes have not been reported for camera-based systems.

For jumping, the within-rater reliability of kinematic parameters in the sagittal and frontal planes was good-to-excellent, except for maximum hip angle in the sagittal plane, where SEM reached $7^{\circ}$, and the within-rater reliability of kinematic parameters in the transverse plane was fair-to-excellent. The within-rater reliability was slightly higher than that reported for drop jumps captured with a camera-based system $[48,49]$.

For jumping, the between-rater reliability of kinematic parameters was fair for the ankle and hip joints in sagittal, frontal, and transverse planes, and slightly worse for the knee joint. The performance of a dynamic task like jumping may have considerable variability across trials [50], and the poor between-rater reliability across planes and joints observed in the jump might be a true reflection of changes in performance, rather than any deficit of the motion capture system. To help us evaluate whether the poor between-rater reliability was due to the motion capture system or true variability in performance we evaluated the reliability of joint angles obtained from the camera-based motion capture system (VICON) in the first and second half of the session. The markers were not moved between the first and second half of the session; however, ICC was between 0.25 and 0.75 (Appendix B, Figures 1 and 2). This indicates that low between-rater reliability observed with the MVN BIOMECH system is likely due to true variability in performance, and is not a deficit of the motion capture system.

\subsection{Concurrent Validity}

The high $\mathrm{CMC}_{2}$ and LFM $R^{2}$ values in the sagittal plane indicate that, in this plane of movement, the joint angle waveforms obtained from the MVN BIOMECH system were similar to those obtained from the VICON system. This supports previous work in healthy participants, which also found a high similarity of joint angle waveforms in the sagittal plane between MVN BIOMECH and an Optotrak motion capture system [18,19], and studies that compared kinematic data estimated from Xsens IMUs using custom-developed techniques to an optoelectronic motion capture system [10,13]. Our results extend these previous findings by including a more challenging dynamic task; the vertical jump. The high similarity between MVN BIOMECH and VICON joint angle waveforms in the jump task indicates that the impact that occurs upon initial contact with the ground when landing from the jump 
did not adversely affect the kinematics provided by the MVN BIOMECH system, and suggests that the MVN BIOMECH system can be used in a dynamic task such as jumping.

Previous studies have reported CMC values for data in frontal and transverse planes [10,18,19], but for the majority of joints and activities we were not able to report CMC in these planes. In line with Ferrari et al. [10], we only report CMC values for joints and planes where all values were real numbers. In the frontal and transverse planes, $\mathrm{CMC}$ was a non-real number for at least one participant for most joints and activities. This is likely because of small range of motion in these planes for the activities studied. An alternative measure of similarity between waveforms can be obtained from the LFM $R^{2}$ value [41]. There was a good-to-strong relation between frontal plane data from the two systems for the hip during walking and the ankle during squatting, and a moderate relation in frontal and transverse planes for the hip during squatting and jumping, the knee during all activities, and the ankle during jumping. These results are in line with previous reports of a moderate-to-strong relation between waveforms obtained from the MVN BIOMECH system and an optoelectronic system for hip and knee angles during walking [19], stair ascent and descent [19], and manual handling tasks [18]. We found a poor relation $\left(R^{2}=0.1\right)$ for the ankle in the transverse and frontal planes during walking, which may be due to the small range of motion in these planes or differences in the anatomical biomechanical definitions between the two systems.

A challenge when validating any new technology is the choice of 'gold-standard' against which to validate. This is particularly relevant in movement analysis where there are several biomechanical models available. We chose to validate against the conventional gait model, which is commercially implemented as Plug-in Gait in VICON Nexus software. One limitation of this model is the effect of errors in markers placement on calculated joint angles [51-55]. For example, a small error in the placement of the thigh maker, which is used to define the internal/external rotation of the femur about the line between the hip joint centre, causes appreciable errors in knee joint kinematics, particularly in the frontal and transverse planes (cf. Figure 11.3; [55]). The high values of knee external rotation obtained from Plug-in Gait in this study (see Figure 7) appear non-physiological, and likely indicate errors in thigh marker placement. There are similar potential effects of errors in shank marker placement on the ankle joint kinematics. The validity results, particularly in the frontal and transverse planes, must be interpreted in light of this limitation. The influence of errors in thigh marker placement can be minimised by using a medial epicondyle marker or knee alignment device, and those of errors in the shank marker placement by using a medial malleolus marker, and future studies may consider these techniques. The CMC values for ankle and hip joint angles in frontal and transverse planes during walking in the current study are lower than those reported by Ferrari et al. [10] and Zhang et al. [19]. In addition to the potential influence marker placement errors, this may be impacted by the biomechanical models used by the systems under comparison. Ferrari et al. [10] compared joint angles obtained from IMUs used with a biomechanical model called Outwalk to those obtained from an optoelectronic system (VICON) used with the Calibration Anatomical System Technique [56]. Our comparison for IMU data was VICON Plug-in Gait. Plug-in Gait estimates joint angles based on Kadaba et al. [26], using segment frames that are constructed from anatomical locations identified by optical markers. By contrast, MVN BIOMECH determines segment frames and changes in body posture based on the segment orientations related to an initial neutral pose (the calibration posture), and calculates joint angles directly from the measured segment orientations. The similarity between biomechanical models used by VICON Plug-In-Gait and MVN BIOMECH is lower than that between the two systems used by Ferrari et al. [10], and this may contribute to the lower waveform similarity.

In the current study, there was a difference (offset) in joint angles provided by the MVN BIOMECH and VICON systems that was particularly noticeable for hip flexion/extension and knee and ankle internal/external rotation. This was constant across participants and activities (see Figures 5 and 7 and small confidence intervals in Figure 8), indicating that it is more likely attributable to differences in the biomechanical models, errors in marker placement or deviation of the body position (posture) during calibration from that required by the system than technical issues such as magnetic field. 
Another possible explanation is that as calibration data for the two systems were collected at different points in the quiet stance trial (see Section 2.4), the posture of the participant may have been slightly different. However, as the data were collected in the same trial, and the participant did not visibly move throughout this trial, we believe that any difference in posture would have been small.

In summary, there was good similarity in joint angle waveforms between the MVN BIOMECH system and VICON Plug-In Gait for all activities, across all joints and planes of motion, with the exception of the ankle joint during walking in the frontal and transverse planes. Despite the similarity in waveforms, the absolute values reported at discrete time points were not similar, particularly at hip and ankle joints. This is because an offset exists between the systems, likely as a result of different biomechanical models employed by the two systems and/or errors in marker placement. This means that the kinematic outputs from the two systems cannot be used interchangeably; for example, hip flexion cannot be compared between one individual who was measured with the MVN BIOMECH system and another individual who was measured with the VICON Plug-in Gait system.

\subsection{Limitations}

Participants in this study were able-bodied adults who attended a single laboratory. Further research is needed to assess the within- and between-rater reliability of the MVN BIOMECH system in pathological populations. The retroreflective markers were placed before the MTw2 trackers, and were not removed until the end of the session (see Section 2.3). This means that the markers were present when both raters placed the MTw2 trackers. The instructions for placement of the MTw2 trackers do not reference anatomical landmarks, therefore we considered the potential impact of the markers being present to be minimal; however, it remains possible that the markers influenced the attachment of the trackers, and thus influenced the inter-rater reliability. We did not use either a medial knee marker or knee alignment device to minimise errors in knee joint angles caused by possible inaccurate thigh marker placement. Our results must be interpreted in with this in mind. Finally, to provide a measure of how repeatable the system is in a clinical environment, participants were not provided with any specific instructions on how to perform the task. This resulted in performance strategy that varied across trials, particularly for the jump. Low reliability in this task is likely influenced by true variations in performance rather than being a reflection of the motion capture system used. This is a clinical strength as the data provide reliability of the system when performance of the task is not constrained.

\subsection{Clinical Implications and Future Research Directions}

Although the reliability and criterion validity of any biomechanical motion analysis system is important, it is not sufficient to recommend transfer of the technology into the clinic. Future work should identify what information is relevant to clinicians, the type of motion capture system that is preferable to clinicians, how the information provided by quantitative motion analysis can best be presented to clinicians, and if such information has any impact on diagnosis or clinical decision making.

\section{Conclusions}

The good between-rater reliability for walk and squat demonstrate that the MVN BIOMECH system provided joint kinematics that were independent of the rater. This is important as the two raters had different backgrounds and expertise: one was a clinically experienced MSKP with no experience of biomechanical motion analysis and the other was an experienced clinical movement scientist. Our results therefore indicate that the data provided by the MVN BIOMECH system are independent of user expertise and the system does not need to be used by an experienced movement scientist. The good within-rater reliability supports the use of this system across multiple participants or sessions. There was excellent similarity between joint angle waveforms obtained from the MVN BIOMECH and VICON systems in the sagittal plane, and acceptable similarity in the frontal and transverse planes in all three tasks. This extends previous reports and indicates that MVN BIOMECH 
system can be used in a dynamic task such as a jump. However, it must be noted that the MVN BIOMECH and VICON Plug-in Gait systems cannot be used interchangeably. Together, these results indicate that the commercially available MVN BIOMECH system is suited for clinical movement analysis in clinical practice. Future work should evaluate reliability across centres and in pathological populations, and explore the utility of the information that can be provided by this system to clinicians.

Acknowledgments: The authors would like to thank the volunteers for their time, enthusiasm, and feedback. J.D. and K.N. are funded by Arthritis Research UK (Grant No. 18461).

Author Contributions: M.A.-A. conceived the initial study design and all authors contributed to the final study design. M.A.-A. and K.N. recruited and enrolled participants and collected data. M.A.-A., K.N. and J.D. processed the data. M.A.-A. and J.L.D. analysed the data and produced figures and tables. M.A.-A., K.N., K.B., L.S. and J.L.D. interpreted the data. M.A.-A. and J.L.D. drafted the manuscript. All authors read and approved the final manuscript.

Conflicts of Interest: The MTw2 hardware and MVN BIOMECH software were provided on a temporary basis at no cost by Xsens Technologies (B.V., The Netherlands). The authors received no financial contribution from Xsens Technologies. Training on how to use the MTw2 hardware and MVN BIOMECH software was provided by Xsens Technologies. The final draft of this article was reviewed by Monique Paulich (Senior Product Specialist in Biomechanics, Xsens Technologies) to ensure accuracy of technical details provided on the MVN BIOMECH system. Xsens Technologies had no input on the data interpretation, data analysis, or manuscript writing. None of the authors have any financial interest in Xsens Technologies. 


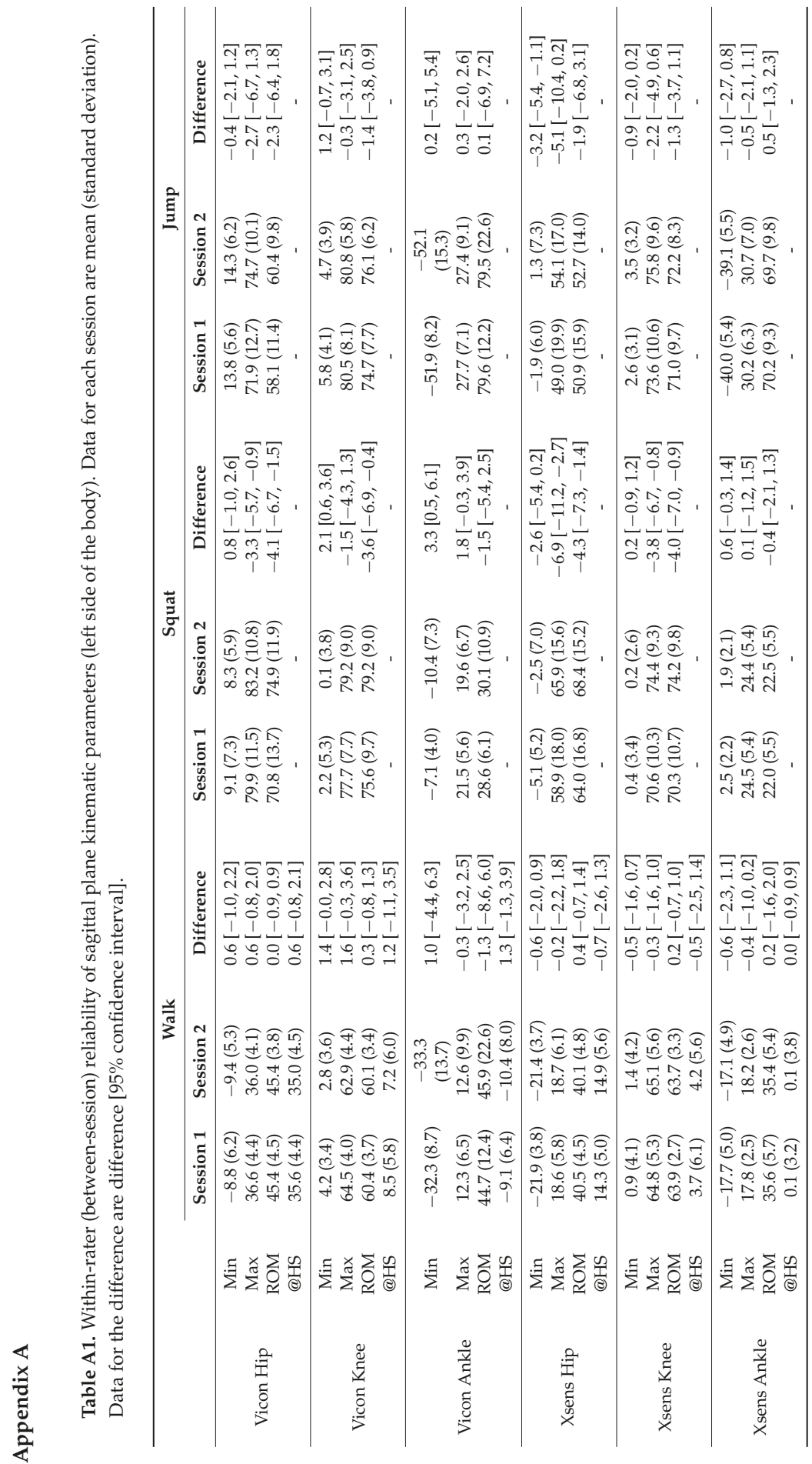




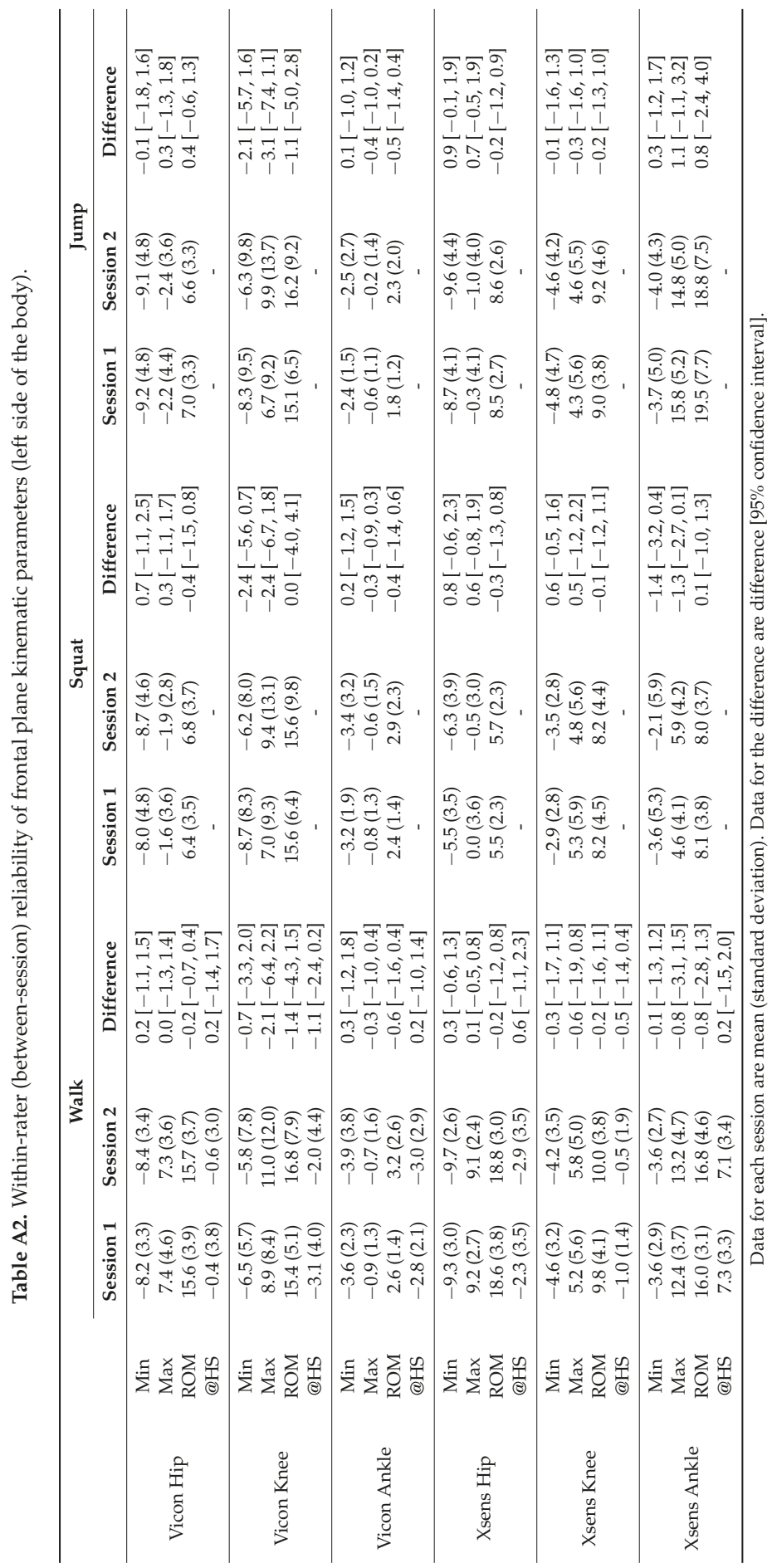




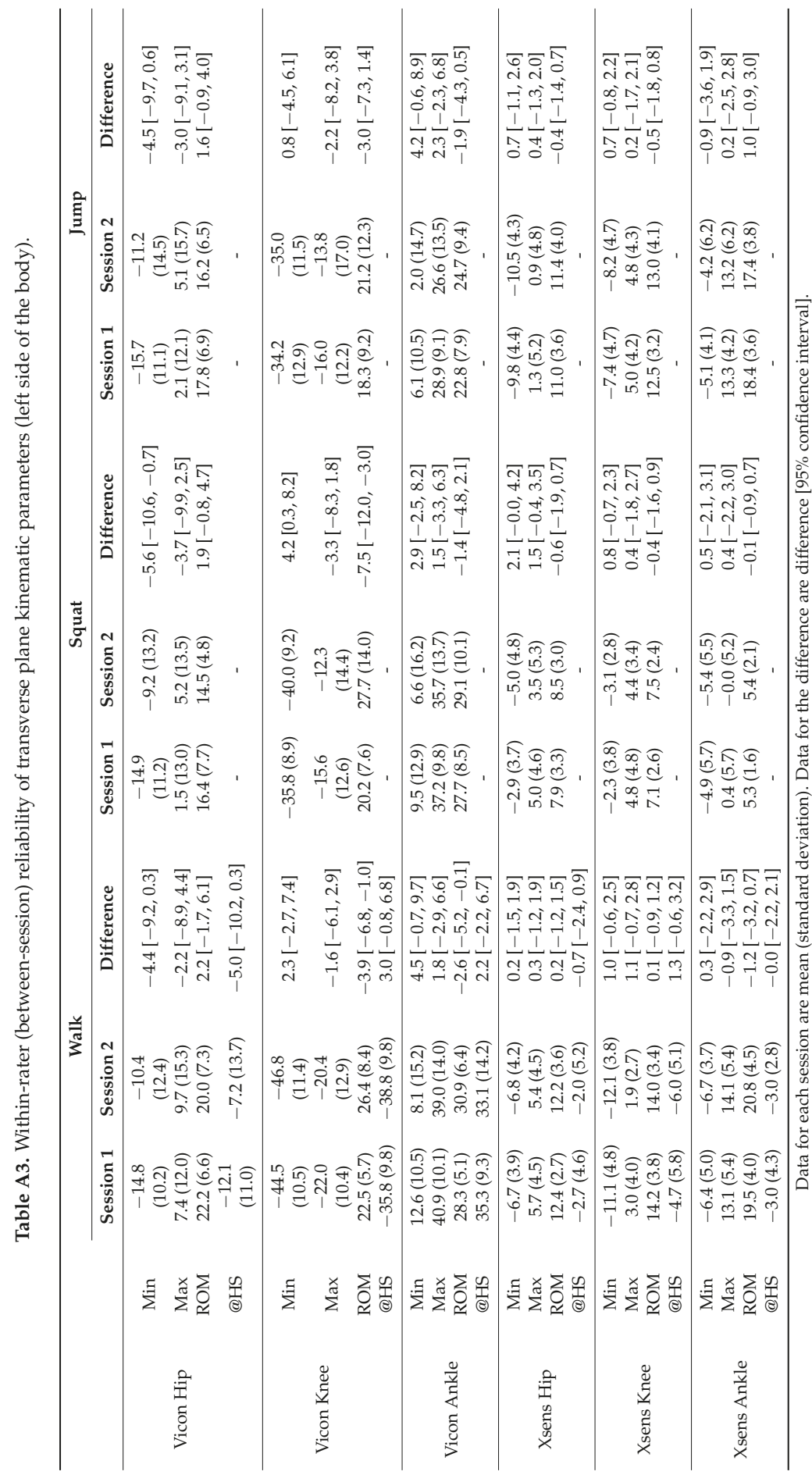




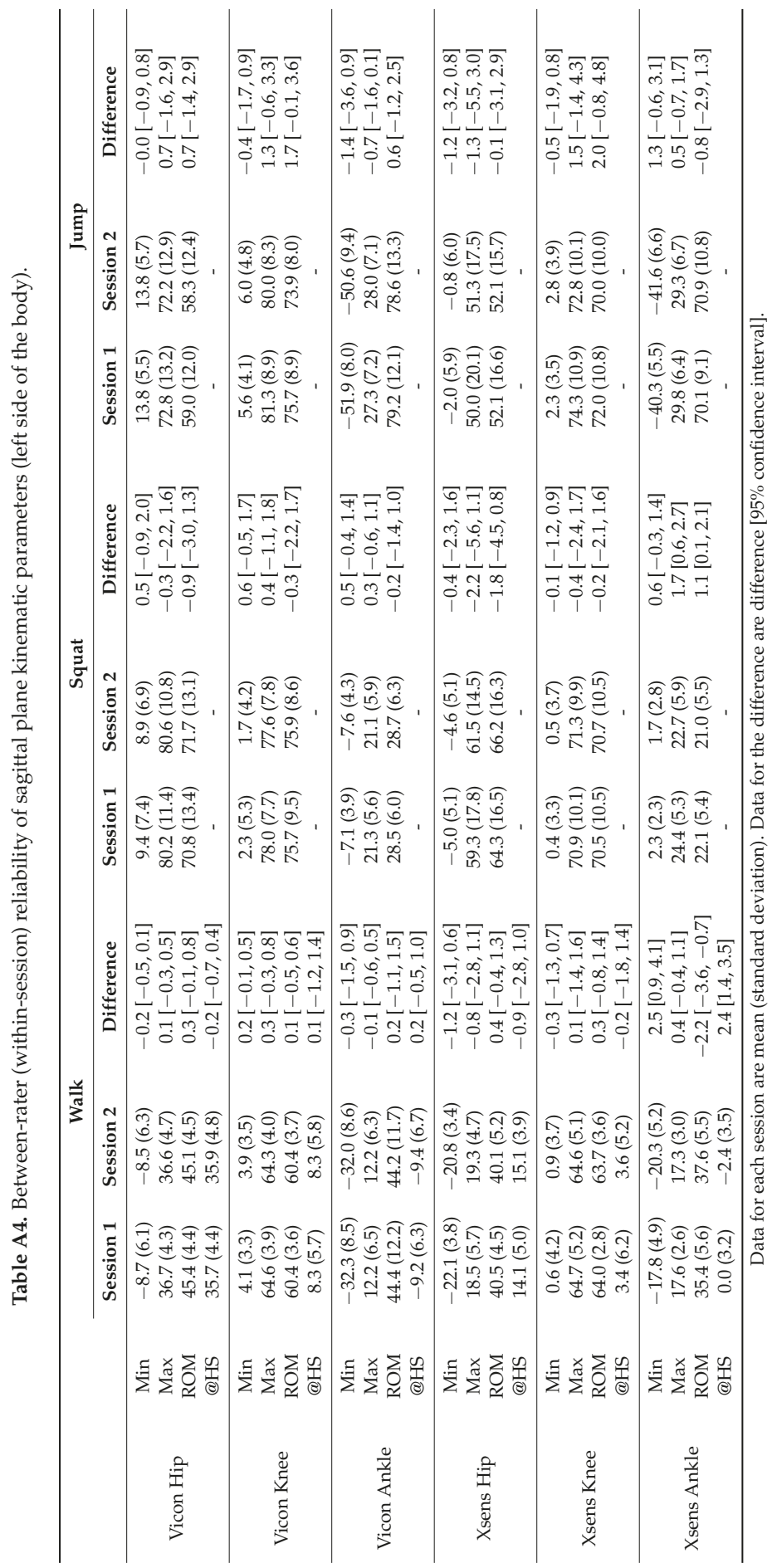




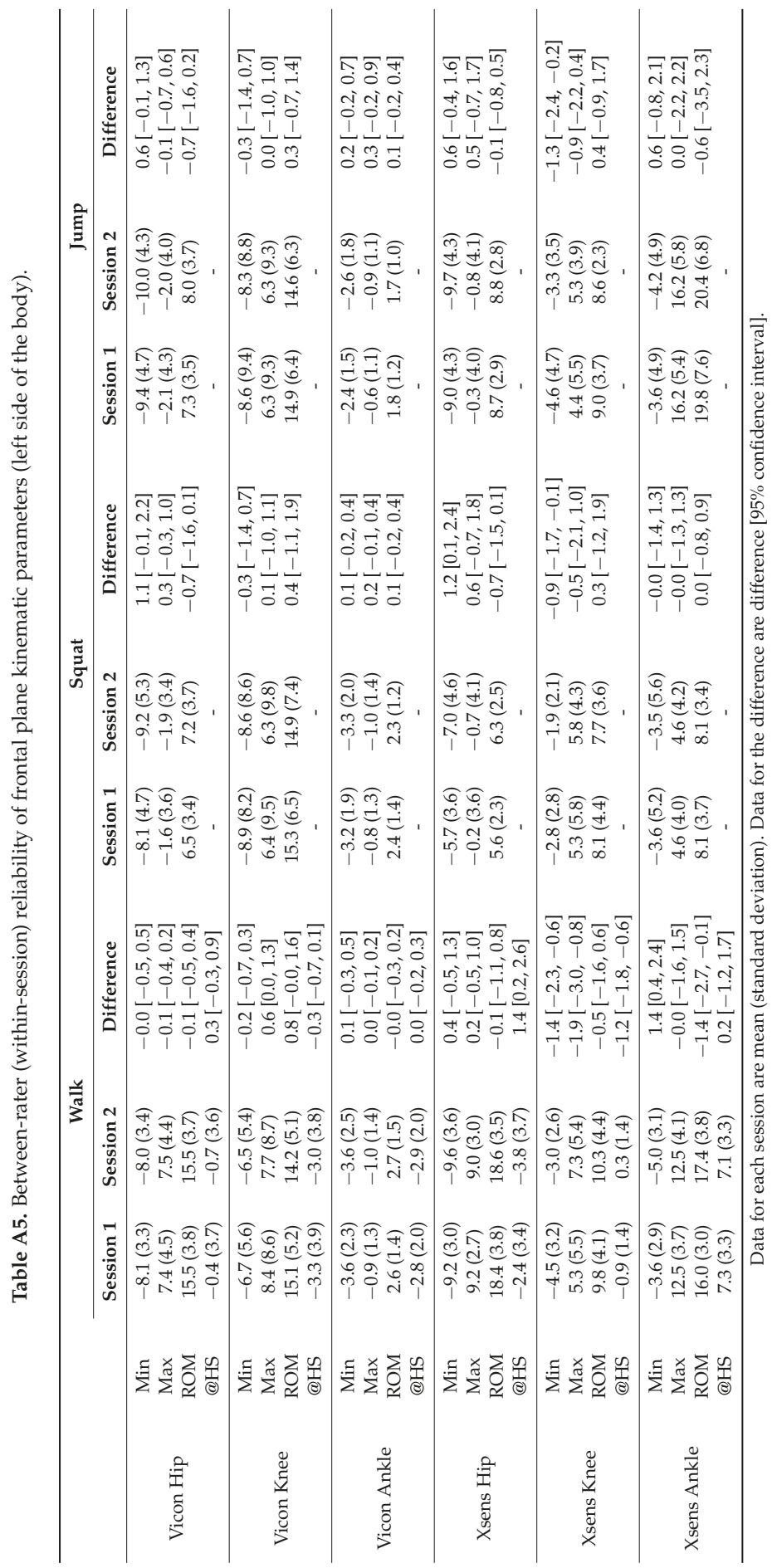




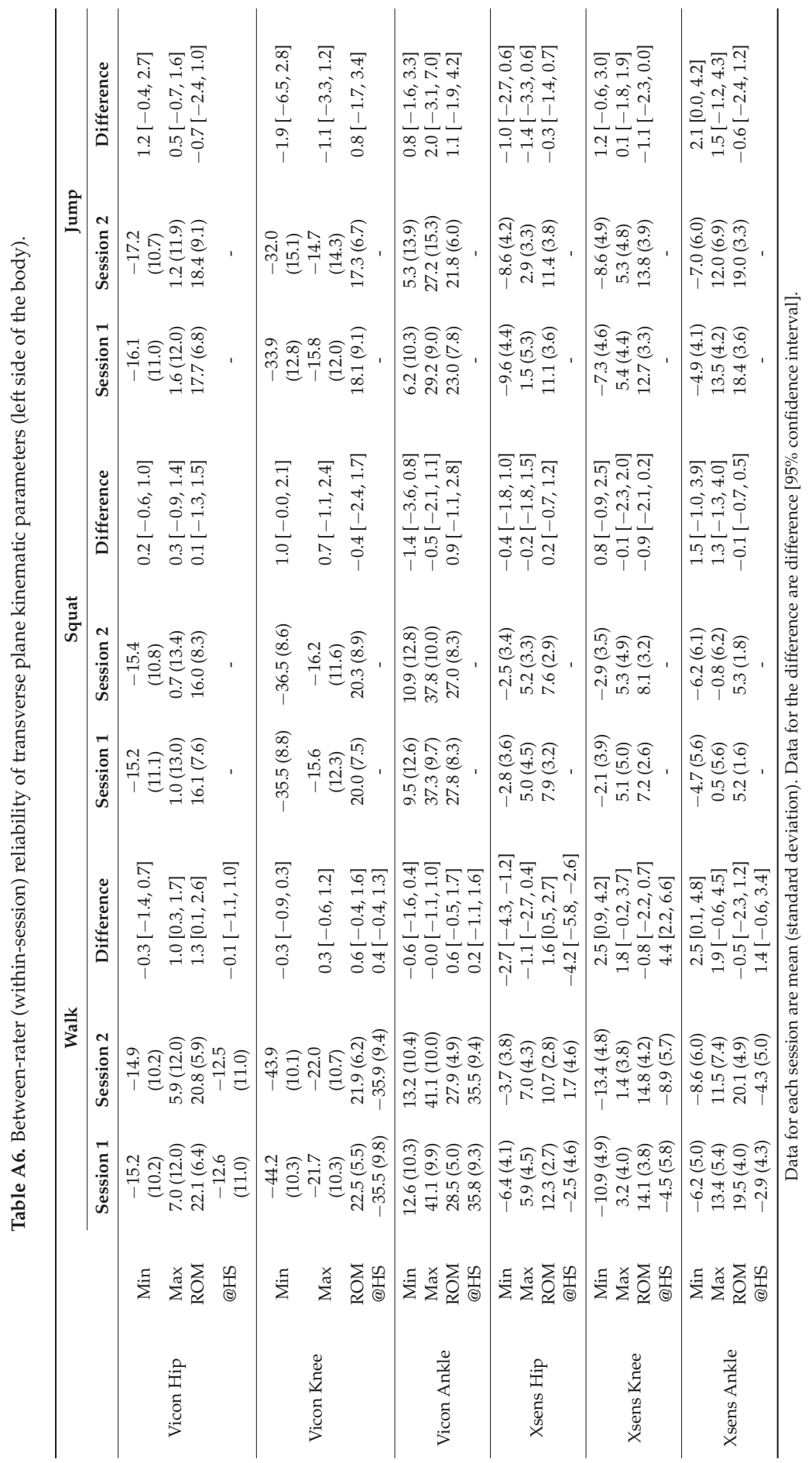


B.
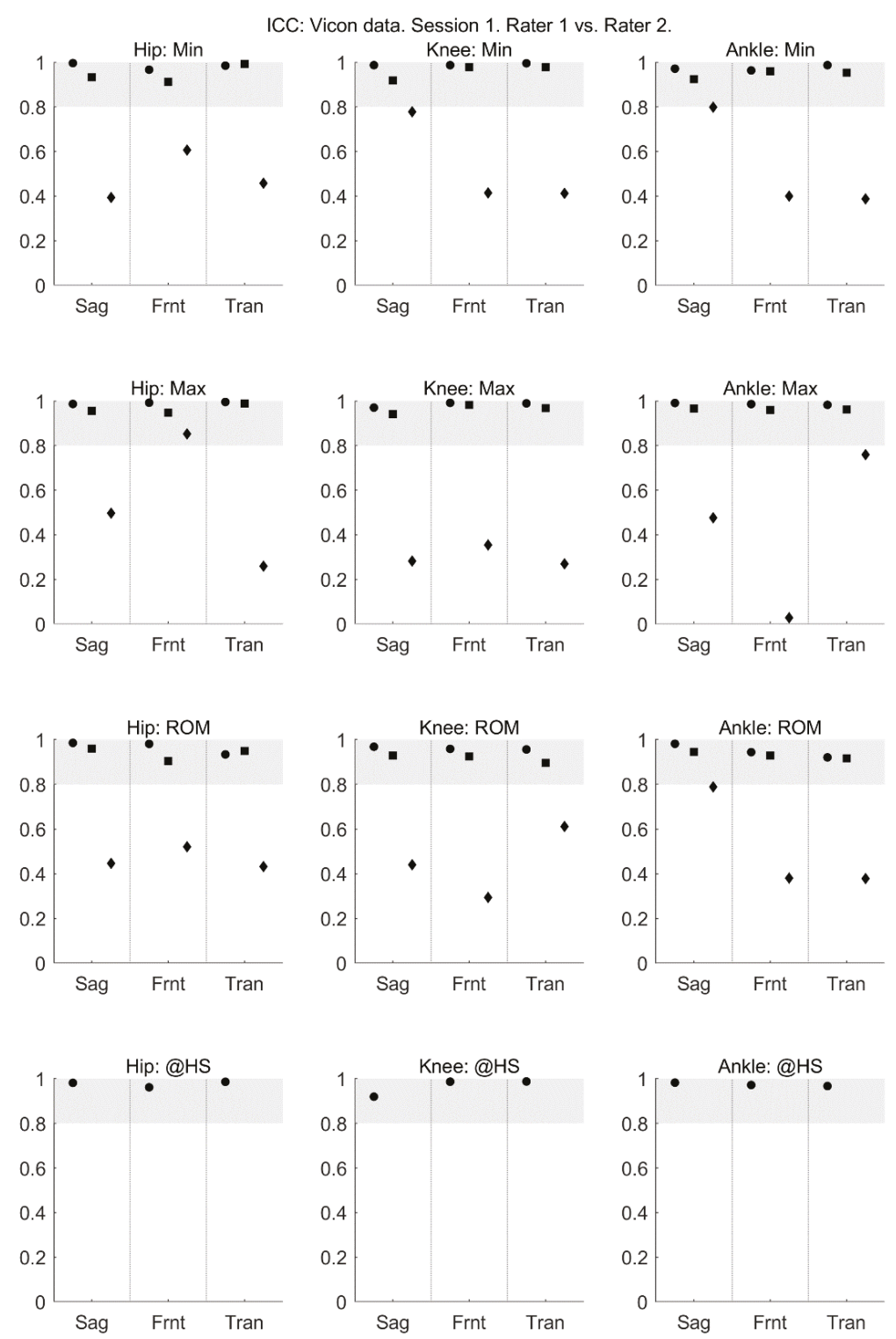

Figure 1. Within-session intraclass correlation coefficient of VICON data collected in session 1. Intraclass correlation coefficient is shown for the minimum angle (min; top row), maximum angle (max; second row), range of motion (ROM; third row) and angle at heel strike (@HS; bottom row) for the hip (left-most column), knee (centre column) and ankle (right-most column) joints in the sagittal (Sag), frontal (Frnt) and transverse (Tran) planes of movement. The three data points in each plane correspond to the walk (left-most point; circle), squat (centre point; square) and jump (right-most point; diamond) tasks. Grey shading indicates values considered to indicate excellent reliability. 


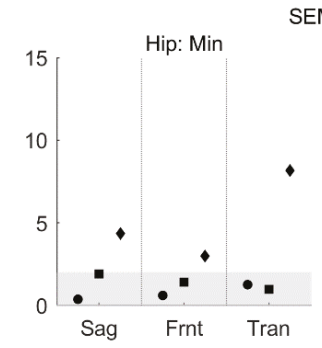

SEM: Vicon data. Session 1. Rater 1 vs. Rater 2.
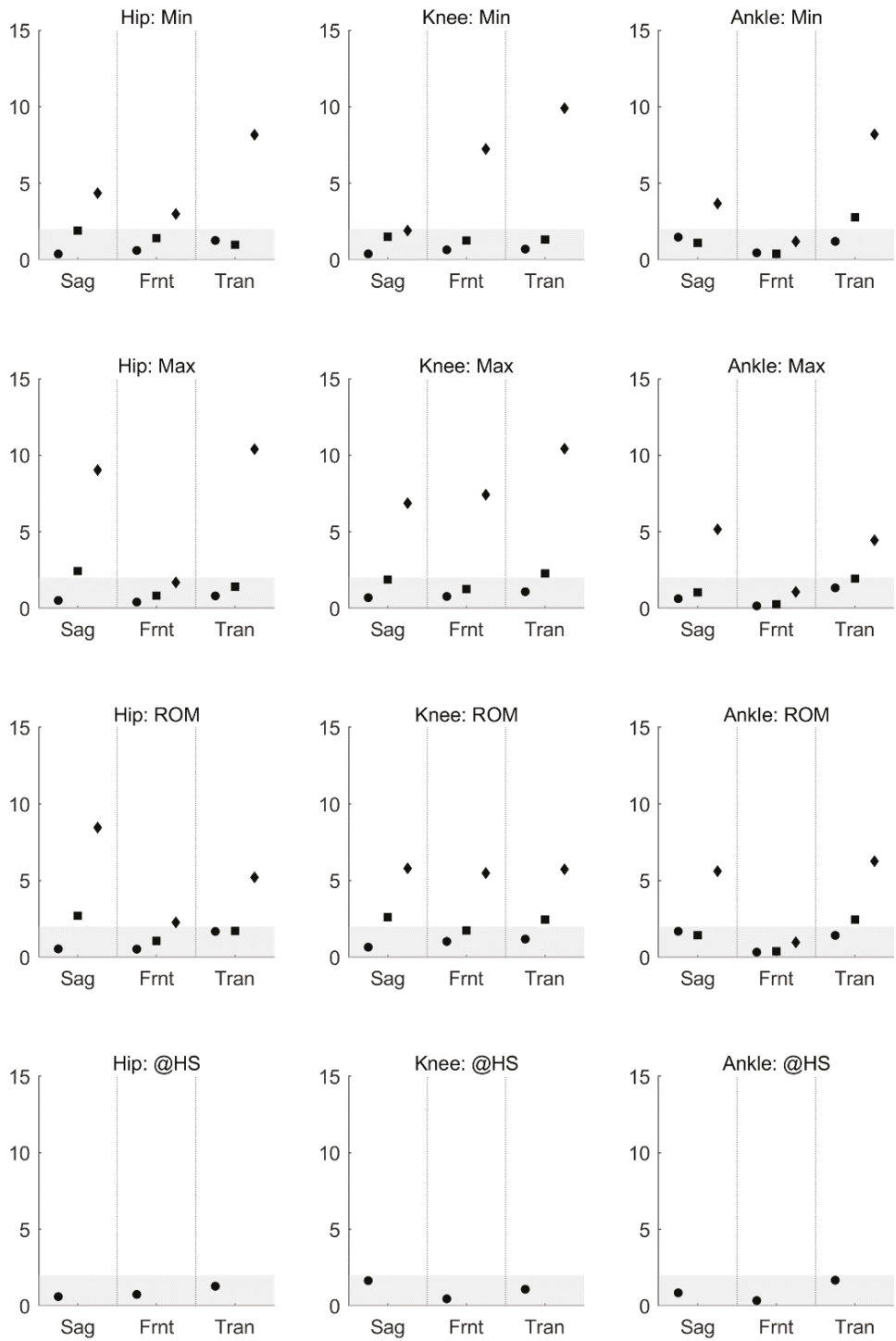

Figure 2. Within-session standard error of measurement of VICON data collected in session 1. Standard error of measurement is shown for the minimum angle (min; top row), maximum angle (max; second row), range of motion (ROM; third row) and angle at heel strike (@HS; bottom row) for the hip (left-most column), knee (centre column) and ankle (right-most column) joints in the sagittal (Sag), frontal (Frnt) and transverse (Tran) planes of movement. The three data points in each plane correspond to the walk (left-most point; circle), squat (centre point; square) and jump (right-most point; diamond) tasks. Grey shading indicates values considered to indicate excellent reliability. 


\section{References}

1. Whatman, C.; Hing, W.; Hume, P. Physiotherapist agreement when visually rating movement quality during lower extremity functional screening tests. Phys. Ther. Sport 2012, 13, 87-96. [CrossRef] [PubMed]

2. Baker, R. Gait analysis methods in rehabilitation. J. Neuroeng. Rehabil. 2006, 3, 4. [CrossRef] [PubMed]

3. Sabatini, A.M. Variable-state-dimension Kalman-based filter for orientation determination using inertial and magnetic sensors. Sensors 2012, 12, 8491-8506. [CrossRef] [PubMed]

4. Sabatini, A.M. Kalman-filter-based orientation determination using inertial/magnetic sensors: Observability analysis and performance evaluation. Sensors 2011, 11, 9182-9206. [CrossRef] [PubMed]

5. Luinge, H.J.; Veltink, P.H. Measuring orientation of human body segments using miniature gyroscopes and accelerometers. Med. Biol. Eng. Comput. 2005, 43, 273-282. [CrossRef] [PubMed]

6. Favre, J.; Aissaoui, R.; Jolles, B.M.; de Guise, J.A.; Aminian, K. Functional calibration procedure for 3D knee joint angle description using inertial sensors. J. Biomech. 2009, 42, 2330-2335. [CrossRef] [PubMed]

7. Favre, J.; Jolles, B.M.; Aissaoui, R.; Aminian, K. Ambulatory measurement of 3D knee joint angle. J. Biomech. 2008, 41, 1029-1035. [CrossRef] [PubMed]

8. Picerno, P.; Cereatti, A.; Cappozzo, A. Joint kinematics estimate using wearable inertial and magnetic sensing modules. Gait Posture 2008, 28, 588-595. [CrossRef] [PubMed]

9. Liu, T.; Inoue, Y.; Shibata, K. Development of a wearable sensor system for quantitative gait analysis. Measurement 2009, 42, 978-988. [CrossRef]

10. Ferrari, A.; Cutti, A.G.; Garofalo, P.; Raggi, M.; Heijboer, M.; Cappello, A.; Davalli, A. First in vivo assessment of "outwalk": A novel protocol for clinical gait analysis based on inertial and magnetic sensors. Med. Biol. Eng. Comput. 2010, 48, 1-15. [CrossRef] [PubMed]

11. Seel, T.; Raisch, J.; Schauer, T. IMU-based joint angle measurement for gait analysis. Sensors 2014, 14, 6891-6909. [CrossRef] [PubMed]

12. Picerno, P. 25 years of lower limb joint kinematics by using inertial and magnetic sensors: A review of methodological approaches. Gait Posture 2017, 51, 239-246. [CrossRef] [PubMed]

13. Van Den Noort, J.C.; Ferrari, A.; Cutti, A.G.; Becher, J.G.; Harlaar, J. Gait analysis in children with cerebral palsy via inertial and magnetic sensors. Med. Biol. Eng. Comput. 2013, 51, 377-386. [CrossRef] [PubMed]

14. Roetenberg, D.; Luinge, H.; Slycke, P. Xsens MVN: Full 6DOF human motion tracking using miniature inertial sensors. Xsens Technol. 2009, 8, 1-7.

15. Godwin, A.; Agnew, M.; Stevenson, J. Accuracy of Inertial Motion Sensors in Static, Quasistatic, and Complex Dynamic Motion. J. Biomech. Eng. 2009, 131, 114501. [CrossRef] [PubMed]

16. Lebel, K.; Boissy, P.; Hamel, M.; Duval, C. Inertial measures of motion for clinical biomechanics: Comparative assessment of accuracy under controlled conditions-Changes in accuracy over time. PLoS ONE 2015, 10. [CrossRef] [PubMed]

17. Lebel, K.; Boissy, P.; Hamel, M.; Duval, C. Inertial measures of motion for clinical biomechanics: Comparative assessment of accuracy under controlled conditions-Effect of velocity. PLoS ONE 2013, 8. [CrossRef] [PubMed]

18. Robert-Lachaine, X.; Mecheri, H.; Larue, C.; Plamondon, A. Validation of inertial measurement units with an optoelectronic system for whole-body motion analysis. Med. Biol. Eng. Comput. 2017, 55, 609-619. [CrossRef] [PubMed]

19. Zhang, J.T.; Novak, A.C.; Brouwer, B.; Li, Q. Concurrent validation of Xsens MVN measurement of lower limb joint angular kinematics. Physiol. Meas. 2013, 34. [CrossRef] [PubMed]

20. Walter, S.D.; Eliasziw, M.; Donner, A. Sample size and optimal designs for reliability studies. Stat. Med. 1998, 17, 101-110. [CrossRef]

21. Xsens Technologies B.V. MVN User Manual: User Guide MVN, MVN BIOMECH MVN Link, MVN Awinda. Available online: https: / / fccid.io/QILMTW2-3A7G6/User-Manual/Users-Manual-2695756 (accessed on 28 June 2017).

22. Vicon Motion System. Plug-in Gait Product Guide-Foundation Notes Revision 2.0. Available online: https://www.vicon.com/downloads/documentation/plug-in-gait-product-guide (accessed on 27 February 2018).

23. Wu, G.; Cavanagh, P.R. ISB Recommendations in the Reporting for Standardization of Kinematic Data. J. Biomech. 1995, 28, 1257-1261. [CrossRef] 
24. Grood, E.S.; Suntay, W.J. A Joint Coordinate System for the Clinical Description of Three-Dimensional Motions: Application to the Knee. J. Biomech. Eng. 1983, 105, 136. [CrossRef] [PubMed]

25. Kadaba, M.P.; Ramakrishnan, H.K.; Wooten, M.E.; Gainey, J.; Gorton, G.; Cochran, G.V.B. Repeatability of Kinematic, Kinetic, and EMG Data in Normal Adult Gait.pdf. J. Orthop. Res. 1989, 7, 849-860. [CrossRef] [PubMed]

26. Kadaba, M.P.; Ramakrishnan, H.K.; Wootten, M.E. Measurement of lower extremity kinematics during level walking. J. Orthop. Res. 1990, 8, 383-392. [CrossRef] [PubMed]

27. Davis, R.B.; Ounpuu, S.; Tyburski, D.; Gage, J.R. A gait analysis data collection and reduction technique. Hum. Mov. Sci. 1991, 10, 575-587. [CrossRef]

28. Button, K.; Roos, P.E.; Van Deursen, R.W.M. Activity progression for anterior cruciate ligament injured individuals. Clin. Biomech. 2014, 29, 206-212. [CrossRef] [PubMed]

29. Roos, P.E.; Button, K.; Van Deursen, R.W.M. Motor control strategies during double leg squat following anterior cruciate ligament rupture and reconstruction: An observational study. J. Neuroeng. Rehabil. 2014, 11. [CrossRef] [PubMed]

30. Roos, P.E.; Button, K.; Sparkes, V.; van Deursen, R.W.M. Altered biomechanical strategies and medio-lateral control of the knee represent incomplete recovery of individuals with injury during single leg hop. J. Biomech. 2014, 47, 675-680. [CrossRef] [PubMed]

31. Button, K.; van Deursen, R.; Price, P. Recovery in functional non-copers following anterior cruciate ligament rupture as detected by gait kinematics. Phys. Ther. Sport 2008, 9, 97-104. [CrossRef] [PubMed]

32. Zeni, J.A.; Richards, J.G.; Higginson, J.S. Two simple methods for determining gait events during treadmill and overground walking using kinematic data. Gait Posture 2008, 27, 710-714. [CrossRef] [PubMed]

33. Mokkink, L.B.; Prinsen, C.A.C.; Bouter, L.M.; de Vet, H.C.W.; Terwee, C.B. The COnsensus-based standards for the selection of health measurement INstruments (COSMIN) and how to select an outcome measurement instrument. Braz. J. Phys. Ther. 2016, 20, 105-113. [CrossRef] [PubMed]

34. Mokkink, L.B.; Terwee, C.B.; Knol, D.L.; Stratford, P.W.; Alonso, J.; Patrick, D.L.; Bouter, L.M.; De Vet, H.C.W. Protocol of the COSMIN study: COnsensus-based Standards for the selection of health Measurement INstruments. BMC Med. Res. Methodol. 2006, 6. [CrossRef] [PubMed]

35. Shrout, P.E.; Fleiss, J.L. Intraclass correlations: Uses in assessing rater reliability. Psychol. Bull. 1979, 86, 420-428. [CrossRef] [PubMed]

36. Bruton, A.; Conway, J.H.; Holgate, S.T. Reliability: What is it, and how is it measured? Physiotherapy 2000, 86, 94-99. [CrossRef]

37. Atkinson, G.; Nevill, A.M. Statistical methods for assessing measurement error (reliability) in variables relevant to sports medicine. Sport. Med. 1998, 26, 217-238. [CrossRef]

38. McGinley, J.L.; Baker, R.; Wolfe, R.; Morris, M.E. The reliability of three-dimensional kinematic gait measurements: A systematic review. Gait Posture 2009, 29, 360-369. [CrossRef] [PubMed]

39. Ferrari, A.; Cutti, A.G.; Cappello, A. A new formulation of the coefficient of multiple correlation to assess the similarity of waveforms measured synchronously by different motion analysis protocols. Gait Posture 2010, 31, 540-542. [CrossRef] [PubMed]

40. Røislien, J.; Skare, O.; Opheim, A.; Rennie, L. Evaluating the properties of the coefficient of multiple correlation (CMC) for kinematic gait data. J. Biomech. 2012, 45, 2014-2018. [CrossRef] [PubMed]

41. Iosa, M.; Cereatti, A.; Merlo, A.; Campanini, I.; Paolucci, S.; Cappozzo, A. Assessment of waveform similarity in clinical gait data: The linear fit method. Biomed Res. Int. 2014, 2014. [CrossRef] [PubMed]

42. Al-Amri, M.; Al Balushi, H.; Mashabi, A. Intra-rater repeatability of gait parameters in healthy adults during self-paced treadmill-based virtual reality walking. Comput. Methods Biomech. Biomed. Eng. 2017, 1-9. [CrossRef] [PubMed]

43. Kaufman, K.; Miller, E.; Kingsbury, T.; Russell Esposito, E.; Wolf, E.; Wilken, J.; Wyatt, M. Reliability of 3D gait data across multiple laboratories. Gait Posture 2016, 49, 375-381. [CrossRef] [PubMed]

44. Wilken, J.M.; Rodriguez, K.M.; Brawner, M.; Darter, B.J. Reliability and minimal detectible change values for gait kinematics and kinetics in healthy adults. Gait Posture 2012, 35, 301-307. [CrossRef] [PubMed]

45. Charlton, I.W.; Tate, P.; Smyth, P.; Roren, L. Repeatability of an optimised lower body model. Gait Posture 2004, 20, 213-221. [CrossRef] [PubMed] 
46. Meldrum, D.; Shouldice, C.; Conroy, R.; Jones, K.; Forward, M. Test-retest reliability of three dimensional gait analysis: Including a novel approach to visualising agreement of gait cycle waveforms with Bland and Altman plots. Gait Posture 2014, 39, 265-271. [CrossRef] [PubMed]

47. Krause, D.A.; Boyd, M.S.; Hager, A.N.; Smoyer, E.C.; Thompson, A.T.; Hollman, J.H. Reliability and accuracy of a goniometer mobile device application for video measurement of the functional movement screen deep squat test. Int. J. Sports Phys. Ther. 2015, 10, 37-44.

48. Ford, K.R.; Myer, G.D.; Hewett, T.E. Reliability of landing 3D motion analysis: Implications for longitudinal analyses. Med. Sci. Sports Exerc. 2007, 39, 2021-2028. [CrossRef] [PubMed]

49. Mok, K.M.; Petushek, E.; Krosshaug, T. Reliability of knee biomechanics during a vertical drop jump in elite female athletes. Gait Posture 2016, 46, 173-178. [CrossRef] [PubMed]

50. Chappell, J.D.; Limpisvasti, O. Effect of a neuromuscular training program on the kinetics and kinematics of jumping tasks. Am. J. Sports Med. 2008, 36, 1081-1086. [CrossRef] [PubMed]

51. Kirtley, C. Sensitivity of the modified helen hayes model to marker placement errors. In Proceedings of the Seventh International Symposium on the 3-D Analysis of Human Movement, Newcastle, UK, 10-12 July 2002.

52. Leardini, A.; Chiari, A.; Della Croce, U.; Cappozzo, A. Human movement analysis using stereophotogrammetry Part 3. Soft tissue artifact assessment and compensation. Gait Posture 2005, 21, 212-225. [CrossRef] [PubMed]

53. Piazza, S.J.; Cavanagh, P.R. Measurement of the screw-home motion of the knee is sensitive to errors in axis alignment. J. Biomech. 2000, 33, 1029-1034. [CrossRef]

54. Ferrari, A.; Benedetti, M.G.; Pavan, E.; Frigo, C.; Bettinelli, D.; Rabuffetti, M.; Crenna, P.; Leardini, A. Quantitative comparison of five current protocols in gait analysis. Gait Posture 2008, 28, 207-216. [CrossRef] [PubMed]

55. Baker, R. Measuring Walking: A Handbook of Clinical Gait Analysis; Mac Keith Press: London, UK, 2013; ISBN 9780874216561.

56. Cappozzo, A.; Catani, F.; Della Croce, U.; Leardini, A. Position and orientation in space of bones during movement: Anatomical frame definition and determination. Clin. Biomech. 1995, 10, 171-178. [CrossRef]

(C) 2018 by the authors. Licensee MDPI, Basel, Switzerland. This article is an open access article distributed under the terms and conditions of the Creative Commons Attribution (CC BY) license (http:/ / creativecommons.org/licenses/by/4.0/). 


\title{
Article \\ Motor Planning Error: Toward Measuring Cognitive Frailty in Older Adults Using Wearables
}

\author{
He Zhou ${ }^{1}$, Hyoki Lee ${ }^{1}$, Jessica Lee ${ }^{1}$, Michael Schwenk ${ }^{2}$ and Bijan Najafi ${ }^{1, *}$ \\ 1 Interdisciplinary Consortium on Advanced Motion Performance (iCAMP), Michael E. DeBakey Department \\ of Surgery, Baylor College of Medicine, Houston, TX 77030, USA; he.zhou2@bcm.edu (H.Z.); \\ leehyoki@gmail.com (H.L.); jcl1@bcm.edu (J.L.) \\ 2 Network Aging Research, Heidelberg University, 69115 Heidelberg, Germany; \\ schwenk@nar.uni-heidelberg.de \\ * Correspondence: najafi.bijan@gmail.com or bijan.najafi@bcm.edu; Tel.: +1-713-798-7536
}

Received: 18 January 2018; Accepted: 16 March 2018; Published: 20 March 2018

\begin{abstract}
Practical tools which can be quickly administered are needed for measuring subtle changes in cognitive-motor performance over time. Frailty together with cognitive impairment, or 'cognitive frailty', are shown to be strong and independent predictors of cognitive decline over time. We have developed an interactive instrumented trail-making task (iTMT) platform, which allows quantification of motor planning error (MPE) through a series of ankle reaching tasks. In this study, we examined the accuracy of MPE in identifying cognitive frailty in older adults. Thirty-two older adults (age $=77.3 \pm 9.1$ years, body-mass-index $=25.3 \pm 4.7 \mathrm{~kg} / \mathrm{m}^{2}$, female $=38 \%$ ) were recruited. Using either the Mini-Mental State Examination or Montreal Cognitive Assessment (MoCA), 16 subjects were classified as cognitive-intact and 16 were classified as cognitive-impaired. In addition, 12 young-healthy subjects (age $=26.0 \pm 5.2$ years, body-mass-index $=25.3 \pm 3.9 \mathrm{~kg} / \mathrm{m}^{2}$, female $=33 \%$ ) were recruited to establish a healthy benchmark. Subjects completed the iTMT, using an ankle-worn sensor, which transforms ankle motion into navigation of a computer cursor. The iTMT task included reaching five indexed target circles (including numbers 1-to-3 and letters A\&B placed in random order) on the computer-screen by moving the ankle-joint while standing. The ankle-sensor quantifies MPE through analysis of the pattern of ankle velocity. MPE was defined as percentage of time deviation between subject's maximum ankle velocity and the optimal maximum ankle velocity, which is halfway through the reaching pathway. Data from gait tests, including single task and dual task walking, were also collected to determine cognitive-motor performance. The average MPE in young-healthy, elderly cognitive-intact, and elderly cognitive-impaired groups was $11.1 \pm 5.7 \%$, $20.3 \pm 9.6 \%$, and $34.1 \pm 4.2 \%(p<0.001)$, respectively. Large effect sizes (Cohen's $d=1.17-4.56)$ were observed for discriminating between groups using MPE. Significant correlations were observed between the MPE and MoCA score $(r=-0.670, p<0.001)$ as well as between the MPE and dual task stride velocity $(r=-0.584, p<0.001)$. This study demonstrated feasibility and efficacy of estimating MPE from a practical wearable platform with promising results in identifying cognitive-motor impairment and potential application in assessing cognitive frailty. The proposed platform could be also used as an alternative to dual task walking test, where gait assessment may not be practical. Future studies need to confirm these observations in larger samples.
\end{abstract}

Keywords: cognitive frailty; wearable; cognitive-motor impairment; Alzheimer's disease; motor planning error; instrumented trail-making task; ankle reaching task; dual task walking

\section{Introduction}

Recent demographic changes have led to emergence of the so-called "dementia epidemic" [1]. Dementia causes great stress to medical, social, and informal care and is currently affecting 
approximately 47.5 million persons worldwide [2-4]. This number is projected to increase to 75.6 million by 2030 and 135.5 million by 2050 [3]. This has created an urgent need for a robust and quickly-administered cognitive assessment tool capable of identifying individuals in the earliest stages of cognitive decline and measuring subtle changes in cognitive-motor performance over time [5]. Early diagnosis of mild cognitive impairment (MCI) and detection (or 'measuring') of its subtle progression over time may allow early intervention and prevention of further cognitive and functional decline [6]. In this context, frailty together with cognitive impairment (known as 'cognitive frailty' [7]) has been shown to be a strong and independent predictor of cognitive decline over time $[1,8,9]$.

The concept of 'frailty' is used to identify homeostenotic older adults with low physiological reserves and vulnerability to illness and high risk of disability, institutionalization, and death $[10,11]$. A recent systematic review identified 67 frailty instruments total; of these, nine were highly-cited ( $\geq 200$ citations) [12]. Among these, the Physical Frailty Phenotype [10] was the most used frailty instrument in the research literature, followed by the Deficit Accumulation Index [11]. The Physical Frailty Phenotype is based on five criteria (weight loss, exhaustion, low physical activity, weak grip strength, and slow walking speed) [10]. The Deficit Accumulation Index is based on a count of 70 accumulated deficits, including the presence and/or severity of diseases, ability in activities of daily living, and physical signs from clinical and neurological examinations [11]. While both assessments have shown to be accurate for identifying older adults with low resilience and high vulnerability independent of comorbidities and disability $[13,14]$, they all neglect assessing cognitive performance, including working memory and executive function. The cognitive assessment could add complementary information (in addition to frailty) to determine healthy aging trajectory as well as post intervention health outcomes. For example, previous studies have suggested that cognitive performance is an independent risk factor influencing adverse health outcomes after major surgical intervention $[15,16]$.

A recent study by Kelaiditi et al. [9] demonstrated that measures of frailty can be used to identify individuals with Alzheimer's disease (AD) who have a greater risk of cognitive decline and loss of independence. An association between cognitive impairment and specific frailty criteria such as gait speed has been demonstrated [17]. Similarly, several studies have suggested that cognitive and motor declines are independent key predictors of dementia in the geriatric population $[8,18,19]$.

Despite this evidence described above, cognitive impairment and frailty are still assessed and studied separately, mainly because conventional cognitive performance assessment tools do not take into account motor performance (an indicator of frailty), and vice versa. In addition, the current motor or cognitive performance assessment tools often neglect characterization of anticipatory motor planning, an important factor that influences ability to accomplish instrumental activities of daily living (iADL) [20-22].

Studies based on motor learning paradigms highlight the importance of characterizing anticipatory motor planning as an important factor that influences daily motor skills and cognitive performance [23,24]. Most of iADLs rely on the individual's anticipatory planning abilities to appropriately plan movements prior to executing them. For example, to successfully accomplish a behavioral goal such as reaching for a piece of fruit, two problems must be solved: to select which fruit to reach for and to specify the parameters of the reach, such as its direction and extent [24]. Studies on primary motor cortex, premotor cortex, and supplementary motor area have demonstrated that human brains can plan and control physical movement ahead of physical body action [25-27]. It is well established that anticipatory planning abilities improve as a function of age, reaching adult-like levels in late childhood to early adolescence. It then rapidly declines to levels observed in young children, in very old adults, particularly in those with frailty and/or cognitive impairment [28]. It has been postulated that the decrease in anticipatory motor planning abilities in older adults is associated with decline in cognitive skills [28], a theory supported by brain imaging research in which an association between deterioration in frontal and parietal white matter and fine motor dexterity was reported [29,30]. Recently, Stockel and colleagues [28] have demonstrated that up to $64 \%$ of 
the variance in motor planning performance across age groups can be explained by the cognitive functions of processing speed, response planning, and cognitive flexibility. They have postulated that anticipatory motor planning abilities are strongly influenced by cognitive control processes, which seem to be key mechanisms compensating for age related decline.

To address the practical barriers for clinical use of cognitive-motor assessment tools as well as incorporating a practical method to quantify motor planning, we have developed a novel cognitive frailty measurement tool call instrumented trail-making task (iTMT). The iTMT is based on the combination of a low-cost wearable sensor attached to patient's lower shin and interactive interface technology. The design of the system is inspired by the conventional trail-making test (TMT), which is a useful test to determine cognitive impairment [31]. The second component of the system is an ankle reaching test performed in a virtual environment, which allows for quantification of motor performance and motor planning error. The proposed system provides a unique opportunity to determine cognitive frailty by enabling simultaneous and rapid measurement of both cognitive and motor performance. In our previous study [32], we have demonstrated the effectiveness of the iTMT to determine cognitive impairment by measuring the time duration needed to complete the test. In addition, we have demonstrated that by measuring the ankle velocity during the reaching task, we can also distinguish pre-frail individuals and frail individuals from non-frail individuals. In the present study, we extended our iTMT approach by quantifying anticipatory motor planning error. We extracted the motor planning error (MPE) through measuring ankle velocity patterns during the ankle reaching task. Our hypothesis was that; due to anticipatory motor planning performance decline associated with cognitive impairment, older adults with cognitive impairment would have iTMT MPEs larger than those of elderly without cognitive impairment as well as those of young-healthy subjects.

\section{Methods}

\subsection{Study Population}

Thirty-two ambulatory older adults (age 65 years or older) were recruited in this study. According to the Mini-Mental State Examination (MMSE) (cutoff score of 23 or less [33]) or Montreal Cognitive Assessment (MoCA) (cutoff score of 25 or less [34]), 16 subjects were classified as cognitive-intact, and 16 were classified as cognitive-impaired. To compare the iTMT results between young and older adults, as well as to establish a healthy benchmark, 12 young-healthy ambulatory subjects with ages ranging from 21 to 34 years were also recruited. Subjects were excluded from the study if they were unable to walk a distance of $20 \mathrm{~m}$ with or without walking assistance; had visual problem, limiting their ability to interact with a computer screen; had lower extremity problems, limiting their ability to perform ankle movement needed for the purpose of this study; or had severe balance impairment, limiting their ability to independently stand. Those who could stand behind a chair and perform the iTMT test by holding the chair were not excluded. All subjects signed a consent form for this study. This study was approved by the local institutional review boards.

\subsection{Demographic Information}

All subjects' demographics were collected, including age, gender, body mass, height, body-mass-index (BMI), history of falls, and history of depression. Subjects self-reported any fall incidents and number of falls in the past year. The Center for Epidemiologic Studies Depression (CES-D) short-version scale was used to measure self-reported depression symptoms. A cutoff of CES-D score of 16 or greater was used to identify subjects with depression.

\subsection{Instrumented Trail-Making Task (iTMT)}

We designed the iTMT platform (Figure 1) based on a wearable sensor (LEGSys ${ }^{\mathrm{TM}}$, Biosensics LLC, Watertown, MA, USA), which has a triaxial accelerometer, triaxial gyroscope, and triaxial magnetometer for the purpose of estimating angles and positions. The wearable sensor was attached 
to the lower shin for tracking three dimensional ankle motion (Figure 1). The real-time data was acquired and transmitted at $100-\mathrm{Hz}$ frequency to an interactive computer interface (developed based on MATLAB ${ }^{\circledR} 2013 \mathrm{~b}$ and Psychophysics Toolbox Version 2.54) installed on a standard computer (Figure 1). The subject could navigate a cursor from a home circle to target circles appearing on the computer screen using ankle rotation.

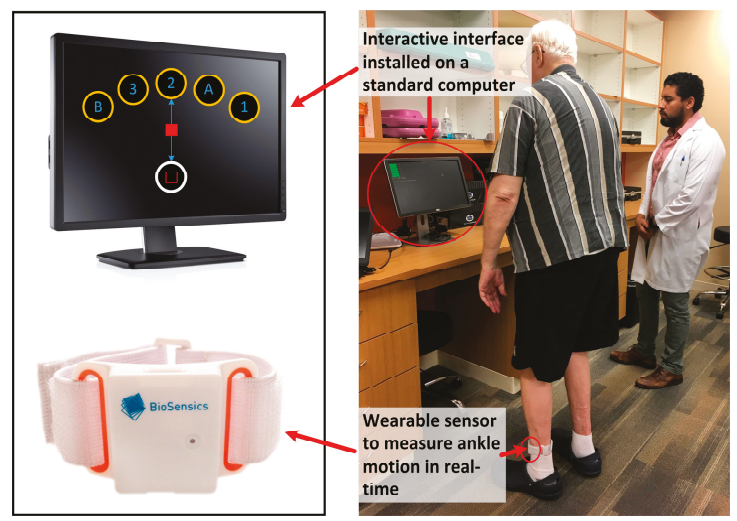

Figure 1. An illustration of the instrumented trail-making task (iTMT) platform with both numbers $(1,2$, and 3$)$ and letters (A and B) randomized in the target circles. One inertial sensor-including a triaxial accelerometer, a triaxial gyroscope, and a triaxial magnetometer-was attached to the subject's lower shin using a comfortable elastic band. The sensor allows measurement of three-dimensional motion of the ankle joint in real time. The instantaneous measured joint angle with a sample frequency of $100-\mathrm{Hz}$ was wirelessly transferred to a computer, using low-power Bluetooth, to create an interactive interface for the purpose of the interactive iTMT test. For safety purposes, a research coordinator was in the room supervising the iTMT test at all times. After starting the iTMT test, the research coordinator did not provide any guidance. The interactive interface provided the necessary guidance and instruction to complete the test.

In the iTMT test, six circles appeared on the screen: one home circle in white and five target circles in yellow. The target circles were located in a fanwise position in front of the home circle (Figure 1). The five target circles were randomly marked with numbers (1, 2, and 3 ) and letters (A and B). At the beginning of the iTMT test, the cursor position was automatically calibrated to the center of the home circle. The iTMT required weight shifting in forward/backward or sideward/diagonal direction, while standing with both feet on the ground in front of the computer screen (Figure 1). To perform the iTMT, the subject would control the movement of ankle joints while maintaining balance. This could be achieved by moving the hip joint in opposite direction of ankle motion (reciprocal compensatory strategy) [35]. Ankle motions on the left foot and right foot are consistent, therefore by attaching just one sensor on one lower shin, three dimensional ankle motion can be tracked. By rotating the ankle joint, the subject could navigate the cursor to the center of target circles with alternating numbers and letters. Specifically, the subject navigated the cursor to the center of the first target circle (with the number ' 1 ' inside). The target circle would explode with a reward sound once the cursor stopped in the middle of the target circle. Then the subject navigated the cursor back to the center of the home circle, then to the second target circle (with the letter ' $A$ '), then back to the home circle, then to the third target circle (with the number ' 2 '), and so forth until the end. To efficiently accomplish the test with minimum time to reach all targets, the subject should have optimum motor planning prior to executing the task. For example, to successfully reach a target, two problems should be solved: to select which target to reach for (i.e., working memory task) and to specify the parameters of the reach, such as its direction and extent. In addition, to minimize the time to reach a target, the subject 
should determine optimum time to decelerate ankle movement so as stop perfectly at the center of the target without re-adjustment.

\section{4. iTMT Motor Planning Error}

We extracted the motor planning error based on ankle velocity pattern measurements during the ankle reaching task. MPE was defined as percentage of time deviation between subject's maximum ankle velocity and the optimal maximum ankle velocity, which is halfway through the reaching pathway (Figure 2). In summary, to minimize the iTMT time, which requires bringing a computer cursor (square shape) from a home circle and stopping at the middle of the target circle (which was defined as the end of a reaching task), the subject should reach maximum ankle velocity at the middle of the reaching pathway and then decelerate to be able to stop at the middle of the target without the need for re-adjustment. Therefore, the ideal ankle velocity curve is a bell shape (indicator of a healthy feedforward performance). Based on our definition, the iTMT MPE can range from 0 to $50 \%$. $0 \%$ indicates that the peak ankle velocity appears at the mid-pathway (optimum motor planning). 50\% indicates that the peak ankle velocity appears at the beginning or end of target reaching. Figure $2 \mathrm{~A}-\mathrm{C}$ show ankle velocity curves of a typical young-healthy subject, elderly cognitive-motor intact subject, and elderly cognitive-motor impaired subject, respectively. For the typical young-healthy subject with good cognitive perception coordinated with good motor performance, the peak ankle velocity appears very close to the mid-pathway, and the iTMT MPE is very small (Figure 2A). For the typical elderly cognitive-motor intact subject, the peak ankle velocity is lower than the young-healthy subject, and the iTMT MPE is moderate (Figure 2B). For the typical elderly cognitive-motor impaired subject with poor cognition and uncoordinated motion, the peak ankle velocity appears very early or very late, and the iTMT MPE is large. Multiple ankle velocity peaks can be observed in Figure 2C.
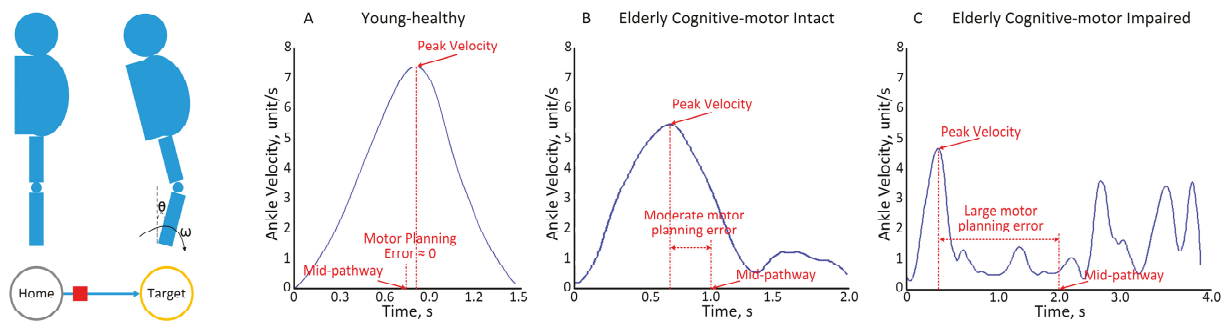

Figure 2. An illustration of the iTMT MPE. (A) Ankle velocity curve of a typical young-healthy subject during the ankle reaching, (B) ankle velocity curve of a typical elderly cognitive-motor intact subject during the ankle reaching, (C) ankle velocity curve of a typical elderly cognitive-motor impaired subject during the ankle reaching.

\section{5. iTMT Time}

The method for measuring the iTMT time has been described in detail in [32]. In summary, at the beginning of the iTMT test, when the cursor position is automatically calibrated to the center of the home circle, the computer program starts recording the time. At the end of the iTMT test, when the cursor position stops at the center of the last target circle (with the number ' 3 '), the computer program stops recording the time. The recorded time period is defined as the iTMT time, which represents the total time a subject uses to complete reaching to all five indexed target circles in the correct order. Our previous study suggests that the iTMT time result is correlated with MoCA test result $(r=-0.598$, $p=0.001)$ and is effective at discriminating between those with and without cognitive impairment with large effect size (Cohen's $d$ effect size $=1.02, p=0.015$ ) [32]. We have also demonstrated that the iTMT time represents mainly cognitive performance and corresponds poorly with motor performance 
(e.g., gait speed) [32]. To compare this marker with the innovative iTMT MPE proposed in this study, iTMT time was collected for all subjects.

\subsection{Walking Test}

For all subjects, wearable sensors were attached to both the left and right lower legs (LegSys ${ }^{\mathrm{TM}}$, BioSensics, Watertown, MA, USA) to measure single task and dual task gait performances. Subjects were asked to walk with their habitual gait speed for $20 \mathrm{~m}$ with no cognitive task (single task walking). Then, they were asked to repeat the test while loudly counting backward from a random number (dual task walking: walking + working memory test) [2,36]. Gait speed was calculated using validated algorithms [37-39].

\subsection{Statistical Analysis}

Age, height, body mass, BMI, single task walking stride velocity, and dual task walking stride velocity for each group were described using both mean \pm standard deviation. Gender, history of falls, and history of depression were expressed as count (percentage). The Shapiro-Wilk test was applied to test normality of the data. One-way analysis of variance was employed for pairwise comparison according to the scale of the investigated variable and the distribution of the data. Analysis of covariance was used to compare the iTMT MPE and the iTMT time, with and without adjustment for age and BMI, among groups. Fisher's least significant difference-based post hoc test was performed for pairwise comparison to explore significant main effects and interactions. Cohen's $d$ effect size was calculated to assess the magnitude of difference between each group. Values ranging from 0.20 to 0.49 indicate small, and values between 0.50 and 0.79 indicate medium. Values ranging from 0.80 to 1.29 indicate large effects, and values above 1.30 indicate very large effects. Values less than 0.20 are considered as having no noticeable effect. Spearman's correlation coefficients were used to evaluate the degree of association between the iTMT MPEs and conventional dual task walking stride velocity, used as a reference. $p<0.050$ was considered statistically significant. All statistical analyses were performed using IBM SPSS Statistics 24 (IBM, Chicago, IL, USA).

Sample Size Calculation

We estimated prior sample size and power by performing a post hoc analysis based on the observed effect size in the previous iTMT study [32]. Considering the observed effect size of 1.02 for the iTMT time difference between cognitive-intact and cognitive-impaired in [32], to achieve a minimum power of $80 \%$, a minimum sample size of 13 subjects per group are required to observe a statistical significant of $5 \%$ or lower using two tails independent sample comparisons.

\section{Results}

\subsection{Study Population}

Table 1 summarizes demographic and clinical data. The elderly subjects' ages ranged from 60 to 93 years, while the young-healthy subjects' ages ranged from 21 to 34 years. No difference between the elderly cognitive-intact group and elderly cognitive-impaired group was observed for demographic information, including gender, age, height, body mass, and BMI $(p>0.050)$. For the clinical examinations including history of fall and depression, no between-group difference was observed $(p>0.050)$. Motor performance based on single task walking did not show significant difference between the elderly cognitive-intact group and elderly cognitive-impaired group. The only significant difference between the two groups was the dual task walking stride velocity $(p=0.031)$. Compared to the elderly cognitive-intact group, the young-healthy group had significantly increased single task and dual task walking stride velocities $(p<0.001$ and $p=0.001)$. 
Table 1. General characteristics of the study population.

\begin{tabular}{cccccc}
\hline & $\begin{array}{c}\text { Elderly Cognitive- } \\
\text { Intact (N) }\end{array}$ & $\begin{array}{c}\text { Elderly Cognitive- } \\
\text { Impaired (P) }\end{array}$ & $\begin{array}{c}p \text {-Value } \\
\text { (P vs. N) }\end{array}$ & $\begin{array}{c}\text { Young- } \\
\text { Healthy (H) }\end{array}$ & $\begin{array}{c}p \text {-Value } \\
\text { (N vs. H) }\end{array}$ \\
\hline Number of subject, $\mathrm{n}$ & 16 & 16 & - & 12 & - \\
Female, n (\%) & $7.0(44)$ & $5.0(31)$ & 0.481 & $4.0(33)$ & 0.593 \\
Age, years & $75.6 \pm 9.5$ & $79.0 \pm 8.6$ & 0.292 & $26.0 \pm 5.2$ & $<0.001$ \\
Height, cm & $170.0 \pm 9.8$ & $168.5 \pm 12.8$ & 0.723 & $170.9 \pm 9.4$ & 0.798 \\
Body mass, kg & $69.9 \pm 14.6$ & $77.7 \pm 20.9$ & 0.240 & $74.3 \pm 15.3$ & 0.450 \\
BMI, kg/m m $^{2}$ & $24.0 \pm 3.4$ & $26.7 \pm 5.5$ & 0.113 & $25.3 \pm 3.9$ & 0.387 \\
History of fall, n (\%) & $4.0(25)$ & $7.0(44)$ & 0.279 & - & - \\
Depression, $\mathrm{n}(\%)$ & $4.0(25)$ & $2.0(13)$ & 0.381 & - & - \\
STW SV, m/s & $1.00 \pm 0.18$ & $0.87 \pm 0.21$ & 0.075 & $1.27 \pm 0.15$ & $<0.001$ \\
DTW SV, m/s & $0.85 \pm 0.21$ & $0.68 \pm 0.22$ & $\mathbf{0 . 0 3 1}$ & $1.14 \pm 0.21$ & $\mathbf{0 . 0 0 1}$ \\
\hline
\end{tabular}

BMI: Body-Mass-Index; STW: Single Task Walking; DTW: Dual Task Walking; SV: Stride Velocity. Depression was assessed by Center for Epidemiologic Studies Depression (CES-D) score with a cutoff of 16 or greater. Significant difference between groups were indicated in bold.

\section{2. iTMT Motor Planning Error and iTMT Time among Groups}

Table 2 summarizes the iTMT MPE and the iTMT time for different groups, including young-healthy subjects, elderly cognitive-intact subjects, as well as elderly cognitive-impaired subjects. Table 3 and Figure 3 summarize between-group comparisons with and without adjustment for age and BMI. The iTMT MPE generated during the test was $20.3 \pm 9.6 \%$ in the elderly cognitive-intact group and was significantly increased on average by $68 \%$ in the elderly cognitive-impaired group $(d=1.86$, $p<0.001$, Figure $3 \mathrm{~A}$ ). The iTMT time spent by the elderly cognitive-intact group to complete the test was $25.2 \pm 7.9$ s. It was significantly increased on average by $100 \%$ in the elderly cognitive-impaired group $(d=1.21, p<0.001$, Figure $3 \mathrm{~B})$. When compared with the elderly groups, a much lower iTMT MPE (11.1 $\pm 5.7 \%$, Figure $3 \mathrm{~A})$ and a much shorter iTMT time (18.5 \pm 2.1 s, Figure $3 \mathrm{~B})$ was observed for the young-healthy group. When compared with the iTMT time, the iTMT MPE had much larger effect sizes to discriminate between the three groups (Table 3). After adjusting the results for age and BMI, the between-group difference achieved statistical significance only when comparing between the elderly cognitive-intact and elderly cognitive-impaired groups for both iTMT MPE $(d=1.26, p<0.001)$ and iTMT time $(d=0.98, p<0.001)$ (Table 3$)$.

Table 2. iTMT derived parameters for different groups.

\begin{tabular}{ccccc}
\hline & Young-Healthy & $\begin{array}{c}\text { Elderly } \\
\text { Cognitive-Intact }\end{array}$ & $\begin{array}{c}\text { Elderly } \\
\text { Cognitive-Impaired }\end{array}$ & $p$-Value \\
\hline iTMT Motor & $11.1 \pm 5.7$ & $20.3 \pm 9.6$ & $34.1 \pm 4.2$ & $<0.001$ \\
Planning Error, $\%$ & $18.5 \pm 2.1$ & $25.2 \pm 7.9$ & $50.4 \pm 28.3$ & $<0.001$ \\
\hline iTMT Time, $\mathrm{s}$ & $18.5 \pm$
\end{tabular}

iTMT: instrumented trail-making task. Significant difference between groups were indicated in bold. 
Sensors 2018, 18, 926

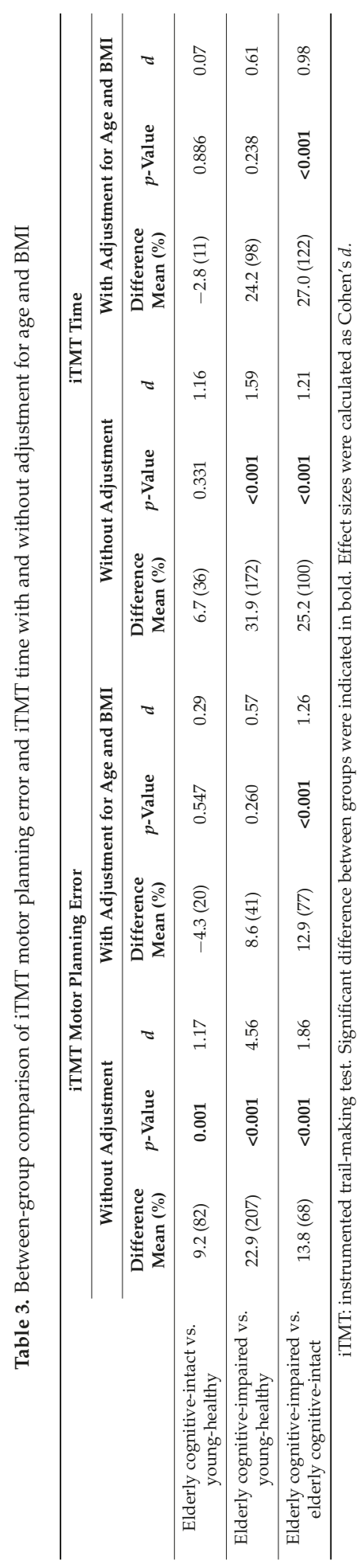


A

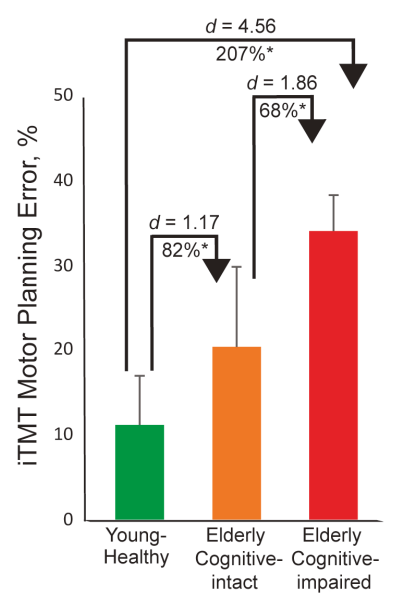

B

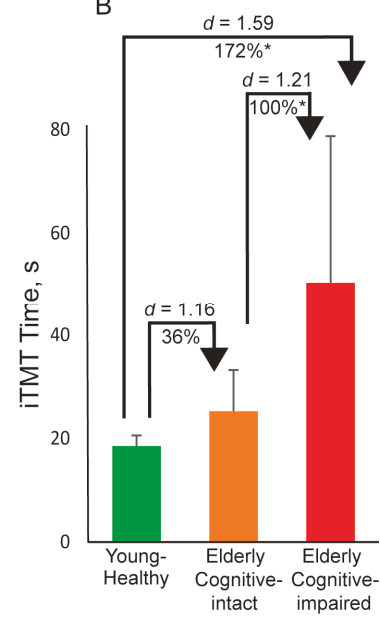

Figure 3. The iTMT derived parameters for all groups, including young-healthy group, elderly cognitive-intact group, and elderly cognitive-impaired group. (A) The iTMT MPE comparison; (B) the iTMT time comparison. Error bar represents the standard error. ' ${ }^{\prime \prime}$ denotes when the pairwise group comparison achieved a statistically significant level $(p<0.050)$. $d$ denotes Cohen's $d$ effect size.

\subsection{Association between iTMT Motor Planning Errors and Conventional Cognitive Assessment}

Figure 4 illustrates the correlation between the iTMT platform and conventional cognitive assessments. In summary, a high correlation was observed between the iTMT MPE and MoCA score $(r=-0.670, p<0.001$, Figure 4A). On the same note, a high correlation was also observed between the iTMT MPE and dual task walking stride velocity ( $r=-0.584, p<0.001$, Figure 4B), indicating that the iTMT MPE may be used as a surrogate for the dual task walking test.

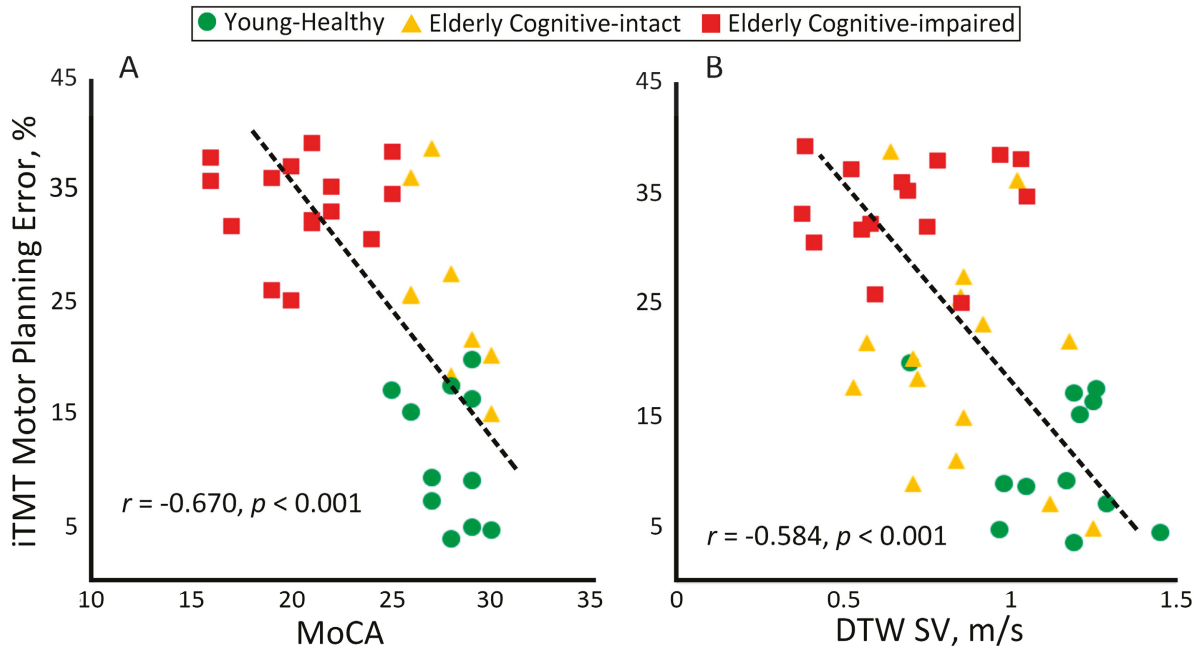

Figure 4. Correlation between the iTMT MPE and (A) MoCA test score and (B) dual task walking stride velocity (DTW SV). 


\section{Discussion}

Cognitive and motor impairments are independent key predictors of further decline in cognitive performance, life independency, and Alzheimer's disease among geriatric population [8,18,19]. In this study, we investigated the feasibility and efficacy of estimating motor planning error from a practical wearable platform with promising results in identifying cognitive-motor impairment in older adults. We were able to confirm our hypothesis that the iTMT MPE is able to discriminate between elderly cognitive-intact and elderly cognitive-impaired groups. The iTMT MPE value is derived from the ankle velocity signals during trail-making and ankle-reaching tasks for examination of both cognitive and motor abilities. In other words, the ankle velocity pattern can provide information on both cognition and motor ability of patients. For example, the velocity pattern of the elderly cognitive-impaired group fluctuated more than that of the elderly cognitive-intact group (Figure 2). This may be due to the fact that both trail-making and ankle-reaching tasks require performing automatic corrections under voluntary control by the brain in order to sharpen target localization [40]. Therefore, the noisy velocity pattern signifies the decrease of motor planning skills in geriatric populations with cognitive impairment.

Cognitive impairment is a symptom that makes it difficult for patients to remember, learn new things, concentrate, or make decisions. While cognitive impairment can be identified with pencil- and paper-based screening tools, such as the MoCA, MMSE, and conventional TMT, these assessments are semi-subjective, time consuming, and insensitive to subtle changes in cognitive frailty. Their accuracy is highly dependent on the examiner's experience and the patient's education level [32,41]. Computerized versions of conventional cognitive screening tools have improved the utility of such measurements. However, they are not capable of monitoring motor performance (an essential component of frailty) and anticipatory planning abilities (an essential component for successfully accomplishment of instrumental activities of daily living) thus are not able to detect cognitive frailty, a known predictor of speed of cognitive decline over time. The dual task paradigm can be used to screen for both cognitive and motor performance and identify both cognitive impairment and frailty [2,36]. The most stablished dual task paradigm is dual task walking. Dual task walking can isolate the cognitive control component of locomotion and expose cognitive deficits through the evaluation of activities that simultaneously demand attentional resources. In addition, dual task walking test is sensitive in determining frailty [36]. However, many older adults have mobility impairment and high risk of falling, making the administration of walking test challenging, in particular in busy clinical settings. In addition, limits on space and time in busy clinics can influence the execution of such tests [42]. Even if gait is assessed over short walking distance to accommodate space limitation, it is debated whether dual-task walking test is reliable over a short walking distance (less than $20 \mathrm{~m}$ ) [38,43]. The proposed ankle reaching task derived MPE addresses the aforementioned limitations associated with penciland paper-based screening tools as well as dual task gait. The iTMT platform consists of a low-cost wearable sensor combined with an interactive interface installable on any standard computer [32]. It is simple, short, safe, and easy to manage by non-expert users [32]. The iTMT platform does not require walking test. It allows long-term quantification of anticipatory motor planning regardless of setting.

In an early study [32], we demonstrated that the iTMT time is an efficient marker to discriminate among age-matched healthy, amnestic mild cognitive impairment, and Alzheimer's disease groups. However, the iTMT time is a global marker of the trail-making performance. It is the total time that a subject needs to complete the iTMT test and can be influenced by many factors such as the time a subject needs to search and locate targets, the speed a subject can rotate the ankle, the number of mistakes a subject makes during the test, etc. Therefore, the iTMT time is not a specific maker of cognitive-motor impairment. It may not be as sensitive as the iTMT MPE in identifying cognitive-motor impairment and assessing cognitive frailty. The iTMT MPE is derived from ankle velocity measurements during the trail-making test. It is a specific marker which can evaluate both cognitive and motor abilities equally and simultaneously. We collected both iTMT markers in the present study and compared them in Tables 2 and 3. Although Table 2 shows that both iTMT markers possessed significant differences 
between the three groups $(p<0.001)$, Table 3 shows that the iTMT MPE had a larger effect size than the iTMT time for discriminating among the three groups, mainly because of relatively lower intra group variation, in particular among the elderly group with cognitive impairment. For instance, when examining the difference between older adults with and without cognitive impairment, the effect size was increased from 1.21 (large effect size) by using the iTMT time to 1.86 (very large effect size) by using the iTMT MPE. In addition, only the iTMT MPE can discriminate between young-healthy and elderly cognitive-intact without adjustment for age. This may be because the major difference between these two groups exists in the motor domain caused by aging. Thus, we may speculate that the iTMT MPE is a cognitive-motor sensitive metric, and may be able to determine motor deficit between groups with comparable cognitive functions. Before adjusting for age and BMI, the iTMT MPE was able to discriminate between all three groups. After adjusting for age and BMI, the difference between young-healthy and older adults was diminished irrespective of cognitive status. However, when comparing between older adults with and without cognitive impairment, the between-group difference remained significant after adjustment. This may suggest that the iTMT MPE is sensitive to age but also able to determine cognitive impairment in the same age categories.

One of the unique opportunities of the iTMT platform is its ability to determine frailty status. In another study, we demonstrated that iTMT parameters were also able to identify presence and absence of frailty phenotypes (Cohen's $d$ effect size $=0.81-1.56$ ). Specifically, the iTMT maximum ankle reaching velocity was associated with slowness, changes in ankle velocity from the first reaching task to the last reaching task was an indicator of exhaustion, ankle reaching moment was a representation of weakness, and jerkiness of ankle movement was associated with inactivity. These parameters allow identification of non-frail, pre-frail, and frail older adults, as identified by the Physical Frailty Phenotype [10], with large to very large effect sizes $(d=1.04-3.14)$. Together with results of the current study in which we demonstrated that motor planning error is an indicator of cognitive impairment, it is postulated that the iTMT platform is able to determine cognitive frailty, a strong predictor of elderly dependency in the near future [8]. Recent studies suggest that frailty is also a significant predictor of cognitive decline over a short period of time. For example, Kelaiditi et al. [9] demonstrated that frail patients with $\mathrm{AD}$ have almost twice the decline in cognitive performance over one year follow up than AD patients without frailty. The same study concluded that a one-unit ( 0.033 points) increase in the frailty index (indicator of cumulative deficits) corresponds to significant and clinically relevant cognitive decline, after adjusting for age, sex, and years of education (0.63-4.63 points on the MMSE, $p=0.010)$.

Another advantage of the iTMT platform is that it uses wearable technology, instead of camera or force platform, to evaluate motor-cognitive performance in older adults. Unlike low-cost camera-based motion tracking system (e.g., Microsoft Kinect), wearable sensors do not require a continuous unobstructed sightline; and thus the test could be administrated behind of a chair to provide support if needed. In addition, the examiner could be next to the subject to provide safety, unlike with the camera-based system. This safety feature is especially important during the iTMT in older adults, in particular, those with cognitive impairment and dementia, who have increased fall risk. The force platform is similar to wearable sensor, which is not limited by continuous unobstructed sightline. However it has other limitations making it unsuitable for motor planning error assessment. For instance, commercially available and low-cost force platforms (e.g., Nintendo Wii Fit) restrict the base of support during testing, which is unsuitable for obese patients or those who need large base of support to maintain balance during dynamic tasks [44]. Furthermore, wearable sensors enable measuring kinematics of body joint of interest and thus diversify the tests. Force platforms, however, are limited to measure only center of pressure or ground reaction force without any information from joint angles. 


\section{Limitations and Future Directions}

Despite the advantages of the iTMT platform enabling us to prescreen for cognitive-motor impairment and cognitive frailty, there are some limitations in this study. Our subjects were recruited from specialized outpatient clinics (e.g., cancer clinic, vascular clinic, dementia clinic, etc.) instead of older adults dwelling communities. Thus our sample may not represent general elderly population. The instruments (MMSE or MoCA) used to identify cognitive impairment in different clinics were not identical. Future study should be done on different days and be administered by different examiners in order to assess test-retest reliability. In addition, this study analyzed the iTMT MPE while standing, which disqualifies bedbound and non-ambulatory patients. This study invites further analysis of the motor planning error while sitting or lying down in order to identify cognitive-motor impairment in bedbound patients.

\section{Conclusions}

In conclusion, we have demonstrated that motor planning error, which could be estimated from a low-cost and simple wearable platform, is able to simultaneously determine cognitive and motor impairments. Based on our observations, the test is feasible and practical even for those with severe cognitive impairment (MoCA < 17) and poor motor performance (single task walking stride velocity $<0.5 \mathrm{~m} / \mathrm{s}$ ). The test is fast (taking less than one minute on average, excluding preparation and sensor attachment), it requires a single inertial sensor (i.e., gyroscope) and low-cost standard computer or tablet, and is reliable as demonstrated in our previous study [32]. Despite the simplicity, it can provide important clinical information about cognitive-motor impairment and cognitive frailty in geriatric populations. The proposed iTMT platform could also be used as an alternative test for dual task gait, which is often impractical in busy clinics and homes, where the space for gait assessment is not available, and for patients with limited mobility (e.g., need to walk with walking accessories). Thus, it can easily be used for both community dwelling elderly as well as those in busy hospital settings.

Acknowledgments: Partial support was provided by the National Institutes of Health/National Institute on Aging (award number 2R42AG032748), the National Institutes of Health/National Cancer Institute (award number 1R21CA190933-01A1), Baylor College of Medicine, and Michael E. DeBakey Department of Surgery. The content is solely the responsibility of the authors and does not necessarily represent the official views of sponsors. We thank Ana Enriquez and Ivan Marin for assisting with data collection and coordination of this research study between involved key investigators.

Author Contributions: H.Z. wrote the manuscript, developed the code for extracting motor planning error, and contributed in data collection and data analysis. H.L., J.L., and M.S. contributed in drafting the manuscript. B.N. contributed in study design and supervising the study. All authors contributed in interpretation of results and critical revision of the study.

Conflicts of Interest: The iTMT is protected by a patent pending. H.Z. and B.N. are listed as co-inventors. However, they do not claim any financial conflict of interest relevant to this study.

\section{References}

1. Kelaiditi, E.; Cesari, M.; Canevelli, M.; van Kan, G.A.; Ousset, P.J.; Gillette-Guyonnet, S.; Ritz, P.; Duveau, F.; Soto, M.E.; Provencher, V.; et al. Cognitive frailty: Rational and definition from an (I.A.N.A./I.A.G.G.) international consensus group. J. Nutr. Health Aging 2013, 17, 726-734. [CrossRef] [PubMed]

2. Bahureksa, L.; Najafi, B.; Saleh, A.; Sabbagh, M.; Coon, D.; Mohler, M.J.; Schwenk, M. The Impact of Mild Cognitive Impairment on Gait and Balance: A Systematic Review and Meta-Analysis of Studies Using Instrumented Assessment. Gerontology 2017, 63, 67-83. [CrossRef] [PubMed]

3. World Health Organization (WHO). Mental Health and Older Adults; WHO: Geneva, Switzerland, 2016.

4. Cornelis, E.; Gorus, E.; Beyer, I.; Bautmans, I.; De Vriendt, P. Early diagnosis of mild cognitive impairment and mild dementia through basic and instrumental activities of daily living: Development of a new evaluation tool. PLoS Med. 2017, 14, e1002250. [CrossRef] [PubMed]

5. Brooks, L.G.; Loewenstein, D.A. Assessing the progression of mild cognitive impairment to Alzheimer's disease: Current trends and future directions. Alzheimers Res. Ther. 2010, 2, 28. [CrossRef] [PubMed] 
6. Dierckx, E.; Engelborghs, S.; De Raedt, R.; De Deyn, P.P.; Ponjaert-Kristoffersen, I. Mild cognitive impairment: What's in a name? Gerontology 2007, 53, 28-35. [CrossRef] [PubMed]

7. Shimada, H.; Makizako, H.; Tsutsumimoto, K.; Doi, T.; Lee, S.; Suzuki, T. Cognitive Frailty and Incidence of Dementia in Older Persons. J. Prev. Alzheimers Dis. 2018, 5, 42-48. [CrossRef] [PubMed]

8. Ruan, Q.; Yu, Z.; Chen, M.; Bao, Z.; Li, J.; He, W. Cognitive frailty, a novel target for the prevention of elderly dependency. Ageing Res. Rev. 2015, 20, 1-10. [CrossRef] [PubMed]

9. Kelaiditi, E.; Canevelli, M.; Andrieu, S.; Del Campo, N.; Soto, M.E.; Vellas, B.; Cesari, M. Impact of Cholinergic Treatment Use Study/DSA Group. Frailty Index and Cognitive Decline in Alzheimer's Disease: Data from the Impact of Cholinergic Treatment USe Study. J. Am. Geriatr. Soc. 2016, 64, 1165-1170. [CrossRef] [PubMed]

10. Fried, L.P.; Tangen, C.M.; Walston, J.; Newman, A.B.; Hirsch, C.; Gottdiener, J.; Seeman, T.; Tracy, R.; Kop, W.J.; Burke, G.; et al. Frailty in older adults: Evidence for a phenotype. J. Gerontol. Ser. A Biol. Sci. Med. Sci. 2001, 56, M146-M156. [CrossRef]

11. Rockwood, K.; Andrew, M.; Mitnitski, A. A comparison of two approaches to measuring frailty in elderly people. J. Gerontol. Ser. A Biol. Sci. Med. Sci. 2007, 62, 738-743. [CrossRef]

12. Buta, B.J.; Walston, J.D.; Godino, J.G.; Park, M.; Kalyani, R.R.; Xue, Q.L.; Bandeen-Roche, K.; Varadhan, R. Frailty assessment instruments: Systematic characterization of the uses and contexts of highly-cited instruments. Ageing Res. Rev. 2016, 26, 53-61. [CrossRef] [PubMed]

13. Clegg, A.; Young, J.; Iliffe, S.; Rikkert, M.O.; Rockwood, K. Frailty in elderly people. Lancet 2013, 381, 752-762. [CrossRef]

14. Walston, J. Frailty in older adults. In Oxford Textbook of Geriatric Medicine; Oxford University Press: Oxford, UK, 2017; p. 429.

15. Montero, M.; Najafi, B.; Hinko, V.; Hoegliner, S.; Rahemi, H.; Enriquez, A.; Chung, J.; Barshes, N.; Gilani, R.; Mills, J. Using Frailty and Cognitive Assessment to Predict Adverse Events After Major Vascular Intervention: Application of Wearable Technologies. J. Vasc. Surg. 2017, 66, e56. [CrossRef]

16. Millar, K.; Asbury, A.; Murray, G. Pre-existing cognitive impairment as a factor influencing outcome after cardiac surgery. Br. J. Anaesth. 2001, 86, 63-67. [CrossRef] [PubMed]

17. Fougère, B.; Daumas, M.; Lilamand, M.; Sourdet, S.; Delrieu, J.; Vellas, B.; van Kan, G.A. Association between frailty and cognitive impairment: Cross-sectional data from Toulouse frailty day hospital. J. Am. Med. Dir. Assoc. 2017, 18, 990.e1-990.e5. [CrossRef] [PubMed]

18. Verghese, J.; Robbins, M.; Holtzer, R.; Zimmerman, M.; Wang, C.; Xue, X.; Lipton, R.B. Gait dysfunction in mild cognitive impairment syndromes. J. Am. Geriatr. Soc. 2008, 56, 1244-1251. [CrossRef] [PubMed]

19. Boyle, P.A.; Capurso, C.; D’Introno, A.; Colacicco, A.M.; Capurso, A.; Solfrizzi, V. Mild cognitive impairment: risk of Alzheimer disease and rate of cognitive decline. Neurology 2006, 67, 441-445. [CrossRef] [PubMed]

20. Patterns of loss of basic activities of daily living in Alzheimer patients: A cross-sectional study of the French REAL cohort. Dement. Geriatr. Cogn. Disord. 2010, 29, 46-54. [CrossRef]

21. Liang, F.W.; Chan, W.; Chen, P.J.; Zimmerman, C.; Waring, S.; Doody, R. Cognitively-Related Basic Activities of Daily Living Impairment Greatly Increases the Risk of Death in Alzheimers Disease. PLoS ONE 2016, 11, e0160671. [CrossRef] [PubMed]

22. Nguyen, H.; Lebel, K.; Bogard, S.; Goubault, E.; Boissy, P.; Clinicians, Q.P.N.; Duval, C. Using Inertial Sensors to Automatically Detect and Segment Activities of Daily Living in People with Parkinson's Disease. IEEE Trans. Neural Syst. Rehabil. Eng. 2017. Available online: http://ieeexplore.ieee.org/stamp/stamp.jsp? arnumber $=8016621$ (accessed on 20 March 2018).

23. Seidler, R.D.; Kwak, Y.; Fling, B.W.; Bernard, J.A. Neurocognitive mechanisms of error-based motor learning. Adv. Exp. Med. Biol. 2013, 782, 39-60. [CrossRef] [PubMed]

24. Cisek, P. Integrated neural processes for defining potential actions and deciding between them: A computational model. J. Neurosci. 2006, 26, 9761-9770. [CrossRef] [PubMed]

25. Tanji, J.; Shima, K. Role for supplementary motor area cells in planning several movements ahead. Nature 1994, 371, 413-416. [CrossRef] [PubMed]

26. Halsband, U.; Ito, N.; Tanji, J.; Freund, H.-J. The role of premotor cortex and the supplementary motor area in the temporal control of movement in man. Brain 1993, 116, 243-266. [CrossRef] [PubMed]

27. Shibasaki, H.; Sadato, N.; Lyshkow, H.; Yonekura, Y.; Honda, M.; Nagamine, T.; Suwazono, S.; Magata, Y.; Ikeda, A.; Miyazaki, M. Both primary motor cortex and supplementary motor area play an important role in complex finger movement. Brain 1993, 116, 1387-1398. [CrossRef] [PubMed] 
28. Stockel, T.; Wunsch, K.; Hughes, C.M.L. Age-Related Decline in Anticipatory Motor Planning and Its Relation to Cognitive and Motor Skill Proficiency. Front. Aging Neurosci. 2017, 9, 283. [CrossRef] [PubMed]

29. Raz, N.; Gunning-Dixon, F.; Head, D.; Rodrigue, K.M.; Williamson, A.; Acker, J.D. Aging, sexual dimorphism, and hemispheric asymmetry of the cerebral cortex: Replicability of regional differences in volume. Neurobiol. Aging 2004, 25, 377-396. [CrossRef]

30. Fazekas, F.; Ropele, S.; Enzinger, C.; Gorani, F.; Seewann, A.; Petrovic, K.; Schmidt, R. MTI of white matter hyperintensities. Brain 2005, 128, 2926-2932. [CrossRef] [PubMed]

31. Tombaugh, T.N. Trail Making Test A and B: Normative data stratified by age and education. Arch. Clin. Neuropsychol. 2004, 19, 203-214. [CrossRef]

32. Zhou, H.; Sabbagh, M.; Wyman, R.; Liebsack, C.; Kunik, M.E.; Najafi, B. Instrumented Trail-Making Task to Differentiate Persons with No Cognitive Impairment, Amnestic Mild Cognitive Impairment, and Alzheimer Disease: A Proof of Concept Study. Gerontology 2017, 63, 189-200. [CrossRef] [PubMed]

33. Cockrell, J.R.; Folstein, M.F. Mini-mental state examination. In Principles and Practice of Geriatric Psychiatry; Wiley: Chichester, UK, 2002; pp. 140-141.

34. Nasreddine, Z.S.; Phillips, N.A.; Bedirian, V.; Charbonneau, S.; Whitehead, V.; Collin, I.; Cummings, J.L.; Chertkow, H. The Montreal Cognitive Assessment, MoCA: A brief screening tool for mild cognitive impairment. J. Am. Geriatr. Soc. 2005, 53, 695-699. [CrossRef] [PubMed]

35. Najafi, B.; Horn, D.; Marclay, S.; Crews, R.T.; Wu, S.; Wrobel, J.S. Assessing postural control and postural control strategy in diabetes patients using innovative and wearable technology. J. Diabetes Sci. Technol. 2010, 4, 780-791. [CrossRef] [PubMed]

36. Schwenk, M.; Howe, C.; Saleh, A.; Mohler, J.; Grewal, G.; Armstrong, D.; Najafi, B. Frailty and technology: A systematic review of gait analysis in those with frailty. Gerontology 2014, 60, 79-89. [CrossRef] [PubMed]

37. Grewal, G.; Sayeed, R.; Yeschek, S.; Menzies, R.A.; Talal, T.K.; Lavery, L.A.; Armstrong, D.G.; Najafi, B. Virtualizing the assessment: A novel pragmatic paradigm to evaluate lower extremity joint perception in diabetes. Gerontology 2012, 58, 463-471. [CrossRef] [PubMed]

38. Najafi, B.; Helbostad, J.L.; Moe-Nilssen, R.; Zijlstra, W.; Aminian, K. Does walking strategy in older people change as a function of walking distance? Gait Posture 2009, 29, 261-266. [CrossRef] [PubMed]

39. Aminian, K.; Najafi, B.; Bula, C.; Leyvraz, P.F.; Robert, P. Spatio-temporal parameters of gait measured by an ambulatory system using miniature gyroscopes. J. Biomech. 2002, 35, 689-699. [CrossRef]

40. Gaveau, V.; Pisella, L.; Priot, A.E.; Fukui, T.; Rossetti, Y.; Pélisson, D.; Prablanc, C. Automatic online control of motor adjustments in reaching and grasping. Neuropsychologia 2014, 55, 25-40. [CrossRef] [PubMed]

41. Lin, F.; Vance, D.E.; Gleason, C.E.; Heidrich, S.M. Caring for older adults with mild cognitive impairment: an update for nurses. J. Gerontol. Nurs. 2012, 38, 22-35. [CrossRef] [PubMed]

42. Razjouyan, J.; Grewal, G.S.; Rishel, C.; Parthasarathy, S.; Mohler, J.; Najafi, B. Activity monitoring and heart rate variability as indicators of fall risk: Proof-of-concept for application of wearable sensors in the acute care setting. J. Gerontol. Nurs. 2017, 43, 53-62. [CrossRef] [PubMed]

43. Lindemann, U.; Najafi, B.; Zijlstra, W.; Hauer, K.; Muche, R.; Becker, C.; Aminian, K. Distance to achieve steady state walking speed in frail elderly persons. Gait Posture 2008, 27, 91-96. [CrossRef] [PubMed]

44. Gerling, K.M.; Schild, J.; Masuch, M. Exergaming for elderly: Analyzing player experience and performance. In Proceedings of the Mensch \& Computer, Chemnitz, Germany, 11-14 September 2011; p. 401.

(C) 2018 by the authors. Licensee MDPI, Basel, Switzerland. This article is an open access article distributed under the terms and conditions of the Creative Commons Attribution (CC BY) license (http://creativecommons.org/licenses/by/4.0/). 
Article

\title{
Nondestructive Estimation of Muscle Contributions to STS Training with Different Loadings Based on Wearable Sensor System
}

\author{
Kun Liu, Yong Liu, Jianchao Yan * and Zhenyuan Sun \\ School of Mechanical Science and Engineering, Jilin University, Changchun 130025, China; \\ kunliu@jlu.edu.cn (K.L.); liuyong16@mails.jlu.edu.cn (Y.L.); zysun17@mails.jlu.edu.cn (Z.S.) \\ * Correspondence: yanjc16@mails.jlu.edu.cn
}

Received: 26 January 2018; Accepted: 21 March 2018; Published: 25 March 2018

\begin{abstract}
Partial body weight support or loading sit-to-stand (STS) rehabilitation can be useful for persons with lower limb dysfunction to achieve movement again based on the internal residual muscle force and external assistance. To explicate how the muscles contribute to the kinetics and kinematics of STS performance by non-invasive in vitro detection and to nondestructively estimate the muscle contributions to STS training with different loadings, a wearable sensor system was developed with ground reaction force (GRF) platforms, motion capture inertial sensors and electromyography (EMG) sensors. To estimate the internal moments of hip, knee and ankle joints and quantify the contributions of individual muscle and gravity to STS movement, the inverse dynamics analysis on a simplified STS biomechanical model with external loading is proposed. The functional roles of the lower limb individual muscles (rectus femoris (RF), gluteus maximus (GM), vastus lateralis (VL), tibialis anterior (TA) and gastrocnemius (GAST)) during STS motion and the mechanism of the muscles' synergies to perform STS-specific subtasks were analyzed. The muscle contributions to the biomechanical STS subtasks of vertical propulsion, anteroposterior (AP) braking and propulsion for body balance in the sagittal plane were quantified by experimental studies with EMG, kinematic and kinetic data.
\end{abstract}

Keywords: nondestructive; joint moment; partial weight loading; muscle contributions; sit-to-stand training

\section{Introduction}

Sit-to-stand (STS) movement is a common, but critical action that almost every independent person performs every day, but millions of people have difficulty in STS motion due to neurological pathologies globally [1]. STS movement is also a complex dynamic task that requires the regulation of lower limb muscles to drive the human body and maintain the dynamic balance while performing subtasks, such as leaning forward movement of the HAT (head-arms-trunk segments) in the chair, vertical stretching movement of the whole body and balancing movement of the whole body off the chair [2-5]. Patients with a decrease in leg muscle function experience difficulties in achieving STS movement, but improvement may occur with proper training [6-9]. STS movement requires coordination of several muscle groups to guarantee human balance while achieving the task. However, for the elderly and dependent people who have lost part of the lower limb functionality, the activity becomes tiring and cannot be accomplished without the help of external assistance [10]. To restore muscle strength and improve STS movement coordination, repetitive STS training with appropriate external support is very important for the patients with lower limb motion impairments. Consequently, assistant systems, such as exoskeleton/orthosis or a partial body weight support (PBWS) rehabilitation robot, can be used to provide and control external assistance [11-14].

A greater understanding of the biomechanics of STS and muscle contributions to STS has important implications for rehabilitation training. It is valuable for clinicians to make a targeted, 
effective and efficient physical therapy plan in individual STS rehabilitation training with motor disability. In common STS movement, the primary mechanism to regulate joint moments is muscle force generation. Muscles accelerate body segments and generate ground reaction forces that alter joint moments about the segments' center-of-mass (COM) to restore and maintain dynamic stability. In addition, gravity contributes to whole-body angular momentum through its contribution to the ground reaction force (GRF) [15]. During STS rehabilitation training, since certain muscles of the lower limb are weak due to neurological pathologies or surgery injury, the patients need external assistant forces (EAF), such as PBWS force on the shoulder or assistant torque on the knee joint. Accordingly the patient is driven to stand up by lower limb muscles and EAF cooperatively. However, because the individual situation varies, it is hard to quantitatively evaluate the muscles' rehabilitation phase and to accurately estimate the muscle contributions to dynamic body support without intrusive observation and measurement. Few previous studies have quantified which muscles primarily contributed to whole-body angular momentum of STS. Identification of muscles' responsibilities and contributions in STS motion has important implications for diagnosis and treatment of balance and movement disorders and the design of effective rehabilitation therapies that target specific muscle groups. Gravity acts throughout the segment, but can be located at the COM for calculation purposes. The gravity contributes to joint reaction forces, and negative joint moments with the moment arms from each segment's COM to the corresponding joint consumed the joint moments that are offered by muscle synergies. There have been many studies on the biomechanics of STS, and many papers have reported findings related to electromyographic (EMG) activity during the action [16-18]. The applications of computational biomechanics for orthopedic treatment and rehabilitation have attracted much attention to provide better quality of life for patients. Especially a greater understanding of the biomechanics and muscle activity of STS movement has important implications to design safe, effective and efficient rehabilitation training guidelines for individuals with motor disability [19-21]. Therefore, non-invasive estimation of joint moments, joint forces and muscle contributions to body support with loading for STS training using a wearable sensor system is very important for STS rehabilitation.

McGowan et al. [22] offered a method to estimate lower limb muscles' contributions to body support and forward propulsion. However, they just analyzed independent effects of weight and mass on plantar flexor activity during walking, not STS movement. Neptune et al. [23] analyzed the muscle contributions to whole-body sagittal plane angular momentum during walking. These results may provide insight into balance and movement disorders and provide a basis for developing locomotor therapies that target specific muscle groups, but there was no practical significance for STS rehabilitation. Wang et al. predicted the joint moments using a neural network model of muscle activations from EMG signals [24], but the method was used to calculate the elbow joint moments, not the lower limb joint moments from the EMG signals of ten flexor and extensor muscles. Thomas Stieglitz et al. presented a noninvasive measurement of torque development in the rat foot, but it is based on electrode implantation in the plantar/dorsiflexion and medial/lateral rotation plane. In a sense, it is not really a noninvasive measurement [25]. Furukawa et al. [26] introduced a newly-developed biosignal-based vertical weight support system, which was composed of pneumatic artificial muscles (PAMs) and an EMG measurement device. It proved that external assistance force can be varied based on measured muscle activities and can be used to instruct STS rehabilitation training. Bonnet et al. [27] investigated the possibility of estimating hip and knee joint angles using a single inertial measurement unit, but they did not pay attention to the rehabilitation of lower limb muscles. Zlatko et al. analyzed and compared kinematics, kinetics and EMG patterns of STS transfer [28] and proved they can be used for STS training. Tatsuya analyzed an EMG-based predictive control model for physical human-robot interaction, but it was not suitable for STS training [29]. Nicole et al. analyzed muscle contribution and coordination during stair ascent using a 26-camera optoelectronic motion capture system. The insightful studies provided a detailed understanding of the biomechanics during stair ascent at the joint level, but it' is not a real-time and wearable method for analyzing joint moments [30]. 
In this paper, the differences of muscle excitation intensity in different phases during STS are identified. The purpose of the study is to identify the functional roles of the lower limb individual muscles during STS movement and the mechanisms by which muscles work together to perform STS-specific subtasks. The muscle contributions to STS motion at the individual muscle level were analyzed by experimental studies with EMG data, kinematic and kinetic data. The STS kinetics and kinematics were non-invasively estimated and analyzed using developed wearable inertial sensors. The patterns of five groups of the lower limb muscles during standing up were characterized by processed EMG to examine the relationship between the muscle activities and STS kinetics. Furthermore, to identify individual muscle contributions to the biomechanical STS subtasks of vertical propulsion, anteroposterior (AP) braking and propulsion and to analyze how individual muscles, EAF and gravity contribute to STS motion and body balance in the sagittal plane, a partial-muscle-actuated STS movement was generated with different loadings on the HAT of the subject. Based on inverse dynamics analysis of the STS biomechanical model, we estimated hip, knee and ankle joint moments from accelerations, angular velocities and angles measured on the lower limb segments (HAT, thigh and shank) using inertial sensors. The results showed insight into the movement coordination of STS and had implications for the ongoing development and testing of more effective STS training techniques in the clinic.

\section{Methods}

Compared to horizontal walking during which the COM of the body is predominantly propelled, STS motion requires an individual to propel the COM significantly more vertically and less horizontally. In addition, it is obvious that dynamic balance is more difficult to maintain after the body left the seat (seat-off). These differences suggest that STS motion likely requires altered muscle contributions relative to different phases before and after seat-off. To estimate the complex relationships between muscle excitation and resultant force, the muscle contributions to accelerate joints and segments in the STS biomechanical task should be analyzed based on the STS dynamic biomechanical model as shown in Figure 1. The subjects rise from sitting in an essentially sagittal symmetric manner; therefore, a two-dimensional three-link segmental model is used in the analysis of the STS task under the assumption of bilateral symmetry in the sagittal plane.
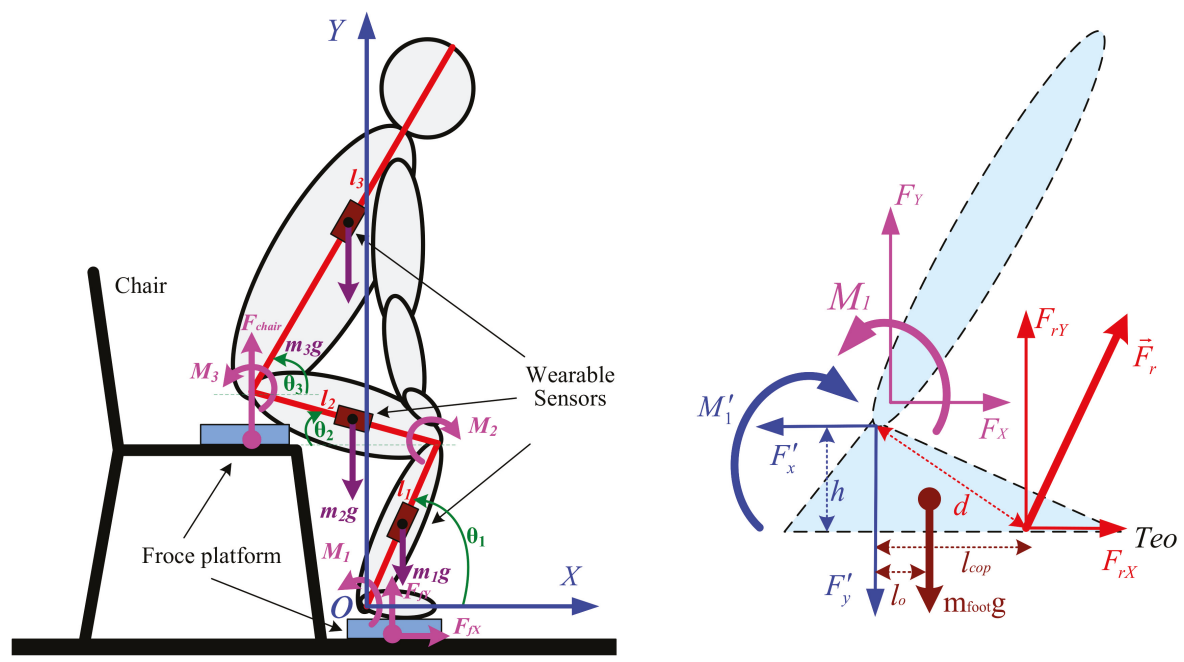

Figure 1. Link segment model of dynamic sit-to-stand (STS) biomechanical model. 
In most tasks involving kinetic measurements of the STS subject, a direct measurement of internal moments acting on lower limb joints was not feasible for PBWS rehabilitation robot users. A dynamic STS biomechanical model of a trainee consisting of the HAT, thighs, shanks and feet is needed to estimate and analyze joint moments with external GRFs, loading forces and kinetic and kinematic parameters (inertial effects). In addition, initial feet position in the STS biomechanical model directly affects the distance that HAT must move forward in STS motion. It was proven that the amplitude of displacement and velocity at the hip could be significantly optimized when the feet are behind the knee in the AP direction [31,32]. Thus, the STS movement is divided into four phases [33,34]: in the first phase, the subject in the sitting position with feet under the knees bends forward the HAT. In the second phase, the subject lifts off the chair. In the third phase, the subject extends the knee joint and the whole body stretches. Then, in the final phase, the subject is full upright in the stand-up position. Therefore, the human body can be represented by three different parts, shank-foot, thigh and HAT, and can be modeled for the STS movements as a triple inverted pendulum model in the sagittal plane articulated around the ankle, knee and hip joints. In our presented work [35], we had non-invasively estimated the joint moments using the developed wearable sensor system and analyzed the kinematic and kinetic profiles underlying the STS movement. As a further analysis of STS using wearable sensors, we recalculated the joint moments $M i(i=1,2,3)$ based on the inertial and static effects of STS motion as follows.

Hip joint moment is shown with Equations (1)-(3):

$$
\begin{gathered}
\vec{M}_{3-I N E R T I A}=\frac{1}{m} \cdot\left(\frac{1}{2} \cdot J_{3} \cdot \vec{\alpha}_{3}+\vec{r}_{33} \times\left(\frac{1}{2} \cdot m_{3} \cdot \vec{a}_{3}\right)\right) \\
\vec{M}_{3-\text { STATIC }}=\frac{1}{m} \cdot\left(\vec{r}_{33} \times\left(\frac{1}{2} \cdot m_{3} \cdot \vec{g}\right)\right) \\
\vec{M}_{3}=\vec{M}_{3-I N E R T I A}+\vec{M}_{3-\text { STATIC }}
\end{gathered}
$$

Knee joint moment is shown with Equations (4)-(6):

$$
\begin{gathered}
\vec{M}_{2-\text { INERTIA }}=\frac{1}{m} \cdot\left(-\frac{1}{2} \cdot J_{3} \cdot \vec{\alpha}_{3}-J_{2} \cdot \vec{\alpha}_{2}-\vec{r}_{23} \times\left(\frac{1}{2} \cdot m_{3} \cdot \vec{a}_{3}\right)-\vec{r}_{22} \times\left(\frac{1}{2} \cdot m_{2} \cdot \vec{a}_{2}\right)\right) \\
\vec{M}_{2-\text { STATIC }}=\frac{1}{m} \cdot\left(-\vec{r}_{23} \times\left(\frac{1}{2} \cdot m_{3} \cdot \vec{g}\right)-\vec{r}_{22} \times\left(m_{2} \cdot \vec{g}\right)\right) \\
\vec{M}_{2}=\vec{M}_{2-I N E R T I A}+\vec{M}_{2-\text { STATIC }}
\end{gathered}
$$

Ankle joint moment is shown with Equations (7)-(9):

$$
\begin{gathered}
\vec{M}_{1-I N E R T I A}=\frac{1}{m} \cdot\left(\frac{1}{2} \cdot J_{3} \cdot \vec{\alpha}_{3}+J_{2} \cdot \vec{\alpha}_{2}+J_{1} \cdot \vec{\alpha}_{1}+\vec{r}_{13} \times\left(\frac{1}{2} \cdot m_{3} \cdot \vec{a}_{3}\right)+\vec{r}_{12} \times\left(m_{2} \cdot \vec{a}_{2}\right)+\vec{r}_{11} \times\left(m_{1} \cdot \vec{a}_{1}\right)\right) \\
\vec{M}_{1-\text { STATIC }}=\frac{1}{m} \cdot\left(\vec{r}_{13} \times\left(\frac{1}{2} \cdot m_{3} \cdot \vec{g}\right)+\vec{r}_{12} \times\left(m_{2} \cdot \vec{g}\right)+\vec{r}_{11} \times\left(m_{1} \cdot \vec{g}\right)\right) \\
\vec{M}_{1}=\vec{M}_{1-I N E R T I A}+\vec{M}_{1-S T A T I C}
\end{gathered}
$$

where $\vec{M}_{i}$ is the joint moment vector about joint $i$; $J_{j}$ the moment of inertia of segment $j$ about the center of mass; $\vec{\alpha}_{j}$ the angular acceleration vector of segment $j$ about the center of mass; $\vec{r}_{i j}$ the position vector from joint $i$ to the center of gravity of segment $j ; m_{j}$ the mass of segment $j$; $m$ the whole body mass with loading on the HAT; $\vec{a}_{j}$ the acceleration vector of the center of gravity of segment $j$.

To quantify the causal relationships between the lower limb muscle excitation inputs and the resulting STS performance, the musculoskeletal modeling with explicit equations for the neuromuscular and musculoskeletal system dynamics should be utilized. Previous studies have 
used simulations to identify individual muscle contributions to tasks such as walking [36] and wheelchair propulsion [37]. Recently, Lin et al. [38] performed a simulation analysis to determine the contributions of five muscle groups to body support, forward propulsion and balance control during stair ascent by analyzing whole-body COM accelerations. The insightful studies in [30] provided a detailed understanding of the biomechanics of stair ascent. It was obvious that the five lower limb muscles (rectus femoris (RF), gluteus maximus (GM), vastus lateralis (VL), tibialis anterior (TA) and gastrocnemius (GAST)) among the analyzed fifteen muscles made the main contributions to the vertical propulsion. Therefore, to nondestructively identify the functional roles of the lower limb individual muscles during STS training movement, our research focused on the five lower limb muscles' contributions to the biomechanical STS subtasks of vertical propulsion, AP braking and propulsion in the sagittal plane. Meanwhile, to develop a wearable succedaneum of the bulky camera optoelectronic motion capture system for detailed understanding of how individual muscles, EAF and gravity contribute to STS motion and body balance, a wearable sensor system was developed.

The mass and dimension of each segment of the subject were estimated based on the average current Chinese male inertia parameters of body segments according to Chinese national standards, as shown in Table 1. The moment of inertia of each segment was estimated based on the height and the total mass of the subject [39] and shown in Table 1. All segments were assumed to be rigid, and the STS movement was performed only in the sagittal plane.

Table 1. Average inertia parameters of body segments of current Chinese male adults according to Chinese national standards [39]. HAT, head-arms-trunk.

\begin{tabular}{lcccc}
\hline \multicolumn{1}{c}{ Segments (Definition) } & $\begin{array}{c}\text { Segment Length/ } \\
\text { Height (\%) }\end{array}$ & $\begin{array}{c}\text { Segment Mass/ } \\
\text { Whole Body Mass (\%) }\end{array}$ & $\begin{array}{c}\text { Center of Mass/ } \\
\text { Segment Length Distal }\end{array}$ & $\begin{array}{c}\text { Moment of } \\
\text { Inertia (kg. m² }\end{array}$ \\
\hline $\begin{array}{l}\text { Foot (lateral malleolus/ } \\
\text { head metatarsal) }\end{array}$ & 14.77 & 3.6 & 0.5 & 0.0044 \\
\hline $\begin{array}{l}\text { Shank (femoral condyles/ } \\
\text { medial malleolus) }\end{array}$ & 23.86 & 10.6 & 0.567 & 0.0385 \\
\hline $\begin{array}{l}\text { Thigh (greater trochanter/ } \\
\text { femoral condyles) }\end{array}$ & 28.13 & 22.7 & 0.567 & 0.1978 \\
\hline $\begin{array}{l}\text { HAT (greater trochanter/ } \\
\text { glenohumeral joint) }\end{array}$ & 50.17 & 63.1 & 0.374 & 0.9180 \\
\hline
\end{tabular}

\section{Experiment}

A sensor system composed of two force plates, inertial sensor modules (IMUs) and EMG electrodes was developed. To measure the kinematic parameters of the segments for calculating joint moments according to Equations (1)-(9), three customized IMUs (wearable sensor JY-901B, $1.1 \times 1.1 \times 0.5$ inches with battery and Bluetooth communication) were attached on the position of COM of the shank, thigh and HAT with Velcro, as shown in Figure 1. A microcontroller (Arduino UNO) was used to capture accelerations and angular velocities from the IMUs at a $100-\mathrm{Hz}$ sampling rate, store data and communicate with a PC in real time. The two force plates were developed with pressure sensors (YZC-1B), and the forces were sampled at a 100-Hz rate using the microcontroller (Arduino UNO). Force Plate A was fixed on the chair to measure the vertical chair reaction force (VCRF) before seat-off. Force Plate B was placed under subjects' feet to measure the vertical ground reaction force (VGRF). During the initial calibration, the force plates were positioned horizontally with calibration errors of $0.74 \%$ and $0.68 \%$ of the measured vertical forces. To relate each IMU's orientation to the corresponding segment's orientation, a sensor-to-segment calibration procedure is performed as presented in [40]. The X-axis of each IMU was repeatedly adjusted to coincide with the axial direction of its corresponding segment in the segment coordinate frames based on the recommendations of the International Society of Biomechanics [41]. The wearable sensor system has been verified in our previous research work [35] to be available for non-invasive estimation of joint moments and analysis of STS kinematics. 
EMG signals from five lower limb muscles of the right leg, RF, GM, VL, TA and GAST, were monitored by bipolar surface electrodes (Meditrace 0.11 -m self-adhesive $\mathrm{Ag} / \mathrm{AgCl}$ electrodes). The electrode positions are indicated in Figure 2. The proximal electrode was positioned above either the bulkiest part or the middle of the muscle belly with a 0.01-m standard interelectrode distance according to [42]. At the electrode locations, the skin was shaved, cleaned with alcohol and lightly abrased to further reduce skin resistance. Electrical silence in all five muscles was achieved prior to data collection. The EMG signals were processed by means of two customized self-developed 3-channel amplifiers as shown in Figure 3 with a band-pass filter from $10 \mathrm{~Hz}-500 \mathrm{~Hz}$. The signal was amplified $(1 \mathrm{~K})$ and converted from the analog-to-digital form via an analog-to-digital interface board (PCI 1713U) at a sampling frequency of $1000 \mathrm{~Hz}$. To procure a relative impression of the degree of activity, all EMG signals were full-wave rectified and low-pass filtered.
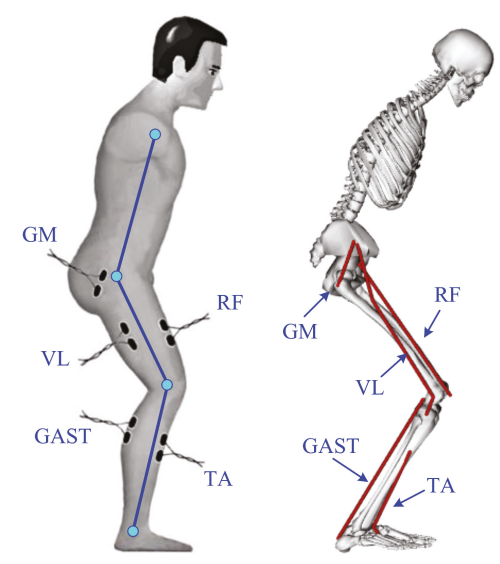

Figure 2. Schematic presentation of the EMG electrodes' placement and muscles in the sagittal plane. GM, gluteus maximus; VL, vastus lateralis; GAST, gastrocnemius; RF, rectus femoris; $\mathrm{TA}$, tibialis anterior.

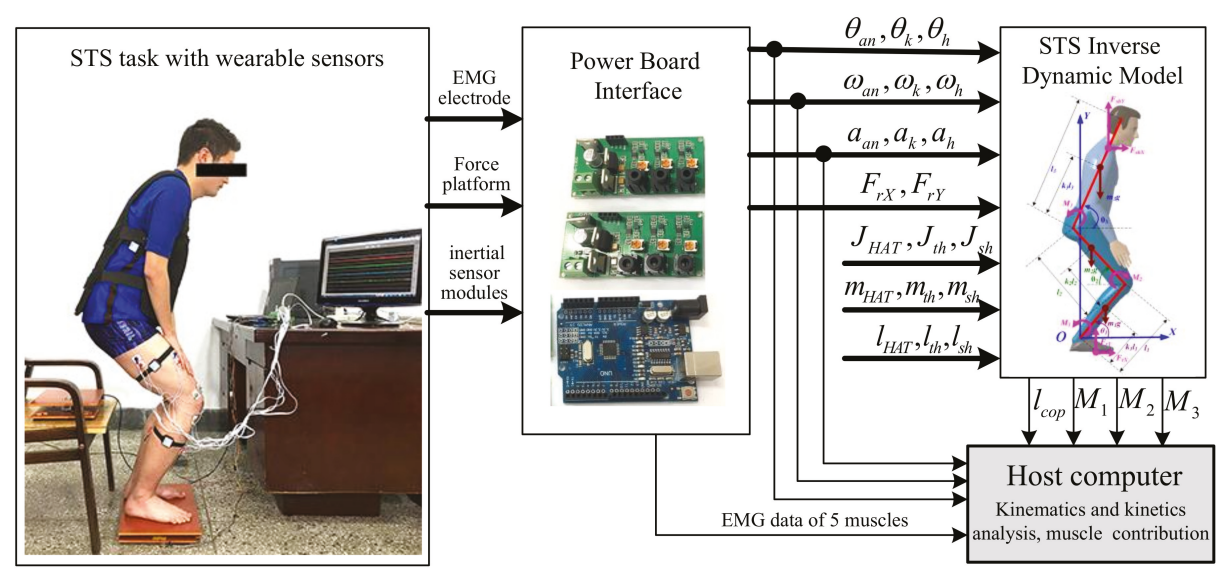

Figure 3. Schematic diagram of the proposed method.

Ten healthy male subjects (age $26 \pm 2$ years, height $1.74 \pm 0.05 \mathrm{~m}$, weight $66 \pm 6.5 \mathrm{~kg}$ ) without any known musculoskeletal or neurological dysfunction participated in this study and received informed 
consent. The experimental protocol was approved by the Human Ethical Review Committee of Jilin University. After familiarization and practice, the subjects reported no serious impediment of the developed sensor system. Then, each subject performed three STS trials as a task and five STS tasks with different loadings $(0 \mathrm{~kg}, 5 \mathrm{~kg}, 10 \mathrm{~kg}, 15 \mathrm{~kg}$ and $20 \mathrm{~kg})$ on the HAT at a self-selected preferred speed and movement pattern without any support. In each initial sitting posture of the STS trials, the feet position were located behind the knee in the anteroposterior direction with self-selected appropriate distance of about half the length of the foot. Therefore, 150 trials ( 3 trials $\times 5$ groups of tasks per subject $\times 10$ subjects) were achieved. Although a task of three STS trials was performed by one subject, the STS time and the amount of captured data of each trial could not be absolutely the same. Three events provided reference points for kinematic data: movement onset (when the angular velocity of the COM of HAT being greater than $0.01 \mathrm{rad} / \mathrm{s}$ ), seat-off (zero VCRF) and movement end (when the angular velocity of the COM of thigh was less than $0.01 \mathrm{rad} / \mathrm{s}$ or VCRF approximately equaled the subject's weight). The three events enabled the action to be divided into two phases: a pre-stretch phase (movement onset to seat-off) and a stretch phase (seat-off to movement end). Therefore, the time of seat-off (zero VCRF) was designated as the referenced standard point (RSP) within whole STS to synchronize the kinematic, kinetic and EMG data derived from the three STS trials of a task to the same percentage metric, then the ensemble averages of a task were obtained. The kinematic data, VGRF, VCRF and the calculated joint moments were normalized to the subject's height and weight, then were used to analyze the muscle contributions to body support in different-loading STS training compared with the five groups of EMG data captured from RF, GM, VL, TA and GAST.

\section{Results}

The wearable sensor system has been verified in our earlier research work [35]. It was available for non-invasive estimation of joint moments and analysis of STS kinematics. All signals captured by the developed sensor system were off-line processed by MATLAB. A typical group of the ensemble averages of the synchronized kinetic and kinematic parameters and EMG signals derived from five different loading STS tasks performed by one healthy subject is shown in Figures 4-8. In each figure, the first column shows the calculated hip, knee and ankle joint angles and moments (green, red and blue lines, correspondingly), angular velocities and angular accelerations of the HAT, thigh and shank (green, red and blue lines, correspondingly) and VCRF and VGRF (blue and red lines, correspondingly). The second column shows the surface EMG signals of the five lower limb muscles (TA, GAST, RF, VL and GM). The third column shows the bar graph of the analysis of the muscle contribution index (MCI) based on the surface EMG signal after further calculation.

Voluntary muscle activity detection from EMG signals can be problematic due to spurious involuntary spikes produced by physiological and extrinsic/accidental origins [43]. Accurate detection of surface EMG signals for the lower limb muscle activities is important to understand how muscles made the contributions to STS motion. However, the EMG signal distortion is unavoidable with the removal of the spike contamination, thus impeding reliable measurement of weak EMG signals. The study in [44] demonstrated the usefulness of the integrated profile of EMG for muscle activity detection using surface EMG signals in the presence of spurious background spike contamination. The integrated profile method was used to determinate the onset of muscle contraction and contribution to STS motion without removing spurious background spikes from raw surface EMG signals. In this paper, as further processing of the integrated profile of EMG, MCI is defined to quantitatively indicate the five lower limb muscles' contributions to the biomechanical STS subtasks of vertical propulsion, AP braking and propulsion in the sagittal plane. The MCI of each muscle was calculated from the ensemble averages of the synchronized integrated profile of EMG of the three STS trials in the same task and was quantified by 50 integers in a whole STS cycle by percentage metric. 

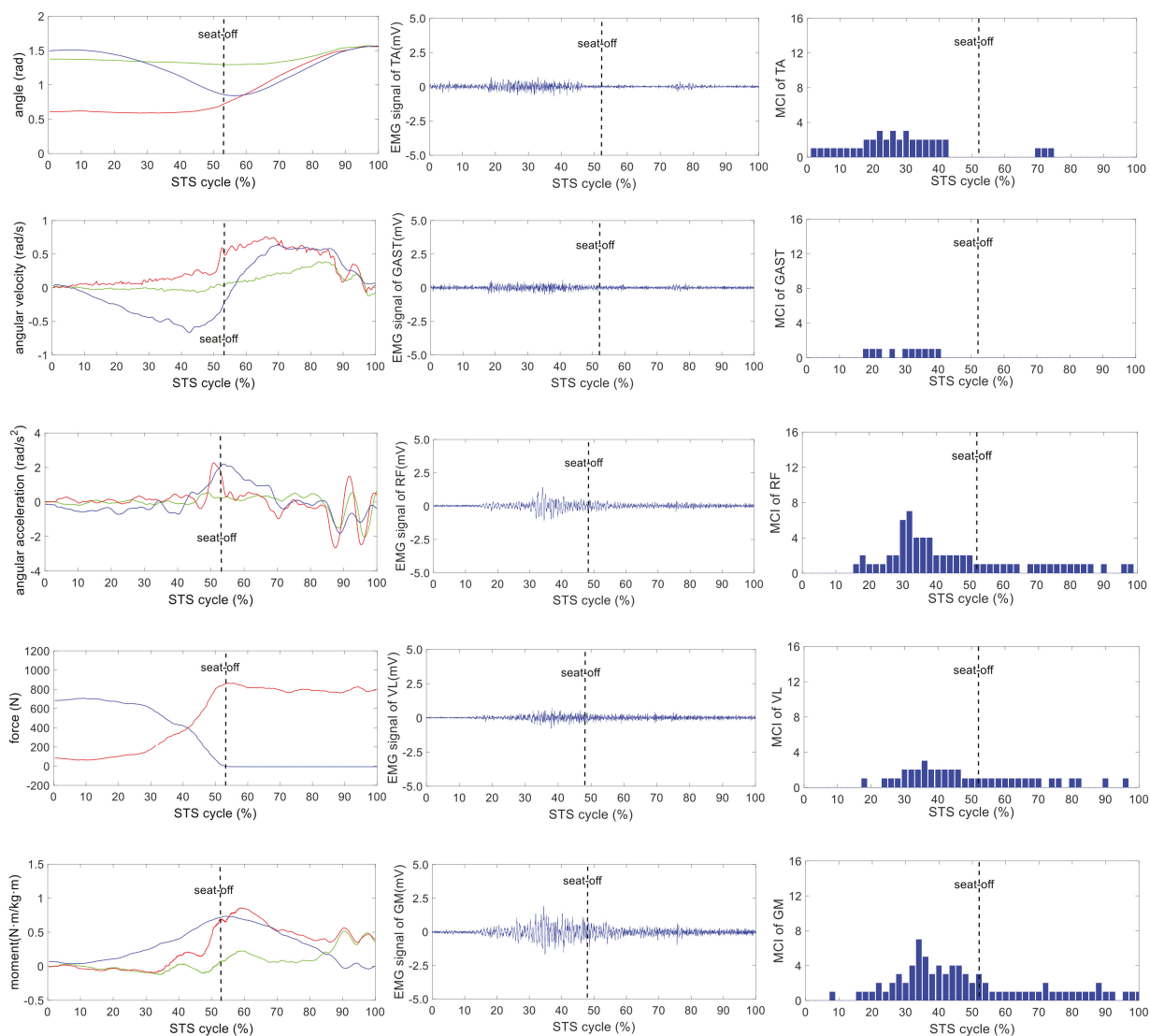

Figure 4. The analysis result of a typical STS task with no loadings: the left column shows the hip, knee and ankle joint angles and moments (green, red and blue lines, correspondingly), angular velocities and angular accelerations of the HAT, thigh and shank (green, red and blue lines, correspondingly), and vertical chair reaction force (VCRF) and vertical ground reaction force (VGRF) (blue and red lines, correspondingly). The middle column shows the surface EMG signals of the five lower limb muscles (TA, GAST, RF, VL and GM). The third column shows the muscle contribution indexes (MCI) of the five lower limb muscles (TA, GAST, RF, VL and GM).
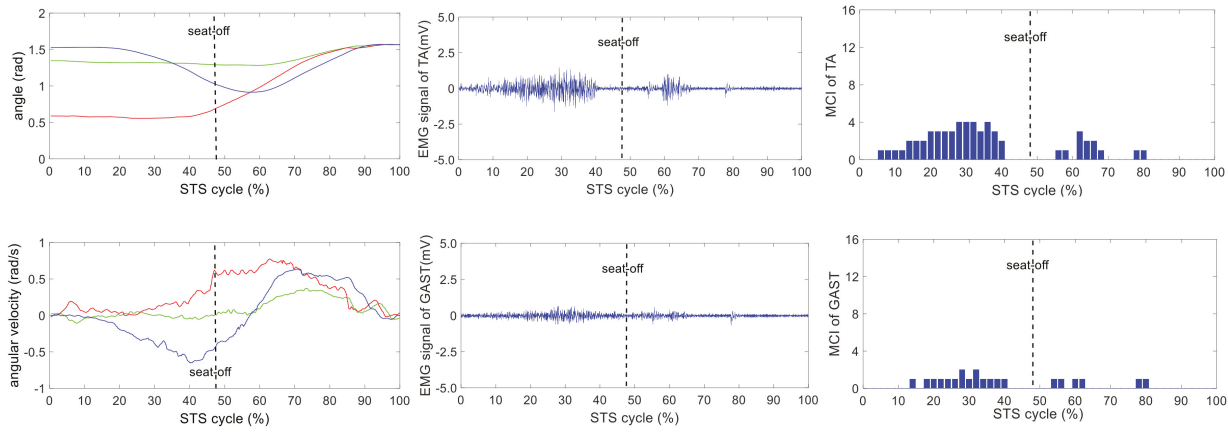

Figure 5. Cont. 

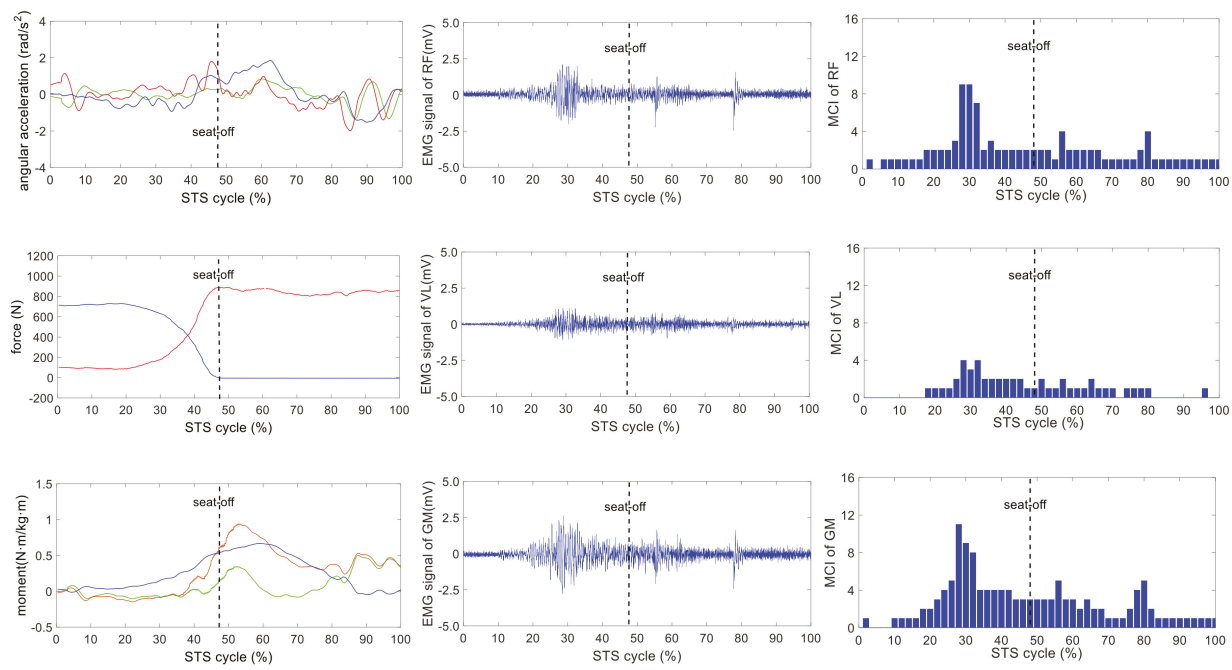

Figure 5. The analysis result of a typical STS task with 5-kg loadings: the left column shows the hip, knee and ankle joint angles and moments (green, red and blue lines, correspondingly), angular velocities and angular accelerations of the HAT, thigh and shank (green, red and blue lines, correspondingly) and VCRF and VGRF (blue and red lines, correspondingly). The middle column shows the surface EMG signals of the five lower limb muscles (TA, GAST, RF, VL and GM). The third column shows the muscle contribution indexes (MCI) of the five lower limb muscles (TA, GAST, RF, VL and GM).
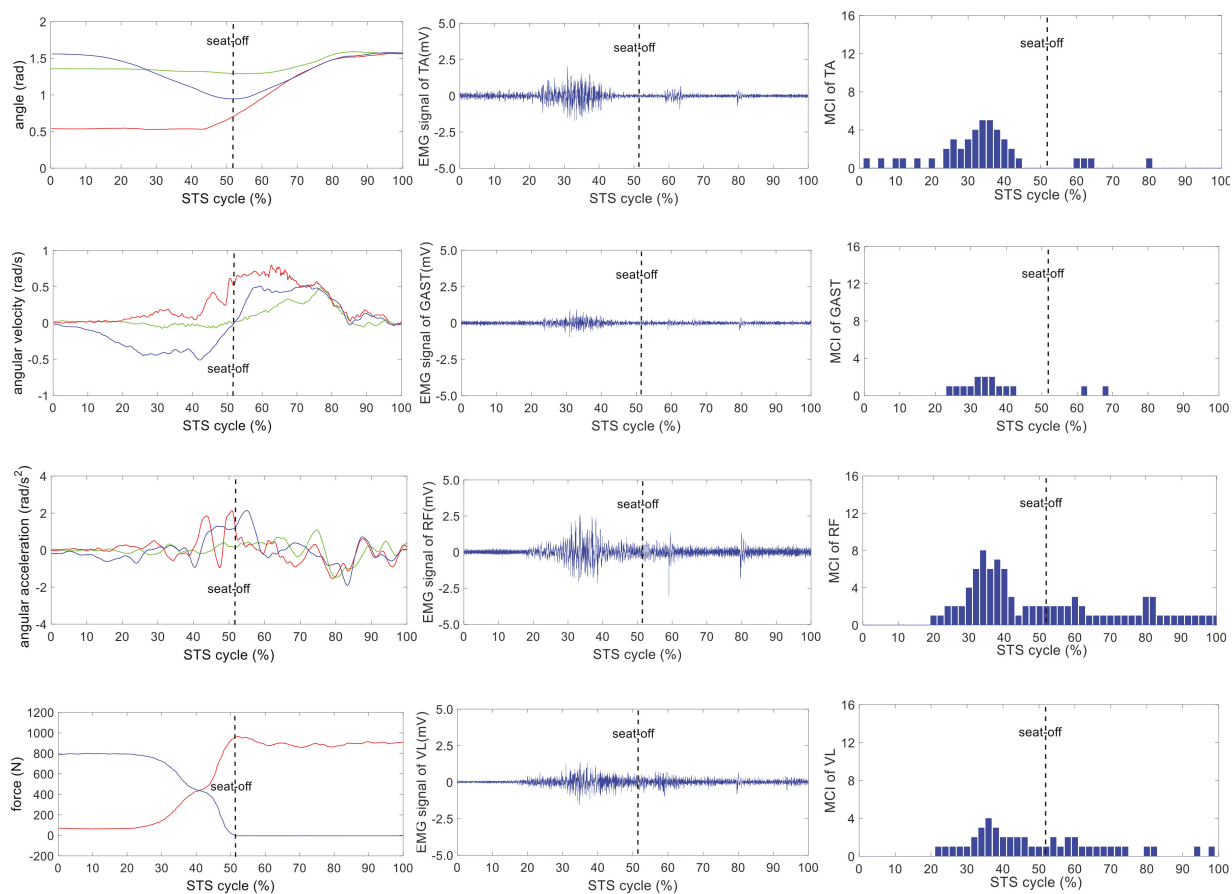

Figure 6. Cont. 

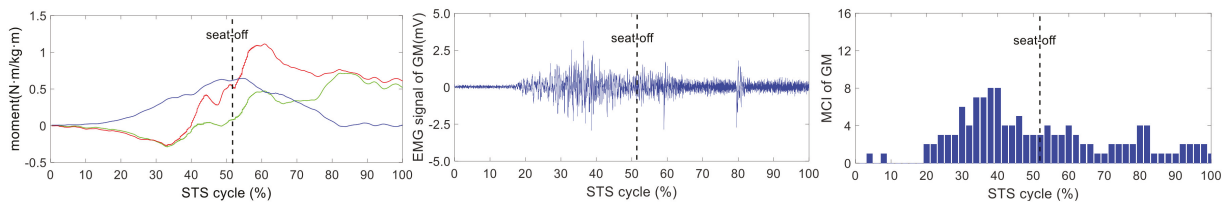

Figure 6. The analysis result of a typical STS task with 10-kg loadings: the left column shows the hip, knee and ankle joint angles and moments (green, red and blue lines, correspondingly), angular velocities and angular accelerations of the HAT, thigh and shank (green, red and blue lines, correspondingly) and VCRF and VGRF (blue and red lines, correspondingly). The middle column shows the surface EMG signals of the five lower limb muscles (TA, GAST, RF, VL and GM). The third column shows the muscle contribution indexes (MCI) of the five lower limb muscles (TA, GAST, RF, VL and GM).
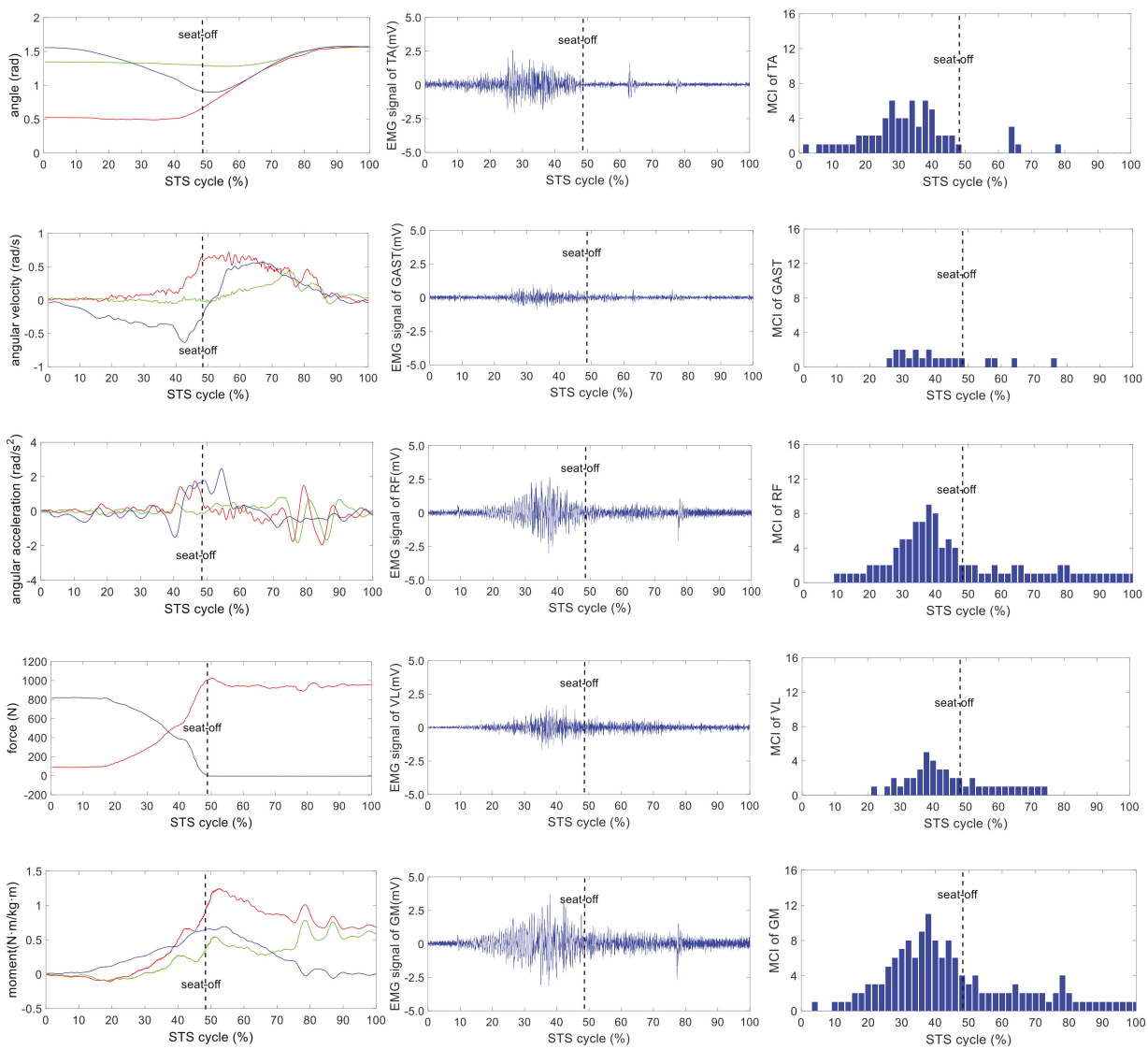

Figure 7. The analysis result of a typical STS task with 15-kg loadings: the left column shows the hip, knee and ankle joint angles and moments (green, red and blue lines, correspondingly), angular velocities and angular accelerations of the HAT, thigh and shank (green, red and blue lines, correspondingly) and VCRF and VGRF (blue and red lines, correspondingly). The middle column shows the surface EMG signals of the five lower limb muscles (TA, GAST, RF, VL and GM). The third column shows the muscle contribution indexes (MCI) of the five lower limb muscles (TA, GAST, RF, VL and GM). 

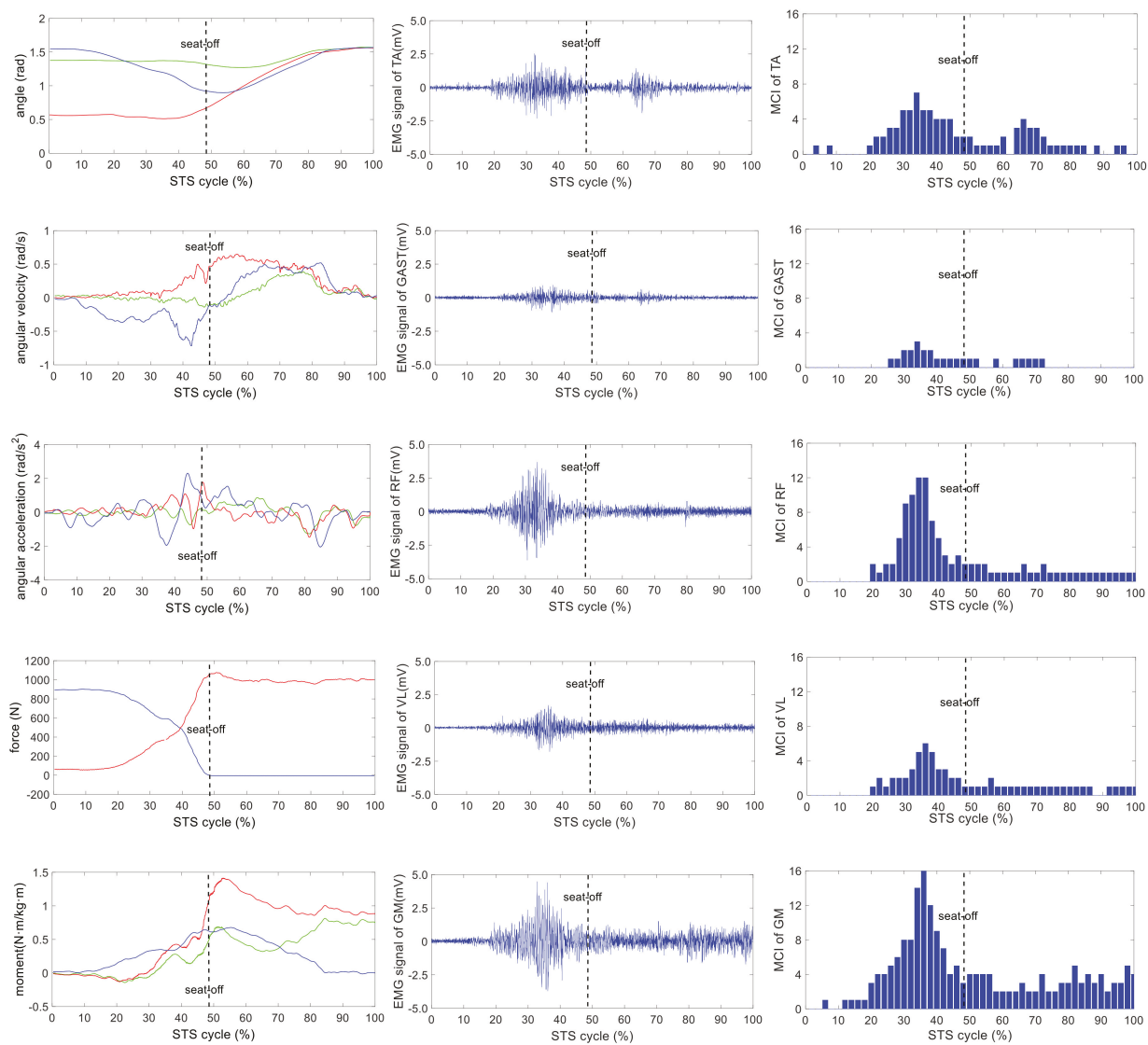

Figure 8. The analysis result of a typical STS task with 20-kg loadings: the left column shows the hip, knee and ankle joint angles and moments (green, red and blue lines, correspondingly), angular velocities and angular accelerations of the HAT, thigh and shank (green, red and blue lines, correspondingly) and VCRF and VGRF (blue and red lines, correspondingly). The middle column shows the surface EMG signals of the five lower limb muscles (TA, GAST, RF, VL and GM). The third column shows the muscle contribution indexes (MCI) of the five lower limb muscles (TA, GAST, RF, VL and GM).

\section{Discussion}

As indicated in [35], faster and more fluent STS movements resulted in better accuracy of the kinetic and kinematic analysis of STS using the developed inertial sensor system. Since it was more difficult to firmly fix the IMUs on the soft human body segments than on a rigid body without any slight movement, the systematic error was predictable, but inevitable. Especially in lower speed STS motion, the long duration of skin motion artifact due to impact loading and muscle activation and body-sway motion in non-fluent STS trials would inevitably contaminate the measured original angular velocities, accelerations and the raw EMG signals.

The difference of the five muscles was due to their relative contributions to the horizontal and vertical GRFs and joint moments resulting in different kinematics of STS motions with different loadings. Referring to the joint angles of the hip, knee and ankle in the first column of Figures 4-8, it is suggested that the HAT took action first among the three segments of the subject. When the HAT was still swinging forward before the hip joint angle reached the maximum, the knee joint angle had begun to increase since seat-off; 
meanwhile, the HAT continued to swing forward into the stretch phase (after seat-off), then the ankle joint began to increase in the terminal stretch phase. Compared with the EMG signals, MCIs and the kinetic and kinematic parameters in Figures 4-8, the highest levels of the five muscles' excitation intensities (indicated by maximum MCI) all occurred before seat-off. Meanwhile, as shown in Figure 9, the percentages of each muscle's accumulated MCI before seat-off were all no less than $58 \%$ of the total contributions to whole STS motion, which suggests that the muscles' contributions to the whole STS motion primarily concentrated before seat-off compared to those after seat-off. However the max knee joint moment appeared after seat-off, and the HAT still swung forward after seat-off into the stretch phase. It is, therefore, quite clear that the muscles' co-contractions were recruited ahead of the stretch subtask before seat-off. The superimposition before seat-off could be intended as a synergic action of muscles for controlling weight bearing as static joint moments, then the muscle synergies continued to increase until the inertial joint moments were adequate to promote vertical propulsion, anteroposterior (AP) braking and propulsion for performing a balanced body support of STS motion.

In addition, with regard to the sum of MCI of each muscle before seat-off in different loading tasks in Figure 10, the absolute accumulated MCI of GAST before seat-off was the least among the five muscles in any task (no more than 18 in the $20-\mathrm{kg}$ task, minimum MCI = 10 in the $0-\mathrm{kg}$ task), and as shown in Figures 4-8, the MCI of GAST was even weaker or not detected after seat-off. This suggested that GAST made the least contribution to STS motion among the five muscles in any loading task, but almost all of its contributions were made before seat-off (100\% in the $0-\mathrm{kg}$ task in Figure 10$)$. It is, therefore, quite clear that GAST primarily contributed to the preparation of a stable stretch phase by holding the shank before seat-off and mainly contributed to the ankle joint extension and holding after seat-off. Compared the MCIs of TA and GAST in Figures 4-8, TA had the same characteristic as GAST to contribute synergy for holding the shank and ankle joint extension before seat-off, but it was obvious that TA contracted earlier (almost from the beginning) and contributed more synergy in duration and intensity than GAST. Compared with the onset times of the five muscles in each loading task, GAST and VL contracted relatively the latest of the five muscles (almost after 20\% of the STS cycle). This once again suggested that the primary contributions of GAST (including VL here) were to vertical STS propulsion preparation and balance holding of the lower limb, but not to the swing of HAT before seat-off. Besides, with regard to the onset time and MCI of each muscle in Figures 4-8, the muscles' activities in the stretch phase were recognized as typical activations of RF muscle as knee extensors, GM muscle as hip extensors and TA muscle as ankle extensors. Comparing the joint angles and moments with the corresponding MCIs of GM, TA and RF in the same task in Figures 4-8, the high correlation of the onset time and amplitude suggested that the GM, TA and RF were the primary contributors to forward propulsion of HAT from the beginning, then all five muscles co-contracted to apply sufficient synergies for vertical propulsion until the subject seated off. Obviously, as shown in Figure 11, the GM contributed the most among the five muscles in duration and intensity to accelerate HAT forwards in the pre-stretch phase and keep stable hip joint extension in the stretch phase in any loading task. For example, in Figure 10, before seat-off in each task, the accumulated MCI of GM was 56, 73, 77, 98 and 113, respectively, any of which was the biggest of the five muscles in the same loading task. It is obvious in Figure 10 that, with the addition of loadings, the muscles' contributions indicated by MCI also increased. This fully illustrates the causal relationship between muscle force and loadings. For example, in the successful STS task with the 20-kg loading, as shown in Figure 8, all of the MCIs of the five muscles indicated the maximum contributions compared with other loading tasks. On the contrary, this also suggested that deficits in the neuromuscular control of the five selected muscles could adversely influence balance recovery, which may be important targets in rehabilitation to improve balance recovery performance. As the MCIs of VL, RF and GM in each task show, their synergic action after seat-off was still obvious. This suggests that the synergies were presented to assist knee and hip extensions and develop muscle tension for weight and external loading acceptance in the stretch phase, meanwhile providing the majority of braking accelerations for the balance recovery in the terminal stretch phase before movement end. 


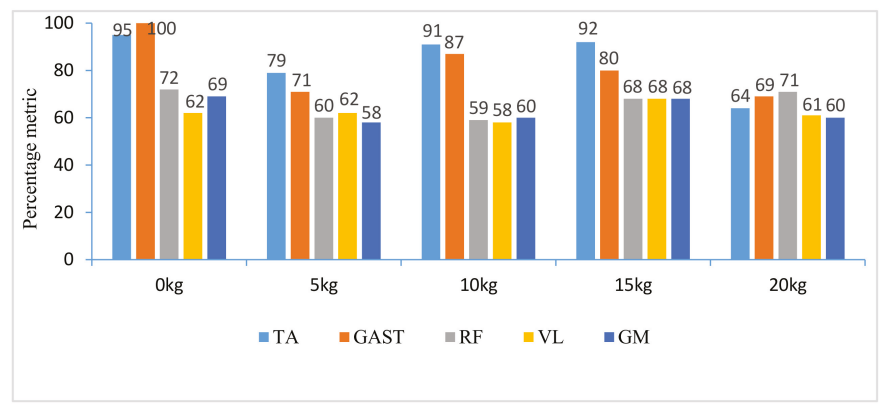

Figure 9. Percentage metric of each muscle's accumulated contribution before seat-off in different tasks.

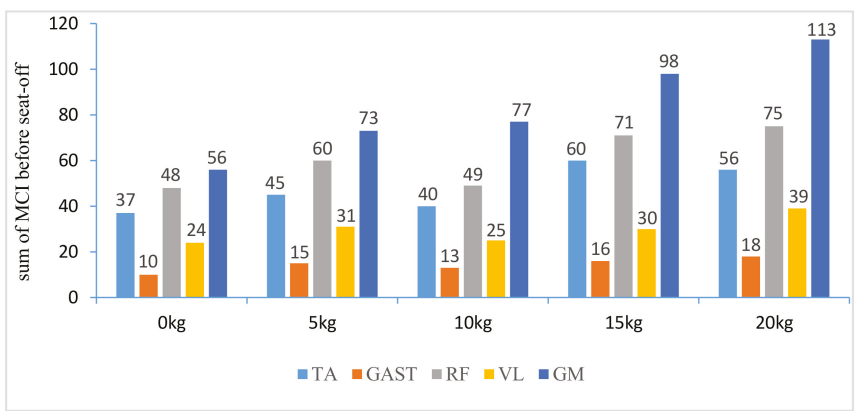

Figure 10. The sum of each muscle's MCI before seat-off in different tasks.

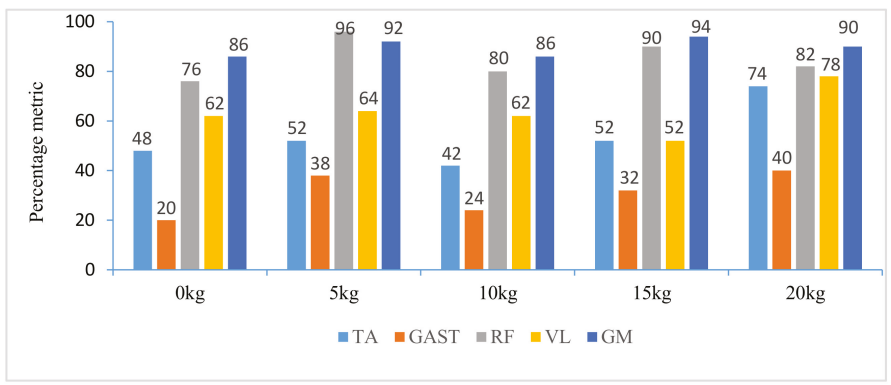

Figure 11. Percentage metric of each muscle's contraction duration in whole STS cycle of different loading tasks.

Kinetics analysis in the stretch phase: To control STS balance, the antagonistic muscles spanning the hip, knee and ankle joints distributed power from the leg to the remaining body segments. This also suggested that the uniarticular hip and knee extensors (GM and VL) and ankle dorsiflexors (TA) generated backward angular momentum while the ankle plantar flexors (GAS) generated forward momentum; therefore, the whole body began vertical propulsion with enough kinetic energy into the stretch phase, then the muscles' co-contractions obviously reduced with less MCIs after seat-off. That RF and VL overlapped their activities in the early stretch phase just after seat-off (refer to the third column of Figures 4-8) was likely synergizing for modulating rapid knee flexion, although in less than $10 \%$ of the whole STS cycle and with low levels of MCI. 
In particular, there are still some uncommon and variable activities of the TA, RF and GM indicated by significant MCIs in the middle and terminal stretch phases before movement end (in the third column of Figures 4-8), probably because with the stretching of the body segments and the vertical propulsion of the COM after seat-off, the primary work of lower limb muscles had converted to support body balance. RF and VL supposedly contributed to knee flexion and patella stabilization in the middle and terminal stretch phase; RF and GM participated in hip stabilization; and TA and GAST contributed to ankle flexion and stabilization. According to the consultancies with subjects, as evidence, the deficiency of sufficient stability of the self-made force plate indeed caused an instantaneous stretch balance adjustment in the terminal stretch phase. Especially in the tasks with more loadings (15 kg and $20 \mathrm{~kg}$ ), TA, RF and GM should instantaneously recruit obvious muscle synergies for stretch balance. Compared with the MCIs of TA, RF and GM in the stretch phases of the five loading tasks in Figures 4-8, the MCIs of the three muscles became higher with more obvious fluctuation as the loadings increased, so this confirmed that more loadings would induce more muscle activities and co-contractions of TA, RF and GM for balance control in the stretch phase.

The results and discussion could provide insight into STS balance and movement disorders and offer a reference for developing locomotor therapies that target specific muscle groups; meanwhile, it may provide further rationale for developing targeted STS rehabilitation robot control strategies to address patient-specific deficits in STS training. Although the selected lower limb muscles' relative contributions to STS motion were compared and analyzed based on the muscle excitation intensities and durations and the kinetics and kinematics of STS motion, outcomes on muscle co-contraction and absolute contributions of more lower limb muscles should be confirmed and supported by further analysis. In addition, differences in designated loadings and self-selected STS speeds across subjects could introduce variability in muscle onset-offset times and co-contraction. Thus, STS speed and loadings could be acknowledged as a limitation of the study.

\section{Conclusions}

Quantification of lower limb muscles' co-contraction contributing to STS propulsion movement with different loadings was analyzed based on a self-developed wearable sensor system. How the muscles generate, absorb and / or transfer mechanical power between body segments to accelerate the whole-body COM was identified by quantitative assessment of five lower limb muscles' contributions during able-bodied STS motion, in terms of variability of onset-offset muscular activation, excitation intensity indicated by MCI and the percentage occurrence of EMG signals and kinetic and kinematic information derived from the wearable sensor system. The results would contribute to further understanding of more muscles' contributions and coordination during STS movement and provide additional insight for the development of effective, targeted rehabilitation programs aimed at improving an individual's ability to STS rehabilitation training and useful in the clinical context for designing future STS or gait studies. In the future, partial body weight support or more specified loading would be tested on patients in rehabilitation by more motion models, including assisted standing motion with hands or weight support rehabilitation robots. Finally, the presented method of nondestructive estimation of muscle contributions to STS motion based on the wearable sensor system is expected to be applied to weight support or loading rehabilitation robot control or evaluation of STS rehabilitation therapy.

Acknowledgments: The authors wish to acknowledge the support of the volunteer subjects of the Robotics and Dynamics Research Lab in Jilin University. The work was partly supported by the Youth Research Fund Project of the Department of Science and Technology of Jilin province (20160520066JH) and the Graduate Innovation Fund of Jilin University.

Author Contributions: Kun Liu, Jianchao Yan, Yong Liu and Zhenyuan Sun conceived of and designed the experiments. Kun Liu and Zhenyuan Sun helped to perform the experiments. Kun Liu, Jianchao Yan and Yong Liu analyzed the data. Kun Liu, Jianchao Yan and Yong Liu wrote the paper. In addition, Kun Liu and Jianchao Yan were responsible for the implementation of the proposed scheme.

Conflicts of Interest: The authors declare no conflict of interest. 


\section{References}

1. Cheng, Y.Y.; Hsieh, W.L. Principles of rehabilitation for common chronic neurologic diseases in the elderly. Clin. Gerontol. Geriatr. 2012, 3, 5-13. [CrossRef]

2. Enoka, R.M. Neuromechanics of human movements. Percept. Motor Skills 2001, 93, 575.

3. Janssen, W.G.; Bussman, H.B.; Stam, H.J. Determinants of the sit-to-stand movement: A review. Phys. Ther. 2002, 82, 866-879. [PubMed]

4. Kawagoe, S.; Tajima, N.; Chosa, E. Biomechanical analysis of effects of foot placement with varying chair height on the motion of standing up. J. Orthop. Sci. 2000, 5, 124-133. [CrossRef] [PubMed]

5. Kralj, A.; Jaeger, R.J.; Munih, M. Analysis of standing up and sitting down in humans: Definitions and normative data presentation. J. Biomech. 1990, 23, 1123-1138. [CrossRef]

6. Schot, P.K.; Knutzen, K.M.; Poole, S.M.; Mrotek, L.A. Sit-to-stand performance of older adults following strength training. Res. Q. Exerc. Sport 2003, 74, 1-8. [CrossRef] [PubMed]

7. Maren, S.; Fragala, H.; Fukuda, R. Muscle quality index improves with resistance exercise training in older adults. Exp. Gerontol. 2014, 53, 1-6.

8. Arnold, P.; Bautmans, I. The influence of strength training on muscle activation in elderly persons: A systematic review and meta-analysis. Exp. Gerontol. 2014, 58, 58-68. [CrossRef] [PubMed]

9. Paul, A.; Andrew, P.; Peake, J.M.; Cameron-Smith, D. Effect of exercise training on skeletal muscle cytokine expression in the elderly. Brain Behav. Immun. 2014, 39, 80-86.

10. Mefoued, S.; Mohammed, S.; Amirat, Y.; Fried, G. Sit-To-Stand Movement Assistance Using an Actuated Knee Joint Orthosis. In Proceedings of the Fourth IEEE RAS/EMBS International Conference on Biomedical Robotics and Biomechatronics, Roma, Italy, 24-27 June 2012; pp. 1753-1758.

11. Shinsuke, Y.; Akinori, N.; Hay, D.C.; Fukashiro, S. The minimum required muscle force for a sit-to-stand task. J. Biomech. 2012, 45, 699-705.

12. Van der Heijden, M.M.; Meijer, K.; Willems, P.J.; Savelberg, H.H. Muscles limiting the sit-to-stand movement: An experimental simulation of muscle weakness. Gait Posture 2009, 30, 110-114. [CrossRef] [PubMed]

13. Yan, T.F.; Marco, C.; Oddo, C.M.; Vitiello, N. Review of assistive strategies in powered lower-limb orthoses and exoskeletons. Robot. Auton. Syst. 2015, 64, 120-136. [CrossRef]

14. Wesley, R.; Kubica, E. Modeling and Control Considerations for Powered Lower-Limb Orthoses: A Design Study for Assisted STS. J. Med. Devices 2006, 1, 126-139.

15. Annachiara, S.; Alessandro, M.; Sandro, F. Surface-EMG analysis for the quantification of thigh Muscle dynamic co-contractions during normal gait. Gait Posture 2017, 51, 228-233.

16. Lancini, M.; Serpelloni, M.; Pasinetti, S.; Guanziroli, E. Healthcare sensor system exploiting instrumented crutches for force measurement during assisted gait of exoskeleton users. IEEE Sens. J. 2016, 16, 8228-8237. [CrossRef]

17. Winslow, J.; Martinez, A.; Thomas, C.K. Automatic Identification and Classification of Muscle Spasms in Long-term EMG Recordings. IEEE J. Biomed. Health Inform. 2015, 19, 464-470. [CrossRef] [PubMed]

18. Bonato, P.; Boissy, P.; Della, C.U.; Roy, S.H. Changes in the surface EMG signal and the biomechanics of motion during a repetitive lifting task. Neural Syst. Rehabilit. Eng. 2002, 10, 38-47. [CrossRef] [PubMed]

19. Khemlani, M.M.; Carr, J.H.; Crosbie, W.J. Muscle synergies and joint linkages in sit-to-stand under two initial foot positions. Clin. Biomech. 1999, 14, 236-246. [CrossRef]

20. An, Q.; Ishikawa, Y.; Shinya, A. Analysis of Muscle Synergy Contribution on Human Standing-up Motion Using a Neuro-Musculoskeletal Model. In Proceedings of the 2015 IEEE International Conference on Robotics and Automation (ICRA), Seattle, WA, USA, 26-30 May 2015; pp. 5885-5890.

21. Hanawa, H.; Kubota, K.; Kokubun, T. Muscle synergies underlying sit-to-stand tasks in elderly people and their relationship with kinetic characteristics. J. Electromyogr. Kinesiol. 2017, 37, 15-20. [CrossRef] [PubMed]

22. McGowan, C.P.; Neptune, R.R.; Kram, R. Independent effects of weight and mass on plantar flexor activity during walking: Implications for their contributions to body support and forward propulsion. J. Appl. Physiol. 2008, 105, 486-494. [CrossRef] [PubMed]

23. Neptune, R.R.; McGowan, C.P. Muscle contributions to whole-body sagittal plane angular momentum during walking. J. Biomech. 2011, 44, 6-12. [CrossRef] [PubMed]

24. Wang, L.; Buchanan, T.S. Prediction of joint moments using a neural network model of muscle activations from EMG signals. Neural Syst. Rehabilit. Eng. 2002, 10, 30-37. [CrossRef] [PubMed] 
25. Stieglitz, T.; Schuettler, M.; Schneider, A.; Valderrama, E.; Navarro, X. Noninvasive measurement of torque development in the rat foot: Measurement setup and results from stimulation of the sciatic nerve with polyimide-based cuff electrodes. Neural Syst. Rehabilit. Eng. 2003, 11, 427-437. [CrossRef] [PubMed]

26. Furukawa, J.; Noda, T.; Teramae, T.; Morimoto, J. An EMG-Driven Weight Support System with Pneumatic Artificial Muscles. IEEE Syst. J. 2014, 10, 1026-1034. [CrossRef]

27. Bonnet, V.; Joukov, V.; Kulic, D.; Fraisse, P.; Ramdani, N.; Venture, G. Monitoring of Hip and Knee Joint Angles Using A Single Inertial Measurement Unit During Lowe-limb Rehabilitation. IEEE Sens. J. 2016, 16, 1557-1564. [CrossRef]

28. Matjacic, Z.; Zadravec, M.; Oblak, J. Sit-to-stand trainer: An apparatus for training "normal-like" sit to stand movement. IEEE Trans. Neural Syst. Rehabilit. Eng. 2016, 24, 639-649. [CrossRef] [PubMed]

29. Tatsuya, T.; Noda, T.; Morimoto, J. EMG-based model predictive control for physical human-robot interaction application for assist-as-needed control. IEEE Robot. Autom. Lett. 2018, 3, 210-217.

30. Nicole, G.; Harper, J.M.; Wilken, R.R. Muscle Function and Coordination of Stair Ascent. J. Biomech. Eng. 2018, 140. [CrossRef]

31. Shepherd, R.B.; Koh, H.P. Some biomechanical consequences of varying foot placement in sit-to-stand in young women. Scand. J. Rehabilit. Med. 1996, 28, 79-88.

32. Fleckenstein, S.J.; Kirby, R.L.; MacLeod, D.A. Effect of limited knee-fexion range on peak hip moments of force while transferring from sitting to standing. J. Biomech. 1998, 21, 915-918. [CrossRef]

33. Schenkman, M.; Berger, R.A.; Riley, P.O.; Mann, R.W.; Hodge, W.A. Whole-body movements during rising to standing from sitting. Phys. Ther. 1990, 70, 638-648. [CrossRef] [PubMed]

34. Riener, R.; Fuhr, T. Patient-driven control of FES-supported standingup: A simulation study. IEEE Trans. Rehabilit. Eng. 2002, 8, 523-529. [CrossRef]

35. Liu, K.; Yan, J.C.; Liu, Y.; Ye, M. Non-Invasive Estimation of Joint Moments with Inertial Sensor System for Analysis of STS. Rehabilitation Training. J. Healthc. Eng. 2018, 2018, 6570617. [CrossRef]

36. Pandy, M.G.; Lin, Y.C.; Kim, H.J. Muscle Coordination of Mediolateral Balance in Normal Walking. J. Biomech. 2010, 43, 2055-2064. [CrossRef] [PubMed]

37. Rankin, J.W.; Richter, W.M.; Neptune, R.R. Individual Muscle Contributions to Push and Recovery Subtasks during Wheelchair Propulsion. J. Biomech. 2011, 44, 1246-1252. [CrossRef] [PubMed]

38. Lin, Y.C.; Fok, L.A.; Schache, A.G.; Pandy, M.G. Muscle Coordination of Support, Progression and Balance during Stair Ambulation. J. Biomech. 2015, 48, 340-347. [CrossRef] [PubMed]

39. General Administration of Quality Supervision, Inspection and Quarantine of the People's Republic of China; Standardization Administration of the People's Republic of China. Inertial Parameters of Adult Human Body; Standardization Administration of the People's Republic of China: Beijing, China, 2004; GB/T 17245-2004.

40. Karatsidis, A.; Bellusci, G.; Schepers, H.M.; Andersen, M.S.; Veltink, P.H. Estimation of ground reaction forces and moments during gait using only inertial motion capture. Sensors 2017, 17, 75. [CrossRef] [PubMed]

41. Wu, G.; Siegler, S.; Allard, P. ISB recommendation on definitions of joint coordinate system of various joints for the reporting of human joint motion-Part I: Ankle, hip, and spine. J. Biomech. 2002, 35, 543-548. [CrossRef]

42. Williamson, D.A.; Epstein, L.H.; Lombardo, T.W. EMG measurement as a function of electrode placement and lever of EMG. Psychophysiology 1980, 17, 279-282. [CrossRef] [PubMed]

43. Liu, J.; Ying, D.; Zhou, P. Wiener filtering of surface EMG with a priori SNR estimation toward myoelectric control for neurological injury patients. Med. Eng. Phys. 2014, 36, 1711-1715. [CrossRef] [PubMed]

44. Liu, J.; Liu, R. Use of the integrated profile for voluntary muscle activity detection using EMG signals with spurious background spikes: A study with incomplete spinal cord injury. Biomed. Signal Process. Control 2016, 24, 19-24. [CrossRef]

(C) 2018 by the authors. Licensee MDPI, Basel, Switzerland. This article is an open access article distributed under the terms and conditions of the Creative Commons Attribution (CC BY) license (http:/ / creativecommons.org/licenses/by/4.0/). 
Article

\title{
The Height-Adaptive Parameterized Step Length Measurement Method and Experiment Based on Motion Parameters
}

\author{
Yanshun Zhang ${ }^{1}$, Yingyue Li ${ }^{1, *}$, Chuang Peng ${ }^{1}$, Dong Mou ${ }^{2}$, Ming $\mathrm{Li}^{1}$ and Wei Wang ${ }^{1}$ \\ 1 School of Instrumentation and Optoelectronic Engineering, Beihang University, \\ Beijing 100191, China; zhangyanshun@buaa.edu.cn (Y.Z.); pengchuang@buaa.edu.cn (C.P.); \\ liliyalm@buaa.edu.cn (M.L.); wangwei_b10@163.com (W.W.) \\ 2 Electronic Engineering Research Institute, China Academy of Engineering Physics, \\ Mianyang 621900, China; md1015@126.com \\ * Correspondence: shixiliufang316@163.com; Tel.: +86-10-8233-8438 or +86-130-5158-1752; \\ Fax: +86-10-8231-6813
}

Received: 8 February 2018; Accepted: 22 March 2018; Published: 30 March 2018

\begin{abstract}
In order to tackle the inaccurate step length measurement of people with different heights and in different motion states, a height-adaptive method of step length measurement based on motion parameters is proposed in this paper. This method takes people's height, stride frequency, and changing accelerometer output while walking into integrated consideration, and builds a dynamic and parameterized model of their step length. In this study, these parameters were calibrated with thirty sets of experiment data from people with different heights and in different motion states, which were then verified experimentally by motion data of randomly selected subjects, regardless of speed and height. The experiment results indicate that the height-adaptive step length measurement was realized, thus eliminating the influence of height exerted on step length measurement.
\end{abstract}

Keywords: motion parameters; gait analysis; step length; self-adaptation

\section{Introduction}

As one of the important parameters reflecting people's motion characteristics, step length can be used in the research of measurements of body motion parameters, disease diagnosis and treatment, health monitoring, rehabilitation training, and pedestrian navigation [1-5]. Motion parameters measured by small Micro Electro Mechanical Systems (MEMS) inertial sensors, at a low cost and with high precision, render step length measurement feasible and effective $[3,6]$.

Step length measurement bas been an important aspect of gait analysis. Many authors employ foot-mounted or leg-mounted IMU which is the abbreviation of "Inertial measurement unit" and can be used as measuring device to get data. However, this can cause a change in angle of the IMU, because of the transformation of the foot or leg when the subjects walk. We employed awaist-mounted IMU to measure the data, so that no matter how the angle of the foot changed, the orientation of the IMU would stay the same; this way, we were able to reduce the parameters of the algorithm [7-10].

Different body characteristics and motion parameters lead to unavoidable errors in step length measurement. This problem can be solved by adopting the corresponding step length measurement under different circumstances. At present, there are three main methods of step length measurement:

The first method calculates the step length based on geometry models. Cavagna et al. (among other scholars) believe that the displacement of the horizontal direction in a single foot motion during walking can be described as an inverted pendulum model, providing the mathematic relationship between the displacement of body's center of gravity in the horizontal direction, and that in the 
vertical direction [11-13]. A modified pendulum model is proposed by González et al. to estimate real-time step length, with each step divided into the single foot support phase and the double foot support phase [14]. Without the prophase training, Kun-Chan Lan et al. also bring up a step length measurement method based on the Pythagorean theorem [15]. However, because of the integral operation involved, the methods based on geometry models mentioned above can easily result in drift errors.

The second method resorts to the nonlinear empirical formula of the step length. By studying human walking, Weinberg proposes the nonlinear step length measurement method based on the peak values and the valley values of the acceleration in the center of gravity [16]. Due to its simplicity and easy application, this formula is used by a group of scholars researching pedestrian navigation, either directly or indirectly $[3,5,17,18]$. Parameter recalibration is required when dealing with a variety of pedestrians.

The third method is based on the linear combination: Levi et al. utilize a constant and stride frequency [19], while Ladetto takes both stride frequency and acceleration variance into consideration $[2,20,21]$. If applied in pedestrians with different heights, these two methods require parameter recalibration, which lacks wide adaptability. Another linear combination method based on height and stride frequency is presented by Renaudin et al., with the measurement precision of step length increased [22]. The measurement precision, however, deteriorates when there are strenuous motions during human walking.

Summarizing advantages and disadvantages of the methods mentioned above, this paper introduces a novel step length measurement method with the integration of pedestrians' height, stride frequency, and acceleration variance during walking, by analyzing the gait characteristics. Meanwhile, the corresponding experimental research is performed.

\section{Analysis of Gait Characteristics}

When people walk, there is a motion in virtually each part of body (feet, legs, waist, etc.) [23,24], which means the acceleration speed and angular velocity are constantly changing. The motion of feet and legs are relatively strenuous, with apparent acceleration and angular velocity change, making it easy to extract useful information from them. It is convenient to wear and fix the sensors at the waist, and there is only a little influence exerted on the body motion because of the gentle waist motion. When wearing sensors at the waist, the change of the acceleration and angular velocity in the vertical direction is more obvious than that in other directions, thus facilitating the analysis, extraction, and estimation of the human motion status. The vertical waist acceleration is shown in Figure 1.

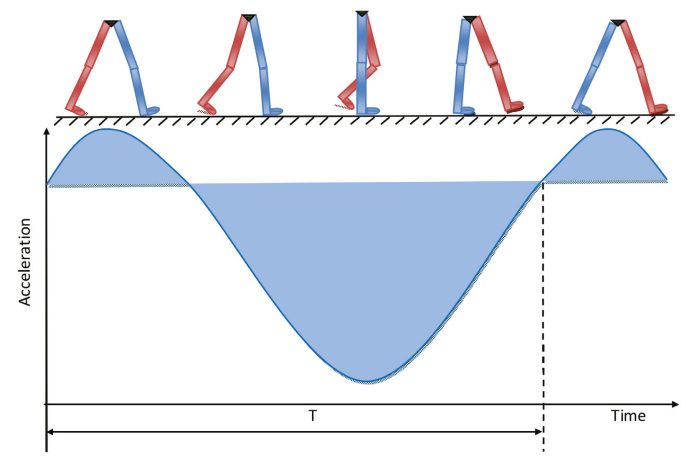

Figure 1. The relationship between the vertical acceleration and gait.

From the figure above, it is seen that the center of gravity changes periodically up and down with each step. In addition, the vertical acceleration of the center of gravity changes periodically too, 
which leads to a different step length resulting from different walking habits, body characteristics, and walking status. Here, "step length" is the distance between the footsteps of the left and right foot. As shown in Figure 2, the step length is the distance between the blue foot and the red foot. The relationship between the step length and the changing vertical acceleration needs to be studied. The corresponding parameters have to be identified and extracted from the accelerometer or gyroscope for real-time calculation.

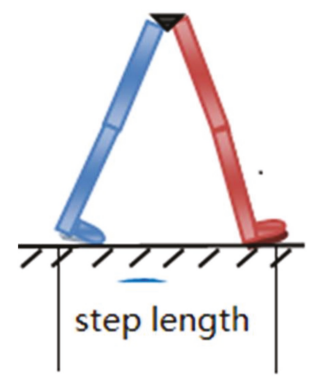

Figure 2. The illustration of the step length.

In practical applications, as shown in Figure 3, there are sensor noises, different periodical peak values, and false peak values from the accelerator output resulting from sensor detection errors and step inconformity.

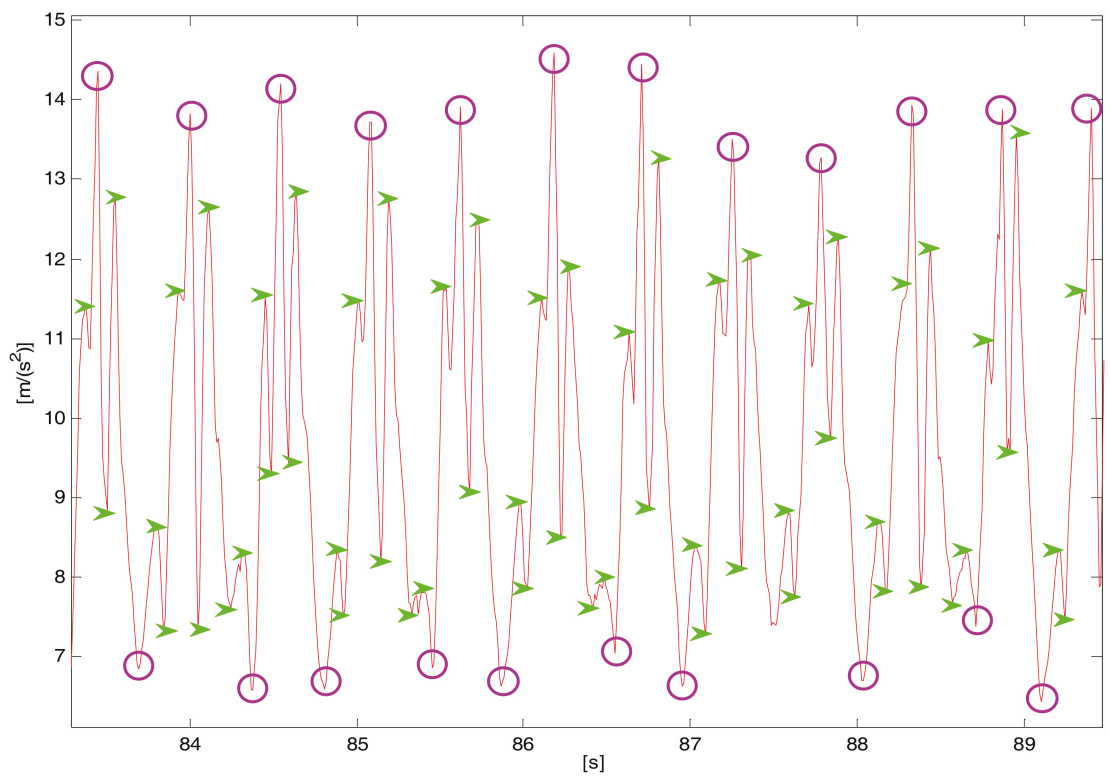

Figure 3. The change of vertical acceleration during walking.

The overlay of walking speed, the periodical change of center of gravity, and the variation in the heaviness of step generates the vertical acceleration. All of these factors reflect walking status from which the step length is calculated. It is feasible to study the relationship between these factors and the step length, and then conduct the step length measurement. 


\section{Step Length Measurement Method}

\subsection{The Step Length Measurement Method Based on Stride Frequency and Acceleration Variance}

By analyzing different walking speeds and types of gait per person during walking, Ladetto proposes the step length measurement based on the linear relation of the step length, stride frequency, and acceleration variance [18], which can be expressed as follows:

$$
S L_{i}=A \cdot f_{i}+B \cdot \operatorname{var}_{i}+C
$$

where $A$ is the coefficient of the stride frequency, $B$ is the coefficient of the vertical acceleration variance, and $C$ is a constant. $A, B, C$ are parameters calculated by the least square method, which is a form of mathematical optimization technology. It finds the best function match of the data by minimizing the sum of the square of the error; $f_{i}$ stands for the stride frequency, indicating how fast the pedestrian walks; var represents the acceleration variance during walking, describing whether the step is heavy or light: it can be calculated by Equation (4). These two factors indicate the pedestrian's step length indirectly. SL is the short name of Step Length. In the positioning of the same walking person, this model is frequently applied in a precise manner [22-24]. However, when the application extends to different people, a lack of consideration about differences between individuals deteriorates the accuracy of the step length measurement.

\subsection{The Step Length Measurement Method Based on Height, Stride Frequency and Acceleration Variance}

For pedestrians with different heights, it is found that the step length is also different even when $f_{i}$ and $v_{a r}$ are the same. According to the method mentioned in Section 3.1, when applied to different people, calibration is once again required for each individual to improve measurement accuracy, which limits its application. According to the kinetics of the human body, the step length is proportional to the leg length as well as the body height under normal circumstances.

By analyzing the research and methods described in Section 3.1, a novel step length measurement model based on height, stride frequency, and acceleration variance is proposed:

$$
S L_{i}=h \cdot\left(A \cdot f_{i}+B \cdot \operatorname{var}_{i}+C\right)+D
$$

where $i$ represents the $i$ th step during walking; $h, f_{i}$ and $v a r_{i}$ stand for height, stride frequency, and vertical acceleration variance during the $i$ th step, respectively. $A, B, C, D$ are the corresponding model coefficients.

Inputs of this method are height $h$, stride frequency $f_{i}$, and acceleration variance var $i . h$ is a fixed constant and remains unchanged during different walking processes of the same individual. $f_{i}$ can be obtained by Equation (3):

$$
f_{i}=\frac{1}{t_{i}-t_{i-1}}
$$

where $t_{i}$ and $t_{i-1}$ represent the corresponding moments when detecting two adjacent steps [25]. In addition, the vertical acceleration variance of each step during walking can be calculated by Equation (4):

$$
\operatorname{var}_{i}=\frac{1}{N-1} \sum_{t=t_{i-1}}^{t_{i}}\left(a_{t}-\bar{a}_{i}\right)^{2}
$$

where $a_{t}, \bar{a}_{i}, N$ stand for the acceleration at the moment of $t$, the average acceleration, and the number of sampling points within one step, respectively.

According to Equations (2)-(4), the step length measurement is realized. In Figure 3, the flow chart of the step length measurement based on the low-cost MEMS inertial system is presented.

As shown in Figure 4, before calculating step length, errors of inertial sensors and parameters of the step length model are calibrated by the method presented in the reference paper [26]. Once the 
height of an individual is entered, the height-adaptive step length is calculated according to the program. Once calculations begin, data is read, and then the number of steps is detected. For every single step, the sensors calculate stride frequency $f_{i}$, acceleration variance $v a r_{i}$, and the step length $S L_{i}$. $S L_{i}$ serves as the input to the practical application.

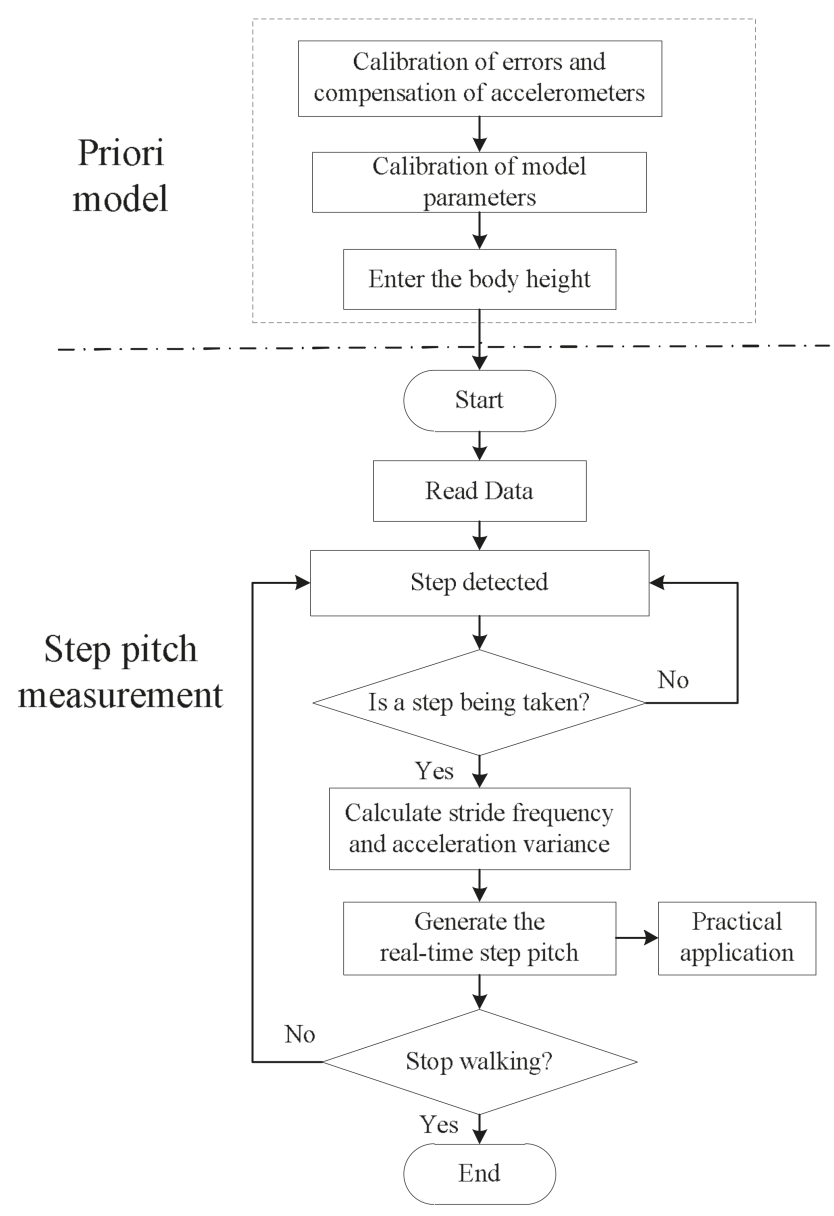

Figure 4. The flow chart of the step length measurement.

\section{Experiment Research and Analysis}

In order to verify the proposed method in this paper, the following experiments have been conducted. The experiments consist of two parts:

(1) The model parameter calibration of the height-adaptive and parameterized step length model;

(2) An evaluation of the accuracy of the step length measurement based on a walking experiment.

As a consequence of the experimental complexity and the unavailability of a high-speed synchronous camera shooting, the step length was measured, and its accuracy was verified indirectly, by walking along one fixed route several times over. 


\subsection{Experimental Equipment}

The experimental equipment consisted of a signal acquisition and transmission module, a laptop, and a wearable device. As shown in Figure 5, the acceleration output of MPU6050 is acquired by the microprocessor STM32 in the signal acquisition and transmission module, which is then sent to the laptop through a serial interface. Once the raw data is received, the step length is calculated, and the DR navigation is conducted. DR is the abbreviation of "Dead reckoning". Dead reckoning algorithm uses inertial navigation algorithm to predict motion position MPU-6050 is a MEMS inertial sensor with a 3-axis accelerometer and a 3-axis gyroscope, the advantages of which are its small size, its low cost, and its high precision. MEMS (Micro-Electro-Mechanical System) is also called micro-electromechanical system, micro-system, micro-mechanics, etc. It refers to high-tech devices with a size of a few millimeters or even less. The accelerometer features a measurement range of $\pm 2 \mathrm{~g}$, a zero bias of $50 \mathrm{mg}$, and random error of $0.4 \mathrm{mg}$. The random error of the gyroscope is due to the random variation of gyroscope output, which changes with time. It is expressed by the mean square error of the output data during idle state. As for the gyroscope, the measurement range is $\pm 1000^{\circ} / \mathrm{s}$, the zero bias is $0.4^{\circ} / \mathrm{s}$, and the random error is $0.05^{\circ} / \mathrm{s}$. The wearable device is tied up at the waist, as Figure 6 demonstrates.

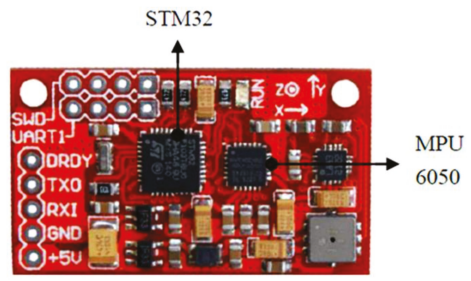

Figure 5. The signal collection and transmission module.

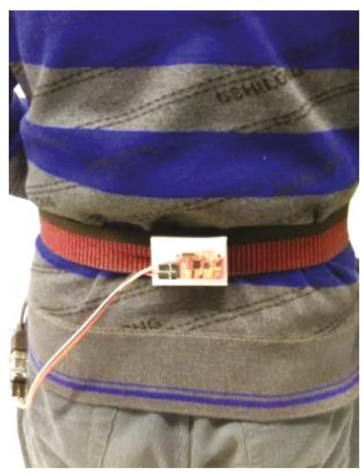

Figure 6. The wearing method.

\subsection{Calibration of Experiment Parameters for the Step Length Model}

Before measuring step length using Equation (2), the parameters $A, B, C, D$ need to be calibrated. The height was entered and the result was calculated. For more accurate calibrated parameters, the subjects of different heights such as $1.60 \mathrm{~m}, 1.63 \mathrm{~m}, 1.71 \mathrm{~m}, 1.78 \mathrm{~m}$ and $1.83 \mathrm{~m}$ were selected to walk along one fixed route at different speeds. As shown in Figure 7, each subject was asked to walk a certain distance $(24 \mathrm{~m})$ along a flat road at a slow speed, at a preferred speed and at a fast speed. Each trajectory was conducted twice. In total, there are 30 sets of data in Table 1 . The walking speed during each set of the experiment was made to be as stable and as consistent as possible. In order to reduce random errors, 
the average step length, the stride frequency and the vertical acceleration variance for one step were measured and calculated. Besides the walking data, the subject's height is also added, thus enhancing the experimental data to include different heights, different step frequencies, different variances, and different step lengths. Finally, the model parameters of the step length measurement proposed in this paper were calibrated by the least square method. The variable was written as $a=\left[\begin{array}{cccc}1 & f_{1} & \operatorname{var}_{1} & h_{1} \\ 1 & f_{2} & \operatorname{var}_{2} & h_{2} \\ 1 & f_{3} & \operatorname{var}_{3} & h_{3}\end{array}\right]$, the coefficient as $b=\left[\begin{array}{c}A \\ B \\ C \\ D\end{array}\right]$, and the $S L$ as $S L=\left[\begin{array}{l}S L_{1} \\ S L_{2} \\ S L_{3}\end{array}\right]$. Then the formula was minimized to $\|a b-S L\|_{2}$, in order to calibrate the coefficient $A, B, C, D$.

The step length model in Section 3.1 has nothing to do with height. For a better comparison and analysis, the experimental data of the subject with the height of $1.71 \mathrm{~m}$ was calibrated.

Table 1. The data for parameter calibration.

\begin{tabular}{ccccc}
\hline Groups & Height $(\mathbf{m})$ & Stride Frequency $\mathbf{m}(\mathbf{H z})$ & Acceleration Variance & Step Length $(\mathbf{m})$ \\
\hline 1 & 1.60 & 1.6418 & 0.1662 & 0.5455 \\
2 & 1.60 & 1.5842 & 0.1253 & 0.5217 \\
3 & 1.60 & 1.8242 & 0.4685 & 0.6154 \\
4 & 1.60 & 1.8470 & 0.3424 & 0.6000 \\
5 & 1.60 & 1.8377 & 0.5645 & 0.6316 \\
6 & 1.60 & 1.9035 & 0.8991 & 0.6857 \\
7 & 1.63 & 1.5820 & 0.2578 & 0.6154 \\
8 & 1.63 & 1.5016 & 0.2138 & 0.6000 \\
9 & 1.63 & 1.7242 & 0.6021 & 0.7102 \\
10 & 1.63 & 1.7566 & 0.5718 & 0.7059 \\
11 & 1.63 & 1.8346 & 1.2402 & 0.8000 \\
12 & 1.63 & 1.8831 & 1.1853 & 0.8000 \\
13 & 1.71 & 1.7640 & 0.5182 & 0.6667 \\
14 & 1.71 & 1.7624 & 0.3912 & 0.6667 \\
15 & 1.71 & 1.8278 & 0.9619 & 0.7273 \\
16 & 1.71 & 1.8471 & 0.8618 & 0.7273 \\
17 & 1.71 & 1.9942 & 1.4286 & 0.8000 \\
18 & 1.71 & 2.0901 & 1.8048 & 0.8276 \\
19 & 1.78 & 1.4914 & 0.2503 & 0.6667 \\
20 & 1.78 & 1.6307 & 0.3343 & 0.7059 \\
21 & 1.78 & 1.7328 & 0.5587 & 0.7500 \\
22 & 1.78 & 1.7476 & 0.5065 & 0.7500 \\
23 & 1.78 & 1.9508 & 1.2965 & 0.8889 \\
24 & 1.78 & 1.9738 & 1.2014 & 0.8571 \\
25 & 1.83 & 1.4564 & 0.2006 & 0.6486 \\
26 & 1.83 & 1.6707 & 0.2367 & 0.6857 \\
27 & 1.83 & 1.7334 & 0.4753 & 0.7500 \\
28 & 1.83 & 1.7614 & 0.5075 & 0.7500 \\
29 & 1.83 & 2.0869 & 1.2029 & 0.9600 \\
30 & 1.83 & 2.0604 & 1.2742 & \\
\hline
\end{tabular}




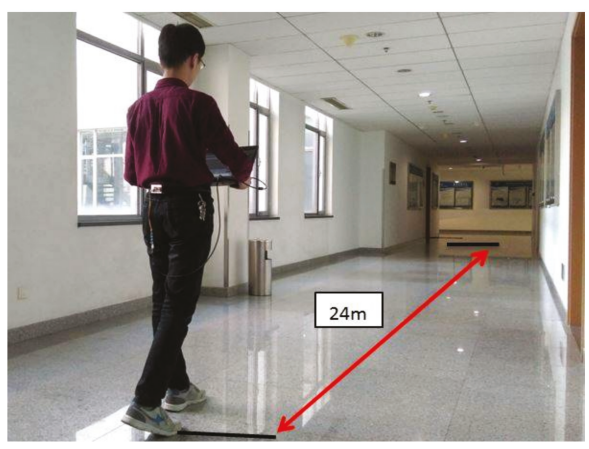

Figure 7. The parameter calibration experiment.

\subsection{Walking Experiments}

During walking, the pedestrian's step length is not technically consistent for each step, leading to the unobtainability of precise measurements. Consequently, walking experiments of a certain distance are performed to verify the feasibility and accuracy of the step length measurement method proposed in this paper. Three other subjects were chosen to walk along the standard track in the playground of Beihang University three times over, from which the mean value was calculated. The experiment was conducted as follows: (1) the signal acquisition and transmission module was attached to the subject's waist with a belt; (2) the walking experiment was conducted and the motion data was acquired during walking; (3) the step length was measured by both the method explored in this paper and the method based on stride frequency and variance separately; (4) the experiment data was analyzed, and the results of the two different methods were compared.

The step length during walking was summed up to obtain the total walking distance, which was compared with the actual path length. The number of steps, the mean step length, the total walking distance, and the error rate are listed in Table 2.

The actual path length is the length of the track, namely, $400 \mathrm{~m}$, but the length of the track of a few groups ended up being $453 \mathrm{~m}$, because there were students taking physical education class when we were conducting our experiment. Therefore, the subjects were walking on the playground's outer ring, the actual path length of the track thus being $453 \mathrm{~m}$. The walking distance was calculated based on the estimated step length in real time.

Experimental results indicate that the precision of the step length measurement of the method proposed in this paper is superior to that of the method based on stride frequency and variance. By comparing the average error and the standard deviation, we can conclude that the method in this paper can be used for subjects with different heights, the error is more constant than the method based on the frequency and acceleration variance during walking. More specifically, when adopting the step length measurement method based on stride frequency and variance, the user has to be the same person or at least someone with similar physical characteristics. A calibration of parameters had to be conducted again to achieve favorable results for different users, thus restricting its wide application. In this paper, the body height is added to the proposed step length measurement model. Despite the different heights of users, the step length measurement accuracy is relatively high. 


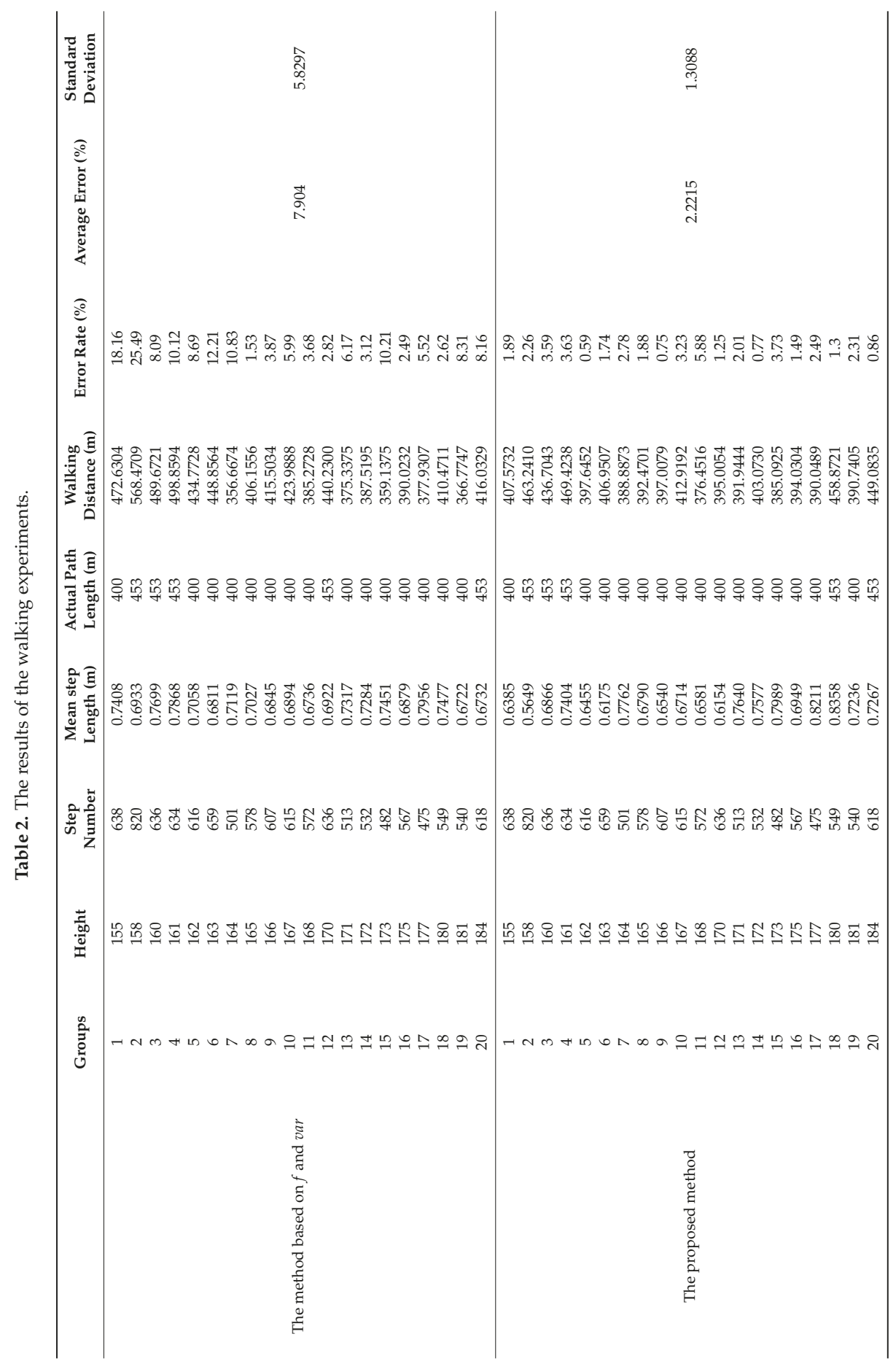




\section{Conclusions}

This paper proposes a height-adaptive step length measurement method based on the low-cost MEMS inertial system. Taking the height, the stride frequency, and the vertical acceleration variance into account, the step length was estimated with the motion data measured by the output of the accelerometer worn at the pedestrian's waist. Without any parameter calibration, this method is highly height-adaptive, that is to say, different users just need to input different heights for the step length to be properly measured. In addition, a series of walking experiments were performed, the results of which prove that this method can measure the step length accurately, giving rise to a great application prospect in fields such as auxiliary medical treatment, exercise rehabilitation, and more.

Acknowledgments: This work is supported by the National Natural Science Foundation of China under Grant 61473019 , the Beijing Natural Science Foundation under Grant 4172036, the Beijing Science and Technology Plan under Grant D171100006217003, the Key Research and Development Project of the Ministry of Science, and Technology of China under Grant 2016YFB051600.

Author Contributions: Yanshun Zhang conceived and designed the experiments and contributed materials. Yingyue Li performed the experiments; Chuang Peng wrote the paper. Yingyue Li, Dong Mou, Ming Li and Wei Wang analyzed the data.

Conflicts of Interest: The authors declare no conflict of interest.

\section{References}

1. Durie, N.D.; Farley, R.L. An apparatus for step length measurement. J. Biomed. Eng. 1980, 2, 38-40. [CrossRef]

2. Shin, S.H.; Chan, G.P. Adaptive step length estimation algorithm using optimal parameters and movement status awareness. Med. Eng. Phys. 2011, 33, 1064-1071. [CrossRef] [PubMed]

3. Sayeed, T.; Samã, A.; Catalã, A.; Rodríguez-Molinero, A.; Cabestany, J. Adapted step length estimators for patients with Parkinson's disease using a lateral belt worn accelerometer. Technol. Health Care Off. J. Eur. Soc. Eng. Med. 2015, 23, 179-194.

4. Juen, J.; Cheng, Q.; Schatz, B. A natural walking monitor for pulmonary patients using mobile phones. IEEE J. Biomed. Health Inform. 2015, 19, 1399-1405. [CrossRef] [PubMed]

5. Zhuang, Y.; Lan, H.; Li, Y.; El-Sheimy, N. PDR/INS/WiFi integration based on handheld devices for indoor pedestrian navigation. Micromachines 2015, 6, 793-812. [CrossRef]

6. Renaudin, V.; Combettes, C. Magnetic, Acceleration Fields and Gyroscope Quaternion (MAGYQ)-Based Attitude Estimation with Smartphone Sensors for Indoor Pedestrian Navigation. Sensors 2014, 14, 22864-22890. [CrossRef] [PubMed]

7. Müller, P.; Seel, T.; Schauer, T. Experimental Evaluation of a Novel Inertial Sensor Based Realtime Gait Phase Detection Algorithm. In Proceedings of the European Conference on Technically Assisted Rehabilitation-TAR 2015, Berlin, Germany, 12-13 March 2015.

8. Gao, Y.; Jiang, Z.; Ni, W.; Vasic, Z.L.; Cifrek, M.; Du, M.; Vai, M.I.; Pun, S.H. A Novel Gait Detection Algorithm Based on Wireless Inertial Sensors. In CMBEBIH 2017; Springer: Singapore, 2017; pp. 300-304.

9. Seel, T.; Graurock, D.; Schauer, T. Realtime assessment of foot orientation by accelerometers and gyroscopes. Curr. Dir. Biomed. Eng. 2015, 1, 466-469. [CrossRef]

10. Brahms, C.M.; Zhao, Y.; Gerhard, D.; Barden, J.M. Stride length determination during overground running using a single foot-mounted inertial measurement unit. J. Biomech. 2018. [CrossRef] [PubMed]

11. Cavagna, G.A.; Thys, H.; Zamboni, A. The sources of external work in level walking and running. J. Physiol. (Lond.) 1976, 262, 639-657. [CrossRef]

12. Zijlstra, W.; Hof, A. Assessment of spatio-temporal gait parameters from trunk accelerations during human walking. Gait Posture 2003, 18, 1-10. [CrossRef]

13. Brandes, M.; Zijlstra, W.; Heikens, S.; van Lummel, R.; Rosenbaum, D. Accelerometry based assessment of gait parameters in children. Gait Posture 2006, 24, 482-486. [CrossRef] [PubMed]

14. González, R.C.; Alvarez, D.; López, A.M.; Alvarez, J.C. Modified pendulum model for mean step length estimation. In Proceedings of the International Conference of the IEEE Engineering in Medicine and Biology Society (EMBS 2007), Lyon, France, 22-26 August 2007; pp. 1371-1374. 
15. Lan, K.C.; Shih, W.Y. Using smart-phones and floor plans for indoor location tracking. IEEE Trans. Hum. Mach. Syst. 2014, 44, 211-221.

16. Weinberg, H. Using the ADXL202 in Pedometer and Personal Navigation Applications. Analog Devices AN-602 Application Note. Available online: http://www.docin.com/p-1743985325.html (accessed on 23 March 2018).

17. Ho, N.H.; Truong, P.H.; Jeong, G.M. Step-Detection and Adaptive Step-Length Estimation for Pedestrian Dead-Reckoning at Various Walking Speeds Using a Smartphone. Sensors 2016, 16, 1423. [CrossRef] [PubMed]

18. Zhu, Y.; Zhang, R.; Xia, W.; Jia, Z.; Shen, L. A hybrid step model and new azimuth estimation method for pedestrian dead reckoning. In Proceedings of the Sixth International Conference on Wireless Communications and Signal Processing, Hefei, China, 23-25 October 2014; pp. 1-5.

19. Levi, R.W.; Judd, T. Dead Reckoning Navigational System Using Accelerometer to Measure Foot Impacts. U.S. Patent US5,583,776, 10 December 1996.

20. Ladetto, Q. On Foot Navigation: Continuous Step Calibration Using Both Complementary Recursive Prediction and Adaptive Kalman Filtering. In Proceedings of the ION GPS, Salt Lake City, UT, USA, 19-22 September 2000; pp. 1735-1740.

21. Qian, J.; Pei, L.; Zou, D.; Qian, K.; Liu, P. Optical flow based step length estimation for indoor pedestrian navigation on a smartphone. In Proceedings of the Position, Location and Navigation Symposium (PLANS 2014), Monterey, CA, USA, 5-8 May 2014; pp. 205-211.

22. Renaudin, V.; Susi, M.; Lachapelle, G. Step length estimation using handheld inertial sensors. Sensors 2012, 12,8507-8525. [CrossRef] [PubMed]

23. Shibuya, N.; Nukala, B.T.; Rodriguez, A.I.; Tsay, J.; Nguyen, T.Q.; Zupancic, S.; Lie, D.Y. A real-time fall detection system using a wearable gait analysis sensor and a Support Vector Machine (SVM) classifier. In Proceedings of the Eighth International Conference on Mobile Computing and Ubiquitous Networking, Hakodate, Japan, 20-22 January 2015; pp. 66-67.

24. Kauw-A-Tjoe, R.; Thalen, J.; Marin-Perianu, M.; Havinga, P. SensorShoe: Mobile Gait Analysis for Parkinson's Disease Patients. In Proceedings of the UbiComp 2007 Workshops, University of Innsbruck, Innsbruck, Austria, 16-19 September 2017.

25. Zhang, Y.; Xiong, Y.; Wang, Y.; Li, C.; Wang, Z. An Adaptive Dual-Window Step Detection Method for a Waist-Worn Inertial Navigation System. J. Navig. 2016, 69, 659-672. [CrossRef]

26. Zhang, Y.; Xu, Y.; Xing, X.; Wang, Z.; Xiong, Y. The Standing Calibration Method of MEMS Gyro Bias for Autonomous Pedestrian Navigation System. J. Navig. 2016, 70, 607-617. [CrossRef]

(C) 2018 by the authors. Licensee MDPI, Basel, Switzerland. This article is an open access article distributed under the terms and conditions of the Creative Commons Attribution (CC BY) license (http:/ / creativecommons.org/licenses/by/4.0/). 
Article

\title{
Motor Subtypes of Parkinson's Disease Can Be Identified by Frequency Component of Postural Stability
}

\author{
Saba Rezvanian ${ }^{1}$, Thurmon Lockhart ${ }^{1, *}$, Christopher Frames ${ }^{1,2}$, Rahul Soangra ${ }^{3}$ and \\ Abraham Lieberman ${ }^{2}$ \\ 1 School of Biological and Health Systems Engineering, Arizona State University, Tempe, AZ 85281, USA; \\ saba.rezvanian@asu.edu (S.R.); cframes@asu.edu (C.F.) \\ 2 Barrow Neurological Institute, Phoenix, AZ 85013, USA; Abe.Lieberman@dignityhealth.org \\ 3 Department of Physical Therapy, Crean College of Health and Behavioral Sciences, Chapman University, \\ Orange, CA 92866, USA; soangra@chapman.edu \\ * Correspondence: thurmon.lockhart@asu.edu
}

Received: 26 February 2018; Accepted: 4 April 2018; Published: 5 April 2018

\begin{abstract}
Parkinson's disease (PD) can be divided into two subtypes based on clinical features-namely tremor dominant (TD) and postural instability and gait difficulty (PIGD). This categorization is important at the early stage of PD, since identifying the subtypes can help to predict the clinical progression of the disease. Accordingly, correctly diagnosing subtypes is critical in initiating appropriate early interventions and tracking the progression of the disease. However, as the disease progresses, it becomes increasingly difficult to further distinguish those attributes that are relevant to the subtypes. In this study, we investigated whether a method using the standing center of pressure (COP) time series data can separate two subtypes of PD by looking at the frequency component of COP (i.e., COP position and speed). Thirty-six participants diagnosed with PD were evaluated, with their bare feet on the force platform, and were instructed to stand upright with their arms by their sides for $20 \mathrm{~s}$ (with their eyes open and closed), which is consistent with the traditional COP measures. Fast Fourier transform (FFT) and wavelet transform (WT) were performed to distinguish between the motor subtypes using the COP measures. The TD group exhibited larger amplitudes at the frequency range of $3-7 \mathrm{~Hz}$ when compared to the PIGD group. Both the FFT and WT methods were able to differentiate the subtypes. COP time series information can be used to differentiate between the two motor subtypes of PD, using the frequency component of postural stability.
\end{abstract}

Keywords: Parkinson's disease (PD); tremor dominant (TD); postural instability and gait difficulty (PIGD); center of pressure (COP); fast Fourier transform (FFT); wavelet transform (WT)

\section{Introduction}

In 2010, approximately 630,000 people in the U.S. were diagnosed with Parkinson's disease (PD) - a number that is estimated to double by 2040 [1]. PD is a progressive neurodegenerative disorder that includes motor and non-motor features [2]. PD can be further divided into two subtypes based on clinical features-namely tremor dominant (TD) and postural instability and gait difficulty (PIGD) [2-5]. This categorization is important at the early stage of PD, since identifying the PD subtypes can help to predict the clinical progression of the disease. Several studies have confirmed that the PIGD subtype has a faster disease progression and greater motor function impairment [6], and is less responsive to levodopa and deep brain stimulation when compared to the TD subtype $[3,5,7,8]$. It has also been reported that there is a correlation between the freezing of gait (FOG) score and the 
PIGD score [7]. Additionally, the PIGD subtype can place PD patients at a higher risk of falling when compared to TD patients [9]. It has been shown that PIGD patients have worse postural control when compared to TD patients $[9,10]$. Accordingly, correctly diagnosing subtypes can help caregivers to initiate early amenable interventions and track the progression of the disease. It should be noted that the diagnosis would not lead to a different medical treatment. However, another treatment needs to be taken alongside the medical treatment for PIGD patients in order to reduce the loss of balance and falling, since dopaminergic medications may result in limited improvement in postural instability and gait $[11,12]$. Thus, the diagnosis leads to the specific path that should be taken for the patient to manage the symptoms.

The differentiation of TD from PIGD is currently based on sub-scores of the Unified Parkinson's Disease Rating Scale (UPDRS) [3,11]. The UPDRS is scored by clinicians, and thus is subjective and prone to error [12]. Subtype-specific biomarkers may improve the accuracy of the diagnoses that are relevant to the PD subtypes and progression.

The center of pressure (COP) measure is widely employed in assessing postural control, and has been utilized for analyzing the disease-related features in PD patients [13-16]. The results of different studies have indicated that COP was more variable for PD patients, relative to the control participants [14,15], and that COP-derived velocities were abnormally large for PD patients with FOG when compared to the patients without FOG [13]. Thus, COP is considered as a good measure for representing PD disease-related postural characteristics.

PD tremor is present while resting, and is typically dampened with kinetic movement. Therefore, in order to distinguish between the two subtypes, proposing a static test appears to be more appropriate than a dynamic task [17]. Several studies have reported a frequency range of 3-7 Hz for PD tremor [17-19]. It has also been demonstrated that the whole-body COP signal has a frequency lower than $2 \mathrm{~Hz}$ [20-22]. Subtype-specific postural instability in PD may be better identified by the frequencies that make up the COP signal. We hypothesized that the whole-body COP frequency may be a better and more objective means of identifying the PD subtypes. The most common method to investigate the tremor in PD is fast Fourier transformation (FFT) [5,6]. FFT transfers a signal from the time domain to the frequency domain. In this method, the time information is lost after the transformation. Therefore, a method such as wavelet transformation (WT) - which includes both the time and frequency information of the signal [7]—might help to diagnose the subtypes better than FFT. Based on the importance of correct PD subtype diagnosis and the lack of an objective method among the current diagnosis techniques, this study aims to develop an objective method to diagnose PD motor subtypes by employing COP data and using the FFT and WT methods.

\section{Materials and Methods}

\subsection{Participants}

Thirty-six participants that were diagnosed with PD by specialists at the Muhammad Ali Parkinson Center at the Barrow Neurological Institute (Phoenix, AZ, USA) were recruited for this study. The participants' demographic information is presented in Table 1. The Movement Disorder Society Unified Parkinson's Disease Rating Scale (MDS-UPDRS) was employed to identify the TD and PIGD groups [23]. The designated items for TD (kinetic and postural tremor in both the right and left hand; tremor-while at rest—of either the face and lips or the chain, arms, and legs) and PIGD (freezing, walking, posture, gait, and postural stability) were used to calculate the mean TD and PIGD scores. The ratio of the mean TD score to the mean PIGD score was used to identify the TD group. The patients with a ratio greater than or equal to 1.5 were classified as TD, while those with a ratio less than or equal to 1.0 were classified as PIGD. The patients with ratios ranging from 1.0 to 1.5 were classified as mixed-type, and were considered as an exclusionary criterion for this study $[5,11,24]$. 
Table 1. Demographics of the tremor dominant (TD) and postural instability and gait difficulty (PIGD) groups (mean \pm standard deviation-SD). MDS-UPDRS: Movement Disorder Society Unified Parkinson's Disease Rating Scale.

\begin{tabular}{ccc}
\hline & TD $(\boldsymbol{n}=\mathbf{1 3})$ & PIGD $(\boldsymbol{n}=\mathbf{2 3})$ \\
\hline Gender (F:M) & $0: 13$ & $9: 14$ \\
Age (years) & $59.92 \pm 9.63(34-71)$ & $70.43 \pm 6.18(59-81)$ \\
Disease duration (months) & $20.23 \pm 19.14(4-60)$ & $37.78 \pm 54.69(1-216)$ \\
MDS-UPDRS III (ON) & $14.85 \pm 9.85$ & $15.08 \pm 8.48$ \\
\hline
\end{tabular}

The study was approved by the Institutional Review Board at the Barrow Neurological Institute and Arizona State University, Tempe, AZ, USA. The participants provided informed consent prior to their inclusion in the study. All of the assessments were performed while subjects were in the "on" medication status-approximately 1 to $1.5 \mathrm{~h}$ after taking the PD medication.

\subsection{Experimental Procedure}

The participants were placed with their bare feet on the force platform and were instructed to stand upright with their feet shoulder width apart and their arms by their sides for $20 \mathrm{~s}$, and look straight ahead during the experiment. They were instructed not to talk or bend their knees throughout the experimental trials. Harnesses were fitted onto the participants to avoid falls. This task was performed under two conditions-namely eyes open and eyes closed. For the eyes closed condition, the subjects were asked to close their eyes during the experiment. Each participant performed the experiment under both conditions. Each condition had three trials.

\subsection{Data Analysis}

COP data were derived using force plate data sampled at $100 \mathrm{~Hz}$. Both anterior-posterior (AP) and medial-lateral (ML) COP data were low-pass-filtered using a fourth-order, zero lag Butterworth filter with a cut-off frequency of $10 \mathrm{~Hz}$. Five traditional COP measures were calculated to assess whether or not the two subtypes of PD can be distinguished by using the time domain information. The measures included the following: $\mathrm{COP}$ range (the range of $\mathrm{COP}$ displacement), resultant $\mathrm{COP}$ path length (the total COP trajectory length), resultant mean velocity (the resultant path length divided by the total duration), and a $95 \%$ confidence ellipse area (the smallest ellipse that will cover $95 \%$ of the points of the COP diagram). Based on previous studies, these traditional parameters are good indicators of postural instability $[14,25,26]$ and were considered as variables that might help us to distinguish PIGD from TD. All of the analyses were performed in MATLAB version 2015a.

\subsection{TD vs. PIGD Detection Method}

In order to distinguish between the TD and PIGD subtypes, the following two methods were utilized: fast Fourier transform (FFT) and wavelet transform (WT). In the FFT method, the PD subtypes were identified by the frequency spectra of COP signals. Two frequency bands were introduced [27-29]: the COP band and the tremor band. The COP and tremor bands were defined as the frequency components from $0-3 \mathrm{~Hz}$ to $3-7 \mathrm{~Hz}$, respectively. The detection method was defined as the ratio of the area under the power spectra of the tremor band to the summation of the areas under the power spectra of the COP band and the tremor band.

$\mathrm{COP}$ data were transformed into the wavelet domain using daubechies mother wavelet (db6). It was chosen because it has been widely employed in different human posture and movement studies $[27,30]$. Various mother wavelets were also applied to ensure that the optimal selection was made appropriately. The results supported the notion that daubechies mother wavelet was the best choice. In the WT method, the COP and tremor bands were defined as the scales that corresponded to the frequency ranges of $0-3 \mathrm{~Hz}$ and $3-7 \mathrm{~Hz}$, respectively. The detection method was defined in 
a similar manner to the way it was defined in the FFT method: the ratio of the averaged WT coefficients of the tremor band to the summation of the averaged WT coefficients of the COP band and the tremor band. This ratio was unitless because it was a ratio of values with the same unit. In both methods, the defined ratio was multiplied by 100 in order to obtain a value between 0 and 100 . Values that were closer to 100 indicated a higher possibility of the TD subtype, while the possibility of the PIGD subtype increased as the values approached 0 . The first time derivative of COP time series was defined as $\mathrm{COP}$ velocity $(\mathrm{V}-\mathrm{COP})$. The ratio that was defined above was applied to $\mathrm{COP}\left(\mathrm{R}_{\mathrm{COP}}\right)$ and $\mathrm{COP}$ velocity $\left(\mathrm{R}_{\mathrm{VCOP}}\right)$ in both the AP and ML directions.

\subsection{Statistical Analysis}

Analysis of variance (ANOVA) with repeated measures on the traditional COP measures and the proposed detection ratio (using both the FFT and WT methods) were performed. Different factors - such as condition (two levels: eyes open (EO) and eyes closed (EC)) and group (or subtype) of PD (two levels: TD and PIGD)—were considered as within-subject and between-subject factors, respectively. Comparisons of interest exhibiting statistically significant differences $(p<0.05)$ were further analyzed using post hoc tests with Bonferroni corrections. In all analyses, sphericity assumptions were tested (Greenhouse-Geisser analysis). The diagnostic performance of the proposed method-or the accuracy of a test to discriminate between the subtypes-was further evaluated using receiver operating characteristic (ROC) curve analysis [31] for the directions and factors of both methods. In a ROC curve, the true positive rate (sensitivity) is plotted as a function of the false positive rate (100-specificity) at different cut-off points. Therefore, each point on the ROC curve corresponds to a sensitivity/specificity pair for a particular decision threshold. Therefore, the upper-left corner denotes a test with perfect discrimination (no overlap in the two distributions) in a ROC curve analysis. Accordingly, the closer the ROC curve is to the upper-left corner, the higher the overall accuracy of the test [31]. In this study, PD subtypes were diagnosed by utilizing UPDRS and were considered as a correct diagnosis. All of the statistical analyses were performed based on this assumption. In all tests, $p<0.05$ was considered as a significant level. Statistical analyses were performed using IBM SPSS Statistics 22.

\section{Results}

The results of the traditional COP measures-under both the eyes open and eyes closed conditions-are provided in Table 2. All of the variables had larger values in the eyes closed condition compared to the eyes open condition. Because these parameters did not have a normal distribution, a Box-Cox transformation was applied and parametric methods were performed. There was no significant difference between the two groups for all the variables. However, there was a significant difference between the conditions for all of the parameters (range AP: $\mathrm{F}_{(1,34)}=4.252, p=0.047$; range ML: $\mathrm{F}_{(1,34)}=60.34, p=0.001$; path length: $\mathrm{F}_{(1,34)}=29.797, p=0.001$; mean velocity: $\mathrm{F}_{(1,34)}=29.795$, $p=0.001$; area: $\left.\mathrm{F}_{(1,34)}=11.847, p=0.002\right)$.

Table 2. Selected postural stability parameters. Range anterior-posterior (AP): center of pressure (COP) range in the AP direction, range medial-lateral (ML): COP range in the ML direction, path length: resultant COP path length, mean velocity: resultant COP mean velocity, and area: $95 \%$ ellipse area. The symbols * or ** denote which of the two variables were significantly different at each parameter $(p<0.05)$.

\begin{tabular}{ccccccc}
\hline & & Range AP $(\mathbf{c m})$ & Range ML $(\mathbf{c m})$ & $\begin{array}{c}\text { Mean Velocity } \\
(\mathbf{c m} / \mathbf{s})\end{array}$ & $\begin{array}{c}\text { Path Length } \\
(\mathbf{c m})\end{array}$ & $\begin{array}{c}\text { Area }(\mathbf{c m})^{2} \\
\text { Eyes open }\end{array}$ \\
& TD & $0.81 \pm 0.15^{*}$ & $1.49 \pm 0.10^{*}$ & $1.46 \pm 0.27^{*}$ & $29.28 \pm 5.49^{*}$ & $0.92 \pm 0.22^{*}$ \\
& PIGD & $1.06 \pm 0.13^{* *}$ & $1.81 \pm 0.16^{* *}$ & $1.48 \pm 0.23^{* *}$ & $29.62 \pm 4.57^{* *}$ & $1.53 \pm 0.32^{* *}$ \\
\hline \multirow{2}{*}{ Eyes closed } & TD & $1.15 \pm 0.24^{*}$ & $2.81 \pm 0.39^{*}$ & $2.56 \pm 0.66^{*}$ & $51.23 \pm 13.16^{*}$ & $2.95 \pm 1.05^{*}$ \\
& PIGD & $1.14 \pm 0.14^{* *}$ & $2.75 \pm 0.36^{* *}$ & $2.01 \pm 0.20^{* *}$ & $40.23 \pm 4.01^{* *}$ & $2.51 \pm 0.45^{* *}$ \\
\hline
\end{tabular}


A power spectral analysis of the COP and COP velocity of a TD patient and a PIGD patient are plotted in Figure 1, revealing that both patients had frequency components ranging from 0 to $2 \mathrm{~Hz}$ in their COP and COP velocity signals. However, only the TD patient had an increase in power spectrum in the frequency band of 3-7 Hz. This increase was larger in the ML direction.
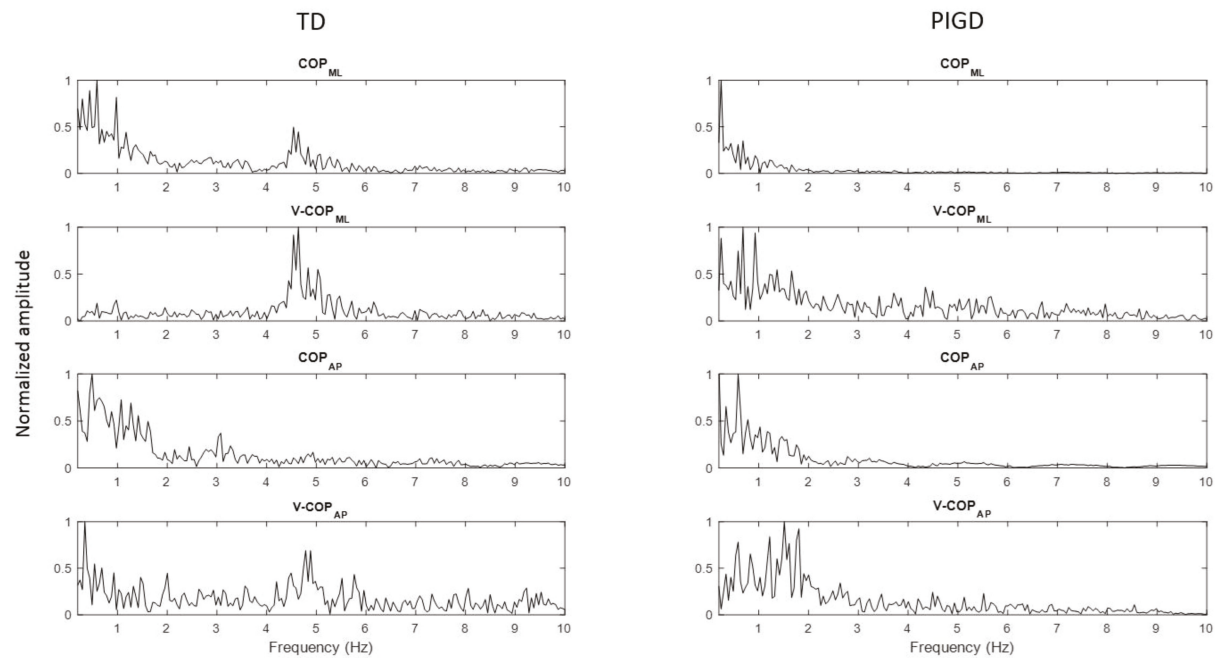

Figure 1. Power spectrum of COP and COP velocity of a tremor dominant (TD) patient and a postural instability and gait difficulty (PIGD) patient for both the medial-lateral (ML) and anterior-posterior (AP) directions. The graphs on the left and right sides of the page present the power spectrum signal of a TD patient and a PIGD patient, respectively. $\mathrm{COP}_{\mathrm{ML}}$ : $\mathrm{COP}$ in the ML direction, $\mathrm{COP}_{\mathrm{AP}}$ : $\mathrm{COP}$ in the $\mathrm{AP}$ direction, $\mathrm{V}-\mathrm{COP}_{\mathrm{ML}}$ : $\mathrm{COP}$ velocity in the $\mathrm{ML}$ direction, and $\mathrm{V}-\mathrm{COP}_{\mathrm{AP}}$ : $\mathrm{COP}$ velocity in the AP direction.

The WT of COP and COP velocity of a TD patient and a PIGD patient in both the ML and AP directions are plotted in Figure 2. The horizontal white lines in each figure indicate the PD tremor scale range corresponding to the frequency range of 3-7 Hz. The WT coefficients in Figure 2 display relatively larger values in the PD tremor scale range (i.e., lighter blue values appeared in between two horizontal white lines) for the TD patient when compared to the PIGD patient. Similar to the power spectral analysis (Figure 1), these increases were larger in the ML direction.

The results of the proposed detection ratio for COP and its velocity in both directions using FFT are presented in Figure 3. Neither the ratio of $\mathrm{COP}\left(\mathrm{R}_{\mathrm{COP} \_\mathrm{ML}}\right)$ nor its velocity $\left(\mathrm{R}_{\mathrm{VCOP}} \mathrm{ML}\right)$ in the $\mathrm{ML}$ direction were significantly different across the different conditions $\left(\mathrm{R}_{\mathrm{COP} \_\mathrm{ML}}: \mathrm{F}_{(1,34)}=2.006, p=0.112\right.$; $\left.\mathrm{R}_{\mathrm{VCOP} \_\mathrm{ML}}: \mathrm{F}_{(1,34)}=2.67, p=0.112\right)$. However, a statistically significant difference in $\mathrm{R}_{\mathrm{VCOP} \_\mathrm{ML}}$ across the groups $\left(\mathrm{F}_{(1,34)}=7.978, p=0.008\right)$ was observed, although no significant difference was found in $\mathrm{R}_{\mathrm{COP} \_\mathrm{ML}}\left(\mathrm{F}_{(1,34)}=3.449, p=0.072\right)$. In both $\mathrm{R}_{\mathrm{COP} \_\mathrm{ML}}$ and $\mathrm{R}_{\mathrm{VCOP} \_\mathrm{ML}}$, there was no significant interaction between the condition and the group $\left(\mathrm{R}_{\mathrm{COP} \_\mathrm{ML}}: \mathrm{F}_{(1,34)}=1.181, p=0.285 ; \mathrm{R}_{\mathrm{VCOP} \_\mathrm{ML}}: \mathrm{F}_{(1,34)}=2.037\right.$, $p=0.163)$. $\mathrm{R}_{\mathrm{VCOP} \_M L}$ was larger for the TD group than for the PIGD group (Figure 3A,B). This indicated that there were larger amplitudes in the frequency range of 3-7 Hz in this group. In the AP direction, there was no significant difference across the groups $\left(\mathrm{R}_{\mathrm{COP} \_\mathrm{AP}}: \mathrm{F}_{(1,34)}=0.498, p=0.485\right.$; $\mathrm{R}_{\mathrm{VCOP} \_\mathrm{AP}}$ : $\left.\mathrm{F}_{(1,34)}=0.628, p=0.433\right)$ and the conditions ( $\mathrm{R}_{\mathrm{COP} \_\mathrm{AP}}: \mathrm{F}_{(1,34)}=1.306, p=0.201 ; \mathrm{R}_{\mathrm{VCOP} \_A P}: \mathrm{F}_{(1,34)}=3.45$, $p=0.08)$ in both $\mathrm{R}_{\mathrm{COP} \_\mathrm{AP}}$ and $\mathrm{R}_{\mathrm{VCOP} \_\mathrm{AP}}$.

The explained WT method was applied to COP and its velocity in both directions. The results are presented in Figure 4 . We found a significant difference between the groups for $\mathrm{R}_{\mathrm{COP}-\mathrm{ML}}$ and 
$\mathrm{R}_{\mathrm{VCOP} \_\mathrm{ML}}\left(\mathrm{R}_{\mathrm{COP} \_\mathrm{ML}}: \mathrm{F}_{(1,34)}=7.589, p=0.009 ; \mathrm{R}_{\mathrm{VCOP} \_\mathrm{ML}}: \mathrm{F}_{(1,34)}=10.066, p=0.003\right)$, but no significant difference between the conditions $\left(\mathrm{R}_{\mathrm{COP} \_\mathrm{ML}}: \mathrm{F}_{(1,34)}=0.373, p=0.814 ; \mathrm{R}_{\mathrm{VCOP} \_\mathrm{ML}}: \mathrm{F}_{(1,34)}=2.5, p=0.123\right)$. There was no significant interaction between the conditions and the groups $\left(\mathrm{R}_{\mathrm{COP}} \mathrm{ML}: \mathrm{F}_{(1,34)}=3.044\right.$, $\left.p=0.09 ; \mathrm{R}_{\mathrm{VCOP} \_\mathrm{ML}}: \mathrm{F}_{(1,34)}=2.828, p=0.102\right)$. Both $\mathrm{R}_{\mathrm{COP} \_\mathrm{ML}}$ and $\mathrm{R}_{\mathrm{VCOP}} \mathrm{ML}$ had larger values for the TD group than for the PIGD group (Figure 3A,B). These increases occurred because of the larger amplitude values in the scales corresponding to the frequency range of 3-7 Hz. In the AP direction, there were no significant differences across the groups $\left(\mathrm{R}_{\mathrm{COP} \_A P}: \mathrm{F}_{(1,34)}=0.004, p=0.952 ; \mathrm{R}_{\mathrm{VCOP} \_A P}: \mathrm{F}_{(1,34)}=0.854\right.$, $p=0.362$ ) or conditions ( $\mathrm{R}_{\mathrm{COP} \_\mathrm{AP}}: \mathrm{F}_{(1,34)}=0.011, p=0.916$; $\left.\mathrm{R}_{\mathrm{VCOP} \_\mathrm{AP}}: \mathrm{F}_{(1,34)}=3.047, p=0.091\right)$ in both $\mathrm{R}_{\mathrm{COP} \_\mathrm{AP}}$ and $\mathrm{R}_{\mathrm{VCOP} \_\mathrm{AP}}$.
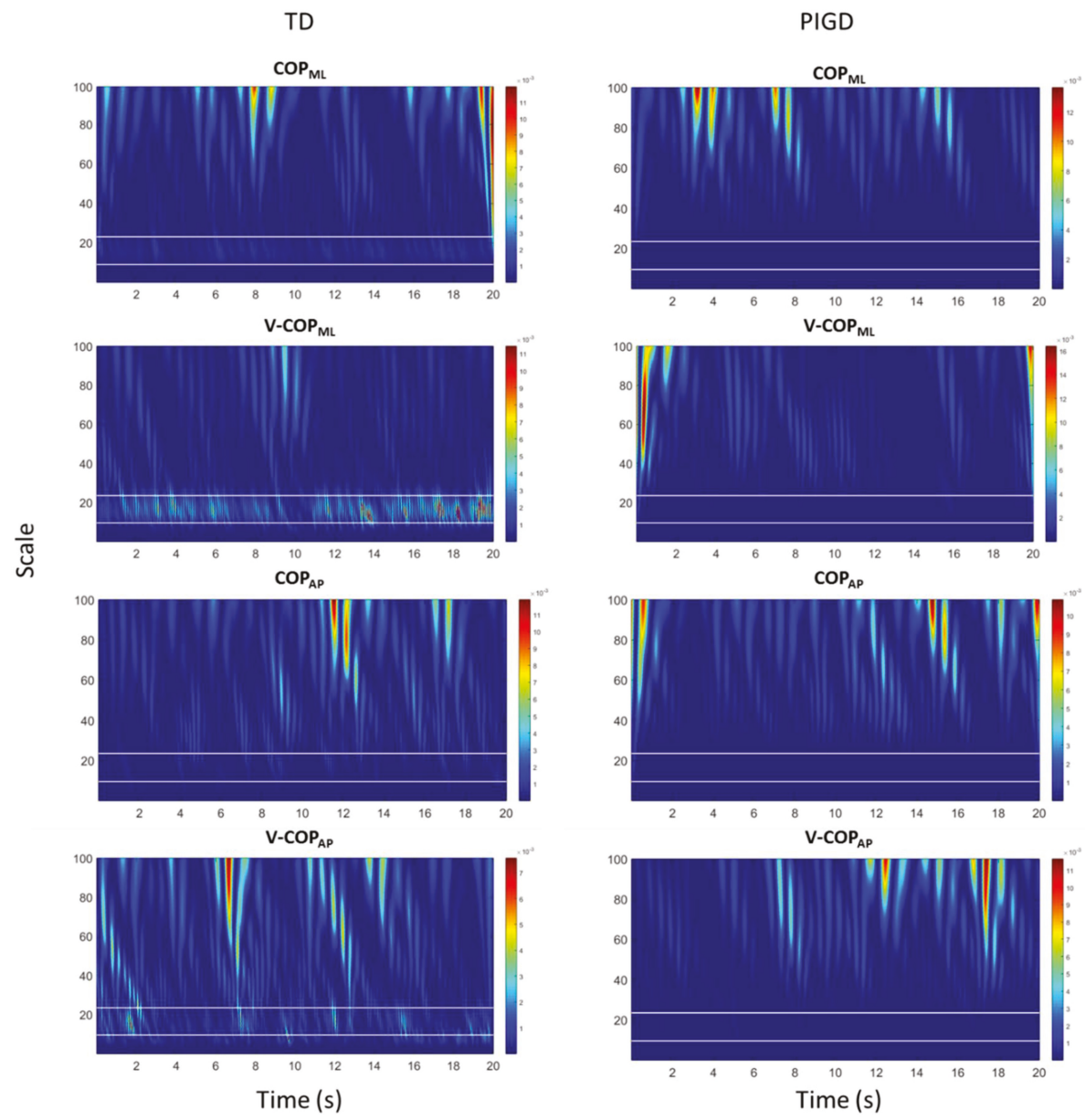

Figure 2. Wavelet transform (WT) of COP and COP velocity of a TD patient and a PIGD patient for both the ML and AP directions. The horizontal white lines in each plot indicate the PD tremor scale range corresponding to the frequency range of $3-7 \mathrm{~Hz}$. The frequencies of $3 \mathrm{~Hz}$ and $7 \mathrm{~Hz}$ correspond to the scales of 24 and 10, respectively. $\mathrm{COP}_{\mathrm{ML}}$ : $\mathrm{COP}$ in the $\mathrm{ML}$ direction, $\mathrm{COP}_{\mathrm{AP}}$ : $\mathrm{COP}$ in the $\mathrm{AP}$ direction,

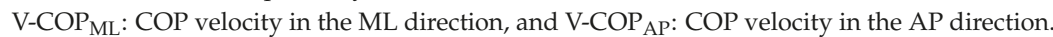


(A)

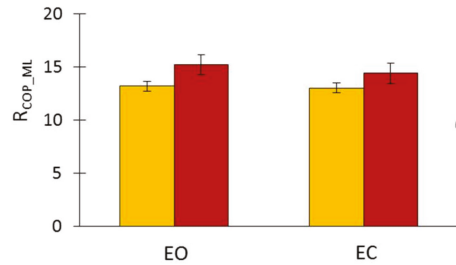

(C)

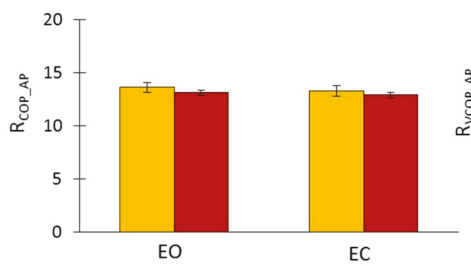

(B)

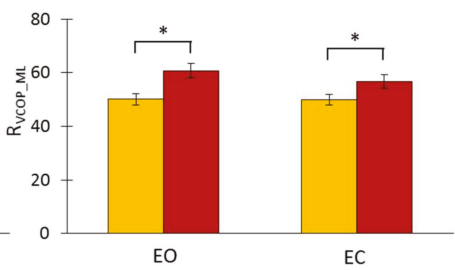

(D)

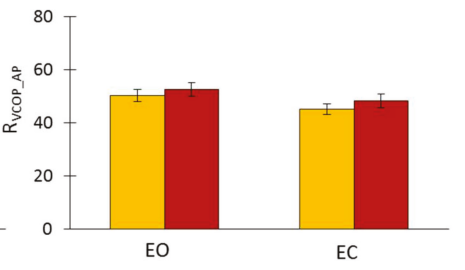

$\square$ PIGD $\square$ TD

Figure 3. Fast Fourier transform (FFT) results of the proposed detection ratio for COP and its velocity

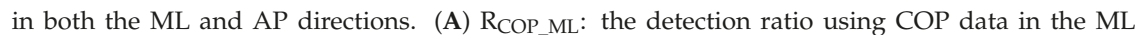
direction, (B) $\mathrm{R}_{\mathrm{VCOP} \_\mathrm{ML}}$ : the detection ratio using COP velocity data in the ML direction, $(\mathbf{C}) \mathrm{R}_{\mathrm{COP} \_\mathrm{AP}}$ : the detection ratio using COP data in the AP direction, and (D) $\mathrm{R}_{\mathrm{VCOP} \_\mathrm{AP}}$ : the detection ratio using $\mathrm{COP}$ velocity data in the AP direction. The asterisks $\left({ }^{*}\right)$ placed over the vertical bars denote a significant difference $(p<0.05)$. EC: eyes closed condition; EO: eyes open condition.

(A)

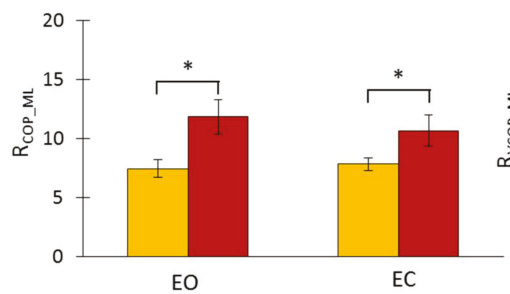

(C)

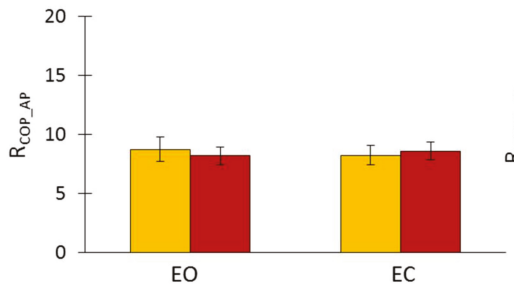

(B)

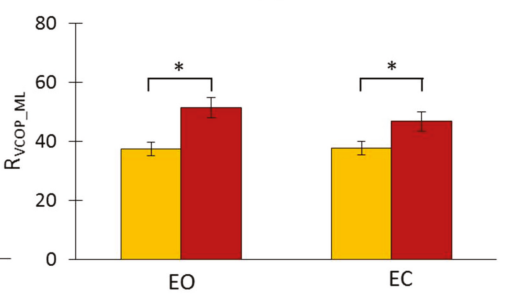

(D)

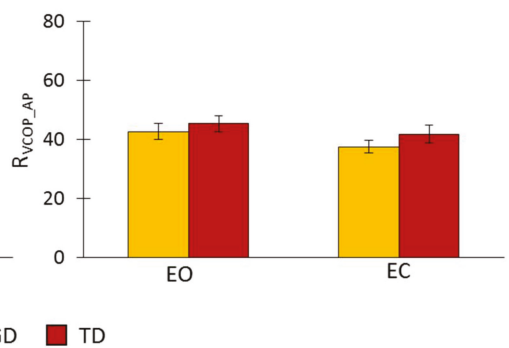

Figure 4. WT results of the proposed detection ratio for COP and its velocity in both the ML and AP directions. (A) $\mathrm{R}_{\mathrm{COP} \_\mathrm{ML}}$ : the detection ratio using COP data in the ML direction, $(\mathbf{B}) \mathrm{R}_{\mathrm{VCOP} \_\mathrm{ML}}$ : the detection ratio using COP velocity data in the $\mathrm{ML}$ direction, $(\mathrm{C}) \mathrm{R}_{\mathrm{COP}} \mathrm{AP}$ : the detection ratio using $\mathrm{COP}$ data in the AP direction, and (D) $\mathrm{R}_{\mathrm{VCOP}} \mathrm{AP}$ : the detection ratio using COP velocity data in the AP direction. The asterisks $\left({ }^{*}\right)$ placed over the vertical bars denote a significant difference $(p<0.05)$. 
The ROC curves of the proposed detection ratio for COP and its velocity in both directions and under both conditions are plotted in Figures 5 and 6 for the FFT and WT methods, respectively. In both methods, the ROC curves were closer to the upper-left corner in the ML direction than they were in the AP direction, which indicated a higher overall accuracy of the test in the ML direction [31].

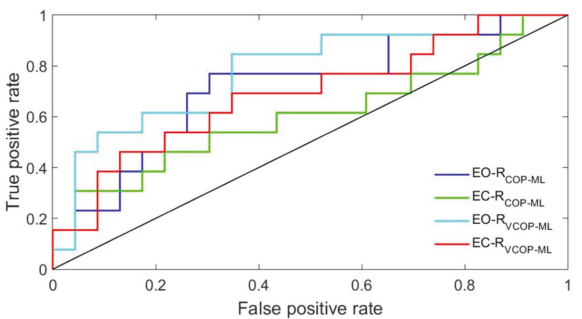

(A)

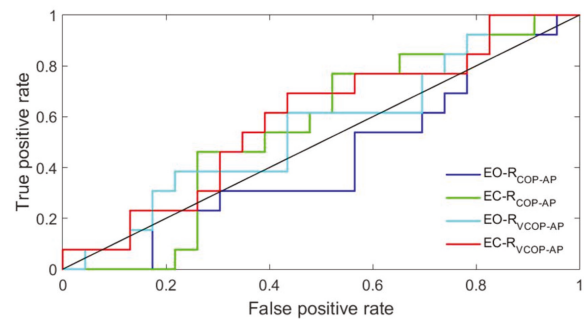

(B)

Figure 5. Receiver operating characteristic (ROC) curves of the proposed detection ratio using the FFT method for COP and its velocity: (A) ML direction and (B) AP direction. EO-R $\mathrm{COP}_{\mathrm{ML}}$ : the detection ratio using $\mathrm{COP}$ data in the $\mathrm{ML}$ direction under the eyes open condition, $\mathrm{EC}-\mathrm{R}_{\mathrm{COP} \_\mathrm{ML}}$ : the detection ratio using $\mathrm{COP}$ data in the $\mathrm{ML}$ direction under the eyes closed condition, EO-R $\mathrm{VCOP}_{\mathrm{ML}}$ : the detection ratio using COP velocity data in the ML direction under the eyes open condition, EC-R $\mathrm{R}_{\mathrm{VCOP} \_\mathrm{ML}}$ : the detection ratio using COP velocity data in the ML direction under the eyes closed condition, EO- $\mathrm{R}_{\mathrm{COP}} \mathrm{AP}$ : the detection ratio using $\mathrm{COP}$ data in the AP direction under the eyes open condition, EC-R $\mathrm{ROP}_{\mathrm{C}} \mathrm{AP}$ : the detection ratio using $\mathrm{COP}$ data in the AP direction under the eyes closed condition, EO- $\mathrm{R}_{\mathrm{VCOP}} \mathrm{AP}$ : the detection ratio using $\mathrm{COP}$ velocity data in the AP direction under the eyes open condition, and EC-R $\mathrm{VCOP} \_\mathrm{AP}$ : the detection ratio using COP velocity data in the AP direction under the eyes closed condition.

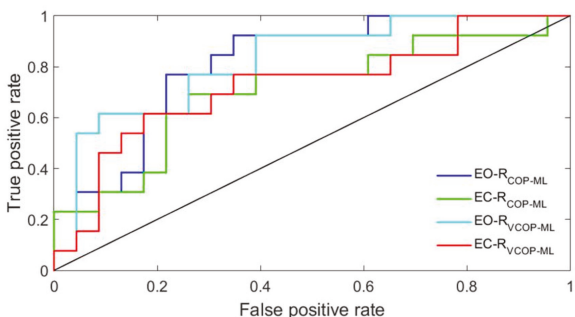

(A)

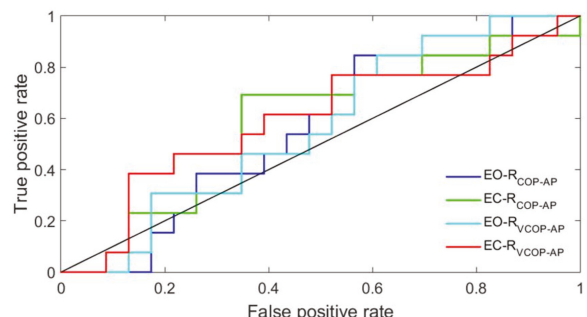

(B)

Figure 6. ROC curves of the proposed detection ratio using the WT method for COP and its velocity: (A) ML direction and (B) AP direction. EO- $\mathrm{R}_{\mathrm{COP} \_\mathrm{ML}}$ : the detection ratio using COP data in the ML direction under the eyes open condition, $\mathrm{EC}-\mathrm{R}_{\mathrm{COP} \_\mathrm{ML}}$ : the detection ratio using COP data in the ML direction under the eyes closed condition, $\mathrm{EO}-\mathrm{R}_{\mathrm{VCOP}} \mathrm{ML}$ : the detection ratio using COP velocity data in the $\mathrm{ML}$ direction under the eyes open condition, $\mathrm{EC}-\mathrm{R}_{\mathrm{VCOP}} \mathrm{ML}$ : the detection ratio using COP velocity data in the ML direction under the eyes closed condition, EO-R $\mathrm{COP}_{\mathrm{AP}}$ : the detection ratio using COP data in the AP direction under the eyes open condition, EC-R $\mathrm{R}_{\mathrm{COP} \_\mathrm{AP}}$ : the detection ratio using COP data in the AP direction under the eyes closed condition, EO-R $\mathrm{R}_{\mathrm{VCOP}} \mathrm{AP}$ : the detection ratio using COP velocity data in the AP direction under the eyes open condition, and EC-R $\mathrm{VCOP}_{\mathrm{AP}}$ : the detection ratio using COP velocity data in the AP direction under the eyes closed condition.

The ROC curves were further analyzed by calculating the areas under each curve. The results are presented in Table 3. Only COP velocity data in the ML direction could significantly distinguish between the two subtypes using the FFT method. The results of the area under the ROC curves also 
revealed that the WT method could significantly distinguish between the two subtypes by using either $\mathrm{COP}$ or COP velocity data in the ML direction, regardless of the conditions.

Table 3. The area under the receiver operating characteristic (ROC) curves of the proposed detection ratio, using both the FFT and WT methods, for COP and its velocity in the ML and AP directions under the two conditions (eyes open (EO) and eyes closed (EC)). The $p$-values of each parameter are presented in parentheses. The asterisks $\left(^{*}\right)$ indicate that the area under the ROC curve was significantly different from $0.5(p<0.05)$.

\begin{tabular}{|c|c|c|c|c|c|}
\hline & & \multicolumn{2}{|c|}{ FFT } & \multicolumn{2}{|c|}{ WT } \\
\hline & & COP & V_COP & COP & V_COP \\
\hline \multirow{2}{*}{ ML-Direction } & $\mathrm{EO}$ & $\begin{array}{c}0.689 \\
(p=0.05)\end{array}$ & $\begin{array}{c}0.779 * \\
(p=0.001)\end{array}$ & $\begin{array}{c}0.809 * \\
(p=0.001)\end{array}$ & $\begin{array}{c}0.823 * \\
(p=0.001)\end{array}$ \\
\hline & $\mathrm{EC}$ & $\begin{array}{c}0.602 \\
(p=0.343)\end{array}$ & $\begin{array}{c}0.712 * \\
(p=0.023)\end{array}$ & $\begin{array}{c}0.706^{*} \\
(p=0.033)\end{array}$ & $\begin{array}{c}0.726^{*} \\
(p=0.016)\end{array}$ \\
\hline \multirow{2}{*}{ AP-Direction } & $\mathrm{EO}$ & $\begin{array}{c}0.562 \\
(p=0.542)\end{array}$ & $\begin{array}{c}0.555 \\
(p=0.5873)\end{array}$ & $\begin{array}{c}0.562 \\
(p=0.529)\end{array}$ & $\begin{array}{c}0.569 \\
(p=0.482)\end{array}$ \\
\hline & $\mathrm{EC}$ & $\begin{array}{c}0.555 \\
(p=0.578)\end{array}$ & $\begin{array}{c}0.592 \\
(p=0.358)\end{array}$ & $\begin{array}{c}0.579 \\
(0.442)\end{array}$ & $\begin{array}{c}0.595 \\
(p=0.363)\end{array}$ \\
\hline
\end{tabular}

\section{Discussion}

This study addressed subtype-specific biomarkers in order to classify the inherent heterogeneity of PD. This categorization can help to predict the clinical progression of the disease. Thus, the correct diagnosis of the subtypes can assist caregivers in initiating early amenable interventions and managing symptoms. The COP time series of PD patients were analyzed to distinguish between the two subtypes of PD. To the best of our knowledge, this study is the first to attempt to objectively diagnose the TD and PIGD subtypes of PD. Postural stability is maintained through neuromuscular feedback loops and open loop control processes that constantly adapt to internal and external perturbations $[32,33]$. Utilizing specific statistical and numerical tools, these control mechanisms can be quantified to identify neuromuscular changes that occur with pathology. Thus, traditional linear postural measures and Fourier transformation were applied to the COP time series and the increment of the COP time series in both the AP and ML directions. Furthermore, in order to quantify the changes in COP dynamics that occur at multiple timescales, a wavelet transform was employed to infer the underlying nature and control mechanisms involved in balance maintenance and the disease state.

In the traditional measures of postural sway, the parameters that denoted the magnitude of the postural movements were unable to discriminate between the TD and PIGD subtypes (Table 2). However, when visual information was occluded, a coincident decrease in postural stability was reflected in both subtypes for the linear postural measures (i.e., COP range, mean velocity, path length, and a 95\% confidence ellipse area). These results were consistent with previous investigations regarding postural stability in PD patients [34].

Both the power spectral density and the WT of the COP time series and its velocity (Figures 1 and 2) revealed an increase in the 3-7 Hz frequency range of the TD group, a frequency spectra that is reportedly symptomatic of parkinsonian tremor [17-19]. In fact, the ML COP data exhibited a greater frequency content than the AP COP data, which was consistent with previous investigations, which reported that PD patients exhibited increased ML sway amplitude, decreased AP sway amplitude, and possibly postural inflexibility in the AP direction [15,35-37]. In this context, the preponderance of the ML frequency in the ML direction, coupled with the impaired movement in the AP direction, suggested an underlying postural inflexibility in PD patients, where the tremor reflected in the ML time domain might be a consequence of the AP direction's inability to contain movements in a higher frequency range $[35,38,39]$. Our proposed ratio was not able to show a statistically significant difference 
between the TD and PIGD patients in the AP direction using either of the methods—even accounting for both COP and COP velocity. The reason was that the tremor frequency had a larger amplitude in the ML direction than it did in the AP direction (as shown in Figures 1 and 2). However, both the FFT and WT methods were able to distinguish the TD patients from the PIGD patients using the ML-COP velocity signal, while only the WT method was able to specify the subtype with the COP position time series. This could be explained by the fact that the FFT method used only the frequency information from the signals, while the WT method employed both the frequency and time components. The information from the signals that was utilized by WT enabled us to specify the subtypes of PD using both COP and COP velocity. Additionally, FFT displayed significant results when it employed COP velocity-as opposed to COP in itself-because the velocity of the signal was a first time derivative of the signal, which captured more variation of the signal. Hence, FFT could assess more information about the signals when it employed COP velocity. The results of the proposed method were consistent across both conditions (EO and EC) in both methods (FFT and WT). This consistency indicated the strength of the proposed diagnostic method using the proposed ratio. Although the proposed method can distinguish the TD from the PIGD subtypes, further studies are required to define the threshold value ranges that can classify the patients.

Acknowledgments: This research was supported by the NSF-Information and Intelligent Systems (IIS) and Smart and Connected Health (1065442, and 1547466, and secondary 1065262).

Author Contributions: Saba Rezvanian and Thurmon Lockhart conceived and designed the experiments; Saba Rezvanian and Christopher Frames performed the experiment; Saba Rezvanian analyzed the data and wrote the manuscript with support from Thurmon Lockhart, Abraham Lieberman and Rahul Soangra. All authors discussed the results and contributed to the final manuscript.

Conflicts of Interest: The authors declare no conflicts of interest.

Ethical Statements: All subjects gave their informed consent for inclusion before they participated in the study. The study was conducted in accordance with the Declaration of Helsinki, and the protocol was approved by the St. Joseph's Hospital and Medical Center (PHX-16-0227-71-04).

\section{References}

1. Kowal, S.L.; Dall, T.M.; Chakrabarti, R.; Storm, M.V.; Jain, A. The current and projected economic burden of Parkinson's disease in the United States. Mov. Disord. 2013, 28, 311-318. [CrossRef] [PubMed]

2. Fahn, S.; Jankovic, J.; Hallett, M. Principles and Practice of Movement Disorders; Elsevier Health Sciences: New York, NY, USA, 2011.

3. Chen, H.M.; Wang, Z.J.; Fang, J.P.; Gao, L.Y.; Ma, L.Y.; Wu, T.; Hou, Y.N.; Zhang, J.R.; Feng, T. Different Patterns of Spontaneous Brain Activity between Tremor-Dominant and Postural Instability/Gait Difficulty Subtypes of Parkinson's Disease: A Resting-State fMRI Study. CNS Neurosci. Ther. 2015, 21, 855-866. [CrossRef] [PubMed]

4. Thenganatt, M.A.; Jankovic, J. Parkinson disease subtypes. JAMA Neurol. 2014, 71, 499-504. [CrossRef] [PubMed]

5. Jankovic, J.; McDermott, M.; Carter, J.; Gauthier, S.; Goetz, C.; Golbe, L.; Huber, S.; Koller, W.; Olanow, C.; Shoulson, I. Variable expression of Parkinson's disease: A base-line analysis of the DATATOP cohort. The Parkinson Study Group. Neurology 1990, 40, 1529-1534. [CrossRef] [PubMed]

6. Jankovic, J.; Kapadia, A.S. Functional decline in Parkinson disease. Arch. Neurol. 2001, 58, 1611-1615. [CrossRef] [PubMed]

7. Rajput, A.H.; Pahwa, R.; Pahwa, P.; Rajput, A. Prognostic significance of the onset mode in parkinsonism. Neurology 1993, 43, 829-830. [CrossRef] [PubMed]

8. Mehanna, R.; Lai, E.C. Deep brain stimulation in Parkinson's disease. Transl. Neurodegener. 2013, 2, 22. [CrossRef] [PubMed]

9. Rudzińska, M.; Marona, M.; Bukowczan, S.; Banaszkiewicz, K.; Mirek, E.; Szczudlik, A. Falls in different types of Parkinson's disease. Neurol. Neurochir. Pol. 2007, 41, 395-403.

10. Herman, T.; Rosenberg-Katz, K.; Jacob, Y.; Auriel, E.; Gurevich, T.; Giladi, N.; Hausdorff, J.M. White Matter Hyperintensities in Parkinson's Disease: Do They Explain the Disparity between the Postural Instability Gait Difficulty and Tremor Dominant Subtypes? PLoS ONE 2013, 8, e55193. [CrossRef] [PubMed] 
11. Stebbins, G.T.; Goetz, C.G.; Burn, D.J.; Jankovic, J.; Khoo, T.K.; Tilley, B.C. How to identify tremor dominant and postural instability/gait difficulty groups with the movement disorder society unified Parkinson's disease rating scale: Comparison with the unified Parkinson's disease rating scale. Mov. Disord. 2013, 28, 668-670. [CrossRef] [PubMed]

12. Mds, T.; Goetz, C.G.; Poewe, W.; Rascol, O.; Christina, S. The Unified Parkinson's Disease Rating Scale (UPDRS): Status and Recommendations. Mov. Disord. 2003, 18, 738-750.

13. Schlenstedt, C.; Muthuraman, M.; Witt, K.; Weisser, B.; Fasano, A.; Deuschl, G. Postural control and freezing of gait in Parkinson's disease. Park. Relat. Disord. 2016, 24, 107-112. [CrossRef] [PubMed]

14. Schmit, J.M.; Riley, M.A.; Dalvi, A.; Sahay, A.; Shear, P.K.; Shockley, K.D.; Pun, R.Y.K. Deterministic center of pressure patterns characterize postural instability in Parkinson's disease. Exp. Brain Res. 2006, 168, 357-367. [CrossRef] [PubMed]

15. Rocchi, L.; Chiari, L.; Cappello, A.; Horak, F.B. Identification of distinct characteristics of postural sway in Parkinson's disease: A feature selection procedure based on principal component analysis. Neurosci. Lett. 2006, 394, 140-145. [CrossRef] [PubMed]

16. Diab, K.S.; Hale, L.A.; Waters, D.L.; Skinner, M.A. Factors contributing to postural instability in patients with idiopathic Parkinson's disease. Phys. Ther. Rev. 2014, 19, 302-327. [CrossRef]

17. Hallett, M. Overview of human tremor physiology. Mov. Disord. 1998, 13 (Suppl. S3), 43-48. [CrossRef] [PubMed]

18. Lemstra, A.W.; Verhagen Metman, L.; Lee, J.I.; Dougherty, P.M.; Lenz, F.A. Tremor-frequency (3-6 Hz) activity in the sensorimotor arm representation of the internal segment of the globus pallidus in patients with Parkinson's disease. Neurosci. Lett. 1999, 267, 129-132. [CrossRef]

19. Timmermann, L.; Gross, J.; Dirks, M.; Volkmann, J.; Freund, H.-J.; Schnitzler, A. The cerebral oscillatory network of parkinsonian resting tremor. Brain 2003, 126, 199-212. [CrossRef] [PubMed]

20. Freitas, S.M.S.F.; Wieczorek, S.A.; Marchetti, P.H.; Duarte, M. Age-related changes in human postural control of prolonged standing. Gait Posture 2005, 22, 322-330. [CrossRef] [PubMed]

21. Vieira, T.M.M.; Oliveira, L.F.; Nadal, J. Estimation procedures affect the center of pressure frequency analysis. Braz. J. Med. Biol. Res. 2009, 42, 665-673. [CrossRef] [PubMed]

22. Kanekar, N.; Lee, Y.-J.; Aruin, A.S. Frequency analysis approach to study balance control in individuals with multiple sclerosis. J. Neurosci. Methods 2014, 222, 91-96. [CrossRef] [PubMed]

23. Goetz, C.G.; Tilley, B.C.; Shaftman, S.R.; Stebbins, G.T.; Fahn, S.; Martinez-Martin, P.; Poewe, W.; Sampaio, C.; Stern, M.B.; Dodel, R.; et al. Movement Disorder Society-Sponsored Revision of the Unified Parkinson's Disease Rating Scale (MDS-UPDRS): Scale presentation and clinimetric testing results. Mov. Disord. 2008, 23, 2129-2170. [CrossRef] [PubMed]

24. Van der Heeden, J.F.; Marinus, J.; Martinez-Martin, P.; Rodriguez-Blazquez, C.; Geraedts, V.J.; van Hilten, J.J. Postural instability and gait are associated with severity and prognosis of Parkinson disease. Neurology 2016, 86, 2243-2250. [CrossRef] [PubMed]

25. Cavalheiro, G.L.; Almeida, M.F.S.; Pereira, A.A.; Andrade, A.O. Study of age-related changes in postural control during quiet standing through linear discriminant analysis. Biomed. Eng. Online 2009, 8, 35. [CrossRef] [PubMed]

26. Lafond, D.; Corriveau, H.; Hébert, R.; Prince, F. Intrasession reliability of center of pressure measures of postural steadiness in healthy elderly people. Arch. Phys. Med. Rehabil. 2004, 85, 896-901. [CrossRef] [PubMed]

27. Rezvanian, S.; Lockhart, T. Towards Real-Time Detection of Freezing of Gait Using Wavelet Transform on Wireless Accelerometer Data. Sensors 2016, 16, 475. [CrossRef] [PubMed]

28. Mojtahedi, K.; Fu, Q.; Santello, M. Extraction of Time and Frequency Features from Grip Force Rates during Dexterous Manipulation. IEEE Trans. Biomed. Eng. 2015, 62, 1363-1375. [CrossRef] [PubMed]

29. Rezvanian, S.; Lockhart, T.; Frames, C.; Soangra, R. Toward an objective method to classify tremor dominant and postural instability and gait difficulty subtypes of parkinson's disease: A pilot study. Biomed. Sci. Instrum. 2017, 53, 138-142.

30. Lockhart, T.E.; Soangra, R.; Zhang, J.; Wu, X. Wavelet based automated postural event detection and activity classification with single imu-biomed 2013. Biomed. Sci. Instrum. 2013, 49, 224-233. [PubMed]

31. Zweig, M.; Campbell, G. Receiver-operating characteristic (ROC) plots: A fundamental evaluation tool in clinical medicine. Clin. Chem. 1993, 39, 561-577. [PubMed] 
32. Pai, Y.-C.; Wening, J.D.; Runtz, E.F.; Iqbal, K.; Pavol, M.J. Role of feedforward control of movement stability in reducing slip-related balance loss and falls among older adults. J. Neurophysiol. 2003, 90, 755-762. [CrossRef] [PubMed]

33. Nallegowda, M.; Singh, U.; Handa, G.; Khanna, M.; Wadhwa, S.; Yadav, S.L.; Kumar, G.; Behari, M. Role of Sensory Input and Muscle Strength in Maintenance of Balance, Gait, and Posture in Parkinson's disease: A pilot study. Am. J. Phys. Med. Rehabil. 2004, 83, 898-908. [CrossRef] [PubMed]

34. Błaszczyk, J.W.; Orawiec, R.; Duda-Kłodowska, D.; Opala, G. Assessment of postural instability in patients with Parkinson's disease. Exp. Brain Res. 2007, 183, 107-114. [CrossRef] [PubMed]

35. Mitchell, S.L.; Collin, J.J.; De Luca, C.J.; Burrows, A.; Lipsitz, L.A. Open-loop and closed-loop postural control mechanisms in Parkinson's disease: Increased mediolateral activity during quiet standing. Neurosci. Lett. 1995, 197, 133-136. [CrossRef]

36. Van Wegen, E.E.H.; van Emmerik, R.E.A.; Wagenaar, R.C.; Ellis, T. Stability Boundaries and Lateral Postural Control in Parkinson's Disease. Motor Control 2001, 5, 254-269. [CrossRef] [PubMed]

37. Viitasalo, M.K.; Kampman, V.; Sotaniemi, K.A.; Leppävuori, S.; Myllylä, V.V.; Korpelainen, J.T. Analysis of sway in Parkinson's disease using a new inclinometry-based method. Mov. Disord. 2002, 17, 663-669. [CrossRef] [PubMed]

38. Horak, F.B.; Nutt, J.G.; Nashner, L.M. Postural inflexibility in parkinsonian subjects. J. Neurol. Sci. 1992, 111, 46-58. [CrossRef]

39. Schieppati, M.; Hugon, M.; Grasso, M.; Nardone, A.; Galante, M. The limits of equilibrium in young and elderly normal subjects and in parkinsonians. Electroencephalogr. Clin. Neurophysiol. 1994, 93, 286-298. [CrossRef]

(C) 2018 by the authors. Licensee MDPI, Basel, Switzerland. This article is an open access article distributed under the terms and conditions of the Creative Commons Attribution (CC BY) license (http:/ / creativecommons.org/licenses/by/4.0/). 
Article

\title{
Analysis of a Smartphone-Based Architecture with Multiple Mobility Sensors for Fall Detection with Supervised Learning
}

\author{
José Antonio Santoyo-Ramón, Eduardo Casilari * and José Manuel Cano-García \\ Departamento de Tecnología Electrónica, Universidad de Málaga, ETSI Telecomunicación, 29071 Málaga, Spain; \\ jasantoyo@uma.es (J.A.S.-R.); jcgarcia@uma.es (J.M.C.-G.) \\ * Correspondence: ecasilari@uma.es
}

Received: 15 February 2018; Accepted: 4 April 2018; Published: 10 April 2018

\begin{abstract}
This paper describes a wearable Fall Detection System (FDS) based on a body-area network consisting of four nodes provided with inertial sensors and Bluetooth wireless interfaces. The signals captured by the nodes are sent to a smartphone which simultaneously acts as another sensing point. In contrast to many FDSs proposed by the literature (which only consider a single sensor), the multisensory nature of the prototype is utilized to investigate the impact of the number and the positions of the sensors on the effectiveness of the production of the fall detection decision. In particular, the study assesses the capability of four popular machine learning algorithms to discriminate the dynamics of the Activities of Daily Living (ADLs) and falls generated by a set of experimental subjects, when the combined use of the sensors located on different parts of the body is considered. Prior to this, the election of the statistics that optimize the characterization of the acceleration signals and the efficacy of the FDS is also investigated. As another important methodological novelty in this field, the statistical significance of all the results (an aspect which is usually neglected by other works) is validated by an analysis of variance (ANOVA).
\end{abstract}

Keywords: fall detection system; inertial sensors; smartphones; accelerometers; machine learning algorithms; supervised learning; ANOVA analysis

\section{Introduction}

According to some forecasts [1], it is expected that between 2015 and 2050 the over-60 years of age world population will grow from 900 to 2000 million. This dramatic demographic change will undoubtedly give rise to a series of challenges in the health systems that must be faced in order to sustain and increase the quality of life of the citizens. The present study focuses on one of the biggest public health problems faced by the world society: falls.

A fall can result in minor consequences such as bruises, lacerations or abrasions, but it can also lead to more serious and dangerous situations such as bone fractures or psychological disorders (such as the so-called Fear of Falling or FoF syndrome). The World Health Organization has reported that falls are the second worldwide cause of mortality provoked by accidental or unintentional injuries. 37.3 million falls requiring medical attention occur annually, while it has been estimated that around 646,000 people die every year due to falls [2] A remarkable fact is that people over 65 years old experience the greatest number of falls. Approximately $28-35 \%$ of people over 65 suffer a fall each year, whereas this percentage climbs with age, reaching $32-42 \%$ for people over 70 [3]. Risk groups are not limited to the older people. Other population groups are exposed to endure severe falls during their work or leisure time (cyclists, mountaineers, firemen, antenna installers, cable layers, etc.).

The probability of survival after a serious fall is strongly related to the rapidness of the medical assistance. As mentioned in [4], 50\% of people affected by a bad fall who remain without assistance for 
more than an hour die before the six months following the accident. Consequently, the research on reliable and cost-effective systems for the automatic detection of falls has gained attention during the last decade.

\section{State of the Art on Wearable Fall Detection Systems and Multisensory Architectures}

Fall Detection Systems (FDS) are intended to permanently monitor the mobility of a target user or patient in order to automatically alert his/her relatives or the medical staff whenever a fall occurrence is identified. The goal of a FDS is to maximize the possibility of detecting actual falls while minimizing the number of false alarms, i.e., the conventional movements or Activities of Daily Living (ADLs) that are mistaken as falls.

FDSs can be generically classified into two large groups. On the one hand, in context-aware systems, the detection decision is based on the signals captured by a network of environmental sensors (microphones, cameras, vibration sensors, etc.) located in a specific area around the user to be monitored. These FDSs have clear drawbacks provoked by their high installation and maintenance costs, their limitation in terms of the area where the user can be monitored or their high probability of spurious interference caused by external factors such as changes in the light, noises, presence of another individual, displacement of furniture, domestic pets or other falling objects, etc. In addition, under a context-aware tracking solution, the user can feel their privacy invaded because of the continuous use of audiovisual equipment.

In contrast, wearable systems use sensors that are integrated into the patient's clothing. Hence, these sensors only track magnitudes unambiguously linked to the patient mobility, such as the acceleration or angular velocity of the body. Wearable systems offer some clear advantages over the context-aware solutions since the restrictions about the monitoring area are eliminated, as long as the sensors always accompany the patient. Besides, they are also less intrusive, more economical and less vulnerable to the effects of external factors. As disadvantages, wearable FDSs can adversely affect the patients' comfort during their daily routines (especially if they are too bulky). They can also be useless if the user forgets to wear it or to recharge the corresponding battery or if the FDS is unintentionally misplaced or not fit properly on the patient's body. In addition, in certain environments such as toilets or bathrooms or during certain activities (such as showers) the monitoring activity may be inviable.

Due to the fact that most present smartphones integrate the inertial sensors that are required by a wearable FDS (mainly an accelerometer but also a gyroscope and a magnetometer), smartphone technology has been proposed as a basis to deploy cost-effective FDSs [5,6]. The use of smartphones can benefit from the plummeting costs, increasing hardware capabilities and the widespread popularity of these personal devices. Although the access to a mobile phone is lower for older age groups, smartphone usage among people over 60 years old in the United States was about $81 \%$ in 2005 , while $60 \%$ of the elderly above 60 declare to own a smartphone [7]. Moreover, these percentages tend to increase. As predicted in [8], almost the entire population over 60 years old in Western countries is expected to use cell phones in the next 10 years.

As a result of the easiness of developing a FDS on a smartphone, most works of the recent related literature on wearable FDS have focused on the study of 'smartphone-only' based detectors, i.e., architectures that employ the smartphone as the unique element in the system, simultaneously acting as a sensing unit, data processor node and communication gateway [9]. As smartphones are natively provided with cellular $(3 \mathrm{G} / 4 \mathrm{G})$ communications, the patient can be tracked almost ubiquitously (at least in urban scenarios) and fall detection alarms can be seamlessly integrated with emergency response systems by means of a SMS, an automatic phone call to a predefined set of phone numbers, a notification through a remote server on the Internet, etc. The use of smartphones as mobility monitoring units have been even proposed to characterize the gait speed as a predictor of post-operative morbidity and mortality in elderly cardiovascular disease [10].

However, it has been have shown [11] that the position of the sensor that monitors the movements of the subject is crucial for the effectiveness of the FDS. In this regard, recommended locations such 
as the chest or waist are not comfortable positions to place a smartphone (which is conventionally transported in a hand-bag or in a loose pocket, where the phone may exhibit a certain freedom of movements that can affect the representativeness of the mobility measurements provided by its sensors). Furthermore, the range of the sensors integrated in the smartphone were not originally conceived to quantify the intensity of the movements that a fall can produce. Thus, the use of smaller and specific sensors that can be easily incorporated into the patient's clothing seems advisable for the sake of ergonomics. Nowadays there is a great variety of low-cost sensing motes that can be used for this purpose. These devices embed inertial sensors accelerometers as well as wireless communication interfaces (Bluetooth, 802.15.4 ...) that can be utilized to send the monitored signals to devices with a greater processing capacity such as a smartphone.

Multisensory Body Area Networks (BANs) have been proposed in works such as [12-14] to recognize and differentiate diverse activities of daily life. These networks are composed of a set of sensing nodes located on different parts of the body of the subjects under test. Nonetheless, in these works the network was never employed to identify falls and in most of them the importance of the position of the sensors to identify the activities was not specifically addressed as a research issue.

The work by Hyunh et al. in [15] (whose results are commented by the same authors in [16]) investigated the optimal position of a fall detection sensor, concluding that the sensor should be attached to the center of the chest.

An architecture with multiple sensors for fall detection has been recently investigated by Turan in the interesting work presented in [17]. This author analyzed the performance of a FDS built on a BAN consisting of six wireless motes placed at six positions (head, chest, waist, wrist, thigh and ankle). After recording a dataset of falls and movements of daily life executed by a group of experimental subjects, the effectiveness of different detection algorithms to discriminate falls is assessed taking into account the positions of the motes that are considered to produce the detection decision. In the deployed testbed, the network did not employ any smartphone (the signals are sent via ZigBee to a computer) while the ADLs emulated by the volunteers did not include activities (such as hopping or climbing stairs) that can be easily mistaken as falls. The statistical significance of the results was not evaluated either. In fact, except for a very reduced set of works (such as [18], in which a $p$-value analysis is carried out to examine if the election of the samples influences the efficacy of the detection method), the literature on FDSs disregards the analysis of the statistical significance of the metrics that are obtained to compare the performance of the detection algorithms.

The present study will extend a previous work [11] to systematically evaluate the application of different learning machine strategies when they are applied to the traces captured by a hybrid multisensory FDS architecture (consisting of a set of sensing motes and a smartphone). The study tries to optimize the election of the acceleration statistics that characterize the body mobility as well as to identify the sensor placement combinations that produce the best detection performance. In all the cases, the validity of the conclusions will be contrasted through an analysis of variance (ANOVA) of the results.

\section{Description of the Experimental Testbed}

In order to characterize the movements of a set of experimental subjects, we developed a monitoring system, sketched in Figure 1, based on a smartphone which is wirelessly connected to a set of four sensing nodes or 'motes', which are located on different parts of the body of the subject (chest, waist, wrist and ankle). The motes measure the acceleration (expressed in $g$-or g-force-), angular velocity (in ${ }^{\circ} / \mathrm{s}$ ) and flux density of the magnetic field (in $\mu \mathrm{T}$ ).

The sensing nodes were deployed on CC2650 SimpleLink ${ }^{\mathrm{TM}}$ Bluetooth low energy/Multi-standard SensorTag modules [19], manufactured by Texas Instruments. Every SensorTag incorporates a CC2650 ARM microcontroller and a set of MEMS sensors, including an InveSense MPU-9250 Inertial Measurement Unit (IMU) which integrates three triaxial sensors: an accelerometer, a gyroscope and a magnetometer. The SensorTag modules, which are powered through a CR2032-type battery, 
support different wireless communications, which avoids wiring and provides the user with a complete freedom of movement. In particular, the sensing motes embed a multi-standard $2.4 \mathrm{GHz}$ ultra-low power wireless MCU compatible with ZigBee, 6LowPAN or Bluetooth Low Energy (BLE) communications. In our prototype, benefiting from the fact that most current smartphones support BLE, a smartphone was selected as the central node of a BLE piconet. The piconet follows a typical star topology. Thus, the smartphone, which plays the role of the piconet master, receives all the packets containing the measurements captured by four SensorTags, which act as slaves in the BLE piconet.

The best election of the sensor sampling rate is a still open issue in the area of fall detection system (see the works by Medrano in [20] and Fudickar in [21] for a further analysis of the importance of the sampling rate in the efficacy of a FDS). As a matter of fact, sampling frequencies ranging from $5 \mathrm{~Hz}$ to $256 \mathrm{~Hz}$ have been used by the related literature to generate benchmarking datasets to compare fall detection algorithms. Aiming at avoiding saturation problems in the Bluetooth network, the sampling rate of the sensors was set to $20 \mathrm{~Hz}$. For that purpose, the original firmware of the SensorTags was modified, so that the 9 values periodically collected by the three IMU triaxial sensors could be transmitted every $50 \mathrm{~ms}$ through the BLE connection. In addition, as the smartphone in turn also integrates an IMU, we used the mobile phone, which was located in a trouser pocket for all the experiments, as a fifth sensor to describe the mobility registered on the subject's thigh. The employed sampling frequency of the smartphone measurement was $200 \mathrm{~Hz}$.

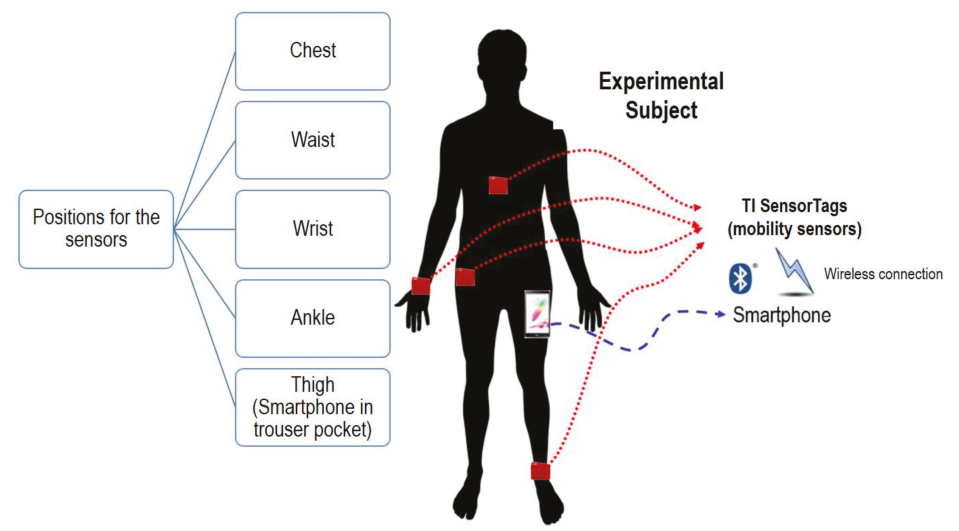

Figure 1. Basic architecture of the testbed.

Besides, a specific Android application (app) was programmed to receive and save the information captured by all the sensors, so that a repository with the generated mobility traces could be progressively generated. The app is in charge of storing the measurements transmitted from the four SensorTags as well as those captured by the smartphone in a CSV (Comma Separated Value)-format log file. Every sample received from any of the mobility sensors and any source (the four SensorTags and the smartphone) is recorded in an independent line of the log file, together with a timestamp generated by the app. No further synchronization between the sensing nodes is needed as long as a default value of $30 \mathrm{~ms}$ is selected by Android OS for the polling BLE Connection Interval (the time between two consecutive transmissions from the same slave to the Bluetooth master). So, the communication delay that may be introduced by BLE technology can be considered negligible when compared to the observation window that is required to detect a fall event.

For each experiment the program creates a new log file that stores the signals obtained from the subject during the execution of a certain movement (ADL or fall) for a fixed period of time (15 s). The log file also includes the personal characteristics of the experimental subject (weight, age, gender, height) as well as the typology of the experiment performed movement. In the log files, whenever 
a sample is received from a certain sensor, the app adds a timestamp and the MAC address of the mote (so that the sensor that has sent the information can be identified). Finally, in every log file, the app includes the employed sampling rate and additional information of the sensing device (manufacturer, range, etc.).

The final resulting dataset (called UMAFall) containing all the log files has been made publicly available in Internet [22] (together with some video clips that illustrate the activity of the subjects) as a benchmarking tool for the research on fall detection systems.

With regard to energy consumption, the batteries of the sensors allowed generating the dataset without being replaced. Similarly, the battery of the smartphone supported experimental one-day sessions of continuous use without having to be recharged.

Battery-life is obviously a key issue for the feasibility of any wearable monitoring system. However, the detailed study of the consumption of the implemented architecture is out of the analysis of this work (which focuses on evaluating the performance of the detection algorithms). Refer to [23-25] for a further study of the challenges caused by mobile sensing to battery-life in wearables and smart personal devices.

As it respects to the employed methodology to validate the detection strategies, due to the obvious difficulties of utilizing measurements from real-world falls, we followed the procedure that is commonly observed by the related literature. Thus, a group of 19 experimental subjects, whose basic characteristics are presented in Table 1, systematically performed a series of predefined movements, including 11 types of Activities of Daily Life (ADL) and 3 types of emulated falls, which were executed on a mattress to avoid injuries. Table 2 in Section 5 includes a description of each type of movement as well as the number of samples collected from the subjects. For the selection of the typology of ADLs and falls in the testbed we considered the following criteria:

- To include the falls and ADLs that are typically considered in similar datasets in the literature (see [26] for a detailed analysis of these existing datasets). This typical ADLs encompass from basic operation (walking, sitting down) to daily activities which may cause very specific patterns of the acceleration measurements (applauding, going upstairs or downstairs).

- To incorporate some 'sporting activities' (hopping, running), normally neglected by all these datasets, which may cause strong acceleration peaks that must be discriminated from those originated during a fall event.

Table 1. Personal characteristics of the participants in the testbed.

\begin{tabular}{ccccc}
\hline Subject ID & Gender & Age & Height (cm) & Weight (kg) \\
\hline Subject 1 & Female & 67 & 156 & 76 \\
Subject 2 & Female & 22 & 167 & 63 \\
Subject 3 & Male & 68 & 168 & 97 \\
Subject 4 & Male & 27 & 173 & 90 \\
Subject 5 & Male & 24 & 179 & 68 \\
Subject 6 & Male & 24 & 175 & 79 \\
Subject 7 & Male & 28 & 195 & 81 \\
Subject 8 & Female & 22 & 167 & 57 \\
Subject 9 & Male & 55 & 170 & 83 \\
Subject 10 & Male & 19 & 178 & 68 \\
Subject 11 & Male & 26 & 176 & 73 \\
Subject 12 & Female & 51 & 155 & 55 \\
Subject 13 & Female & 18 & 159 & 50 \\
Subject 14 & Female & 22 & 164 & 52 \\
Subject 15 & Male & 26 & 179 & 67 \\
Subject 16 & Male & 21 & 173 & 77 \\
Subject 17 & Female & 27 & 166 & 66 \\
Subject 18 & Male & 24 & 177 & 66 \\
Subject 19 & Female & 23 & 163 & 93 \\
Mean value & & 170.53 & 71.63 \\
Standard deviation & & 91.26 & 170 & 13.52 \\
Median value & & 24 &
\end{tabular}


Table 2. Typology and number of the movements executed by the experimental subjects during the testbed. The table also indicates the number of samples utilized for both training and testing phases during the validation of the machine learning algorithms.

\begin{tabular}{|c|c|c|c|c|c|c|c|c|}
\hline \multirow{2}{*}{$\begin{array}{c}\text { Movement Type } \\
\text { Applauding }\end{array}$} & \multirow{2}{*}{$\begin{array}{c}\begin{array}{c}\text { No. of } \\
\text { Executions }\end{array} \\
42\end{array}$} & \multirow{2}{*}{$\begin{array}{c}\begin{array}{c}\text { No. of Training } \\
\text { Samples }\end{array} \\
14\end{array}$} & \multicolumn{6}{|c|}{ No. of Test Samples (for the 6 Sub-Sets) } \\
\hline & & & 4 & 4 & 5 & 5 & 5 & 5 \\
\hline Raising both arms & 43 & 14 & 5 & 5 & 4 & 5 & 5 & 5 \\
\hline Emulating a phone call & 46 & 14 & 5 & 5 & 5 & 5 & 6 & 6 \\
\hline Opening a door & 43 & 14 & 5 & 5 & 5 & 5 & 4 & 5 \\
\hline $\begin{array}{l}\text { Sitting on a chair and } \\
\text { getting up }\end{array}$ & 64 & 14 & 9 & 9 & 8 & 8 & 8 & 8 \\
\hline Walking & 63 & 14 & 8 & 9 & 8 & 8 & 8 & 8 \\
\hline Bending & 59 & 14 & 7 & 7 & 8 & 8 & 8 & 7 \\
\hline Hopping & 53 & 14 & 6 & 6 & 6 & 7 & 7 & 7 \\
\hline $\begin{array}{c}\text { Lying down on/standing } \\
\text { up from a bed }\end{array}$ & 57 & 14 & 7 & 7 & 7 & 7 & 7 & 8 \\
\hline $\begin{array}{l}\text { Going upstairs and } \\
\text { downstairs }\end{array}$ & 40 & 7 & 6 & 5 & 5 & 5 & 6 & 6 \\
\hline Jogging & 28 & 9 & 3 & 3 & 3 & 3 & 4 & 3 \\
\hline Forwards fall & 71 & 13 & 10 & 10 & 10 & 10 & 9 & 9 \\
\hline Backwards fall & 73 & 14 & 9 & 10 & 10 & 10 & 10 & 10 \\
\hline Lateral fall & 64 & 14 & 8 & 8 & 9 & 9 & 8 & 8 \\
\hline Total & 746 & 183 & 92 & 93 & 93 & 95 & 95 & 95 \\
\hline
\end{tabular}

The divergence in the mobility patterns of actual falls suffered by older people and falls mimicked by young and healthy adults on a cushioning surface is still under discussion (see, for example, the studies in [27-29]) but that issue is beyond the scope of this paper. In any case, as it can be noticed from Table 1, two experimental subjects older than 65 participated in our testbed although they did not perform falling movements for safety reasons.

In all the trials, the starting position of the subject performing the movement (ADL or fall) was standing with hands on hips. All the experiments took place in a home environment. As it can be contemplated in Figure 2, the motes were tightly attached to the subject's body by means of elastic bands. For all the tests, the accelerometer axes of the SensorTag modules and the Smartphone were oriented and aligned as depicted in Figure 3. Thus, the components of the measurements of the tri-axial sensors are always associated to the same actual direction of the body position.

The log files obtained (one for each experiment) were formatted and stored in the internal memory of the smartphone. After the experiments were finished, the data were extracted and moved to a PC where they are processed in an offline way with Matlab scripts that implement the different machine learning algorithms to be tested.

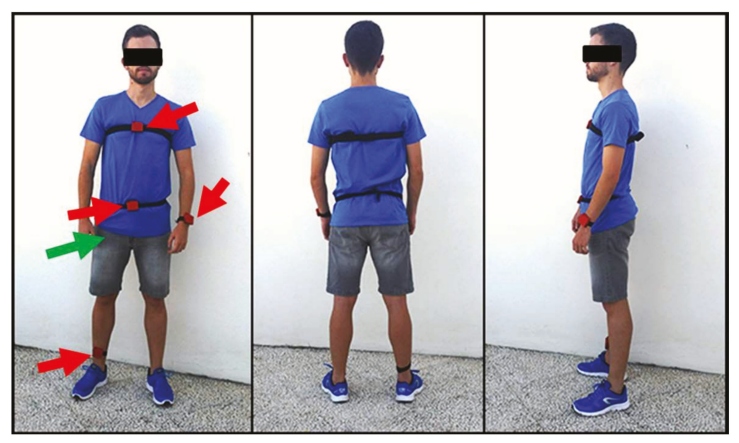

Figure 2. Example of the distribution of the sensors worn by an experimental subject. The green arrow indicates the position of the smartphone. Red arrows correspond to the SensorTag modules, which are attached to the user's body by means of elastic bands. 


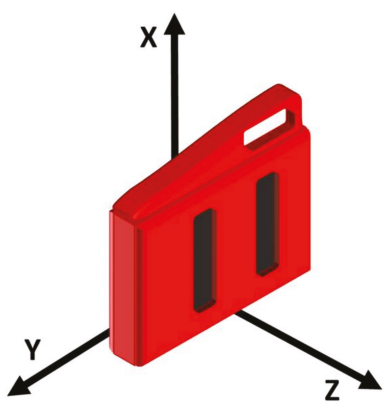

(a)

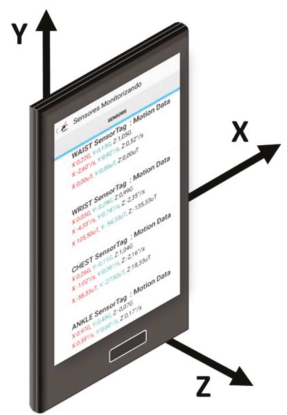

(b)

Figure 3. Representation of the spatial reference system of the employed sensing devices (devices are firmly attached to the subjects' body to guarantee that the reference system does not change during the experiments). (a) SensorTag (b) Smartphone.

\section{Machine Learning Algorithms and Selection of the Input Features}

The data collected through the developed system are processed and subsequently utilized to study the behavior of four supervised learning classification algorithms. Such algorithms (whose operational procedure is sketched in Figure 4) need to be trained before being applied to the test data. This training is achieved by providing the algorithm with a series of features (statistics that characterize the movements and which are computed from the mobility traces) that are extracted from a set of training samples as well as with the decisions that the detector should make (ADL or fall) for every sample. From the training phase, the algorithm builds a mathematical model that is later employed with the test data to decide the data 'class', that is to say, to discriminate falls from ADLs [30].

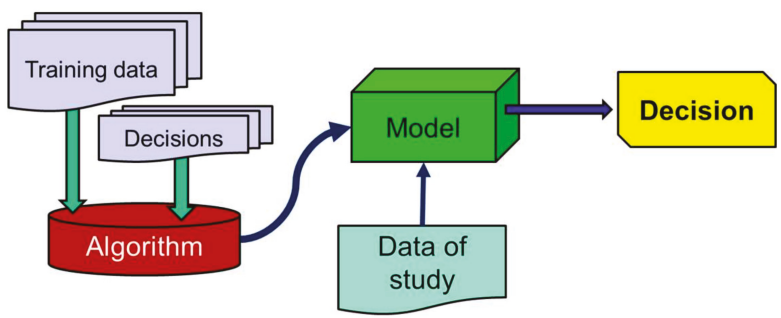

Figure 4. Typical Flow chart of a supervised learning classification algorithm.

\subsection{Feature Extraction: Selection of the Input Statistics of the Machine Learning Algorithms}

In the development of any machine learning algorithm, a proper selection of the input characteristics is a key aspect.

As in most works in the literature, the statistical characterization of the movements will be based on the measurements $\left(A_{X_{i}}, A_{Y_{i}}, A_{Z_{i}}\right)$ of the acceleration captured by the tri-axial accelerometers embedded in the five sensing points: chest, wrist, waist, ankle and thigh (pocket). Future studies should contemplate the information provided by the gyroscope and the magnetometer.

The statistics to be studied as the input of the supervised algorithms are derived from the samples of $15 \mathrm{~s}$ generated through the experimental testbed. Falls are typically associated to a brusque decay (which tends to $0 \mathrm{~g}$ ) of the acceleration components (during an initial free-fall period) followed by one or several acceleration peaks caused by the impact (or impact) against the floor [31]. Thus, we focus our analysis of the signal on the period of the signal where the difference between the 'peaks' and the 
valleys' in the acceleration is maximized. In particular, to analyze the acceleration measurements in every axis $(x, y$ or $z)$, we utilize a sliding window of duration $t_{W}=0.5 \mathrm{~s}$ or $N_{W}$ samples, where:

$$
N_{W}=t_{W} \cdot f_{S}
$$

being $f_{s}$ the sampling rate of the sensors $(20 \mathrm{~Hz}$ for the SensorTags and $200 \mathrm{~Hz}$ for the smartphone). This value of $0.5 \mathrm{~s}$ is coherent with other related studies on context aware and wearable systems for fall detection, which also employ a sliding window to detect the falls: $0.4 \mathrm{~s}$ [32], $0.6 \mathrm{~s}$ [33], $0.75 \mathrm{~s}$ [34], $1 \mathrm{~s}$ in $[35,36]$ or $2 \mathrm{~s}$ in $[17,37,38]$. In [39] authors claim that an observation interval of 0.5 is the best compromise between effectiveness, complexity and low power consumption to track the acceleration measurements in a fall detection system.

In order to locate the interval where the acceleration components suffer the highest variation, we compute for each window the module of the maximum variation of the acceleration components in the three axes. For the $j$-th window, this parameter $\left(A_{w_{\text {diff }}}(j)\right)$ is calculated as:

$$
A_{w_{\text {diff }}}(j)=\sqrt{\left(A_{X_{\max _{j}}}-A_{X \min _{j}}\right)^{2}+\left(A_{Y_{\max _{j}}}-A_{Y_{\min _{j}}}\right)^{2}+\left(A_{Z_{\max _{j}}}-A_{Z \min _{j}}\right)^{2}}
$$

where $A_{X_{\text {max }_{j}}}, A_{Y_{\max _{j}}}$ and $A_{Z_{\max _{j}}}$ indicate the maximum values of the acceleration components in the $x$, $y$ and $z$ - axes during the $j$-th sliding window (e.g., $A_{X_{\max _{j}}}=\max \left(A_{X_{i}}\right) \forall i \in\left[j, j+N_{W}-1\right]$ ).

Thus, the analysis interval will be defined as the subset of consecutive samples $\left[k_{o}, k_{o}+N_{W}-1\right]$ where the maximum value of $A_{w_{\text {diff }}}(j)$ is found to be:

$$
\left.A_{w_{\text {diff }(\max )}}=A_{w_{\text {diff }}}\left(k_{o}\right)=\max \left(A_{w_{\text {diff }}}(j)\right) \forall j \in[1, N-j]\right)
$$

where $k_{o}$ is the first sample of the analysis interval while $N$ indicates the total number of samples of the trace (for each axis).

The rest of the input features for the detection algorithms are computed just taking into account the values of the acceleration components during this analysis interval. We consider as candidate features to feed the machine learning algorithms the following statistics:

- $\quad$ Mean Signal Magnitude Vector $\left(\mu_{S M V}\right)$, which describes the mean motion or agitation level of the body throughout the movement. This variable is computable as the mean module of the acceleration vector during the analysis interval:

$$
\mu_{S M V}=\frac{1}{N_{W}} \cdot \sum_{i=k_{o}}^{k_{o}+N_{W}-1} S M V_{i}
$$

where $S M V_{i}$ represents the Signal Magnitude Vector for the $i$-th measurement of the accelerometer:

$$
S M V_{i}=\sqrt{A_{X_{i}}^{2}+A_{Y_{i}^{2}}^{2}+A_{Z_{i}^{2}}^{2}}
$$

- The standard deviation of the Signal Magnitude Vector $\left(\sigma_{S M V}\right)$, which informs about the variability of the acceleration

$$
\sigma_{S M V}=\sqrt{\frac{1}{N_{W}} \cdot \sum_{i=k_{o}}^{k_{0}+N_{W}-1}\left(S M V_{i}-\mu_{S M V}\right)^{2}}
$$

This parameter may be clearly affected by the presence of 'valleys' and 'peaks' in the evolution of the acceleration. 
- $\quad$ The sudden fluctuation of the mobility during a fall can be also described by the mean absolute difference $\left(\mu_{S M V_{\text {diff }}}\right)$ between consecutive samples of the acceleration module [40]:

$$
\mu_{S M V_{\text {diff }}}=\frac{1}{N_{W}} \cdot \sum_{i=k_{o}}^{k_{o}+N_{W}-1}\left|S M V_{i+1}-S M V_{i}\right|
$$

- As a fall occurrence usually implies a change in the orientation of the body, we also consider the mean rotation angle $\left(\mu_{\theta}\right)$, computable as [40]:

$$
\mu_{\theta}=\frac{1}{N_{W}} \cdot \sum_{i=k_{0}}^{k_{o}+N_{W}-1}\left(\cos ^{-1}\left[\frac{A_{X i} \cdot A_{X i+1}+A_{Y i} \cdot A_{Y i+1}+A_{Z i} \cdot A_{Z i+1}}{S M V_{i} \cdot S M V_{i+1}}\right]\right)
$$

- While the subject remains in an upright position, the effect of the gravity strongly determines the value of the acceleration component in the direction which is perpendicular to the floor plane. As a consequence, the inclination of the body caused by the falls normally provokes a remarkable modification of the acceleration components that defines the plane parallel to the floor when the subject is standing. Thus, to characterize this phenomenon, we utilize as a new feature the mean module $\left(\mu_{A p}\right)$ of these acceleration components $\left(A_{Y}\right.$ and $A_{Z}$ in the case of the SensorTag motes, and $A_{X}$ and $A_{Z}$ for the smartphone, as it can be appreciated from the resting upright position depicted in Figure 2).

$$
\begin{aligned}
& \mu_{A p}=\frac{1}{N_{W}} \cdot \sum_{i=k_{o}}^{k_{o}+N_{W}-1} \sqrt{A_{Y_{i}}^{2}+A_{Z_{i}}^{2}} \text { for the SensorTags } \\
& \mu_{A p}=\frac{1}{N_{W}} \cdot \sum_{i=k_{o}}^{k_{o}+N_{W}-1} \sqrt{A_{X_{i}}{ }^{2}+A_{Z_{i}}^{2}} \text { for the smartphone }
\end{aligned}
$$

\subsection{Employed Supervised Learning Classification Algorithm}

As the detection techniques, we compare four supervised learning classification algorithms that are commonly employed by the related literature (refer to [5,8,41-45] for a state-of-the-art on the detection techniques used in FDS): Support Vector Machine, $k$-Nearest Neighbors, Naives Bayes and Decision Tree, which are briefly presented in the following sub-sections (see $[30,46]$ to gain a much deeper insight into this set of techniques).

\subsubsection{Support Vector Machine (SVM)}

Support Vector Machine (SVM) is perhaps the most popular learning algorithm employed in the fall detection system literature [31,47-51]. According to the SVM algorithm, the input space defined trough the features of the different training samples is converted into a multi-dimensional space by means of a non-linear mapping.

Thus, as reflected in Figure 5, from the training dataset the algorithm is capable of building a 'maximum margin hyperplane' that acts as a decision boundary to categorize and discriminate the samples. The hyperplane, which separates two multi-dimensional regions, is defined so that the distances to the nearest points (from both sides) are maximized [30]. Once that the system is trained, the classification of the samples is directly based on the region where the sample is included. 


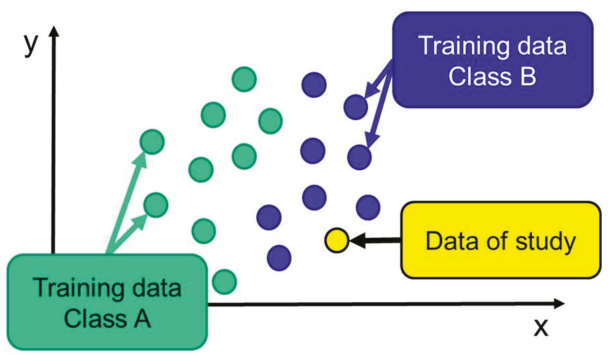

(a)

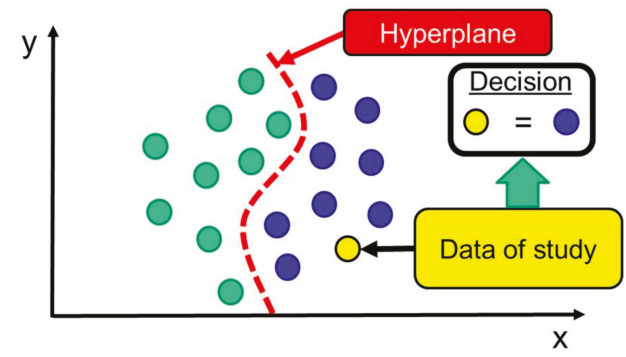

(b)

Figure 5. Example of the performance of the SVM algorithm for a two-dimensional space (samples characterized by two input features): (a) distribution of the training data on the two-dimensional space (b) creation of the hyperplane and classification decision for a certain test data.

\subsection{2. $k$-Nearest Neighbors $(k-\mathrm{NN})$}

This instance-based classifier has been utilized as a decision algorithm in works such as [17,50-54]. The typical operation of $k$-NN is represented in Figure 6, utilizes the training dataset in a very simple way: whenever a new activity has to be classified, $k$-NN searches for the $k$ already classified samples that are closest to this new uncategorized data. After this, the classification decision is based on the most popular class that is found among these neighbors. Although other techniques (such as Manhattan and city-block distances) have been suggested [42], we compute the Euclidean distance between the numerical features to estimate the distance between the training samples and the sample to be classified.

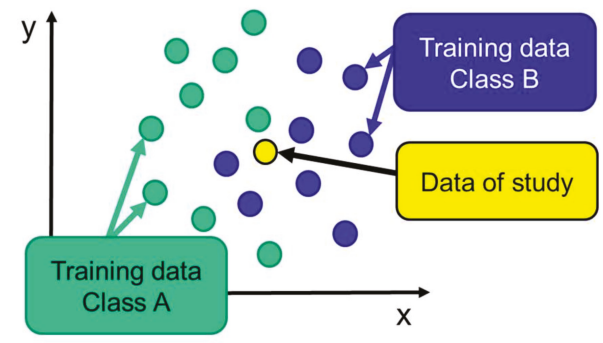

(a)

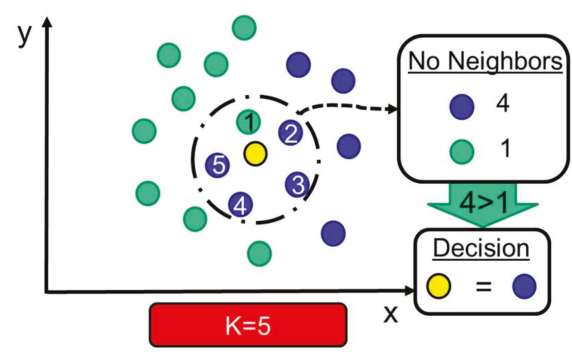

(b)

Figure 6. Operation of $k$-NN classifier: (a) the data under study is located among the training patterns aiming at finding the $k$ nearest neighbor (in the example $k=5$ ); (b) after detecting the $k$ nearest samples, the new sample is classified considering the 'majority vote' (most common class) of its neighbors.

\subsubsection{Naive Bayes}

This probabilistic classifier (used for FDS in papers such as $[50,55]$ ) calculates the probabilities of a particular sample to belong to the existing classes as a function of its associated multi-variate features. By applying the Bayes Theorem, these probabilities are estimated from the prior probability of the classes and the features, as well as the so-called likelihood (conditional probability of the features given a class). For this purpose, the statistical characterization of these variables must be previously computed from the training data, 'naively' assuming that the features are mutually independent. From these probabilities, the class of an unclassified pattern is decided taking into account the most likely hypothesis. 


\subsubsection{Decision Tree}

The use of Decision Trees has also been considered by the related literature [50,54,56-58]. The basic operation of the algorithm is exemplified in Figure 7, makes use of the training data to create a decision tree that will allow assigning a class to the unclassified mobility patterns. For this purpose, the algorithm selects a certain feature as a decision 'root' of the tree while different 'branches' are progressively created depending on the values of the other features. The tree is completed when all the features have been considered or when all the training samples in the same branch belong to the same class. The selection criteria of this flowchart-like structure are established to maximize the probability of assigning the correct class to the training data when the different classification rules of the branches are applied.

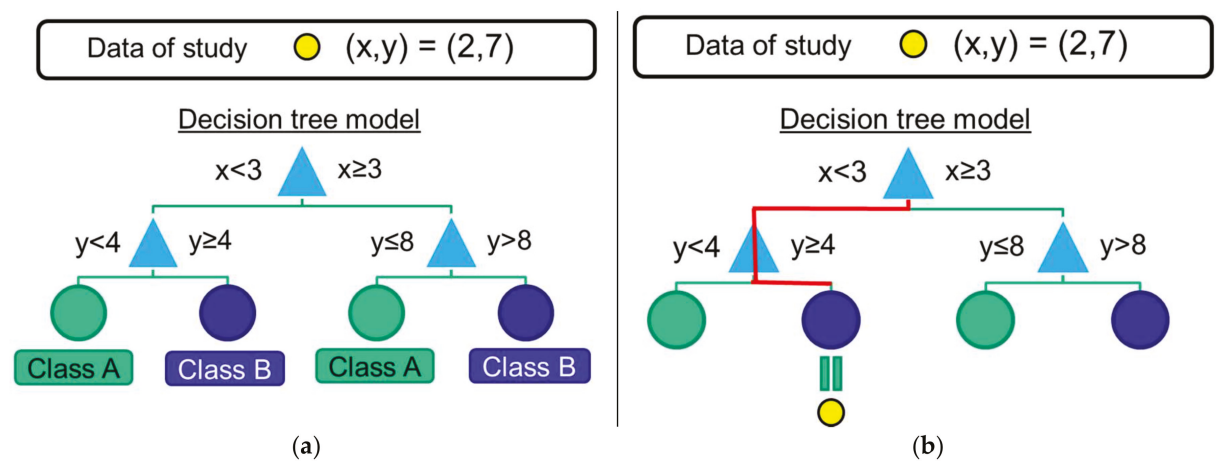

Figure 7. Example of an operation of a decision-tree algorithm: (a) after the training phase, the branches and the decision rules the tree are configured; (b) during the test phase, the features of an unclassified sample are utilized to apply the decision rules and determine the sample class.

\section{Result and Discussion}

In this section we analyze the performance of the four afore-described algorithms when they are employed to detect falls in the datasets obtained from the testbed. Apart from comparing the results of the four machine learning techniques, the goal is to explore two elements that impact on the precision of the fall detection process: (1) the selection of the input characteristics for the machine learning techniques, (2) the number and positions of the sensors that are more relevant for the fall identification.

As performance metrics, we utilize the recall or sensitivity $(S e)$ and the specificity $(S p)$, which are commonly employed to evaluate the effectiveness of binary classification systems. In contrast with other metrics used in pattern recognition (such as precision or accuracy), Se and Sp are not affected by the unbalance between the number of existing samples of both types (in this case, falls and ADLs) which are employed to test the detection algorithms.

The sensitivity describes the capacity of the classificatory to correctly identify an event of the 'positive' class (here: falls) when this event actually occurs. So, it can be computed as the true positive rate:

$$
S e=\frac{T P}{T P+F N}
$$

where TP indicates the number of 'True Positives', i.e., the number of falls correctly labeled by the classifier, while FN is the number of 'False Negatives' or the number of falls that are misidentified as ADLs. The sum TP + TN corresponds to the total number of falls in the testing dataset. 
Similarly, the specificity informs about the efficacy of the detection system to avoid false alarms (or 'False Positives') by properly classifying the actual ADLs. This true negative rate is defined as it follows:

$$
S p=\frac{T N}{T N+F P}
$$

where TN and FP represent the number of 'True Negatives' (ADLs which are well identified) and 'False Positives' (ADLs mistaken as falls), respectively.

In all the detection schemes, a higher specificity is attained at the cost of decreasing the sensitivity of the system. Therefore, as a trade-off between specificity and sensitivity must be achieved in a FDS, we consider the geometric mean of these two parameters $(\sqrt{S p \cdot S e})$ as a global metric to characterize the goodness of the detection process.

Once that the performance metric is defined, for the study of these elements we utilize a $2^{k}$ factorial design, where $k$ designs the number of possible factors (selected input statistic or sensor position) that can affect the detection process.

Every comparison is repeated for the four considered machine-learning based detection techniques. Thus the effectiveness of the algorithms to detect the falls is also compared in a systematic way under a wide range of circumstances.

To investigate the statistical representativeness of the comparison, the differences in the different results that the alternative use of the factors entails are assessed by means of an ANOVA test. The ANOVA test allows to decide if the means of two or more different populations are equal or not. The test is aimed at determining if different treatments in an experiment cause significant differences in the final results, and consequently, it permits evaluating the importance of the different factors that may alter the operation of the fall detector.

In particular, the factorial ANOVA analyzes the series of residuals resulting from subtracting the values of the obtained observations and the mean of the observations. ANOVA assumes that residuals are independent and follow a normal distribution with a common finite variance (homoscedasticity) [59].

Table 2 details the number of experiments employed for every type of movement (ADLs and falls) as well as the distribution in groups employed for both training and testing with an ANOVA analysis.

To proceed with the supervised learning of the algorithms, the 746 samples obtained from the testbed were divided in a training and a test dataset of 183 and 563 samples, respectively. The number of samples selected to define the training datasets for each type of fall or ADL are described in Table 2. Within the same type, the samples for each phase (training and testing) were randomly chosen.

To apply the ANOVA test, we also divided at random the test dataset into six different 'blocks' or 'subsets' of approximately 93 samples. We decided to divide the samples into six subsets as long as this number would allow representing the Gaussian nature of the residuals with six points while keeping a population of almost 100 samples in each subset for an adequate estimation of the performance metric.

The number of samples of the same movement type is almost homogeneous for the six sub-sets (as also indicated in Table 2). The values of the specificity (Sp) and sensitivity (Se) were computed after separately applying the four detection algorithms to the six testing sub-sets for all the possible combinations of the impacting factors (input characteristics and sensor positions). Thus, by replicating the test with the 6 sub-sets, the variance of the series of the performance metric $(\sqrt{S p \cdot S e})$ measured for the subsets can be investigated.

\subsection{Analysis of the Impact of the Selection of the Acceleration-Based Features on the Fall Detection Performance}

We firstly investigate which characteristic (or set of characteristics) should be selected as input statistics of the machine learning algorithms to maximize the accuracy of the detection decision. For that purpose, we compare the algorithms by analyzing the performance metrics that are obtained when different sets of the input features (described in Section 4.1) are considered. For each set of 
characteristics, the global specificity and sensitivity are computed by averaging the metrics that are obtained when a specific combination of body sensors are employed. Thus, the specificity $\left(S p_{i}\right)$ and sensitivity $\left(S e_{i}\right)$ for the $i$-th combination of characteristics are calculated as:

$$
\begin{aligned}
& S p_{i}=\frac{1}{N_{c S}} \sum_{j=1}^{N_{c S}} S p_{i j} \\
& S e_{i}=\frac{1}{N_{c S}} \sum_{j=1}^{N_{c S}} S e_{i j}
\end{aligned}
$$

where $S p_{i j}$ and $S e_{i j}$ indicate the specificity and the sensitivity estimated for the $i$-th combination of input characteristics and the $j$-th combination of employed sensors, respectively. In the equations $N_{c s}$ represents the total number of the possible 31 combinations of the five body sensors, as the algorithms may consider the signals from just one single device to the five sensors $\left(N_{c s}=2^{5}-1=31\right)$.

To proceed with the ANOVA analysis of the results, we focus the comparison on the series of the geometric means of the specificity and the sensitivity $\left(\sqrt{S p_{i} \cdot S e_{i}}\right)$ which are computed for the different sets of test samples (defined in Table 2 ) and for every combination of input characteristic and considered sensors. The goal is to identify the combination of input features that lead to the best detection performance in a statistically significant way. In case that the best options do not differ significantly, the combination with a lower dimension (i.e., the lower number of features) should be preferred in order to reduce the computational complexity of the system (which is normally intended to be implemented on a wearable device, such as a smartwatch or a smartphone, which can exhibit memory and computation restrictions). This procedure is repeated for the four machine-learning algorithms, whose results are discussed in the following sub-sections.

\subsubsection{Results for Support Vector Machine (SVM) Algorithm}

We focus on the analysis of the performance of the SVM algorithm when it is alternatively fed with the different possible combinations $\left(2^{6}-1=63\right)$ of the six 'candidate' input features.

Firstly, to prove the validity of the ANOVA analysis of the results, we check the assumptions of normality and homogeneity of variance of the residuals (differences between the obtained metrics for every sample sub-test and the global mean value of the metric for each combination of input features).

Figure 8a shows that the Cumulative Distributed Function (CDF) of the residuals can be reasonably approximated by a normal distribution. Similarly, Figure $8 \mathrm{~b}$ depicts the scatterplot of residuals versus the predicted values (estimated means) of the series of performance metrics obtained for every combination of inputs. The figure illustrates that the variability of the series is only clearly different for those input combinations for which the algorithm presents a very poor behavior. In these cases, where the input features do not feed the algorithm with a convenient characterization of the movements, a very low value of the performance metric (with values of the specificity or the sensitivity close to zero) is achieved, and, consequently residuals also tend to zero. Conversely, this problem does not appear for the rest of combinations, for which the variability of the residuals is homogeneous. This lack of homoscedasticity is not critical for the ANOVA test, provided that the experiments are balanced (all series have the same size) and that the ratio of the maximum to the minimum variances of the series does not exceed a proportion of 4 to 1 [59]. 


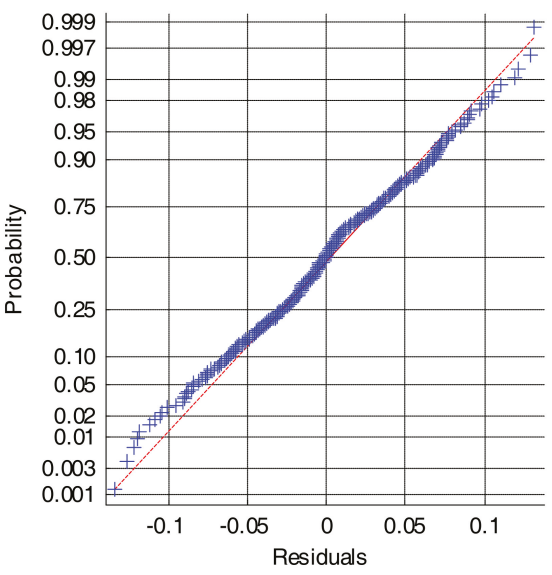

(a)

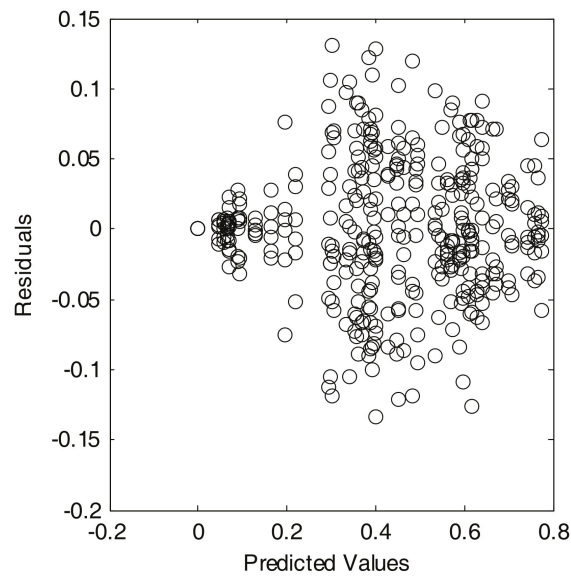

(b)

Figure 8. Results for the SVM algorithm. Analysis of the residuals: (a) Normal probability plot (crosses: empirical data, dashed line: theoretical normal fit); (b) Values of the residuals versus the predicted (or fitted) values.

In fact, different data transformations that are recommended by the literature $[59,60]$ when the criterion of a common variance is not completely met were also applied to the data series. As the conclusions drawn with these transformations were basically the same for all the cases, for the sake of clarity, we only show the results obtained with the untransformed series and in the original units.

Figure 9 shows the post hoc multiple comparison of the computed means based on the results generated by the one-way ANOVA analysis. The central marks in the bars of the graph represents the estimated mean while the lines expand to the corresponding comparison interval for a $95 \%$ confidence level.

In the figure the blue mark and line refers to the optimal combination, i.e., that which minimizes the number of required input features while achieving an estimated mean of the performance metric which is not significantly lower than those obtained with the rest of the combinations. The gray color of the marks and lines indicates those combinations that offer a similar performance to the optimal case but requiring a higher number of input features. The red color is in turn utilized to illustrate the results of employing those feature combinations that underperform (when compared to the optimal case) in a statistically significant way. From the figure, we can observe that the optimal results are generated by the combination that employs $\mu_{S M V}, A_{w_{\text {diff }(\max )}}, \sigma_{S M V}$, and $\mu_{A p}(\mathrm{~A}=\mathrm{B}=\mathrm{C}=\mathrm{F}=1$, $\mathrm{D}=\mathrm{E}=0$ ), without considering $\mu_{\theta}$ and $\mu_{S M V_{\text {diff }}}$. For the combination of these four inputs, the achieved value of the geometric mean of the specificity and the sensitivity lies in the interval [0.735-0.785], which is significantly different from the result attained with the use of 59 out of the other 62 possible combinations of input features. 


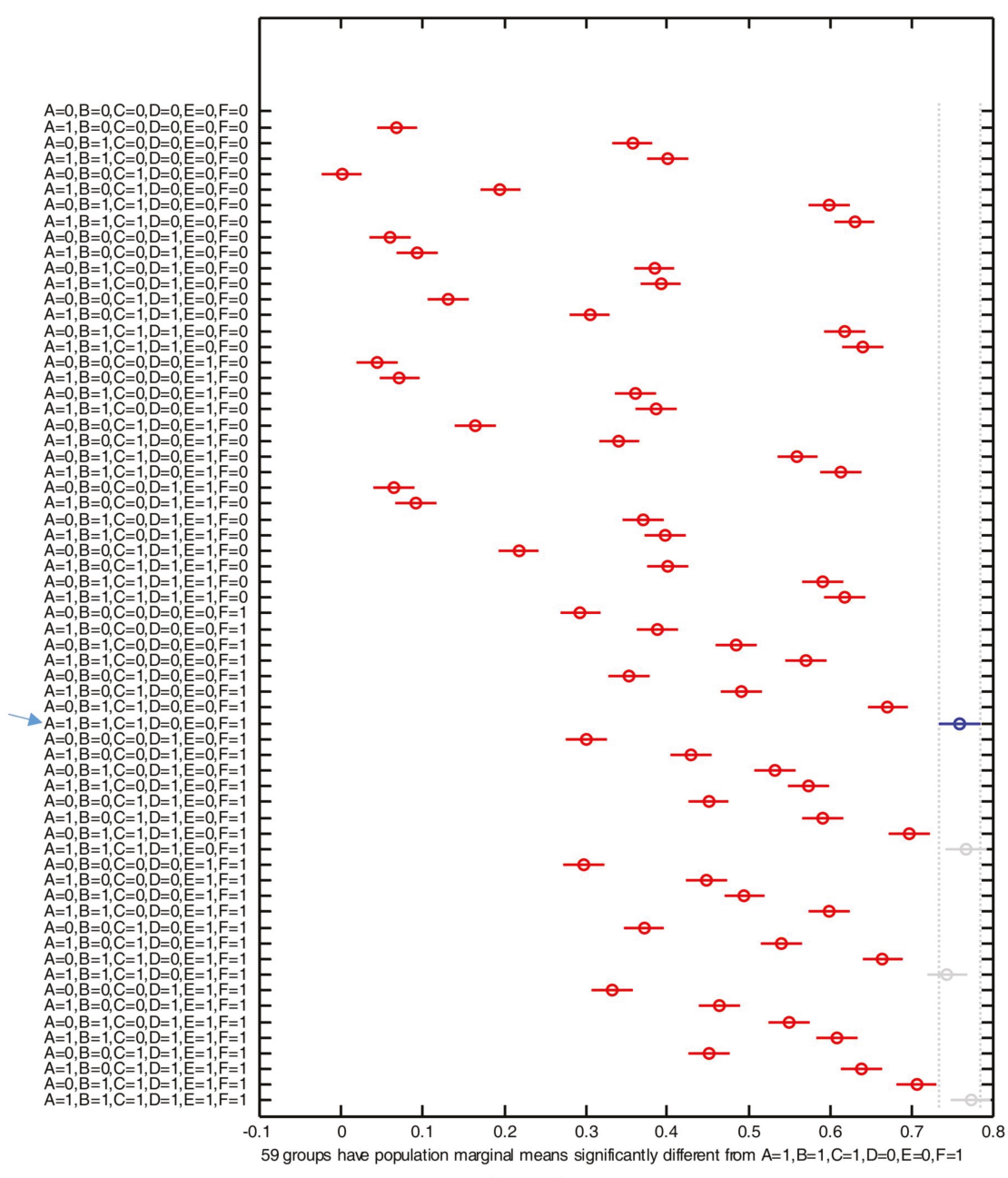

$\sqrt{S p \cdot S e}$

Figure 9. Comparison of the means of the performance metric $(\sqrt{S p \cdot S e}$ or geometric mean of the sensitivity and the specificity) obtained with the SVM algorithm for all the possible combinations of

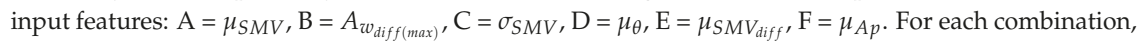
the $y$-axis of the figure indicates with ' 1 ' or ' 0 ' whether the corresponding feature is considered (' 1 ') or not $\left({ }^{\prime} 0\right.$ '). The combination with the best results is indicated with a blue arrow.

According to the ANOVA analysis, Table 3 shows the relative variation (expressed as a percentage) that every single input feature produces in the results, with respect to the global mean of the metric. The Table also includes the same values for the combinations of two inputs $\left(\mu_{S M V}\right.$ and $A_{w_{\text {diff }(\max )}}$, and

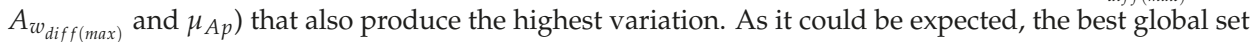
of inputs is that resulting from combining the four inputs that individually have a higher impact on the result, especially $A_{w_{\text {diff }(\max )}}\left(42.312 \%\right.$ of variation) $\mu_{A p}(25.807 \%)$ and $\sigma_{S M V}(14.971 \%)$. The interaction of the inputs $A_{w_{\text {diff }(\max )}}$ and $\mu_{A p}$ adds an increase of $3.032 \%$. To characterize the robustness of the results attained by the algorithm, Table 3 also includes the error reported by the ANOVA analysis, which is $5.218 \%$. This error, which should be minimized, may be explained by other factors that have 
not been considered as input features, such as the small differences in the composition of the test subsets (type and number of executed movements, election and particularities of the experimental subjects for each subset, etc.).

Table 3. Error reported by the ANOVA analysis and relative influence of the election of the input features on the global result when the Support Vector Machine (SVM) algorithm is applied. Inputs: $\mathrm{A}=\mu_{S M V}, \mathrm{~B}=A_{w_{\text {diff(max) }}}, \mathrm{C}=\sigma_{S M V}, \mathrm{D}=\mu_{A p \theta}, \mathrm{E}=\mu_{S M V_{\text {diff }}}, \mathrm{F}=\mu_{A p}$.

\begin{tabular}{cc}
\hline Feature & $\%$ \\
\hline A & 3.332 \\
B & 42.312 \\
C & 14.971 \\
D & 0.701 \\
E & 0.207 \\
F & 25.807 \\
A\&B & 0.785 \\
B\&F & 3.032 \\
Error & 5.218 \\
\hline
\end{tabular}

\subsubsection{Results for the $k$-Nearest Neighbors $(k-\mathrm{NN})$ Algorithm}

The previous analysis is repeated for the case in which the detection decision is based on the $k$-Nearest Neighbors $(k-N N)$ algorithm. For the application of the algorithm, we set the value of $k$ to 9 (nine neighbors). This value, which is selected after different tests, improves the behavior of the algorithm for the employed datasets, as it is close to the number of samples of the different movement types which are employed during the initial training phase of the algorithm.

The results (not depicted here for reasons of space) illustrate again that the CDF of the residuals can be reasonably approximated by a normal distribution while no relevant variation the variability of the residuals is detected for the whole range of predicted values. Consequently, the ANOVA analysis can be considered valid. The post hoc multiple comparison of the performance metric obtained for the different combination of input features indicate that the best performance for the $k$-NN algorithm is achieved when the chosen input characteristics are $\mu_{S M V}, \sigma_{S M V}, \mu_{\theta}$ and $\mu_{A p}$. The use of this particular set of characteristics outperforms the results of 59 combinations of the six possible input statistics in a statistically significant way. The particular contribution of the six statistics in the global performance of the detection process is described in Table 4 . As it can be observed, the best combination includes the two factors $\left(C=\sigma_{S M V}, \mathrm{~F}=\mu_{A p}\right)$ with the highest impact in the results $(21.329 \%$ and $19.393 \%$, respectively). The high value $(6.064 \%)$ of the percentage computed for $A_{w_{\text {diff }(\max )}}$, which is not included in the best combination of input characteristics, can be actually explained by the negative impact (reduction of the performance metric) that is the achieved if this factor is considered. The table with the conclusions of the ANOVA analysis also informs that the error due to other factors (not considered as inputs) amounts to $21.25 \%$.

Table 4. Error reported by the ANOVA analysis and relative influence of the election of the input features on the global result when the $k$-Nearest Neighbor $(k-N N)$ algorithm is applied. Inputs: $\mathrm{A}=\mu_{S M V}, \mathrm{~B}=A_{w_{\text {diff(max) }},}, \mathrm{C}=\sigma_{S M V}, \mathrm{D}=\mu_{\theta}, \mathrm{E}=\mu_{S M V_{\text {diff }}}, \mathrm{F}=\mu_{A p}$.

\begin{tabular}{cc}
\hline Feature & $\%$ \\
\hline A & 0.954 \\
B & 6.065 \\
C & 21.329 \\
D & 4.120 \\
E & 1.334 \\
F & 19.393 \\
Error & 21.250 \\
\hline
\end{tabular}




\subsubsection{Results for the Naïve Bayes Algorithm}

As in the previous cases, the visual inspection of the CDF and the variability of the residuals show that the conditions of the normal distribution of the population and the homogeneity of the variances are reasonably met. Again, we assume that the small divergences between the actual CDF and the Gaussian fit can be neglected if we take into account that the model is balanced as the number of samples in each test group is basically the same. Thus, we assume the ANOVA analysis can be reasonably applied as a valid tool. Besides, the post hoc comparison of the method when the different combinations of input features are considered indicate that, for the Naïve Bayes algorithm, the best

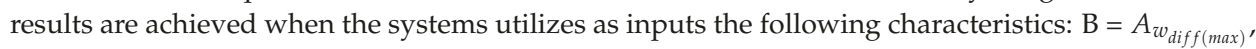
$\mathrm{C}=\sigma_{S M V}, \mathrm{D}=\mu_{\theta}$ and $\mathrm{F}=\mu_{A p}$. The performance of the algorithm for this combination of inputs (which yields a global metric in the interval [0.671-0.745]) significantly differs from those obtained with other 48 combinations. Table 5 in turn specifies the individual influence of each possible input characteristics as well as the four combinations of two inputs that presents the highest impact on the results. The table reveals the importance of selecting $\mu_{A p}$ and $A_{w_{\text {diff (max) }}}$ (and their corresponding combination) on the global performance. On the other hand, the error (due to the presence of other external components that affect the evaluation) computed by the ANOVA analysis is $13.484 \%$.

Table 5. Error reported by the ANOVA analysis and relative influence of the election of the input features on the global result when the Naïve Bayes algorithm is applied. Inputs: $\mathrm{A}=\mu_{S M V}$, $\mathrm{B}=A_{w_{\text {diff }(\max )}}, \mathrm{C}=\sigma_{S M V}, \mathrm{D}=\mu_{\theta}, \mathrm{E}=\mu_{S M V_{\text {diff }}}, \mathrm{F}=\mu_{A p}$.

\begin{tabular}{cc}
\hline Feature & $\%$ \\
\hline $\mathrm{A}$ & 0.977 \\
$\mathrm{~B}$ & 11.552 \\
$\mathrm{C}$ & 7.472 \\
$\mathrm{D}$ & 12.604 \\
$\mathrm{E}$ & 2.143 \\
$\mathrm{~F}$ & 32.782 \\
$\mathrm{~A} \& \mathrm{~F}$ & 1.668 \\
$\mathrm{~B} \& \mathrm{D}$ & 1.494 \\
$\mathrm{~B} \& \mathrm{~F}$ & 4.067 \\
$\mathrm{C} \& \mathrm{~F}$ & 1.361 \\
Error & 13.484 \\
\hline
\end{tabular}

\subsubsection{Results for the Decision Tree Algorithm}

The same analysis procedure of the Sections 5.1.1-5.1.3 is applied to the results achieved by the Decision Tree learning method. The normality and the homoscedasticity of the residuals are contrasted and again, the graphs (not depicted here) seem to hold the conditions of the ANOVA test.

From the comparison of the mean performance metrics achieved for the different combinations of input features, we conclude that the best performance of the Decision Tree algorithm takes place when $\mu_{S M V}, A_{w_{\text {diff(max) }}} \sigma_{S M V}$, and $\mu_{A p}$ are used as input variables to characterize the mobility of the individuals. For this combination, the performance metric (the geometric mean of the specificity and sensitivity) is estimated to be in the range [0.801-0.849], which is significantly higher than the intervals computed for most other combinations (53 out of 62).

Table 6 describes the contribution of the different statistics (and the most impacting combinations of two factors) to the global performance of the algorithm. The table highlights the importance of employing, as input parameters, the variance of the standard deviation of the acceleration module $\left(\sigma_{S M V}\right)$ and, in the second place, $\mu_{A p}$ to discriminate falls from ADLs.

The error due to non-considered factors is computed to be $12.110 \%$. 
Table 6. Error reported by the ANOVA analysis and relative influence of the election of the input features on the global result when the Decision Tree algorithm is applied. Inputs: $\mathrm{A}=\mu_{S M V}$, $\mathrm{B}=A_{w_{\text {diff(max })}}, \mathrm{C}=\sigma_{S M V}, \mathrm{D}=\mu_{\theta}, \mathrm{E}=\mu_{S M V_{\text {diff }}}, \mathrm{F}=\mu_{A p}$.

\begin{tabular}{cc}
\hline Feature & $\%$ \\
\hline $\mathrm{A}$ & 3.709 \\
$\mathrm{~B}$ & 6.775 \\
$\mathrm{C}$ & 41.181 \\
$\mathrm{D}$ & 1.450 \\
$\mathrm{E}$ & 1.158 \\
$\mathrm{~F}$ & 14.913 \\
$\mathrm{C \& F}$ & 2.178 \\
D\&E & 1.979 \\
Error & 12.110 \\
\hline
\end{tabular}

5.1.5. Impact of the Election of the Input Characteristics: Summary of the Results

From the study performed in the four previous subsections we can draw the following conclusions:

- The use of a higher number of input characteristics does not necessarily correlate with an enhancement in the behavior of the decision algorithms. For the four strategies under study, no statistically significant improvement (in the geometric mean of the specificity and the sensitivity) is achieved by using more than four inputs. In fact, the performance of some machine learning strategies even deteriorates when the number of considered inputs increases.

- $\quad$ The most frequent statistics in the combinations that yield the best performances of the algorithms are $\sigma_{S M V}$ (which describes the variability of the acceleration module) and $\mu_{A p}$ (which is linked to the changes in the perpendicularity of the body with respect to the floor plane). The use of $A_{w_{\text {diff }(\max )}}$ (which identifies the presence of sudden and brusque changes of the acceleration module) also increases the effectiveness of all the algorithms (except for $k$-NN). Conversely, $\mu_{S M V_{\text {diff }}}$ (which characterizes the mean variation of two consecutive samples of the acceleration module during a certain time window) is not included in any of the best combinations of statistics for any algorithm. In any case, the optimal election of the input characteristics clearly depends on the particularities of the employed algorithm. As it can be noted from Table 7, which summarizes the error and the relative impact of the six possible input characteristics on the algorithm's performance, the importance of the election of each parameter strongly varies from one algorithm to another. A universal set of parameters cannot be proposed to characterize the mobility with independence of the underlying AI technique selected to identify the fall patterns. Thus, results indicate that input characteristics must be carefully designed and individualized for each detection policy.

- $\quad$ SVM obtains the best results, notably outperforming the other algorithms (especially $k$-NN). The next subsection thoroughly investigates this comparison between the machine-learning strategies when other impacting factor (the selected position of the sensors) is considered.

Table 7. Error reported by the ANOVA analysis and relative influence of the election of the six considered input features on the global result for the four learning machine strategies. The symbol $(\checkmark)$ or (-) indicates if the corresponding feature is included (or not) in the combination that achieves the (statistically significant) best performance.

\begin{tabular}{|c|c|c|c|c|c|c|c|c|c|c|c|c|c|}
\hline \multirow[b]{2}{*}{ SVM } & \multicolumn{2}{|c|}{${ }_{-S M V}$} & \multicolumn{2}{|c|}{$A_{w_{d i f f(\max )}}$} & \multicolumn{2}{|c|}{$\propto_{S M V}$} & \multicolumn{2}{|c|}{-} & \multicolumn{2}{|c|}{$\bar{S}_{S M V_{\text {diff }}}$} & \multicolumn{2}{|c|}{${ }^{-} A p$} & \multirow{2}{*}{$\begin{array}{r}\text { Error } \\
5.218 \%\end{array}$} \\
\hline & $\checkmark$ & $3.332 \%$ & $\checkmark$ & $42.312 \%$ & $\checkmark$ & $14.971 \%$ & - & $0.701 \%$ & - & $0.207 \%$ & $\checkmark$ & $25.807 \%$ & \\
\hline$k-\mathrm{NN}$ & $\checkmark$ & $0.954 \%$ & - & $6.065 \%$ & $\checkmark$ & $21.329 \%$ & $\checkmark$ & $4.120 \%$ & - & $1.334 \%$ & $\checkmark$ & $19.393 \%$ & $21.250 \%$ \\
\hline Naive Bayes & - & $0.977 \%$ & $\checkmark$ & $11.552 \%$ & $\checkmark$ & $7.472 \%$ & $\checkmark$ & $12.604 \%$ & - & $2.143 \%$ & $\checkmark$ & $32.782 \%$ & $13.484 \%$ \\
\hline Decision Tree & $\checkmark$ & $3.709 \%$ & $\checkmark$ & $6.775 \%$ & $\checkmark$ & $41.181 \%$ & - & $1.450 \%$ & - & $1.158 \%$ & $\checkmark$ & $14.913 \%$ & $12.110 \%$ \\
\hline
\end{tabular}




\subsection{Study of the Importance of the Sensor Position for the Decision of Machine-Learning Fall Detection Algorithms}

Taking advantage from the multisensory Body Area Network developed for the testbed, this section investigates the importance of the election of the sensor positions for an adequate detection decision. In particular, we try to assess if the simultaneous use of several wearable sensors may introduce any improvement. This analysis, which is aimed at minimizing the number of sensors that should be transported in a real application scenario, will be carried out for each of the four considered supervised learning algorithms. The input statistics that will be taken into account for each algorithm in order to classify the samples will be those (summarized in Table 7) that were proved to induce a better discrimination between ADLs and falls.

In all the tests, we evaluate the performance of the algorithms (expressed again in terms of the geometric mean of the specificity and the sensitivity) for all the 31 possible combinations of the five sensors of the body area network (from the simplest case where only the signals captured by just one single sensor are considered, to the case where the measurements of the five sensors are utilized as the input for the machine learning technique).

In the comparison, the algorithms are always individually applied for each considered position. Thus, when more than one sensor is employed, an 'AND' policy is applied, that is to say, a fall is assumed to have occurred only if it is simultaneously detected by the algorithm in all the considered positions. From some tentative experiments, we checked that the detection based on an 'OR' operation (i.e., a fall is presumed if just one sensor detects it, with independence of the results at the other sensing points) is proved to dramatically increase the number of false positives.

The same procedure of the previous section is again utilized to assess the influence of the position and number of sensors. Firstly, it is confirmed that the series of performance metrics that have been obtained meet the assumptions required by the ANOVA analysis. Then, the results of the analysis itself are presented.

\subsubsection{Results for the SVM Algorithm}

Figure 10 again illustrates the two graphical tests that can be used to check if the data meet the assumptions of normality and equality of variance. At first glance it is verified that the series do not strictly comply with these conditions. This fact is perceptible in Figure 10b, where the assumption homoscedasticity is shown not to be met completely.

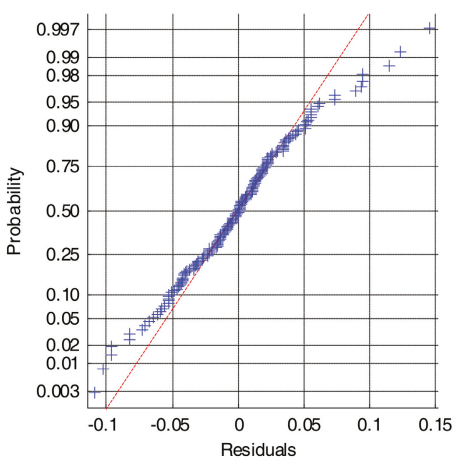

(a)

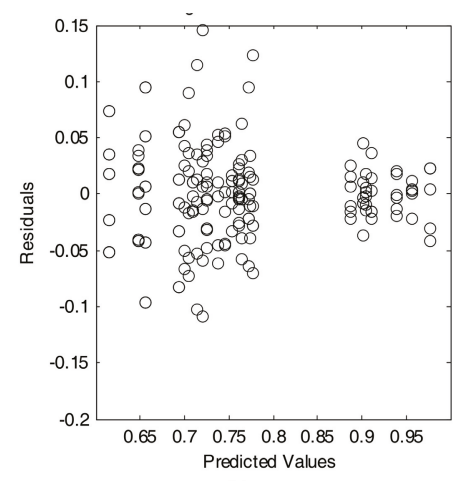

(b)

Figure 10. Results for the SVM algorithm. Analysis of the residuals: (a) Normal probability plot (crosses: empirical data, dashed line: theoretical normal fit); (b) Values of the residuals versus the predicted (or fitted) values. 
As discussed in the previous sections, there are some possible solutions to reduce this problem. In our case, as recommended in $[59,60]$, we have proved to transform the data $(y)$ by applying an exponential function of the type $y^{\alpha}$, where $\alpha$ is a constant. This transformation is intended to improve the normality and equality of variance of the numerical series. However, as this experiment is unbalanced, the variations obtained after this transformation do not meaningfully vary. Thus, so we will continue with the ANOVA analysis with the original data to simplify their understanding.

Figure 11 displays the post hoc multiple comparison of the computed means of the performance metric that resulted from the ANOVA analysis (for which an error of $14.162 \%$ was computed), when all the 31 possible combinations of the five BAN sensors are considered. As in the previous section, the figure indicates the estimated mean by depicting a circle in the corresponding bar, which in turn expands to a comparison interval for a $95 \%$ confidence level. The blue color is again employed to underline the combination which maximizes the performance, while the red lines correspond to those combinations whose behavior is significantly worse than the optimal case. Gray lines are utilized to identify those combinations whose results present a confidence interval which partially overlap the optimal case (and, consequently, cannot be considered significantly different).

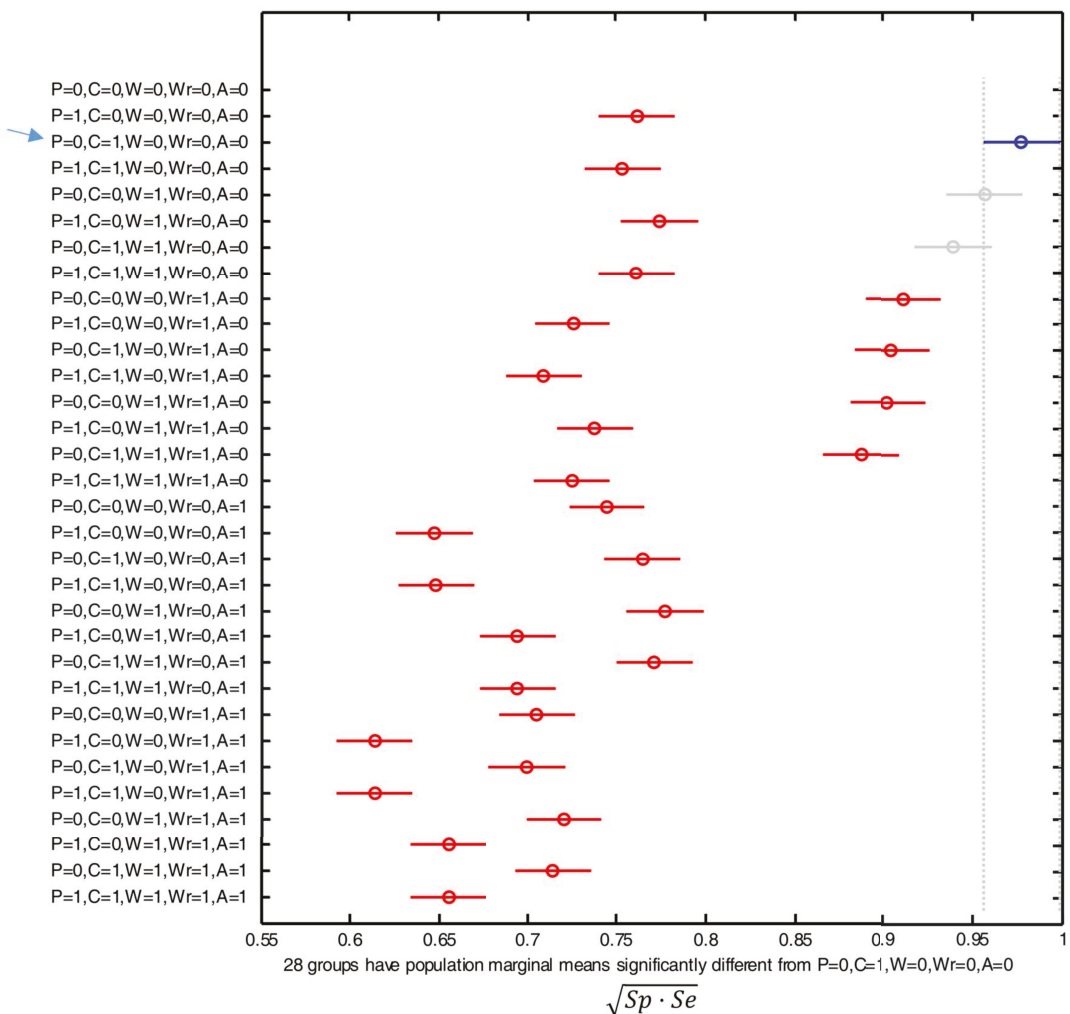

Figure 11. Comparison of the means of the performance metric $(\sqrt{S p \cdot S e}$ or geometric mean of the sensitivity and the specificity) obtained with the SVM algorithm for all the possible combinations and positions of the sensors. The positions are indicated as: $\mathrm{P}$ (Pocket), C (Chest), W (Waist), Wr (Wrist) or A (Ankle). For each combination, the y-axis of the figure indicates with ' 1 ' or ' 0 ' whether the corresponding sensor is considered (' 1 ') or not (' 0 ') for the detection decision. The combination with the best results is indicated with a blue arrow. 
From the results we can infer that the best performance is achieved by using the sensors attached to the waist and chest. Thus, the utilization of any of these two devices as the unique sensor of the system or the combination of both sensors clearly improves the efficiency of the algorithm, as their results significantly differ from the rest. For these three configurations (only-waist, only-chest or the combined use of the sensors on the waist and chest) the computed geometric mean of the sensitivity and the specificity is around 0.95 (which implies false negative and false positive rates lower than $5 \%$ ). This outperformance could be attributed to the fact that the chest and waist are the sensing points which are closest to the gravity center of the body so that they are less affected by spurious and sudden movements of the limbs. Consequently, the sensors on these positions probably characterize better the mobility of the whole body. Contrariwise, the utilization of the signals captured by the other three devices (the phone in the pocket and the SensorTags on the wrist and on the ankle) do not contribute to the system efficacy and even degrade the algorithm performance. This is particularly true for the case of the sensor on the ankle, as the performance metric plummets below 0.75 for any combination that employs the measurements of the accelerometer located on this point.

\subsubsection{Results for the $k$-NN Algorithm}

In the case of studying the residuals plots computed when the ANOVA test is applied to the performance metric obtained with the $k$-NN algorithm, we can conclude that the assumptions of normality and homoscedasticity are met. The small variations of the variance as a function of the range of the predicted values can be neglected due to the balanced design of the experiment.

The ANOVA analysis results in an error of $22.857 \%$, which can be justified by the presence of factors different from the position of the sensor, which cause a deviation from the overall average of the experiment, such as the physiognomics and mobility differences of the subjects

From the multiple comparison of the mean values of the performance metric and their corresponding $95 \%$ confidence intervals for the different combinations of sensors, we obtain that the best results are again achieved by using the acceleration signals measured at the chest and waist. The differences of the results for these three configurations (only-waist, only-chest and waist-chest) combinations are not statistically significant although they are slightly higher (close to 0.975 ) than those obtained with the SVM algorithm. Again, the consideration of a more complex BAN (by combining the detection decision on other locations) does not introduce any benefit in the system. Similarly, the use of the signals at the ankle particularly deteriorates the system behavior.

\subsubsection{Results for the Naive Bayes Algorithm}

The same conclusions as for the previous algorithms can be drawn if we employ a Naïve Bayes strategy to detect the falls. Firstly, the conditions of normality and homoscedasticity of the residuals present a similar behavior to those registered for SVM and $k$-NN algorithms.

The study of the performance metric also reveals that the best option is to consider the detection carried out by the algorithm at the chest, which yields a value of the geometric mean of $S p$ and $S e$ in the interval [0.922-0.981]. Similarly, the 95\% confidence intervals of the results obtained with the sensor at the waist and with a combination of the sensors on the waist and chest overlaps with that achieved on the chest. Thus, they cannot be considered as poorer. Conversely, the worst performance is again attained whenever the detection process takes into account the application of the algorithm to the signals captured at the ankle. The error of the ANOVA analysis for this algorithm was estimated to be $22.857 \%$ (higher than for the precedent algorithms).

\subsubsection{Results for the Decision Tree Algorithm}

We repeat the testing procedure when the detection algorithm is based on a Decision Tree learning mechanism. We observe again that the criteria of normality and equality of variance of the residuals are not completely met. However, as in the previous experiments, although the data do not strictly 
comply with these assumptions, the balanced nature of the experiment allows assuming that their influence on the validity of the final results will be limited.

The multiple comparison in turn shows that the optimal operation of the algorithm (with a performance metric in the interval [0.938-0.991]) is also achieved by the use of the signals captured on the chest followed by those of the waist, the combination of waist and chest, the wrist and the combination of wrist and chest. On the other hand, the detection based on the sensors located on the pocket and/or the ankle remarkably deteriorates the effectiveness of the algorithm. Besides, the computed error of the ANOVA analysis was $40.620 \%$ (higher than for the precedent algorithms). This is a sign that there are a number of external factors-not considered in the analysis as system variables-that could notably impact on the results.

\subsubsection{Summary and Discussion of the Results}

In the previous subsections we have assessed the behavior of the four considered machine-learning strategies to detect falls when they are individually applied to the signals produced by the 31 possible combinations of sensors employed in our testbed.

Table 8 summarizes the results obtained by the best combinations that lead to the best results of the geometric mean of the specificity and the sensitivity, as well as the results that are obtained when just a single sensor is employed. The table indicates the sensors that are considered for each combination and highlights in bold those cases in which the performance metric is above 0.9 (which implies that the specificity and sensitivity simultaneously reach values higher than $94.8 \%$ ). From these results we can draw the following conclusions:

- The particular location of the sensors on the user's body has a noteworthy influence on the effectiveness of the fall detector with independence of the chosen algorithm.

- The best results are always achieved when the detection algorithm employs the acceleration measurements captured on the chest (or trunk) or waist, the two points that are closest to the gravity center of the human body. This conclusion is coherent with the results of previous works $[17,33,61-66]$ that compared the performance of the FDS when operating on two or more positions. In this regard, we cannot forget that ergonomics is a key aspect in the design of any wearable system. The analysis of the state-of-the art on FDSs performed by Thilo et al. in [67] has shown that all the aspects related to ergonomics are almost permanently ignored by the related literature. Thus, for example, these authors prove that the research on FDS prototypes rarely takes into account the opinion of the elderly (the main target of this type of emergency systems). In this vein, chest may constitute a quite unnatural place to locate a FDS as attaching or fixing a sensor to the chest most probably results in some discomfort to the user. Conversely, a belt on the waist (to which the sensors can be easily bonded or stitched) may introduce a more ergonomic alternative.

- For all the algorithms, the worst results are associated to the use of the sensor at the ankle. This can be justified by the fact that the mobility of the ankle does not describe the global stability of the body. Hence, many ADLs can be misidentified as falls (and vice versa) if this location is used as a sensing point.

- The consideration of a trouser pocket as a location for the sensor does not seem to introduce any improvement in the results. The outcome of the detection process is negatively affected if the algorithms take into account the measurements of the accelerometer embedded in a smartphone in the pocket. Pockets may provide users with a comfortable solution to carry small sensors. However, the variability of the typology of the pockets (width, height, ergonomics, internal space, attachment to the body) among the users may impact on the capability of this location to characterize the mobility of the body. Authors in [58] already showed that smartphone-based fall detectors are prone to errors if the device shifts within the pocket. Thus, the use of loose pockets to transport the smartphone may clearly undermine the reliability of this device to implement a FDS. In addition, the motion of the thigh during falls can likely be mistaken by that registered during 
the execution of certain types of ADLs. This bad behavior of this position forces to reconsider the role of the smartphone as a wearable sensor in fall detection systems. Although many studies have proposed its use in FDSs (taking advantage of its popularity and ease of programming), its effectiveness would be very limited unless it is transported in an uncomfortable (e.g., on a belt) or unnatural place (some works have even tested its effectiveness in FDSs when it is firmly attached to the chest). In the case of the smartphones employed in this study, the sampling frequency of its acceleration sensor was higher than that of the sensor motes $(200 \mathrm{~Hz}$ vs. $20 \mathrm{~Hz})$, so it can also be deduced that a higher sampling rate does not correlate with a better performance. The simultaneous application of the algorithm to the signals sent by more than two sensors never increases the global performance metric (i.e., the possible increase of the specificity never compensates the loss of sensitivity caused by the fact that the fall must be detected in several points). As a consequence, the increase of the complexity of the body sensor network (which undoubtedly affects its ergonomics and cost) is not connected to a higher efficacy of the detection decision and should not be contemplated. The number of sensors is much less important than the election of the sensor position. A single-sensor architecture (with an accelerometer attached to the chest) seems to be enough to maximize the system effectiveness. The use of a second 'backup' sensor on the waist does not degrade the performance while it could help to avoid false positives in case of any anomaly suffered by the device on the chest. In this regard, the use of a smartwatch on the wrist as a more natural backup detection point could be also contemplated. In fact, the combined use of a smartwatch and a smartphone has been proved to outperform the effectiveness of the detection decision when compared to a "stand-alone" smartphone-based FDS [68-70]. Özdemir has shown in [17] that a fall detection algorithm based on the measurements captured by a sensor on the wrist may lead to a sensitivity higher than $97 \%$. If we compare the results achieved by the sensor on the waist (used as a single sensor) for the different algorithms, we can conclude that the best performance is attained by the SVM algorithm (although the confidence intervals obtained for the four machine learning strategies partly overlap). For the case of the SVM, the confidence interval of the performance metric is in the interval [0.956-0.999] with a mean value of 0.9775 , which entails minimum mean values for the specificity and the sensitivity higher than $95.5 \%$. In addition, SVM is the algorithm that produces the lowest error (14.162\%) for the ANOVA analysis. This lower error reduces the possible impact of other possible factors that have not been considered (e.g., differences in the mobility patterns or physical characteristics of the subject). In any case, the impact of inter subject variability on the results should be studied in future studies. Thus, the ANOVA analysis should be developed taking into account the individual performance of the algorithms when applied to each subject separately. For that purpose, a longer dataset with a higher number of samples per subject is required in order to obtain a better characterization of the performance metrics (sensitivity and specificity) for each participant. 
Table 8. Summary of the results of the study on the impact of the sensor position. For every combination, the tick symbol $(\checkmark)$ identifies those sensors that are employed by the algorithm.

\begin{tabular}{|c|c|c|c|c|c|c|}
\hline & Trouser Pocket & Chest & Waist & Wrist & Ankle & $\sqrt{S e \cdot S p}$ \\
\hline \multirow{6}{*}{ SVM Error $=14.162 \%$} & $\checkmark$ & - & - & - & - & {$[0.741-0.783]$} \\
\hline & - & $\checkmark$ & - & - & - & [0.956-0.999] \\
\hline & - & - & $\checkmark$ & - & - & {$[0.935-0.978]$} \\
\hline & - & - & - & $\checkmark$ & - & [0.890-0.933] \\
\hline & - & - & - & - & $\checkmark$ & {$[0.724-0.766]$} \\
\hline & - & $\checkmark$ & $\checkmark$ & - & - & {$[0.918-0.961]$} \\
\hline \multirow{6}{*}{$k$-NN Error $=22.857 \%$} & $\checkmark$ & - & - & - & - & [0.833-0.875] \\
\hline & - & $\checkmark$ & - & - & - & [0.950-0.993] \\
\hline & - & - & $\checkmark$ & - & - & [0.950-0.992] \\
\hline & - & - & - & $\checkmark$ & - & {$[0.903-0.946]$} \\
\hline & - & - & - & - & $\checkmark$ & {$[0.797-0.840]$} \\
\hline & - & $\checkmark$ & $\checkmark$ & - & - & {$[0.926-0.969]$} \\
\hline \multirow{6}{*}{ Naive Bayes Error = 16.967\% } & $\checkmark$ & - & - & - & - & [0.792-0.853] \\
\hline & - & $\checkmark$ & - & - & - & {$[0.922-0.981]$} \\
\hline & - & - & $\checkmark$ & - & - & {$[0.903-0.963]$} \\
\hline & - & - & - & $\checkmark$ & - & [0.863-0.899] \\
\hline & - & - & - & - & $\checkmark$ & {$[0.614-0.675]$} \\
\hline & - & $\checkmark$ & $\checkmark$ & - & - & {$[0.863-0.923]$} \\
\hline \multirow{6}{*}{ Decision Tree Error $=40.620 \%$} & $\checkmark$ & - & - & - & - & {$[0.787-0.840]$} \\
\hline & - & $\checkmark$ & - & - & - & {$[0.938-0.991]$} \\
\hline & - & - & $\checkmark$ & - & - & {$[0.913-0.965]$} \\
\hline & - & - & - & $\checkmark$ & - & [0.890-0.943] \\
\hline & - & - & - & - & $\checkmark$ & {$[0.825-0.878]$} \\
\hline & - & $\checkmark$ & $\checkmark$ & - & - & {$[0.896-0.949]$} \\
\hline
\end{tabular}

\section{Conclusions}

This article has presented a systematic assessment of the performance of different supervised learning algorithms when they are utilized for the automatic discrimination of falls and ADLs in wearable fall detection system. The study is based on the analysis of a dataset (UMAFall) obtained through a testbed with experimental subjects. In order to generate the mobility data traces, the subjects were requested to executed a series of predefined movements (ADLs and mimicked falls) while wearing a multisensory wireless Body Area Network, consisting of a Bluetooth network with five inertial mobility units (embedded in four commercial sensing motes and a smartphone) located on five different parts of the body. In our framework, the quality metric selected to weigh up the efficiency of the algorithms to detect falls was the geometric mean of the specificity and variance, which can be considered a good trade-off between the needs of avoiding false negatives (falls identified as ADLs) and false positives (ADLs mistaken as falls).

The purpose of the analysis was twofold. Firstly, we intended to assess the impact of the election of the statistics employed (as input variables for the algorithms) to describe the evolution of the subjects' mobility during the movements. Secondly, we evaluated the importance of the location of the sensors for the detection effectiveness of the machine learning strategies.

The final results enable quantifying the contribution of the different factors involved in the detection of a fall and discerning if they lead to significant changes in the final performance of the system.

As it refers to the characterization of the accelerometer signals, it has been shown that each detection mechanism needs a different combination of input characteristics to achieve its best performance. Nevertheless, there are statistics that augment the effectiveness of all the algorithms whenever they are considered: the standard deviation of the acceleration module (measured during a time window around the acceleration peak), which describes the variability of the human mobility, and the mean module of the non-vertical acceleration components, which characterizes the alterations of the perpendicularity of the subjects' body with respect to the floor plane. 
In any case, results suggest that a greater number of input features does not necessarily lead to a better operation of the machine learning algorithms. In fact, in many cases, this increases in the complexity of the system and even produces a decrease in the measured performance metric.

Besides, it has been evidenced that the location of the employed sensors strongly influences the efficacy of the FDS. In this regard, for all the studied algorithms, the best results correspond to the detection based on the signals captured on the chest and/or waist (the two considered sensing points which are closest to the center of mass and gravity of the human body). In addition, it has also been inferred that the combined use of several sensors (aimed at decreasing the number of false alarms) does not improve the performance, since it heavily decreases the sensitivity. The study has also shown that a sensor in a trouser pocket (the position that is conventionally utilized by for many users to transport a smartphone) is not the best option for an adequate discrimination rate. Consequently, a fall detection system merely based on a smartphone (as it is massively proposed by many papers in the literature) is not feasible as long as the phone should be constantly fixed to a belt or 'worn' in a quite unnatural way (firmly attached to the chest).

Another important contribution of the paper is the employed methodology as the validity of all the comparisons was evaluated through an ANOVA analysis. Thus, it has been possible to determine the statistical significance of the differences obtained when the algorithms were tested under different configurations of the detection process (input variables, selection of the sensors). The study of the statistical significance of the results (an aspect that is normally neglected by the existing bibliography about FDSs) is completed by the information reported by the error computed by the ANOVA analysis. This error can be used as a metric to evaluate the robustness of the algorithms with respect to the presence of other impacting factors that have not been taken into consideration for the analysis (such as the personal characteristics of the subjects).

Many mid- and low-end smartphones are still manufactured without gyroscopes. Thus, as the smartphone is a key element in our architecture, this study has only focused on the discrimination of falls based on signals captured by the accelerometers. However, the trunk angle velocity and trunk orientation have been proved to be two key parameters to detect anomalies in the mobility of the body during the moments immediately preceding the impact [71]. Our future research plans to extend this study by incorporating metrics derived from the signals measured by the gyroscope embedded in the sensors. So, the contribution of the information on the changes of the body orientation to the fall detection process could also be evaluated.

The analysis of the performance of the fall detection schemes of this work was developed in an offline mode (by processing the samples on a computer using Matlab scripts), as the general goal was to assess the 'abstract' effectiveness of the machine learning strategies under different circumstances. Therefore, the problems related to the implementation of this type of architectures in a wearable system (with inherent hardware and software limitations) were outside the scope of our study. However, these aspects (which are normally ignored by the literature) should also be systematically investigated.

Future studies should analyze in detail the implementability of systems that combine wireless communications with external sensors and complex artificial intelligence techniques that must make decisions in real time.

Battery-powered wearable units acting as the core of a Body Area Network for a FDS may have serious shortcomings to provide the computational resources (computing speed, memory, etc.) required by the communication dynamics and the algorithm of the fall detection service. In the case of using a conventional smartphone as the center of the sensor network, the practical coexistence of the detection application and the other typical activities executed by the device (making calls, messaging, browsing, etc.) should be also studied in detail. Otherwise, the use of a smartphone as a specific tool exclusively intended for fall detections (as it is conceived in those architectures where the phone is firmly attached to the user's chest) is pointless. In this context, battery drain due to FDS applications should be also studied in detail. 
Acknowledgments: This work was supported by Universidad de Málaga, Campus de Excelencia Internacional Andalucia Tech, Málaga, Spain.

Author Contributions: J.A.S.-R. deployed the testbed, executed the tests to collect the dataset, proposed the ANOVA analysis methodology, programmed and performed the off-line study of the algorithms, analyzed the results and co-wrote the paper. E.C.-P. designed the experimental setup, defined the comparison procedure and the machine-learning algorithms to be compared, co-analyzed the results, elaborated the critical review, wrote and revised the paper. J.M.C.-G. helped in the collection of datasets and gave technical advice regarding the implementation of the testbed.

Conflicts of Interest: The authors declare no conflict of interest.

\section{References}

1. World Health Organization (WHO). Ageing and Health-Fact Sheet No 404 (September 2015). Available online: http:/ / www.who.int/mediacentre/factsheets/fs404/en/ (accessed on 17 January 2018).

2. World Health Organisation (WHO). Falls—Fact Sheet (Updated August 2017). Available online: http: / / www.who.int/mediacentre/factsheets/fs344/en/ (accessed on 17 January 2018).

3. World Health Organization. Ageing \& Life Course Unit. WHO Global Report on Falls Prevention in Older Age; World Health Organization: Geneva, Switzerland, 2008.

4. Lord, S.R.; Sherrington, C.; Menz, H.B.; Close, J.C.T. Falls in older people: risk factors and strategies for prevention; Cambridge University Press: Cambridge, UK, 2007.

5. Habib, M.A.; Mohktar, M.S.; Kamaruzzaman, S.B.; Lim, K.S.; Pin, T.M.; Ibrahim, F. Smartphone-based solutions for fall detection and prevention: Challenges and open issues. Sensors 2014, 14, 7181-7208. [CrossRef] [PubMed]

6. Khan, S.S.; Hoey, J.R. Review of fall detection techniques: A data availability perspective. Med. Eng. Phys. 2016, 39, 12-22. [CrossRef] [PubMed]

7. Board of Governors of the Federal Reserve System. Consumers and Mobile Financial Services 2016, March 2016; Board of Governors of the Federal Reserve System: Washington, DC, USA, 2016.

8. Casilari, E.; Luque, R.; Morón, M. Analysis of android device-based solutions for fall detection. Sensors 2015, 15, 17827-17894. [CrossRef] [PubMed]

9. Zhang, T.; Wang, J.; Liu, P.; Hou, J. Fall detection by embedding an accelerometer in cellphone and using KFD algorithm. Int. J. Comput. Sci. Netw. Secur. 2006, 6, 277-284.

10. Soangra, R.; Lockhart, T.E. Agreement in gait speed from smartphone and stopwatch for five meter walk in laboratory and clinical environments. Biomed. Sci. Instrum. 2014, 50, 254-264. [PubMed]

11. Casilari, E.; Santoyo-Ramón, J.A.; Cano-García, J.M. Analysis of a Smartphone-Based Architecture with Multiple Mobility Sensors for Fall Detection. PLoS ONE 2016, 11, e0168069. [CrossRef] [PubMed]

12. Banos, O.; Damas, M.; Pomares, H.; Prieto, A.; Rojas, I. Daily living activity recognition based on statistical feature quality group selection. Expert Syst. Appl. 2012, 39, 8013-8021. [CrossRef]

13. Zhang, L.; Liu, T.; Zhu, S.; Zhu, Z. Human Activity Recognition based on Triaxial Accelerometer. In Proceedings of the 2012 7th International Conference on Computing and Convergence Technology (ICCCT), Seoul, Korea, 3-5 December 2012; pp. 261-266.

14. Wang, L.; Gu, T.; Tao, X.; Lu, J. A hierarchical approach to real-time activity recognition in body sensor networks. Pervasive Mob. Comput. 2012, 8, 115-130. [CrossRef]

15. Huynh, Q.T.; Nguyen, U.D.; Tran, S.V.; Tran, B.Q. Optimum location for sensors in fall detection. In Proceedings of the International Conference on Green and Human Information Technology (ICGHIT), Hanoi, Vietnam, 27 February-1 March 2013.

16. Huynh, Q.T.; Nguyen, U.D.; Irazabal, L.B.; Ghassemian, N.; Tran, B.Q. Optimization of an Accelerometer and Gyroscope-Based Fall Detection Algorithm. J. Sens. 2015, 2015, 452078. [CrossRef]

17. Özdemir, A.; Turan, A. An Analysis on Sensor Locations of the Human Body for Wearable Fall Detection Devices: Principles and Practice. Sensors 2016, 16, 1161. [CrossRef] [PubMed]

18. Igual, R.; Medrano, C.; Plaza, I. A comparison of public datasets for acceleration-based fall detection. Med. Eng. Phys. 2015, 37, 870-878. [CrossRef] [PubMed]

19. Texas Instruments SimpleLink ${ }^{\mathrm{TM}}$. Bluetooth Low Energy/Multi-Standard SensorTag. Available online: http:/ / www.ti.com/ww/en/wireless_connectivity/sensortag/ (accessed on 6 March 2017). 
20. Medrano, C.; Igual, R.; Plaza, I.; Castro, M. Detecting falls as novelties in acceleration patterns acquired with smartphones. PLoS ONE 2014, 9, e94811. [CrossRef] [PubMed]

21. Fudickar, S.; Lindemann, A.; Schnor, B. Threshold-based Fall Detection on Smart Phones. In Proceedings of the 7th International Conference on Health Informatics (HEALTHINF'2014), Angers, France, 3-6 March 2014.

22. Casilari, E.; Santoyo-Ramón, J.A. UMAFall: Fall Detection Dataset (Universidad de Malaga). Available online: https:/ / figshare.com/articles/UMA_ADL_FALL_Dataset_zip/4214283 (accessed on 5 April 2018).

23. Rawassizadeh, R.; Momeni, E.; Dobbins, C.; Gharibshah, J.; Pazzani, M. Scalable Daily Human Behavioral Pattern Mining from Multivariate Temporal Data. IEEE Trans. Knowl. Data Eng. 2016, 28, 3098-3112. [CrossRef]

24. Figueiredo, I.N.; Leal, C.; Pinto, L.; Bolito, J.; Lemos, A. Exploring smartphone sensors for fall detection. mUX J. Mob. User Exp. 2016, 5, 2. [CrossRef]

25. Min, C.; Kang, S.; Yoo, C.; Cha, J.; Choi, S.; Oh, Y.; Song, J. Exploring current practices for battery use and management of smartwatches. In Proceedings of the 2015 ACM International Symposium on Wearable Computers-ISWC '15, Osaka, Japan, 7-11 September 2015; ACM Press: New York, NY, USA, 2015; pp. 11-18.

26. Casilari, E.; Santoyo-Ramón, J.-A.; Cano-García, J.-M. Analysis of public datasets for wearable fall detection systems. Sensors 2017, 17, 1513. [CrossRef] [PubMed]

27. Bagalà, F.; Becker, C.; Cappello, A.; Chiari, L.; Aminian, K.; Hausdorff, J.M.; Zijlstra, W.; Klenk, J. Evaluation of accelerometer-based fall detection algorithms on real-world falls. PLoS ONE 2012, 7, e37062. [CrossRef] [PubMed]

28. Jämsä, T.; Kangas, M.; Vikman, I.; Nyberg, L.; Korpelainen, R. Fall detection in the older people: From laboratory to real-life. Proc. Est. Acad. Sci. 2014, 63, 341-345. [CrossRef]

29. Kangas, M.; Vikman, I.; Nyberg, L.; Korpelainen, R.; Lindblom, J.; Jämsä, T. Comparison of real-life accidental falls in older people with experimental falls in middle-aged test subjects. Gait Posture 2012, 35, 500-505. [CrossRef] [PubMed]

30. Kotsiantis, S.B.; Zaharakis, I.D.; Pintelas, P.E. Machine learning: A review of classification and combining techniques. Artif. Intell. Rev. 2006, 26, 159-190. [CrossRef]

31. Liu, S.H.; Cheng, W.C. Fall detection with the support vector machine during scripted and continuous unscripted activities. Sensors 2012, 12, 12301-12316. [CrossRef] [PubMed]

32. Yoshida, T.; Mizuno, F.; Hayasaka, T.; Tsubota, K.; Wada, S.; Yamaguchi, T. A wearable computer system for a detection and prevention of elderly users from falling. In Proceedings of the 12th International Conference on Biomedical Engineering (ICBME), Singapore, 7-10 December 2005; Magjarevic, R.R., Nagel, J.H., Eds.; Springer: Singapore, 2005.

33. Kangas, M.; Konttila, A.; Winblad, I.; Jamsa, T. Determination of simple thresholds for accelerometry-based parameters for fall detection. In Proceedings of the 29th Annual International Conference of the IEEE Engineering in Medicine and Biology Society (EMBS 2007), Lyon, France, 22-26 August 2007; pp. 1367-1370.

34. Ngu, A.; Wu, Y.; Zare, H.; Polican, A.; Yarbrough, B.; Yao, L. Fall Detection using Smartwatch Sensor Data with Accessor Architecture. In Proceedings of the International Conference, ICSH 2017, Hong Kong, China, 26-27 June 2017.

35. Sucerquia, A.; López, J.D.; Vargas-Bonilla, F. Real-Life/Real-Time Elderly Fall Detection with a Triaxial Accelerometer. Preprints 2018. [CrossRef]

36. Chen, J.; Kwong, K.; Chang, D.; Luk, J.; Bajcsy, R. Wearable sensors for reliable fall detection. In Proceedings of the IEEE Engineering in Medicine and Biology 27th Annual Conference (EMBC), Shanghai, China, 1-4 September 2005; pp. 3551-3554.

37. Rodriguez, J.; Mercuri, M.; Karsmakers, P.; Soh, P.J.; Leroux, P.; Schreurs, D. Automatic fall detector based on sliding window principle. In Proceedings of the 34th WIC Symposium on Information Theory in the Benelux and the Third Joint WIC/IEEE SP Symposium on Information Theory and Signal Processing in the Benelux, Leuven, Belgium, 30-31 May 2013; pp. 215-219.

38. Kozina, S.; Gjoreski, H.; Gams, M.; Luštrek, M. Efficient Activity Recognition and Fall Detection Using Accelerometers. In Evaluating AAL Systems Through Competitive Benchmarking; Botía, J.A., Álvarez-García, J.A., Barsocchi, P., Kaori, F., Riedel, T., Eds.; Springer: Berlin/Heidelberg, Germany, 2013; pp. 13-23.

39. Lombardi, A.; Ferri, M.; Rescio, G.; Grassi, M.; Malcovati, P. Wearable wireless accelerometer with embedded fall-detection logic for multi-sensor ambient assisted living applications. In Proceedings of the 2009 IEEE Sensors, Christchurch, New Zealand, 25-28 October 2009; pp. 1967-1970. 
40. Chen, K.-H.; Yang, J.-J.; Jaw, F.-S. Accelerometer-based fall detection using feature extraction and support vector machine algorithms. Instrum. Sci. Technol. 2016, 44, 333-342. [CrossRef]

41. Kerdegari, H.; Samsudin, K.; Ramli, A.R.; Mokaram, S. Evaluation of fall detection classification approaches. In Proceedings of the 4th International Conference on Intelligent and Advanced Systems (ICIAS 2012), Kuala Lumpur, Malaysia, 12-14 June 2012; Volume 1, pp. 131-136.

42. Delahoz, Y.S.; Labrador, M.A. Survey on fall detection and fall prevention using wearable and external sensors. Sensors 2014, 14, 19806-19842. [CrossRef] [PubMed]

43. Schwickert, L.; Becker, C.; Lindemann, U.; Maréchal, C.; Bourke, A.; Chiari, L.; Helbostad, J.L.; Zijlstra, W.; Aminian, K.; Todd, C. Fall detection with body-worn sensors: A systematic review. Z. Gerontol. Geriatr. 2013, 46, 706-719. [CrossRef] [PubMed]

44. Igual, R.; Medrano, C.; Plaza, I. Challenges, issues and trends in fall detection systems. Biomed. Eng. Online 2013, 12, 66. [CrossRef] [PubMed]

45. Mubashir, M.; Shao, L.; Seed, L. A survey on fall detection: Principles and approaches. Neurocomputing 2013, 100, 144-152. [CrossRef]

46. Kotsiantis, S.B.; Zaharakis, I.; Pintelas, P. Supervised machine learning: A review of classification techniques. Emerg. Artif. Intell. Appl. Comput. Eng. 2007, 160, 3-24.

47. Zhang, T.; Wang, J.; Xu, L.; Liu, P. Fall Detection by Wearable Sensor and One-Class SVM Algorithm. In Intelligent Computing in Signal Processing and Pattern Recognition; Springer: Berlin/Heidelberg, Germany, 2006; pp. 858-863.

48. Salgado, P.; Afonso, P. Body Fall Detection with Kalman Filter and SVM. In Proceedings of the 11th Portuguese Conference on Automatic Control (CONTROLO'2014), Porto, Portugal, 21-23 July 2014; Springer: Cham, Switzerland, 2015; pp. 407-416.

49. Wang, Y.; Wu, K.; Ni, L.M. WiFall: Device-Free Fall Detection by Wireless Networks. IEEE Trans. Mob. Comput. 2017, 16, 581-594. [CrossRef]

50. Albert, M.V.; Kording, K.; Herrmann, M.; Jayaraman, A. Fall classification by machine learning using mobile phones. PLoS ONE 2012, 7, e36556. [CrossRef] [PubMed]

51. Putra, I.P.E.S.; Vesilo, R. Window-size impact on detection rate of wearable-sensor-based fall detection using supervised machine learning. In Proceedings of the 2017 IEEE Life Sciences Conference (LSC), Sydney, NSW, Australia, 13-15 December 2017; pp. 21-26.

52. Gunale, K.G.; Mukherji, P. Fall detection using k-nearest neighbor classification for patient monitoring. In Proceedings of the IEEE International Conference on Information Processing (ICIP 2015), Pune, India, 16-19 December 2015.

53. Liu, C.-L.; Lee, C.-H.; Lin, P.-M. A fall detection system using k-nearest neighbor classifier. Expert Syst. Appl. 2010, 37, 7174-7181. [CrossRef]

54. Genoud, D.; Cuendet, V.; Torrent, J. Soft Fall Detection Using Machine Learning in Wearable Devices. In Proceedings of the 2016 IEEE 30th International Conference on Advanced Information Networking and Applications (AINA), Crans-Montana, Switzerland, 23-25 March 2016; pp. 501-505.

55. Yang, X.; Dinh, A.; Chen, L. A wearable real-time fall detector based on Naive Bayes classifier. In Proceedings of the 23rd Canadian Conference on Electrical and Computer Engineering (CCECE), Calgary, AB, Canada, 2-5 May 2010.

56. Ojetola, O.; Gaura, E.I.; Brusey, J. Fall Detection with Wearable Sensors-Safe (Smart Fall Detection). In Proceedings of the 7th International Conference on Intelligent Environments; Nottingham, UK, 25-28 July 2011; pp. 318-321.

57. Stone, E.E.; Skubic, M. Fall Detection in Homes of Older Adults Using the Microsoft Kinect. IEEE J. Biomed. Heal. Inform. 2015, 19, 290-301. [CrossRef] [PubMed]

58. Aguiar, B.; Rocha, T.; Silva, J.; Sousa, I. Accelerometer-based fall detection for smartphones. In Proceedings of the IEEE International Symposium on Medical Measurements and Applications (MeMeA), Lisbon, Portugal, 11-12 June 2014; pp. 1-6.

59. Montgomery, D. Design and Analysis of Experiments; John Wiley and Sons: New York, NY, USA, 2001; Volume 5.

60. Jaeger, T.F. Categorical data analysis: Away from ANOVAs (transformation or not) and towards logit mixed models. J. Mem. Lang. 2008, 59, 434-446. [CrossRef] [PubMed] 
61. Fang, S.-H.; Liang, Y.-C.; Chiu, K.-M. Developing a mobile phone-based fall detection system on android platform. In Proceedings of the Computing, Communications and Applications Conference (ComComAp), Hong Kong, China, 11-13 January 2012; BenLetaief, K., Zhang, Q., Eds.; pp. 143-146.

62. Dai, J.; Bai, X.; Yang, Z.; Shen, Z.; Xuan, D. Mobile phone-based pervasive fall detection. Pers. Ubiquitous Comput. 2010, 14, 633-643. [CrossRef]

63. Dai, J.; Bai, X.; Yang, Z.; Shen, Z.; Xuan, D. PerFallD: A pervasive fall detection system using mobile phones. In Proceedings of the 8th IEEE International Conference on Pervasive Computing and Communications Workshops (PERCOM Workshops), Mannheim, Germany, 29 March-2 April 2010; Becker, C., Conti, M., Eds.; pp. 292-297.

64. Gannapathy, V.R.; Ibrahim, A.; Zakaria, Z.B.; Othman, A.R.B.; Latiff, A.A. Zigbee-Based Smart Fall Detection and Notification System with Wearable Sensor (e-SAFE). Int. J. Res. Eng. Technol. 2013, 2, 337-344.

65. Bourke, A.K.; O'Brien, J.V.; Lyons, G.M. Evaluation of a threshold-based tri-axial accelerometer fall detection algorithm. Gait Posture 2007, 26, 194-199. [CrossRef] [PubMed]

66. Mao, L.; Liang, D.; Ning, Y.; Ma, Y.; Gao, X.; Zhao, G. Pre-impact and Impact Detection of Falls Using Built-In Tri-accelerometer of Smartphone. In Proceedings of the Third International Conference on Health Information Science (HIS 2014), Shenzhen, China, 22-23 April 2014; Springer: Cham, Switzerland, 2014; pp. 167-174.

67. Thilo, F.J.S.; Hürlimann, B.; Hahn, S.; Bilger, S.; Schols, J.M.G.A.; Halfens, R.J.G. Involvement of older people in the development of fall detection systems: A scoping review. BMC Geriatr. 2016, 16, 42. [CrossRef] [PubMed]

68. Vilarinho, T.; Farshchian, B.; Bajer, D.G.; Dahl, O.H.; Egge, I.; Hegdal, S.S.; Lones, A.; Slettevold, J.N.; Weggersen, S.M. A Combined Smartphone and Smartwatch Fall Detection System. In Proceedings of the 2015 IEEE International Conference on Computer and Information Technology; Ubiquitous Computing and Communications; Dependable, Autonomic and Secure Computing; Pervasive Intelligence and Computing (CIT/IUCC/DASC/PICOM), Liverpool, UK, 26-28 October 2015; pp. 1443-1448.

69. Maglogiannis, I.; Ioannou, C.; Spyroglou, G.; Tsanakas, P. Fall Detection Using Commodity Smart Watch and Smart Phone. In Artificial Intelligence Applications and Innovations; Maglogiannis, I.L., Papadopoulos, H., Sioutas, S., Makris, C., Eds.; Springer: Berlin/Heidelberg, Germany, 2014; pp. 70-78.

70. Casilari, E.; Oviedo-Jiménez, M.A. Automatic fall detection system based on the combined use of a smartphone and a smartwatch. PLOS ONE 2015, 10, e0140929. [CrossRef] [PubMed]

71. Liu, J.; Lockhart, T.E. Development and Evaluation of a Prior-to-Impact Fall Event Detection Algorithm. IEEE Trans. Biomed. Eng. 2014, 61, 2135-2140. [PubMed]

(C) 2018 by the authors. Licensee MDPI, Basel, Switzerland. This article is an open access article distributed under the terms and conditions of the Creative Commons Attribution (CC BY) license (http:/ / creativecommons.org/licenses/by/4.0/). 


\title{
Article \\ "What Is a Step?" Differences in How a Step Is Detected among Three Popular Activity Monitors That Have Impacted Physical Activity Research
}

\author{
Dinesh John ${ }^{1, *}$, Alvin Morton ${ }^{2}$, Diego Arguello ${ }^{1}$, Kate Lyden ${ }^{3}$ and David Bassett ${ }^{2}$ \\ 1 Department of Health Sciences, Northeastern University, Boston, MA 02115, USA; \\ arguello.d@husky.neu.edu \\ 2 Department of Kinesiology, Recreation, and Sport Studies, University of Tennessee, Knoxville, TN 37996 \\ USA; amorto16@vols.utk.edu (A.M.); dbassett@utk.edu (D.B.) \\ 3 KAL Research/Consulting, Denver, CO 80206, USA; katelyden6@gmail.com \\ * Correspondence: d.john@neu.edu; Tel.: 617-373-5695
}

Received: 28 February 2018; Accepted: 12 April 2018; Published: 15 April 2018

\begin{abstract}
Background: This study compared manually-counted treadmill walking steps from the hip-worn DigiwalkerSW200 and OmronHJ720ITC, and hip and wrist-worn ActiGraph GT3X+ and GT9X; determined brand-specific acceleration amplitude (g) and/or frequency (Hz) step-detection thresholds; and quantified key features of the acceleration signal during walking. (2) Methods: Twenty participants (Age: $26.7 \pm 4.9$ years) performed treadmill walking between 0.89 -to- $1.79 \mathrm{~m} / \mathrm{s}(2-4 \mathrm{mph})$ while wearing a hip-worn DigiwalkerSW200, OmronHJ720ITC, GT3X+ and GT9X, and a wrist-worn GT3X+ and GT9X. A DigiwalkerSW200 and OmronHJ720ITC underwent shaker testing to determine device-specific frequency and amplitude step-detection thresholds. Simulated signal testing was used to determine thresholds for the ActiGraph step algorithm. Steps during human testing were compared using bias and confidence intervals. (3) Results: The OmronHJ720ITC was most accurate during treadmill walking. Hip and wrist-worn ActiGraph outputs were significantly different from the criterion. The DigiwalkerSW200 records steps for movements with a total acceleration of $\geq 1.21 \mathrm{~g}$. The OmronHJ720ITC detects a step when movement has an acceleration $\geq 0.10 \mathrm{~g}$ with a dominant frequency of $\geq 1 \mathrm{~Hz}$. The step-threshold for the ActiLife algorithm is variable based on signal frequency. Acceleration signals at the hip and wrist have distinctive patterns during treadmill walking. (4) Conclusions: Three common research-grade physical activity monitors employ different step-detection strategies, which causes variability in step output.
\end{abstract}

Keywords: Step-detection; ActiGraph; Pedometer; acceleration; physical activity

\section{Introduction}

Wearable activity monitors are commonly used to detect steps to quantify physical activity (PA) [1]. While this seemingly standard metric should enable a direct comparison of PA among studies using step-counters, validation studies report discrepancies in steps from different commercial activity monitors [2-5]. This may be attributable to variability in criteria used by the devices to translate human movement into steps.

Furthermore, there may be within-brand differences in step-output. For example, steps using ActiGraph's ActiLife software on data from newer devices (i.e., model GT1M and higher), yield step-outputs that are not comparable to the older $7164[6,7]$ and location of wear (hip vs. wrist) impacts ActiGraph step-output [8]. A substantial proportion of current knowledge on associations between objectively measured PA and health results from the nationally representative 2003-04/2005-06 National Health and Nutrition Examination Survey (NHANES), which used the ActiGraph 7164 [9-11]. 
However, the latest cycle of the NHANES (2011-12/2013-14) used a wrist-worn GT3X+ [12]. Additionally, while steps computed using ActiLife is most common in PA research, real-time step output is also available to a user via the device display screen in the new GT9X. However, the latter is computed using a real-time step-counting algorithm that is different from that in ActiLife. The real-time algorithm on-board the GT9X uses tri-axial (as opposed to uni-axial acceleration data in ActiLife), and filters out steps that are accumulated in bouts lasting less than 2 and $10 \mathrm{~s}$ on the hip and wrist, respectively. Thus, in the context of objectively measuring PA using motion sensors, the answer to the question "what is a step?" may be contingent on device-specific proprietary strategies used to detect and translate movement acceleration into steps.

Thus, our study examined the abovementioned question by first investigating step-output comparability among three devices that have significantly impacted PA research. This was followed-up with a determination of device-specific parameters used to define and detect a step. The three devices were the mechanical Yamax Digiwalker SW200, the piezoelectric cantilever accelerometer-based Omron HJ720ITC, and MEMs capacitive sensor-based ActiGraph monitors. A reason for selecting these three devices was that unlike various consumer-based devices that are currently available, these three brands of devices have significantly influenced the creation of commonly used step goals (e.g., 10,000 steps/day) [13-15] and/or have been widely used in both population-level studies (e.g., NHANES) [16-20] and in clinical trials [15,21-23]. However, these devices also use unknown proprietary strategies to define a step. Differences in proprietary strategies that dictate how a step is detected, may result in the invalidation of a step as a universal metric of PA and compromise inter-study comparisons of this metric. To our knowledge, no study has systematically determined device-specific step-detection parameters among these three brands of activity monitors. Such an examination will provide an improved understanding of "why" movements recorded as a step by one device are not classified as such by another device.

Thus, Aim 1 of our study compared manually-counted steps during treadmill walking to those from the hip-worn Digiwalker SW200 and Omron HJ720ITC, and steps from hip and wrist-worn GT3X+ and GT9X monitors processed using ActiLife software. Aim 2 determined device-specific raw acceleration amplitude (g-value) and/or frequency (Hz) thresholds to record steps. Results of Aim 2 may explain likely differences in step outputs among devices in Aim 1. A secondary aim of our study was to quantify features of high-resolution acceleration signals detected at the hip and the wrist and identify/describe visually distinctive patterns in the signals during walking. Such findings may further explain variability in output from Aim 1 and inform the development of superior non-proprietary step-detection methods when using accelerometer-based step-counters.

\section{Materials and Methods}

Twenty participants (Age: $26.7 \pm 4.9$ years; BMI: $26.1 \pm 3.5 \mathrm{~kg} / \mathrm{m}^{2} ; 12$ males and 8 females; all right-handed) with no gait abnormalities and who were experienced in using a treadmill volunteered for this study. The study was approved by the Northeastern University Institutional Review Board and all participants provided written informed consent prior to commencing study participation. Figure 1 is a conceptual outline of study aims and associated experimental protocols, which are detailed below (Figure 1). 


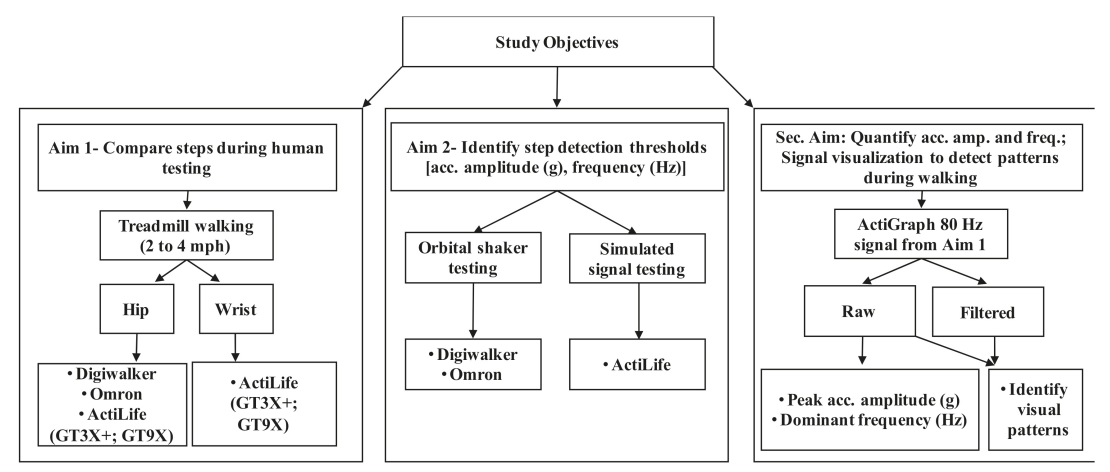

Figure 1. Conceptual outline of study aims and associated experimental protocols (acc. = acceleration; amp. = amplitude; freq.= frequency).

\subsection{Devices}

The hip-worn Digiwalker SW200 is a coiled spring-suspended pendulum-based mechanical device, which counts steps when vertical acceleration during hip movement sufficiently displaces the pendulum to complete an electrical circuit (Figure 2). The Omron HJ720ITC is typically worn on the hip, has two piezoelectric sensors that are placed perpendicular to each other on its circuit board, and uses proprietary criteria to detect steps in real-time. The ActiGraph GT3X+ and GT9X can be worn on both the hip and wrist. Data from these capacitive accelerometer-based devices [24] can be post-processed to obtain steps using ActiLife software (ActiGraph LLC., Pensacola, FL, USA).

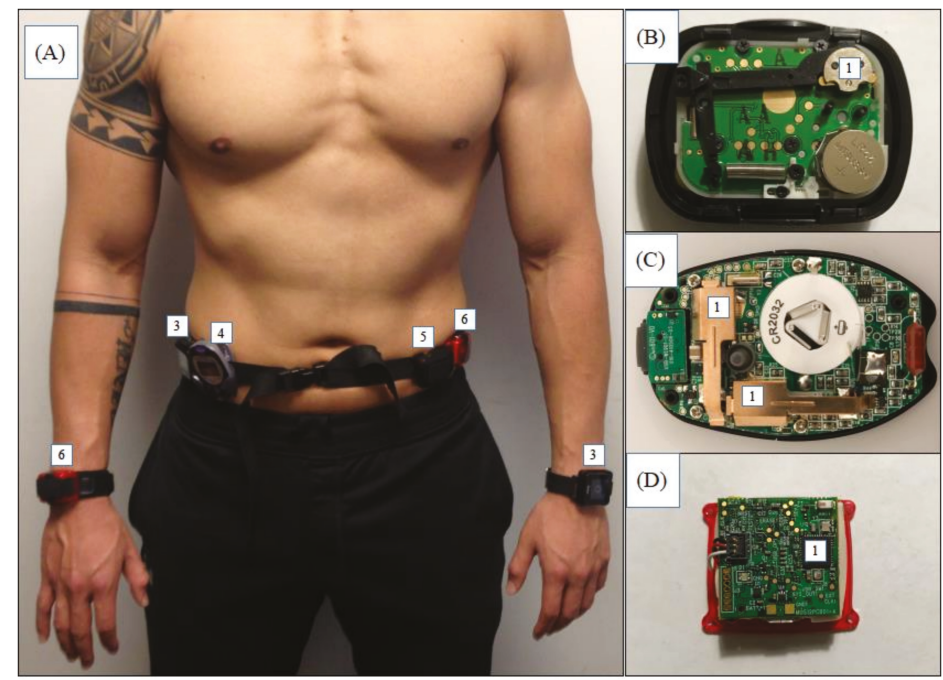

Figure 2. Participant with the ActiGraph GT3X+ (\#6), GT9X (\#3), the Omron HJ720ITC (\#4), and the Digiwalker SW300 (\#5). Figures (B-D) depict the sensing elements (\#1) inside the three devices.

\subsection{Aim 1-Human Treadmill Testing}

Participants wore a single Digiwalker SW200, Omron HJ720ITC, GT3X+, and GT9X at the waist using a snug elastic belt (Figure 2). The GT3X+ and the GT9X were positioned in line with the anterior 
axillary line on the left and right hip. The Omron HJ720ITC and Digiwalker SW200 were placed medially adjacent to the ActiGraph monitors. A GT3X+ and GT9X were worn at the most distal location on the left and right wrist. Monitor locations (left and right) were counterbalanced among participants to account for any confounding due to placement. Device placement was based on typical procedures followed in PA research, i.e., as per the manufacturer's recommendation (Figure 2).

The experimental protocol consisted of treadmill walking at 11 speeds ranging between 0.89 and $1.79 \mathrm{~m} / \mathrm{s}$ ( 2 to $4 \mathrm{mph}$ ) in increments of $0.089 \mathrm{~m} / \mathrm{s}(0.2 \mathrm{mph})$. The protocol yielded steady-state step-counts for one minute at each speed. A researcher manually counted steps (criterion) during the protocol. A researcher verified each treadmill speed using a handheld tachometer (Shimpo DT-105A, Nidec-Shimpo America Corp., Itasca, IL., USA).

\subsection{Aim 2-Testing to Examine Step-Detection Thresholds for Acceleration Amplitude (g) and Frequency (Hz)}

Processing simulated signals of known frequency and amplitude using device-specific algorithms lends maximum control and accuracy in determining the minimum acceleration required to detect a step (i.e., step-counting threshold). However, the Omron HJ720ITC and the Digiwalker SW200 do not have associated software or software features that facilitate such processing. Hence, to determine step-detection thresholds, we conducted shaker-testing for the Omron HJ720ITC and Digiwalker SW200, and simulated sinusoidal signal testing for the ActiGraph algorithm.

\subsubsection{Shaker Testing (Omron HJ720ITC and Digiwalker SW200)}

Our orbital shaker (Model 1231A89, Thomson Scientific, London, UK) maintains a monitor in a fixed spatial orientation, while subjecting the device to a circular motion in the horizontal plane at adjustable frequencies and radii ranging from 0.25 to $8.0 \mathrm{~Hz}$ (15 to $480 \mathrm{rpm}$ ) and 1.6 to $16 \mathrm{~cm}$, respectively. Both devices were tested at 4 different radii: $2.1,2.6,4.5$, and $12.0 \mathrm{~cm}$. Since, acceleration thresholds required to commence step counting may be different for these devices, an initial trial was conducted to determine monitor-specific testing frequencies. Testing frequencies were increased at the rate of $0.016 \mathrm{~Hz}(1 \mathrm{rpm})$. Each monitor then underwent 10 trials that yielded a minute of steady-state shaker testing at each testing frequency. The Omron HJ720ITC was tested from 0.97 to $1.17 \mathrm{~Hz}$ ( 58 to $70 \mathrm{rpm}$ ) at each of the testing radii. The Digiwalker SW200 was tested from 3.73 to $3.83 \mathrm{~Hz}$ (224 to $230 \mathrm{rpm}$ ) at $2.1 \mathrm{~cm}$, from 3.35 to $3.45 \mathrm{~Hz}$ (201 to $207 \mathrm{rpm}$ ) at $2.6 \mathrm{~cm}$, from 2.53 to $2.63 \mathrm{~Hz}$ (152 to $158 \mathrm{rpm}$ ) at $4.5 \mathrm{~cm}$, and from 1.50 to $1.63 \mathrm{~Hz}$ (90 to $98 \mathrm{rpm}$ ) at $12 \mathrm{~cm}$.

Devices were fastened to the shaker plate such that there was no movement artifact due to placement and each brand of monitor was tested separately. The protocol was video-recorded using a stationary high-speed camera placed at a height of approximately $30-\mathrm{cm}$ from the shaker plate. Time-stamped video recordings were immediately used to retrieve and record steps/min at each frequency from the display screens of the two devices.

\subsubsection{Simulated Sinusoidal Signal Testing for ActiGraph}

Simulated signal testing has been used to test characteristics of ActiLife software [25-27]. Signals were simulated at a sampling rate of $80 \mathrm{~Hz}$ for frequencies ranging from 0.2 to $2.4 \mathrm{~Hz}$, in increments of $0.2 \mathrm{~Hz}$. For each frequency, signals were generated at amplitudes ranging between 0.01 to $0.2 \mathrm{~g}$ in increments of $0.01 \mathrm{~g}$. Simulated signals were generated using MATLAB (R2016B, MathWorks, Natick, MA., USA) and were processed using ActiLife v6.13.3 to generate step-output [25,26].

\subsection{Secondary Aim-Quantifying Selected Signal Features and Analysis of Acceleration Waveforms (Raw/Filtered) during Treadmill Walking}

We quantified specific amplitude and frequency characteristics of the high-resolution acceleration signal from a GT3X+ worn at the hip and wrist. Walking is rhythmic and produces a relatively consistent waveform pattern with acceleration peaks during a step. Average peak acceleration amplitudes were derived from steady-state walking steps at each treadmill speed. Frequency 
characteristics included the dominant and 2nd dominant signal frequencies derived using Fourier Transform in MATLAB. We also report visually detectable time-domain patterns in the acceleration signal during walking for raw and filtered acceleration $(0.25-2.5 \mathrm{~Hz})$. This bandwidth was selected for this demonstration as previous work has shown that it is sufficient to capture movement related to human ambulation [28].

\subsection{Data Analyses}

Step outputs during human, shaker, and simulated signal testing were compiled into a Microsoft Excel database. All ActiGraph data ( $80 \mathrm{~Hz}$ sampling rate) were processed using ActiLife v6.13.3.

For the treadmill protocol (aim 1), mean steps and estimation bias (95\% confidence intervals) were derived using IBM ${ }^{\circledR}$ SPSS $^{\circledR}$ Statistics software (v. 23, Armonk, NY, USA). Bias was defined as steps from direct observation minus monitor output. Acceleration amplitude and/or frequency thresholds required by a device to detect steps were derived from shaker and sinusoidal signal testing (aim 2). Orbital shaker testing subjected the Digiwalker SW200 and the Omron HJ720ITC to sinusoidal acceleration in the $x$ and $y$-axes of the device where each revolution of the orbital shaker generates a curve that contains the two extrema of the acceleration sine wave with a zero-g value crossing. Hence, one revolution is one step. Details on computing peak acceleration amplitude during orbital shaker testing can be found elsewhere [29].

\section{Results}

\subsection{Aim 1-Human Treadmill Testing}

Table 1 depicts mean criterion and estimated steps and estimation bias (95\% confidence intervals) from hip and wrist-worn devices during the treadmill protocol. Statistically significant underestimations were found for the hip-worn Omron HJ720ITC at $1.61 \mathrm{~m} / \mathrm{s}(3.6 \mathrm{mph})$ and $1.79 \mathrm{~m} / \mathrm{s}$ (4.0 mph), the hip-worn Digiwalker SW200 at $0.89 \mathrm{~m} / \mathrm{s}(2.0 \mathrm{mph}), 0.98 \mathrm{~m} / \mathrm{s}(2.2 \mathrm{mph})$, and $1.16 \mathrm{~m} / \mathrm{s}$ $(2.6 \mathrm{mph})$, and both hip and wrist outputs from the GT3X+ and GT9X at all speeds. ActiGraph monitors were least accurate in detecting steps (Table 1).

\subsection{Aim 2-Testing to Examine Step-Count Thresholds for Acceleration Amplitude (g) and Frequency (Hz)}

\subsubsection{Digiwalker SW200}

Table 2 contains mean steps detected during the shaker testing protocol for the Digiwalker SW200 and the corresponding acceleration amplitude computed at the testing frequencies. Our findings suggest that a total acceleration amplitude of at least $1.21 \mathrm{~g}$ is required to sufficiently displace the spring-levered mechanism in the Digiwalker SW200 and count a step. We used the lowest g-force when a step is detected to represent step-detection threshold. This accounts for marginal decrements in the g-force produced (during few to several rotations) that is attributable to friction and other environmental factors that impact the rotation of the shaker plate.

\subsubsection{Omron HJ720ITC}

The Omron HJ720ITC counts a step when the detected motion satisfies both an acceleration amplitude and frequency threshold. Movement with a minimum acceleration amplitude of $0.10 \mathrm{~g}$ is required to detect steps (Table 2). At a radius of $2.1 \mathrm{~cm}$, step counting did not commence until a frequency of $1.10 \mathrm{~Hz}(66 \mathrm{rpm})$, which generates an acceleration of $0.10 \mathrm{~g}$. At all other radii, i.e., 2.6, 4.5 , and $12 \mathrm{~cm}$, acceleration amplitude at $1 \mathrm{~Hz}(60 \mathrm{rpm})$ and above were greater or equal to $0.10 \mathrm{~g}$ (range: 0.10 to $0.65 \mathrm{~g}$ ). Our findings suggest that the Omron HJ720ITC records steps for those peaks in the acceleration waveform that have a minimum amplitude threshold of $\pm 0.10 \mathrm{~g}$, but only when the waveform has a dominant frequency $\geq 1 \mathrm{~Hz}$ (Table 2). 

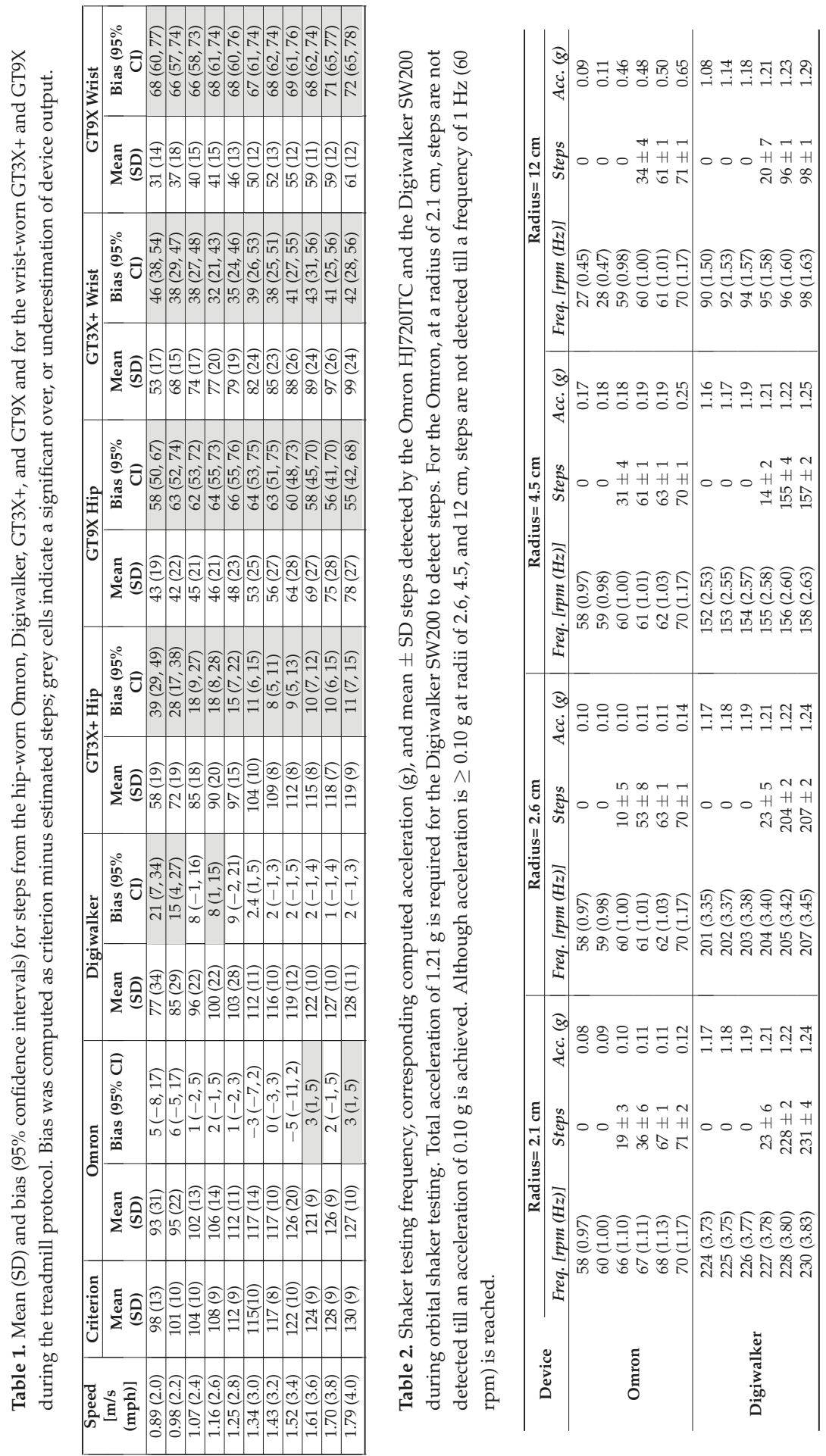


\subsubsection{ActiGraph's ActiLife Algorithm}

Sinusoidal signal testing demonstrated that the step-detection response of the ActiLife algorithm is variable based on both acceleration amplitude and frequency. Table 3 is a grid that shows the relationship between raw acceleration amplitude and frequency for the ActiLife step counting algorithm. The algorithm was most sensitive between frequencies of 0.7 and $0.8 \mathrm{~Hz}$ and required an acceleration amplitude of at least $0.07 \mathrm{~g}$ to detect a step (Table 3).

Table 3. Variable combinations of acceleration frequency and amplitude thresholds necessary to commence step counting using ActiLife software. ' $x$ ' indicates step detection, while ' 0 ' indicates the absence of step detection.

\begin{tabular}{|c|c|c|c|c|c|c|c|c|c|c|c|c|c|}
\hline Freq. (Hz.) & $0.06 \mathrm{~g}$ & $0.07 \mathrm{~g}$ & $0.08 \mathrm{~g}$ & $0.09 \mathrm{~g}$ & $0.1 \mathrm{~g}$ & $0.11 \mathrm{~g}$ & $0.12 \mathrm{~g}$ & $0.13 \mathrm{~g}$ & $0.14 \mathrm{~g}$ & $0.15 \mathrm{~g}$ & $0.16 \mathrm{~g}$ & $0.17 \mathrm{~g}$ & $0.18 \mathrm{~g}$ \\
\hline 0.2 & 0 & 0 & 0 & 0 & 0 & 0 & 0 & 0 & 0 & $x$ & $x$ & $x$ & $x$ \\
\hline 0.4 & 0 & 0 & 0 & $x$ & $x$ & $x$ & $x$ & $x$ & $x$ & $x$ & $x$ & $x$ & $x$ \\
\hline 0.6 & 0 & $x$ & $x$ & $x$ & $x$ & $x$ & $x$ & $x$ & $x$ & $x$ & $x$ & $x$ & $x$ \\
\hline 0.8 & 0 & $x$ & $x$ & $x$ & $x$ & $x$ & $x$ & $x$ & $x$ & $x$ & $x$ & $x$ & $x$ \\
\hline 1.0 & 0 & 0 & $x$ & $x$ & $x$ & $x$ & $x$ & $x$ & $x$ & $x$ & $x$ & $x$ & $x$ \\
\hline 1.2 & 0 & 0 & $x$ & $x$ & $x$ & $x$ & $x$ & $x$ & $x$ & $x$ & $x$ & $x$ & $x$ \\
\hline 1.4 & 0 & 0 & 0 & $x$ & $x$ & $x$ & $x$ & $x$ & $x$ & $x$ & $x$ & $x$ & $x$ \\
\hline 1.6 & 0 & 0 & 0 & 0 & $x$ & $x$ & $x$ & $x$ & $x$ & $x$ & $x$ & $x$ & $x$ \\
\hline 1.8 & 0 & 0 & 0 & 0 & 0 & $x$ & $x$ & $x$ & $x$ & $x$ & $x$ & $x$ & $x$ \\
\hline 2.0 & 0 & 0 & 0 & 0 & 0 & 0 & 0 & $x$ & $x$ & $x$ & $x$ & $x$ & $x$ \\
\hline 2.2 & 0 & 0 & 0 & 0 & 0 & 0 & 0 & 0 & 0 & $x$ & $x$ & $x$ & $x$ \\
\hline 2.4 & 0 & 0 & 0 & 0 & 0 & 0 & 0 & 0 & 0 & 0 & 0 & $x$ & $x$ \\
\hline
\end{tabular}

3.3. Secondary Aim-Quantifying Selected Signal Features and Visual Analysis of Acceleration Waveforms (Raw/Filtered) during Treadmill Walking

Overall, peak acceleration and the dominant signal frequency at the hip and wrist increased with speed (Figure 3A,B). Supplementary Table S1 contains values for the 2nd dominant frequency of hip and wrist movement (Figure 3).

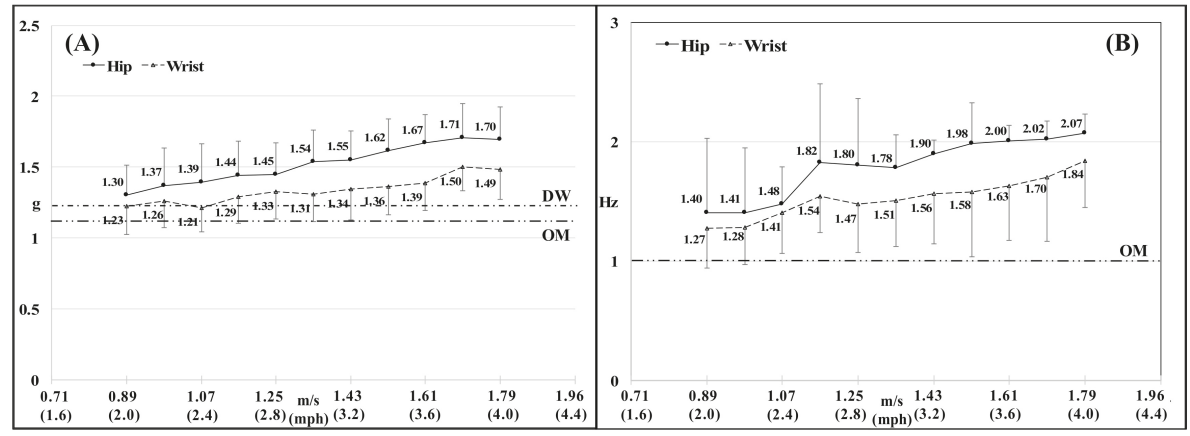

Figure 3. Mean $\pm \mathrm{SD}$ of peak high-resolution $(80 \mathrm{~Hz}$ ) acceleration detected in the $y$-axis of the GT3X+at the hip (A) and wrist (B) during walking at various speeds. Dotted lines in 3A indicate step-detection total acceleration thresholds for the hip-worn Digiwalker SW200 (1.21 g) (DW) and the Omron HJ720 ITC $(0.10 \mathrm{~g})(\mathrm{OM})$. Please note that although the piezoelectric Omron HJ720 ITC is not sensitive to the force of gravity, we added the gravity component to denote the threshold for the Omron because, the figure depicts total acceleration (gravity component + movement acceleration). Dotted line in 3B is the frequency threshold for the Omron HJ720 ITC $(1 \mathrm{~Hz})$. We do not depict thresholds for ActiLife as it is variable based on signal frequency due to bandpass filtering. Additionally, specifications of the filter are proprietary and hence it is not possible to scale the filtered signal thresholds identified in the study to an unfiltered acceleration (g) value in Figure 3A. 
Signal visualization ( $y$-axis and tri-axial vector magnitude) revealed a consistent pattern where the shape of the acceleration waveforms for consecutive steps looked different, but alternate step patterns were similar. Figure 4 demonstrates this pattern using the signal detected in the $y$-axis of a GT9X at the hip and wrist. To further explore this phenomenon, we conducted video analyses of five participants who wore a GT9X at the dominant hip and the non-dominant wrist while walking at $1.61 \mathrm{~m} / \mathrm{s}(3.6 \mathrm{mph})$ on a treadmill. Time-stamped videos were synchronized with the $y$-axis waveform from the GT9X at each site. For the hip, we defined an ipsilateral step as that by the leg on the same side as the hip on which the device was worn. The contralateral step was that taken by the opposite leg. Ipsilateral steps taken in concert with a forward arm swing of the non-dominant wrist generated acceleration curves that were larger than contralateral steps and the backward arm swing, respectively. Additionally, compared to raw data, filtered data are smoother and resemble sine-waves. The latter may simplify the detection of movements associated with a step. Thus, it is likely that monitor manufacturers may employ some form of acceleration signal filtering when detecting steps (Figure 4).

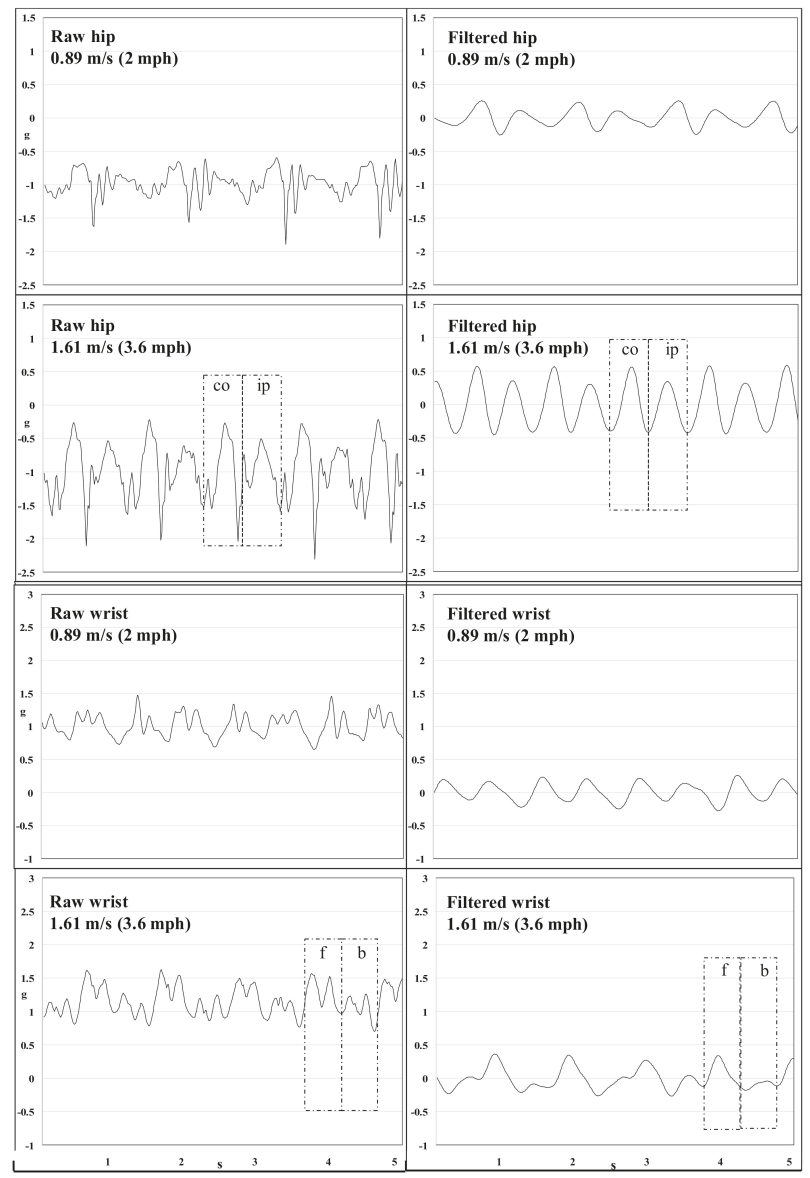

Figure 4. $y$-axis acceleration $(5 \mathrm{~s} ; 80 \mathrm{~Hz})$ from a hip and wrist GT9X on a single participant and their corresponding bandpass filtered signals $(0.25-2.5 \mathrm{~Hz})$ processed using a fourth-order Butterworth filter. Dotted boxes indicate the same steps in the raw and filtered signals. ' $\mathrm{co}^{\prime}=$ contralateral step (step taken by the leg on the same side as the hip-worn device); 'ip'= ipsilateral step (step taken by the leg on the opposite side as the hip-worn device); ' $\mathrm{f}$ '= forward arm swing; ' $\mathrm{b}$ '= backward arm swing. 


\section{Discussion}

The overarching goal of this study was to examine how inter-device differences in proprietary step-detection strategies impact the comparability of step outputs during human movement. For this, we used devices that have significantly contributed to existing knowledge on the impact of steps/day on health. We first present a discussion that integrates findings from human testing in our study (Aim 1) and previous work with findings from our investigation of step-detection strategies in the three brands of activity monitors (Aim 2). This is followed by a discussion on observations from the secondary aim.

4.1. Aims 1 and 2-Human Treadmill Testing and Examining Step-Count Thresholds for Acceleration Amplitude $(\mathrm{g})$ and Frequency $(\mathrm{Hz})$

\subsubsection{Digiwalker SW200}

The device in our study required a total raw g-force threshold of $1.21 \mathrm{~g}$ to count a step. We placed the device flat on the shaker table, and subjected it to orbital motion in the horizontal plane. Such a placement eliminates the gravity component $(1 \mathrm{~g})$ that is exerted on the spring-based pendulum when the device is worn on the body (vertical plane). Thus, the additional vertical acceleration (beyond gravity) required to count a step is $0.21 \mathrm{~g}$. Our empirically determined threshold of $0.21 \mathrm{~g}$ for the Digiwalker SW200 is lower than the previously reported threshold of $0.35 \mathrm{~g}$ [30]. Supporting evidence for our estimate can be inferred from the raw acceleration detected by the ActiGraph at the hip (Figure 3A). At speeds between $0.89 \mathrm{~m} / \mathrm{s}(2 \mathrm{mph})$ and $1.25 \mathrm{~m} / \mathrm{s}(2.8 \mathrm{mph})$, step-detection accuracy increased from 79 to $92 \%$. At these speeds, mean \pm SD of peak acceleration (including the gravity component) ranged between $1.30 \pm 0.21$ to $1.45 \pm 0.22 \mathrm{~g}$. Given that (i) peak acceleration increases with increasing speed; (ii) there is considerable variability in peak acceleration at each speed $(\sim 0.21 \mathrm{~g}$, Figure 3A); and (iii) contralateral steps generate lower acceleration than ipsilateral steps (Figure 4); increasing walking speed results in an incremental proportion of steps that satisfy the acceleration threshold to detect a step, thereby resulting in a corresponding increase in step-detection accuracy (Table 1).

\subsubsection{Omron HJ720ITC}

The acceleration amplitude threshold of $0.10 \mathrm{~g}$ in the Omron HJ720ITC does not include the gravity component, which is attributable to the use of piezoelectric sensors to detect steps. Piezoelectric sensors do not detect static acceleration and produce an electric voltage that is proportional to movement only. We found that beyond the $0.10 \mathrm{~g}$ threshold, subsequent increases in the amplitude of acceleration did not alter the frequency threshold of $1 \mathrm{~Hz}(60 \mathrm{rpm})$. Thus, the hip-worn Omron HJ720ITC may cause larger step-detection errors during slow walking that may be attributable to a low step frequency rather than an insufficient acceleration amplitude because during walking, it is improbable that acceleration at the hip will not exceed a threshold of $0.10 \mathrm{~g}$. Correspondingly, Jehn et al. reported that a step-frequency between 80-90 steps/min was necessary for the Omron HJ720ITC to accurately detect steps [31].

\subsubsection{ActiLife}

The ActiLife step-counting algorithm is based on proprietary thresholding of the bandpass filtered uni-axial (y-axis) acceleration signal. Here, the filtered acceleration waveform needs to cross both positive and negative values of a fixed proprietary acceleration amplitude threshold to qualify as a step. Consecutive positive and negative crossings of the amplitude threshold are equidistantly separated by a zero-g crossing, which is used as an event that marks a valid step [32]. Bandpass filtering eliminates the gravity component, which yields a zero-g crossing and a low amplitude threshold for step-detection (see Figure 4).

Variability in acceleration thresholds: Although step-counting in ActiLife is based on a fixed acceleration amplitude threshold, we found that at different signal frequencies, the step-detection 
amplitude threshold is variable (Table 3). This is due to bandpass filtering of the raw acceleration signal that occurs prior to signal thresholding to detect steps (described above). ActiGraph's bandpass filter is maximally sensitive at $0.75 \mathrm{~Hz}$. As frequency components in the signal move away from $0.75 \mathrm{~Hz}$ within the passband $(0.25$ to $2.5 \mathrm{~Hz})$, the amplitude of the signal is reduced symmetrically [27]. At 0.29 and $1.66 \mathrm{~Hz}$, signal amplitude is reduced to $70.7 \%$ of that at $0.75 \mathrm{~Hz}$ [27]. This filter response explains peak sensitivity to detect steps between 0.7 and $0.8 \mathrm{~Hz}$ and the need for increasing acceleration amplitude (g-value) with increasing frequency to detect steps (table 3). During commonly preferred walking speeds of approximately 1.34 to $1.56 \mathrm{~m} / \mathrm{s}$ ( 3 to $3.5 \mathrm{mph}$ ) in normal adults [33,34], the dominant frequencies in the acceleration signal at both the hip and wrist in our study were close to or greater than $1.66 \mathrm{~Hz}[1.78 \pm 0.28 \mathrm{~Hz}$ at $1.34 \mathrm{~m} / \mathrm{s}(3 \mathrm{mph})$ at the hip; $1.63 \pm 0.46 \mathrm{~Hz}$ at $1.52 \mathrm{~m} / \mathrm{s}$ ( $3.4 \mathrm{mph}$ ) at the wrist (Figure 3B)].

Effect of monitor location on step-counting: We are unaware of the reasons causing differences in step output between the hip-worn GT3X+ and GT9X. However, we discuss causes for differences between hip and wrist-worn ActiGraph devices and between wrist-worn GT3X+ and GT9X devices.

The ActiLife step-counting algorithm was developed for hip-worn devices and uses acceleration from only the $y$-axis to detect steps. Acceleration detected at the wrist are smaller in magnitude than those at the hip during walking at the same speed (Figure 4). This will result in fewer instances where the acceleration waveform satisfies thresholding criteria, especially at slower walking speeds. However, during a 24-h period under free-living conditions, a wrist-worn GT3X+ resulted in higher step counts than a hip-worn device [8]. This paradoxical finding may be explained by the fact that many activities of daily living involve hand movements that result in the detection of several false-positive steps.

A significant source of error between a wrist-worn GT3X+ and GT9X is a discrepancy in the orientation of the tri-axial coordinate system due to a dissimilarity in "form-factor" (Figure 5A,B). This inter-device discrepancy in axis orientations will significantly impact step counting accuracy between the GT9X and other ActiGraph devices at the wrist (Figure 5).

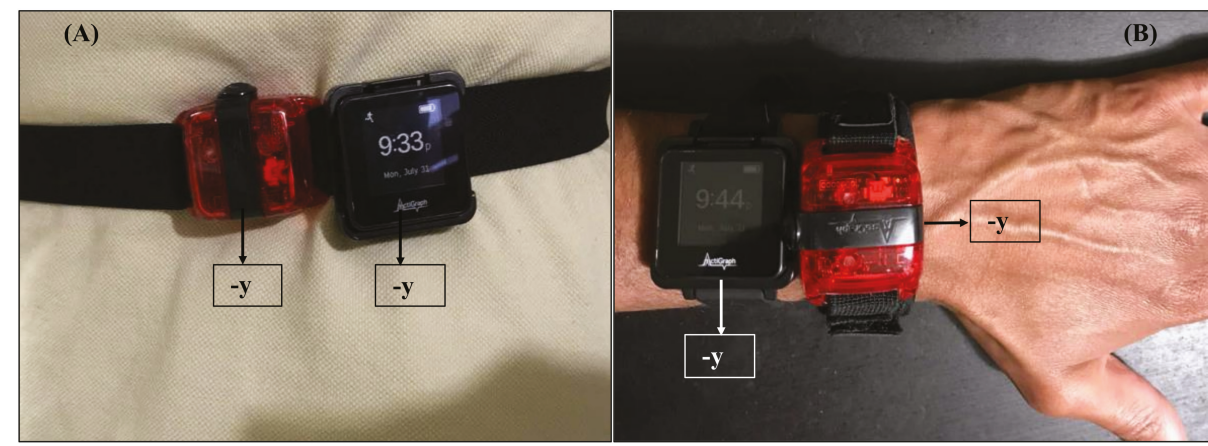

Figure 5. Orientation of the axes in the GT3X+ and GT9X when worn on the hip (A) and wrist (B), respectively.

Differences in individual gait, location of wear, and walking speed may impact thresholding of the signal in ActiLife, and thereby step-counting accuracy. These factors may yield a filtered signal that may not (i) cross both negative and positive thresholds required to count a step, or (ii) produce a zero crossing.

\subsection{Secondary Aim-Quantifying Selected Signal Features and Visual Analysis of Acceleration Waveforms} (Raw/Filtered) during Treadmill Walking

Although stepping involves displacement of the body's center of mass, which results in hip movement, the magnitude of peak-to-peak acceleration between two consecutive steps varies for 
hip-worn devices. During the contralateral step, i.e., a step taken by the left leg when the device is worn on the right hip, and vice versa, the sensor captures attenuated accelerations (Figure 4). This is because the device is not on the hip that is causing the step. At slower walking speeds, smaller accelerations detected during the contralateral step may not satisfy the step-detection criteria of the Digiwalker SW200 and ActiGraph, which may yield fewer step-counts (Figure 4).

At the wrist, the step waveform during the forward swing of the arm, tends to produce a distinctly larger waveform as compared to that during the backward arm swing. However, during walking, arm movement may not always reflect whole body movement (e.g., when holding an umbrella). Thus, arm swing patterns during walking may be less consistent than those observed at the hip. Signal processing techniques that are not limited to simple thresholding, but which also identify frequency features that are inherent in the signal during walking, may be useful to detect steps when wrist movement is restricted during ambulation.

\subsection{Strengths and Limitations}

A strength of this study is the use of three proprietary devices that have significantly influenced PA research. These devices use different hardware and strategies to detect steps, a universal metric of PA. Our study used human, mechanical, and software-based testing to demonstrate variability in step-detection parameters among tested devices, and how variability translates to discrepancies in steps. Weaknesses include limiting human testing to treadmill walking that may not represent natural over-ground walking, and exclusion of popular consumer wearables.

\section{Conclusions}

The Digiwalker SW200, the Omron HJ720ITC and the ActiGraph devices have significantly influenced the understanding of the dose-response between steps/day and health outcomes. However, the parameters to define and detect a step in all three devices are different.

- The Digiwalker SW200 has a movement acceleration step-detection threshold of $0.21 \mathrm{~g}$.

- The Omron HJ720ITC detects a step when movement acceleration peak is $\geq 0.10 \mathrm{~g}$, but only when the dominant frequency of the signal is $\geq 1 \mathrm{~Hz}$. The ActiLife algorithm primarily relies on thresholding of the band-pass filtered acceleration signal from a single axis. A step is detected only when the signal has a zero-crossing and crosses both a positive and negative threshold on either side of the zero-crossing.

Step-detection threshold for the ActiGraph's ActiLife algorithm is variable based on signal frequency due to signal filtering.

Our findings suggest that current step-detection strategies in ActiLife may be invalid for step detection using devices worn at both the hip and the wrist.

\subsection{Implications of Variability in Proprietary Step-Detection Parameters}

Similar to our study, previous work found that steps from hip-worn ActiGraphs and Digiwalker perform poorly at slower walking speeds $[<\sim 1.12 \mathrm{~m} / \mathrm{s}(2.5 \mathrm{mph})]$, while Omron devices detect most steps at speeds above $0.89 \mathrm{~m} / \mathrm{s}(2 \mathrm{mph})[3,35,36]$. Another study reported that the Omron HJ720ITC was unreliable at slow walking speeds $<0.83 \mathrm{~m} / \mathrm{s}(1.85 \mathrm{mph}$.). Thus, the proprietary step detection thresholds in these three devices may be inappropriate to detect steps during slow ambulation, particularly when used in populations where gait speed is markedly reduced (e.g., older adults, disabled populations). Studies comparing the hip-worn Omron HJ720 ITC, the Digiwalker SW200, and ActiGraph devices to a criterion ankle-worn step-counter have reported significant underestimations of steps during 24 -h free living [3,37,38]. Additionally, existing evidence that the Omron HJ720ITC does not count steps accumulated in durations lasting less than $4 \mathrm{~s}$ [38] in combination with findings on the frequency threshold from our study suggest that the step-counting 
algorithm in the Omron HJ720ITC was designed to capture purposeful, rhythmic bouts of walking. However, activities of daily living often involve short/intermittent stepping bouts that may last few to several seconds $[39,40]$. Thus, several of these steps may go undetected by the Omron HJ720ITC.

From a public health perspective, our findings may explain differences in steps/day estimates for the US population $[20,41]$. One study using a spring-levered pedometer reported that adults averaged approximately 5117 steps/day [41]. Conversely, based on NHANES 2005-2006 accelerometer data, American adults accumulate 9676 steps/day. This nearly 2-fold difference in steps/day from two US population-level studies may be attributable to differences in step-detection parameters in the two devices used in these studies. Given a rapid turnover of new models for each brand of popular consumer wearables, such devices are likely more suitable for end-users in the general population. However, the adoption of consumer wearables in PA and public health research is quickly increasing $[42,43]$. Most of these devices use proprietary methodologies to detect steps and hence, differences may exist in the types of movements that are captured as steps. For example, one study examining steps during numerous simulated activities of daily living (including ambulation) demonstrated significant differences in step outputs between the Apple watch and ground truth [44]. Another study using a wrist-worn Fitbit reported that the device underestimated free-living steps by up to $30 \%$ as compared to directly-observed ground truth [45]. Recent work found that proprietary methods in the Apple Watch and the FitBit Blaze yield significantly different steps during 19 different activities and during free-living conditions [46]. One factor may be that the Apple watch likely uses a summarized acceleration derived from each axis of a triaxial accelerometer as compared to the FitBit Blaze, which uses individual information from each of the three axis [46].

\subsection{Outlook for the Future}

Devices and methodologies that use proprietary and/or rudimentary acceleration threshold-based strategies may insufficiently capture steps during purposeful ambulation and activities with intermittent movements. Furthermore, to establish steps as a standard metric of PA, it may be necessary for the research community to have a consensus on movements (and related acceleration signal characteristics) required to represent a step. Such a consensus framework may then be used to expedite the development of a non-proprietary, open-source step-detection methodology that does not rely solely on simple acceleration thresholds, but considers multiple acceleration features to classify movements as a step. This may help to standardize the step as a metric of PA. Similar approaches may be necessary when considering new methods for clinical populations to ensure uniformity across devices used in a specific pathological condition that alters gait.

Supplementary Materials: The following are available online at www.mdpi.com/1424-8220/18/4/1206/s1, Table S1: Mean \pm SD of the second dominant frequencies $(\mathrm{Hz})$ detected in the acceleration signal during the walking protocol.

Author Contributions: All authors were involved in the conception of the study, interpretation of data and findings, and drafting/editing of the article. Alvin Morton and Diego Arguello were involved in collecting data for the study.

Conflicts of Interest: The authors declare no conflict of interest.

\section{References}

1. Bravata, D.M.; Smith-Spangler, C.; Sundaram, V.; Gienger, A.L.; Lin, N.; Lewis, R.; Stave, C.D.; Olkin, I.; Sirard, J.R. Using pedometers to increase physical activity and improve health: A systematic review. JAMA 2007, 298, 2296-2304. [CrossRef] [PubMed]

2. Crouter, S.E.; Schneider, P.L.; Karabulut, M.; Bassett, D.R., Jr. Validity of 10 electronic pedometers for measuring steps, distance, and energy cost. Med. Sci. Sports Exerc. 2003, 35, 1455-1460. [CrossRef] [PubMed] 
3. Hickey, A.; John, D.; Sasaki, J.E.; Mavilia, M.; Freedson, P. Validity of Activity Monitor Step Detection Is Related to Movement Patterns. J. Phys. Act. Health 2016, 13, 145-153. [CrossRef] [PubMed]

4. Schneider, P.L.; Crouter, S.; Bassett, D.R. Pedometer measures of free-living physical activity: Comparison of 13 models. Med. Sci. Sports Exerc. 2004, 36, 331-335. [CrossRef] [PubMed]

5. Tyo, B.M.; Fitzhugh, E.C.; Bassett, D.R., Jr.; John, D.; Feito, Y.; Thompson, D.L. Effects of body mass index and step rate on pedometer error in a free-living environment. Med. Sci. Sports Exerc. 2011, 43, 350-356. [CrossRef] [PubMed]

6. Lee, K.Y.; Macfarlane, D.; Cerin, E. Do three different generations of the Actigraph accelerometer provide the same output? Med. Sci. Sports Exerc. 2010, 42, 476. [CrossRef]

7. Lohne-Seiler, H.; Hansen, B.H.; Kolle, E.; Anderssen, S.A. Accelerometer-determined physical activity and self-reported health in a population of older adults (65-85 years): A cross-sectional study. BMC Public Health 2014, 14, 284. [CrossRef] [PubMed]

8. Tudor-Locke, C.; Barreira, T.V.; Schuna, J.M., Jr. Comparison of step outputs for waist and wrist accelerometer attachment sites. Med. Sci. Sports Exerc. 2015, 47, 839-842. [CrossRef] [PubMed]

9. Clark, B.K.; Healy, G.N.; Winkler, E.A.; Gardiner, P.A.; Sugiyama, T.; Dunstan, D.W.; Matthews, C.E.; Owen, N. Relationship of television time with accelerometer-derived sedentary time: NHANES. Med. Sci. Sports Exerc. 2011, 43, 822-828. [CrossRef] [PubMed]

10. Healy, G.N.; Matthews, C.E.; Dunstan, D.W.; Winkler, E.A.; Owen, N. Sedentary time and cardio-metabolic biomarkers in US adults: NHANES 2003-06. Eur. Heart J. 2011, 32, 590-597. [CrossRef] [PubMed]

11. Wolff-Hughes, D.L.; Fitzhugh, E.C.; Bassett, D.R.; Churilla, J.R. Total Activity Counts and Bouted Minutes of Moderate-to-Vigorous Physical Activity: Relationships with Cardiometabolic Biomarkers Using 2003-2006 NHANES. J. Phys. Act. Health 2015, 12, 694-700. [CrossRef] [PubMed]

12. Troiano, R.P.; McClain, J.J.; Brychta, R.J.; Chen, K.Y. Evolution of accelerometer methods for physical activity research. Br. J. Sports Med. 2014, 48, 1019-1023. [CrossRef] [PubMed]

13. Hatano, Y. Prevalence and use of pedometer. Res. J. Walk. 1997, 1, 45-54.

14. Hatano, Y. Use of the pedometer for promoting daily walking exercise. Int. Counc. Health Phys. Educ. Recreat. 1997, 29, 4-8.

15. Yamanouchi, K.; Shinozaki, T.; Chikada, K.; Nishikawa, T.; Ito, K.; Shimizu, S.; Ozawa, N.; Suzuki, Y.; Maeno, H.; Kato, K. Daily walking combined with diet therapy is a useful means for obese NIDDM patients not only to reduce body weight but also to improve insulin sensitivity. Diabetes Care 1995, 18, 775-778. [CrossRef] [PubMed]

16. De Craemer, M.; Lateva, M.; Iotova, V.; De Decker, E.; Verloigne, M.; De Bourdeaudhuij, I.; Androutsos, O.; Socha, P.; Kulaga, Z.; Moreno, L.; et al. Differences in energy balance-related behaviours in European preschool children: The ToyBox-study. PLoS ONE 2015, 10, e0118303. [CrossRef] [PubMed]

17. Duncan, M.J.; Birch, S.L.; Eyre, E.; Bryant, E.; Rutten, C.; Boen, F.; Seghers, J. Comparisons in ambulatory physical activity in children from the United Kingdom and Belgium. Ann. Hum. Biol. 2015, 42, 290-292. [CrossRef] [PubMed]

18. Matthiessen, J.; Andersen, E.W.; Raustorp, A.; Knudsen, V.K.; Sorensen, M.R. Reduction in pedometer-determined physical activity in the adult Danish population from 2007 to 2012. Scand. J. Public Health 2015, 43, 525-533. [CrossRef] [PubMed]

19. Pelclova, J.; Fromel, K.; Repka, E.; Blaha, L.; Suchomel, A.; Fojtik, I.; Feltlova, D.; Valach, P.; Horak, S.; Nykodym, J.; et al. Is Pedometer-Determined Physical Activity Decreasing in Czech Adults? Findings from 2008 to 2013. Int. J. Environ. Res. Public Health 2016, 13, 1040. [CrossRef] [PubMed]

20. Tudor-Locke, C.; Johnson, W.D.; Katzmarzyk, P.T. Accelerometer-determined steps per day in US adults. Med. Sci. Sports Exerc. 2009, 41, 1384-1391. [CrossRef] [PubMed]

21. Pahor, M.; Guralnik, J.M.; Ambrosius, W.T.; Blair, S.; Bonds, D.E.; Church, T.S.; Espeland, M.A.; Fielding, R.A.; Gill, T.M.; Groessl, E.J.; et al. Effect of structured physical activity on prevention of major mobility disability in older adults: The LIFE study randomized clinical trial. JAMA 2014, 311, 2387-2396. [CrossRef] [PubMed]

22. Piette, J.D.; Richardson, C.; Himle, J.; Duffy, S.; Torres, T.; Vogel, M.; Barber, K.; Valenstein, M. A randomized trial of telephonic counseling plus walking for depressed diabetes patients. Med. Care 2011, 49, 641-648. [CrossRef] [PubMed] 
23. Sigal, R.J.; Kenny, G.P.; Boule, N.G.; Wells, G.A.; Prud'homme, D.; Fortier, M.; Reid, R.D.; Tulloch, H.; Coyle, D.; Phillips, P.; et al. Effects of aerobic training, resistance training, or both on glycemic control in type 2 diabetes: A randomized trial. Ann. Intern. Med. 2007, 147, 357-369. [CrossRef] [PubMed]

24. John, D.; Freedson, P. ActiGraph and Actical physical activity monitors: A peek under the hood. Med. Sci. Sports Exerc. 2012, 44 (Suppl. S1), S86-S89. [CrossRef] [PubMed]

25. Brond, J.C.; Andersen, L.B.; Arvidsson, D. Generating ActiGraph Counts from Raw Acceleration Recorded by an Alternative Monitor. Med. Sci. Sports Exerc. 2017, 49, 2351-2360. [CrossRef] [PubMed]

26. Brond, J.C.; Arvidsson, D. Sampling frequency affects the processing of Actigraph raw acceleration data to activity counts. J. Appl. Physiol. 2016, 120, 362-369. [CrossRef] [PubMed]

27. Tryon, W.W.; Williams, R. Fully proportional actigraphy: A new instrument. Behav. Res. Methods 1996, 28, 392-403. [CrossRef]

28. John, D.; Miller, R.; Kozey-Keadle, S.; Caldwell, G.; Freedson, P. Biomechanical examination of the 'plateau phenomenon' in ActiGraph vertical activity counts. Physiol. Meas. 2012, 33, 219-230. [CrossRef] [PubMed]

29. John, D.; Sasaki, J.; Staudenmayer, J.; Mavilia, M.; Freedson, P.S. Comparison of raw acceleration from the GENEA and ActiGraph GT3X+ activity monitors. Sensors 2013, 13, 14754-14763. [CrossRef] [PubMed]

30. Tudor-Locke, C.; Ainsworth, B.E.; Thompson, R.W.; Matthews, C.E. Comparison of pedometer and accelerometer measures of free-living physical activity. Med. Sci. Sports Exerc. 2002, 34, 2045-2051. [CrossRef] [PubMed]

31. Jehn, M.; Schmidt-Trucksaess, A.; Schuster, T.; Hanssen, H.; Weis, M.; Halle, M.; Koehler, F. Accelerometer-based quantification of 6-minute walk test performance in patients with chronic heart failure: Applicability in telemedicine. J. Card. Fail. 2009, 15, 334-340. [CrossRef] [PubMed]

32. Nguyen, J.; ActiGraph LLC., Pensacola FL. USA. Personal communication, 2017.

33. Browning, R.C.; Baker, E.A.; Herron, J.A.; Kram, R. Effects of obesity and sex on the energetic cost and preferred speed of walking. J. Appl. Physiol. 2006, 100, 390-398. [CrossRef] [PubMed]

34. Rose, J.; Ralston, J.; Gamble, G. Energetics of walking. In Human Walking; Rose, J., Gamble, J.G., Eds.; Williams \& Wilkins: Baltimore, MD, USA, 1994; pp. 45-72.

35. Hasson, R.E.; Haller, J.; Pober, D.M.; Staudenmayer, J.; Freedson, P.S. Validity of the Omron HJ-112 pedometer during treadmill walking. Med. Sci. Sports Exerc. 2009, 41, 805-809. [CrossRef] [PubMed]

36. Lee, J.A.; Williams, S.M.; Brown, D.D.; Laurson, K.R. Concurrent validation of the Actigraph gt3x+, Polar Active accelerometer, Omron HJ-720 and Yamax Digiwalker SW-701 pedometer step counts in lab-based and free-living settings. J. Sports Sci. 2015, 33, 991-1000. [CrossRef] [PubMed]

37. Feito, Y.; Bassett, D.R.; Thompson, D.L. Evaluation of activity monitors in controlled and free-living environments. Med. Sci. Sports Exerc. 2012, 44, 733-741. [CrossRef] [PubMed]

38. Silcott, N.A.; Bassett, D.R., Jr.; Thompson, D.L.; Fitzhugh, E.C.; Steeves, J.A. Evaluation of the Omron HJ-720ITC pedometer under free-living conditions. Med. Sci. Sports Exerc. 2011, 43, 1791-1797. [CrossRef] [PubMed]

39. Dall, P.M.; McCrorie, P.R.; Granat, M.H.; Stansfield, B.W. Step accumulation per minute epoch is not the same as cadence for free-living adults. Med. Sci. Sports Exerc. 2013, 45, 1995-2001. [CrossRef] [PubMed]

40. Orendurff, M.S.; Schoen, J.A.; Bernatz, G.C.; Segal, A.D.; Klute, G.K. How humans walk: Bout duration, steps per bout, and rest duration. J. Rehabil. Res. Dev. 2008, 45, 1077-1089. [CrossRef] [PubMed]

41. Bassett, D.R., Jr.; Wyatt, H.R.; Thompson, H.; Peters, J.C.; Hill, J.O. Pedometer-measured physical activity and health behaviors in U.S. adults. Med. Sci. Sports Exerc. 2010, 42, 1819-1825. [CrossRef] [PubMed]

42. Althoff, T.; Sosic, R.; Hicks, J.L.; King, A.C.; Delp, S.L.; Leskovec, J. Large-scale physical activity data reveal worldwide activity inequality. Nature 2017, 547, 336-339. [CrossRef] [PubMed]

43. Fitbit Selected for National Institutes of Health (NIH) Precision Medicine Research Program with the Scripps Research Institute (TSRI). Available online: https://investor.fitbit.com/press/press-releases/pressrelease-details / 2017 / Fitbit-Selected-for-National-Institutes-of-Health-NIH-Precision-Medicine-ResearchProgram-with-The-Scripps-Research-Institute-TSRI/default.aspx (accessed on 24th March 2018).

44. Lee, B.; John, D. "How 'bout Them Apples?" Validating Step Counts From The Apple Watch: 1350 Board\# 25 June 19. Med. Sci. Sports Exerc. 2017, 49, 365. 
45. Toth, L.P.; Park, S.; Springer, C.M.; Feyerabend, M.; Steeves, J.A.; Bassett, D.R. Video-Recorded Validation of Wearable Step Counters under Free-living Conditions. Med. Sci. Sports Exerc. 2018. [CrossRef] [PubMed]

46. Lee, B.; Arguello, D.; John, D. Validity of the Fitbit Blaze and the Apple watch during 19 different activities and in the free-living environment. J. Meas. Phys. Behav. 2018, unpublished work.

(C) (2)

(C) 2018 by the authors. Licensee MDPI, Basel, Switzerland. This article is an open access article distributed under the terms and conditions of the Creative Commons Attribution (CC BY) license (http:/ / creativecommons.org/licenses/by/4.0/). 


\title{
Article \\ Older Adults with Weaker Muscle Strength Stand up from a Sitting Position with More Dynamic Trunk Use
}

\author{
Rob C. van Lummel ${ }^{1,2, *}$, Jordi Evers ${ }^{1}$, Martijn Niessen ${ }^{1}$, Peter J. Beek ${ }^{2}$ and Jaap H. van Dieën ${ }^{2}$ \\ 1 McRoberts, Raamweg 43, 2596 HN The Hague, The Netherlands; J.Evers@mcroberts.nl (J.E.); \\ M.Niessen@mcroberts.nl (M.N.) \\ 2 Department of Human Movement Sciences, Faculty of Behavioural and Movement Sciences, \\ Vrije Universiteit Amsterdam, Amsterdam Movement Sciences, Van der Boechorststraat 9, 1081 BT \\ Amsterdam, The Netherlands; p.j.beek@vu.nl (P.J.B.); j.van.dieen@vu.nl (J.H.v.D.) \\ * Correspondence: r.vanlummel@mcroberts.nl; Tel.: +31-70-310-6462
}

Received: 16 March 2018; Accepted: 12 April 2018; Published: 17 April 2018

\begin{abstract}
The ability to stand up from a sitting position is essential for older adults to live independently. Body-fixed inertial sensors may provide an approach for quantifying the sit-to-stand (STS) in clinical settings. The aim of this study was to determine whether measurements of STS movements using body-fixed sensors yield parameters that are informative regarding changes in STS performance in older adults with reduced muscle strength. In twenty-seven healthy older adults, handgrip strength was assessed as a proxy for overall muscle strength. Subjects were asked to stand up from a chair placed at three heights. Trunk movements were measured using an inertial sensor fixed to the back. Duration, angular range, and maximum angular velocity of STS phases, as well as the vertical velocity of the extension phase, were calculated. Backwards elimination using Generalized Estimating Equations was used to determine if handgrip strength predicted the STS durations and trunk kinematics. Weaker subjects (i.e., with lower handgrip strength) were slower during the STS and showed a larger flexion angular range and a larger extension angular range. In addition, weaker subjects showed a greater maximum angular velocity, which increased with lower seat heights. Measurements with a single inertial sensor did reveal that older adults with lower handgrip strength employed a different strategy to stand up from a sitting position, involving more dynamic use of the trunk. This effect was greatest when elevating body mass. Trunk kinematic parameters were more sensitive to reduced muscle strength than durations.
\end{abstract}

Keywords: physical function; physical performance test; chair stand; sit to stand transfer; wearables; inertial sensors; accelerometers; gyroscopes

\section{Introduction}

The ability to stand up from a sitting position is essential for older adults to live independently and maintain an adequate level of physical activity. Older adults with a better sit-to-stand (STS) performance compared to age-matched controls showed shorter sitting periods, longer standing periods, and a higher number of locomotion periods in daily life, which indicates a more active lifestyle [1]. In community-dwelling older adults, sedentary behavior caused in part by difficulty to stand up was associated with increased risk of sarcopenia [2] and mortality [3-5].

Previous research clarified the dynamics of the STS movement to better understand its dynamics. Schenkman et al. distinguished four STS phases, which include the flexion momentum phase, the momentum transfer phase, the vertical extension phase, and the stabilization phase [6]. Riley et al. found indications that the momentum transfer phase, which starts with the lift-off from the chair seat, 
represents the most demanding phase [7]. The STS involves a transition from an intrinsically stable three-point support to a dynamically stable two-point support [7]. Difficulty in rising from a seated position may directly increase the risk of injury since STS transfers were found to be responsible for $41 \%$ of all falls in nursing homes [8].

Muscle strength represents one of the most important factors contributing to success in rising from a chair [9] and has been identified as a factor determining the lowest chair height from which the functionally impaired elderly can rise [10]. STS transitions require the development of substantial muscle power and consequently many older adults perform such transitions close to their maximal ability $[10,11]$. In addition, functionally limited elderly individuals with lower quadriceps muscle strength showed a lower dynamic stability when performing the STS at their preferred speed [12]. Several studies have reported different strategies for standing up [13-15]. In general, functionally impaired elderly stand up with greater flexion of the upper body [16], possibly to achieve better postural stability [13]. Age-related reduction in muscle strength may determine the choice of STS strategy [17]. Using a muscle-actuated optimal control model, Bobbert et al. [18] found that optimizing the STS strategy can reduce the mechanical demands on all muscles by $45 \%$ compared to a normal STS. In particular, an STS strategy with greater trunk flexion led to reduced demands on the knee extensors. This finding is in line with the effects that are observed when subjects are instructed to increase trunk flexion [14]. Overall, the existing body of evidence suggests that older adults, especially adults with muscle weakness, may show adaptations in the manner in which they perform the STS.

In clinical practice, STS performance is usually evaluated by measuring the time it takes to perform a series of STS movements. Since the early 1990s, body-fixed inertial sensors have been used to quantify STS movements [19-24]. Single sensor instrumentation can be easily applied in an unobtrusive manner with high measurement reliability in a geriatric setting [25]. Instrumented measurements of STS performance yield several additional parameters that may provide a basis for a quantitative assessment of STS performance in a clinical practice setting [26]. Repeated STS movements with automatically detected sub-durations were proven to have stronger associations with health status, functional status, and physical activity than manually recorded STS durations, which implies greater clinical relevance outcomes of instrumented analysis than manually recorded durations [27]. Furthermore, instrumented analysis allowed for the assessment of the test's dynamic phases, which are likely more informative than the static sitting and standing phases [27].

The aim of this study was to investigate whether measurements of STS movements using body-fixed sensors yield parameters that are informative regarding changes in the STS kinematics in older adults with reduced muscle strength by using handgrip strength (HGS) as a proxy for overall muscle strength. HGS is an easy to use method to measure muscle strength in a clinical practice setting and has frequently been associated with overall and lower extremity muscle strength [28-30]. It was hypothesized that weaker adults (i.e., with lower handgrip strength) would be inclined to use an STS strategy involving a more dynamic use of the trunk.

\section{Materials and Methods}

\subsection{Participants}

Twenty-seven healthy older adults, living in sheltered housing or in the community (12 females; mean age: $73.8 \pm 7.9$ years; mean weight: $77.1 \pm 13.2 \mathrm{~kg}$; mean height: $173.4 \pm 7.4 \mathrm{~cm}$ ) participated in this cross-sectional study. The protocol was approved by the ethics committee of the Department of Human Movement Sciences of the Vrije Universiteit Amsterdam (ECB 2014-3M) before it was conducted. Prior to testing, all participants provided written informed consent.

\subsection{Instrumentation and Data Acquisition}

Trunk movements during STS were measured using a small and light $(87 \times 45 \times 14 \mathrm{~mm}, 74 \mathrm{~g})$ inertial sensor measurement system (DynaPort Hybrid, McRoberts, The Hague, The Netherlands), 
which was fixed with an elastic belt around the waist and placed over the spine. The experimental set-up is depicted in Figure 1.

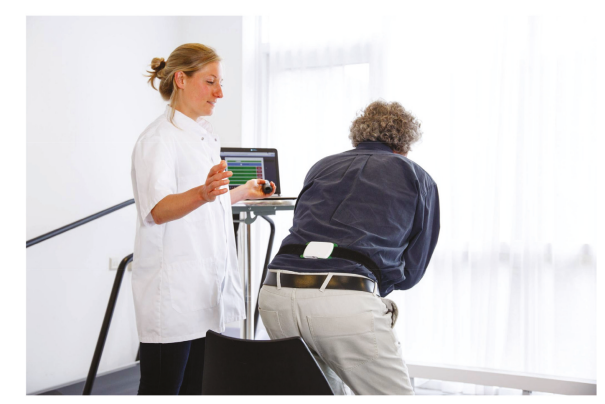

Figure 1. The experimenter stayed in close proximity to the patient. The protocol was implemented on a computer. With a remote control, the start and end of every STS was marked and stored with the raw data.

This sensor measured acceleration and angular velocity in three directions at a rate of 100 samples/s (see Figure 2). A single sensor was used because this is more practical for clinical use than multiple sensors. A position near the center of mass was chosen to obtain an adequate reflection of the whole body movement [31]. Moreover, at this position the sensor was unobtrusive, easy to fasten, and did not hamper the participant's movements.

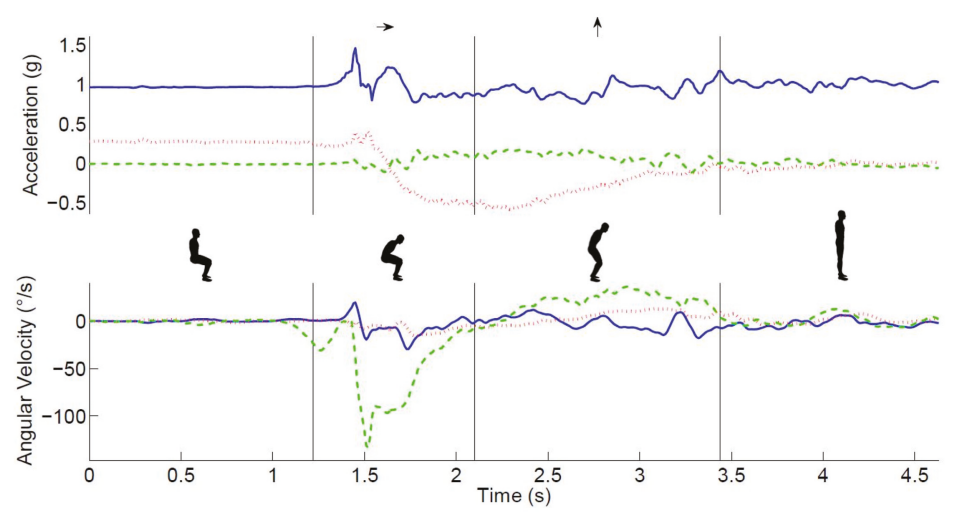

Figure 2. The main phases of the STS movement. The top panel shows the time series of raw acceleration (green/dashed-mediolateral, red/dotted anteroposterior, and blue/solid vertical), the lower panel shows the angular velocity (green/dashed-pitch, red/dotted-roll, and blue/solid -yaw) signals, and the insets illustrate the main phases of the STS movement, which are separated by vertical lines in both graphs.

\subsection{Test Protocol}

Participants were asked to stand up at their preferred speed from a height adjustable chair because effects of muscle strength are likely to be greater when standing up from lower chairs. Practice was allowed if needed. After standing up, participants were required to stand still for $10 \mathrm{~s}$. Each participant performed two STS movements from three different chair heights at $100 \%, 90 \%$, and $80 \%$. The $100 \%$ chair height was defined according to the operational definition used by Schenkman [6] with the chair adjusted in such a manner that the participant's thighs were horizontal with shanks and feet 
situated in a preferred orientation to stand up. The three chair heights were determined prior to performing the trials. The STS protocol was implemented on a computer, which randomly assigned the order of the height conditions. Markers set with the remote control at the start and end of each trial were stored with the raw signals to enable automatic data analysis. The three height conditions were performed with the arms folded in front of the trunk. When participants were unable to perform a certain condition, it was skipped and the next condition was offered.

\subsection{Signal Analysis}

The measurement of three-dimensional accelerations and angular velocities of the trunk allowed for a detailed analysis of the STS movement's different phases (see Figure 2). The acceleration and the angular velocity in the sagittal plane were used to calculate the trunk pitch angle [32]. Drift and noise were removed from the trunk pitch angle using the discrete wavelet transformation [19]. The dips in the trunk angle were used to detect a change in the trunk rotation direction. The start of the STS was defined as the end of the plateau before the first dip in the trunk pitch angle. Similarly, the end of the sit-to-stand was defined as the start of the plateau after the first dip in the trunk pitch angle. 'True vertical acceleration' was estimated by removing the influence of the trunk pitch angle from the vertical acceleration signal. Subsequently, the vertical velocity was derived by integrating this signal.

The following events were defined using commercially available software (MoveTest ${ }^{\circledR}$, McRoberts ${ }^{\circledR}$, The Hague, The Netherlands): the start of trunk movement, the end of the trunk flexion phase, and the end of the trunk rising phase [33] (see Figure 2). After automated identification of these phases, the duration, angular range, and maximum angular velocity of these phases as well as the vertical velocity of the extension phase were calculated. The mean of the two repetitions was used for statistical analysis.

\subsection{Handgrip Strength (HGS)}

HGS of both hands was measured using a digital handgrip dynamometer (Takei A5401) following a standardized protocol. Participants were standing in an upright position with their arms alongside their body. The handle was adjusted to fit their hand size. They were asked to squeeze the dynamometer as forcefully as possible.

Maximal HGS was measured twice for both hands with brief pauses between each measurement. The mean of all four measurements was used to estimate HGS. To assess the maximal HGS, at least three attempts are needed if HGS is considered to be a continuous variable while two attempts are sufficient to assess age-associated loss of muscle strength not caused by neurological or muscular diseases (dynapenia) [34].

\subsection{Statistical Analysis}

Normally distributed characteristics were presented as the mean and standard deviation (SD). Skewed (non-Gaussian) distributed continuous variables were presented as the median and interquartile range (IQR).

\subsubsection{Checks for Normality}

STS parameters were checked for normality using the Kolmogorov-Smirnov test. Out of a total of 24 parameters, 8 were not normally distributed. Total STS duration and the duration of the flexion phase did not meet the criteria for normality for all three seat heights. For these duration parameters, skewness and kurtosis ranged from 2.0 to 2.1 and from 3.0 to 5.9, respectively. Also non-normal distribution included the maximum angular velocity during the flexion phase at the $80 \%$ chair height $(D(24)=0.187$, $p<0.05)$ and the duration of the extension phase at the $90 \%$ seat height $(\mathrm{D}(25)=0.206, p<0.05)$. 


\subsubsection{Analysis}

All outcome variables were analyzed using Generalized Estimating Equations (GEE) to examine whether they were associated with HGS. GEE was chosen instead of a conventional least-squares regression because it can cope with missing values and takes into account that observations within a data set are dependent. In our study, these were the different seat heights for all subjects. In addition, body length, weight, age, and gender were treated as potential confounders. The main effects were calculated for HGS and these potential confounders. Because the effect of handgrip force was of interest and an interaction with task difficulty was plausible, the interaction between handgrip strength and seat height was also included in the initial model. The first iteration of the GEE analysis included HGS, HGS*seat height, and all potential confounders. A backward elimination method was used to eliminate non-significant predictors. Seat height was always included in the model since this was the within-subject variable. All analyses were conducted in SPSS v. 21.0.

\section{Results}

From the 27 participants, 24 completed the entire protocol. One participant was unable to stand up from the $80 \%$ chair height while two participants were unable to stand up from both the $90 \%$ and $80 \%$ chair heights. All participants (i.e., also the three participants that did not complete the entire protocol) were included in the analysis. Participant characteristics and descriptive statistics are presented in Table 1.

Table 1. Descriptive statistics of study population.

\begin{tabular}{cc}
\hline $\mathbf{n}$ & $\mathbf{2 7}$ \\
\hline percentage male $(\mathrm{n})$ & $55.6 \%(15)$ \\
Age (years) & $70.0(67-83)$ \\
Height $(\mathrm{cm})$ & $173.4 \pm 7.4$ \\
Weight $(\mathrm{kg})$ & $77.1 \pm 13.2$ \\
BMI $\left(\mathrm{kg} / \mathrm{m}^{2}\right)$ & $25.6 \pm 3.8$ \\
Hand Grip strength $(\mathrm{kg})$ & $29.2 \pm 10.1$ \\
\hline
\end{tabular}

Note: Data represents the mean \pm SD or median $(Q 1-Q 3)$.

The results of the durations and kinematics of the three seat heights and the model effects of GEE are presented in Table 2 .

\subsection{Sit-To-Stand}

There was no significant effect of seat height on STS duration ( $p=0.097)$. HGS was the only significant covariate showing that weaker subjects had a longer STS duration $(p=0.015)$.

\subsection{Flexion Phase}

There was no significant effect of seat height on flexion duration $(p=0.071)$. Angular range increased with lower seat height $(p=0.003)$, weaker subjects used a greater angular range $(p<0.001)$, and taller subjects used a greater angular range $(p=0.019)$. Age was the only covariate showing a significant effect on maximum angular velocity $(p=0.004)$ since angular velocity decreased with age. 
Sensors 2018, 18, 1235

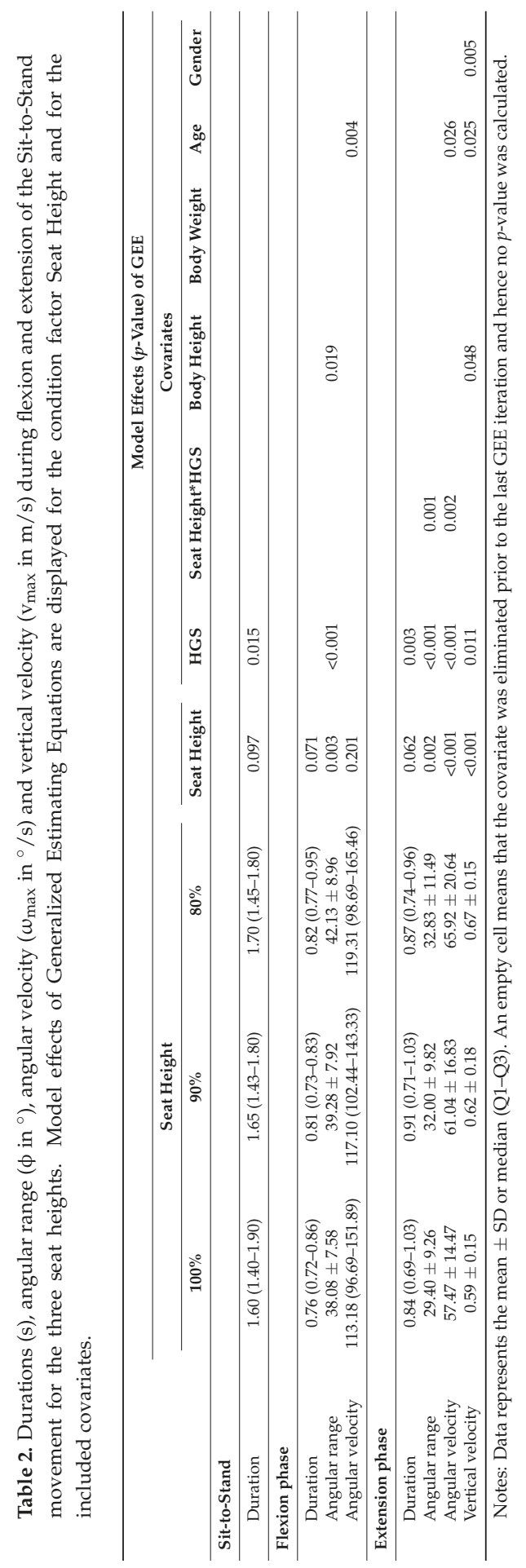




\subsection{Extension Phase}

The duration of the extension phase tended to be longer for lower seat heights $(p=0.062)$ and was significantly longer for lower HGS $(p=0.003)$. The angular range was larger for lower seat heights $(p=0.002)$ and for lower HGS $(p<0.001)$ in the presence of a significant interaction effect of seat height and HGS $(p<0.001)$. Maximum angular velocity was higher for lower seat height $(p<0.001)$ and for lower HGS $(p<0.001)$. In addition, there was a significant seat height ${ }^{*}$ HGS interaction $(p=0.002)$. Older subjects showed a lower angular velocity $(p=0.026)$. Vertical velocity was higher for lower seat heights $(p<0.001)$ and for higher HGS $(p=0.011)$. Furthermore, vertical velocity was higher for taller subjects $(p=0.048)$, lower for older ages $(p=0.025)$, and higher for males $(p<0.005)$.

The most important outcomes are visualized in the scatterplots depicted in Figure 3 . The four panels show the relation between handgrip strength (horizontal axes) and the STS duration, the flexion angular range, the extension angular range, and the extension angular velocity, respectively. As is evident from panel A, stronger subjects (i.e., with greater handgrip strength) performed the STS faster. Panel B shows that stronger subjects had a smaller flexion angular range while panel $\mathrm{C}$ shows that the weaker subjects had a much larger extension angular range, which increased with lower seat heights. Panel D shows a comparable effect for the maximum angular velocity during extension.
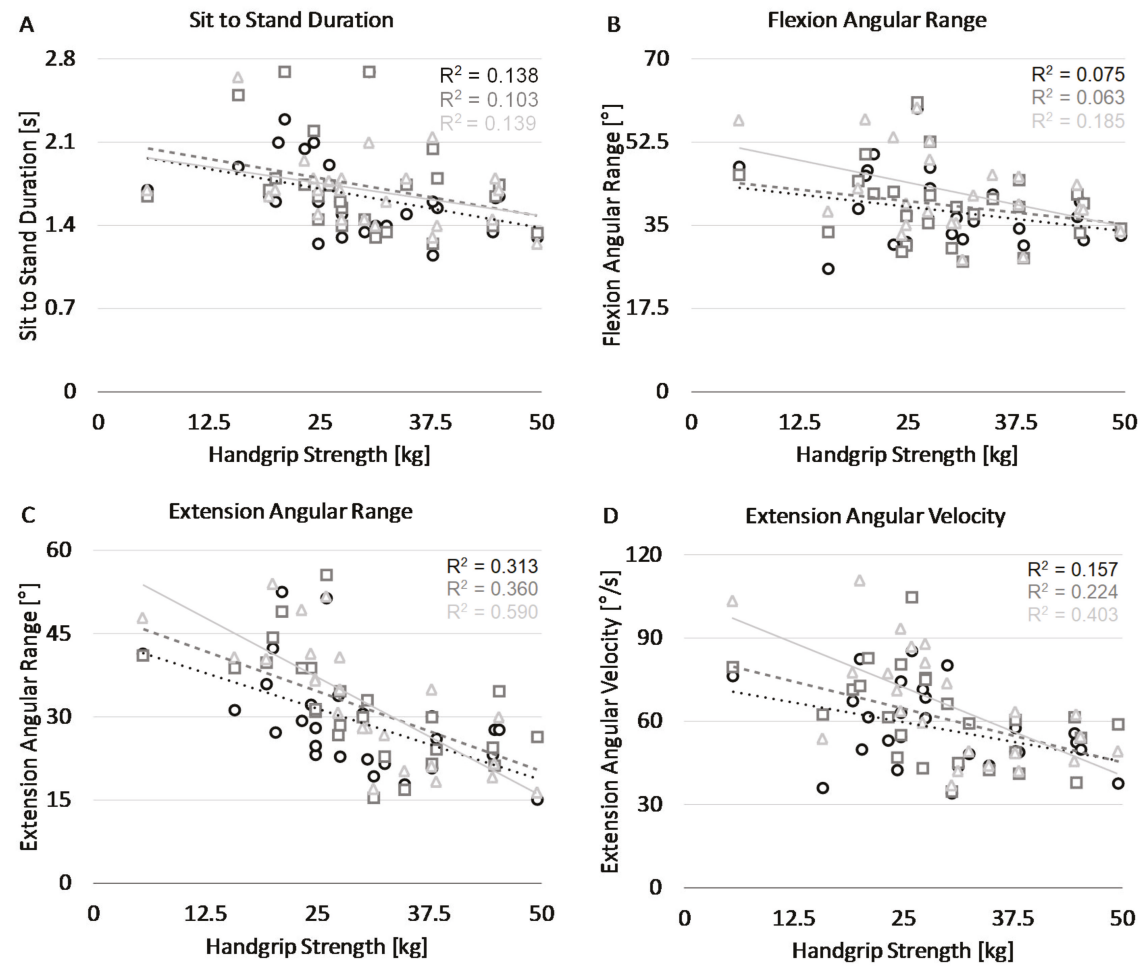

Seat Height: $\circ 100 \%, \square 90 \%, \triangle 80 \%$

Linear fit: ... 100\%, - - 90\%, - 80\%

Figure 3. Scatter plots display values of the four STS parameters and handgrip strength at three different seat heights. Panel (A) shows the total duration of the SiSt phase. Panel (B) shows the angular range during the STS flexion phase. Panel $(\mathbf{C})$ shows the angular range during the STS extension phase. Panel (D) shows the maximal angular velocity during the STS extension phase. 


\section{Discussion}

The results showed that older adults with lower handgrip strength stand up with greater flexion of the trunk than older adults with higher handgrip strength. The weaker subjects also showed greater trunk extension with a higher angular velocity after seat-off, indicating a more dynamic use of the trunk. The hypothesis that older adults with lower muscle strength stand up with more dynamic use of the trunk was, therefore, confirmed. This finding is consistent with the results of Hughes et al. showing that functionally impaired elderly rising from chairs below knee height significantly increased peak hip flexion velocity and the time to rise when compared to rising from a knee height chair. In addition, they significantly decreased their mean center of mass/base of support (COM/BOS) separation at lift-off [16] in line with the suggestion that this strategy may facilitate balance control [13].

The single module instrumentation can be easily attached over undergarments beneath outer clothes in a manner that is unobtrusive to the subject. The participant's awareness of being assessed is low because the instrumentation is not visible for the participant. Data collection is fast and, with the remote control, the test leader can stay in close contact with the participant. Moreover, the online connection of the remote control makes it possible for a single test leader to simultaneously collect data and watch over the participant since it is no longer necessary to read out the stopwatch and write down the times. The automated analysis of the data provides detailed insight into the quality of the movements.

Physical performance tests that include the STS such as the Timed Up and Go [35] and the repeated STS as a sub-test of the Short Physical Performance Battery [36] measure the duration of the total test and provide no information about the STS sub-durations and kinematics. The duration of the static periods (standing and sitting) during the $5 \times$ repeated STS represents, at least for some older adults, a great part of the total duration [27]. The total STS duration as measured with a stopwatch provides incomplete information about the dynamic phases of the STS, which are particularly relevant. The present findings demonstrate that kinematic parameters of standing up might be useful for clinical practice. Trunk range of motion, maximum angular velocity, and vertical velocity might be used to identify STS strategies in clinical practice and to evaluate interventions.

Chair height has a major influence on the ability to successfully stand up from a sitting position. A previous study reported that older adults found that, in general, the use of hands during STS transfer from different bed heights made the performance less challenging. All participants reported that the lowest position was the most challenging position to perform the task [37].

Using backwards elimination from all covariates, HGS was proven to be the strongest predictor of the durations as well as the kinematic outcomes. Older adults with lower handgrip strength, therefore, seemed to resort to a different STS strategy, which involved a more dynamic use of the trunk. The present and previous studies have shown that muscle strength is likely an important determinant of successfully standing up from a sitting position [10,12,38,39]. However, besides strength training, the training of STS transfer skills may well form a critical element in interventions aimed at preventing loss of mobility in older adults and patients [40]. Improvement of the technical performance of the STS might be more sustainable than strength training. Analysis of STS strategy using body fixed sensors in combination with easy-to-use strength measures will help to objectively measure changes over time and better understand how older adults could improve their STS ability.

Several determinants like chair seat height and lower limb strength have a major influence on the ability to successfully stand up from a sitting position. Increasing lower limb strength is a possible solution. Seat height may need to be more closely scrutinized in areas frequented by frail elderly people. Augmentation of seat height by small increments facilitates chair rise performance. In the present study, we manipulated seat height. Standing up from a lower seat height commonly occurs in daily life such as when standing up from a sofa. However, the seating area of a sofa is longer and it includes a backward slope and a compliant seat [41]. Therefore, standing up from a sofa might be even more difficult. 
Adaptations of STS behavior have also been described in patients with other health conditions. Patients with knee osteoarthritis primarily shifted their COM closer to the base of support provided by the feet in an attempt to achieve stability at and after seat-off [42]. Additionally, individuals with Parkinson's disease have difficulty rising from a chair as a result of reduced muscle strength, particularly at the hip amongst other factors [43]. In patients with a stroke, longer total time as well as changes at the initial phase and at the end of hip and knee extension phase were observed. The maximal hip flexion was lower during the rising phase from a seated position on the affected side in the stroke group [44]. Patients with end stage renal disease undergoing hemodialysis have high morbidity and mortality due to multiple causes. The results indicated that patients were slower to get out of the chair, which was measured by trunk flexion angular accelerations, time to peak trunk flexion, and overall STW completion time following the dialysis therapy session [45]. The present results add to these findings by showing that healthy older adults with reduced muscle strength also show significant adaptations in STS behavior, which is characterized by a more dynamic use of the trunk.

A limitation of the present study involves not measuring lower extremity strength and instead using HGS as a proxy for global muscle strength even though lower extremity muscle power was proven no better than knee extension torque or handgrip strength in the early identification of poor mobility [46]. Nevertheless, the change to a more dynamic STS strategy should be tested in a study protocol including lower leg strength measurements. To date, only a few studies have demonstrated the added value of using the instrumented STS in interventions [47,48]. The cross-sectional design of the present study precluded an analysis of how the STS strategy changes over time as muscle strength changes. It would be useful to examine whether older adults do revert to another STS strategy after an intervention is aimed at increasing lower leg strength. Longitudinal studies are needed to shed light on this issue. Finally, in the present study, using the arms during the STS procedure was prohibited by the protocol. The reason for this limitation was due to a focus specifically on the role of muscle strength and we, therefore, wished to eliminate the use of arm support or movement to compensate for lower leg strength. Future research could focus on how arm use might affect the STS dynamics.

\section{Conclusions}

Older adults with lower handgrip strength employed a different strategy to stand up from a sitting position than older adults with higher handgrip strength, which was characterized by more trunk flexion and a more dynamic use of the trunk during the extension phase. Changing to another strategy seems to be an adaptation of older adults to their reduced muscle strength. Measures of trunk kinematics were more sensitive to muscle strength than durations of STS phases.

Acknowledgments: We are very grateful to the older adults who volunteered to join this study and we thank Gijs van Cappelle for his support in the data collection.

Author Contributions: R.C.v.L., J.E., and J.H.v.D. conceived and designed the experiments. J.E. and R.C.v.L. performed the experiments. J.E. and M.N. analyzed the data. J.H.v.D., J.E., and R.C.v.L. contributed reagents/ materials/analysis tools. R.C.v.L., J.E., J.H.v.D., and P.J.B. wrote the paper.

Conflicts of Interest: R.C.v.L. is the owner and J.E. and M.N. are employees of the company McRoberts B.V., which is the manufacturer of the DynaPort.

\section{References}

1. Van Lummel, R.C.; Walgaard, S.; Pijnappels, M.; Elders, P.J.M.; Garcia-Aymerich, J.; van Dieën, J.H.; Beek, P.J. Physical performance and physical activity in older adults: Associated but separate domains of physical function in Old Age. PLoS ONE 2015, 10, e0144048. [CrossRef] [PubMed]

2. Gianoudis, J.; Bailey, C.A.; Daly, R.M. Associations between sedentary behaviour and body composition, muscle function and sarcopenia in community-dwelling older adults. Osteoporos. Int. 2015, 26, 571-579. [CrossRef] [PubMed]

3. Van der Ploeg, H.P.; Chey, T.; Korda, R.J.; Banks, E. A. Bauman. Sitting time and all-cause mortality risk in 222.497 Australian adults. Arch. Intern. Med. 2012, 172, 494-500. [CrossRef] [PubMed] 
4. Chau, J.Y.; Grunseit, A.C.; Chey, T.; Stamatakis, E.; Brown, W.J.; Matthews, C.E.; Bauman, A.E.; van der Ploeg, H.P. Daily sitting time and all-cause mortality: A meta-analysis. PLoS ONE 2013, 8, 1-14. [CrossRef] [PubMed]

5. Pulsford, R.M.; Stamatakis, E.; Britton, A.R.; Brunner, E.J.; Hillsdon, M. Associations of sitting behaviours with all-cause mortality over a 16-year follow-up: The Whitehall II study. Int. J. Epidemiol. 2015, 44, 1909-1916. [CrossRef] [PubMed]

6. Schenkman, M.L.; Berger, R.A.; Riley, P.O.; Mann, R.W.; Hodge, W.A. Whole-body movements during rising to standing from sitting. Phys. Ther. 1990, 70, 638-648. [CrossRef] [PubMed]

7. Riley, P.O.; Schenkman, M.L.; Mann, R.W.; Hodge, W.A. Mechanics of a constrained chair-rise. J. Biomech. 1991, 24, 77-85. [CrossRef]

8. Büchele, G.; Becker, C.; Cameron, I.D.; König, H.H.; Robinovitch, S.; Rapp, K. Predictors of serious consequences of falls in residential aged care: Analysis of more than 70,000 falls from residents of Bavarian nursing homes. J. Am. Med. Dir. Assoc. 2014, 15, 559-563. [CrossRef] [PubMed]

9. Alexander, N.B.; Schultz, A.B.; Ashton-Miller, J.A.; Gross, M.M.; Gordiani, B. Muscle strength and rising from a chair in older adults. Muscle Nerve 1997, 20, 56-59. [CrossRef]

10. Hughes, M.; Myers, B.S.; Schenkman, M.L. The role of strength in rising from a chair in the functionally impaired elderly. J. Biomech. 1996, 29, 1509-1513. [CrossRef]

11. Hortobágyi, T.; Mizelle, C.; Beam, S.; DeVita, P. Old adults perform activities of daily living near their maximal capabilities. J. Gerontol. A Biol. Sci. Med. Sci. 2003, 58, M453-M460. [CrossRef] [PubMed]

12. Scarborough, D.M.; Krebs, D.E.; Harris, B.A. Quadriceps muscle strength and dynamic stability in elderly persons. Gait Posture 1999, 10, 10-20. [CrossRef]

13. Hughes, M.A.; Weiner, D.K.; Schenkman, M.L.; Studenski, M. Chair rise strategies in the elderly. Clin. Biomech. 1994, 9, 187-192. [CrossRef]

14. Doorenbosch, G.; Harlaar, C.; Roebroeck, J.; Lankhorst, M. Two strategies of transferring from sit-to-stand; the activation of monoarticular and biarticular muscles. J. Biomech. 1994, 27, 1299-1307. [CrossRef]

15. Scarborough, D.M.; McGibbon, C.A.; Krebs, D.E. Chair rise strategies in older adults with functional limitations. J. Rehabil. Res. Dev. 2007, 4, 33-42. [CrossRef]

16. Hughes, M.A.; Schenkman, M.L. Chair rise strategy in the functionally impaired elderly. J. Rehabil. Res. Dev. 1996, 33, 409-412. [CrossRef] [PubMed]

17. Gross, M.M.; Stevenson, P.J.; Charette, S.L.; Pyka, G.; Marcus, R. Effect of muscle strength and movement speed on the biomechanics of rising from a chair in healthy elderly and young women. Gait Posture 1998, 8, 175-185. [CrossRef]

18. Bobbert, M.F.; Kistemaker, D.A.; Vaz, M.A.; Ackermann, M. Searching for strategies to reduce the mechanical demands of the sit-to-stand task with a muscle-actuated optimal control model. Clin. Biomech. 2016, 37, 83-90. [CrossRef] [PubMed]

19. Najafi, B.; Aminian, K.; Loew, F.; Blanc, Y.; Robert, P.A.; Member, S. Measurement of stand-sit and sit-stand transitions using a miniature gyroscope and its application in fall risk evaluation in the elderly. IEEE Trans. Biomed. Eng. 2002, 49, 843-851. [CrossRef] [PubMed]

20. Janssen, W.G.M.; Bussman, J.B.J.; Horemans, H.L.D.; Stam, H.J. Analysis and decomposition of accelerometric signals of trunk and thigh obtained during the sit-to-stand movement. Med. Biol. Eng. Comput. 2005, 43, 265-272. [CrossRef] [PubMed]

21. Boonstra, M.C.; van der Slikke, R.M.A.; Keijsers, N.L.W.; van Lummel, R.C.; de Waal Malefijt, M.C.; Verdonschot, N. The accuracy of measuring the kinematics of rising from a chair with accelerometers and gyroscopes. J. Biomech. 2006, 39, 354-358. [CrossRef] [PubMed]

22. Zijlstra, W.; Bisseling, R.W.; Schlumbohm, S.; Baldus, H. A body-fixed-sensor-based analysis of power during sit-to-stand movements. Gait Posture 2010, 31, 272-278. [CrossRef] [PubMed]

23. Millor, N.; Lecumberri, P.; Gomez, M.; Martinez-Ramirez, A.; Rodriguez-Manas, L.; Garcia-Garcia, F.J.; Izquierdo, M. Automatic evaluation of the 30-s chair stand test using inertial/magnetic-based technology in an older prefrail population. IEEE J. Biomed. Health Inf. 2013, 17, 820-827. [CrossRef] [PubMed]

24. Soangra, R.; Lockhart, T.E. A comparative study for performance evaluation of sit-to-stand task with body worn sensor and existing laboratory methods. Biomed. Sci. Instrum. 2012, 48, 407-414. [PubMed]

25. Schwenk, M.; Gogulla, S.; Englert, S.; Czempik, A.; Hauer, K. Test-retest reliability and minimal detectable change of repeated sit-to-stand analysis using one body fixed sensor in geriatric patients. Physiol. Meas. 2012, 33, 1931-1946. [CrossRef] [PubMed] 
26. Van Lummel, R.C.; Ainsworth, E.; Lindemann, U.; Zijlstra, W.; Chiari, L.; van Campen, P.; Hausdorff, J.M. Automated approach for quantifying the repeated sit-to-stand using one body fixed sensor in young and older adults. Gait Posture 2013, 38, 153-156. [CrossRef] [PubMed]

27. Van Lummel, R.C.; Walgaard, S.; Maier, A.B.; Ainsworth, E.; Beek, P.J.; van Dieën, J.H. The instrumented sit-to-Stand sest (iSTS) has greater clinical relevance than the manually recorded sit-to-stand test in older adults. PLoS ONE 2016, 11, e0157968. [CrossRef] [PubMed]

28. Pijnappels, M.; van der Burg, P.; Reeves, N.D.; van Dieën, J.H. Identification of elderly fallers by muscle strength measures. Eur. J. Appl. Physiol. 2008, 102, 585-592. [CrossRef] [PubMed]

29. Aadahl, M.; Beyer, N.; Linneberg, A.; Thuesen, B.H.; Jorgensen, T. Grip strength and lower limb extension power in 19-72-year-old Danish men and women: The Health2006 study. BMJ Open 2011, 1, e000192. [CrossRef] [PubMed]

30. Geusens, P.; Vandevyver, C.; Vanhoof, J.; Cassiman, J.; Boonen, S.; Raus, J. Quadriceps and grip strength are related to Vitamin D receptor genotype in elderly nonobese women. J. Bone Miner. Res. 1997, 12, 2082-2088. [CrossRef] [PubMed]

31. Giansanti, D.; Maccioni, G. Physiological motion monitoring: A wearable device and adaptative algorithm for sit-to-stand timing detection. Physiol. Meas. 2006, 27, 713-723. [CrossRef] [PubMed]

32. Williamson, R.; Andrews, B.J. Detecting absolute human knee angle and angular velocity using accelerometers and rate gyroscopes. Med. Biol. Eng. Comput. 2001, 39, 294-302. [CrossRef] [PubMed]

33. Walgaard, S.; Faber, G.S.; van Lummel, R.C.; van Dieën, J.H.; Kingma, I. The validity of assessing temporal events, sub-phases and trunk kinematics of the sit-to-walk movement in older adults using a single inertial sensor. J. Biomech. 2016, 49, 1933-1937. [CrossRef] [PubMed]

34. Reijnierse, E.M.; de Jong, N.; Trappenburg, M.C.; Blauw, G.J.; Butler-Browne, G.; Gapeyeva, H.; Hogrel, J.; McPhee, J.S.; Narici, M.V. Assessment of maximal handgrip strength: How many attempts are needed? J. Cachexia Sarcopenia Muscle 2017, 8, 466-474. [CrossRef] [PubMed]

35. Podsiadlo, D.; Richardson, S. The Timed Up \& Go: A test of basic functional mobility for frail elderly persons. J. Am. Geriatr. Soc. 1991, 39, 142-148. [PubMed]

36. Guralnik, J.M.; Ferrucci, L.; Simonsick, E.M.; Salive, M.E.; Wallace, R.B. Short Physical Performance Battery protocol and score sheet. 1994, pp. 1-8. Available online: http://hdcs.fullerton.edu/csa/Research/ documents/SPPBInstructions_ScoreSheet.pdf (accessed on 15 January 2018).

37. Lindemann, U.; van Oosten, L.; Evers, J.; Becker, C.; van Dieen, J.H.; van Lummel, R.C. Effect of bed height and use of hands on trunk angular velocity during the sit-to-stand transfer. Ergonomics 2014, 57, 1536-1540. [CrossRef] [PubMed]

38. Schenkman, M.; Hughes, O.M.A.; Samsa, M.S.G.; Studenski, S. The relative importance of strength and balance in chair rise by functionally impaired older individuals. J. Am. Geriatr. Soc. 1996, 44, 1441-1446. [CrossRef] [PubMed]

39. Janssen, W.G.M.; Bussmann, H.B.J.; Stam, H.J. Determinants of the sit-to-stand movement: A review. Phys. Ther. 2002, 82, 866-879. [PubMed]

40. Lord, S.R.; Murray, S.M.; Chapman, K.; Munro, B.; Tiedemann, A. Sit-to-stand performance depends on sensation, speed, balance, and psychological status in addition to strength in older people. J. Gerontol. A Biol. Sci. Med. Sci. 2002, 57, M539-M543. [CrossRef] [PubMed]

41. Alexander, N.B.; Koester, D.J.; Grunawalt, J.A. Chair design affects how older adults rise from a chair. J. Am. Geriatr. Soc. 1996, 44, 356-362. [CrossRef] [PubMed]

42. Anan, M.; Shinkoda, K.; Suzuki, K.; Yagi, M.; Ibara, T.; Kito, N. Do patients with knee osteoarthritis perform sit-to-stand motion efficiently? Gait Posture 2015, 41, 488-492. [CrossRef] [PubMed]

43. Inkster, L.M.; Eng, J.J.; MacIntyre, D.L.; Stoessl, A.J. Leg muscle strength is reduced in Parkinson's disease and relates to the ability to rise from a chair. Mov. Disord. 2003, 18, 157-162. [CrossRef] [PubMed]

44. Mao, Y.R.; Wu, X.Q.; Zhao, J.L.; Lo, W.L.A.; Chen, L.; Ding, M.H.; Xu, Z.Q.; Bian, R.H.; Huang, D.F.; $\mathrm{Li}$, L. The crucial changes of sit-to-stand phases in subacute stroke survivors identified by movement decomposition analysis. Front. Neurol. 2018, 9, 185. [CrossRef] [PubMed]

45. Soangra, R.; Lockhart, T.E.; Lach, J.; Abdel-Rahman, E.M. Effects of hemodialysis therapy on sit-to-walk characteristics in end stage renal disease patients. Ann. Biomed. Eng. 2013, 41, 795-805. [CrossRef] [PubMed] 
46. Lauretani, F.; Russo, C.R.; Bandinelli, S.; Bartali, B.; Cavazzini, C.; di Iorio, A.; Corsi, A.M.; Rantanen, T.; Guralnik, J.M.; Ferrucci, L. Age-associated changes in skeletal muscles and their effect on mobility: An operational diagnosis of sarcopenia. J. Appl. Physiol. 2003, 95, 1851-1860. [CrossRef] [PubMed]

47. Millor, N.; Lecumberri, P.; Gómez, M.; Martínez-Ramírez, A.; Izquierdo, M. An evaluation of the 30-s chair stand test in older adults: Frailty detection based on kinematic parameters from a single inertial unit. J. Neuroeng. Rehabil. 2013, 10, 86. [CrossRef] [PubMed]

48. Regterschot, G.R.H.; Zhang, W.; Baldus, H.; Stevens, M.; Zijlstra, W. Sensor-based monitoring of sit-to-stand performance is indicative of objective and self-reported aspects of functional status in older adults. Gait Posture 2015, 41, 935-940. [CrossRef] [PubMed]

(C) 2018 by the authors. Licensee MDPI, Basel, Switzerland. This article is an open access article distributed under the terms and conditions of the Creative Commons Attribution (CC BY) license (http://creativecommons.org/licenses/by/4.0/). 
MDPI

St. Alban-Anlage 66

4052 Basel

Switzerland

Tel. +41616837734

Fax +41 613028918

www.mdpi.com

Sensors Editorial Office

E-mail: sensors@mdpi.com www.mdpi.com/journal/sensors

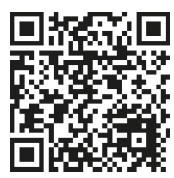



MDPI

St. Alban-Anlage 66

4052 Basel

Switzerland

Tel: +41 616837734

Fax: +41 613028918 